\title{
Develop an ONIOM-G3B3 Method to Accurately Predict C-H and N-H Bond Dissociation Enthalpies of Ribonucleosides and Deoxyribonucleosides
}

\author{
Min-Jie Li, Lei Liu,* Yao Fu, Qing-Xiang Guo*
}

\section{Index}

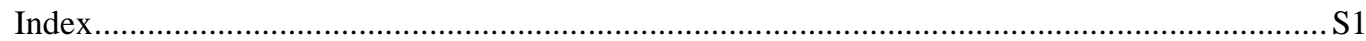

Part 1 The Cartesian Coordinates of molecules discussed in the text. .................................. S2

(1) The Cartesian Coordinates of the $56 \mathrm{C}-\mathrm{H}$ molecules....................................................S2

(2) The Cartesian Coordinates of the $10 \mathrm{~N}-\mathrm{H}$ molecules. ...............................................S126

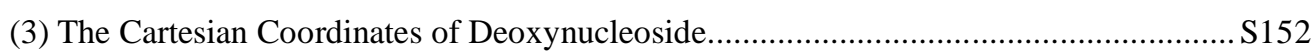

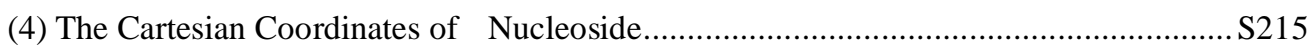

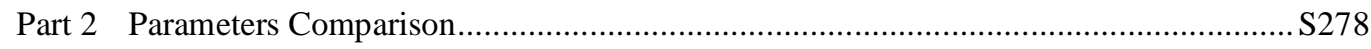

(1) Comparison Between the Parameters in the Previous Work and Mine of

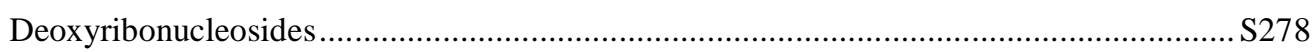

(2) Comparison Between the Parameters in the Previous Work ${ }^{[1,3,4]}$ and Mine of

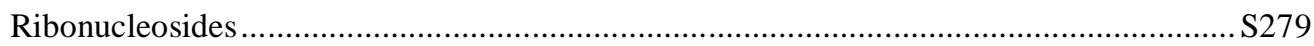

Part 3 The BDEs of $56 \mathrm{C}-\mathrm{H}$ and 10 N-H Molecules with B3LYP ..................................... S280

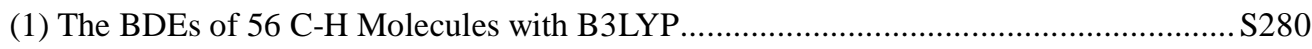

(2) The BDEs of $10 \mathrm{~N}-\mathrm{H}$ Molecules with B3LYP .......................................................... S284

Part 4 The Core Layer Division of Deoxynucleoside and Nucleoside Labeled With Red. ..... S285

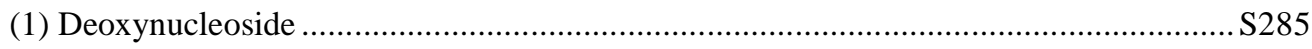

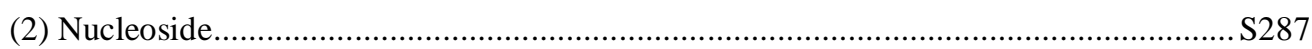

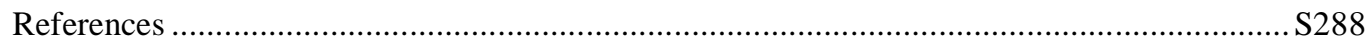




\section{Part 1 The Cartesian Coordinates of molecules discussed in the text.}

\section{(1) The Cartesian Coordinates of the $56 \mathrm{C}-\mathrm{H}$ molecules.}

No.1<smiles>Cc1c2ccccc2c(C=O)c2ccccc12</smiles>

neutral molecule

Charge $=0$ Multiplicity $=1$ for low level calculation on real system.

Charge $=0$ Multiplicity $=1$ for high level calculation on model system .

Charge $=0$ Multiplicity $=1$ for low level calculation on model system.

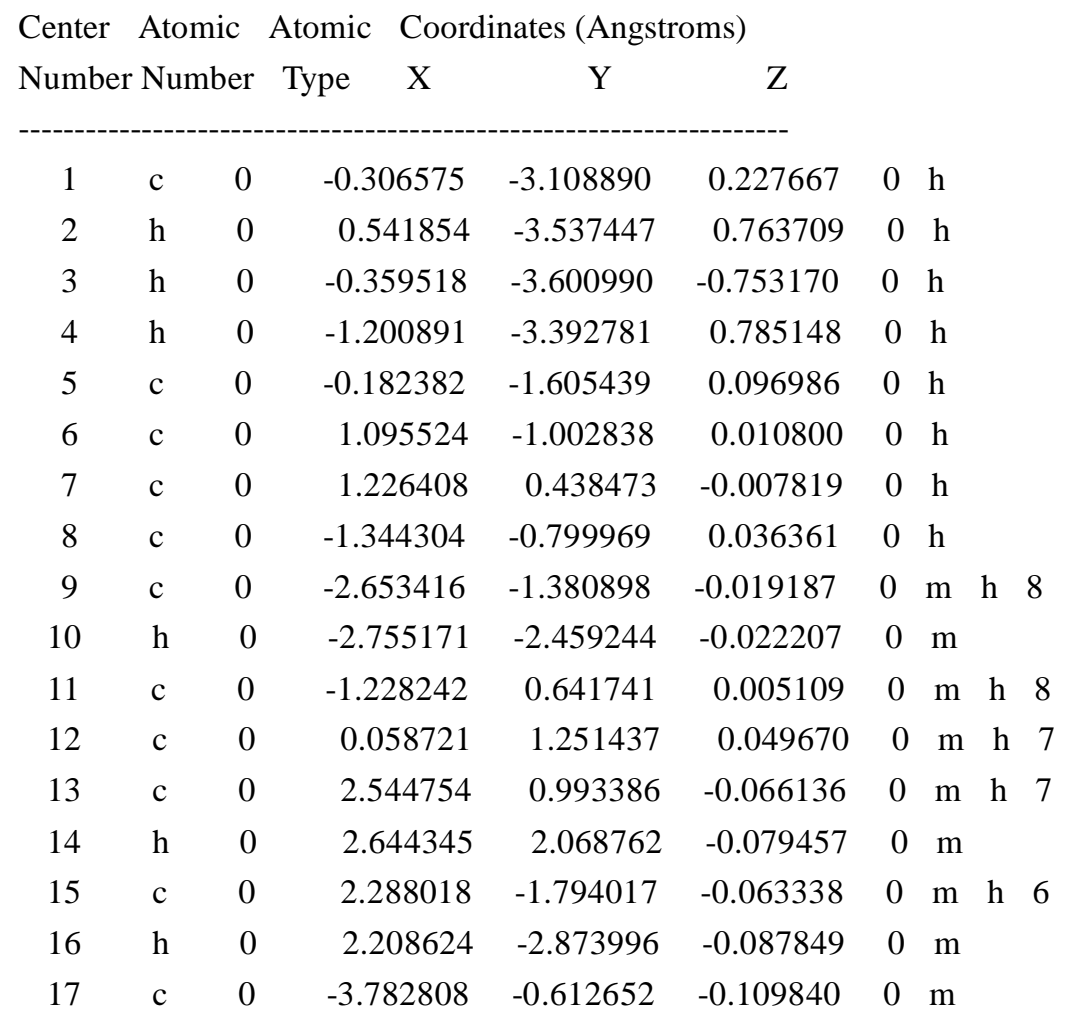




$\begin{array}{lllrrrrc}18 & \mathrm{~h} & 0 & -4.760972 & -1.082213 & -0.162512 & 0 & \mathrm{~m} \\ 19 & \mathrm{c} & 0 & -3.670153 & 0.799496 & -0.159958 & 0 & \mathrm{~m} \\ 20 & \mathrm{~h} & 0 & -4.563680 & 1.410118 & -0.256929 & 0 & \mathrm{~m} \\ 21 & \mathrm{c} & 0 & -2.439165 & 1.400262 & -0.110463 & 0 & \mathrm{~m} \\ 22 & \mathrm{~h} & 0 & -2.393880 & 2.477829 & -0.201510 & 0 & \mathrm{~m} \\ 23 & \mathrm{c} & 0 & 0.133356 & 2.718506 & 0.236168 & 0 & \mathrm{~m} \\ 24 & \mathrm{~h} & 0 & -0.805557 & 3.179201 & 0.595073 & 0 & \mathrm{~m} \\ 25 & \mathrm{o} & 0 & 1.105074 & 3.444422 & 0.081014 & 0 & \mathrm{~m} \\ 26 & \mathrm{c} & 0 & 3.655540 & 0.191412 & -0.129264 & 0 & \mathrm{~m} \\ 27 & \mathrm{~h} & 0 & 4.642040 & 0.644237 & -0.183090 & 0 & \mathrm{~m} \\ 28 & \mathrm{c} & 0 & 3.529215 & -1.220502 & -0.136930 & 0 & \mathrm{~m} \\ 29 & \mathrm{~h} & 0 & 4.415272 & -1.845897 & -0.204278 & 0 & \mathrm{~m}\end{array}$

radical

Charge $=0$ Multiplicity $=2$ for low level calculation on real system .

Charge $=0$ Multiplicity $=2$ for high level calculation on model system .

Charge $=0$ Multiplicity $=2$ for low level calculation on model system .

\begin{tabular}{|c|c|c|c|c|c|c|c|c|}
\hline Center & Atomic & & Atomic & ates (Angstro & & & & \\
\hline Number & Numbe & & Type & $\mathrm{Y}$ & Z & & & \\
\hline 1 & $\mathrm{c}$ & 0 & 0.260277 & -2.899178 & 0.840801 & 0 & $\mathrm{~h}$ & \\
\hline 2 & $\mathrm{~h}$ & 0 & -0.621387 & -3.483585 & 1.076521 & 0 & $\mathrm{~h}$ & \\
\hline 3 & $\mathrm{~h}$ & 0 & 1.212160 & -3.345151 & 1.104597 & 0 & $\mathrm{~h}$ & \\
\hline 4 & $\mathrm{c}$ & 0 & 0.173569 & -1.653034 & 0.308142 & 0 & $\mathrm{~h}$ & \\
\hline 5 & $\mathrm{c}$ & 0 & -1.126592 & -1.027145 & 0.015880 & 0 & $\mathrm{~h}$ & \\
\hline 6 & $\mathrm{c}$ & 0 & -1.233397 & 0.397512 & -0.025370 & 0 & $\mathrm{~h}$ & \\
\hline 7 & $\mathrm{c}$ & 0 & 1.373336 & -0.840204 & 0.052745 & 0 & $\mathrm{~h}$ & \\
\hline 8 & $\mathrm{c}$ & 0 & 2.635502 & -1.433092 & -0.124295 & 0 & $\mathrm{~m}$ & $\mathrm{~h}$ \\
\hline 9 & $\mathrm{~h}$ & 0 & 2.718411 & -2.515051 & -0.121424 & 0 & $\mathrm{~m}$ & \\
\hline 10 & $\mathrm{c}$ & 0 & 1.254919 & 0.580695 & -0.010981 & 0 & $\mathrm{~m}$ & $\mathrm{~h}$ \\
\hline 11 & $\mathrm{c}$ & 0 & -0.044253 & 1.206084 & 0.126256 & 0 & $\mathrm{~m}$ & $\mathrm{~h}$ \\
\hline 12 & $\mathrm{c}$ & 0 & -2.517360 & 0.972162 & -0.226032 & 0 & $\mathrm{~m}$ & $\mathrm{~h}$ \\
\hline 13 & $\mathrm{~h}$ & 0 & -2.601416 & 2.049298 & -0.239089 & 0 & $\mathrm{~m}$ & \\
\hline 14 & $\mathrm{c}$ & 0 & -2.277348 & -1.805919 & -0.193018 & 0 & $\mathrm{~m}$ & $\mathrm{~h}$ \\
\hline 15 & $\mathrm{~h}$ & 0 & -2.190570 & -2.887449 & -0.214365 & 0 & $\mathrm{~m}$ & \\
\hline 16 & $\mathrm{c}$ & 0 & 3.771379 & -0.668371 & -0.345192 & 0 & $\mathrm{~m}$ & \\
\hline 17 & $\mathrm{~h}$ & 0 & 4.732598 & -1.153101 & -0.490618 & 0 & $\mathrm{~m}$ & \\
\hline 18 & $\mathrm{c}$ & 0 & 3.665397 & 0.728575 & -0.411152 & 0 & $\mathrm{~m}$ & \\
\hline 19 & $\mathrm{~h}$ & 0 & 4.544581 & 1.334836 & -0.610207 & 0 & $\mathrm{~m}$ & \\
\hline 20 & $\mathrm{c}$ & 0 & 2.433297 & 1.337108 & -0.254506 & 0 & $\mathrm{~m}$ & \\
\hline 21 & $\mathrm{~h}$ & 0 & 2.370698 & 2.412960 & -0.365862 & 0 & $\mathrm{~m}$ & \\
\hline
\end{tabular}




$\begin{array}{lllrrrrc}22 & \mathrm{c} & 0 & -0.099341 & 2.632922 & 0.461186 & 0 & \mathrm{~m} \\ 23 & \mathrm{~h} & 0 & 0.872662 & 3.069258 & 0.757669 & 0 & \mathrm{~m} \\ 24 & \mathrm{o} & 0 & -1.095217 & 3.351340 & 0.489617 & 0 & \mathrm{~m} \\ 25 & \mathrm{c} & 0 & -3.633925 & 0.177292 & -0.414384 & 0 & \mathrm{~m} \\ 26 & \mathrm{~h} & 0 & -4.601395 & 0.642313 & -0.582678 & 0 & \mathrm{~m} \\ 27 & \mathrm{c} & 0 & -3.516522 & -1.220315 & -0.408467 & 0 & \mathrm{~m} \\ 28 & \mathrm{~h} & 0 & -4.388219 & -1.845610 & -0.581170 & 0 & \mathrm{~m}\end{array}$

No.2<smiles>Cc1c2ccccc2c(C#N)c2ccccc12</smiles>

neutral molecule Charge $=0$ Multiplicity $=1$ for low level calculation on real system.

Charge $=0$ Multiplicity $=1$ for high level calculation on model system.

Charge $=0$ Multiplicity $=1$ for low level calculation on model system.

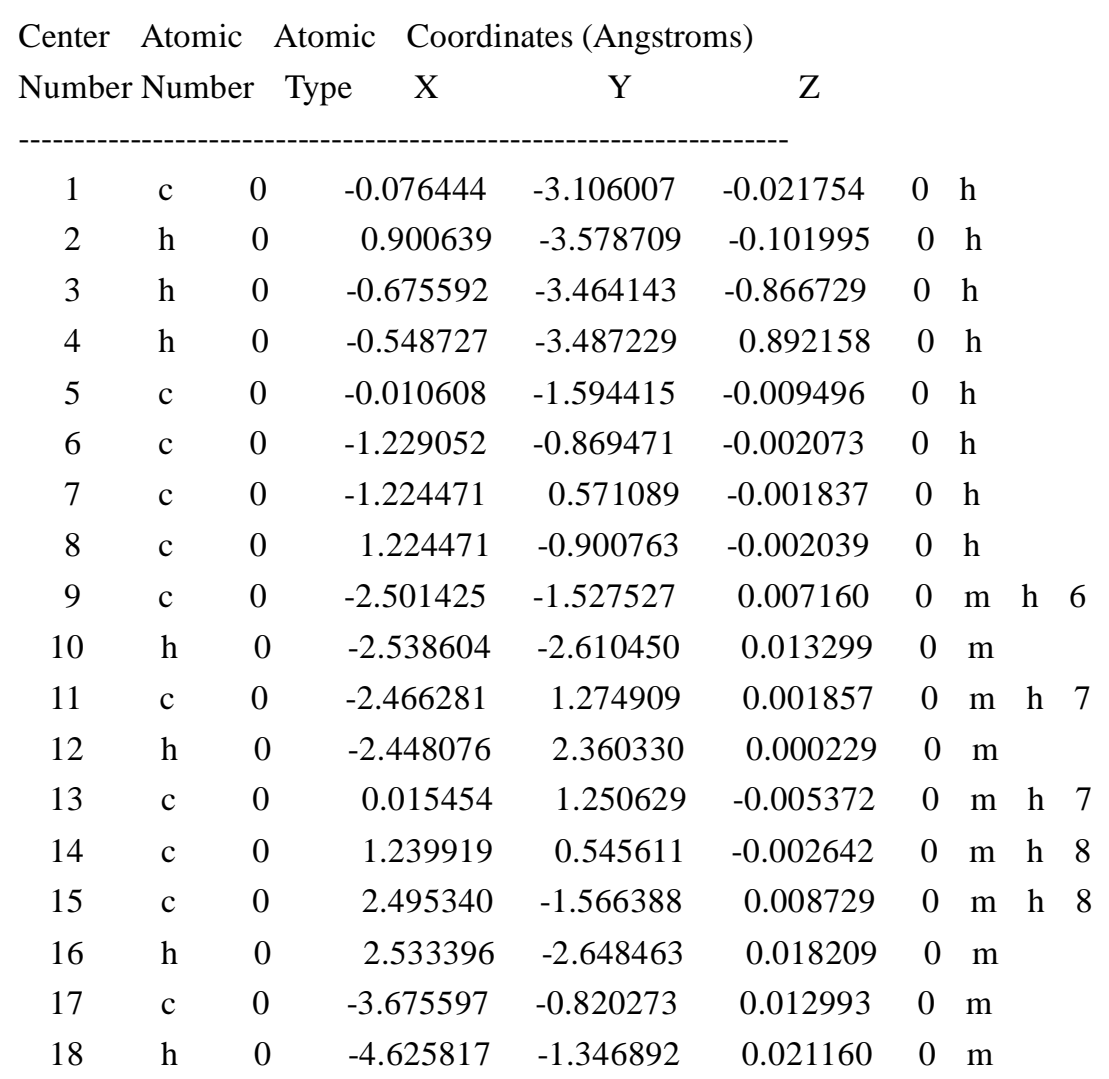




$\begin{array}{lllrrrrr}19 & \mathrm{c} & 0 & -3.658675 & 0.600256 & 0.008885 & 0 & \mathrm{~m} \\ 20 & \mathrm{~h} & 0 & -4.594859 & 1.151280 & 0.012241 & 0 & \mathrm{~m} \\ 21 & \mathrm{c} & 0 & 0.028469 & 2.680740 & -0.008648 & 0 & \mathrm{~m} \\ 22 & \mathrm{n} & 0 & 0.036564 & 3.845814 & -0.009057 & 0 & \mathrm{~m} \\ 23 & \mathrm{c} & 0 & 2.491094 & 1.234650 & 0.000030 & 0 & \mathrm{~m} \\ 24 & \mathrm{~h} & 0 & 2.483938 & 2.320134 & -0.002483 & 0 & \mathrm{~m} \\ 25 & \mathrm{c} & 0 & 3.675725 & 0.549216 & 0.007671 & 0 & \mathrm{~m} \\ 26 & \mathrm{~h} & 0 & 4.617869 & 1.089931 & 0.010387 & 0 & \mathrm{~m} \\ 27 & \mathrm{c} & 0 & 3.675518 & -0.871707 & 0.013803 & 0 & \mathrm{~m} \\ 28 & \mathrm{~h} & 0 & 4.619253 & -1.409777 & 0.023328 & 0 & \mathrm{~m}\end{array}$

radical

Charge $=0$ Multiplicity $=2$ for low level calculation on real system.

Charge $=0$ Multiplicity $=2$ for high level calculation on model system.

Charge $=0$ Multiplicity $=2$ for low level calculation on model system.

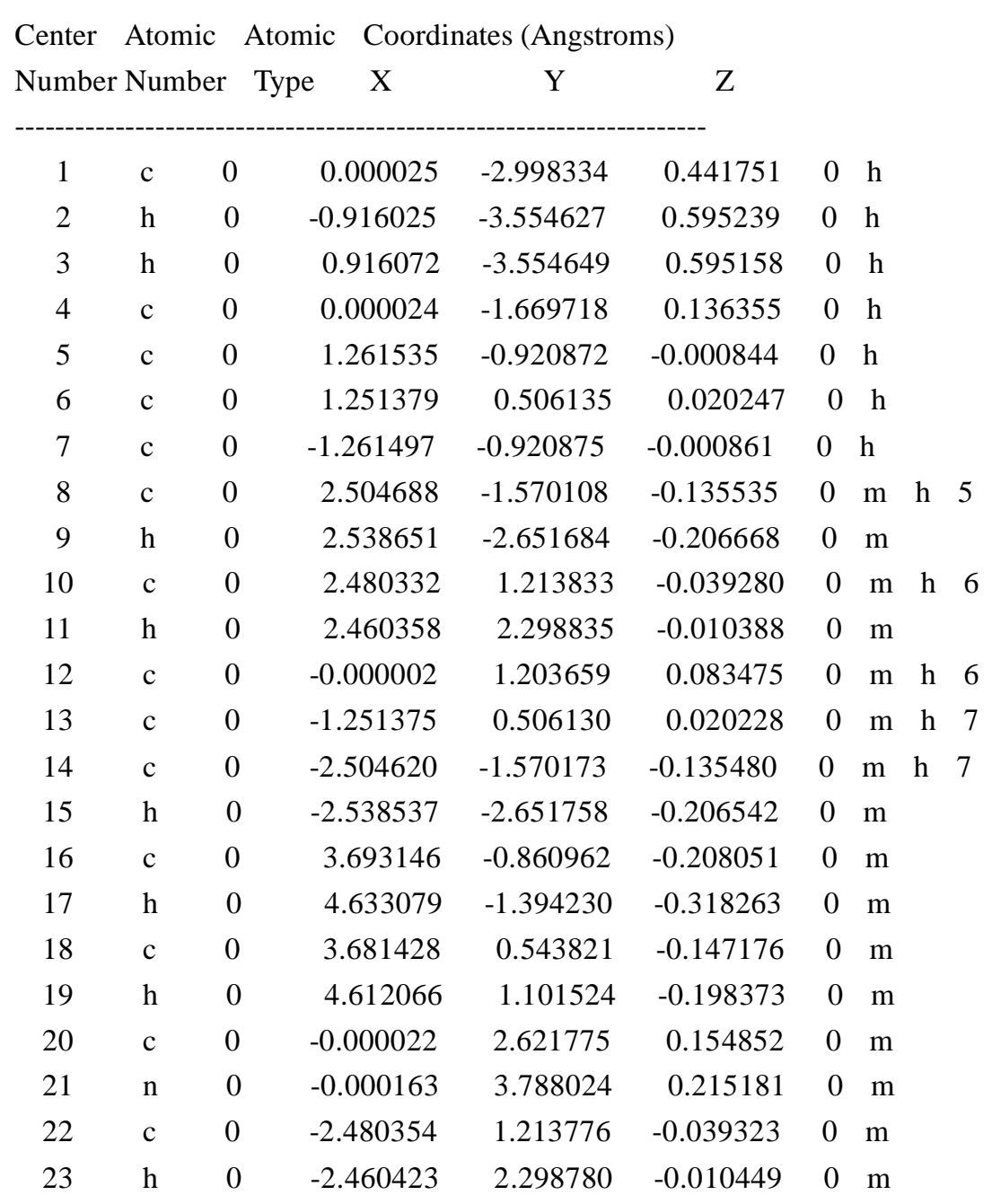




$\begin{array}{llllllll}24 & \mathrm{c} & 0 & -3.681424 & 0.543740 & -0.147237 & 0 & \mathrm{~m} \\ 25 & \mathrm{~h} & 0 & -4.612075 & 1.101421 & -0.198450 & 0 & \mathrm{~m} \\ 26 & \mathrm{c} & 0 & -3.693099 & -0.861062 & -0.208015 & 0 & \mathrm{~m} \\ 27 & \mathrm{~h} & 0 & -4.633021 & -1.394363 & -0.318171 & 0 & \mathrm{~m}\end{array}$

No 3<smiles>[CH]c1c2ccccc2c(C)c2ccccc12</smiles>

neutral molecule

Charge $=0$ Multiplicity $=1$ for low level calculation on real system.

Charge $=0$ Multiplicity $=1$ for high level calculation on model system .

Charge $=0$ Multiplicity $=1$ for low level calculation on model system.

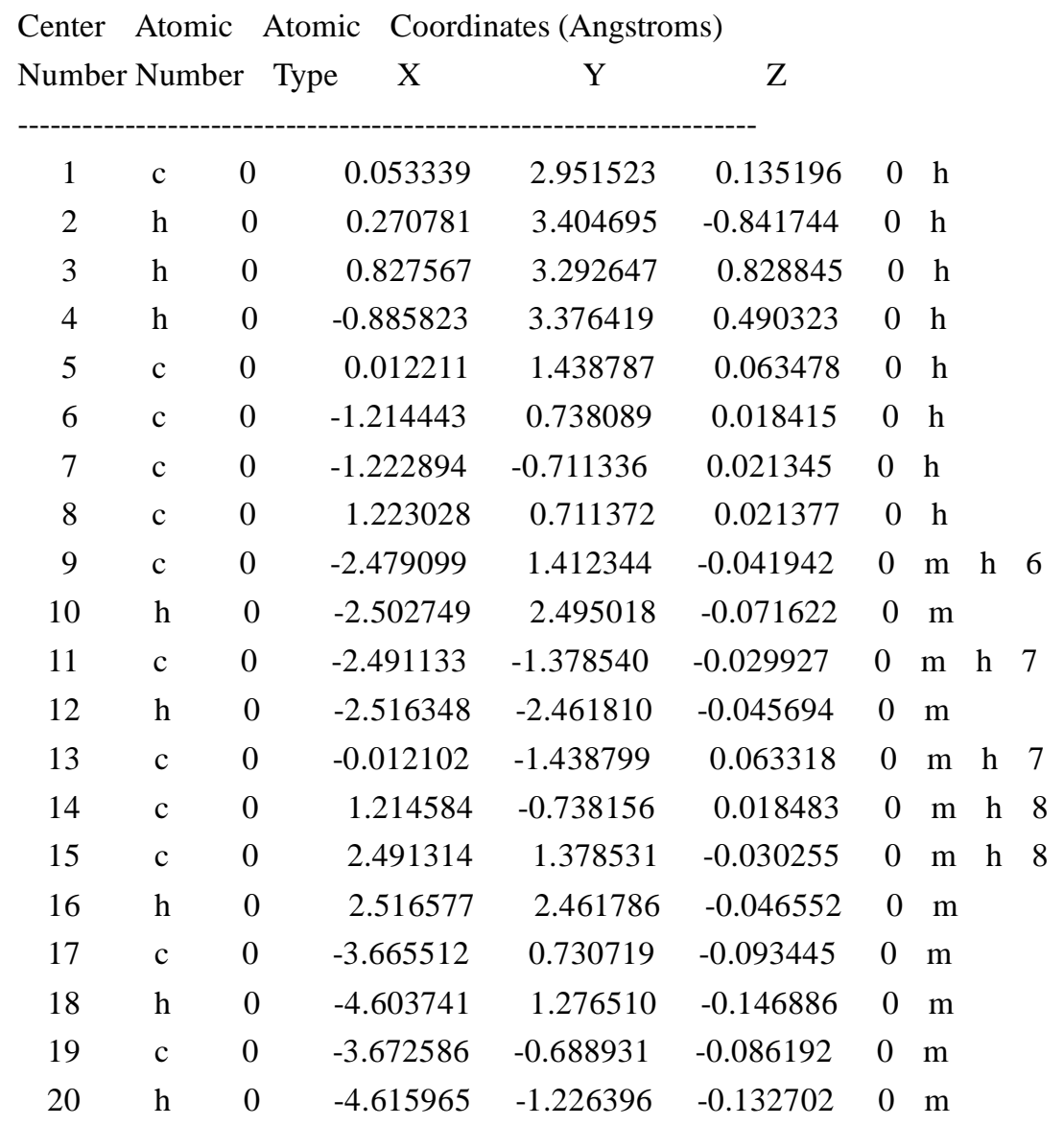




$\begin{array}{lllrrrrc}21 & \mathrm{c} & 0 & -0.054127 & -2.951517 & 0.134784 & 0 & \mathrm{~m} \\ 22 & \mathrm{~h} & 0 & -0.824938 & -3.291959 & 0.832669 & 0 & \mathrm{~m} \\ 23 & \mathrm{~h} & 0 & -0.277740 & -3.404010 & -0.841057 & 0 & \mathrm{~m} \\ 24 & \mathrm{~h} & 0 & 0.886470 & -3.377754 & 0.484311 & 0 & \mathrm{~m} \\ 25 & \mathrm{c} & 0 & 2.479353 & -1.412340 & -0.041576 & 0 & \mathrm{~m} \\ 26 & \mathrm{~h} & 0 & 2.503160 & -2.495011 & -0.070930 & 0 & \mathrm{~m} \\ 27 & \mathrm{c} & 0 & 3.665745 & -0.730715 & -0.093172 & 0 & \mathrm{~m} \\ 28 & \mathrm{~h} & 0 & 4.603978 & -1.276518 & -0.146375 & 0 & \mathrm{~m} \\ 29 & \mathrm{c} & 0 & 3.672763 & 0.688959 & -0.086440 & 0 & \mathrm{~m} \\ 30 & \mathrm{~h} & 0 & 4.616127 & 1.226426 & -0.133261 & 0 & \mathrm{~m}\end{array}$

radical

Charge $=0$ Multiplicity $=2$ for low level calculation on real system.

Charge $=0$ Multiplicity $=2$ for high level calculation on model system.

Charge $=0$ Multiplicity $=2$ for low level calculation on model system.

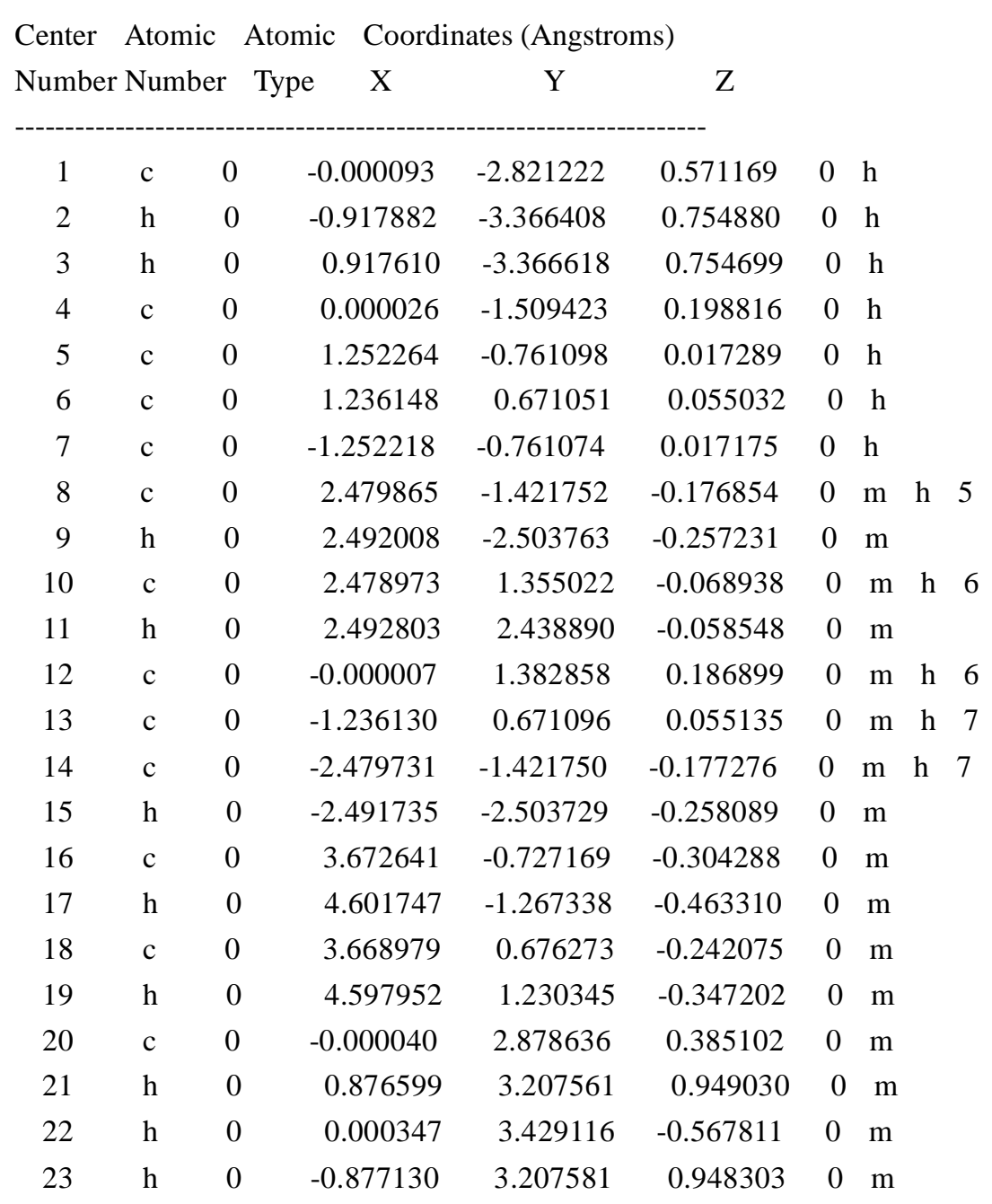




$\begin{array}{llllllll}24 & \mathrm{c} & 0 & -2.479048 & 1.355003 & -0.068532 & 0 & \mathrm{~m} \\ 25 & \mathrm{~h} & 0 & -2.493012 & 2.438874 & -0.057837 & 0 & \mathrm{~m} \\ 26 & \mathrm{c} & 0 & -3.669004 & 0.676195 & -0.241837 & 0 & \mathrm{~m} \\ 27 & \mathrm{~h} & 0 & -4.597999 & 1.230280 & -0.346714 & 0 & \mathrm{~m} \\ 28 & \mathrm{c} & 0 & -3.672571 & -0.727211 & -0.304552 & 0 & \mathrm{~m} \\ 29 & \mathrm{~h} & 0 & -4.601629 & -1.267403 & -0.463759 & 0 & \mathrm{~m}\end{array}$

\section{No 4}<smiles>Cc1c2ccccc2c([N+](=O)[O-])c2ccccc12</smiles>

neutral molecule

Charge $=0$ Multiplicity $=1$ for low level calculation on real system.

Charge $=0$ Multiplicity $=1$ for high level calculation on model system.

Charge $=0$ Multiplicity $=1$ for low level calculation on model system.

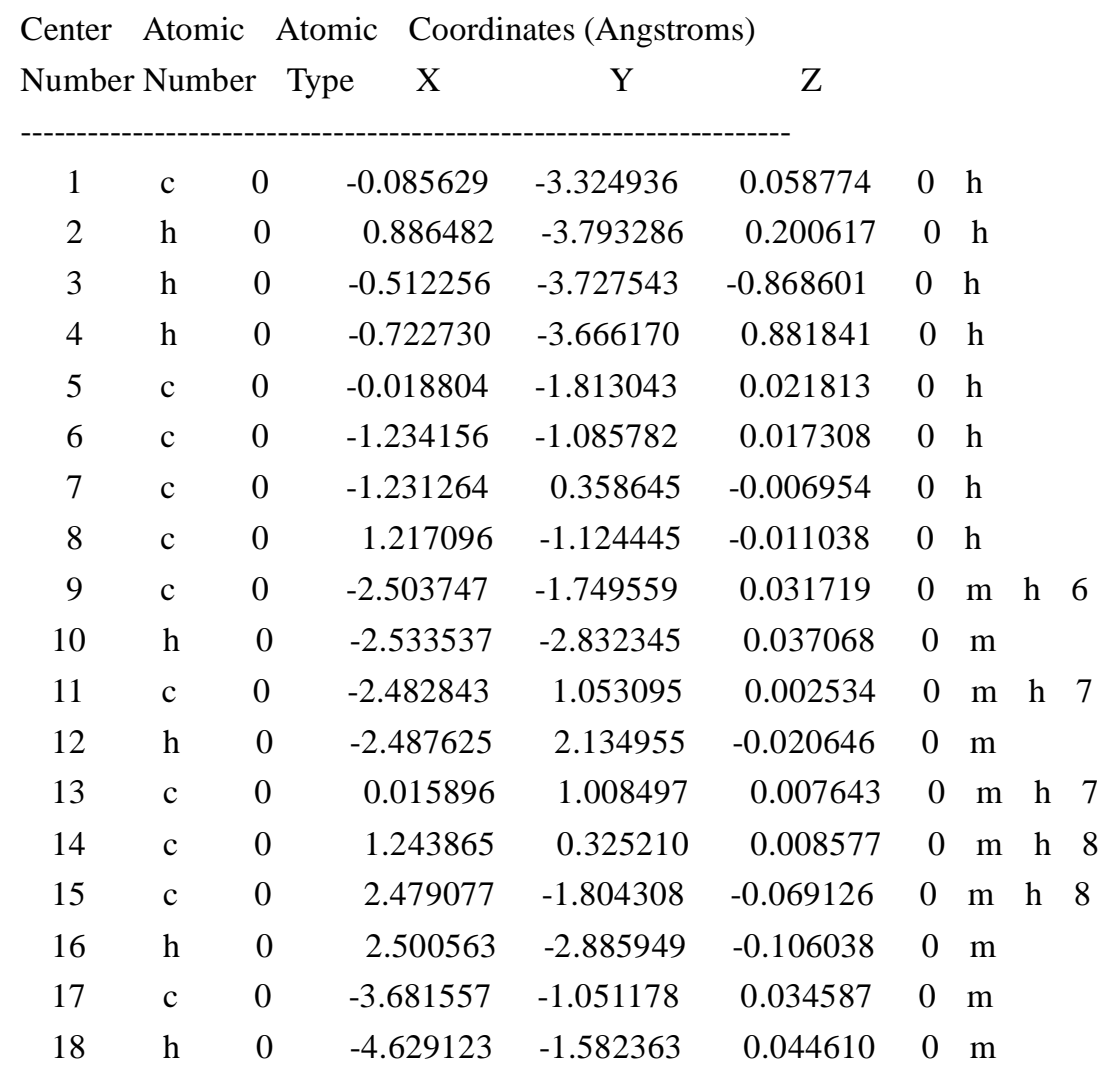




$\begin{array}{lllrrrcc}19 & \mathrm{c} & 0 & -3.668322 & 0.368585 & 0.025774 & 0 & \mathrm{~m} \\ 20 & \mathrm{~h} & 0 & -4.606012 & 0.916956 & 0.033259 & 0 & \mathrm{~m} \\ 21 & \mathrm{n} & 0 & 0.034662 & 2.480814 & 0.017227 & 0 & \mathrm{~m} \\ 22 & \mathrm{o} & 0 & -0.578897 & 3.069314 & -0.874314 & 0 & \mathrm{~m} \\ 23 & \mathrm{o} & 0 & 0.661655 & 3.042112 & 0.916761 & 0 & \mathrm{~m} \\ 24 & \mathrm{c} & 0 & 2.508654 & 0.996625 & -0.018716 & 0 & \mathrm{~m} \\ 25 & \mathrm{~h} & 0 & 2.532592 & 2.078061 & 0.009708 & 0 & \mathrm{~m} \\ 26 & \mathrm{c} & 0 & 3.680790 & 0.293658 & -0.073367 & 0 & \mathrm{~m} \\ 27 & \mathrm{~h} & 0 & 4.627662 & 0.825469 & -0.097387 & 0 & \mathrm{~m} \\ 28 & \mathrm{c} & 0 & 3.666930 & -1.126128 & -0.100352 & 0 & \mathrm{~m} \\ 29 & \mathrm{~h} & 0 & 4.603375 & -1.674497 & -0.149662 & 0 & \mathrm{~m}\end{array}$

radical

Charge $=0$ Multiplicity $=2$ for low level calculation on real system.

Charge $=0$ Multiplicity $=2$ for high level calculation on model system.

Charge $=0$ Multiplicity $=2$ for low level calculation on model system.

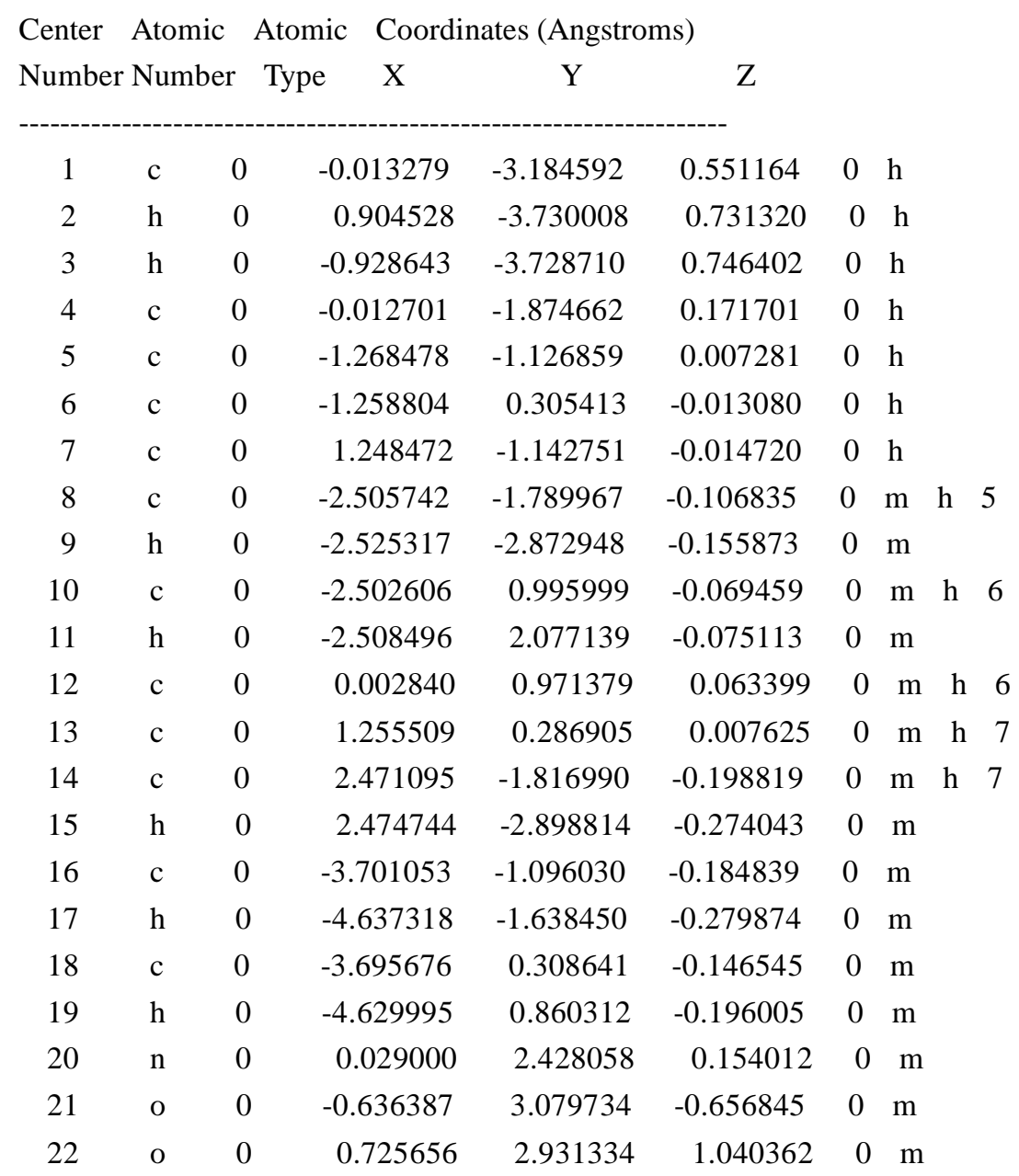




$\begin{array}{llllllll}23 & \mathrm{c} & 0 & 2.499939 & 0.968100 & -0.104942 & 0 & \mathrm{~m} \\ 24 & \mathrm{~h} & 0 & 2.520314 & 2.049358 & -0.068339 & 0 & \mathrm{~m} \\ 25 & \mathrm{c} & 0 & 3.679593 & 0.271816 & -0.264018 & 0 & \mathrm{~m} \\ 26 & \mathrm{~h} & 0 & 4.615509 & 0.814999 & -0.357114 & 0 & \mathrm{~m} \\ 27 & \mathrm{c} & 0 & 3.668260 & -1.132440 & -0.322166 & 0 & \mathrm{~m} \\ 28 & \mathrm{~h} & 0 & 4.593330 & -1.681607 & -0.472065 & 0 & \mathrm{~m}\end{array}$

No. 5<smiles>COc1c2ccccc2c(C)c2ccccc12</smiles>

neutral molecule

Charge $=0$ Multiplicity $=1$ for low level calculation on real system.

Charge $=0$ Multiplicity $=1$ for high level calculation on model system.

Charge $=0$ Multiplicity $=1$ for low level calculation on model system.

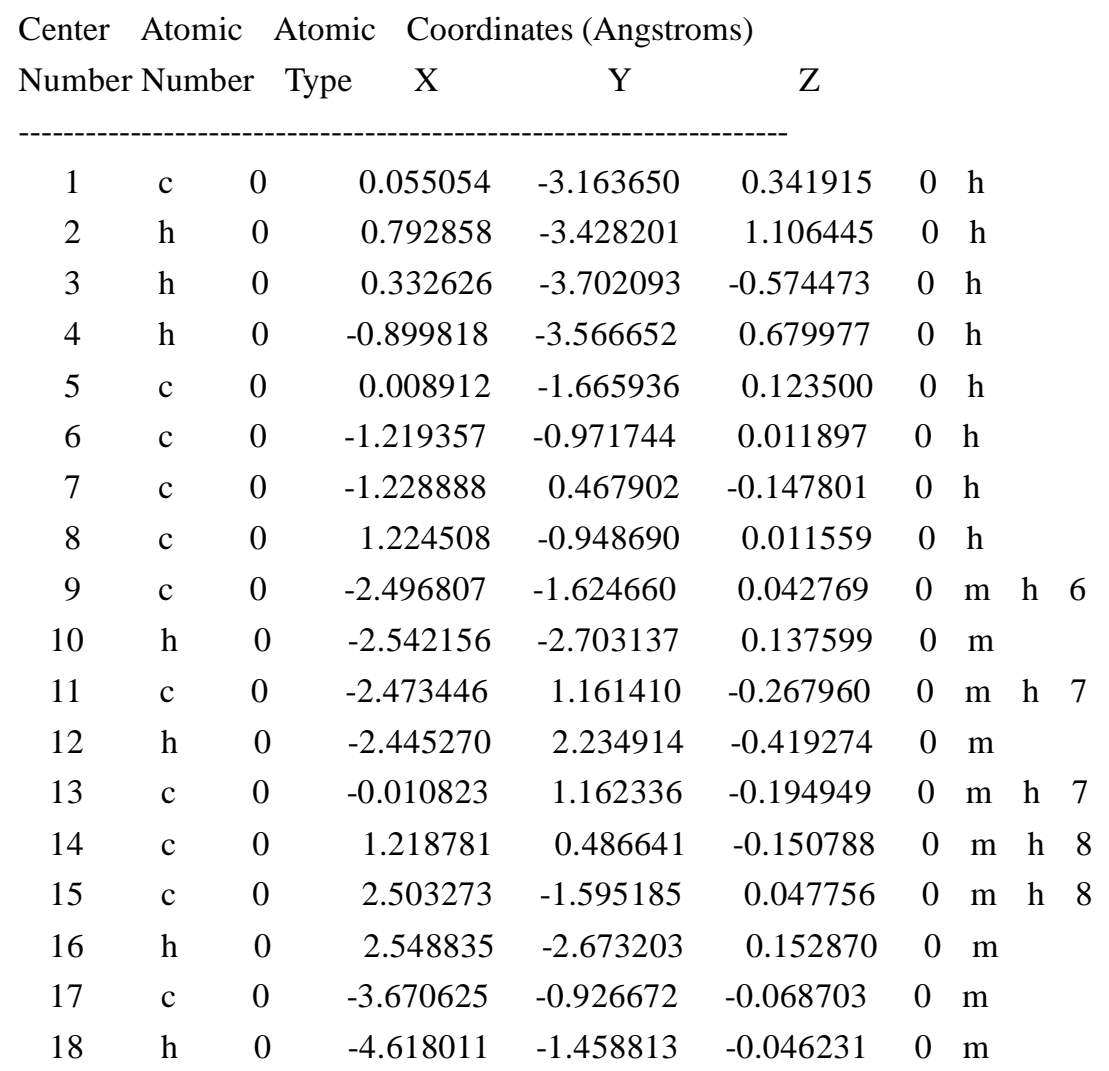




$\begin{array}{lllrrrrc}19 & \mathrm{c} & 0 & -3.663279 & 0.486926 & -0.228000 & 0 & \mathrm{~m} \\ 20 & \mathrm{~h} & 0 & -4.602420 & 1.024160 & -0.328739 & 0 & \mathrm{~m} \\ 21 & \mathrm{o} & 0 & -0.020891 & 2.538700 & -0.332743 & 0 & \mathrm{~m} \\ 22 & \mathrm{c} & 0 & -0.021746 & 3.252944 & 0.906437 & 0 & \mathrm{~m} \\ 23 & \mathrm{~h} & 0 & 0.874757 & 3.024520 & 1.496654 & 0 & \mathrm{~m} \\ 24 & \mathrm{~h} & 0 & -0.912689 & 3.014459 & 1.501074 & 0 & \mathrm{~m} \\ 25 & \mathrm{~h} & 0 & -0.028333 & 4.315017 & 0.649643 & 0 & \mathrm{~m} \\ 26 & \mathrm{c} & 0 & 2.456114 & 1.190407 & -0.274722 & 0 & \mathrm{~m} \\ 27 & \mathrm{~h} & 0 & 2.420280 & 2.263367 & -0.428892 & 0 & \mathrm{~m} \\ 28 & \mathrm{c} & 0 & 3.651809 & 0.524155 & -0.233347 & 0 & \mathrm{~m} \\ 29 & \mathrm{~h} & 0 & 4.586327 & 1.068904 & -0.336982 & 0 & \mathrm{~m} \\ 30 & \mathrm{c} & 0 & 3.672409 & -0.888133 & -0.067274 & 0 & \mathrm{~m} \\ 31 & \mathrm{~h} & 0 & 4.624798 & -1.411138 & -0.041458 & 0 & \mathrm{~m}\end{array}$

radical

Charge $=0$ Multiplicity $=2$ for low level calculation on real system.

Charge $=0$ Multiplicity $=2$ for high level calculation on model system.

Charge $=0$ Multiplicity $=2$ for low level calculation on model system.

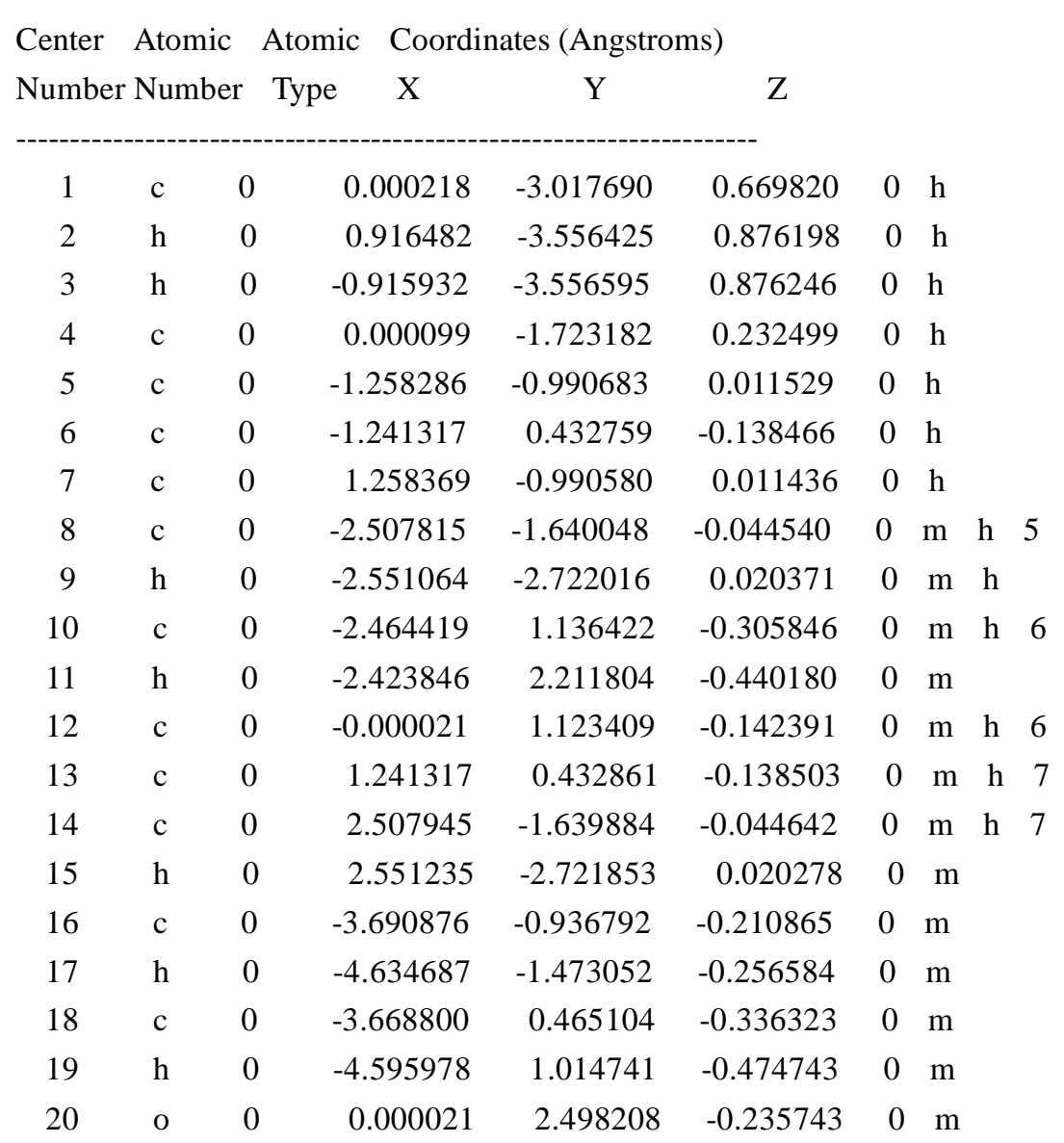




$\begin{array}{lllrrrrr}21 & \mathrm{c} & 0 & -0.000443 & 3.177248 & 1.025446 & 0 & \mathrm{~m} \\ 22 & \mathrm{~h} & 0 & 0.893480 & 2.925030 & 1.609423 & 0 & \mathrm{~m} \\ 23 & \mathrm{~h} & 0 & -0.895050 & 2.925512 & 1.608586 & 0 & \mathrm{~m} \\ 24 & \mathrm{~h} & 0 & -0.000061 & 4.246056 & 0.799110 & 0 & \mathrm{~m} \\ 25 & \mathrm{c} & 0 & 2.464383 & 1.136630 & -0.305685 & 0 & \mathrm{~m} \\ 26 & \mathrm{~h} & 0 & 2.423718 & 2.212046 & -0.439740 & 0 & \mathrm{~m} \\ 27 & \mathrm{c} & 0 & 3.668798 & 0.465402 & -0.336198 & 0 & \mathrm{~m} \\ 28 & \mathrm{~h} & 0 & 4.595951 & 1.015125 & -0.474449 & 0 & \mathrm{~m} \\ 29 & \mathrm{c} & 0 & 3.690975 & -0.936528 & -0.210923 & 0 & \mathrm{~m} \\ 30 & \mathrm{~h} & 0 & 4.634821 & -1.472723 & -0.256658 & 0 & \mathrm{~m}\end{array}$

No. 6<smiles>Cc1c2ccccc2c(-c2ccccc2)c2ccccc12</smiles>

neutral molecule

Charge $=0$ Multiplicity $=1$ for low level calculation on real system.

Charge $=0$ Multiplicity $=1$ for high level calculation on model system .

Charge $=0$ Multiplicity $=1$ for low level calculation on model system.

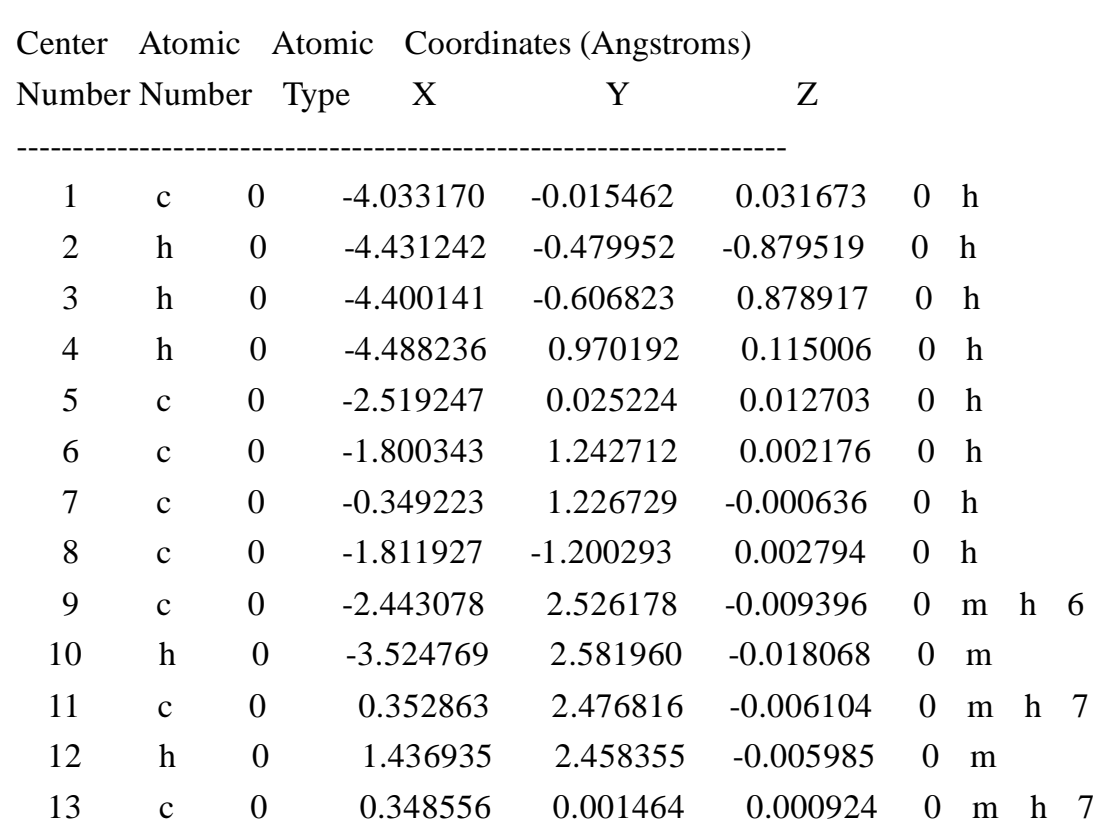




$\begin{array}{lllrllllll}14 & \mathrm{c} & 0 & -0.366980 & -1.215089 & -0.001360 & 0 & \mathrm{~m} & \mathrm{~h} & 8 \\ 15 & \mathrm{c} & 0 & -2.492764 & -2.461458 & -0.005169 & 0 & \mathrm{~m} & \mathrm{~h} & 8 \\ 16 & \mathrm{~h} & 0 & -3.576531 & -2.477086 & -0.008736 & 0 & \mathrm{~m} & & \\ 17 & \mathrm{c} & 0 & -1.731617 & 3.695746 & -0.017417 & 0 & \mathrm{~m} & \\ 18 & \mathrm{~h} & 0 & -2.255854 & 4.647714 & -0.027771 & 0 & \mathrm{~m} & \\ 19 & \mathrm{c} & 0 & -0.310514 & 3.673048 & -0.013792 & 0 & \mathrm{~m} & \\ 20 & \mathrm{~h} & 0 & 0.245895 & 4.606469 & -0.018881 & 0 & \mathrm{~m} & \\ 21 & \mathrm{c} & 0 & 1.847086 & -0.011840 & 0.002719 & 0 & \mathrm{~m} & \\ 22 & \mathrm{c} & 0 & 2.562076 & -0.013881 & 1.210117 & 0 & \mathrm{~m} & \\ 23 & \mathrm{~h} & 0 & 2.016096 & -0.003723 & 2.149797 & 0 & \mathrm{~m} & \\ 24 & \mathrm{c} & 0 & 3.957861 & -0.028592 & 1.213382 & 0 & \mathrm{~m} & \\ 25 & \mathrm{~h} & 0 & 4.494590 & -0.029898 & 2.158618 & 0 & \mathrm{~m} & \\ 26 & \mathrm{c} & 0 & 4.662036 & -0.041641 & 0.008031 & 0 & \mathrm{~m} & \\ 27 & \mathrm{~h} & 0 & 5.748780 & -0.053048 & 0.010023 & 0 & \mathrm{~m} & \\ 28 & \mathrm{c} & 0 & 3.962225 & -0.040100 & -1.199930 & 0 & \mathrm{~m} & \\ 29 & \mathrm{~h} & 0 & 4.502387 & -0.050313 & -2.143154 & 0 & \mathrm{~m} & & \\ 30 & \mathrm{c} & 0 & 2.566402 & -0.025414 & -1.201904 & 0 & \mathrm{~m} & & \\ 31 & \mathrm{~h} & 0 & 2.023885 & -0.024186 & -2.143655 & 0 & \mathrm{~m} & & \\ 32 & \mathrm{c} & 0 & 0.305272 & -2.479973 & -0.006733 & 0 & \mathrm{~m} & & \\ 33 & \mathrm{~h} & 0 & 1.389587 & -2.489503 & -0.007461 & 0 & \mathrm{~m} & & \\ 34 & \mathrm{c} & 0 & -0.389767 & -3.659962 & -0.012471 & 0 & \mathrm{~m} & \\ 35 & \mathrm{~h} & 0 & 0.143816 & -4.606664 & -0.017329 & 0 & \mathrm{~m} & & \\ 36 & \mathrm{c} & 0 & -1.810515 & -3.649873 & -0.013163 & 0 & \mathrm{~m} & & \\ 37 & \mathrm{~h} & 0 & -2.356587 & -4.589536 & -0.020463 & 0 & \mathrm{~m} & \\ & & & & & & & & & \end{array}$

radical

Charge $=0$ Multiplicity $=2$ for low level calculation on real system.

Charge $=0$ Multiplicity $=2$ for high level calculation on model system.

Charge $=0$ Multiplicity $=2$ for low level calculation on model system.

Center Atomic Atomic Coordinates (Angstroms)

Number Number Type $\quad \mathrm{X} \quad \mathrm{Y} \quad$ Z

\begin{tabular}{|c|c|c|c|c|c|c|c|}
\hline 1 & $\mathrm{c}$ & 0 & 3.916104 & -0.000086 & 0.475448 & 0 & $\mathrm{~h}$ \\
\hline 2 & $\mathrm{~h}$ & 0 & 4.470904 & -0.915755 & 0.635690 & 0 & $\mathrm{~h}$ \\
\hline 3 & $\mathrm{~h}$ & 0 & 4.471004 & 0.915532 & 0.635618 & 0 & $\mathrm{~h}$ \\
\hline 4 & $\mathrm{c}$ & 0 & 2.586823 & -0.000031 & 0.154954 & 0 & $\mathrm{~h}$ \\
\hline 5 & $\mathrm{c}$ & 0 & 1.837731 & 1.255983 & 0.006117 & 0 & $\mathrm{~h}$ \\
\hline 6 & $\mathrm{c}$ & 0 & 0.404880 & 1.238570 & -0.013796 & 0 & $\mathrm{~h}$ \\
\hline 7 & $\mathrm{c}$ & 0 & 1.837695 & -1.256001 & 0.006036 & 0 & $\mathrm{~h}$ \\
\hline 8 & $\mathrm{c}$ & 0 & 2.491708 & 2.500198 & -0.104097 & 0 & $\mathrm{~m} \quad \mathrm{~h}$ \\
\hline 9 & $\mathrm{~h}$ & 0 & 3.575097 & 2.531425 & -0.144913 & 0 & $\mathrm{~m}$ \\
\hline
\end{tabular}




\begin{tabular}{|c|c|c|c|c|c|c|c|c|}
\hline 10 & $\mathrm{c}$ & 0 & -0.290025 & 2.480336 & -0.084777 & 0 & $\mathrm{~m}$ & h \\
\hline 11 & $\mathrm{~h}$ & 0 & -1.374177 & 2.469961 & -0.084917 & 0 & $\mathrm{~m}$ & \\
\hline 12 & $\mathrm{c}$ & 0 & -0.306195 & 0.000003 & 0.021874 & 0 & $\mathrm{~m}$ & h \\
\hline 13 & $\mathrm{c}$ & 0 & 0.404854 & -1.238554 & -0.013954 & 0 & $\mathrm{~m}$ & h \\
\hline 14 & $\mathrm{c}$ & 0 & 2.491650 & -2.500222 & -0.104195 & 0 & $\mathrm{~m}$ & $\mathrm{~h}$ \\
\hline 15 & $\mathrm{~h}$ & 0 & 3.575047 & -2.531477 & -0.144863 & 0 & $\mathrm{~m}$ & \\
\hline 16 & $\mathrm{c}$ & 0 & 1.790824 & 3.691597 & -0.190324 & 0 & $\mathrm{~m}$ & \\
\hline 17 & $\mathrm{~h}$ & 0 & 2.329434 & 4.630849 & -0.281026 & 0 & $\mathrm{~m}$ & \\
\hline 18 & $\mathrm{c}$ & 0 & 0.384237 & 3.680168 & -0.167434 & 0 & $\mathrm{~m}$ & \\
\hline 19 & $\mathrm{~h}$ & 0 & -0.170979 & 4.612392 & -0.227546 & 0 & $\mathrm{~m}$ & \\
\hline 20 & $\mathrm{c}$ & 0 & -1.801572 & 0.000010 & 0.045442 & 0 & $\mathrm{~m}$ & \\
\hline 21 & $\mathrm{c}$ & 0 & -2.541006 & 0.000804 & -1.147798 & 0 & $\mathrm{~m}$ & \\
\hline 22 & $\mathrm{~h}$ & 0 & -2.014224 & 0.001420 & -2.098409 & 0 & $\mathrm{~m}$ & \\
\hline 23 & $\mathrm{c}$ & 0 & -3.936630 & 0.000788 & -1.122903 & 0 & $\mathrm{~m}$ & \\
\hline 24 & $\mathrm{~h}$ & 0 & -4.492154 & 0.001394 & -2.057177 & 0 & $\mathrm{~m}$ & \\
\hline 25 & $\mathrm{c}$ & 0 & -4.616433 & 0.000019 & 0.096455 & 0 & $\mathrm{~m}$ & \\
\hline 26 & $\mathrm{~h}$ & 0 & -5.703013 & 0.000023 & 0.116274 & 0 & $\mathrm{~m}$ & \\
\hline 27 & $\mathrm{c}$ & 0 & -3.892642 & -0.000776 & 1.290122 & 0 & $\mathrm{~m}$ & \\
\hline 28 & $\mathrm{~h}$ & 0 & -4.413888 & -0.001394 & 2.243933 & 0 & $\mathrm{~m}$ & \\
\hline 29 & $\mathrm{c}$ & 0 & -2.496997 & -0.000780 & 1.264520 & 0 & $\mathrm{~m}$ & \\
\hline 30 & $\mathrm{~h}$ & 0 & -1.936220 & -0.001400 & 2.195455 & 0 & $\mathrm{~m}$ & \\
\hline 31 & $\mathrm{c}$ & 0 & -0.290073 & -2.480291 & -0.085228 & 0 & $\mathrm{~m}$ & \\
\hline 32 & $\mathrm{~h}$ & 0 & -1.374225 & -2.469888 & -0.085520 & 0 & $\mathrm{~m}$ & \\
\hline 33 & $\mathrm{c}$ & 0 & 0.384170 & -3.680128 & -0.167978 & 0 & $\mathrm{~m}$ & \\
\hline 34 & $\mathrm{~h}$ & 0 & -0.171077 & -4.612319 & -0.228299 & 0 & $\mathrm{~m}$ & \\
\hline 35 & $\mathrm{c}$ & 0 & 1.790751 & -3.691592 & -0.190639 & 0 & $\mathrm{~m}$ & \\
\hline 36 & $\mathrm{~h}$ & 0 & 2.329345 & -4.630851 & -0.281363 & 0 & $\mathrm{~m}$ & \\
\hline
\end{tabular}

No. 7<smiles>[CH]c1c2ccccc2c(C(=O)c2ccccc2)c2ccccc12</smiles>

neutral molecule

Charge $=0$ Multiplicity $=1$ for low level calculation on real system.

Charge $=0$ Multiplicity $=1$ for high level calculation on model system .

Charge $=0$ Multiplicity $=1$ for low level calculation on model system. 


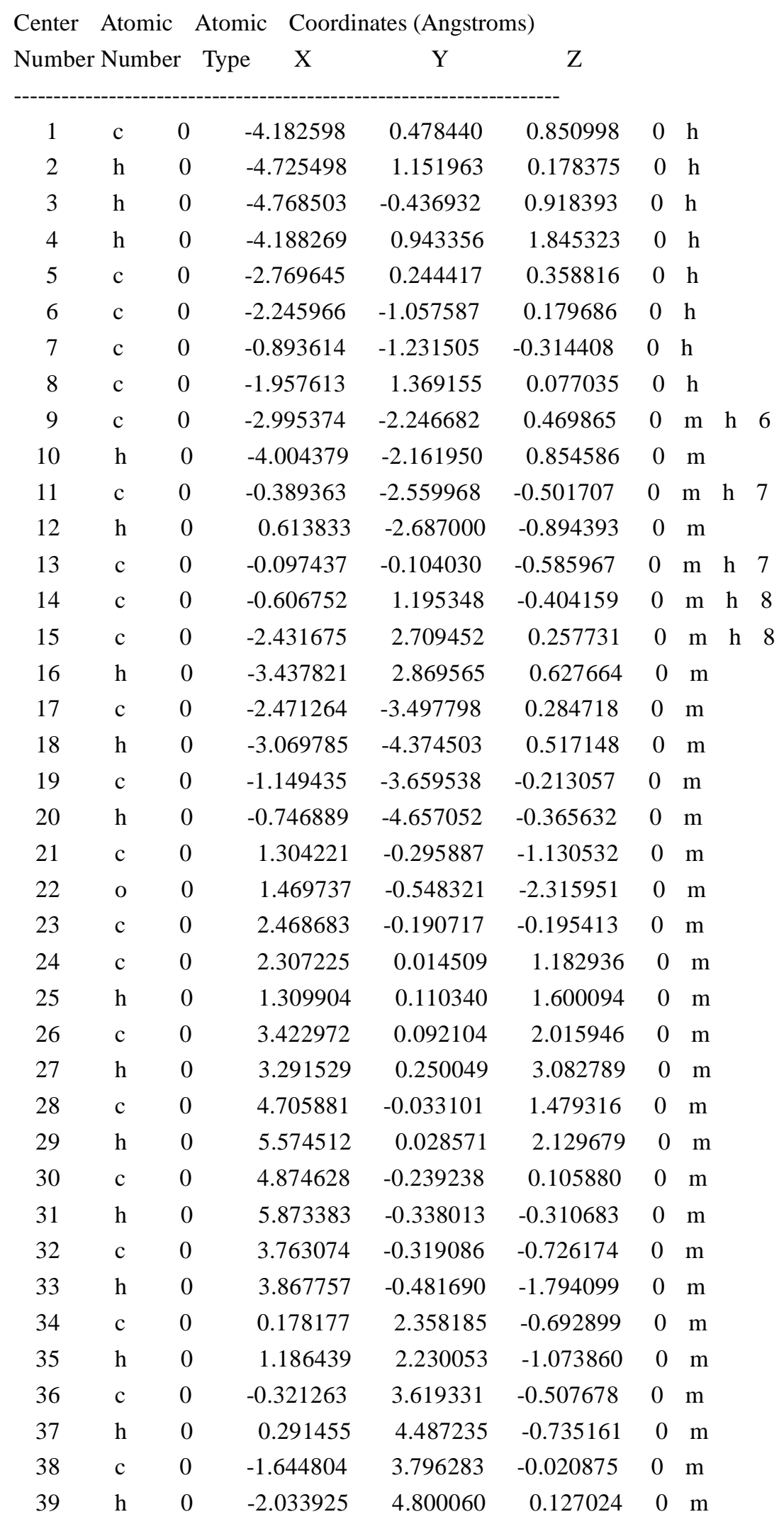


radical

Charge $=0$ Multiplicity $=2$ for low level calculation on real system.

Charge $=0$ Multiplicity $=2$ for high level calculation on model system.

Charge $=0$ Multiplicity $=2$ for low level calculation on model system.

Center Atomic Atomic Coordinates (Angstroms)

Number Number Type X $\quad$ Y $\quad$ Z

\begin{tabular}{|c|c|c|c|c|c|c|c|c|}
\hline 1 & $\mathrm{c}$ & 0 & -3.504403 & 1.371784 & 1.450552 & 0 & & \\
\hline 2 & $\mathrm{~h}$ & 0 & -3.522664 & 2.419005 & 1.727137 & 0 & $\mathrm{~h}$ & \\
\hline 3 & $\mathrm{~h}$ & 0 & -4.246208 & 0.743998 & 1.929082 & 0 & $\mathrm{~h}$ & \\
\hline 4 & $\mathrm{c}$ & 0 & -2.564637 & 0.865481 & 0.603039 & 0 & $\mathrm{~h}$ & \\
\hline 5 & $\mathrm{c}$ & 0 & -2.506968 & -0.570112 & 0.285918 & 0 & $\mathrm{~h}$ & \\
\hline 6 & $\mathrm{c}$ & 0 & -1.303071 & -1.139144 & -0.241064 & 0 & $\mathrm{~h}$ & \\
\hline 7 & $\mathrm{c}$ & 0 & -1.525550 & 1.723804 & 0.015539 & 0 & $\mathrm{~h}$ & \\
\hline 8 & $\mathrm{c}$ & 0 & -3.611133 & -1.414175 & 0.511298 & 0 & $\mathrm{~m}$ & h 5 \\
\hline 9 & $\mathrm{~h}$ & 0 & -4.546015 & -0.984777 & 0.855659 & 0 & $\mathrm{~m}$ & \\
\hline 10 & $\mathrm{c}$ & 0 & -1.257529 & -2.545779 & -0.456566 & 0 & $\mathrm{~m}$ & h \\
\hline 11 & $\mathrm{~h}$ & 0 & -0.355238 & -2.985089 & -0.861677 & 0 & $\mathrm{~m}$ & \\
\hline 12 & $\mathrm{c}$ & 0 & -0.177594 & -0.291797 & -0.514478 & 0 & $\mathrm{~m}$ & h \\
\hline 13 & $\mathrm{c}$ & 0 & -0.325802 & 1.133338 & -0.491655 & 0 & $\mathrm{~m}$ & h \\
\hline 14 & $\mathrm{c}$ & 0 & -1.672073 & 3.122202 & -0.054126 & 0 & $\mathrm{~m}$ & h \\
\hline 15 & $\mathrm{~h}$ & 0 & -2.599052 & 3.576238 & 0.280378 & 0 & $\mathrm{~m}$ & \\
\hline 16 & $\mathrm{c}$ & 0 & -3.546198 & -2.777619 & 0.273833 & 0 & $\mathrm{~m}$ & \\
\hline 17 & $\mathrm{~h}$ & 0 & -4.419280 & -3.400383 & 0.447840 & 0 & $\mathrm{~m}$ & \\
\hline 18 & $\mathrm{c}$ & 0 & -2.352698 & -3.346315 & -0.202880 & 0 & $\mathrm{~m}$ & \\
\hline 19 & $\mathrm{~h}$ & 0 & -2.294210 & -4.414867 & -0.390748 & 0 & $\mathrm{~m}$ & \\
\hline 20 & $\mathrm{c}$ & 0 & 1.134284 & -0.905630 & -0.918603 & 0 & $\mathrm{~m}$ & \\
\hline 21 & o & 0 & 1.203416 & -1.641766 & -1.898849 & 0 & $\mathrm{~m}$ & \\
\hline 22 & $\mathrm{c}$ & 0 & 2.354281 & -0.650543 & -0.083488 & 0 & $\mathrm{~m}$ & \\
\hline 23 & $\mathrm{c}$ & 0 & 2.290032 & -0.080338 & 1.197146 & 0 & $\mathrm{~m}$ & \\
\hline 24 & $\mathrm{~h}$ & 0 & 1.332359 & 0.225367 & 1.605711 & 0 & $\mathrm{~m}$ & \\
\hline 25 & $\mathrm{c}$ & 0 & 3.451302 & 0.086694 & 1.950647 & 0 & $\mathrm{~m}$ & \\
\hline 26 & $\mathrm{~h}$ & 0 & 3.392375 & 0.523327 & 2.943837 & 0 & $\mathrm{~m}$ & \\
\hline 27 & $\mathrm{c}$ & 0 & 4.685840 & -0.307673 & 1.430877 & 0 & $\mathrm{~m}$ & \\
\hline 28 & $\mathrm{~h}$ & 0 & 5.590390 & -0.172931 & 2.018213 & 0 & $\mathrm{~m}$ & \\
\hline 29 & $\mathrm{c}$ & 0 & 4.757949 & -0.879354 & 0.156596 & 0 & $\mathrm{~m}$ & \\
\hline 30 & $\mathrm{~h}$ & 0 & 5.717947 & -1.189039 & -0.247585 & 0 & $\mathrm{~m}$ & \\
\hline 31 & $\mathrm{c}$ & 0 & 3.598991 & -1.055600 & -0.592810 & 0 & $\mathrm{~m}$ & \\
\hline 32 & $\mathrm{~h}$ & 0 & 3.629465 & -1.508734 & -1.578405 & 0 & $\mathrm{~m}$ & \\
\hline 33 & $\mathrm{c}$ & 0 & 0.679264 & 1.990761 & -1.021682 & 0 & $\mathrm{~m}$ & \\
\hline 34 & $\mathrm{~h}$ & 0 & 1.585135 & 1.556128 & -1.429935 & 0 & $\mathrm{~m}$ & \\
\hline
\end{tabular}




$\begin{array}{llllllll}35 & \mathrm{c} & 0 & 0.512428 & 3.360469 & -1.057404 & 0 & \mathrm{~m} \\ 36 & \mathrm{~h} & 0 & 1.291510 & 3.989819 & -1.478406 & 0 & \mathrm{~m} \\ 37 & \mathrm{c} & 0 & -0.676525 & 3.934045 & -0.574944 & 0 & \mathrm{~m} \\ 38 & \mathrm{~h} & 0 & -0.824994 & 5.009074 & -0.624788 & 0 & \mathrm{~m}\end{array}$

No. 8

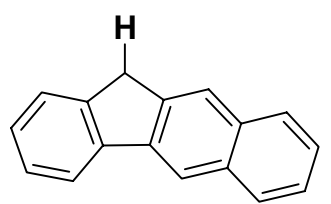

neutral molecule

Charge $=0$ Multiplicity $=1$ for low level calculation on real system.

Charge $=0$ Multiplicity $=1$ for high level calculation on model system .

Charge $=0$ Multiplicity $=1$ for low level calculation on model system .

Center Atomic Atomic Coordinates (Angstroms)

$\begin{array}{ccccccl}\text { Number Number } & \text { Type } & \text { X } & \text { Y } & \text { Z } & \\ 1 & \text { c } & 0 & -1.446488 & 1.929640 & 0.000121 & 0 \mathrm{~h} \\ 2 & \mathrm{~h} & 0 & -1.538517 & 2.582299 & -0.879125 & 0 \mathrm{~h} \\ 3 & \mathrm{~h} & 0 & -1.538440 & 2.581851 & 0.879727 & 0 \mathrm{~h} \\ 4 & \mathrm{c} & 0 & -0.139422 & 1.157886 & -0.000108 & 0 \mathrm{~h} \\ 5 & \mathrm{c} & 0 & -0.408415 & -0.247881 & -0.000049 & 0 \mathrm{~h} \\ 6 & \mathrm{c} & 0 & -1.864879 & -0.437079 & 0.000165 & 0 \mathrm{~h} \\ 7 & \mathrm{c} & 0 & -2.488417 & 0.827809 & -0.000065 & 0 \mathrm{~h} \\ 8 & \mathrm{c} & 0 & 1.149678 & 1.622815 & -0.000137 & 0 \mathrm{~m} \mathrm{~h} 4 \\ 9 & \mathrm{~h} & 0 & 1.363142 & 2.690073 & 0.000176 & 0 \mathrm{~m} \\ 10 & \mathrm{c} & 0 & 0.623292 & -1.160549 & -0.000041 & 0 \mathrm{~m} \mathrm{~h} 5 \\ 11 & \mathrm{~h} & 0 & 0.428521 & -2.230864 & 0.000325 & 0 \mathrm{~m} \\ 12 & \mathrm{c} & 0 & -2.632970 & -1.604228 & 0.000365 & 0 \mathrm{~m} \mathrm{~h} \\ 13 & \mathrm{~h} & 0 & -2.158362 & -2.582192 & 0.000233 & 0 \mathrm{~m} \\ 14 & \mathrm{c} & 0 & -3.875412 & 0.928680 & -0.000305 & 0 \mathrm{~m} \mathrm{~h} 7 \\ 15 & \mathrm{~h} & 0 & -4.362134 & 1.901303 & -0.000401 & 0 \mathrm{~m} \\ 16 & \mathrm{c} & 0 & 2.239223 & 0.704728 & 0.000063 & 0 \mathrm{~m} \\ 17 & \mathrm{c} & 0 & 3.590004 & 1.142201 & 0.000146 & 0 \mathrm{~m} \\ 18 & \mathrm{~h} & 0 & 3.790472 & 2.211370 & 0.000003 & 0 \mathrm{~m} \\ 19 & \mathrm{c} & 0 & 4.629954 & 0.238964 & 0.000031 & 0 \mathrm{~m} \\ 20 & \mathrm{~h} & 0 & 5.657893 & 0.591376 & -0.000313 & 0 \mathrm{~m} \\ 21 & \mathrm{c} & 0 & 4.365142 & -1.152318 & -0.000152 & 0 \mathrm{~m} \\ 22 & \mathrm{~h} & 0 & 5.192065 & -1.857394 & -0.000637 & 0 \mathrm{~m} \\ & & & & & & \end{array}$




$\begin{array}{lllrrrc}23 & \mathrm{c} & 0 & 3.067093 & -1.610946 & 0.000054 & 0 \mathrm{~m} \\ 24 & \mathrm{~h} & 0 & 2.861062 & -2.678983 & -0.000348 & 0 \mathrm{~m} \\ 25 & \mathrm{c} & 0 & 1.969559 & -0.707339 & 0.000127 & 0 \mathrm{~m} \\ 26 & \mathrm{c} & 0 & -4.024457 & -1.497170 & -0.000348 & 0 \mathrm{~m} \\ 27 & \mathrm{~h} & 0 & -4.633768 & -2.397099 & -0.000317 & 0 \mathrm{~m} \\ 28 & \mathrm{c} & 0 & -4.642597 & -0.241401 & 0.000305 & 0 \mathrm{~m} \\ 29 & \mathrm{~h} & 0 & -5.727263 & -0.174612 & -0.000353 & 0 \mathrm{~m}\end{array}$

radical

Charge $=0$ Multiplicity $=2$ for low level calculation on real system.

Charge $=0$ Multiplicity $=2$ for high level calculation on model system.

Charge $=0$ Multiplicity $=2$ for low level calculation on model system.

\begin{tabular}{|c|c|c|c|c|c|c|}
\hline \multirow{2}{*}{$\begin{array}{l}\text { Center } \\
\text { Number }\end{array}$} & & \multirow{2}{*}{$\begin{array}{l}\text { Atomic } \\
\text { r Type }\end{array}$} & \multicolumn{3}{|c|}{ Coordinates (Angstroms) } & \\
\hline & Number & & $\mathrm{X}$ & $\mathrm{Y}$ & Z & \\
\hline 1 & $\mathrm{c}$ & 0 & 1.423630 & 1.838948 & -0.000050 & $0 \mathrm{~h}$ \\
\hline 2 & $\mathrm{~h}$ & 0 & 1.561644 & 2.914305 & 0.000078 & $0 \mathrm{~h}$ \\
\hline 3 & $\mathrm{c}$ & 0 & 0.167857 & 1.160223 & 0.000244 & $0 \mathrm{~h}$ \\
\hline 4 & $\mathrm{c}$ & 0 & 0.425100 & -0.269976 & 0.000270 & $0 \mathrm{~h}$ \\
\hline 5 & $\mathrm{c}$ & 0 & 1.879887 & -0.444311 & 0.000007 & $0 \mathrm{~h}$ \\
\hline 6 & $\mathrm{c}$ & 0 & 2.469048 & 0.863241 & -0.000267 & $0 \mathrm{~h}$ \\
\hline 7 & $\mathrm{c}$ & 0 & -1.136186 & 1.633168 & 0.000108 & $0 \mathrm{~m} \mathrm{~h} 3$ \\
\hline 8 & $\mathrm{~h}$ & 0 & -1.341917 & 2.701365 & 0.000054 & $0 \mathrm{~m}$ \\
\hline 9 & $\mathrm{c}$ & 0 & -0.610929 & -1.163700 & 0.000140 & $0 \mathrm{~m} \mathrm{~h} 4$ \\
\hline 10 & $\mathrm{~h}$ & 0 & -0.431676 & -2.237007 & 0.000087 & $0 \mathrm{~m}$ \\
\hline 11 & $\mathrm{c}$ & 0 & 2.684024 & -1.577286 & 0.000080 & $0 \mathrm{~m} \mathrm{~h} 5$ \\
\hline 12 & $\mathrm{~h}$ & 0 & 2.246722 & -2.572754 & 0.000336 & $0 \mathrm{~m}$ \\
\hline 13 & $\mathrm{c}$ & 0 & 3.867641 & 1.001323 & -0.000079 & $0 \mathrm{~m} \mathrm{~h} 6$ \\
\hline 14 & $\mathrm{~h}$ & 0 & 4.324040 & 1.987952 & 0.000027 & $0 \mathrm{~m}$ \\
\hline 15 & $\mathrm{c}$ & 0 & -2.224638 & 0.719055 & 0.000079 & $0 \mathrm{~m}$ \\
\hline 16 & $\mathrm{c}$ & 0 & -3.573431 & 1.161303 & 0.000004 & $0 \mathrm{~m}$ \\
\hline 17 & $\mathrm{~h}$ & 0 & -3.770098 & 2.231018 & 0.000051 & $0 \mathrm{~m}$ \\
\hline 18 & $\mathrm{c}$ & 0 & -4.619131 & 0.261144 & -0.000127 & $0 \mathrm{~m}$ \\
\hline 19 & $\mathrm{~h}$ & 0 & -5.645188 & 0.618766 & -0.000149 & $0 \mathrm{~m}$ \\
\hline 20 & $\mathrm{c}$ & 0 & -4.360194 & -1.127636 & -0.000188 & $0 \mathrm{~m}$ \\
\hline 21 & $\mathrm{~h}$ & 0 & -5.188130 & -1.831281 & -0.000258 & $0 \mathrm{~m}$ \\
\hline 22 & $\mathrm{c}$ & 0 & -3.058376 & -1.591257 & -0.000097 & $0 \mathrm{~m}$ \\
\hline 23 & $\mathrm{~h}$ & 0 & -2.858504 & -2.660448 & -0.000095 & $0 \mathrm{~m}$ \\
\hline 24 & $\mathrm{c}$ & 0 & -1.962896 & -0.696160 & 0.000069 & $0 \mathrm{~m}$ \\
\hline 25 & $\mathrm{c}$ & 0 & 4.076825 & -1.422498 & 0.000011 & $0 \mathrm{~m}$ \\
\hline 26 & $\mathrm{~h}$ & 0 & 4.714158 & -2.302502 & 0.000200 & $0 \mathrm{~m}$ \\
\hline
\end{tabular}




$\begin{array}{lllllll}27 & \mathrm{c} & 0 & 4.659558 & -0.145190 & -0.000223 & 0 \mathrm{~m} \\ 28 & \mathrm{~h} & 0 & 5.742213 & -0.051755 & -0.000212 & 0 \mathrm{~m}\end{array}$

No. 9<smiles>[CH]c1cc2c3ccccc3ccc2c2ccccc12</smiles>

neutral molecule

Charge $=0$ Multiplicity $=1$ for low level calculation on real system.

Charge $=0$ Multiplicity $=1$ for high level calculation on model system.

Charge $=0$ Multiplicity $=1$ for low level calculation on model system .

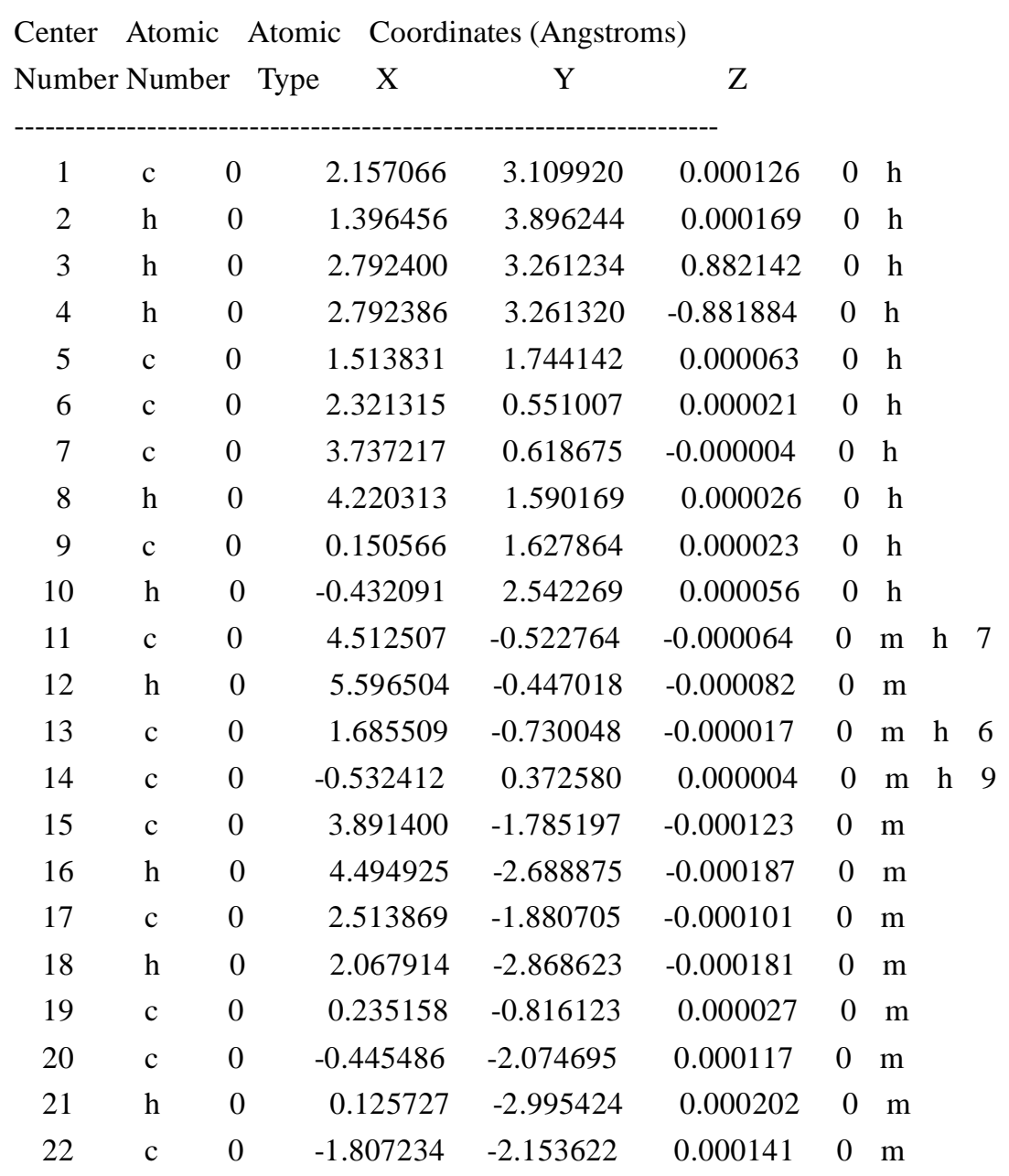




$\begin{array}{lllrrrrr}23 & \mathrm{~h} & 0 & -2.298174 & -3.123883 & 0.000227 & 0 & \mathrm{~m} \\ 24 & \mathrm{c} & 0 & -2.617291 & -0.979516 & 0.000055 & 0 & \mathrm{~m} \\ 25 & \mathrm{c} & 0 & -4.030766 & -1.067854 & 0.000037 & 0 & \mathrm{~m} \\ 26 & \mathrm{~h} & 0 & -4.488970 & -2.054256 & 0.000091 & 0 & \mathrm{~m} \\ 27 & \mathrm{c} & 0 & -4.813555 & 0.066857 & -0.000046 & 0 & \mathrm{~m} \\ 28 & \mathrm{~h} & 0 & -5.897212 & -0.012515 & -0.000059 & 0 & \mathrm{~m} \\ 29 & \mathrm{c} & 0 & -4.197151 & 1.334612 & -0.000136 & 0 & \mathrm{~m} \\ 30 & \mathrm{~h} & 0 & -4.808625 & 2.232980 & -0.000219 & 0 & \mathrm{~m} \\ 31 & \mathrm{c} & 0 & -2.820344 & 1.445802 & -0.000121 & 0 & \mathrm{~m} \\ 32 & \mathrm{~h} & 0 & -2.384210 & 2.438363 & -0.000226 & 0 & \mathrm{~m} \\ 33 & \mathrm{c} & 0 & -1.983755 & 0.300400 & -0.000017 & 0 & \mathrm{~m}\end{array}$

radical

Charge $=0$ Multiplicity $=2$ for low level calculation on real system.

Charge $=0$ Multiplicity $=2$ for high level calculation on model system.

Charge $=0$ Multiplicity $=2$ for low level calculation on model system.

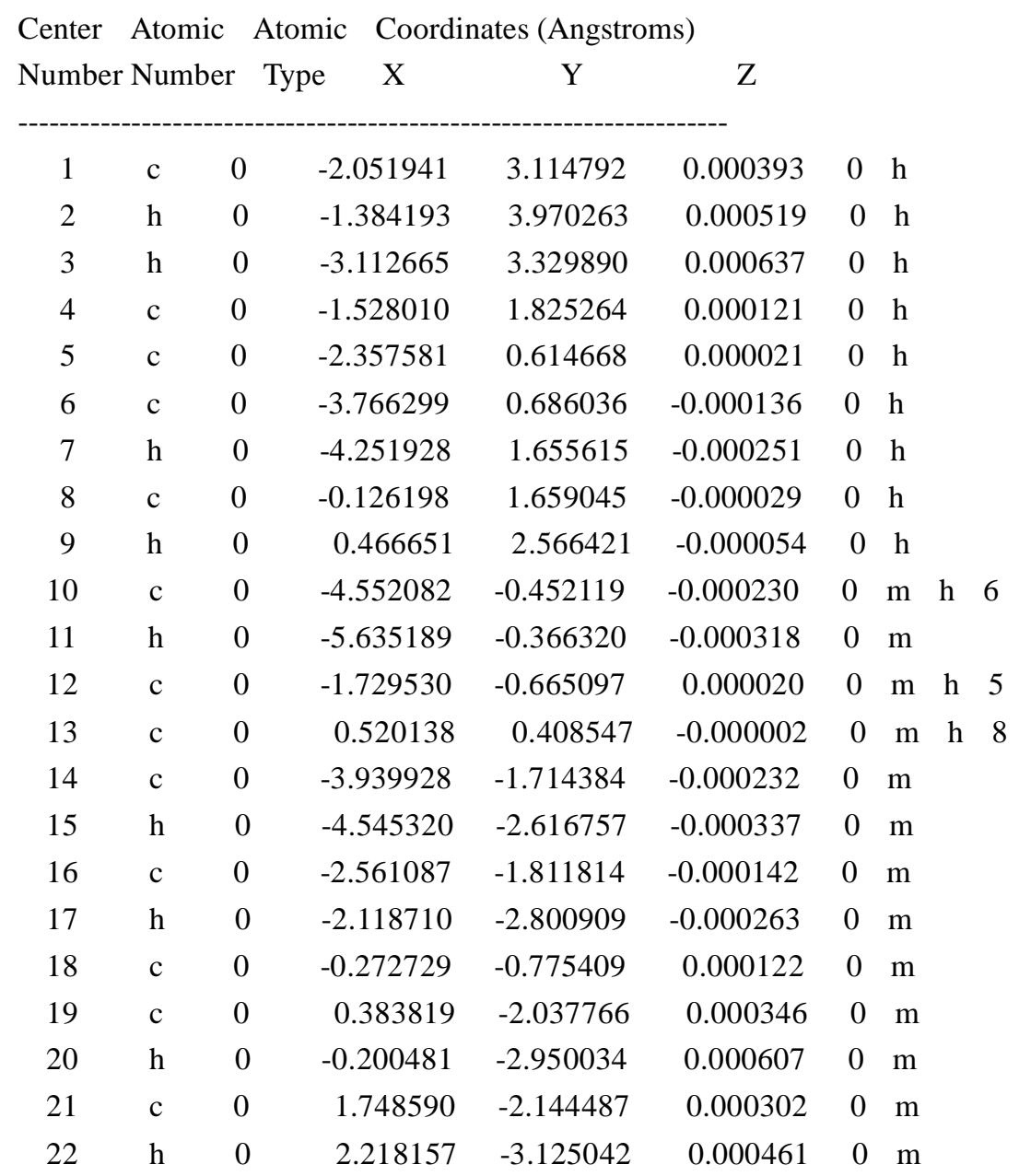




$\begin{array}{lllrrrrr}23 & \mathrm{c} & 0 & 2.582679 & -0.987792 & 0.000072 & 0 & \mathrm{~m} \\ 24 & \mathrm{c} & 0 & 3.993678 & -1.106010 & 0.000007 & 0 & \mathrm{~m} \\ 25 & \mathrm{~h} & 0 & 4.431026 & -2.101759 & 0.000104 & 0 & \mathrm{~m} \\ 26 & \mathrm{c} & 0 & 4.800315 & 0.012697 & -0.000175 & 0 & \mathrm{~m} \\ 27 & \mathrm{~h} & 0 & 5.882045 & -0.089257 & -0.000240 & 0 & \mathrm{~m} \\ 28 & \mathrm{c} & 0 & 4.210391 & 1.291767 & -0.000255 & 0 & \mathrm{~m} \\ 29 & \mathrm{~h} & 0 & 4.839393 & 2.177866 & -0.000365 & 0 & \mathrm{~m} \\ 30 & \mathrm{c} & 0 & 2.834493 & 1.430609 & -0.000195 & 0 & \mathrm{~m} \\ 31 & \mathrm{~h} & 0 & 2.419038 & 2.432084 & -0.000243 & 0 & \mathrm{~m} \\ 32 & \mathrm{c} & 0 & 1.976643 & 0.304444 & -0.000052 & 0 & \mathrm{~m}\end{array}$

No. 10<smiles>c1ccc(COCc2ccccc2)cc1</smiles>

neutral molecule

Charge $=0$ Multiplicity $=1$ for low level calculation on real system.

Charge $=0$ Multiplicity $=1$ for high level calculation on model system.

Charge $=0$ Multiplicity $=1$ for low level calculation on model system .

Center Atomic Atomic Coordinates (Angstroms)

\begin{tabular}{rrrrrrl} 
Number Number & Type & X & Y & Z & \\
\hline- & c & 0 & 1.179638 & 1.374684 & 0.035096 & $0 \mathrm{~h}$ \\
2 & $\mathrm{~h}$ & 0 & 1.188718 & 2.067524 & -0.826264 & $0 \mathrm{~h}$ \\
3 & $\mathrm{~h}$ & 0 & 1.200297 & 2.001620 & 0.942855 & $0 \mathrm{~h}$ \\
4 & $\mathrm{c}$ & 0 & 2.396702 & 0.479103 & -0.008136 & $0 \mathrm{~h}$ \\
5 & $\mathrm{c}$ & 0 & 3.594785 & 0.898554 & 0.580844 & $0 \mathrm{~h}$ \\
6 & $\mathrm{~h}$ & 0 & 3.631373 & 1.850147 & 1.107641 & $0 \mathrm{~h}$ \\
7 & $\mathrm{o}$ & 0 & -0.000013 & 0.592491 & 0.000067 & $0 \mathrm{~h}$ \\
8 & $\mathrm{c}$ & 0 & -1.179631 & 1.374744 & -0.034878 & $0 \mathrm{~h}$ \\
9 & $\mathrm{~h}$ & 0 & -1.200257 & 2.001783 & -0.942567 & $0 \mathrm{~h}$ \\
10 & $\mathrm{~h}$ & 0 & -1.188685 & 2.067474 & 0.826571 & $0 \mathrm{~h}$ \\
11 & $\mathrm{c}$ & 0 & -2.396719 & 0.479194 & 0.008234 & $0 \mathrm{~h}$ \\
12 & $\mathrm{c}$ & 0 & -2.359913 & -0.753275 & 0.670426 & $0 \mathrm{~h}$ \\
13 & $\mathrm{~h}$ & 0 & -1.427982 & -1.085392 & 1.115924 & $0 \mathrm{~h}$ \\
14 & $\mathrm{c}$ & 0 & 2.359759 & -0.753498 & -0.670054 & $0 \mathrm{~m} \mathrm{~h}$ \\
15 & $\mathrm{~h}$ & 0 & 1.427719 & -1.085743 & -1.115224 & $0 \mathrm{~m}$ \\
16 & $\mathrm{c}$ & 0 & 4.741208 & 0.106864 & 0.502885 & $0 \mathrm{~m} \mathrm{~h}$
\end{tabular}




$\begin{array}{lllrrrl}17 & \mathrm{~h} & 0 & 5.664106 & 0.445662 & 0.966452 & 0 \mathrm{~m} \\ 18 & \mathrm{c} & 0 & -3.594646 & 0.898475 & -0.581164 & 0 \mathrm{~m} \mathrm{~h} \\ 19 & \mathrm{~h} & 0 & -3.631145 & 1.849961 & -1.108158 & 0 \mathrm{~m} \\ 20 & \mathrm{c} & 0 & -3.503330 & -1.549970 & 0.740353 & 0 \mathrm{~m} \mathrm{~h} \\ 21 & \mathrm{~h} & 0 & -3.460697 & -2.508108 & 1.252071 & 0 \mathrm{~m} \\ 22 & \mathrm{c} & 0 & 3.503193 & -1.550169 & -0.740131 & 0 \mathrm{~m} \\ 23 & \mathrm{~h} & 0 & 3.460449 & -2.508424 & -1.251622 & 0 \mathrm{~m} \\ 24 & \mathrm{c} & 0 & 4.697887 & -1.121958 & -0.157968 & 0 \mathrm{~m} \\ 25 & \mathrm{~h} & 0 & 5.587590 & -1.743585 & -0.214536 & 0 \mathrm{~m} \\ 26 & \mathrm{c} & 0 & -4.741061 & 0.106746 & -0.503377 & 0 \mathrm{~m} \\ 27 & \mathrm{~h} & 0 & -5.663843 & 0.445414 & -0.967271 & 0 \mathrm{~m} \\ 28 & \mathrm{c} & 0 & -4.697869 & -1.121937 & 0.157765 & 0 \mathrm{~m} \\ 29 & \mathrm{~h} & 0 & -5.587556 & -1.743596 & 0.214217 & 0 \mathrm{~m}\end{array}$

radical

Charge $=0$ Multiplicity $=2$ for low level calculation on real system.

Charge $=0$ Multiplicity $=2$ for high level calculation on model system.

Charge $=0$ Multiplicity $=2$ for low level calculation on model system.

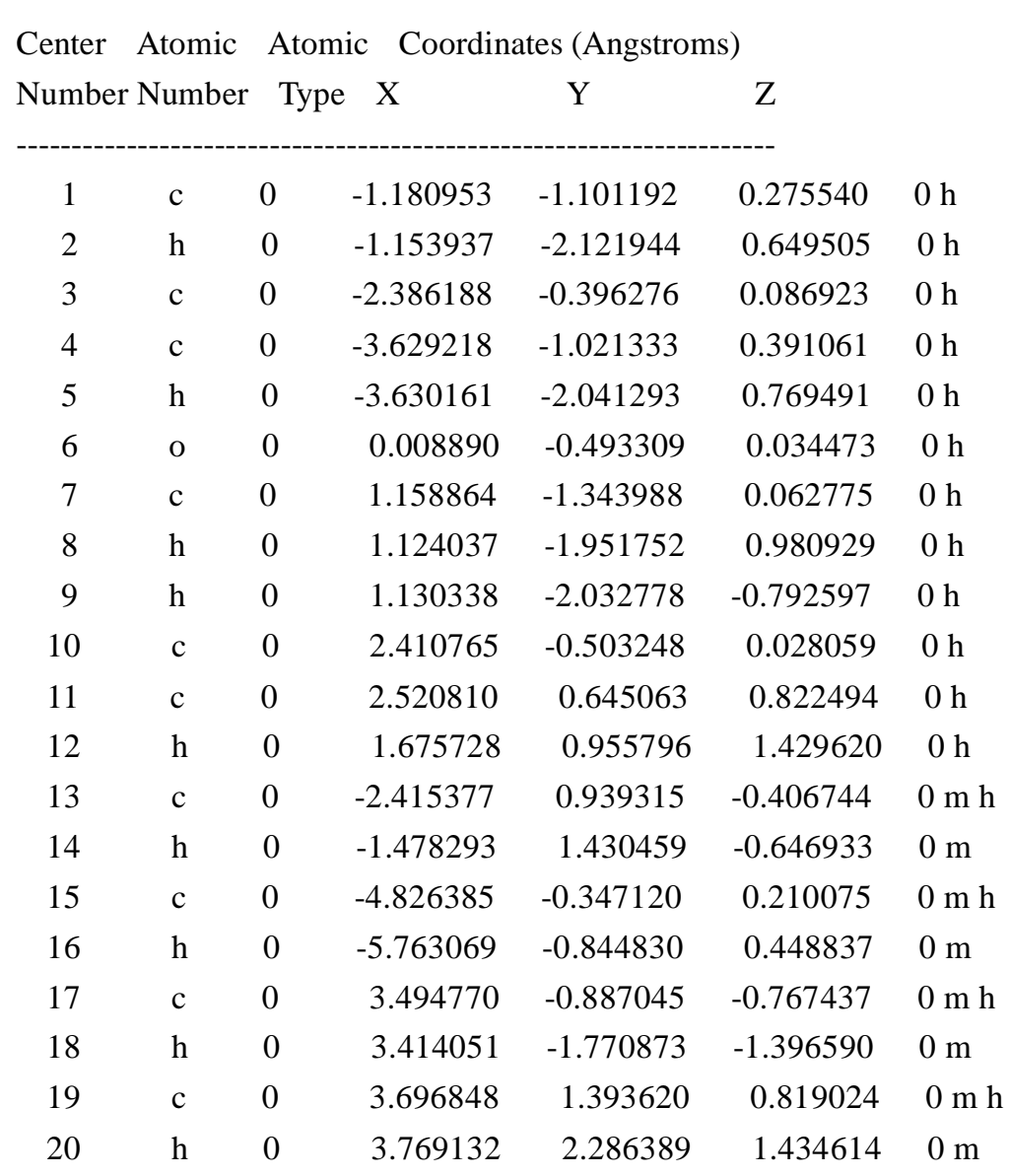




$\begin{array}{lllllll}21 & \mathrm{c} & 0 & -3.623354 & 1.598593 & -0.580762 & 0 \mathrm{~m} \\ 22 & \mathrm{~h} & 0 & -3.624602 & 2.617960 & -0.959391 & 0 \mathrm{~m} \\ 23 & \mathrm{c} & 0 & -4.838046 & 0.968373 & -0.276586 & 0 \mathrm{~m} \\ 24 & \mathrm{~h} & 0 & -5.778947 & 1.492814 & -0.416353 & 0 \mathrm{~m} \\ 25 & \mathrm{c} & 0 & 4.676998 & -0.144117 & -0.763599 & 0 \mathrm{~m} \\ 26 & \mathrm{~h} & 0 & 5.511328 & -0.453592 & -1.387376 & 0 \mathrm{~m} \\ 27 & \mathrm{c} & 0 & 4.779912 & 0.998944 & 0.029358 & 0 \mathrm{~m} \\ 28 & \mathrm{~h} & 0 & 5.696605 & 1.582592 & 0.029382 & 0 \mathrm{~m}\end{array}$

No. 11<smiles>O=C(Cc1ccccc1)c1ccccc1</smiles>

neutral molecule

Charge $=0$ Multiplicity $=1$ for low level calculation on real system.

Charge $=0$ Multiplicity $=1$ for high level calculation on model system.

Charge $=0$ Multiplicity $=1$ for low level calculation on model system.

\begin{tabular}{|c|c|c|c|c|c|c|}
\hline \multicolumn{7}{|c|}{ Center Atomic Atomic Coordinates (Angstroms) } \\
\hline Number & Numbe & er Type & $\mathrm{e}$ & $\mathrm{Y}$ & $\mathrm{Z}$ & \\
\hline 1 & c & 0 & 0.736619 & 0.469123 & 1.077149 & $0 \mathrm{~h}$ \\
\hline 2 & $\mathrm{~h}$ & 0 & 0.852339 & 1.122950 & 1.947534 & $0 \mathrm{~h}$ \\
\hline 3 & $\mathrm{~h}$ & 0 & 0.133107 & -0.397324 & 1.370067 & $0 \mathrm{~h}$ \\
\hline 4 & $\mathrm{c}$ & 0 & 2.093675 & 0.008422 & 0.568506 & $0 \mathrm{~h}$ \\
\hline 5 & $\mathrm{c}$ & 0 & 2.382604 & -1.351111 & 0.400536 & $0 \mathrm{~h}$ \\
\hline 6 & $\mathrm{~h}$ & 0 & 1.629905 & -2.094383 & 0.654383 & $0 \mathrm{~h}$ \\
\hline 7 & $\mathrm{c}$ & 0 & -0.000054 & 1.269235 & 0.000586 & $0 \mathrm{~h}$ \\
\hline 8 & o & 0 & -0.000263 & 2.485387 & 0.001091 & $0 \mathrm{~h}$ \\
\hline 9 & $\mathrm{c}$ & 0 & -0.736467 & 0.469729 & -1.076579 & $0 \mathrm{~h}$ \\
\hline 10 & $\mathrm{~h}$ & 0 & -0.132864 & -0.396495 & -1.369973 & $0 \mathrm{~h}$ \\
\hline 11 & $\mathrm{~h}$ & 0 & -0.852141 & 1.124094 & -1.946561 & $0 \mathrm{~h}$ \\
\hline 12 & $\mathrm{c}$ & 0 & -2.093601 & 0.008613 & -0.568478 & $0 \mathrm{~h}$ \\
\hline 13 & $\mathrm{c}$ & 0 & -3.078589 & 0.952616 & -0.242723 & $0 \mathrm{~h}$ \\
\hline 14 & $\mathrm{~h}$ & 0 & -2.859820 & 2.010963 & -0.360587 & $0 \mathrm{~h}$ \\
\hline 15 & $\mathrm{c}$ & 0 & 3.078096 & 0.952694 & 0.241866 & $0 \mathrm{~m} \mathrm{~h}$ \\
\hline 16 & $\mathrm{~h}$ & 0 & 2.858991 & 2.011019 & 0.359320 & $0 \mathrm{~m}$ \\
\hline 17 & $\mathrm{c}$ & 0 & 3.629453 & -1.762008 & -0.075908 & $0 \mathrm{~m} \mathrm{~h}$ \\
\hline 18 & $\mathrm{~h}$ & 0 & 3.838629 & -2.821939 & -0.194970 & $0 \mathrm{~m}$ \\
\hline
\end{tabular}




$\begin{array}{lllrrrl}19 & \mathrm{c} & 0 & -2.382004 & -1.350967 & -0.400048 & 0 \mathrm{~m} \mathrm{~h} \\ 20 & \mathrm{~h} & 0 & -1.628873 & -2.094031 & -0.653222 & 0 \mathrm{~m} \\ 21 & \mathrm{c} & 0 & -4.323800 & 0.543693 & 0.232629 & 0 \mathrm{~m} \mathrm{~h} \\ 22 & \mathrm{~h} & 0 & -5.077379 & 1.287440 & 0.477994 & 0 \mathrm{~m} \\ 23 & \mathrm{c} & 0 & 4.323256 & 0.544093 & -0.233914 & 0 \mathrm{~m} \\ 24 & \mathrm{~h} & 0 & 5.076394 & 1.288054 & -0.479983 & 0 \mathrm{~m} \\ 25 & \mathrm{c} & 0 & 4.603202 & -0.815256 & -0.394681 & 0 \mathrm{~m} \\ 26 & \mathrm{~h} & 0 & 5.574366 & -1.133191 & -0.764275 & 0 \mathrm{~m} \\ 27 & \mathrm{c} & 0 & -3.628909 & -1.762190 & 0.076013 & 0 \mathrm{~m} \\ 28 & \mathrm{~h} & 0 & -3.837705 & -2.822152 & 0.195454 & 0 \mathrm{~m} \\ 29 & \mathrm{c} & 0 & -4.603218 & -0.815717 & 0.393874 & 0 \mathrm{~m} \\ 30 & \mathrm{~h} & 0 & -5.574425 & -1.133921 & 0.763127 & 0 \mathrm{~m}\end{array}$

radical

Charge $=0$ Multiplicity $=2$ for low level calculation on real system.

Charge $=0$ Multiplicity $=2$ for high level calculation on model system .

Charge $=0$ Multiplicity $=2$ for low level calculation on model system.

\begin{tabular}{ccccccl} 
Center & Atomic & Atomic & \multicolumn{5}{l}{ Coordinates (Angstroms) } \\
Number Number & Type & X & Y & Z & \\
-1 & c & 0 & 0.783569 & 0.044013 & -0.798160 & $0 \mathrm{~h}$ \\
2 & $\mathrm{~h}$ & 0 & 0.346755 & -0.793265 & -1.338721 & $0 \mathrm{~h}$ \\
3 & $\mathrm{c}$ & 0 & 2.099286 & -0.152523 & -0.286659 & $0 \mathrm{~h}$ \\
4 & $\mathrm{c}$ & 0 & 2.738122 & -1.402594 & -0.521746 & $0 \mathrm{~h}$ \\
5 & $\mathrm{~h}$ & 0 & 2.203136 & -2.167279 & -1.079821 & $0 \mathrm{~h}$ \\
6 & $\mathrm{c}$ & 0 & -0.052759 & 1.226795 & -0.680446 & $0 \mathrm{~h}$ \\
7 & $\mathrm{o}$ & 0 & 0.273948 & 2.248858 & -0.066451 & $0 \mathrm{~h}$ \\
8 & $\mathrm{c}$ & 0 & -1.437290 & 1.149438 & -1.342151 & $0 \mathrm{~h}$ \\
9 & $\mathrm{~h}$ & 0 & -1.356834 & 0.699908 & -2.338985 & $0 \mathrm{~h}$ \\
10 & $\mathrm{~h}$ & 0 & -1.784682 & 2.180707 & -1.461591 & $0 \mathrm{~h}$ \\
11 & $\mathrm{c}$ & 0 & -2.413808 & 0.349576 & -0.496790 & $0 \mathrm{~h}$ \\
12 & $\mathrm{c}$ & 0 & -2.741444 & 0.782539 & 0.796984 & $0 \mathrm{~h}$ \\
13 & $\mathrm{~h}$ & 0 & -2.288081 & 1.692432 & 1.182247 & $0 \mathrm{~h}$ \\
14 & $\mathrm{c}$ & 0 & 2.818209 & 0.833333 & 0.447071 & $0 \mathrm{~m} \mathrm{~h}$ \\
15 & $\mathrm{~h}$ & 0 & 2.340817 & 1.786712 & 0.633005 & $0 \mathrm{~m}$ \\
16 & $\mathrm{c}$ & 0 & 4.017607 & -1.655897 & -0.052623 & $0 \mathrm{~m} \mathrm{~h}$ \\
17 & $\mathrm{~h}$ & 0 & 4.484588 & -2.618240 & -0.243709 & $0 \mathrm{~m}$ \\
18 & $\mathrm{c}$ & 0 & -2.994027 & -0.830448 & -0.977027 & $0 \mathrm{~m} \mathrm{~h}$ \\
19 & $\mathrm{~h}$ & 0 & -2.753249 & -1.176197 & -1.980190 & $0 \mathrm{~m}$ \\
20 & $\mathrm{c}$ & 0 & -3.631885 & 0.055392 & 1.585050 & $0 \mathrm{~m} \mathrm{~h}$ \\
21 & $\mathrm{~h}$ & 0 & -3.876887 & 0.405984 & 2.584294 & $0 \mathrm{~m}$
\end{tabular}




$\begin{array}{lllrrrc}22 & \mathrm{c} & 0 & 4.098771 & 0.567360 & 0.910588 & 0 \mathrm{~m} \\ 23 & \mathrm{~h} & 0 & 4.634042 & 1.330341 & 1.469489 & 0 \mathrm{~m} \\ 24 & \mathrm{c} & 0 & 4.706958 & -0.671092 & 0.666982 & 0 \mathrm{~m} \\ 25 & \mathrm{~h} & 0 & 5.710185 & -0.868069 & 1.034999 & 0 \mathrm{~m} \\ 26 & \mathrm{c} & 0 & -3.886334 & -1.560628 & -0.188863 & 0 \mathrm{~m} \\ 27 & \mathrm{~h} & 0 & -4.330541 & -2.472161 & -0.580492 & 0 \mathrm{~m} \\ 28 & \mathrm{c} & 0 & -4.207946 & -1.119638 & 1.094755 & 0 \mathrm{~m} \\ 29 & \mathrm{~h} & 0 & -4.903007 & -1.685493 & 1.709298 & 0 \mathrm{~m}\end{array}$

\section{No. 12}<smiles>CN1c2ccccc2C=Cc2ccccc21</smiles>

neutral molecule

Charge $=0$ Multiplicity $=1$ for low level calculation on real system.

Charge $=0$ Multiplicity $=1$ for high level calculation on model system .

Charge $=0$ Multiplicity $=1$ for low level calculation on model system .

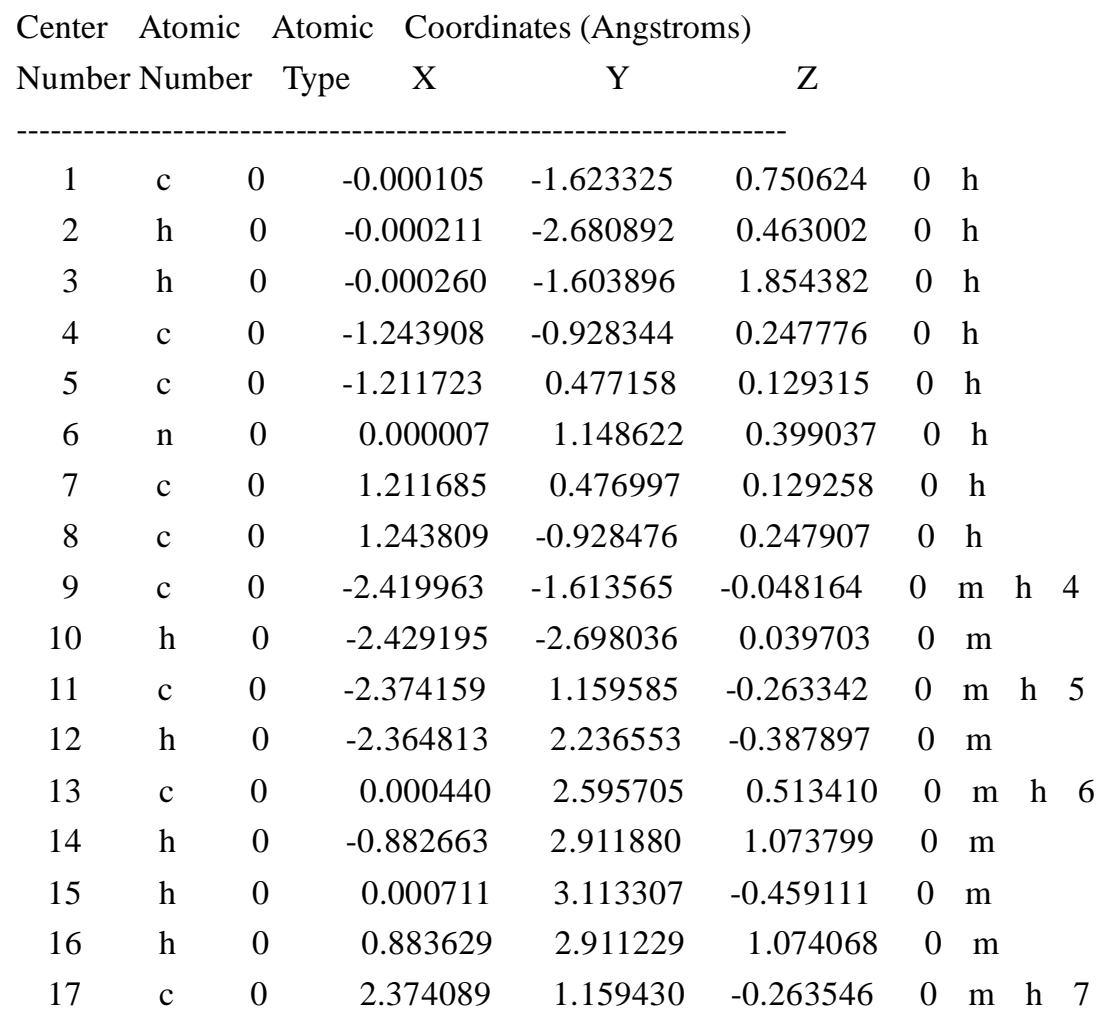




\begin{tabular}{|c|c|c|c|c|c|c|c|}
\hline 18 & $\mathrm{~h}$ & 0 & 2.364618 & 2.236375 & -0.388368 & 0 & $\mathrm{~m}$ \\
\hline 19 & $\mathrm{c}$ & 0 & 2.419883 & -1.613687 & -0.047949 & 0 & $\mathrm{~m} \mathrm{~h}$ \\
\hline 20 & $\mathrm{~h}$ & 0 & 2.429166 & -2.698150 & 0.040020 & 0 & $\mathrm{~m}$ \\
\hline 21 & c & 0 & -3.579467 & -0.935078 & -0.430755 & 0 & $\mathrm{~m}$ \\
\hline 22 & h & 0 & -4.490724 & -1.485220 & -0.646650 & 0 & $\mathrm{~m}$ \\
\hline 23 & $\mathrm{c}$ & 0 & -3.548828 & 0.454372 & -0.529050 & 0 & $\mathrm{~m}$ \\
\hline 24 & $\mathrm{~h}$ & 0 & -4.437822 & 1.001703 & -0.831506 & 0 & $\mathrm{~m}$ \\
\hline 25 & $\mathrm{c}$ & 0 & 3.548787 & 0.454205 & -0.529069 & 0 & $\mathrm{~m}$ \\
\hline 26 & $\mathrm{~h}$ & 0 & 4.437749 & 1.001537 & -0.831626 & 0 & $\mathrm{~m}$ \\
\hline 27 & c & 0 & 3.579322 & -0.935209 & -0.430820 & 0 & $\mathrm{~m}$ \\
\hline 28 & $\mathrm{~h}$ & 0 & 4.490587 & -1.485360 & -0.646658 & 0 & $\mathrm{~m}$ \\
\hline
\end{tabular}

radical

Charge $=0$ Multiplicity $=2$ for low level calculation on real system .

Charge $=0$ Multiplicity $=2$ for high level calculation on model system .

Charge $=0$ Multiplicity $=2$ for low level calculation on model system .

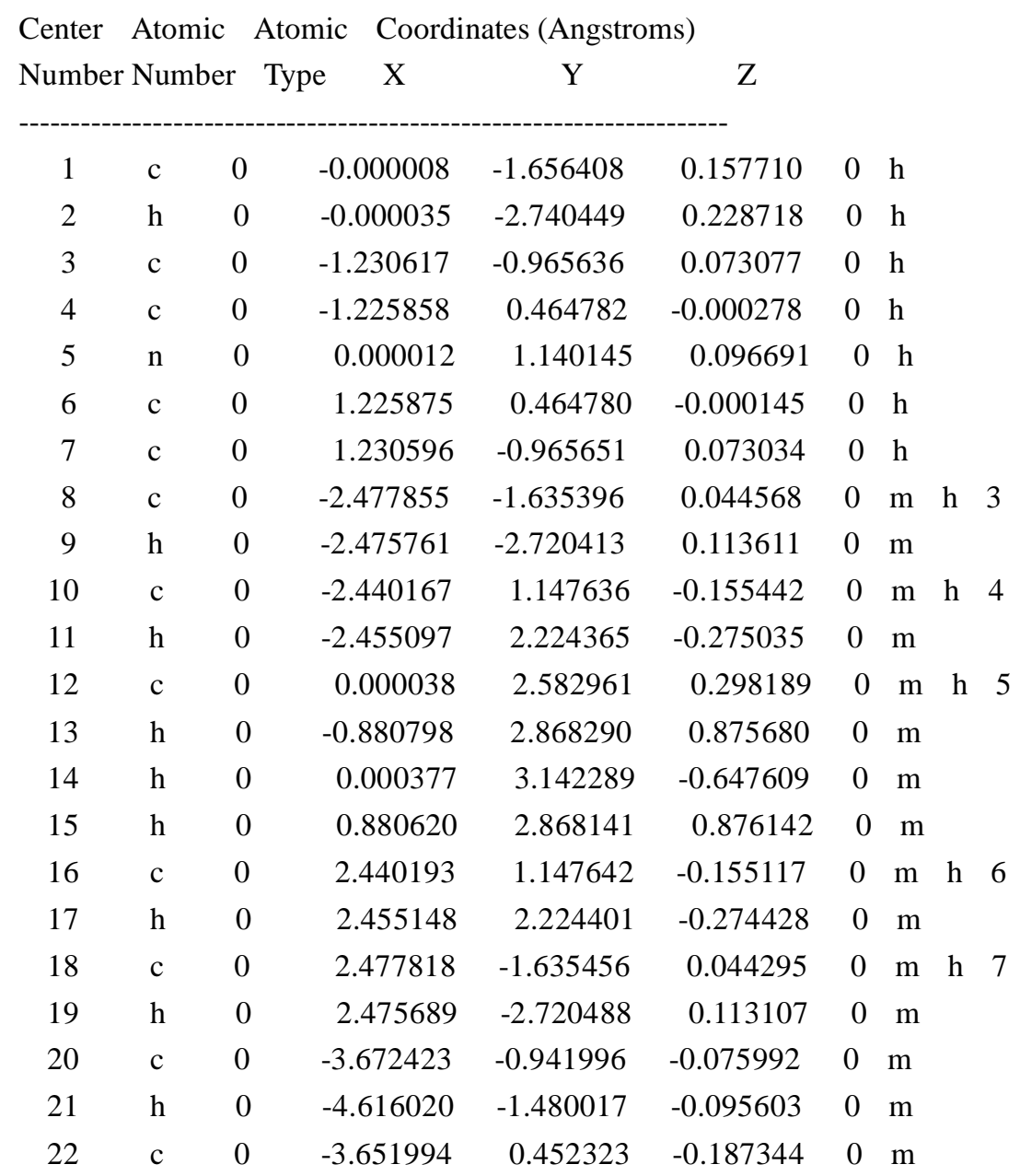




$\begin{array}{lllrrrrc}23 & \mathrm{~h} & 0 & -4.578209 & 1.006934 & -0.307361 & 0 & \mathrm{~m} \\ 24 & \mathrm{c} & 0 & 3.651985 & 0.452321 & -0.187187 & 0 & \mathrm{~m} \\ 25 & \mathrm{~h} & 0 & 4.578225 & 1.006929 & -0.307032 & 0 & \mathrm{~m} \\ 26 & \mathrm{c} & 0 & 3.672383 & -0.942059 & -0.076201 & 0 & \mathrm{~m} \\ 27 & \mathrm{~h} & 0 & 4.615987 & -1.480065 & -0.096031 & 0 & \mathrm{~m}\end{array}$

\section{No. 13}

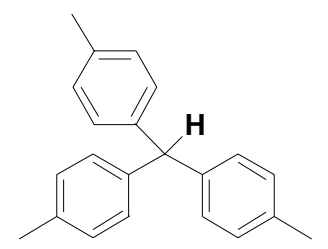

neutral molecule

Charge $=0$ Multiplicity $=1$ for low level calculation on real system.

Charge $=0$ Multiplicity $=1$ for high level calculation on model system .

Charge $=0$ Multiplicity $=1$ for low level calculation on model system.

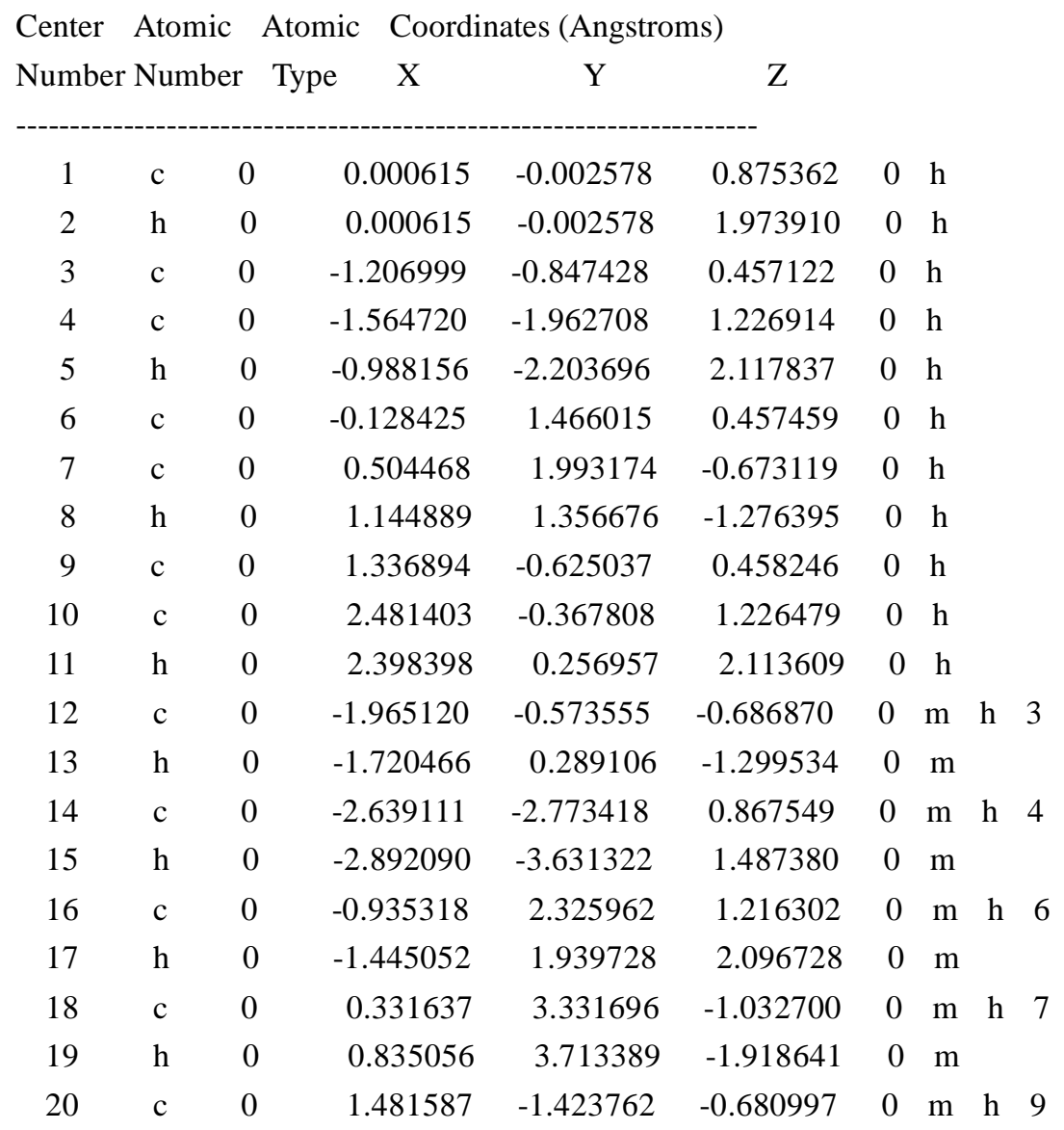




\begin{tabular}{|c|c|c|c|c|c|c|c|}
\hline 21 & $\mathrm{~h}$ & 0 & 0.613657 & -1.650726 & -1.292999 & 0 & $\mathrm{~m}$ \\
\hline 22 & $\mathrm{c}$ & 0 & 3.722525 & -0.888178 & 0.869161 & 0 & $\mathrm{~m} \quad \mathrm{~h} \quad 10$ \\
\hline 23 & $\mathrm{~h}$ & 0 & 4.591768 & -0.670739 & 1.486711 & 0 & $\mathrm{~m}$ \\
\hline 24 & $\mathrm{c}$ & 0 & -3.038774 & -1.389727 & -1.047612 & 0 & $\mathrm{~m}$ \\
\hline 25 & $\mathrm{~h}$ & 0 & -3.608461 & -1.153433 & -1.944117 & 0 & $\mathrm{~m}$ \\
\hline 26 & $\mathrm{c}$ & 0 & -3.400036 & -2.500721 & -0.277169 & 0 & $\mathrm{~m}$ \\
\hline 27 & $\mathrm{c}$ & 0 & -4.588710 & -3.357173 & -0.647355 & 0 & $\mathrm{~m}$ \\
\hline 28 & $\mathrm{~h}$ & 0 & -5.510338 & -2.986688 & -0.178201 & 0 & $\mathrm{~m}$ \\
\hline 29 & $\mathrm{~h}$ & 0 & -4.756966 & -3.362763 & -1.729545 & 0 & $\mathrm{~m}$ \\
\hline 30 & $\mathrm{~h}$ & 0 & -4.454196 & -4.394021 & -0.319986 & 0 & $\mathrm{~m}$ \\
\hline 31 & $\mathrm{c}$ & 0 & -1.103364 & 3.660993 & 0.858146 & 0 & $\mathrm{~m}$ \\
\hline 32 & $\mathrm{~h}$ & 0 & -1.734962 & 4.303470 & 1.468454 & 0 & $\mathrm{~m}$ \\
\hline 33 & $\mathrm{c}$ & 0 & -0.469841 & 4.191473 & -0.274512 & 0 & $\mathrm{~m}$ \\
\hline 34 & $\mathrm{c}$ & 0 & -0.625568 & 5.648472 & -0.643904 & 0 & $\mathrm{~m}$ \\
\hline 35 & $\mathrm{~h}$ & 0 & -1.633911 & 6.014313 & -0.420089 & 0 & $\mathrm{~m}$ \\
\hline 36 & $\mathrm{~h}$ & 0 & -0.435062 & 5.814024 & -1.709598 & 0 & $\mathrm{~m}$ \\
\hline 37 & $\mathrm{~h}$ & 0 & 0.078342 & 6.280305 & -0.085431 & 0 & $\mathrm{~m}$ \\
\hline 38 & $\mathrm{c}$ & 0 & 2.728073 & -1.941911 & -1.039583 & 0 & $\mathrm{~m}$ \\
\hline 39 & $\mathrm{~h}$ & 0 & 2.810434 & -2.558158 & -1.932622 & 0 & $\mathrm{~m}$ \\
\hline 40 & $\mathrm{c}$ & 0 & 3.869834 & -1.689024 & -0.272117 & 0 & $\mathrm{~m}$ \\
\hline 41 & $\mathrm{c}$ & 0 & 5.210564 & -2.280176 & -0.641614 & 0 & $\mathrm{~m}$ \\
\hline 42 & $\mathrm{~h}$ & 0 & 6.031078 & -1.592446 & -0.408337 & 0 & $\mathrm{~m}$ \\
\hline 43 & $\mathrm{~h}$ & 0 & 5.403124 & -3.210894 & -0.090907 & 0 & $\mathrm{~m}$ \\
\hline 44 & $\mathrm{~h}$ & 0 & 5.262608 & -2.517985 & -1.709350 & 0 & $\mathrm{~m}$ \\
\hline
\end{tabular}

radical

Charge $=0$ Multiplicity $=2$ for low level calculation on real system.

Charge $=0$ Multiplicity $=2$ for high level calculation on model system .

Charge $=0$ Multiplicity $=2$ for low level calculation on model system .

Center Atomic Atomic Coordinates (Angstroms)

Number Number Type $\quad \mathrm{X} \quad \mathrm{Y} \quad$ Z

$\begin{array}{rrrrrrrr}1 & \mathrm{c} & 0 & -0.002383 & -0.001896 & -0.002214 & 0 & \mathrm{~h} \\ 2 & \mathrm{c} & 0 & 0.934059 & -1.126515 & 0.000302 & 0 & \mathrm{~h} \\ 3 & \mathrm{c} & 0 & 0.639521 & -2.342309 & -0.661658 & 0 & \mathrm{~h} \\ 4 & \mathrm{~h} & 0 & -0.297241 & -2.436374 & -1.201886 & 0 & \mathrm{~h} \\ 5 & \mathrm{c} & 0 & -1.444738 & -0.248630 & -0.002468 & 0 & \mathrm{~h} \\ 6 & \mathrm{c} & 0 & -2.347599 & 0.618231 & -0.662760 & 0 & \mathrm{~h} \\ 7 & \mathrm{~h} & 0 & -1.958223 & 1.476748 & -1.200826 & 0 & \mathrm{~h} \\ 8 & \mathrm{c} & 0 & 0.505305 & 1.371772 & -0.001089 & 0 & \mathrm{~h} \\ 9 & \mathrm{c} & 0 & 1.703350 & 1.721113 & -0.668318 & 0 & \mathrm{~h}\end{array}$




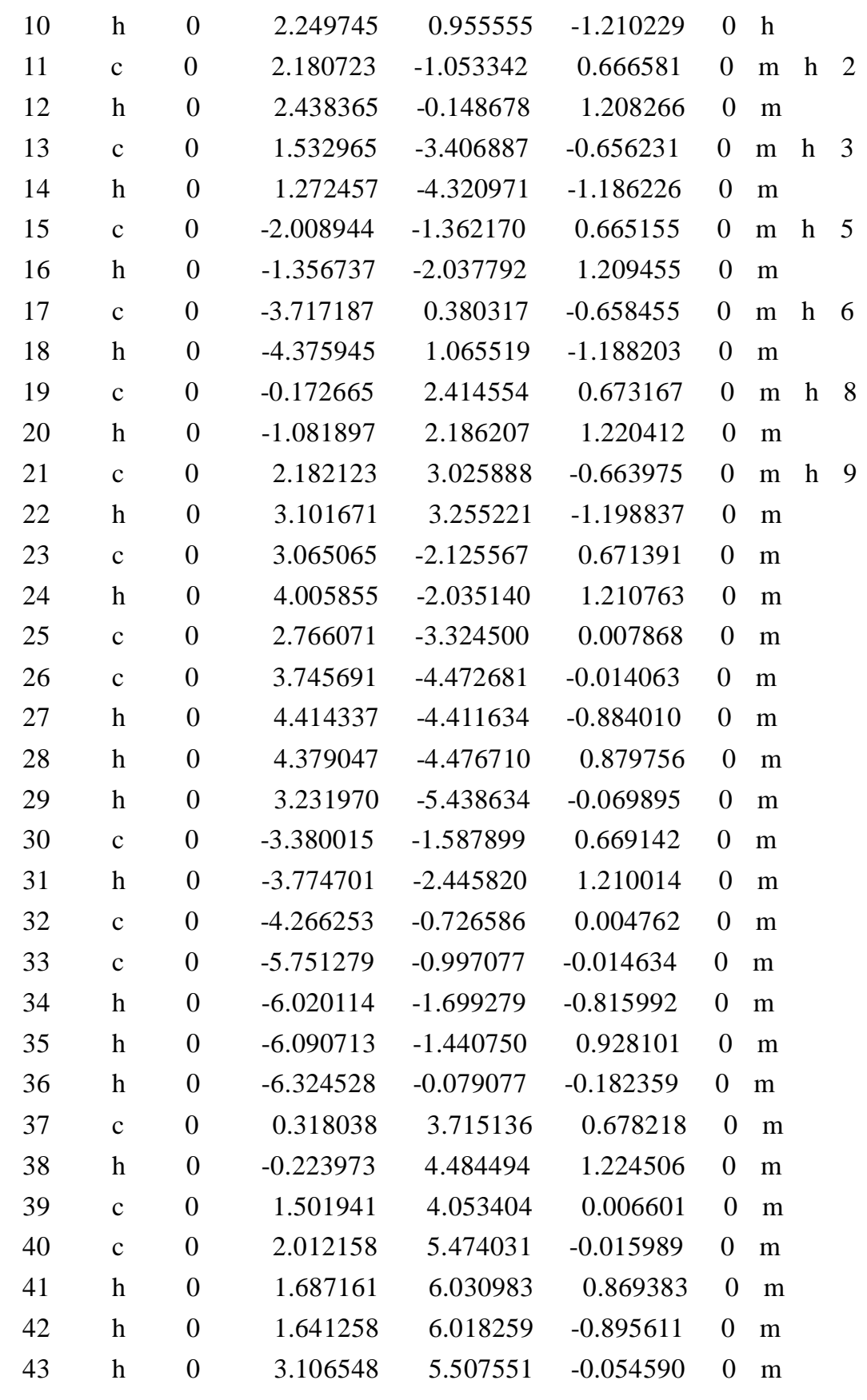

\section{No. 14}<smiles>CC(c1ccccc1)c1ccccc1</smiles>

neutral molecule 
Charge $=0$ Multiplicity $=1$ for low level calculation on real system .

Charge $=0$ Multiplicity $=1$ for high level calculation on model system .

Charge $=0$ Multiplicity $=1$ for low level calculation on model system .

\begin{tabular}{|c|c|c|c|c|c|c|}
\hline \multirow{2}{*}{$\begin{array}{l}\text { Center } \\
\text { Number }\end{array}$} & \multirow{2}{*}{$\begin{array}{l}\text { Atomic } \\
\text { Number }\end{array}$} & \multirow{2}{*}{$\begin{array}{l}\text { Atomic } \\
\text { r Type }\end{array}$} & \multicolumn{3}{|c|}{ Coordinates (Angstroms) } & \\
\hline & & & $\mathrm{X}$ & $\mathrm{Y}$ & $\mathrm{Z}$ & \\
\hline 1 & $\mathrm{c}$ & 0 & 0.021618 & 1.161651 & -0.556590 & $0 \mathrm{~h}$ \\
\hline 2 & $\mathrm{~h}$ & 0 & 0.066789 & 1.333078 & -1.641045 & $0 \mathrm{~h}$ \\
\hline 3 & $\mathrm{c}$ & 0 & 0.080629 & 2.547305 & 0.114856 & $0 \mathrm{~h}$ \\
\hline 4 & $\mathrm{~h}$ & 0 & 0.114884 & 2.465633 & 1.206802 & $0 \mathrm{~h}$ \\
\hline 5 & $\mathrm{~h}$ & 0 & -0.786824 & 3.158676 & -0.158702 & $0 \mathrm{~h}$ \\
\hline 6 & $\mathrm{~h}$ & 0 & 0.986431 & 3.075108 & -0.200302 & $0 \mathrm{~h}$ \\
\hline 7 & $\mathrm{c}$ & 0 & 1.262903 & 0.345255 & -0.192703 & $0 \mathrm{~h}$ \\
\hline 8 & $\mathrm{c}$ & 0 & 2.341248 & 0.264116 & -1.082304 & $0 \mathrm{~h}$ \\
\hline 9 & $\mathrm{~h}$ & 0 & 2.272251 & 0.755966 & -2.050709 & $0 \mathrm{~h}$ \\
\hline 10 & $\mathrm{c}$ & 0 & -1.273513 & 0.391957 & -0.293520 & $0 \mathrm{~h}$ \\
\hline 11 & $\mathrm{c}$ & 0 & -2.181943 & 0.742497 & 0.711884 & $0 \mathrm{~h}$ \\
\hline 12 & $\mathrm{~h}$ & 0 & -1.988287 & 1.603121 & 1.344478 & $0 \mathrm{~h}$ \\
\hline 13 & $\mathrm{c}$ & 0 & 3.501349 & -0.436617 & -0.745588 & $0 \mathrm{~m} \mathrm{~h} 8$ \\
\hline 14 & $\mathrm{~h}$ & 0 & 4.324798 & -0.488926 & -1.453396 & $0 \mathrm{~m}$ \\
\hline 15 & $\mathrm{c}$ & 0 & 1.373454 & -0.299279 & 1.047445 & $0 \mathrm{~m} \mathrm{~h} 7$ \\
\hline 16 & $\mathrm{~h}$ & 0 & 0.540585 & -0.263280 & 1.745137 & $0 \mathrm{~m}$ \\
\hline 17 & $\mathrm{c}$ & 0 & -1.571990 & -0.723249 & -1.092972 & $0 \mathrm{~m} \mathrm{~h} 10$ \\
\hline 18 & $\mathrm{~h}$ & 0 & -0.875423 & -1.014742 & -1.876198 & $0 \mathrm{~m}$ \\
\hline 19 & $\mathrm{c}$ & 0 & -3.351285 & 0.002459 & 0.914654 & $0 \mathrm{~m} \mathrm{~h} 11$ \\
\hline 20 & $\mathrm{~h}$ & 0 & -4.041662 & 0.295798 & 1.701541 & $0 \mathrm{~m}$ \\
\hline 21 & $\mathrm{c}$ & 0 & 3.600212 & -1.070336 & 0.493103 & $0 \mathrm{~m}$ \\
\hline 22 & $\mathrm{~h}$ & 0 & 4.500228 & -1.618963 & 0.757847 & $0 \mathrm{~m}$ \\
\hline 23 & $\mathrm{c}$ & 0 & 2.530502 & -0.998884 & 1.388616 & $0 \mathrm{~m}$ \\
\hline 24 & $\mathrm{~h}$ & 0 & 2.595167 & -1.493925 & 2.354327 & $0 \mathrm{~m}$ \\
\hline 25 & $\mathrm{c}$ & 0 & -2.736128 & -1.461755 & -0.896696 & $0 \mathrm{~m}$ \\
\hline 26 & $\mathrm{~h}$ & 0 & -2.945167 & -2.318825 & -1.531897 & $0 \mathrm{~m}$ \\
\hline 27 & $\mathrm{c}$ & 0 & -3.633725 & -1.100857 & 0.112274 & $0 \mathrm{~m}$ \\
\hline 28 & $\mathrm{~h}$ & 0 & -4.543759 & -1.674300 & 0.267360 & $0 \mathrm{~m}$ \\
\hline
\end{tabular}

radical

Charge $=0$ Multiplicity $=2$ for low level calculation on real system.

Charge $=0$ Multiplicity $=2$ for high level calculation on model system .

Charge $=0$ Multiplicity $=2$ for low level calculation on model system. 


\begin{tabular}{|c|c|c|c|c|c|c|}
\hline Center & Atomic & & Atomic Coordi & ates (Angstro & & \\
\hline Number & Number & & Type & Y & Z & \\
\hline 1 & $\mathrm{c}$ & 0 & -0.012782 & 1.020631 & 0.034350 & $0 \mathrm{~h}$ \\
\hline 2 & $\mathrm{c}$ & 0 & -0.040363 & 2.526469 & 0.133448 & $0 \mathrm{~h}$ \\
\hline 3 & $\mathrm{~h}$ & 0 & -0.419084 & 2.849996 & 1.115311 & $0 \mathrm{~h}$ \\
\hline 4 & $\mathrm{~h}$ & 0 & -0.696178 & 2.980615 & -0.620508 & $0 \mathrm{~h}$ \\
\hline 5 & $\mathrm{~h}$ & 0 & 0.955511 & 2.961161 & 0.030933 & $0 \mathrm{~h}$ \\
\hline 6 & $\mathrm{c}$ & 0 & 1.277275 & 0.335569 & 0.034184 & $0 \mathrm{~h}$ \\
\hline 7 & $\mathrm{c}$ & 0 & 2.415188 & 0.904459 & -0.588678 & $0 \mathrm{~h}$ \\
\hline 8 & $\mathrm{~h}$ & 0 & 2.318330 & 1.843819 & -1.124663 & $0 \mathrm{~h}$ \\
\hline 9 & $\mathrm{c}$ & 0 & -1.274450 & 0.311114 & -0.011184 & $0 \mathrm{~h}$ \\
\hline 10 & $\mathrm{c}$ & 0 & -2.484164 & 0.943228 & 0.384201 & $0 \mathrm{~h}$ \\
\hline 11 & $\mathrm{~h}$ & 0 & -2.457829 & 1.956845 & 0.769866 & $0 \mathrm{~h}$ \\
\hline 12 & $\mathrm{c}$ & 0 & 3.650379 & 0.264947 & -0.569951 & $0 \mathrm{~m} \mathrm{~h} 7$ \\
\hline 13 & $\mathrm{~h}$ & 0 & 4.497612 & 0.723534 & -1.073661 & $0 \mathrm{~m}$ \\
\hline 14 & $\mathrm{c}$ & 0 & 1.462064 & -0.905656 & 0.692825 & $0 \mathrm{~m} \mathrm{~h} 6$ \\
\hline 15 & $\mathrm{~h}$ & 0 & 0.625060 & -1.352351 & 1.219483 & $0 \mathrm{~m}$ \\
\hline 16 & $\mathrm{c}$ & 0 & -1.384852 & -1.014652 & -0.512473 & $0 \mathrm{~m} \mathrm{~h} 9$ \\
\hline 17 & $\mathrm{~h}$ & 0 & -0.496846 & -1.517088 & -0.879862 & $0 \mathrm{~m}$ \\
\hline 18 & $\mathrm{c}$ & 0 & -3.706331 & 0.284883 & 0.314530 & $0 \mathrm{~m} \mathrm{~h} 1$ \\
\hline 19 & $\mathrm{~h}$ & 0 & -4.608235 & 0.797581 & 0.639696 & $0 \mathrm{~m}$ \\
\hline 20 & $\mathrm{c}$ & 0 & 3.805153 & -0.960241 & 0.084162 & $0 \mathrm{~m}$ \\
\hline 21 & $\mathrm{~h}$ & 0 & 4.772017 & -1.455460 & 0.103142 & $0 \mathrm{~m}$ \\
\hline 22 & $\mathrm{c}$ & 0 & 2.701463 & -1.536851 & 0.718731 & $0 \mathrm{~m}$ \\
\hline 23 & $\mathrm{~h}$ & 0 & 2.809490 & -2.480134 & 1.248262 & $0 \mathrm{~m}$ \\
\hline 24 & $\mathrm{c}$ & 0 & -2.610359 & -1.664458 & -0.584864 & $0 \mathrm{~m}$ \\
\hline 25 & $\mathrm{~h}$ & 0 & -2.655257 & -2.674530 & -0.984469 & $0 \mathrm{~m}$ \\
\hline 26 & $\mathrm{c}$ & 0 & -3.782435 & -1.025605 & -0.166124 & $0 \mathrm{~m}$ \\
\hline 27 & $\mathrm{~h}$ & 0 & -4.739308 & -1.537015 & -0.222479 & $0 \mathrm{~m}$ \\
\hline
\end{tabular}

No. 15<smiles>N#CC(Oc1ccccc1)c1ccccc1</smiles>

neutral molecule

Charge $=0$ Multiplicity $=1$ for low level calculation on real system.

Charge $=0$ Multiplicity $=1$ for high level calculation on model system .

Charge $=0$ Multiplicity $=1$ for low level calculation on model system . 


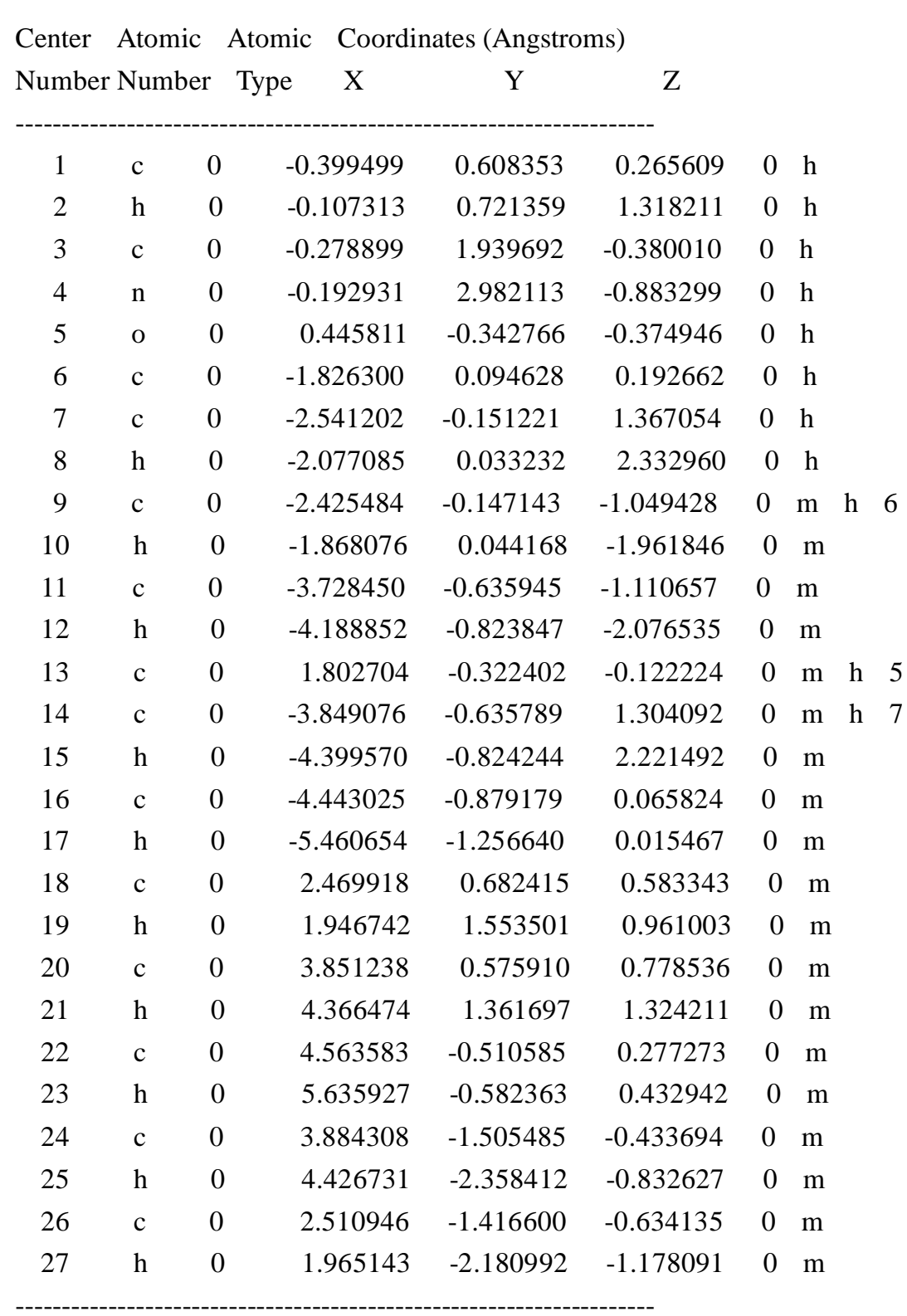

radical

Charge $=0$ Multiplicity $=2$ for low level calculation on real system.

Charge $=0$ Multiplicity $=2$ for high level calculation on model system.

Charge $=0$ Multiplicity $=2$ for low level calculation on model system.

Center Atomic Atomic Coordinates (Angstroms)

Number Number Type $\quad \mathrm{X} \quad \mathrm{Y} \quad \mathrm{Z}$

$\begin{array}{llllllll}1 & \text { c } & 0 & 0.466564 & 0.405504 & -0.286472 & 0 & \mathrm{~h} \\ 2 & \mathrm{c} & 0 & 0.062935 & 1.742897 & -0.466338 & 0 & \mathrm{~h}\end{array}$




\begin{tabular}{|c|c|c|c|c|c|c|c|c|}
\hline 3 & $\mathrm{n}$ & 0 & -0.230574 & 2.869220 & -0.596164 & 0 & $\mathrm{~h}$ & \\
\hline 4 & o & 0 & -0.441334 & -0.607133 & -0.414988 & 0 & $\mathrm{~h}$ & \\
\hline 5 & $\mathrm{c}$ & 0 & 1.838880 & 0.029582 & -0.086417 & 0 & $\mathrm{~h}$ & \\
\hline 6 & $\mathrm{c}$ & 0 & 2.192962 & -1.328380 & 0.100646 & 0 & $\mathrm{~h}$ & \\
\hline 7 & $\mathrm{~h}$ & 0 & 1.415948 & -2.084138 & 0.091014 & 0 & $\mathrm{~h}$ & \\
\hline 8 & $\mathrm{c}$ & 0 & 2.863808 & 1.007232 & -0.070347 & 0 & $\mathrm{~m}$ & h 5 \\
\hline 9 & $\mathrm{~h}$ & 0 & 2.607866 & 2.052592 & -0.215296 & 0 & $\mathrm{~m}$ & \\
\hline 10 & $\mathrm{c}$ & 0 & 4.186133 & 0.634849 & 0.127843 & 0 & $\mathrm{~m}$ & \\
\hline 11 & $\mathrm{~h}$ & 0 & 4.960198 & 1.397127 & 0.138167 & 0 & $\mathrm{~m}$ & \\
\hline 12 & $\mathrm{c}$ & 0 & -1.787740 & -0.418540 & -0.116085 & 0 & & h 4 \\
\hline 13 & $\mathrm{c}$ & 0 & 3.521160 & -1.685464 & 0.295032 & 0 & $\mathrm{~m}$ & h 6 \\
\hline 14 & $\mathrm{~h}$ & 0 & 3.777638 & -2.731757 & 0.437409 & 0 & $\mathrm{~m}$ & \\
\hline 15 & $\mathrm{c}$ & 0 & 4.525329 & -0.711096 & 0.310814 & 0 & $\mathrm{~m}$ & \\
\hline 16 & $\mathrm{~h}$ & 0 & 5.561902 & -0.997092 & 0.464455 & 0 & $\mathrm{~m}$ & \\
\hline 17 & $\mathrm{c}$ & 0 & -2.211184 & 0.313004 & 0.993811 & 0 & $\mathrm{~m}$ & \\
\hline 18 & $\mathrm{~h}$ & 0 & -1.488319 & 0.795772 & 1.642638 & 0 & $\mathrm{~m}$ & \\
\hline 19 & $\mathrm{c}$ & 0 & -3.578699 & 0.402287 & 1.257669 & 0 & $\mathrm{~m}$ & \\
\hline 20 & $\mathrm{~h}$ & 0 & -3.916441 & 0.972504 & 2.118358 & 0 & $\mathrm{~m}$ & \\
\hline 21 & $\mathrm{c}$ & 0 & -4.505139 & -0.238477 & 0.433358 & 0 & $\mathrm{~m}$ & \\
\hline 22 & $\mathrm{~h}$ & 0 & -5.567321 & -0.164653 & 0.647256 & 0 & $\mathrm{~m}$ & \\
\hline 23 & $\mathrm{c}$ & 0 & -4.060424 & -0.976786 & -0.665956 & 0 & $\mathrm{~m}$ & \\
\hline 24 & $\mathrm{~h}$ & 0 & -4.774807 & -1.479283 & -1.311935 & 0 & $\mathrm{~m}$ & \\
\hline 25 & $\mathrm{c}$ & 0 & -2.698362 & -1.069349 & -0.947576 & 0 & $\mathrm{~m}$ & \\
\hline 26 & $\mathrm{~h}$ & 0 & -2.329314 & -1.632119 & -1.798912 & 0 & $\mathrm{~m}$ & \\
\hline
\end{tabular}

No. 16<smiles>O=C(CCc1ccccc1)c1ccccc1</smiles>

neutral molecule

Charge $=0$ Multiplicity $=1$ for low level calculation on real system.

Charge $=0$ Multiplicity $=1$ for high level calculation on model system.

Charge $=0$ Multiplicity $=1$ for low level calculation on model system.

Center Atomic Atomic Coordinates (Angstroms)

Number Number Type $\quad X \quad$ Y $\quad$ Z

$\begin{array}{llllllll}1 & \mathrm{c} & 0 & 0.885895 & -0.733147 & 1.418185 & 0 & \mathrm{~h} \\ 2 & \mathrm{~h} & 0 & 1.293349 & -1.192204 & 2.330166 & 0 & \mathrm{~h}\end{array}$




\begin{tabular}{|c|c|c|c|c|c|c|c|c|}
\hline 3 & $\mathrm{~h}$ & 0 & 0.246228 & -1.502237 & 0.966664 & 0 & & \\
\hline 4 & $\mathrm{c}$ & 0 & 2.042782 & -0.443132 & 0.472202 & 0 & h & \\
\hline 5 & $\mathrm{c}$ & 0 & 2.757771 & -1.533999 & -0.048531 & 0 & $\mathrm{~h}$ & \\
\hline 6 & $\mathrm{~h}$ & 0 & 2.450365 & -2.545049 & 0.213324 & 0 & $\mathrm{~h}$ & \\
\hline 7 & $\mathrm{c}$ & 0 & 2.452955 & 0.847397 & 0.118109 & 0 & $\mathrm{~h}$ & \\
\hline 8 & $\mathrm{~h}$ & 0 & 1.907031 & 1.713445 & 0.476064 & 0 & $\mathrm{~h}$ & \\
\hline 9 & $\mathrm{c}$ & 0 & 3.550629 & 1.038509 & -0.727500 & 0 & $\mathrm{~h}$ & \\
\hline 10 & $\mathrm{~h}$ & 0 & 3.848148 & 2.049732 & -0.993044 & 0 & $\mathrm{~h}$ & \\
\hline 11 & $\mathrm{c}$ & 0 & 0.010231 & 0.457857 & 1.866102 & 0 & $\mathrm{~h}$ & \\
\hline 12 & $\mathrm{~h}$ & 0 & 0.628724 & 1.245030 & 2.306353 & 0 & $\mathrm{~h}$ & \\
\hline 13 & $\mathrm{~h}$ & 0 & -0.663552 & 0.101537 & 2.655465 & 0 & $\mathrm{~h}$ & \\
\hline 14 & $\mathrm{c}$ & 0 & 3.852048 & -1.345705 & -0.888998 & 0 & $\mathrm{~m}$ & h 5 \\
\hline 15 & $\mathrm{~h}$ & 0 & 4.389141 & -2.206944 & -1.278422 & 0 & $\mathrm{~m}$ & \\
\hline 16 & $\mathrm{c}$ & 0 & 4.255424 & -0.052269 & -1.231697 & 0 & $\mathrm{~m}$ & h 9 \\
\hline 17 & $\mathrm{~h}$ & 0 & 5.107640 & 0.099960 & -1.888768 & 0 & $\mathrm{~m}$ & \\
\hline 18 & $\mathrm{c}$ & 0 & -0.814922 & 1.109621 & 0.759242 & 0 & $\mathrm{~m}$ & $\mathrm{~h}$ \\
\hline 19 & o & 0 & -0.522856 & 2.224298 & 0.347638 & 0 & $\mathrm{~m}$ & \\
\hline 20 & $\mathrm{c}$ & 0 & -1.986351 & 0.375554 & 0.174985 & 0 & $\mathrm{~m}$ & \\
\hline 21 & $\mathrm{c}$ & 0 & -2.525020 & -0.790862 & 0.740385 & 0 & $\mathrm{~m}$ & \\
\hline 22 & $\mathrm{~h}$ & 0 & -2.097865 & -1.212695 & 1.644776 & 0 & $\mathrm{~m}$ & \\
\hline 23 & $\mathrm{c}$ & 0 & -3.627539 & -1.415615 & 0.157168 & 0 & $\mathrm{~m}$ & \\
\hline 24 & $\mathrm{~h}$ & 0 & -4.039678 & -2.314557 & 0.607331 & 0 & $\mathrm{~m}$ & \\
\hline 25 & $\mathrm{c}$ & 0 & -4.199820 & -0.885783 & -1.000297 & 0 & $\mathrm{~m}$ & \\
\hline 26 & $\mathrm{~h}$ & 0 & -5.056615 & -1.375132 & -1.455921 & 0 & $\mathrm{~m}$ & \\
\hline 27 & $\mathrm{c}$ & 0 & -3.671126 & 0.276035 & -1.571291 & 0 & $\mathrm{~m}$ & \\
\hline 28 & $\mathrm{~h}$ & 0 & -4.115704 & 0.690596 & -2.471972 & 0 & $\mathrm{~m}$ & \\
\hline 29 & $\mathrm{c}$ & 0 & -2.576326 & 0.903068 & -0.985607 & 0 & $\mathrm{~m}$ & \\
\hline 30 & $\mathrm{~h}$ & 0 & -2.154151 & 1.808961 & -1.407859 & 0 & $\mathrm{~m}$ & \\
\hline
\end{tabular}

radical

Charge $=0$ Multiplicity $=2$ for low level calculation on real system.

Charge $=0$ Multiplicity $=2$ for high level calculation on model system .

Charge $=0$ Multiplicity $=2$ for low level calculation on model system.

Center Atomic Atomic Coordinates (Angstroms)

Number Number Type $\quad \mathrm{X} \quad \mathrm{Y} \quad \mathrm{Z}$

$\begin{array}{llllllll}1 & \mathrm{c} & 0 & 0.970007 & 1.461425 & 0.194893 & 0 & \mathrm{~h} \\ 2 & \mathrm{~h} & 0 & 0.844203 & 2.017911 & 1.121595 & 0 & \mathrm{~h} \\ 3 & \mathrm{c} & 0 & 2.071652 & 0.575579 & 0.114506 & 0 & \mathrm{~h} \\ 4 & \mathrm{c} & 0 & 2.923965 & 0.416893 & 1.248351 & 0 & \mathrm{~h} \\ 5 & \mathrm{~h} & 0 & 2.700449 & 0.983239 & 2.149231 & 0 & \mathrm{~h}\end{array}$




\begin{tabular}{|c|c|c|c|c|c|c|c|c|}
\hline 6 & c & 0 & 2.399324 & -0.181058 & -1.049053 & 0 & h & \\
\hline 7 & $\mathrm{~h}$ & 0 & 1.795334 & -0.079322 & -1.945876 & 0 & h & \\
\hline 8 & $\mathrm{c}$ & 0 & 3.492996 & -1.035413 & -1.064886 & 0 & $\mathrm{~h}$ & \\
\hline 9 & $\mathrm{~h}$ & 0 & 3.719339 & -1.597021 & -1.967731 & 0 & $\mathrm{~h}$ & \\
\hline 10 & $\mathrm{c}$ & 0 & -0.059671 & 1.727764 & -0.862001 & 0 & h & \\
\hline 11 & $\mathrm{~h}$ & 0 & 0.054565 & 1.063188 & -1.723186 & 0 & $\mathrm{~h}$ & \\
\hline 12 & $\mathrm{~h}$ & 0 & 0.021194 & 2.762057 & -1.222864 & 0 & $\mathrm{~h}$ & \\
\hline 13 & $\mathrm{c}$ & 0 & 4.013362 & -0.438225 & 1.220967 & 0 & $\mathrm{~m}$ & h 4 \\
\hline 14 & $\mathrm{~h}$ & 0 & 4.641419 & -0.537444 & 2.102532 & 0 & $\mathrm{~m}$ & \\
\hline 15 & $\mathrm{c}$ & 0 & 4.308604 & -1.175453 & 0.065171 & 0 & $\mathrm{~m}$ & h 8 \\
\hline 16 & $\mathrm{~h}$ & 0 & 5.162959 & -1.845820 & 0.044973 & 0 & $\mathrm{~m}$ & \\
\hline 17 & $\mathrm{c}$ & 0 & -1.477602 & 1.618404 & -0.272177 & 0 & $\mathrm{~m} \quad \mathrm{l}$ & 10 \\
\hline 18 & o & 0 & -2.062077 & 2.633401 & 0.073957 & 0 & $\mathrm{~m}$ & \\
\hline 19 & $\mathrm{c}$ & 0 & -2.108686 & 0.270939 & -0.103362 & 0 & $\mathrm{~m}$ & \\
\hline 20 & $\mathrm{c}$ & 0 & -3.433284 & 0.223408 & 0.362199 & 0 & $\mathrm{~m}$ & \\
\hline 21 & $\mathrm{~h}$ & 0 & -3.932545 & 1.161115 & 0.583185 & 0 & $\mathrm{~m}$ & \\
\hline 22 & $\mathrm{c}$ & 0 & -4.079768 & -0.996442 & 0.531681 & 0 & $\mathrm{~m}$ & \\
\hline 23 & $\mathrm{~h}$ & 0 & -5.105713 & -1.021778 & 0.888972 & 0 & $\mathrm{~m}$ & \\
\hline 24 & $\mathrm{c}$ & 0 & -3.408354 & -2.188781 & 0.243578 & 0 & $\mathrm{~m}$ & \\
\hline 25 & $\mathrm{~h}$ & 0 & -3.911768 & -3.142813 & 0.376564 & 0 & $\mathrm{~m}$ & \\
\hline 26 & $\mathrm{c}$ & 0 & -2.089789 & -2.153355 & -0.213296 & 0 & $\mathrm{~m}$ & \\
\hline 27 & $\mathrm{~h}$ & 0 & -1.563158 & -3.078295 & -0.431676 & 0 & $\mathrm{~m}$ & \\
\hline 28 & $\mathrm{c}$ & 0 & -1.442224 & -0.930621 & -0.389217 & 0 & $\mathrm{~m}$ & \\
\hline 29 & $\mathrm{~h}$ & 0 & -0.412857 & -0.922603 & -0.731496 & 0 & $\mathrm{~m}$ & \\
\hline
\end{tabular}

No. 17<smiles>CCCCCN1C(c2ccccc2)=CC(=O)C1N1CCCCC1</smiles>

neutral molecule

Charge $=0$ Multiplicity $=1$ for low level calculation on real system.

Charge $=0$ Multiplicity $=1$ for high level calculation on model system.

Charge $=0$ Multiplicity $=1$ for low level calculation on model system .

Center Atomic Atomic Coordinates (Angstroms)

$\begin{array}{lllll}\text { Number Number Type } & \text { X } & \text { Y } & \text { Z }\end{array}$ 


\begin{tabular}{|c|c|c|c|c|c|c|}
\hline 1 & $\mathrm{c}$ & 0 & 0.427966 & -0.157452 & -0.276076 & $0 \mathrm{~h}$ \\
\hline 2 & $\mathrm{~h}$ & 0 & 0.181327 & -0.385999 & -1.327292 & $0 \mathrm{~h}$ \\
\hline 3 & $\mathrm{c}$ & 0 & -0.616644 & -0.827818 & 0.653521 & $0 \mathrm{~h}$ \\
\hline 4 & o & 0 & -0.341849 & -1.072120 & 1.819829 & $0 \mathrm{~h}$ \\
\hline 5 & $\mathrm{c}$ & 0 & -2.013879 & -1.062038 & 0.145915 & $0 \mathrm{~h}$ \\
\hline 6 & $\mathrm{c}$ & 0 & -2.954457 & -1.537753 & 1.075324 & $0 \mathrm{~h}$ \\
\hline 7 & $\mathrm{~h}$ & 0 & -2.625695 & -1.693827 & 2.097326 & $0 \mathrm{~h}$ \\
\hline 8 & $\mathrm{n}$ & 0 & 1.791851 & -0.589713 & 0.031126 & $0 \mathrm{~h}$ \\
\hline 9 & $\mathrm{n}$ & 0 & 0.166302 & 1.262214 & -0.091907 & $0 \mathrm{~h}$ \\
\hline 10 & $\mathrm{c}$ & 0 & -2.424009 & -0.859656 & -1.181562 & $0 \mathrm{~m} \mathrm{~h}$ \\
\hline 11 & $\mathrm{~h}$ & 0 & -1.727611 & -0.493578 & -1.927717 & $0 \mathrm{~m}$ \\
\hline 12 & $\mathrm{c}$ & 0 & -4.266103 & -1.800032 & 0.693160 & $0 \mathrm{~m} \mathrm{~h}$ \\
\hline 13 & $\mathrm{~h}$ & 0 & -4.980643 & -2.166555 & 1.425367 & $0 \mathrm{~m}$ \\
\hline 14 & $\mathrm{c}$ & 0 & 1.950941 & -2.048768 & -0.051834 & $0 \mathrm{~m} \mathrm{~h}$ \\
\hline 15 & $\mathrm{~h}$ & 0 & 1.784994 & -2.406142 & -1.091033 & $0 \mathrm{~m}$ \\
\hline 16 & $\mathrm{~h}$ & 0 & 1.199260 & -2.521240 & 0.582674 & $0 \mathrm{~m}$ \\
\hline 17 & $\mathrm{c}$ & 0 & 2.780249 & 0.084908 & -0.817659 & $0 \mathrm{~m} \mathrm{~h}$ \\
\hline 18 & $\mathrm{~h}$ & 0 & 2.628158 & 1.163745 & -0.742609 & $0 \mathrm{~m}$ \\
\hline 19 & $\mathrm{~h}$ & 0 & 2.643394 & -0.189093 & -1.886286 & $0 \mathrm{~m}$ \\
\hline 20 & $\mathrm{c}$ & 0 & 0.632142 & 1.892881 & 1.142600 & $0 \mathrm{~m} \mathrm{l}$ \\
\hline 21 & $\mathrm{~h}$ & 0 & 0.630256 & 1.143442 & 1.936345 & $0 \mathrm{~m}$ \\
\hline 22 & $\mathrm{~h}$ & 0 & 1.675605 & 2.246311 & 1.037837 & $0 \mathrm{~m}$ \\
\hline 23 & $\mathrm{c}$ & 0 & 0.115168 & 2.155765 & -1.243026 & $0 \mathrm{~m} \mathrm{~h}$ \\
\hline 24 & $\mathrm{~h}$ & 0 & 1.118837 & 2.541167 & -1.512217 & $0 \mathrm{~m}$ \\
\hline 25 & $\mathrm{~h}$ & 0 & -0.252063 & 1.595415 & -2.109908 & $0 \mathrm{~m}$ \\
\hline 26 & $\mathrm{c}$ & 0 & -3.738975 & -1.123094 & -1.565265 & $0 \mathrm{~m}$ \\
\hline 27 & $\mathrm{~h}$ & 0 & -4.041165 & -0.960682 & -2.596379 & $0 \mathrm{~m}$ \\
\hline 28 & $\mathrm{c}$ & 0 & -4.662965 & -1.592811 & -0.630808 & $0 \mathrm{~m}$ \\
\hline 29 & $\mathrm{~h}$ & 0 & -5.686738 & -1.797673 & -0.932811 & $0 \mathrm{~m}$ \\
\hline 30 & $\mathrm{c}$ & 0 & 3.346651 & -2.477524 & 0.412046 & $0 \mathrm{~m}$ \\
\hline 31 & $\mathrm{~h}$ & 0 & 3.435963 & -3.567923 & 0.326922 & $0 \mathrm{~m}$ \\
\hline 32 & $\mathrm{~h}$ & 0 & 3.451201 & -2.222943 & 1.474331 & $0 \mathrm{~m}$ \\
\hline 33 & $\mathrm{c}$ & 0 & 4.440816 & -1.775702 & -0.400435 & $0 \mathrm{~m}$ \\
\hline 34 & $\mathrm{~h}$ & 0 & 4.411968 & -2.137015 & -1.439190 & $0 \mathrm{~m}$ \\
\hline 35 & $\mathrm{~h}$ & 0 & 5.435696 & -2.020892 & -0.008327 & $0 \mathrm{~m}$ \\
\hline 36 & $\mathrm{c}$ & 0 & 4.209331 & -0.260301 & -0.384809 & $0 \mathrm{~m}$ \\
\hline 37 & $\mathrm{~h}$ & 0 & 4.920578 & 0.251068 & -1.045913 & $0 \mathrm{~m}$ \\
\hline 38 & $\mathrm{~h}$ & 0 & 4.369549 & 0.127428 & 0.629598 & $0 \mathrm{~m}$ \\
\hline 39 & $\mathrm{c}$ & 0 & -0.267181 & 3.081614 & 1.502824 & $0 \mathrm{~m}$ \\
\hline 40 & $\mathrm{~h}$ & 0 & -1.266508 & 2.704394 & 1.756771 & $0 \mathrm{~m}$ \\
\hline 41 & $\mathrm{~h}$ & 0 & 0.129969 & 3.582245 & 2.395140 & $0 \mathrm{~m}$ \\
\hline 42 & $\mathrm{c}$ & 0 & -0.370931 & 4.069859 & 0.331707 & $0 \mathrm{~m}$ \\
\hline 43 & $\mathrm{~h}$ & 0 & -1.068485 & 4.882240 & 0.570403 & $0 \mathrm{~m}$ \\
\hline
\end{tabular}




$\begin{array}{rrrrrrr}44 & \mathrm{~h} & 0 & 0.611227 & 4.537394 & 0.164243 & 0 \mathrm{~m} \\ 45 & \mathrm{c} & 0 & -0.807704 & 3.346250 & -0.950684 & 0 \mathrm{~m} \\ 46 & \mathrm{~h} & 0 & -0.800067 & 4.034329 & -1.805994 & 0 \mathrm{~m} \\ 47 & \mathrm{~h} & 0 & -1.833793 & 2.973883 & -0.834079 & 0 \mathrm{~m}\end{array}$

radical

Charge $=0$ Multiplicity $=2$ for low level calculation on real system.

Charge $=0$ Multiplicity $=2$ for high level calculation on model system.

Charge $=0$ Multiplicity $=2$ for low level calculation on model system.

\begin{tabular}{|c|c|c|c|c|c|c|c|}
\hline \multicolumn{8}{|c|}{ Center Atomic Atomic Coordinates (Angstroms) } \\
\hline Number & Numbe & & Type & $\mathrm{X}$ & $\mathrm{Y}$ & $\mathrm{Z}$ & \\
\hline 1 & $\mathrm{c}$ & 0 & & -0.603882 & -0.010537 & 0.079031 & $0 \mathrm{~h}$ \\
\hline 2 & $\mathrm{c}$ & 0 & & 0.070680 & -1.273252 & -0.121161 & $0 \mathrm{~h}$ \\
\hline 3 & o & 0 & & -0.587527 & -2.310006 & -0.397876 & $0 \mathrm{~h}$ \\
\hline 4 & $\mathrm{c}$ & 0 & & 1.562847 & -1.389866 & 0.005405 & $0 \mathrm{~h}$ \\
\hline 5 & $\mathrm{c}$ & 0 & & 2.207158 & -2.372077 & -0.765171 & $0 \mathrm{~h}$ \\
\hline 6 & $\mathrm{~h}$ & 0 & & 1.605195 & -2.967269 & -1.444472 & $0 \mathrm{~h}$ \\
\hline 7 & $\mathrm{n}$ & 0 & & -1.939844 & 0.015331 & 0.416863 & $0 \mathrm{~h}$ \\
\hline 8 & $\mathrm{n}$ & 0 & & 0.040251 & 1.213730 & -0.093174 & $0 \mathrm{~h}$ \\
\hline 9 & $\mathrm{c}$ & 0 & & 2.327897 & -0.656685 & 0.927516 & $0 \mathrm{~m} \mathrm{~h}$ \\
\hline 10 & $\mathrm{~h}$ & 0 & & 1.843064 & 0.080129 & 1.559521 & $0 \mathrm{~m}$ \\
\hline 11 & $\mathrm{c}$ & 0 & & 3.578429 & -2.587556 & -0.647846 & $0 \mathrm{~m} \mathrm{~h}$ \\
\hline 12 & $\mathrm{~h}$ & 0 & & 4.060483 & -3.344847 & -1.261213 & $0 \mathrm{~m}$ \\
\hline 13 & $\mathrm{c}$ & 0 & & -2.562980 & -1.004994 & 1.267643 & $0 \mathrm{~m} \mathrm{~h}$ \\
\hline 14 & $\mathrm{~h}$ & 0 & & -1.780512 & -1.646079 & 1.670990 & $0 \mathrm{~m}$ \\
\hline 15 & $\mathrm{~h}$ & 0 & & -3.043684 & -0.481002 & 2.111575 & $0 \mathrm{~m}$ \\
\hline 16 & $\mathrm{c}$ & 0 & & -2.892131 & 0.908774 & -0.249070 & $0 \mathrm{~m} \mathrm{~h}$ \\
\hline 17 & $\mathrm{~h}$ & 0 & & -3.405021 & 1.527732 & 0.504845 & $0 \mathrm{~m}$ \\
\hline 18 & $\mathrm{~h}$ & 0 & & -2.340884 & 1.579790 & -0.909754 & $0 \mathrm{~m}$ \\
\hline 19 & $\mathrm{c}$ & 0 & & -0.344291 & 2.388954 & 0.691751 & $0 \mathrm{~m} \mathrm{~h}$ \\
\hline 20 & $\mathrm{~h}$ & 0 & & -0.844560 & 2.042180 & 1.599482 & $0 \mathrm{~m}$ \\
\hline 21 & $\mathrm{~h}$ & 0 & & -1.066244 & 3.017832 & 0.141394 & $0 \mathrm{~m}$ \\
\hline 22 & $\mathrm{c}$ & 0 & & 0.781104 & 1.516334 & -1.324846 & $0 \mathrm{~m} \mathrm{~h}$ \\
\hline 23 & $\mathrm{~h}$ & 0 & & 0.113197 & 2.047373 & -2.029475 & $0 \mathrm{~m}$ \\
\hline 24 & $\mathrm{~h}$ & 0 & & 1.067194 & 0.574790 & -1.796366 & $0 \mathrm{~m}$ \\
\hline 25 & $\mathrm{c}$ & 0 & & 3.698455 & -0.883674 & 1.057377 & $0 \mathrm{~m}$ \\
\hline 26 & $\mathrm{~h}$ & 0 & & 4.271598 & -0.316843 & 1.787214 & $0 \mathrm{~m}$ \\
\hline 27 & $\mathrm{c}$ & 0 & & 4.331523 & -1.842684 & 0.263476 & $0 \mathrm{~m}$ \\
\hline 28 & $\mathrm{~h}$ & 0 & & 5.400119 & -2.015497 & 0.362068 & $0 \mathrm{~m}$ \\
\hline 29 & $\mathrm{c}$ & 0 & & -3.610807 & -1.833837 & 0.514991 & $0 \mathrm{~m}$ \\
\hline
\end{tabular}




$\begin{array}{lllrrrl}30 & \mathrm{~h} & 0 & -3.081217 & -2.441402 & -0.225078 & 0 \mathrm{~m} \\ 31 & \mathrm{~h} & 0 & -4.105820 & -2.513435 & 1.220603 & 0 \mathrm{~m} \\ 32 & \mathrm{c} & 0 & -4.639623 & -0.925573 & -0.172205 & 0 \mathrm{~m} \\ 33 & \mathrm{~h} & 0 & -5.338581 & -1.521210 & -0.771916 & 0 \mathrm{~m} \\ 34 & \mathrm{~h} & 0 & -5.242851 & -0.404885 & 0.587620 & 0 \mathrm{~m} \\ 35 & \mathrm{c} & 0 & -3.932013 & 0.112369 & -1.053969 & 0 \mathrm{~m} \\ 36 & \mathrm{~h} & 0 & -3.420316 & -0.394342 & -1.882342 & 0 \mathrm{~m} \\ 37 & \mathrm{~h} & 0 & -4.655135 & 0.812429 & -1.493248 & 0 \mathrm{~m} \\ 38 & \mathrm{c} & 0 & 0.883189 & 3.245286 & 1.030623 & 0 \mathrm{~m} \\ 39 & \mathrm{~h} & 0 & 1.544748 & 2.681399 & 1.701012 & 0 \mathrm{~m} \\ 40 & \mathrm{~h} & 0 & 0.553825 & 4.138980 & 1.576085 & 0 \mathrm{~m} \\ 41 & \mathrm{c} & 0 & 1.644386 & 3.635998 & -0.243004 & 0 \mathrm{~m} \\ 42 & \mathrm{~h} & 0 & 1.009075 & 4.293070 & -0.856007 & 0 \mathrm{~m} \\ 43 & \mathrm{~h} & 0 & 2.543620 & 4.212007 & 0.006477 & 0 \mathrm{~m} \\ 44 & \mathrm{c} & 0 & 2.014547 & 2.384078 & -1.050050 & 0 \mathrm{~m} \\ 45 & \mathrm{~h} & 0 & 2.473255 & 2.661787 & -2.007255 & 0 \mathrm{~m} \\ 46 & \mathrm{~h} & 0 & 2.749898 & 1.787585 & -0.497526 & 0 \mathrm{~m}\end{array}$

\section{No. 18}

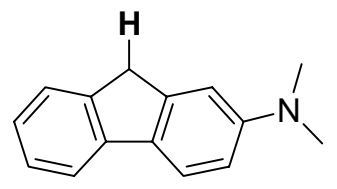

neutral molecule

Charge $=0$ Multiplicity $=1$ for low level calculation on real system.

Charge $=0$ Multiplicity $=1$ for high level calculation on model system.

Charge $=0$ Multiplicity $=1$ for low level calculation on model system .

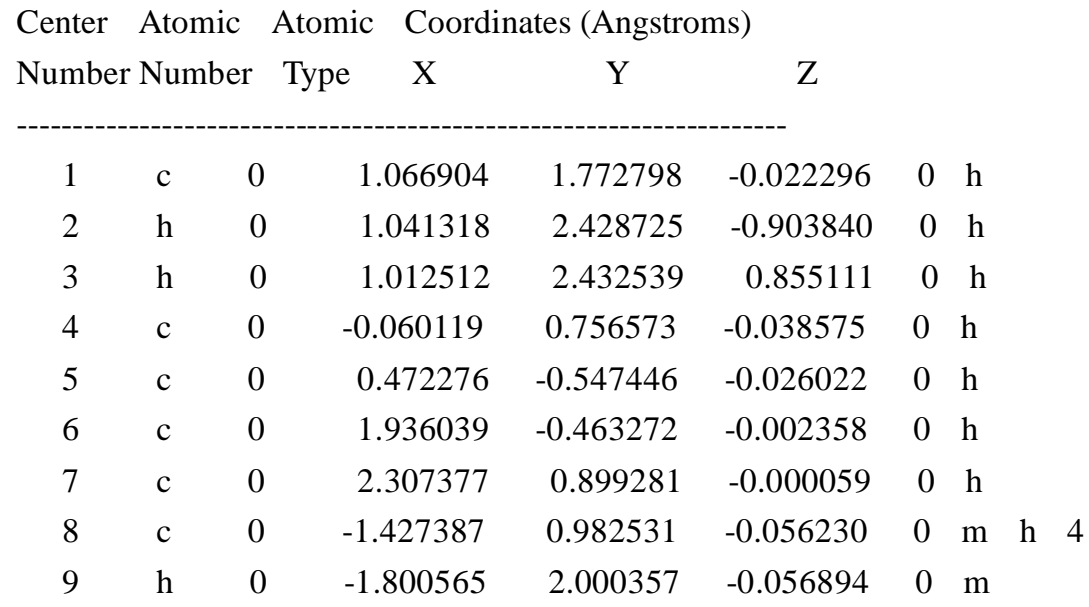




$\begin{array}{lllrrrrrrr}10 & \mathrm{c} & 0 & -0.402301 & -1.634558 & -0.029211 & 0 & \mathrm{~m} & \mathrm{~h} & 5 \\ 11 & \mathrm{~h} & 0 & -0.024771 & -2.654256 & -0.011486 & 0 & \mathrm{~m} & & \\ 12 & \mathrm{c} & 0 & 2.916260 & -1.458600 & 0.016434 & 0 & \mathrm{~m} & \mathrm{~h} & 6 \\ 13 & \mathrm{~h} & 0 & 2.641036 & -2.510484 & 0.014732 & 0 & \mathrm{~m} & & \\ 14 & \mathrm{c} & 0 & 3.647040 & 1.265856 & 0.020368 & 0 & \mathrm{~m} & \mathrm{~h} & 7 \\ 15 & \mathrm{~h} & 0 & 3.936374 & 2.314644 & 0.022042 & 0 & \mathrm{~m} & & \\ 16 & \mathrm{c} & 0 & -2.324982 & -0.113595 & -0.072093 & 0 & \mathrm{~m} & & \\ 17 & \mathrm{n} & 0 & -3.702181 & 0.087473 & -0.120570 & 0 & \mathrm{~m} & \\ 18 & \mathrm{c} & 0 & -4.230852 & 1.423902 & 0.089941 & 0 & \mathrm{~m} & \\ 19 & \mathrm{~h} & 0 & -3.863419 & 2.117248 & -0.676156 & 0 & \mathrm{~m} & \\ 20 & \mathrm{~h} & 0 & -3.968842 & 1.839443 & 1.076887 & 0 & \mathrm{~m} & \\ 21 & \mathrm{~h} & 0 & -5.319385 & 1.394298 & 0.009162 & 0 & \mathrm{~m} & \\ 22 & \mathrm{c} & 0 & -4.593408 & -1.039144 & 0.095185 & 0 & \mathrm{~m} & \\ 23 & \mathrm{~h} & 0 & -5.627080 & -0.696859 & 0.013643 & 0 & \mathrm{~m} & \\ 24 & \mathrm{~h} & 0 & -4.462301 & -1.508493 & 1.083979 & 0 & \mathrm{~m} & \\ 25 & \mathrm{~h} & 0 & -4.442335 & -1.811833 & -0.668042 & 0 & \mathrm{~m} & & \\ 26 & \mathrm{c} & 0 & -1.777716 & -1.420856 & -0.047919 & 0 & \mathrm{~m} & & \\ 27 & \mathrm{~h} & 0 & -2.433116 & -2.283211 & -0.042709 & 0 & \mathrm{~m} & & \\ 28 & \mathrm{c} & 0 & 4.261982 & -1.083765 & 0.037151 & 0 & \mathrm{~m} & & \\ 29 & \mathrm{~h} & 0 & 5.032810 & -1.850168 & 0.051732 & 0 & \mathrm{~m} & & \\ 30 & \mathrm{c} & 0 & 4.627873 & 0.266037 & 0.039119 & 0 & \mathrm{~m} & & \\ 31 & \mathrm{~h} & 0 & 5.679103 & 0.541279 & 0.055218 & 0 & \mathrm{~m} & & \\ ---------------------------------------------------------- & & & & \end{array}$

radical

Charge $=0$ Multiplicity $=2$ for low level calculation on real system.

Charge $=0$ Multiplicity $=2$ for high level calculation on model system.

Charge $=0$ Multiplicity $=2$ for low level calculation on model system.

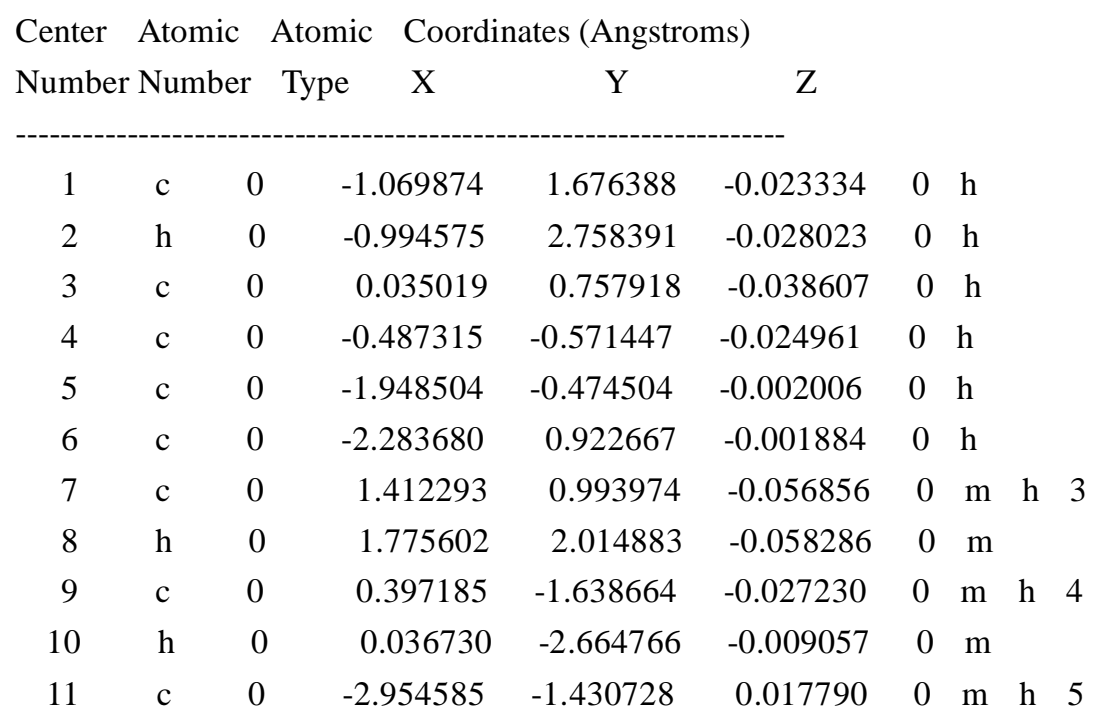




\begin{tabular}{|c|c|c|c|c|c|c|c|}
\hline 12 & $\mathrm{~h}$ & 0 & -2.717385 & -2.491973 & 0.017895 & 0 & $\mathrm{~m}$ \\
\hline 13 & $\mathrm{c}$ & 0 & -3.628459 & 1.324352 & 0.017778 & 0 & $\mathrm{~m} \quad \mathrm{~h} \quad 6$ \\
\hline 14 & $\mathrm{~h}$ & 0 & -3.888093 & 2.380333 & 0.017854 & 0 & $\mathrm{~m}$ \\
\hline 15 & $\mathrm{c}$ & 0 & 2.315049 & -0.096879 & -0.071930 & 0 & $\mathrm{~m}$ \\
\hline 16 & $\mathrm{n}$ & 0 & 3.690985 & 0.114306 & -0.120996 & 0 & $\mathrm{~m}$ \\
\hline 17 & $\mathrm{c}$ & 0 & 4.210938 & 1.454366 & 0.089537 & 0 & $\mathrm{~m}$ \\
\hline 18 & $\mathrm{~h}$ & 0 & 3.948705 & 1.867174 & 1.077479 & 0 & $\mathrm{~m}$ \\
\hline 19 & $\mathrm{~h}$ & 0 & 3.837290 & 2.145597 & -0.675368 & 0 & $\mathrm{~m}$ \\
\hline 20 & $\mathrm{~h}$ & 0 & 5.299392 & 1.431967 & 0.005958 & 0 & $\mathrm{~m}$ \\
\hline 21 & $\mathrm{c}$ & 0 & 4.589516 & -1.006435 & 0.093861 & 0 & $\mathrm{~m}$ \\
\hline 22 & $\mathrm{~h}$ & 0 & 5.620949 & -0.657250 & 0.013783 & 0 & $\mathrm{~m}$ \\
\hline 23 & $\mathrm{~h}$ & 0 & 4.443994 & -1.779115 & -0.670561 & 0 & $\mathrm{~m}$ \\
\hline 24 & $\mathrm{~h}$ & 0 & 4.460234 & -1.477890 & 1.081915 & 0 & $\mathrm{~m}$ \\
\hline 25 & $\mathrm{c}$ & 0 & 1.777859 & -1.407835 & -0.045929 & 0 & $\mathrm{~m}$ \\
\hline 26 & $\mathrm{~h}$ & 0 & 2.440093 & -2.264575 & -0.039306 & 0 & $\mathrm{~m}$ \\
\hline 27 & $\mathrm{c}$ & 0 & -4.294898 & -1.012083 & 0.037497 & 0 & $\mathrm{~m}$ \\
\hline 28 & $\mathrm{~h}$ & 0 & -5.086424 & -1.756742 & 0.052925 & 0 & $\mathrm{~m}$ \\
\hline 29 & $\mathrm{c}$ & 0 & -4.627402 & 0.349907 & 0.037437 & 0 & $\mathrm{~m}$ \\
\hline 30 & $\mathrm{~h}$ & 0 & -5.672256 & 0.647835 & 0.052786 & 0 & $\mathrm{~m}$ \\
\hline
\end{tabular}

No. 19

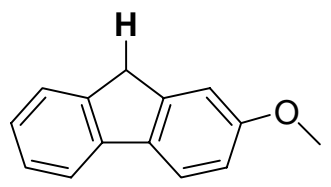

neutral molecule

Charge $=0$ Multiplicity $=1$ for low level calculation on real system.

Charge $=0$ Multiplicity $=1$ for high level calculation on model system .

Charge $=0$ Multiplicity $=1$ for low level calculation on model system.

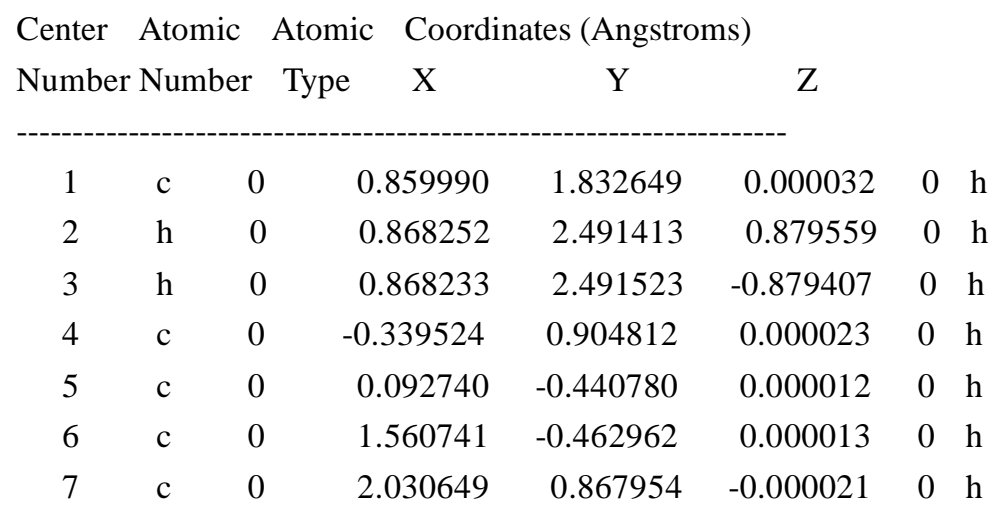




$\begin{array}{rrrrrrrrrr}8 & \mathrm{c} & 0 & -1.686969 & 1.219579 & 0.000040 & 0 & \mathrm{~m} & \mathrm{~h} & 4 \\ 9 & \mathrm{~h} & 0 & -2.044450 & 2.245413 & 0.000095 & 0 & \mathrm{~m} & & \\ 10 & \mathrm{c} & 0 & -0.848311 & -1.467589 & 0.000057 & 0 & \mathrm{~m} & \mathrm{~h} & 5 \\ 11 & \mathrm{~h} & 0 & -0.537187 & -2.509286 & 0.000118 & 0 & \mathrm{~m} & & \\ 12 & \mathrm{c} & 0 & 2.463784 & -1.528611 & 0.000016 & 0 & \mathrm{~m} & \mathrm{~h} & 6 \\ 13 & \mathrm{~h} & 0 & 2.111288 & -2.557133 & 0.000059 & 0 & \mathrm{~m} & & \\ 14 & \mathrm{c} & 0 & 3.394450 & 1.134739 & -0.000074 & 0 & \mathrm{~m} & \mathrm{~h} & 7 \\ 15 & \mathrm{~h} & 0 & 3.760237 & 2.159176 & -0.000101 & 0 & \mathrm{~m} & \\ 16 & \mathrm{c} & 0 & -2.631862 & 0.178335 & 0.000026 & 0 & \mathrm{~m} & \\ 17 & \mathrm{o} & 0 & -3.939273 & 0.577818 & 0.000109 & 0 & \mathrm{~m} & \\ 18 & \mathrm{c} & 0 & -4.948342 & -0.417931 & -0.000182 & 0 & \mathrm{~m} & \\ 19 & \mathrm{~h} & 0 & -5.898833 & 0.119080 & -0.000697 & 0 & \mathrm{~m} & \\ 20 & \mathrm{~h} & 0 & -4.890986 & -1.052635 & -0.894631 & 0 & \mathrm{~m} & \\ 21 & \mathrm{~h} & 0 & -4.891729 & -1.052394 & 0.894519 & 0 & \mathrm{~m} & \\ 22 & \mathrm{c} & 0 & -2.212708 & -1.160304 & 0.000060 & 0 & \mathrm{~m} & & \\ 23 & \mathrm{~h} & 0 & -2.937393 & -1.966373 & 0.000098 & 0 & \mathrm{~m} & & \\ 24 & \mathrm{c} & 0 & 3.833411 & -1.254476 & -0.000015 & 0 & \mathrm{~m} & & \\ 25 & \mathrm{~h} & 0 & 4.545751 & -2.075482 & -0.000013 & 0 & \mathrm{~m} & & \\ 26 & \mathrm{c} & 0 & 4.297692 & 0.065046 & -0.000050 & 0 & \mathrm{~m} & & \\ 27 & \mathrm{~h} & 0 & 5.366533 & 0.261395 & -0.000091 & 0 & \mathrm{~m} & & \\ ------------------------------------------------------------ & & & & \end{array}$

radical

Charge $=0$ Multiplicity $=2$ for low level calculation on real system.

Charge $=0$ Multiplicity $=2$ for high level calculation on model system.

Charge $=0$ Multiplicity $=2$ for low level calculation on model system.

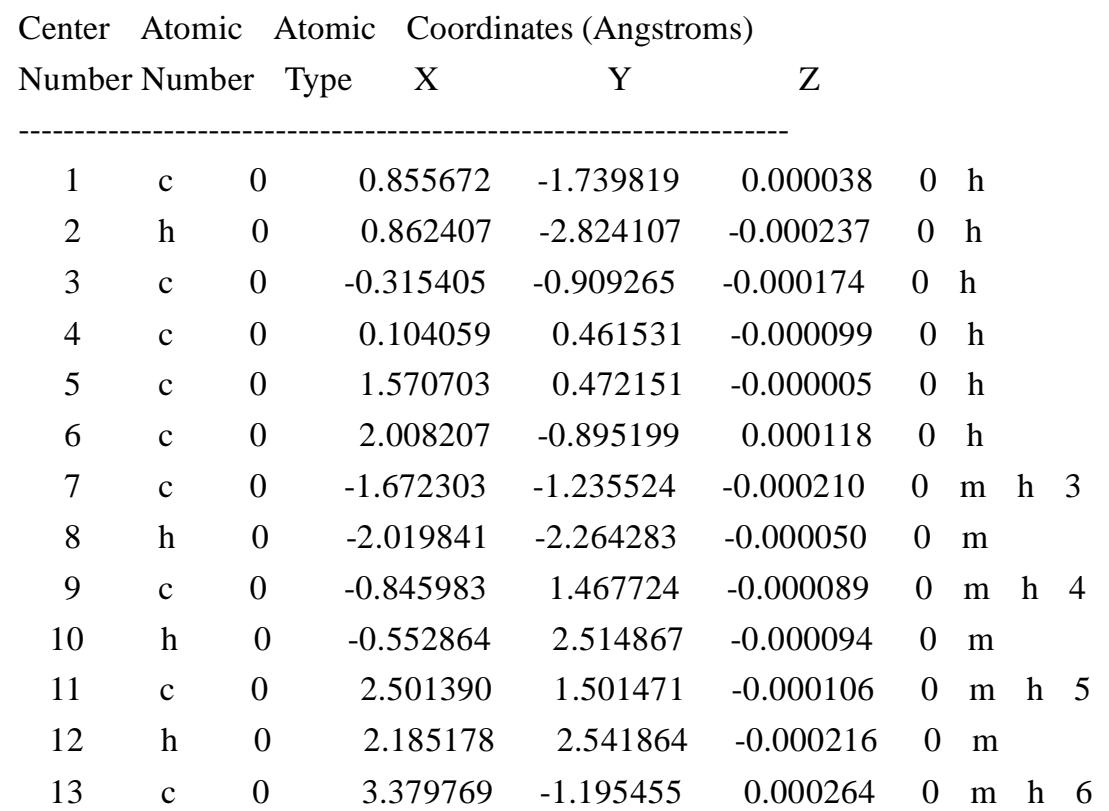




\begin{tabular}{|c|c|c|c|c|c|c|}
\hline 14 & $\mathrm{~h}$ & 0 & 3.717590 & -2.228907 & 0.000456 & 0 \\
\hline 15 & $\mathrm{c}$ & 0 & -2.622172 & -0.200117 & -0.000334 & $0 \mathrm{~m}$ \\
\hline 16 & o & 0 & -3.926940 & -0.608953 & -0.000534 & 0 \\
\hline 17 & $\mathrm{c}$ & 0 & -4.943014 & 0.379588 & 0.000834 & 0 \\
\hline 18 & $\mathrm{~h}$ & 0 & -5.889590 & -0.164209 & 0.001317 & 0 \\
\hline 19 & $\mathrm{~h}$ & 0 & -4.889545 & 1.014040 & 0.895720 & 0 \\
\hline 20 & $\mathrm{~h}$ & 0 & -4.891163 & 1.014911 & -0.893544 & 0 \\
\hline 21 & $\mathrm{c}$ & 0 & -2.214319 & 1.141855 & -0.000272 & 0 \\
\hline 22 & $\mathrm{~h}$ & 0 & -2.946193 & 1.941063 & -0.000220 & 0 \\
\hline 23 & $\mathrm{c}$ & 0 & 3.869416 & 1.184602 & 0.000000 & 0 \\
\hline 24 & $\mathrm{~h}$ & 0 & 4.603132 & 1.986236 & -0.000045 & 0 \\
\hline 25 & $\mathrm{c}$ & 0 & 4.302285 & -0.148909 & 0.000184 & 0 \\
\hline 26 & $\mathrm{~h}$ & 0 & 5.366582 & -0.367652 & 0.000296 & 0 \\
\hline
\end{tabular}

No. 20

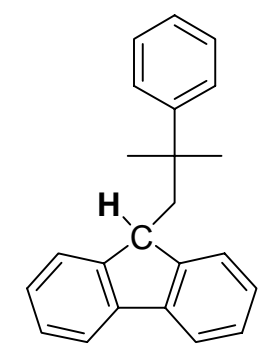

neutral molecule

Charge $=0$ Multiplicity $=1$ for low level calculation on real system.

Charge $=0$ Multiplicity $=1$ for high level calculation on model system.

Charge $=0$ Multiplicity $=1$ for low level calculation on model system.

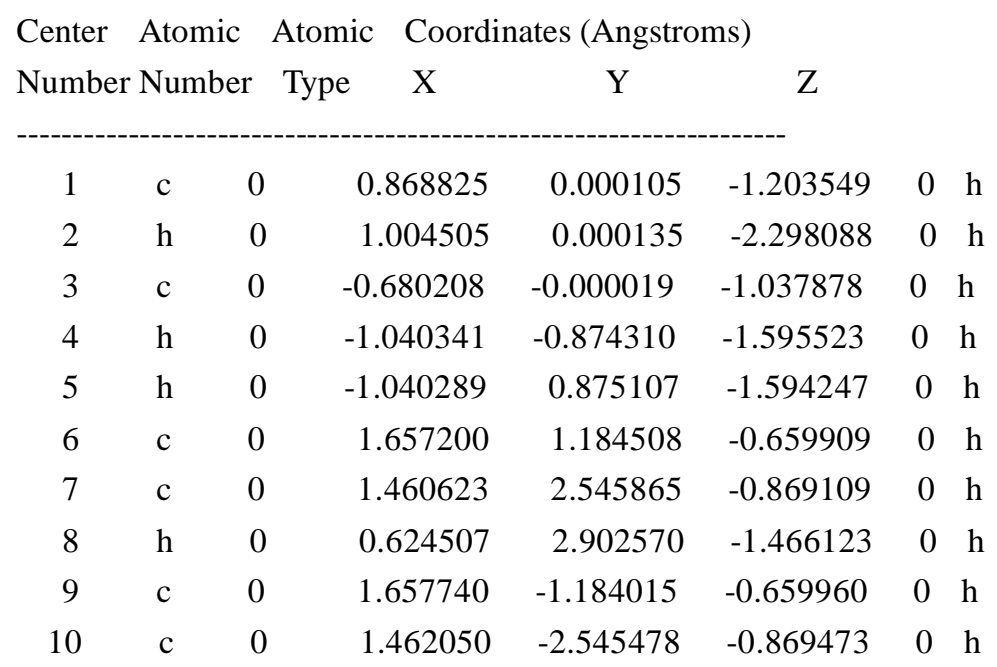




\begin{tabular}{|c|c|c|c|c|c|c|c|c|}
\hline 11 & $\mathrm{~h}$ & 0 & 0.626152 & -2.902578 & -1.466550 & 0 & h & \\
\hline 12 & $\mathrm{c}$ & 0 & -1.383645 & -0.001011 & 0.351775 & 0 & $\mathrm{~m}$ & h \\
\hline 13 & $\mathrm{c}$ & 0 & 2.349899 & 3.460010 & -0.294112 & 0 & $\mathrm{~m}$ & $\mathrm{~h}$ \\
\hline 14 & $\mathrm{~h}$ & 0 & 2.200685 & 4.525565 & -0.446827 & 0 & $\mathrm{~m}$ & \\
\hline 15 & $\mathrm{c}$ & 0 & 2.757494 & 0.734805 & 0.098753 & 0 & $\mathrm{~m}$ & $\mathrm{~h}$ \\
\hline 16 & $\mathrm{c}$ & 0 & 2.757836 & -0.733824 & 0.098706 & 0 & $\mathrm{~m}$ & $\mathrm{~h}$ \\
\hline 17 & $\mathrm{c}$ & 0 & 2.351869 & -3.459179 & -0.294657 & 0 & $\mathrm{~m}$ & $\mathrm{~h}$ \\
\hline 18 & $\mathrm{~h}$ & 0 & 2.203320 & -4.524794 & -0.447597 & 0 & $\mathrm{~m}$ & \\
\hline 19 & $\mathrm{c}$ & 0 & -1.001989 & -1.260017 & 1.166851 & 0 & $\mathrm{~m}$ & \\
\hline 20 & $\mathrm{~h}$ & 0 & 0.064420 & -1.268099 & 1.405166 & 0 & $\mathrm{~m}$ & \\
\hline 21 & $\mathrm{~h}$ & 0 & -1.233340 & -2.177417 & 0.614289 & 0 & $\mathrm{~m}$ & \\
\hline 22 & $\mathrm{~h}$ & 0 & -1.553127 & -1.295211 & 2.111831 & 0 & $\mathrm{~m}$ & \\
\hline 23 & $\mathrm{c}$ & 0 & -1.001002 & 1.256104 & 1.169285 & 0 & $\mathrm{~m}$ & \\
\hline 24 & $\mathrm{~h}$ & 0 & -1.231286 & 2.174795 & 0.618442 & 0 & $\mathrm{~m}$ & \\
\hline 25 & $\mathrm{~h}$ & 0 & 0.065321 & 1.262714 & 1.408019 & 0 & $\mathrm{~m}$ & \\
\hline 26 & $\mathrm{~h}$ & 0 & -1.552299 & 1.290053 & 2.114215 & 0 & $\mathrm{~m}$ & \\
\hline 27 & $\mathrm{c}$ & 0 & -2.923576 & -0.000337 & 0.150616 & 0 & $\mathrm{~m}$ & \\
\hline 28 & $\mathrm{c}$ & 0 & -3.763613 & -0.000692 & 1.279775 & 0 & $\mathrm{~m}$ & \\
\hline 29 & $\mathrm{~h}$ & 0 & -3.328757 & -0.001347 & 2.274995 & 0 & $\mathrm{~m}$ & \\
\hline 30 & $\mathrm{c}$ & 0 & -5.151699 & -0.000247 & 1.165694 & 0 & $\mathrm{~m}$ & \\
\hline 31 & $\mathrm{~h}$ & 0 & -5.763402 & -0.000529 & 2.064492 & 0 & $\mathrm{~m}$ & \\
\hline 32 & $\mathrm{c}$ & 0 & -5.754556 & 0.000556 & -0.093655 & 0 & $\mathrm{~m}$ & \\
\hline 33 & $\mathrm{~h}$ & 0 & -6.837198 & 0.000905 & -0.187791 & 0 & $\mathrm{~m}$ & \\
\hline 34 & $\mathrm{c}$ & 0 & -4.944955 & 0.000879 & -1.225897 & 0 & $\mathrm{~m}$ & \\
\hline 35 & $\mathrm{~h}$ & 0 & -5.391839 & 0.001486 & -2.217081 & 0 & $\mathrm{~m}$ & \\
\hline 36 & $\mathrm{c}$ & 0 & -3.551756 & 0.000440 & -1.103959 & 0 & $\mathrm{~m}$ & \\
\hline 37 & $\mathrm{~h}$ & 0 & -2.965750 & 0.000720 & -2.015274 & 0 & $\mathrm{~m}$ & \\
\hline 38 & $\mathrm{c}$ & 0 & 3.430822 & 3.013543 & 0.474740 & 0 & $\mathrm{~m}$ & \\
\hline 39 & $\mathrm{~h}$ & 0 & 4.112861 & 3.735749 & 0.915634 & 0 & $\mathrm{~m}$ & \\
\hline 40 & $\mathrm{c}$ & 0 & 3.645503 & 1.648381 & 0.672339 & 0 & $\mathrm{~m}$ & \\
\hline 41 & $\mathrm{~h}$ & 0 & 4.494868 & 1.306056 & 1.258251 & 0 & $\mathrm{~m}$ & \\
\hline 42 & $\mathrm{c}$ & 0 & 3.646392 & -1.646990 & 0.672111 & 0 & $\mathrm{~m}$ & \\
\hline 43 & $\mathrm{~h}$ & 0 & 4.495603 & -1.304274 & 1.258017 & 0 & $\mathrm{~m}$ & \\
\hline 44 & $\mathrm{c}$ & 0 & 3.432484 & -3.012245 & 0.474299 & 0 & $\mathrm{~m}$ & \\
\hline 45 & $\mathrm{~h}$ & 0 & 4.114961 & -3.734132 & 0.915042 & 0 & $\mathrm{~m}$ & \\
\hline
\end{tabular}

radical

Charge $=0$ Multiplicity $=2$ for low level calculation on real system.

Charge $=0$ Multiplicity $=2$ for high level calculation on model system.

Charge $=0$ Multiplicity $=2$ for low level calculation on model system.

Center Atomic Atomic Coordinates (Angstroms) 


\begin{tabular}{|c|c|c|c|c|c|c|c|c|}
\hline Number & Nun & & Type & $\mathrm{Y}$ & $\mathrm{Z}$ & & & \\
\hline 1 & $\mathrm{c}$ & 0 & 0.825654 & -0.000006 & -0.375244 & 0 & $\mathrm{~h}$ & \\
\hline 2 & $\mathrm{c}$ & 0 & -0.648205 & -0.000108 & -0.651104 & 0 & $\mathrm{~h}$ & \\
\hline 3 & $\mathrm{~h}$ & 0 & -0.883317 & -0.879776 & -1.263729 & 0 & $\mathrm{~h}$ & \\
\hline 4 & $\mathrm{~h}$ & 0 & -0.883575 & 0.880052 & -1.262910 & 0 & $\mathrm{~h}$ & \\
\hline 5 & $\mathrm{c}$ & 0 & 1.677055 & 1.160822 & -0.261208 & 0 & $\mathrm{~h}$ & \\
\hline 6 & $\mathrm{c}$ & 0 & 1.390814 & 2.532633 & -0.345512 & 0 & $\mathrm{~h}$ & \\
\hline 7 & $\mathrm{~h}$ & 0 & 0.375211 & 2.878506 & -0.515328 & 0 & $\mathrm{~h}$ & \\
\hline 8 & $\mathrm{c}$ & 0 & 1.677412 & -1.160556 & -0.261252 & 0 & $\mathrm{~h}$ & \\
\hline 9 & $\mathrm{c}$ & 0 & 1.391661 & -2.532429 & -0.345754 & 0 & $\mathrm{~h}$ & \\
\hline 10 & $\mathrm{~h}$ & 0 & 0.376178 & -2.878635 & -0.515587 & 0 & $\mathrm{~h}$ & \\
\hline 11 & $\mathrm{c}$ & 0 & -1.620772 & -0.000869 & 0.580898 & 0 & $\mathrm{~m}$ & h 2 \\
\hline 12 & $\mathrm{c}$ & 0 & 2.428940 & 3.455724 & -0.209580 & 0 & $\mathrm{~m}$ & h 6 \\
\hline 13 & $\mathrm{~h}$ & 0 & 2.213997 & 4.518952 & -0.273639 & 0 & $\mathrm{~m}$ & \\
\hline 14 & $\mathrm{c}$ & 0 & 3.026340 & 0.732631 & -0.052311 & 0 & $\mathrm{~m}$ & h 5 \\
\hline 15 & $\mathrm{c}$ & 0 & 3.026585 & -0.731905 & -0.052382 & 0 & $\mathrm{~m}$ & h 8 \\
\hline 16 & $\mathrm{c}$ & 0 & 2.430129 & -3.455177 & -0.210019 & 0 & $\mathrm{~m}$ & h 9 \\
\hline 17 & $\mathrm{~h}$ & 0 & 2.215540 & -4.518469 & -0.274219 & 0 & $\mathrm{~m}$ & \\
\hline 18 & $\mathrm{c}$ & 0 & -1.368005 & -1.261071 & 1.439055 & 0 & $\mathrm{~m}$ & \\
\hline 19 & $\mathrm{~h}$ & 0 & -0.330758 & -1.294809 & 1.786565 & 0 & $\mathrm{~m}$ & \\
\hline 20 & $\mathrm{~h}$ & 0 & -1.568456 & -2.174696 & 0.867922 & 0 & $\mathrm{~m}$ & \\
\hline 21 & $\mathrm{~h}$ & 0 & -2.016698 & -1.276039 & 2.320570 & 0 & $\mathrm{~m}$ & \\
\hline 22 & $\mathrm{c}$ & 0 & -1.367155 & 1.257604 & 1.441305 & 0 & $\mathrm{~m}$ & \\
\hline 23 & $\mathrm{~h}$ & 0 & -1.566113 & 2.172432 & 0.871586 & 0 & $\mathrm{~m}$ & \\
\hline 24 & $\mathrm{~h}$ & 0 & -0.330111 & 1.289549 & 1.789592 & 0 & $\mathrm{~m}$ & \\
\hline 25 & $\mathrm{~h}$ & 0 & -2.016337 & 1.271897 & 2.322465 & 0 & $\mathrm{~m}$ & \\
\hline 26 & $\mathrm{c}$ & 0 & -3.091619 & -0.000141 & 0.108540 & 0 & $\mathrm{~m}$ & \\
\hline 27 & $\mathrm{c}$ & 0 & -4.122233 & 0.000061 & 1.066572 & 0 & $\mathrm{~m}$ & \\
\hline 28 & $\mathrm{~h}$ & 0 & -3.875788 & -0.000164 & 2.124709 & 0 & $\mathrm{~m}$ & \\
\hline 29 & $\mathrm{c}$ & 0 & -5.465623 & 0.000483 & 0.697921 & 0 & $\mathrm{~m}$ & \\
\hline 30 & $\mathrm{~h}$ & 0 & -6.233123 & 0.000628 & 1.467857 & 0 & $\mathrm{~m}$ & \\
\hline 31 & $\mathrm{c}$ & 0 & -5.824895 & 0.000719 & -0.651305 & 0 & $\mathrm{~m}$ & \\
\hline 32 & $\mathrm{~h}$ & 0 & -6.871670 & 0.001065 & -0.943161 & 0 & $\mathrm{~m}$ & \\
\hline 33 & $\mathrm{c}$ & 0 & -4.821528 & 0.000505 & -1.616621 & 0 & $\mathrm{~m}$ & \\
\hline 34 & $\mathrm{~h}$ & 0 & -5.079704 & 0.000666 & -2.672678 & 0 & $\mathrm{~m}$ & \\
\hline 35 & $\mathrm{c}$ & 0 & -3.474827 & 0.000057 & -1.240585 & 0 & $\mathrm{~m}$ & \\
\hline 36 & $\mathrm{~h}$ & 0 & -2.726950 & -0.000095 & -2.025431 & 0 & $\mathrm{~m}$ & \\
\hline 37 & $\mathrm{c}$ & 0 & 3.745182 & 3.027399 & 0.008009 & 0 & $\mathrm{~m}$ & \\
\hline 38 & $\mathrm{~h}$ & 0 & 4.539570 & 3.761300 & 0.113198 & 0 & $\mathrm{~m}$ & \\
\hline 39 & $\mathrm{c}$ & 0 & 4.049816 & 1.660544 & 0.084951 & 0 & $\mathrm{~m}$ & \\
\hline 40 & $\mathrm{~h}$ & 0 & 5.076226 & 1.339452 & 0.245165 & 0 & $\mathrm{~m}$ & \\
\hline 41 & $\mathrm{c}$ & 0 & 4.050406 & -1.659466 & 0.084669 & 0 & $\mathrm{~m}$ & \\
\hline 42 & $\mathrm{~h}$ & 0 & 5.076708 & -1.338026 & 0.244883 & 0 & $\mathrm{~m}$ & \\
\hline
\end{tabular}




$\begin{array}{llllllll}43 & \mathrm{c} & 0 & 3.746249 & -3.026410 & 0.007556 & 0 & \mathrm{~m} \\ 44 & \mathrm{~h} & 0 & 4.540896 & -3.760054 & 0.112591 & 0 & \mathrm{~m}\end{array}$

No. 21

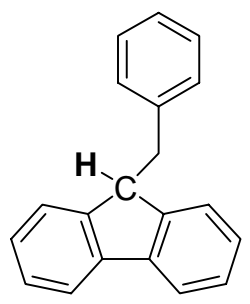

neutral molecule

Charge $=0$ Multiplicity $=1$ for low level calculation on real system.

Charge $=0$ Multiplicity $=1$ for high level calculation on model system .

Charge $=0$ Multiplicity $=1$ for low level calculation on model system .

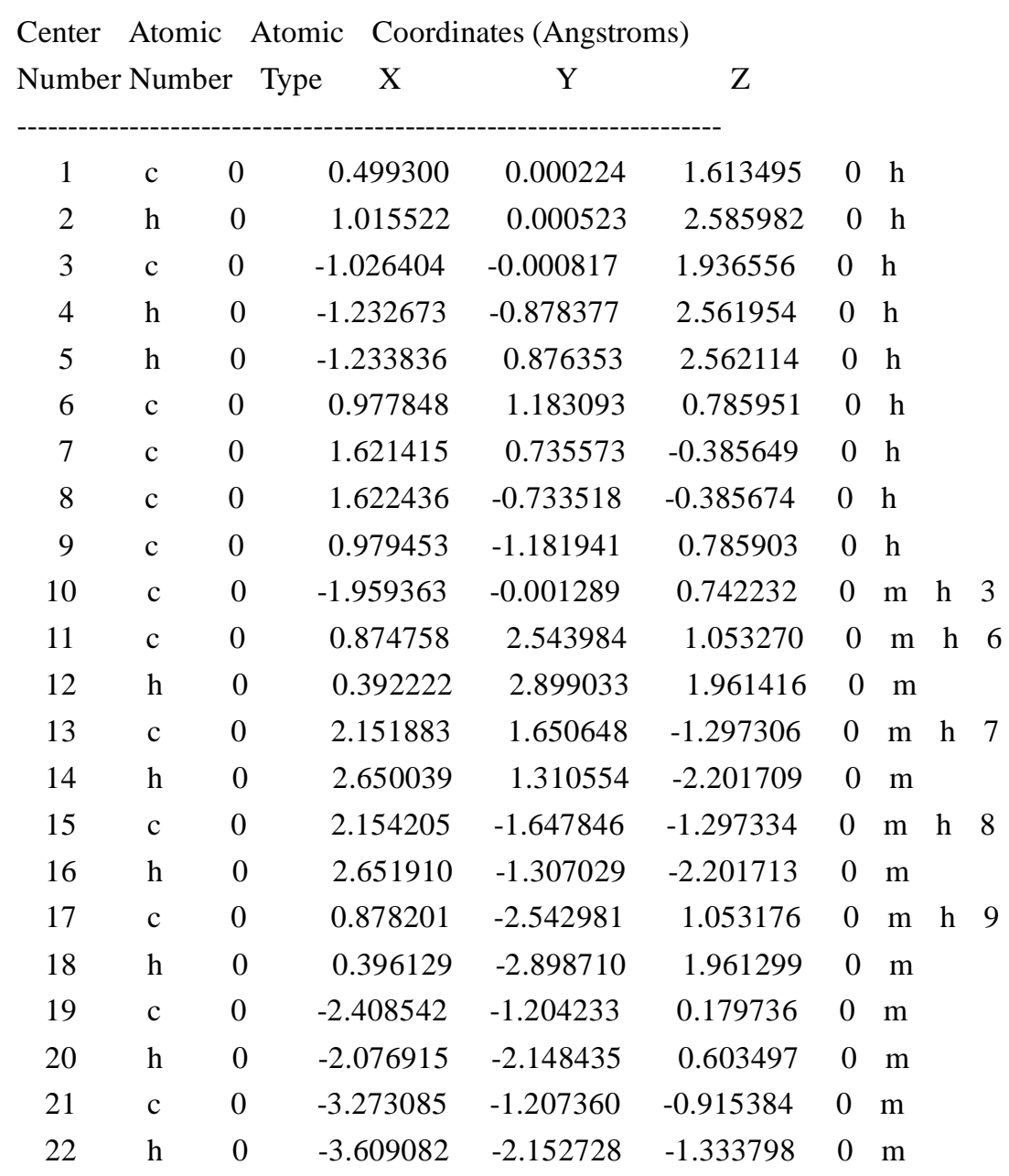




$\begin{array}{llllllll}23 & \mathrm{c} & 0 & -3.709262 & -0.002187 & -1.467773 & 0 & \mathrm{~m} \\ 24 & \mathrm{~h} & 0 & -4.385778 & -0.002551 & -2.318453 & 0 & \mathrm{~m} \\ 25 & \mathrm{c} & 0 & -3.274644 & 1.203441 & -0.915139 & 0 & \mathrm{~m} \\ 26 & \mathrm{~h} & 0 & -3.611841 & 2.148453 & -1.333389 & 0 & \mathrm{~m} \\ 27 & \mathrm{c} & 0 & -2.410038 & 1.201202 & 0.179936 & 0 & \mathrm{~m} \\ 28 & \mathrm{~h} & 0 & -2.079506 & 2.145740 & 0.603794 & 0 & \mathrm{~m} \\ 29 & \mathrm{c} & 0 & 1.406428 & 3.461025 & 0.139075 & 0 & \mathrm{~m} \\ 30 & \mathrm{~h} & 0 & 1.331054 & 4.526657 & 0.338623 & 0 & \mathrm{~m} \\ 31 & \mathrm{c} & 0 & 2.036772 & 3.015979 & -1.027878 & 0 & \mathrm{~m} \\ 32 & \mathrm{~h} & 0 & 2.444821 & 3.739523 & -1.728723 & 0 & \mathrm{~m} \\ 33 & \mathrm{c} & 0 & 2.040950 & -3.013334 & -1.027950 & 0 & \mathrm{~m} \\ 34 & \mathrm{~h} & 0 & 2.450018 & -3.736314 & -1.728782 & 0 & \mathrm{~m} \\ 35 & \mathrm{c} & 0 & 1.411139 & -3.459278 & 0.138989 & 0 & \mathrm{~m} \\ 36 & \mathrm{~h} & 0 & 1.337213 & -4.525018 & 0.338505 & 0 & \mathrm{~m}\end{array}$

radical

Charge $=0$ Multiplicity $=2$ for low level calculation on real system.

Charge $=0$ Multiplicity $=2$ for high level calculation on model system.

Charge $=0$ Multiplicity $=2$ for low level calculation on model system.

\begin{tabular}{|c|c|c|c|c|c|c|c|c|}
\hline Center & Atomic & & Coordi & ates (Angstro & ms) & & & \\
\hline Number & Numbe & & Type & $\mathrm{Y}$ & Z & & & \\
\hline 1 & c & 0 & -0.219639 & -0.211461 & -0.782857 & 0 & $\mathrm{~h}$ & \\
\hline 2 & $\mathrm{c}$ & 0 & 1.063084 & -0.647810 & -1.426709 & 0 & $\mathrm{~h}$ & \\
\hline 3 & $\mathrm{~h}$ & 0 & 0.948285 & -1.673938 & -1.799995 & 0 & $\mathrm{~h}$ & \\
\hline 4 & $\mathrm{~h}$ & 0 & 1.256703 & -0.025851 & -2.311565 & 0 & $\mathrm{~h}$ & \\
\hline 5 & $\mathrm{c}$ & 0 & -0.563856 & 1.137581 & -0.409158 & 0 & $\mathrm{~h}$ & \\
\hline 6 & $\mathrm{c}$ & 0 & -1.875205 & 1.139742 & 0.161470 & 0 & $\mathrm{~h}$ & \\
\hline 7 & $\mathrm{c}$ & 0 & -2.359010 & -0.244079 & 0.154682 & 0 & $\mathrm{~h}$ & \\
\hline 8 & $\mathrm{c}$ & 0 & -1.329668 & -1.053923 & -0.425864 & 0 & $\mathrm{~h}$ & \\
\hline 9 & $\mathrm{c}$ & 0 & 2.284833 & -0.580140 & -0.513181 & 0 & $\mathrm{~m}$ & $\mathrm{~h}$ \\
\hline 10 & $\mathrm{c}$ & 0 & 0.150427 & 2.338507 & -0.528504 & 0 & $\mathrm{~m}$ & $\mathrm{~h}$ \\
\hline 11 & $\mathrm{~h}$ & 0 & 1.154163 & 2.347111 & -0.944282 & 0 & $\mathrm{~m}$ & \\
\hline 12 & $\mathrm{c}$ & 0 & -2.451502 & 2.326270 & 0.594750 & 0 & $\mathrm{~m}$ & $\mathrm{~h}$ \\
\hline 13 & $\mathrm{~h}$ & 0 & -3.448639 & 2.337889 & 1.028285 & 0 & $\mathrm{~m}$ & \\
\hline 14 & $\mathrm{c}$ & 0 & -3.547160 & -0.818615 & 0.583889 & 0 & $\mathrm{~m}$ & $\mathrm{~h}$ \\
\hline 15 & $\mathrm{~h}$ & 0 & -4.333451 & -0.211204 & 1.025557 & 0 & $\mathrm{~m}$ & \\
\hline 16 & $\mathrm{c}$ & 0 & -1.523339 & -2.437951 & -0.561457 & 0 & $\mathrm{~m}$ & $\mathrm{~h}$ \\
\hline 17 & $\mathrm{~h}$ & 0 & -0.752573 & -3.067758 & -0.998866 & 0 & $\mathrm{~m}$ & \\
\hline 18 & $\mathrm{c}$ & 0 & 2.256624 & -1.149831 & 0.767205 & 0 & $\mathrm{~m}$ & \\
\hline 19 & $\mathrm{~h}$ & 0 & 1.343466 & -1.620004 & 1.123570 & 0 & $\mathrm{~m}$ & \\
\hline
\end{tabular}




$\begin{array}{lllrrrrc}20 & \mathrm{c} & 0 & 3.383295 & -1.112412 & 1.587803 & 0 & \mathrm{~m} \\ 21 & \mathrm{~h} & 0 & 3.342776 & -1.558460 & 2.578244 & 0 & \mathrm{~m} \\ 22 & \mathrm{c} & 0 & 4.558666 & -0.502412 & 1.142745 & 0 & \mathrm{~m} \\ 23 & \mathrm{~h} & 0 & 5.435736 & -0.471573 & 1.783654 & 0 & \mathrm{~m} \\ 24 & \mathrm{c} & 0 & 4.597072 & 0.069217 & -0.128963 & 0 & \mathrm{~m} \\ 25 & \mathrm{~h} & 0 & 5.505242 & 0.548587 & -0.485426 & 0 & \mathrm{~m} \\ 26 & \mathrm{c} & 0 & 3.466711 & 0.030081 & -0.948513 & 0 & \mathrm{~m} \\ 27 & \mathrm{~h} & 0 & 3.504599 & 0.476002 & -1.940428 & 0 & \mathrm{~m} \\ 28 & \mathrm{c} & 0 & -0.440988 & 3.524447 & -0.089409 & 0 & \mathrm{~m} \\ 29 & \mathrm{~h} & 0 & 0.103562 & 4.460839 & -0.175386 & 0 & \mathrm{~m} \\ 30 & \mathrm{c} & 0 & -1.727695 & 3.520994 & 0.465466 & 0 & \mathrm{~m} \\ 31 & \mathrm{~h} & 0 & -2.170374 & 4.454677 & 0.801745 & 0 & \mathrm{~m} \\ 32 & \mathrm{c} & 0 & -3.725461 & -2.203303 & 0.442170 & 0 & \mathrm{~m} \\ 33 & \mathrm{~h} & 0 & -4.652560 & -2.661303 & 0.775990 & 0 & \mathrm{~m} \\ 34 & \mathrm{c} & 0 & -2.722997 & -3.002030 & -0.124891 & 0 & \mathrm{~m} \\ 35 & \mathrm{~h} & 0 & -2.882086 & -4.072238 & -0.225134 & 0 & \mathrm{~m} \\ & - & & & & \end{array}$

No. 22

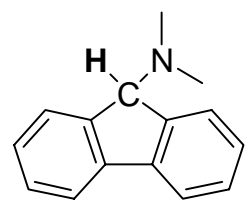

neutral molecule

Charge $=0$ Multiplicity $=1$ for low level calculation on real system.

Charge $=0$ Multiplicity $=1$ for high level calculation on model system.

Charge $=0$ Multiplicity $=1$ for low level calculation on model system .

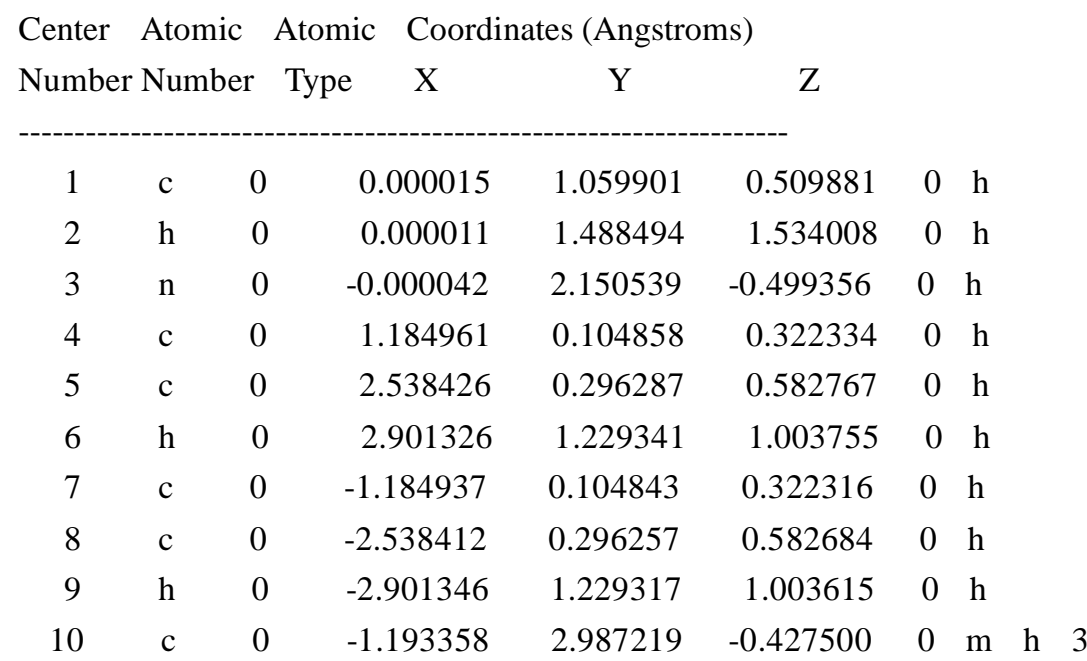




$\begin{array}{lllrrrrrrr}11 & \mathrm{~h} & 0 & -2.083114 & 2.413184 & -0.689167 & 0 & \mathrm{~m} & & \\ 12 & \mathrm{~h} & 0 & -1.098052 & 3.798291 & -1.156133 & 0 & \mathrm{~m} & & \\ 13 & \mathrm{~h} & 0 & -1.350684 & 3.446171 & 0.570246 & 0 & \mathrm{~m} & & \\ 14 & \mathrm{c} & 0 & 1.193368 & 2.987098 & -0.427898 & 0 & \mathrm{~m} & \mathrm{~h} & 3 \\ 15 & \mathrm{~h} & 0 & 1.098346 & 3.797603 & -1.157209 & 0 & \mathrm{~m} & & \\ 16 & \mathrm{~h} & 0 & 1.350602 & 3.446790 & 0.569525 & 0 & \mathrm{~m} & \\ 17 & \mathrm{~h} & 0 & 2.083075 & 2.412732 & -0.688969 & 0 & \mathrm{~m} & & \\ 18 & \mathrm{c} & 0 & 3.443958 & -0.739945 & 0.318633 & 0 & \mathrm{~m} & \mathrm{~h} & 5 \\ 19 & \mathrm{~h} & 0 & 4.501658 & -0.592040 & 0.519189 & 0 & \mathrm{~m} & & \\ 20 & \mathrm{c} & 0 & 0.734143 & -1.148298 & -0.138329 & 0 & \mathrm{~m} & \mathrm{~h} & 4 \\ 21 & \mathrm{c} & 0 & -0.734176 & -1.148265 & -0.138310 & 0 & \mathrm{~m} & \mathrm{~h} & 7 \\ 22 & \mathrm{c} & 0 & -3.443919 & -0.739999 & 0.318538 & 0 & \mathrm{~m} & \mathrm{~h} & 8 \\ 23 & \mathrm{~h} & 0 & -4.501622 & -0.592099 & 0.519081 & 0 & \mathrm{~m} & \\ 24 & \mathrm{c} & 0 & 2.996670 & -1.961826 & -0.189080 & 0 & \mathrm{~m} & & \\ 25 & \mathrm{~h} & 0 & 3.709549 & -2.756623 & -0.392178 & 0 & \mathrm{~m} & & \\ 26 & \mathrm{c} & 0 & 1.634098 & -2.178975 & -0.408543 & 0 & \mathrm{~m} & & \\ 27 & \mathrm{~h} & 0 & 1.283139 & -3.144534 & -0.763673 & 0 & \mathrm{~m} & & \\ 28 & \mathrm{c} & 0 & -1.634152 & -2.178924 & -0.408512 & 0 & \mathrm{~m} & & \\ 29 & \mathrm{~h} & 0 & -1.283214 & -3.144496 & -0.763624 & 0 & \mathrm{~m} & & \\ 30 & \mathrm{c} & 0 & -2.996661 & -1.961786 & -0.189111 & 0 & \mathrm{~m} & & \\ 31 & \mathrm{~h} & 0 & -3.709541 & -2.756586 & -0.392189 & 0 & \mathrm{~m} & & \\ & & & & & & & & \end{array}$

radical

Charge $=0$ Multiplicity $=2$ for low level calculation on real system.

Charge $=0$ Multiplicity $=2$ for high level calculation on model system.

Charge $=0$ Multiplicity $=2$ for low level calculation on model system.

Center Atomic Atomic Coordinates (Angstroms)

Number Number Type $\quad X \quad$ Y $\quad$ Z

\begin{tabular}{|c|c|c|c|c|c|c|c|}
\hline 1 & $\mathrm{c}$ & 0 & -0.004691 & 0.962678 & -0.009330 & 0 & h \\
\hline 2 & $\mathrm{n}$ & 0 & -0.006106 & 2.338390 & -0.017674 & 0 & h \\
\hline 3 & $\mathrm{c}$ & 0 & -1.170551 & 0.100964 & -0.001437 & 0 & h \\
\hline 4 & $\mathrm{c}$ & 0 & -2.552121 & 0.361652 & 0.066732 & 0 & $\mathrm{~h}$ \\
\hline 5 & $\mathrm{~h}$ & 0 & -2.934580 & 1.376027 & 0.106704 & 0 & $\mathrm{~h}$ \\
\hline 6 & $\mathrm{c}$ & 0 & 1.171649 & 0.114953 & -0.011743 & 0 & $\mathrm{~h}$ \\
\hline 7 & $\mathrm{c}$ & 0 & 2.548420 & 0.394903 & -0.090143 & 0 & h \\
\hline 8 & $\mathrm{~h}$ & 0 & 2.914873 & 1.414592 & -0.149907 & 0 & $\mathrm{~h}$ \\
\hline 9 & $\mathrm{c}$ & 0 & 1.007735 & 3.124293 & 0.665443 & 0 & $\begin{array}{lll}m & h & 2\end{array}$ \\
\hline 10 & $\mathrm{~h}$ & 0 & 1.608147 & 2.483748 & 1.310703 & 0 & $\mathrm{~m}$ \\
\hline 11 & $\mathrm{~h}$ & 0 & 1.670048 & 3.649128 & -0.039400 & 0 & $\mathrm{~m}$ \\
\hline 12 & $\mathrm{~h}$ & 0 & 0.521785 & 3.882885 & 1.295510 & 0 & $\mathrm{~m}$ \\
\hline
\end{tabular}




$\begin{array}{lllrrrllll}13 & \mathrm{c} & 0 & -1.065325 & 3.114747 & -0.636778 & 0 & \mathrm{~m} & \mathrm{~h} & 2 \\ 14 & \mathrm{~h} & 0 & -0.622525 & 3.951542 & -1.193855 & 0 & \mathrm{~m} & & \\ 15 & \mathrm{~h} & 0 & -1.763542 & 3.537795 & 0.102192 & 0 & \mathrm{~m} & \\ 16 & \mathrm{~h} & 0 & -1.622050 & 2.495771 & -1.340545 & 0 & \mathrm{~m} & & \\ 17 & \mathrm{c} & 0 & -3.449474 & -0.704185 & 0.102204 & 0 & \mathrm{~m} & \mathrm{~h} & 4 \\ 18 & \mathrm{~h} & 0 & -4.516040 & -0.500644 & 0.151982 & 0 & \mathrm{~m} & & \\ 19 & \mathrm{c} & 0 & -0.719001 & -1.257317 & 0.005227 & 0 & \mathrm{~m} & \mathrm{~h} & 3 \\ 20 & \mathrm{c} & 0 & 0.738280 & -1.248299 & -0.000656 & 0 & \mathrm{~m} & \mathrm{~h} & 6 \\ 21 & \mathrm{c} & 0 & 3.460722 & -0.658663 & -0.113994 & 0 & \mathrm{~m} & \mathrm{~h} & 7 \\ 22 & \mathrm{~h} & 0 & 4.524146 & -0.441314 & -0.171908 & 0 & \mathrm{~m} & & \\ 23 & \mathrm{c} & 0 & -2.997880 & -2.032287 & 0.082398 & 0 & \mathrm{~m} & & \\ 24 & \mathrm{~h} & 0 & -3.715384 & -2.847800 & 0.109366 & 0 & \mathrm{~m} & & \\ 25 & \mathrm{c} & 0 & -1.628711 & -2.310100 & 0.043353 & 0 & \mathrm{~m} & & \\ 26 & \mathrm{~h} & 0 & -1.279503 & -3.339968 & 0.051550 & 0 & \mathrm{~m} & & \\ 27 & \mathrm{c} & 0 & 1.662409 & -2.288982 & -0.027087 & 0 & \mathrm{~m} & & \\ 28 & \mathrm{~h} & 0 & 1.327762 & -3.323686 & -0.021171 & 0 & \mathrm{~m} & & \\ 29 & \mathrm{c} & 0 & 3.027450 & -1.992452 & -0.073521 & 0 & \mathrm{~m} & & \\ 30 & \mathrm{~h} & 0 & 3.756138 & -2.798247 & -0.091526 & 0 & \mathrm{~m} & & \end{array}$

No. 23

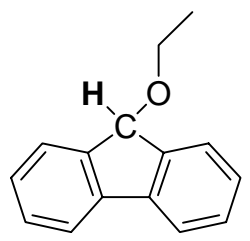

neutral molecule

Charge $=0$ Multiplicity $=1$ for low level calculation on real system.

Charge $=0$ Multiplicity $=1$ for high level calculation on model system.

Charge $=0$ Multiplicity $=1$ for low level calculation on model system.

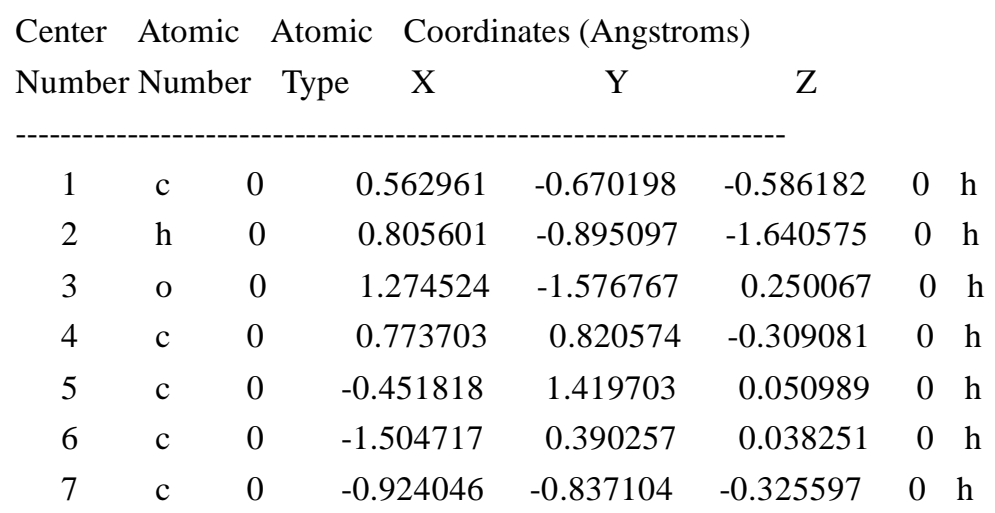




$\begin{array}{rrrrrrrrrr}8 & \mathrm{c} & 0 & 2.641128 & -1.778207 & -0.082725 & 0 & \mathrm{~m} & \mathrm{~h} & 3 \\ 9 & \mathrm{~h} & 0 & 2.744383 & -1.943785 & -1.168999 & 0 & \mathrm{~m} & & \\ 10 & \mathrm{~h} & 0 & 3.239799 & -0.891492 & 0.173894 & 0 & \mathrm{~m} & \\ 11 & \mathrm{c} & 0 & 1.925053 & 1.593311 & -0.410507 & 0 & \mathrm{~m} & \mathrm{~h} & 4 \\ 12 & \mathrm{~h} & 0 & 2.869303 & 1.156139 & -0.722245 & 0 & \mathrm{~m} & & \\ 13 & \mathrm{c} & 0 & -0.521102 & 2.783665 & 0.331581 & 0 & \mathrm{~m} & \mathrm{~h} & 5 \\ 14 & \mathrm{~h} & 0 & -1.464993 & 3.249315 & 0.603438 & 0 & \mathrm{~m} & & \\ 15 & \mathrm{c} & 0 & -2.869593 & 0.468155 & 0.316579 & 0 & \mathrm{~m} & \mathrm{~h} & 6 \\ 16 & \mathrm{~h} & 0 & -3.331265 & 1.412287 & 0.594178 & 0 & \mathrm{~m} & & \\ 17 & \mathrm{c} & 0 & -1.684729 & -1.995507 & -0.392627 & 0 & \mathrm{~m} & \mathrm{~h} & 7 \\ 18 & \mathrm{~h} & 0 & -1.223445 & -2.944676 & -0.651402 & 0 & \mathrm{~m} & & \\ 19 & \mathrm{c} & 0 & 3.138782 & -2.986621 & 0.694814 & 0 & \mathrm{~m} & & \\ 20 & \mathrm{~h} & 0 & 2.564424 & -3.879398 & 0.427447 & 0 & \mathrm{~m} & \\ 21 & \mathrm{~h} & 0 & 4.196663 & -3.172220 & 0.477696 & 0 & \mathrm{~m} & \\ 22 & \mathrm{~h} & 0 & 3.029582 & -2.818986 & 1.771050 & 0 & \mathrm{~m} & \\ 23 & \mathrm{c} & 0 & 1.857909 & 2.962008 & -0.116876 & 0 & \mathrm{~m} & & \\ 24 & \mathrm{~h} & 0 & 2.754994 & 3.571445 & -0.185177 & 0 & \mathrm{~m} & & \\ 25 & \mathrm{c} & 0 & 0.645687 & 3.549649 & 0.252896 & 0 & \mathrm{~m} & & \\ 26 & \mathrm{~h} & 0 & 0.606907 & 4.613417 & 0.472232 & 0 & \mathrm{~m} & \\ 27 & \mathrm{c} & 0 & -3.638650 & -0.696629 & 0.238579 & 0 & \mathrm{~m} & & \\ 28 & \mathrm{~h} & 0 & -4.702987 & -0.652233 & 0.454326 & 0 & \mathrm{~m} & & \\ 29 & \mathrm{c} & 0 & -3.053695 & -1.918959 & -0.108417 & 0 & \mathrm{~m} & & \\ 30 & \mathrm{~h} & 0 & -3.666396 & -2.815157 & -0.156459 & 0 & \mathrm{~m} & & \\ ------------------------------------------------------- & & & & \end{array}$

radical

Charge $=0$ Multiplicity $=2$ for low level calculation on real system.

Charge $=0$ Multiplicity $=2$ for high level calculation on model system.

Charge $=0$ Multiplicity $=2$ for low level calculation on model system.

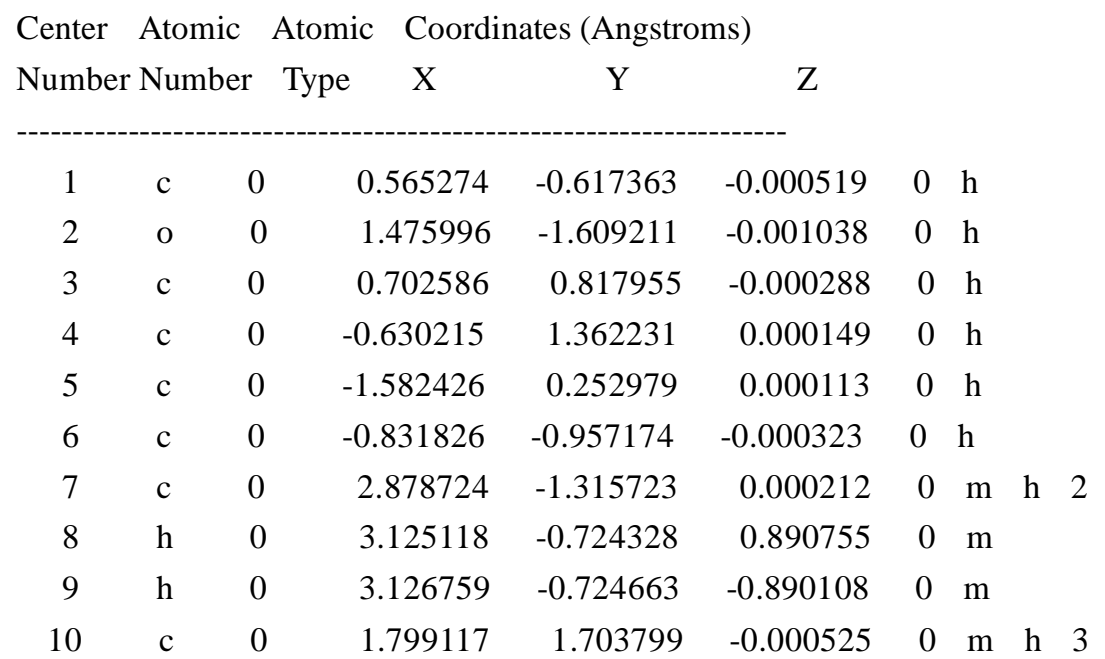




\begin{tabular}{|c|c|c|c|c|c|c|c|c|}
\hline 11 & $\mathrm{~h}$ & 0 & 2.820611 & 1.342056 & -0.000930 & 0 & $\mathrm{~m}$ & \\
\hline 12 & c & 0 & -0.835048 & 2.736350 & 0.000410 & 0 & $\mathrm{~m}$ & h 4 \\
\hline 13 & $\mathrm{~h}$ & 0 & -1.844163 & 3.141166 & 0.000715 & 0 & $\mathrm{~m}$ & \\
\hline 14 & $\mathrm{c}$ & 0 & -2.973249 & 0.209529 & 0.000441 & 0 & $\mathrm{~m}$ & h \\
\hline 15 & $\mathrm{~h}$ & 0 & -3.560091 & 1.124881 & 0.000775 & 0 & $\mathrm{~m}$ & \\
\hline 16 & $\mathrm{c}$ & 0 & -1.476355 & -2.201093 & -0.000436 & 0 & $\mathrm{~m} \quad \mathrm{~h}$ & h 6 \\
\hline 17 & $\mathrm{~h}$ & 0 & -0.898734 & -3.120745 & -0.000764 & 0 & $\mathrm{~m}$ & \\
\hline 18 & $\mathrm{c}$ & 0 & 3.620134 & -2.640349 & 0.001104 & 0 & $\mathrm{~m}$ & \\
\hline 19 & $\mathrm{~h}$ & 0 & 3.364306 & -3.226625 & -0.886934 & 0 & $\mathrm{~m}$ & \\
\hline 20 & $\mathrm{~h}$ & 0 & 3.362734 & -3.226308 & 0.888894 & 0 & $\mathrm{~m}$ & \\
\hline 21 & $\mathrm{~h}$ & 0 & 4.701180 & -2.464341 & 0.002021 & 0 & $\mathrm{~m}$ & \\
\hline 22 & $\mathrm{c}$ & 0 & 1.570384 & 3.078407 & -0.000233 & 0 & $\mathrm{~m}$ & \\
\hline 23 & $\mathrm{~h}$ & 0 & 2.418946 & 3.757319 & -0.000400 & 0 & $\mathrm{~m}$ & \\
\hline 24 & $\mathrm{c}$ & 0 & 0.267860 & 3.597552 & 0.000254 & 0 & $\mathrm{~m}$ & \\
\hline 25 & $\mathrm{~h}$ & 0 & 0.114403 & 4.673057 & 0.000456 & 0 & $\mathrm{~m}$ & \\
\hline 26 & $\mathrm{c}$ & 0 & -3.613077 & -1.035621 & 0.000334 & 0 & $\mathrm{~m}$ & \\
\hline 27 & $\mathrm{~h}$ & 0 & -4.698791 & -1.080468 & 0.000572 & 0 & $\mathrm{~m}$ & \\
\hline 28 & $\mathrm{c}$ & 0 & -2.870234 & -2.227404 & -0.000118 & 0 & $\mathrm{~m}$ & \\
\hline 29 & $\mathrm{~h}$ & 0 & -3.390127 & -3.181760 & -0.000198 & 0 & $\mathrm{~m}$ & \\
\hline
\end{tabular}

No. 24<smiles>c1ccc(OC2c3ccccc3-c3ccccc32)cc1</smiles>

neutral molecule

Charge $=0$ Multiplicity $=1$ for low level calculation on real system.

Charge $=0$ Multiplicity $=1$ for high level calculation on model system .

Charge $=0$ Multiplicity $=1$ for low level calculation on model system.

Center Atomic Atomic Coordinates (Angstroms)

Number Number Type $\quad X \quad$ Y $\quad$ Z

$\begin{array}{lllrrrrr}1 & \mathrm{c} & 0 & 0.070733 & -0.263617 & 0.398309 & 0 & \mathrm{~h} \\ 2 & \mathrm{~h} & 0 & -0.314765 & -0.342935 & 1.424263 & 0 & \mathrm{~h} \\ 3 & \mathrm{o} & 0 & -0.952257 & -0.674940 & -0.515055 & 0 & \mathrm{~h} \\ 4 & \mathrm{c} & 0 & 1.288551 & -1.158217 & 0.247632 & 0 & \mathrm{~h} \\ 5 & \mathrm{c} & 0 & 2.441884 & -0.379809 & 0.045065 & 0 & \mathrm{~h}\end{array}$




\begin{tabular}{|c|c|c|c|c|c|c|c|c|}
\hline 6 & $\mathrm{c}$ & 0 & 2.044433 & 1.036577 & -0.025384 & 0 & & \\
\hline 7 & $\mathrm{c}$ & 0 & 0.646789 & 1.125432 & 0.138946 & 0 & $\mathrm{~h}$ & \\
\hline 8 & $\mathrm{c}$ & 0 & -2.272694 & -0.468799 & -0.215841 & 0 & $\mathrm{~m}$ & $\mathrm{~h}$ \\
\hline 9 & $\mathrm{c}$ & 0 & 1.359812 & -2.542109 & 0.314847 & 0 & $\mathrm{~m}$ & $\mathrm{~h}$ \\
\hline 10 & $\mathrm{~h}$ & 0 & 0.461371 & -3.137127 & 0.454690 & 0 & $\mathrm{~m}$ & \\
\hline 11 & $\mathrm{c}$ & 0 & 3.689732 & -0.991865 & -0.073694 & 0 & $\mathrm{~m}$ & $\mathrm{~h}$ \\
\hline 12 & $\mathrm{~h}$ & 0 & 4.589904 & -0.402054 & -0.225851 & 0 & $\mathrm{~m}$ & \\
\hline 13 & $\mathrm{c}$ & 0 & 2.805851 & 2.184947 & -0.238887 & 0 & $\mathrm{~m}$ & $\mathrm{~h}$ \\
\hline 14 & $\mathrm{~h}$ & 0 & 3.884306 & 2.124381 & -0.360314 & 0 & $\mathrm{~m}$ & \\
\hline 15 & $\mathrm{c}$ & 0 & 0.004299 & 2.355546 & 0.067190 & 0 & $\mathrm{~m}$ & $\mathrm{~h}$ \\
\hline 16 & $\mathrm{~h}$ & 0 & -1.073911 & 2.430316 & 0.170493 & 0 & $\mathrm{~m}$ & \\
\hline 17 & $\mathrm{c}$ & 0 & -2.755596 & 0.251475 & 0.883951 & 0 & $\mathrm{~m}$ & \\
\hline 18 & $\mathrm{~h}$ & 0 & -2.079336 & 0.726481 & 1.585643 & 0 & $\mathrm{~m}$ & \\
\hline 19 & $\mathrm{c}$ & 0 & -4.136105 & 0.373983 & 1.075898 & 0 & $\mathrm{~m}$ & \\
\hline 20 & $\mathrm{~h}$ & 0 & -4.498960 & 0.935252 & 1.933156 & 0 & $\mathrm{~m}$ & \\
\hline 21 & $\mathrm{c}$ & 0 & -5.038685 & -0.203093 & 0.186174 & 0 & $\mathrm{~m}$ & \\
\hline 22 & $\mathrm{~h}$ & 0 & -6.108355 & -0.099216 & 0.342420 & 0 & $\mathrm{~m}$ & \\
\hline 23 & $\mathrm{c}$ & 0 & -4.548036 & -0.914281 & -0.914235 & 0 & $\mathrm{~m}$ & \\
\hline 24 & $\mathrm{~h}$ & 0 & -5.237281 & -1.370364 & -1.620193 & 0 & $\mathrm{~m}$ & \\
\hline 25 & $\mathrm{c}$ & 0 & -3.179177 & -1.049337 & -1.116063 & 0 & $\mathrm{~m}$ & \\
\hline 26 & $\mathrm{~h}$ & 0 & -2.782462 & -1.601903 & -1.962035 & 0 & $\mathrm{~m}$ & \\
\hline 27 & $\mathrm{c}$ & 0 & 2.611426 & -3.156484 & 0.184425 & 0 & $\mathrm{~m}$ & \\
\hline 28 & $\mathrm{~h}$ & 0 & 2.688333 & -4.239404 & 0.228675 & 0 & $\mathrm{~m}$ & \\
\hline 29 & $\mathrm{c}$ & 0 & 3.763820 & -2.386082 & -0.002067 & 0 & $\mathrm{~m}$ & \\
\hline 30 & $\mathrm{~h}$ & 0 & 4.728613 & -2.877317 & -0.097040 & 0 & $\mathrm{~m}$ & \\
\hline 31 & $\mathrm{c}$ & 0 & 2.157164 & 3.421510 & -0.304759 & 0 & $\mathrm{~m}$ & \\
\hline 32 & $\mathrm{~h}$ & 0 & 2.737484 & 4.324400 & -0.475365 & 0 & $\mathrm{~m}$ & \\
\hline 33 & $\mathrm{c}$ & 0 & 0.769844 & 3.506744 & -0.160341 & 0 & $\mathrm{~m}$ & \\
\hline 34 & $\mathrm{~h}$ & 0 & 0.278851 & 4.473865 & -0.225103 & 0 & $\mathrm{~m}$ & \\
\hline
\end{tabular}

radical

Charge $=0$ Multiplicity $=2$ for low level calculation on real system.

Charge $=0$ Multiplicity $=2$ for high level calculation on model system.

Charge $=0$ Multiplicity $=2$ for low level calculation on model system.

\begin{tabular}{|c|c|c|c|c|c|c|}
\hline Center & Atomic & Atomic & Coordi & ates (Angstre & ns) & \\
\hline Number & Numbe & Type & $\mathrm{X}$ & $\mathrm{Y}$ & Z & \\
\hline 1 & $\mathrm{c}$ & 0 & 0.211103 & -0.448207 & -0.361013 & 0 \\
\hline 2 & o & 0 & 0.892821 & -1.162847 & -0.701181 & 0 \\
\hline 3 & $\mathrm{c}$ & 0 & 1.472820 & -1.092819 & -0.151591 & 0 \\
\hline 4 & $\mathrm{c}$ & 0 & 2.445917 & -0.076301 & 0.090365 & 0 \\
\hline
\end{tabular}




\begin{tabular}{|c|c|c|c|c|c|c|c|c|}
\hline 5 & $\mathrm{c}$ & 0 & 1.765065 & 1.219268 & 0.007406 & 0 & & \\
\hline 6 & $\mathrm{c}$ & 0 & 0.381073 & 0.979550 & -0.279372 & 0 & $\mathrm{~h}$ & \\
\hline 7 & $\mathrm{c}$ & 0 & -2.149992 & -0.826070 & -0.220588 & 0 & $\mathrm{~m}$ & h 2 \\
\hline 8 & $\mathrm{c}$ & 0 & 1.831391 & -2.447632 & -0.142140 & 0 & $\mathrm{~m}$ & $\mathrm{~h}$ \\
\hline 9 & $\mathrm{~h}$ & 0 & 1.086088 & -3.215484 & -0.327809 & 0 & $\mathrm{~m}$ & \\
\hline 10 & $\mathrm{c}$ & 0 & 3.766679 & -0.427554 & 0.342640 & 0 & $\mathrm{~m}$ & $\mathrm{~h}$ \\
\hline 11 & $\mathrm{~h}$ & 0 & 4.520162 & 0.332956 & 0.532163 & 0 & $\mathrm{~m}$ & \\
\hline 12 & $\mathrm{c}$ & 0 & 2.238342 & 2.519881 & 0.128731 & 0 & $\mathrm{~m}$ & $\mathrm{~h}$ \\
\hline 13 & $\mathrm{~h}$ & 0 & 3.287091 & 2.710305 & 0.343045 & 0 & $\mathrm{~m}$ & \\
\hline 14 & $\mathrm{c}$ & 0 & -0.498031 & 2.060164 & -0.455985 & 0 & & $\mathrm{~h}$ \\
\hline 15 & $\mathrm{~h}$ & 0 & -1.545607 & 1.894975 & -0.684493 & 0 & $\mathrm{~m}$ & \\
\hline 16 & $\mathrm{c}$ & 0 & -2.349923 & -0.348444 & 1.076401 & 0 & $\mathrm{~m}$ & \\
\hline 17 & $\mathrm{~h}$ & 0 & -1.500621 & -0.182503 & 1.730808 & 0 & $\mathrm{~m}$ & \\
\hline 18 & $\mathrm{c}$ & 0 & -3.651901 & -0.095708 & 1.509914 & 0 & $\mathrm{~m}$ & \\
\hline 19 & $\mathrm{~h}$ & 0 & -3.812502 & 0.276731 & 2.518002 & 0 & $\mathrm{~m}$ & \\
\hline 20 & $\mathrm{c}$ & 0 & -4.740817 & -0.325589 & 0.666318 & 0 & $\mathrm{~m}$ & \\
\hline 21 & $\mathrm{~h}$ & 0 & -5.751097 & -0.128132 & 1.012642 & 0 & $\mathrm{~m}$ & \\
\hline 22 & $\mathrm{c}$ & 0 & -4.522992 & -0.815107 & -0.623767 & 0 & $\mathrm{~m}$ & \\
\hline 23 & $\mathrm{~h}$ & 0 & -5.363670 & -1.000372 & -1.286735 & 0 & $\mathrm{~m}$ & \\
\hline 24 & $\mathrm{c}$ & 0 & -3.227503 & -1.067291 & -1.073753 & 0 & $\mathrm{~m}$ & \\
\hline 25 & $\mathrm{~h}$ & 0 & -3.034966 & -1.447331 & -2.071996 & 0 & $\mathrm{~m}$ & \\
\hline 26 & $\mathrm{c}$ & 0 & 3.161955 & -2.781076 & 0.108390 & 0 & $\mathrm{~m}$ & \\
\hline 27 & $\mathrm{~h}$ & 0 & 3.462036 & -3.825385 & 0.118457 & 0 & $\mathrm{~m}$ & \\
\hline 28 & $\mathrm{c}$ & 0 & 4.120116 & -1.784003 & 0.349032 & 0 & $\mathrm{~m}$ & \\
\hline 29 & $\mathrm{~h}$ & 0 & 5.150968 & -2.067259 & 0.543332 & 0 & $\mathrm{~m}$ & \\
\hline 30 & $\mathrm{c}$ & 0 & 1.349375 & 3.589614 & -0.038631 & 0 & $\mathrm{~m}$ & \\
\hline 31 & $\mathrm{~h}$ & 0 & 1.712536 & 4.609380 & 0.054790 & 0 & $\mathrm{~m}$ & \\
\hline 32 & $\mathrm{c}$ & 0 & -0.001742 & 3.357929 & -0.333114 & 0 & $\mathrm{~m}$ & \\
\hline 33 & $\mathrm{~h}$ & 0 & -0.673466 & 4.201260 & -0.468201 & 0 & $\mathrm{~m}$ & \\
\hline
\end{tabular}

No. 25

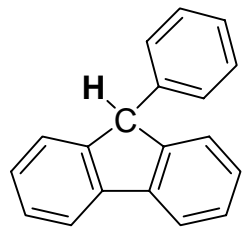

neutral molecule

Charge $=0$ Multiplicity $=1$ for low level calculation on real system.

Charge $=0$ Multiplicity $=1$ for high level calculation on model system .

Charge $=0$ Multiplicity $=1$ for low level calculation on model system. 


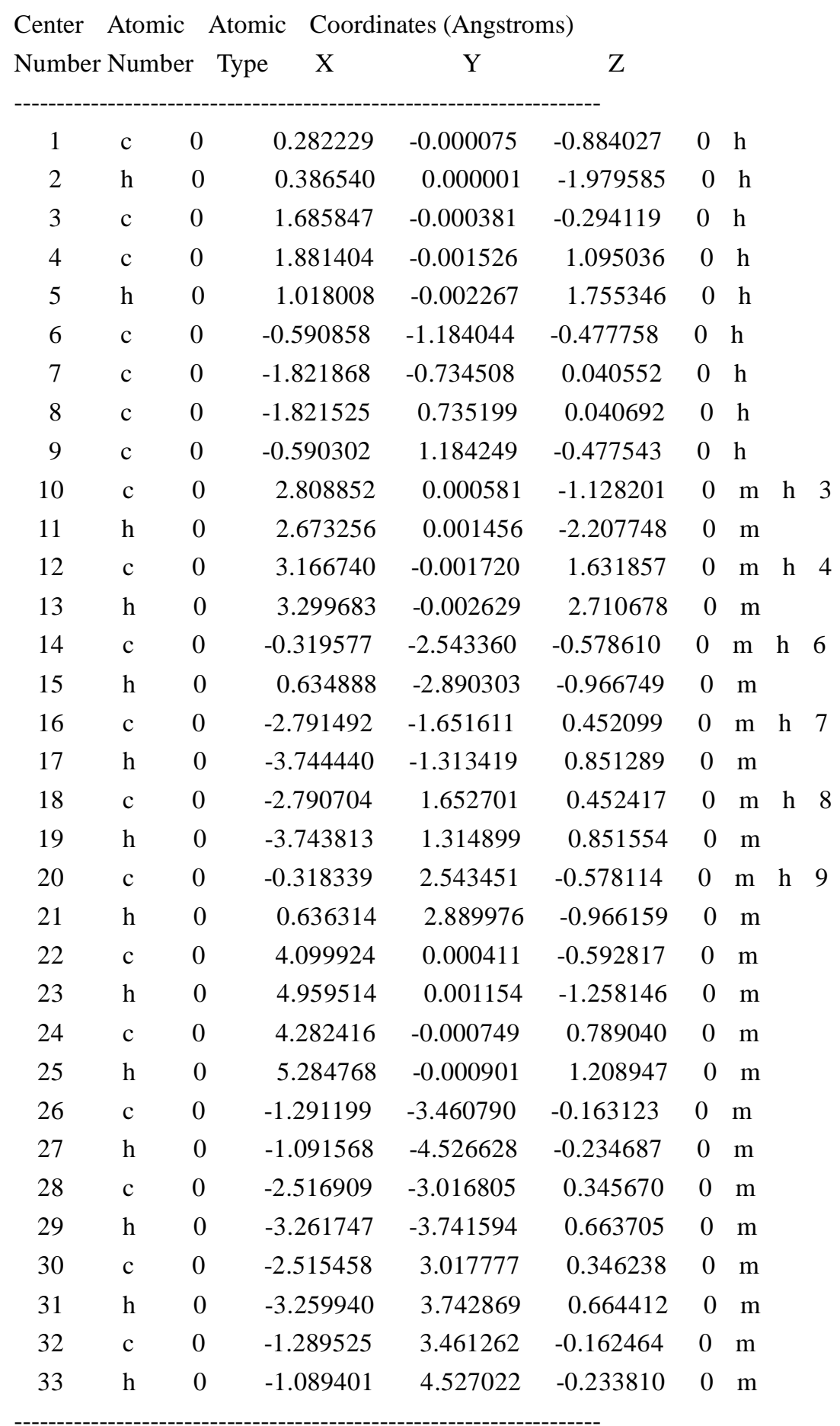

radical

Charge $=0$ Multiplicity $=2$ for low level calculation on real system.

Charge $=0$ Multiplicity $=2$ for high level calculation on model system.

Charge $=0$ Multiplicity $=2$ for low level calculation on model system . 


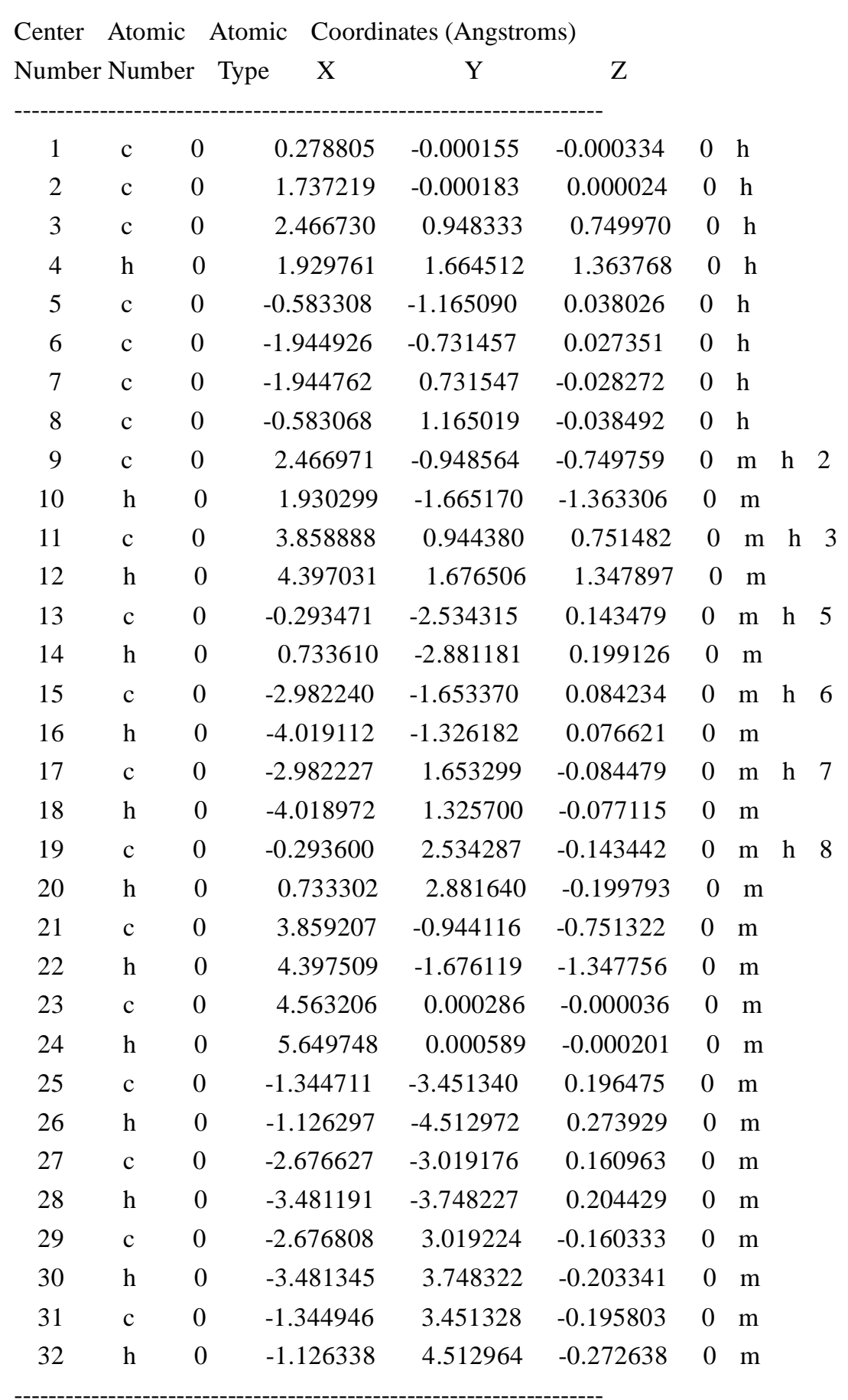


No. 26

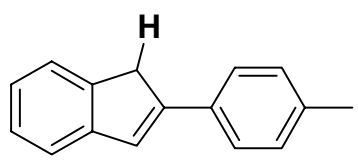

neutral molecule

Charge $=0$ Multiplicity $=1$ for low level calculation on real system.

Charge $=0$ Multiplicity $=1$ for high level calculation on model system .

Charge $=0$ Multiplicity $=1$ for low level calculation on model system.

Center Atomic Atomic Coordinates (Angstroms)

$\begin{array}{lllll}\text { Number Number } & \text { Type } & X & Y & \text { Z }\end{array}$

\begin{tabular}{|c|c|c|c|c|c|c|c|c|}
\hline 1 & $\mathrm{c}$ & 0 & -0.980033 & -1.221042 & 0.001208 & 0 & $\mathrm{~h}$ & \\
\hline 2 & $\mathrm{~h}$ & 0 & -0.769526 & -1.846939 & -0.877562 & 0 & $\mathrm{~h}$ & \\
\hline 3 & $\mathrm{~h}$ & 0 & -0.767566 & -1.844427 & 0.881309 & 0 & $\mathrm{~h}$ & \\
\hline 4 & $\mathrm{c}$ & 0 & -0.142430 & 0.051164 & -0.001485 & 0 & $\mathrm{~h}$ & \\
\hline 5 & $\mathrm{c}$ & 0 & -0.974250 & 1.127348 & -0.002468 & 0 & $\mathrm{~h}$ & \\
\hline 6 & $\mathrm{~h}$ & 0 & -0.668373 & 2.168282 & -0.004613 & 0 & $\mathrm{~h}$ & \\
\hline 7 & $\mathrm{c}$ & 0 & -2.368468 & 0.702779 & -0.000608 & 0 & $\mathrm{~h}$ & \\
\hline 8 & $\mathrm{c}$ & 0 & -2.400779 & -0.708803 & 0.001731 & 0 & $\mathrm{~h}$ & \\
\hline 9 & $\mathrm{c}$ & 0 & 1.322879 & 0.041462 & -0.001908 & 0 & $\mathrm{~m}$ & $\mathrm{~h}$ \\
\hline 10 & $\mathrm{c}$ & 0 & -3.556706 & 1.438295 & -0.000878 & 0 & $\mathrm{~m}$ & h \\
\hline 11 & $\mathrm{~h}$ & 0 & -3.539187 & 2.525374 & -0.002715 & 0 & $\mathrm{~m}$ & \\
\hline 12 & $\mathrm{c}$ & 0 & -3.609974 & -1.389026 & 0.003866 & 0 & $\mathrm{~m}$ & h \\
\hline 13 & $\mathrm{~h}$ & 0 & -3.640656 & -2.476370 & 0.005705 & 0 & $\mathrm{~m}$ & \\
\hline 14 & $\mathrm{c}$ & 0 & 2.039879 & -1.168086 & -0.006533 & 0 & $\mathrm{~m}$ & \\
\hline 15 & $\mathrm{~h}$ & 0 & 1.503793 & -2.112549 & -0.011269 & 0 & $\mathrm{~m}$ & \\
\hline 16 & $\mathrm{c}$ & 0 & 3.433698 & -1.185603 & -0.009375 & 0 & $\mathrm{~m}$ & \\
\hline 17 & $\mathrm{~h}$ & 0 & 3.953347 & -2.141332 & -0.015784 & 0 & $\mathrm{~m}$ & \\
\hline 18 & $\mathrm{c}$ & 0 & 4.175756 & -0.000394 & -0.005491 & 0 & $\mathrm{~m}$ & \\
\hline 19 & $\mathrm{c}$ & 0 & 5.685597 & -0.014492 & 0.013829 & 0 & $\mathrm{~m}$ & \\
\hline 20 & $\mathrm{~h}$ & 0 & 6.079757 & -1.004960 & -0.235451 & 0 & $\mathrm{~m}$ & \\
\hline 21 & $\mathrm{~h}$ & 0 & 6.075794 & 0.254821 & 1.004561 & 0 & $\mathrm{~m}$ & \\
\hline 22 & $\mathrm{~h}$ & 0 & 6.103478 & 0.704397 & -0.700778 & 0 & $\mathrm{~m}$ & \\
\hline 23 & $\mathrm{c}$ & 0 & 3.464431 & 1.210312 & -0.003391 & 0 & $\mathrm{~m}$ & \\
\hline 24 & $\mathrm{~h}$ & 0 & 4.011837 & 2.150765 & -0.005145 & 0 & $\mathrm{~m}$ & \\
\hline 25 & $\mathrm{c}$ & 0 & 2.076279 & 1.233620 & -0.000746 & 0 & $\mathrm{~m}$ & \\
\hline 26 & $\mathrm{~h}$ & 0 & 1.566145 & 2.192452 & -0.000633 & 0 & $\mathrm{~m}$ & \\
\hline 27 & $\mathrm{c}$ & 0 & -4.772062 & 0.748364 & 0.001263 & 0 & $\mathrm{~m}$ & \\
\hline 28 & $\mathrm{~h}$ & 0 & -5.705801 & 1.304815 & 0.001086 & 0 & $\mathrm{~m}$ & \\
\hline 29 & c & 0 & -4.801582 & -0.650129 & 0.003643 & 0 & $\mathrm{~m}$ & \\
\hline
\end{tabular}


radical

Charge $=0$ Multiplicity $=2$ for low level calculation on real system.

Charge $=0$ Multiplicity $=2$ for high level calculation on model system .

Charge $=0$ Multiplicity $=2$ for low level calculation on model system.

Center Atomic Atomic Coordinates (Angstroms)

Number Number Type $\quad \mathrm{X} \quad \mathrm{Y} \quad \mathrm{Z}$

\begin{tabular}{|c|c|c|c|c|c|c|c|c|}
\hline 1 & $\mathrm{c}$ & 0 & 0.992657 & -1.120075 & -0.199792 & 0 & $\mathrm{~h}$ & \\
\hline 2 & $\mathrm{~h}$ & 0 & 0.655231 & -2.128952 & -0.408746 & 0 & $\mathrm{~h}$ & \\
\hline 3 & $\mathrm{c}$ & 0 & 0.159917 & 0.001707 & -0.000581 & 0 & $\mathrm{~h}$ & \\
\hline 4 & $\mathrm{c}$ & 0 & 0.994582 & 1.122274 & 0.199103 & 0 & $\mathrm{~h}$ & \\
\hline 5 & $\mathrm{~h}$ & 0 & 0.658504 & 2.131560 & 0.408262 & 0 & $\mathrm{~h}$ & \\
\hline 6 & $\mathrm{c}$ & 0 & 2.378923 & 0.704175 & 0.125202 & 0 & $\mathrm{~h}$ & \\
\hline 7 & $\mathrm{c}$ & 0 & 2.377773 & -0.704243 & -0.125489 & 0 & $\mathrm{~h}$ & \\
\hline 8 & $\mathrm{c}$ & 0 & -1.311917 & 0.002986 & -0.000899 & 0 & $\mathrm{~m}$ & h 3 \\
\hline 9 & $\mathrm{c}$ & 0 & 3.578357 & 1.394404 & 0.251182 & 0 & $\mathrm{~m}$ & h 6 \\
\hline 10 & $\mathrm{~h}$ & 0 & 3.590710 & 2.464694 & 0.442767 & 0 & $\mathrm{~m}$ & \\
\hline 11 & $\mathrm{c}$ & 0 & 3.576037 & -1.396562 & -0.250841 & 0 & $\mathrm{~m}$ & h 7 \\
\hline 12 & $\mathrm{~h}$ & 0 & 3.586649 & -2.466885 & -0.442365 & 0 & $\mathrm{~m}$ & \\
\hline 13 & $\mathrm{c}$ & 0 & -2.042438 & -1.157608 & 0.313329 & 0 & $\mathrm{~m}$ & \\
\hline 14 & $\mathrm{~h}$ & 0 & -1.511829 & -2.066588 & 0.583854 & 0 & $\mathrm{~m}$ & \\
\hline 15 & $\mathrm{c}$ & 0 & -3.433265 & -1.156013 & 0.310278 & 0 & $\mathrm{~m}$ & \\
\hline 16 & $\mathrm{~h}$ & 0 & -3.966751 & -2.070420 & 0.562430 & 0 & $\mathrm{~m}$ & \\
\hline 17 & $\mathrm{c}$ & 0 & -4.159958 & 0.002822 & -0.000253 & 0 & $\mathrm{~m}$ & \\
\hline 18 & $\mathrm{c}$ & 0 & -5.670566 & -0.004945 & 0.000817 & 0 & $\mathrm{~m}$ & \\
\hline 19 & $\mathrm{~h}$ & 0 & -6.069954 & -0.290926 & 0.982056 & 0 & $\mathrm{~m}$ & \\
\hline 20 & $\mathrm{~h}$ & 0 & -6.069333 & -0.722096 & -0.727938 & 0 & $\mathrm{~m}$ & \\
\hline 21 & $\mathrm{~h}$ & 0 & -6.075262 & 0.981163 & -0.248280 & 0 & $\mathrm{~m}$ & \\
\hline 22 & $\mathrm{c}$ & 0 & -3.434786 & 1.159008 & -0.310093 & 0 & $\mathrm{~m}$ & \\
\hline 23 & $\mathrm{~h}$ & 0 & -3.969057 & 2.072819 & -0.561149 & 0 & $\mathrm{~m}$ & \\
\hline 24 & $\mathrm{c}$ & 0 & -2.041128 & 1.161353 & -0.314128 & 0 & $\mathrm{~m}$ & \\
\hline 25 & $\mathrm{~h}$ & 0 & -1.511883 & 2.071065 & -0.584766 & 0 & $\mathrm{~m}$ & \\
\hline 26 & $\mathrm{c}$ & 0 & 4.787035 & 0.684045 & 0.124318 & 0 & $\mathrm{~m}$ & \\
\hline 27 & $\mathrm{~h}$ & 0 & 5.731145 & 1.213204 & 0.220189 & 0 & $\mathrm{~m}$ & \\
\hline 28 & $\mathrm{c}$ & 0 & 4.785892 & -0.688257 & -0.123409 & 0 & $\mathrm{~m}$ & \\
\hline 29 & $\mathrm{~h}$ & 0 & 5.729133 & -1.219060 & -0.218771 & 0 & $\mathrm{~m}$ & \\
\hline
\end{tabular}


No. 27

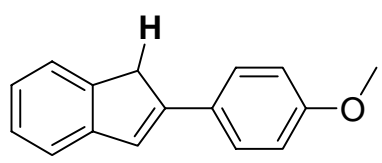

neutral molecule

Charge $=0$ Multiplicity $=1$ for low level calculation on real system.

Charge $=0$ Multiplicity $=1$ for high level calculation on model system .

Charge $=0$ Multiplicity $=1$ for low level calculation on model system.

Center Atomic Atomic Coordinates (Angstroms)

Number Number Type X $\quad$ Y $\quad$ Z

\begin{tabular}{|c|c|c|c|c|c|c|c|c|}
\hline 1 & $\mathrm{c}$ & 0 & 1.446538 & 1.241151 & 0.000176 & 0 & $\mathrm{~h}$ & \\
\hline 2 & $\mathrm{~h}$ & 0 & 1.259847 & 1.873637 & -0.879289 & 0 & $\mathrm{~h}$ & \\
\hline 3 & $\mathrm{~h}$ & 0 & 1.259767 & 1.873443 & 0.879758 & 0 & $\mathrm{~h}$ & \\
\hline 4 & $\mathrm{c}$ & 0 & 0.558716 & 0.003106 & -0.000032 & 0 & $\mathrm{~h}$ & \\
\hline 5 & $\mathrm{c}$ & 0 & 1.347691 & -1.105282 & -0.000214 & 0 & $\mathrm{~h}$ & \\
\hline 6 & $\mathrm{~h}$ & 0 & 1.000994 & -2.133517 & -0.000431 & 0 & $\mathrm{~h}$ & \\
\hline 7 & $\mathrm{c}$ & 0 & 2.757474 & -0.736140 & -0.000126 & 0 & $\mathrm{~h}$ & \\
\hline 8 & $\mathrm{c}$ & 0 & 2.846061 & 0.673179 & 0.000136 & 0 & $\mathrm{~h}$ & \\
\hline 9 & $\mathrm{c}$ & 0 & -0.904080 & 0.074007 & -0.000023 & 0 & $\mathrm{~m} \mathrm{~h}$ & h 4 \\
\hline 10 & $\mathrm{c}$ & 0 & 3.916213 & -1.517364 & -0.000244 & 0 & $\mathrm{~m} \mathrm{~h}$ & h 7 \\
\hline 11 & $\mathrm{~h}$ & 0 & 3.856261 & -2.602986 & -0.000436 & 0 & $\mathrm{~m}$ & \\
\hline 12 & $\mathrm{c}$ & 0 & 4.080943 & 1.305185 & 0.000266 & 0 & $\mathrm{~m}$ & h 8 \\
\hline 13 & $\mathrm{~h}$ & 0 & 4.154306 & 2.390547 & 0.000442 & 0 & $\mathrm{~m}$ & \\
\hline 14 & $\mathrm{c}$ & 0 & -1.573997 & 1.315577 & -0.000282 & 0 & $\mathrm{~m}$ & \\
\hline 15 & $\mathrm{~h}$ & 0 & -0.999822 & 2.237278 & -0.000492 & 0 & $\mathrm{~m}$ & \\
\hline 16 & $\mathrm{c}$ & 0 & -2.958960 & 1.398415 & -0.000298 & 0 & $\mathrm{~m}$ & \\
\hline 17 & $\mathrm{~h}$ & 0 & -3.464275 & 2.359221 & -0.000515 & 0 & $\mathrm{~m}$ & \\
\hline 18 & $\mathrm{c}$ & 0 & -3.736265 & 0.232362 & -0.000053 & 0 & $\mathrm{~m}$ & \\
\hline 19 & o & 0 & -5.088261 & 0.415157 & -0.000090 & 0 & $\mathrm{~m}$ & \\
\hline 20 & $\mathrm{c}$ & 0 & -5.921849 & -0.732729 & 0.000151 & 0 & $\mathrm{~m}$ & \\
\hline 21 & $\mathrm{~h}$ & 0 & -6.947055 & -0.358005 & 0.000073 & 0 & $\mathrm{~m}$ & \\
\hline 22 & $\mathrm{~h}$ & 0 & -5.761058 & -1.348497 & 0.895037 & 0 & $\mathrm{~m}$ & \\
\hline 23 & $\mathrm{~h}$ & 0 & -5.761060 & -1.348870 & -0.894476 & 0 & $\mathrm{~m}$ & \\
\hline 24 & $\mathrm{c}$ & 0 & -3.097601 & -1.015369 & 0.000228 & 0 & $\mathrm{~m}$ & \\
\hline 25 & $\mathrm{~h}$ & 0 & -3.670904 & -1.935563 & 0.000455 & 0 & $\mathrm{~m}$ & \\
\hline 26 & $\mathrm{c}$ & 0 & -1.706795 & -1.080610 & 0.000262 & 0 & $\mathrm{~m}$ & \\
\hline 27 & $\mathrm{~h}$ & 0 & -1.237756 & -2.060092 & 0.000534 & 0 & $\mathrm{~m}$ & \\
\hline 28 & $\mathrm{c}$ & 0 & 5.157992 & -0.875773 & -0.000111 & 0 & $\mathrm{~m}$ & \\
\hline 29 & $\mathrm{~h}$ & 0 & 6.068972 & -1.468862 & -0.000200 & 0 & $\mathrm{~m}$ & \\
\hline
\end{tabular}




$\begin{array}{llllllll}30 & \mathrm{c} & 0 & 5.242983 & 0.520260 & 0.000165 & 0 & \mathrm{~m} \\ 31 & \mathrm{~h} & 0 & 6.217505 & 1.001160 & 0.000265 & 0 & \mathrm{~m}\end{array}$

radical

Charge $=0$ Multiplicity $=2$ for low level calculation on real system.

Charge $=0$ Multiplicity $=2$ for high level calculation on model system.

Charge $=0$ Multiplicity $=2$ for low level calculation on model system.

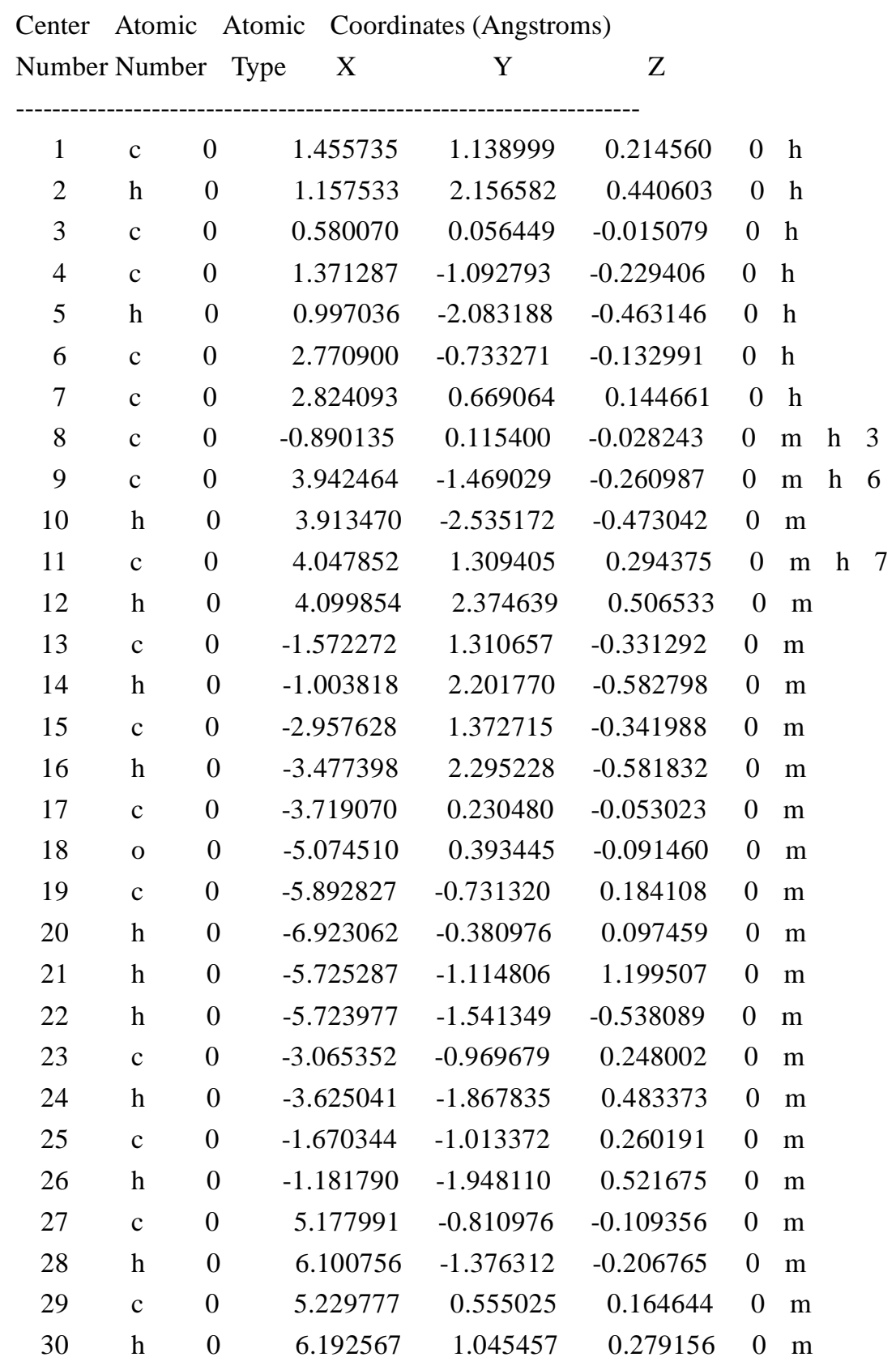


No. 28<smiles>Cc1cccc2ccc3cc4ccccc4cc3c12</smiles>

neutral molecule

Charge $=0$ Multiplicity $=1$ for low level calculation on real system.

Charge $=0$ Multiplicity $=1$ for high level calculation on model system.

Charge $=0$ Multiplicity $=1$ for low level calculation on model system.

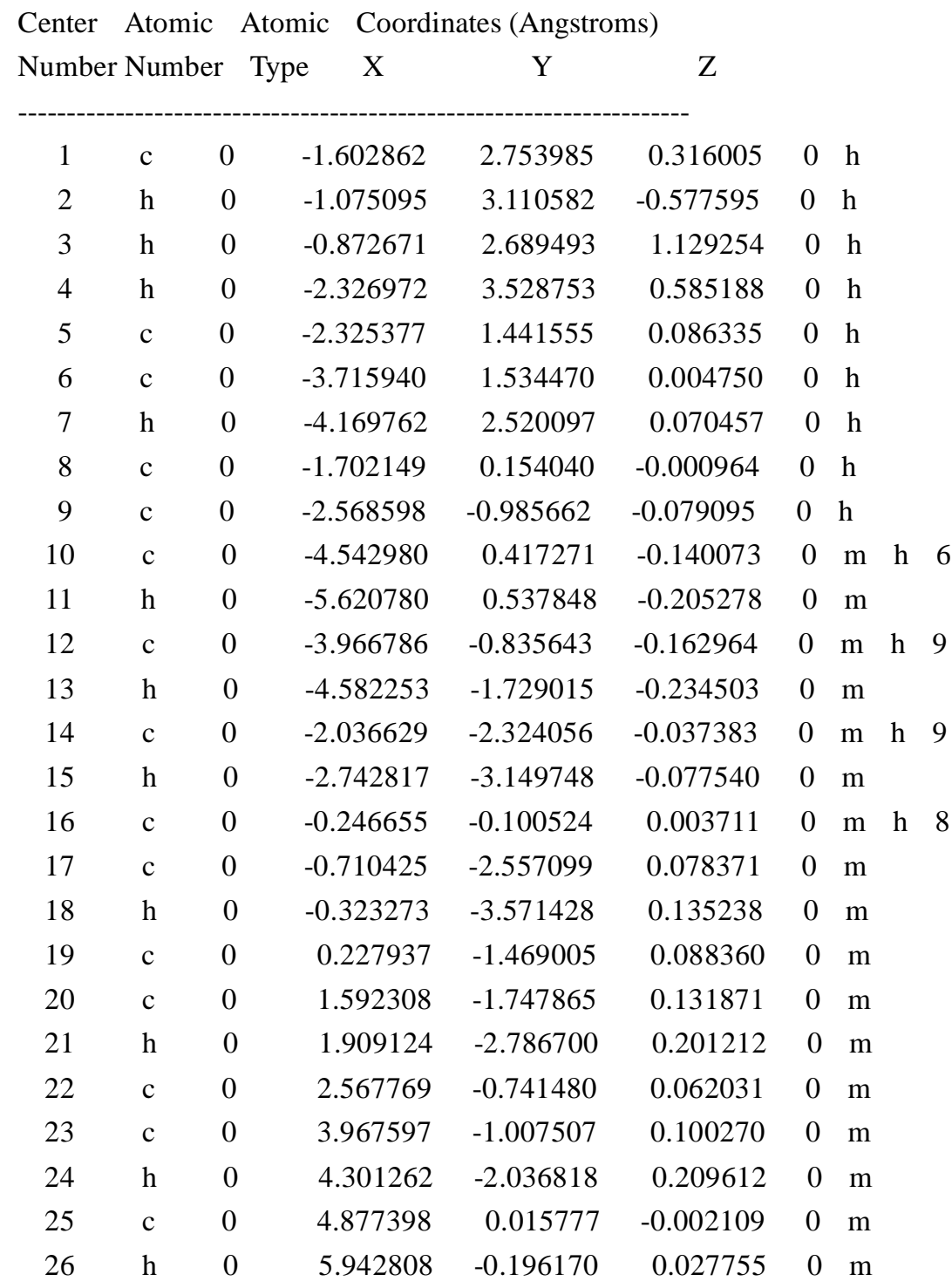




$\begin{array}{llllllll}27 & \mathrm{c} & 0 & 4.434737 & 1.359210 & -0.150625 & 0 & \mathrm{~m} \\ 28 & \mathrm{~h} & 0 & 5.167024 & 2.157858 & -0.232529 & 0 & \mathrm{~m} \\ 29 & \mathrm{c} & 0 & 3.092580 & 1.647858 & -0.190034 & 0 & \mathrm{~m} \\ 30 & \mathrm{~h} & 0 & 2.752784 & 2.675063 & -0.302019 & 0 & \mathrm{~m} \\ 31 & \mathrm{c} & 0 & 2.118481 & 0.612341 & -0.080500 & 0 & \mathrm{~m} \\ 32 & \mathrm{c} & 0 & 0.735610 & 0.887265 & -0.108286 & 0 & \mathrm{~m} \\ 33 & \mathrm{~h} & 0 & 0.464515 & 1.920607 & -0.247283 & 0 & \mathrm{~m}\end{array}$

radical

Charge $=0$ Multiplicity $=2$ for low level calculation on real system.

Charge $=0$ Multiplicity $=2$ for high level calculation on model system.

Charge $=0$ Multiplicity $=2$ for low level calculation on model system .

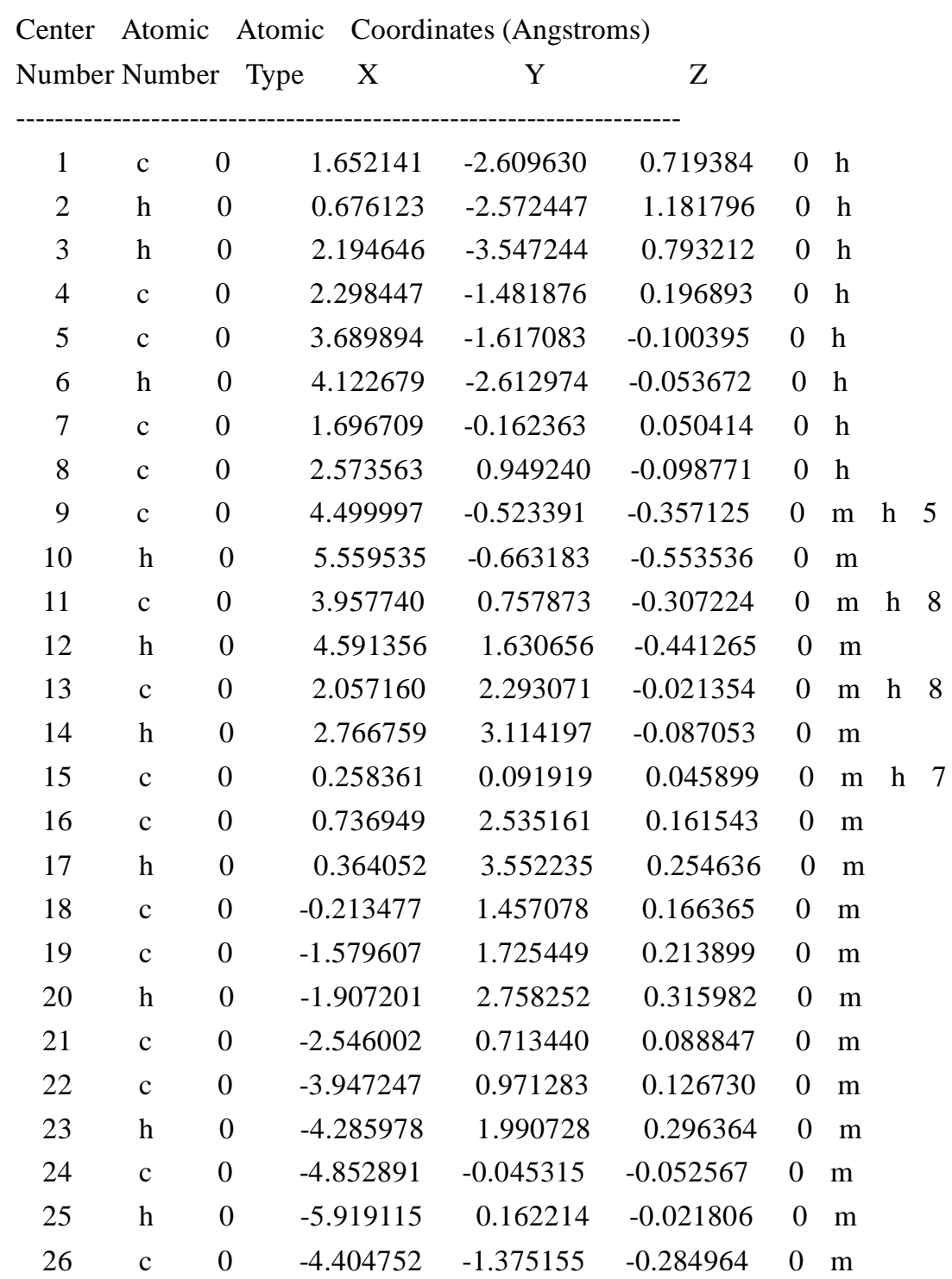




$\begin{array}{llllllll}27 & \mathrm{~h} & 0 & -5.133951 & -2.167741 & -0.429391 & 0 & \mathrm{~m} \\ 28 & \mathrm{c} & 0 & -3.061832 & -1.658354 & -0.326372 & 0 & \mathrm{~m} \\ 29 & \mathrm{~h} & 0 & -2.717099 & -2.674891 & -0.501379 & 0 & \mathrm{~m} \\ 30 & \mathrm{c} & 0 & -2.091104 & -0.631407 & -0.132123 & 0 & \mathrm{~m} \\ 31 & \mathrm{c} & 0 & -0.709044 & -0.899372 & -0.151982 & 0 & \mathrm{~m} \\ 32 & \mathrm{~h} & 0 & -0.401836 & -1.913195 & -0.376472 & 0 & \mathrm{~m}\end{array}$

No. 29

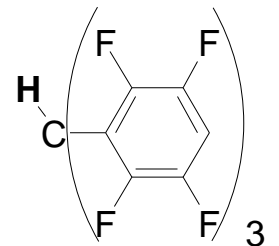

neutral molecule

Charge $=0$ Multiplicity $=1$ for low level calculation on real system.

Charge $=0$ Multiplicity $=1$ for high level calculation on model system .

Charge $=0$ Multiplicity $=1$ for low level calculation on model system .

\begin{tabular}{|c|c|c|c|c|c|c|c|c|}
\hline Center & Atomic & & Coordi & lates (Angstr & & & & \\
\hline Number & Numbe & & Type & $\mathrm{Y}$ & $\mathrm{Z}$ & & & \\
\hline 1 & $\mathrm{c}$ & 0 & 0.000352 & -0.001573 & -0.603440 & 0 & $\mathrm{~h}$ & \\
\hline 2 & $\mathrm{~h}$ & 0 & 0.000352 & -0.001573 & -1.694226 & 0 & $\mathrm{~h}$ & \\
\hline 3 & $\mathrm{c}$ & 0 & -1.079240 & -1.018354 & -0.232553 & 0 & $\mathrm{~h}$ & \\
\hline 4 & $\mathrm{c}$ & 0 & -2.109317 & -1.248548 & -1.151029 & 0 & $\mathrm{~h}$ & \\
\hline 5 & $\mathrm{c}$ & 0 & -0.340710 & 1.441869 & -0.232812 & 0 & $\mathrm{~h}$ & \\
\hline 6 & $\mathrm{c}$ & 0 & -0.031322 & 2.448591 & -1.153739 & 0 & $\mathrm{~h}$ & \\
\hline 7 & $\mathrm{c}$ & 0 & 1.421003 & -0.426847 & -0.232529 & 0 & $\mathrm{~h}$ & \\
\hline 8 & $\mathrm{c}$ & 0 & 2.139534 & -1.195892 & -1.154341 & 0 & $\mathrm{~h}$ & \\
\hline 9 & $\mathrm{c}$ & 0 & -1.112021 & -1.765814 & 0.946828 & 0 & $\mathrm{~m} h ?$ & \\
\hline 10 & $\mathrm{f}$ & 0 & -2.132753 & -0.559159 & -2.306973 & 0 & $\mathrm{~m} \quad \mathrm{~h} \quad 4$ & \\
\hline 11 & $\mathrm{c}$ & 0 & -3.116825 & -2.178408 & -0.912344 & 0 & $\mathrm{~m} \mathrm{~h} 4$ & 4 \\
\hline 12 & $\mathrm{c}$ & 0 & -0.968022 & 1.843552 & 0.948562 & 0 & $\mathrm{~m} \mathrm{~h}$ & 5 \\
\hline 13 & $\mathrm{f}$ & 0 & 0.572818 & 2.124097 & -2.312116 & 0 & $\mathrm{~m} \mathrm{~h}$ & 6 \\
\hline 14 & $\mathrm{c}$ & 0 & -0.335184 & 3.785653 & -0.915338 & 0 & $\mathrm{~m} \mathrm{~h}$ & 6 \\
\hline 15 & $\mathrm{c}$ & 0 & 2.082449 & -0.085150 & 0.949163 & 0 & $\mathrm{~m} h$ & 7 \\
\hline 16 & $\mathrm{f}$ & 0 & 1.556809 & -1.558071 & -2.312532 & 0 & $\mathrm{~m} \mathrm{~h}$ & 8 \\
\hline 17 & $\mathrm{c}$ & 0 & 3.450087 & -1.599561 & -0.916296 & 0 & $\mathrm{~m} \mathrm{~h}$ & 8 \\
\hline 18 & $\mathrm{f}$ & 0 & -0.172684 & -1.600209 & 1.889978 & 0 & $\mathrm{~m}$ & \\
\hline 19 & $\mathrm{c}$ & 0 & -2.117815 & -2.700646 & 1.185917 & 0 & $\mathrm{~m}$ & \\
\hline
\end{tabular}




$\begin{array}{llllllll}20 & \mathrm{f} & 0 & -2.096759 & -3.390497 & 2.336013 & 0 & \mathrm{~m} \\ 21 & \mathrm{c} & 0 & -3.131097 & -2.917420 & 0.262580 & 0 & \mathrm{~m} \\ 22 & \mathrm{~h} & 0 & -3.912758 & -3.642612 & 0.454965 & 0 & \mathrm{~m} \\ 23 & \mathrm{f} & 0 & -4.074698 & -2.353313 & -1.834790 & 0 & \mathrm{~m} \\ 24 & \mathrm{f} & 0 & -1.289433 & 0.947166 & 1.893150 & 0 & \mathrm{~m} \\ 25 & \mathrm{c} & 0 & -1.276194 & 3.181659 & 1.187809 & 0 & \mathrm{~m} \\ 26 & \mathrm{f} & 0 & -1.880588 & 3.507880 & 2.339908 & 0 & \mathrm{~m} \\ 27 & \mathrm{c} & 0 & -0.963474 & 4.167406 & 0.262160 & 0 & \mathrm{~m} \\ 28 & \mathrm{~h} & 0 & -1.202473 & 5.206563 & 0.454436 & 0 & \mathrm{~m} \\ 29 & \mathrm{f} & 0 & -0.015093 & 4.702043 & -1.840891 & 0 & \mathrm{~m} \\ 30 & \mathrm{f} & 0 & 1.465882 & 0.638444 & 1.895104 & 0 & \mathrm{~m} \\ 31 & \mathrm{c} & 0 & 3.395870 & -0.485537 & 1.188126 & 0 & \mathrm{~m} \\ 32 & \mathrm{f} & 0 & 3.979951 & -0.124830 & 2.340448 & 0 & \mathrm{~m} \\ 33 & \mathrm{c} & 0 & 4.094377 & -1.246988 & 0.261594 & 0 & \mathrm{~m} \\ 34 & \mathrm{~h} & 0 & 5.114445 & -1.557787 & 0.453387 & 0 & \mathrm{~m} \\ 35 & \mathrm{f} & 0 & 4.084963 & -2.332722 & -1.842685 & 0 & \mathrm{~m}\end{array}$

radical

Charge $=0$ Multiplicity $=2$ for low level calculation on real system.

Charge $=0$ Multiplicity $=2$ for high level calculation on model system.

Charge $=0$ Multiplicity $=2$ for low level calculation on model system.

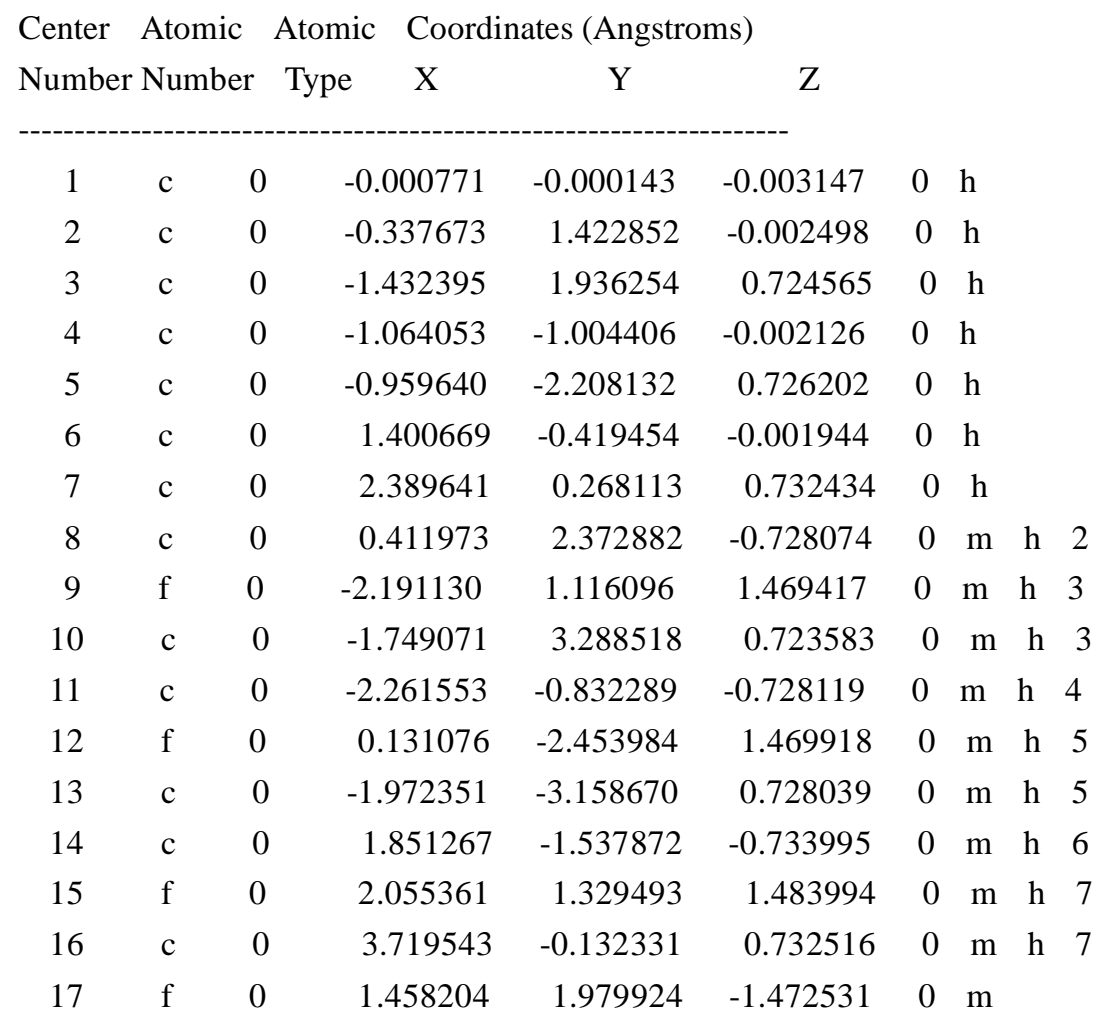




$\begin{array}{lllrrrrc}18 & \text { c } & 0 & 0.090227 & 3.723915 & -0.725264 & 0 & \mathrm{~m} \\ 19 & \mathrm{f} & 0 & 0.840738 & 4.568596 & -1.448011 & 0 & \mathrm{~m} \\ 20 & \mathrm{c} & 0 & -0.993985 & 4.202663 & -0.000388 & 0 & \mathrm{~m} \\ 21 & \mathrm{~h} & 0 & -1.242905 & 5.256969 & 0.000601 & 0 & \mathrm{~m} \\ 22 & \mathrm{f} & 0 & -2.797466 & 3.707227 & 1.448008 & 0 & \mathrm{~m} \\ 23 & \mathrm{f} & 0 & -2.444289 & 0.267780 & -1.476077 & 0 & \mathrm{~m} \\ 24 & \mathrm{c} & 0 & -3.270524 & -1.786612 & -0.722664 & 0 & \mathrm{~m} \\ 25 & \mathrm{f} & 0 & -4.377678 & -1.560982 & -1.445446 & 0 & \mathrm{~m} \\ 26 & \mathrm{c} & 0 & -3.142263 & -2.963267 & 0.004852 & 0 & \mathrm{~m} \\ 27 & \mathrm{~h} & 0 & -3.930650 & -3.706233 & 0.008079 & 0 & \mathrm{~m} \\ 28 & \mathrm{f} & 0 & -1.809797 & -4.274542 & 1.454353 & 0 & \mathrm{~m} \\ 29 & \mathrm{f} & 0 & 0.990438 & -2.243304 & -1.485251 & 0 & \mathrm{~m} \\ 30 & \mathrm{c} & 0 & 3.182745 & -1.933148 & -0.730672 & 0 & \mathrm{~m} \\ 31 & \mathrm{f} & 0 & 3.541866 & -2.999783 & -1.460362 & 0 & \mathrm{~m} \\ 32 & \mathrm{c} & 0 & 4.136764 & -1.237621 & 0.001568 & 0 & \mathrm{~m} \\ 33 & \mathrm{~h} & 0 & 5.174694 & -1.547874 & 0.002679 & 0 & \mathrm{~m} \\ 34 & \mathrm{f} & 0 & 4.603515 & 0.562327 & 1.464145 & 0 & \mathrm{~m} \\ ----------------------------------------------------------------- & & \end{array}$

\section{No. 30}<smiles>CC1c2ccccc2Oc2ccccc21</smiles>

neutral molecule

Charge $=0$ Multiplicity $=1$ for low level calculation on real system.

Charge $=0$ Multiplicity $=1$ for high level calculation on model system.

Charge $=0$ Multiplicity $=1$ for low level calculation on model system.

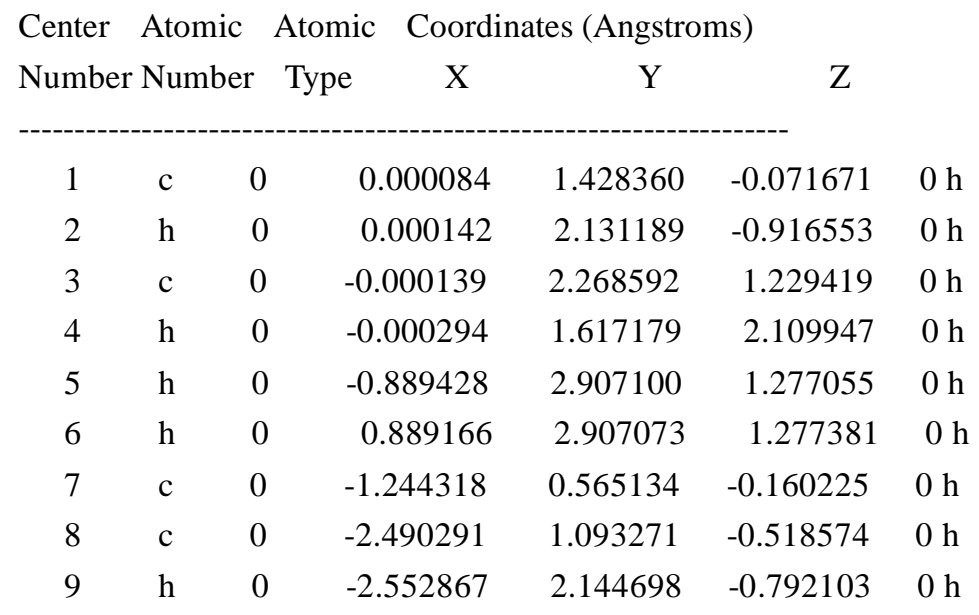




$\begin{array}{lllrrrl}10 & \mathrm{c} & 0 & 1.244358 & 0.565233 & -0.159920 & 0 \mathrm{~h} \\ 11 & \mathrm{c} & 0 & 2.490365 & 1.093343 & -0.518238 & 0 \mathrm{~h} \\ 12 & \mathrm{~h} & 0 & 2.552935 & 2.144799 & -0.791674 & 0 \mathrm{~h} \\ 13 & \mathrm{c} & 0 & -1.184078 & -0.792368 & 0.173215 & 0 \mathrm{~m} \mathrm{~h} \\ 14 & \mathrm{c} & 0 & -3.640709 & 0.306599 & -0.522594 & 0 \mathrm{~m} \mathrm{~h} \\ 15 & \mathrm{~h} & 0 & -4.596182 & 0.740835 & -0.802382 & 0 \mathrm{~m} \\ 16 & \mathrm{c} & 0 & 3.640730 & 0.306630 & -0.522480 & 0 \mathrm{~m} \mathrm{~h} \\ 17 & \mathrm{~h} & 0 & 4.596200 & 0.740897 & -0.802229 & 0 \mathrm{~m} \\ 18 & \mathrm{c} & 0 & 1.184102 & -0.792331 & 0.173261 & 0 \mathrm{~m} \mathrm{~h} \\ 19 & \mathrm{c} & 0 & -2.326220 & -1.596167 & 0.179684 & 0 \mathrm{~m} \\ 20 & \mathrm{~h} & 0 & -2.226253 & -2.643948 & 0.445027 & 0 \mathrm{~m} \\ 21 & \mathrm{c} & 0 & -3.555965 & -1.041729 & -0.163091 & 0 \mathrm{~m} \\ 22 & \mathrm{~h} & 0 & -4.445661 & -1.665350 & -0.160302 & 0 \mathrm{~m} \\ 23 & \mathrm{c} & 0 & 3.555955 & -1.041794 & -0.163315 & 0 \mathrm{~m} \\ 24 & \mathrm{~h} & 0 & 4.445611 & -1.665469 & -0.160816 & 0 \mathrm{~m} \\ 25 & \mathrm{c} & 0 & 2.326206 & -1.596194 & 0.179554 & 0 \mathrm{~m} \\ 26 & \mathrm{~h} & 0 & 2.226110 & -2.644018 & 0.444681 & 0 \mathrm{~m} \\ 27 & \mathrm{o} & 0 & 0.000006 & -1.414308 & 0.507728 & 0 \mathrm{~m}\end{array}$

radical

Charge $=0$ Multiplicity $=2$ for low level calculation on real system.

Charge $=0$ Multiplicity $=2$ for high level calculation on model system.

Charge $=0$ Multiplicity $=2$ for low level calculation on model system .

\begin{tabular}{|c|c|c|c|c|c|c|}
\hline Center & Atomic & & Atomic Coordin & ates (Angstro & $\mathrm{ms})$ & \\
\hline Number & Numbe & & Type & $\mathrm{Y}$ & Z & \\
\hline 1 & $\mathrm{c}$ & 0 & -0.000295 & 1.278993 & -0.045027 & $0 \mathrm{~h}$ \\
\hline 2 & $\mathrm{c}$ & 0 & -0.007214 & 2.781878 & -0.082803 & $0 \mathrm{~h}$ \\
\hline 3 & $\mathrm{~h}$ & 0 & 0.887062 & 3.177896 & -0.571934 & $0 \mathrm{~h}$ \\
\hline 4 & $\mathrm{~h}$ & 0 & -0.050135 & 3.224469 & 0.924189 & $0 \mathrm{~h}$ \\
\hline 5 & $\mathrm{~h}$ & 0 & -0.868560 & 3.164474 & -0.638978 & $0 \mathrm{~h}$ \\
\hline 6 & $\mathrm{c}$ & 0 & 1.228294 & 0.540581 & -0.009083 & $0 \mathrm{~h}$ \\
\hline 7 & $\mathrm{c}$ & 0 & 2.518736 & 1.127801 & 0.049480 & $0 \mathrm{~h}$ \\
\hline 8 & $\mathrm{~h}$ & 0 & 2.606392 & 2.208006 & 0.089971 & $0 \mathrm{~h}$ \\
\hline 9 & $\mathrm{c}$ & 0 & -1.227539 & 0.538076 & -0.009123 & $0 \mathrm{~h}$ \\
\hline 10 & $\mathrm{c}$ & 0 & -2.517732 & 1.125442 & 0.048869 & $0 \mathrm{~h}$ \\
\hline 11 & $\mathrm{~h}$ & 0 & -2.604656 & 2.205824 & 0.088124 & $0 \mathrm{~h}$ \\
\hline 12 & $\mathrm{c}$ & 0 & 1.190101 & -0.879366 & -0.029465 & $0 \mathrm{~m} \mathrm{~h}$ \\
\hline 13 & $\mathrm{c}$ & 0 & 3.672743 & 0.355707 & 0.069216 & $0 \mathrm{~m} \mathrm{~h}$ \\
\hline 14 & $\mathrm{~h}$ & 0 & 4.642646 & 0.843109 & 0.114820 & $0 \mathrm{~m}$ \\
\hline 15 & $\mathrm{c}$ & 0 & -3.671512 & 0.352791 & 0.068991 & $0 \mathrm{~m} \mathrm{~h}$ \\
\hline
\end{tabular}




$\begin{array}{lllrrrl}16 & \mathrm{~h} & 0 & -4.641745 & 0.839562 & 0.114232 & 0 \mathrm{~m} \\ 17 & \mathrm{c} & 0 & -1.188149 & -0.881359 & -0.029323 & 0 \mathrm{~m} \mathrm{~h} \\ 18 & \mathrm{c} & 0 & 2.341162 & -1.658237 & -0.013183 & 0 \mathrm{~m} \\ 19 & \mathrm{~h} & 0 & 2.237292 & -2.738411 & -0.035717 & 0 \mathrm{~m} \\ 20 & \mathrm{c} & 0 & 3.591558 & -1.041346 & 0.034277 & 0 \mathrm{~m} \\ 21 & \mathrm{~h} & 0 & 4.492869 & -1.646861 & 0.047871 & 0 \mathrm{~m} \\ 22 & \mathrm{c} & 0 & -3.589370 & -1.044237 & 0.034857 & 0 \mathrm{~m} \\ 23 & \mathrm{~h} & 0 & -4.490392 & -1.650194 & 0.048830 & 0 \mathrm{~m} \\ 24 & \mathrm{c} & 0 & -2.338758 & -1.660886 & -0.012651 & 0 \mathrm{~m} \\ 25 & \mathrm{~h} & 0 & -2.234653 & -2.741042 & -0.034906 & 0 \mathrm{~m} \\ 26 & \mathrm{o} & 0 & 0.001466 & -1.562732 & -0.074588 & 0 \mathrm{~m}\end{array}$

\section{No. 31}

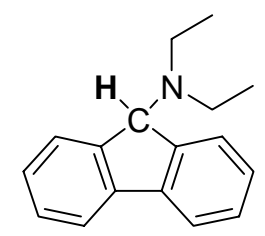

neutral molecule

Charge $=0$ Multiplicity $=1$ for low level calculation on real system.

Charge $=0$ Multiplicity $=1$ for high level calculation on model system .

Charge $=0$ Multiplicity $=1$ for low level calculation on model system.

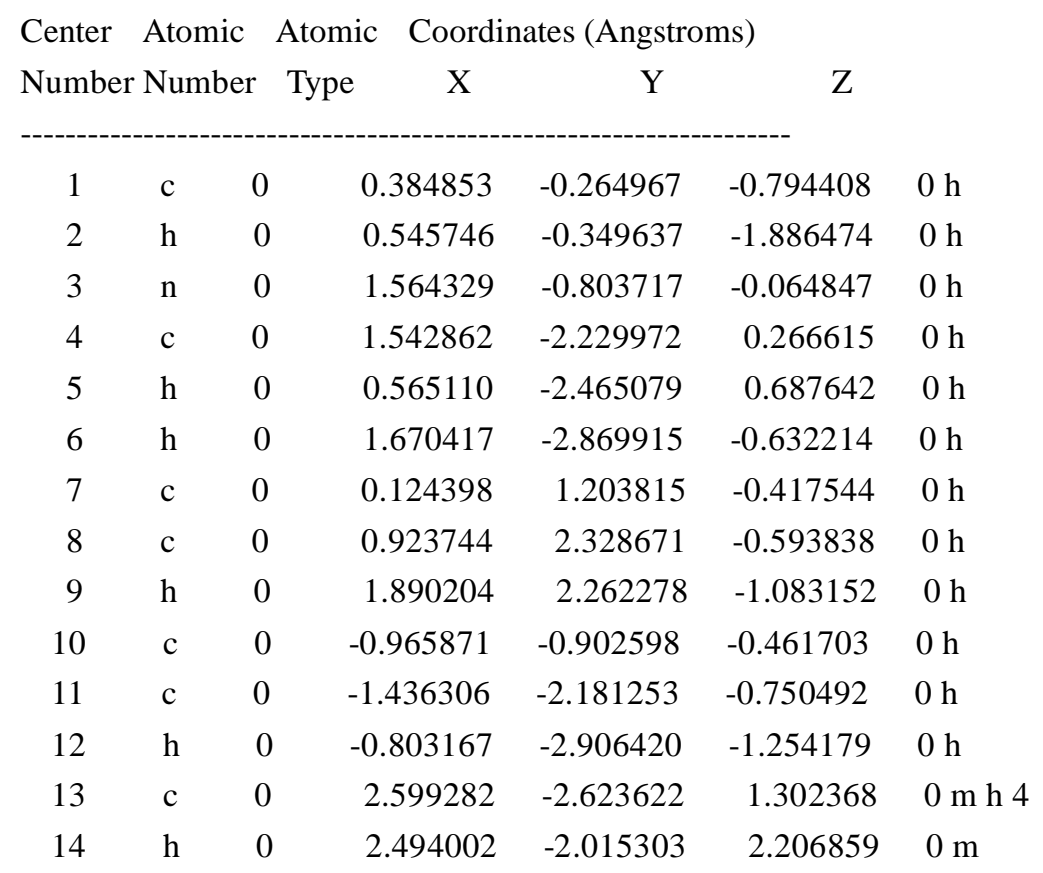




$\begin{array}{lllrrrl}15 & \mathrm{~h} & 0 & 2.468608 & -3.677443 & 1.575114 & 0 \mathrm{~m} \\ 16 & \mathrm{~h} & 0 & 3.621287 & -2.508041 & 0.927337 & 0 \mathrm{~m} \\ 17 & \mathrm{c} & 0 & 2.836641 & -0.387677 & -0.684019 & 0 \mathrm{~m} \mathrm{~h} 3 \\ 18 & \mathrm{~h} & 0 & 3.365127 & -1.270618 & -1.080094 & 0 \mathrm{~m} \\ 19 & \mathrm{~h} & 0 & 2.623516 & 0.235217 & -1.561647 & 0 \mathrm{~m} \\ 20 & \mathrm{c} & 0 & 0.469933 & 3.574281 & -0.141024 & 0 \mathrm{~m} \mathrm{~h} 8 \\ 21 & \mathrm{~h} & 0 & 1.094288 & 4.453383 & -0.276827 & 0 \mathrm{~m} \\ 22 & \mathrm{c} & 0 & -1.145346 & 1.339920 & 0.176937 & 0 \mathrm{~m} \mathrm{~h} 7 \\ 23 & \mathrm{c} & 0 & -1.825684 & 0.039729 & 0.135230 & 0 \mathrm{~m} \mathrm{~h} 10 \\ 24 & \mathrm{c} & 0 & -2.742150 & -2.532520 & -0.388619 & 0 \mathrm{~m} \mathrm{~h} 11 \\ 25 & \mathrm{~h} & 0 & -3.110193 & -3.531273 & -0.607220 & 0 \mathrm{~m} \\ 26 & \mathrm{c} & 0 & 3.764586 & 0.383852 & 0.260852 & 0 \mathrm{~m} \\ 27 & \mathrm{~h} & 0 & 4.064585 & -0.227706 & 1.116411 & 0 \mathrm{~m} \\ 28 & \mathrm{~h} & 0 & 4.674108 & 0.697234 & -0.268133 & 0 \mathrm{~m} \\ 29 & \mathrm{~h} & 0 & 3.264234 & 1.274727 & 0.651275 & 0 \mathrm{~m} \\ 30 & \mathrm{c} & 0 & -0.777939 & 3.697142 & 0.475100 & 0 \mathrm{~m} \\ 31 & \mathrm{~h} & 0 & -1.116898 & 4.669563 & 0.822378 & 0 \mathrm{~m} \\ 32 & \mathrm{c} & 0 & -1.599392 & 2.578688 & 0.630378 & 0 \mathrm{~m} \\ 33 & \mathrm{~h} & 0 & -2.580946 & 2.677931 & 1.087053 & 0 \mathrm{~m} \\ 34 & \mathrm{c} & 0 & -3.127281 & -0.309279 & 0.499298 & 0 \mathrm{~m} \\ 35 & \mathrm{~h} & 0 & -3.789734 & 0.419843 & 0.958723 & 0 \mathrm{~m} \\ 36 & \mathrm{c} & 0 & -3.576707 & -1.606138 & 0.243362 & 0 \mathrm{~m} \\ 37 & \mathrm{~h} & 0 & -4.588328 & -1.891159 & 0.520113 & 0 \mathrm{~m} \\ ---------------------------------------------------- & \end{array}$

radical

Charge $=0$ Multiplicity $=2$ for low level calculation on real system.

Charge $=0$ Multiplicity $=2$ for high level calculation on model system.

Charge $=0$ Multiplicity $=2$ for low level calculation on model system.

\begin{tabular}{|c|c|c|c|c|c|c|c|}
\hline Center & Atomic & & tomic & Coordi & nates (Angstro & ms) & \\
\hline Number & Number & & ype & $X$ & Y & $\mathrm{Z}$ & \\
\hline 1 & $\mathrm{c}$ & 0 & & 0.414456 & -0.163187 & 0.050521 & $0 \mathrm{~h}$ \\
\hline 2 & $\mathrm{n}$ & 0 & & 1.777099 & -0.417334 & 0.090127 & $0 \mathrm{~h}$ \\
\hline 3 & $\mathrm{c}$ & 0 & & 2.263452 & -1.755485 & -0.255669 & $0 \mathrm{~h}$ \\
\hline 4 & $\mathrm{~h}$ & 0 & & 1.550921 & -2.176519 & -0.965570 & $0 \mathrm{~h}$ \\
\hline 5 & $\mathrm{~h}$ & 0 & & 2.268076 & -2.406254 & 0.635586 & $0 \mathrm{~h}$ \\
\hline 6 & $\mathrm{c}$ & 0 & & 0.181298 & 1.162070 & -0.016650 & $0 \mathrm{~h}$ \\
\hline 7 & $\mathrm{c}$ & 0 & & 0.367741 & 2.455594 & -0.083237 & $0 \mathrm{~h}$ \\
\hline 8 & $\mathrm{~h}$ & 0 & & 1.438879 & 2.616025 & -0.075758 & $0 \mathrm{~h}$ \\
\hline 9 & $\mathrm{c}$ & 0 & & 0.674770 & -1.129766 & 0.052688 & $0 \mathrm{~h}$ \\
\hline 10 & $\mathrm{c}$ & 0 & & 0.734581 & -2.533242 & 0.184312 & $0 \mathrm{~h}$ \\
\hline
\end{tabular}




$\begin{array}{lllrrrl}11 & \mathrm{~h} & 0 & -0.159455 & -3.133964 & 0.295372 & 0 \mathrm{~h} \\ 12 & \mathrm{c} & 0 & -3.653012 & -1.795559 & -0.897784 & 0 \mathrm{~m} \mathrm{~h} 3 \\ 13 & \mathrm{~h} & 0 & -3.708740 & -1.132504 & -1.766984 & 0 \mathrm{~m} \\ 14 & \mathrm{~h} & 0 & -3.853321 & -2.819052 & -1.234724 & 0 \mathrm{~m} \\ 15 & \mathrm{~h} & 0 & -4.448507 & -1.524033 & -0.197936 & 0 \mathrm{~m} \\ 16 & \mathrm{c} & 0 & -2.635874 & 0.391274 & 0.975238 & 0 \mathrm{~m} \mathrm{~h} 2 \\ 17 & \mathrm{~h} & 0 & -3.114189 & -0.289105 & 1.696835 & 0 \mathrm{~m} \\ 18 & \mathrm{~h} & 0 & -1.980242 & 1.041826 & 1.557483 & 0 \mathrm{~m} \\ 19 & \mathrm{c} & 0 & 0.478605 & 3.559867 & -0.172263 & 0 \mathrm{~m} \mathrm{~h} 7 \\ 20 & \mathrm{~h} & 0 & 0.048129 & 4.556803 & -0.220501 & 0 \mathrm{~m} \\ 21 & \mathrm{c} & 0 & 1.601226 & 1.015571 & -0.070162 & 0 \mathrm{~m} \mathrm{~h} 6 \\ 22 & \mathrm{c} & 0 & 1.908879 & -0.405892 & -0.027210 & 0 \mathrm{~m} \mathrm{~h} 9 \\ 23 & \mathrm{c} & 0 & 1.970758 & -3.177124 & 0.193088 & 0 \mathrm{~m} \mathrm{~h} 10 \\ 24 & \mathrm{~h} & 0 & 2.002512 & -4.259091 & 0.292307 & 0 \mathrm{~m} \\ 25 & \mathrm{c} & 0 & -3.713313 & 1.239610 & 0.283752 & 0 \mathrm{~m} \\ 26 & \mathrm{~h} & 0 & -4.514168 & 0.633306 & -0.144353 & 0 \mathrm{~m} \\ 27 & \mathrm{~h} & 0 & -4.165312 & 1.919282 & 1.016155 & 0 \mathrm{~m} \\ 28 & \mathrm{~h} & 0 & -3.288612 & 1.841296 & -0.524830 & 0 \mathrm{~m} \\ 29 & \mathrm{c} & 0 & 1.871547 & 3.403888 & -0.206948 & 0 \mathrm{~m} \\ 30 & \mathrm{~h} & 0 & 2.513340 & 4.277932 & -0.276172 & 0 \mathrm{~m} \\ 31 & \mathrm{c} & 0 & 2.435112 & 2.126229 & -0.162793 & 0 \mathrm{~m} \\ 32 & \mathrm{~h} & 0 & 3.514658 & 2.002664 & -0.205150 & 0 \mathrm{~m} \\ 33 & \mathrm{c} & 0 & 3.135182 & -1.063166 & -0.017403 & 0 \mathrm{~m} \\ 34 & \mathrm{~h} & 0 & 4.061610 & -0.496874 & -0.077341 & 0 \mathrm{~m} \\ 35 & \mathrm{c} & 0 & 3.167568 & -2.455898 & 0.083111 & 0 \mathrm{~m} \\ 36 & \mathrm{~h} & 0 & 4.119790 & -2.979108 & 0.089143 & 0 \mathrm{~m} \\ ----------------------------------------------------------- & \end{array}$

\section{No. 32}

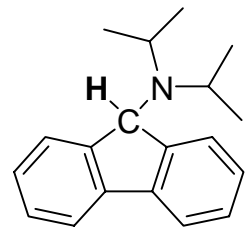

neutral molecule

Charge $=0$ Multiplicity $=1$ for low level calculation on real system.

Charge $=0$ Multiplicity $=1$ for high level calculation on model system .

Charge $=0$ Multiplicity $=1$ for low level calculation on model system .

Center Atomic Atomic Coordinates (Angstroms)

$\begin{array}{lllll}\text { Number Number } & \text { Type } & \text { X } & \text { Y } & \text { Z }\end{array}$




\begin{tabular}{|c|c|c|c|c|c|c|}
\hline 1 & $\mathrm{c}$ & 0 & -0.171361 & -0.065376 & -0.768314 & $0 \mathrm{~h}$ \\
\hline 2 & $\mathrm{~h}$ & 0 & -0.333071 & -0.037674 & -1.855738 & $0 \mathrm{~h}$ \\
\hline 3 & $\mathrm{n}$ & 0 & -1.473915 & -0.313553 & -0.165019 & $0 \mathrm{~h}$ \\
\hline 4 & $\mathrm{c}$ & 0 & 0.602634 & 1.213655 & -0.395097 & $0 \mathrm{~h}$ \\
\hline 5 & $\mathrm{c}$ & 0 & 0.182183 & 2.539181 & -0.396493 & $0 \mathrm{~h}$ \\
\hline 6 & $\mathrm{~h}$ & 0 & -0.842354 & 2.796636 & -0.648634 & $0 \mathrm{~h}$ \\
\hline 7 & $\mathrm{c}$ & 0 & 0.884552 & -1.142897 & -0.493876 & $0 \mathrm{~h}$ \\
\hline 8 & $\mathrm{c}$ & 0 & 0.759457 & -2.521924 & -0.603082 & $0 \mathrm{~h}$ \\
\hline 9 & $\mathrm{~h}$ & 0 & -0.193998 & -2.965888 & -0.878557 & $0 \mathrm{~h}$ \\
\hline 10 & $\mathrm{c}$ & 0 & -2.639509 & -0.270672 & -1.059412 & $0 \mathrm{~m} \mathrm{~h} 3$ \\
\hline 11 & $\mathrm{~h}$ & 0 & -2.284945 & -0.714680 & -2.000519 & $0 \mathrm{~m}$ \\
\hline 12 & $\mathrm{c}$ & 0 & -1.532469 & -0.209535 & 1.305141 & $0 \mathrm{~m} \mathrm{~h} 3$ \\
\hline 13 & $\mathrm{~h}$ & 0 & -0.500889 & 0.012815 & 1.600730 & $0 \mathrm{~m}$ \\
\hline 14 & $\mathrm{c}$ & 0 & 1.093062 & 3.548589 & -0.061026 & $0 \mathrm{~m} \mathrm{~h} 5$ \\
\hline 15 & $\mathrm{~h}$ & 0 & 0.768757 & 4.585919 & -0.054825 & $0 \mathrm{~m}$ \\
\hline 16 & $\mathrm{c}$ & 0 & 1.935497 & 0.897705 & -0.059708 & $0 \mathrm{~m} \mathrm{~h} 4$ \\
\hline 17 & $\mathrm{c}$ & 0 & 2.108994 & -0.562093 & -0.119925 & $0 \mathrm{~m} \mathrm{~h} 7$ \\
\hline 18 & $\mathrm{c}$ & 0 & 1.874190 & -3.326994 & -0.341135 & $0 \mathrm{~m} \mathrm{~h} 8$ \\
\hline 19 & $\mathrm{~h}$ & 0 & 1.790919 & -4.407901 & -0.418853 & $0 \mathrm{~m}$ \\
\hline 20 & $\mathrm{c}$ & 0 & -3.802799 & -1.162771 & -0.598734 & $0 \mathrm{~m}$ \\
\hline 21 & $\mathrm{~h}$ & 0 & -3.463089 & -2.184541 & -0.405942 & $0 \mathrm{~m}$ \\
\hline 22 & $\mathrm{~h}$ & 0 & -4.565384 & -1.197407 & -1.385083 & $0 \mathrm{~m}$ \\
\hline 23 & $\mathrm{~h}$ & 0 & -4.287986 & -0.783576 & 0.306151 & $0 \mathrm{~m}$ \\
\hline 24 & $\mathrm{c}$ & 0 & -3.154830 & 1.140551 & -1.424591 & $0 \mathrm{~m}$ \\
\hline 25 & $\mathrm{~h}$ & 0 & -3.949308 & 1.071708 & -2.178680 & $0 \mathrm{~m}$ \\
\hline 26 & $\mathrm{~h}$ & 0 & -3.562193 & 1.667084 & -0.556126 & $0 \mathrm{~m}$ \\
\hline 27 & $\mathrm{~h}$ & 0 & -2.352128 & 1.752191 & -1.849746 & $0 \mathrm{~m}$ \\
\hline 28 & $\mathrm{c}$ & 0 & -2.385547 & 0.950031 & 1.854298 & $0 \mathrm{~m}$ \\
\hline 29 & $\mathrm{~h}$ & 0 & -3.451614 & 0.818762 & 1.640493 & $0 \mathrm{~m}$ \\
\hline 30 & $\mathrm{~h}$ & 0 & -2.065029 & 1.909150 & 1.436912 & $0 \mathrm{~m}$ \\
\hline 31 & $\mathrm{~h}$ & 0 & -2.277872 & 1.003200 & 2.944415 & $0 \mathrm{~m}$ \\
\hline 32 & $\mathrm{c}$ & 0 & -1.889864 & -1.535171 & 2.008631 & $0 \mathrm{~m}$ \\
\hline 33 & $\mathrm{~h}$ & 0 & -1.705817 & -1.445665 & 3.086728 & $0 \mathrm{~m}$ \\
\hline 34 & $\mathrm{~h}$ & 0 & -1.270140 & -2.350260 & 1.623673 & $0 \mathrm{~m}$ \\
\hline 35 & $\mathrm{~h}$ & 0 & -2.940548 & -1.809434 & 1.877449 & $0 \mathrm{~m}$ \\
\hline 36 & $\mathrm{c}$ & 0 & 2.415696 & 3.232715 & 0.263796 & $0 \mathrm{~m}$ \\
\hline 37 & $\mathrm{~h}$ & 0 & 3.113245 & 4.026264 & 0.518770 & $0 \mathrm{~m}$ \\
\hline 38 & $\mathrm{c}$ & 0 & 2.846821 & 1.904116 & 0.264943 & $0 \mathrm{~m}$ \\
\hline 39 & $\mathrm{~h}$ & 0 & 3.876144 & 1.662225 & 0.517884 & $0 \mathrm{~m}$ \\
\hline 40 & $\mathrm{c}$ & 0 & 3.224118 & -1.363348 & 0.135229 & $0 \mathrm{~m}$ \\
\hline 41 & $\mathrm{~h}$ & 0 & 4.175541 & -0.922392 & 0.422335 & $0 \mathrm{~m}$ \\
\hline 42 & $\mathrm{c}$ & 0 & 3.096637 & -2.749874 & 0.021291 & $0 \mathrm{~m}$ \\
\hline 43 & $\mathrm{~h}$ & 0 & 3.954387 & -3.386985 & 0.220676 & $0 \mathrm{~m}$ \\
\hline
\end{tabular}


radical

Charge $=0$ Multiplicity $=2$ for low level calculation on real system.

Charge $=0$ Multiplicity $=2$ for high level calculation on model system .

Charge $=0$ Multiplicity $=2$ for low level calculation on model system .

\begin{tabular}{|c|c|c|c|c|c|c|}
\hline Center & Atomic & c Atomic & Coordinat & es (Angstrom & & \\
\hline Number & Numbe & er Type & $X$ & Y & $\mathrm{Z}$ & \\
\hline 1 & $\mathrm{c}$ & 0 & 0.095576 & -0.000161 & -0.001281 & $0 \mathrm{~h}$ \\
\hline 2 & $\mathrm{n}$ & 0 & 1.482307 & -0.001602 & -0.001788 & $0 \mathrm{~h}$ \\
\hline 3 & $\mathrm{c}$ & 0 & -0.765528 & 1.169173 & 0.037832 & $0 \mathrm{~h}$ \\
\hline 4 & $\mathrm{c}$ & 0 & -0.489027 & 2.541908 & 0.162280 & $0 \mathrm{~h}$ \\
\hline 5 & $\mathrm{~h}$ & 0 & 0.532670 & 2.904733 & 0.226403 & $0 \mathrm{~h}$ \\
\hline 6 & $\mathrm{c}$ & 0 & -0.766190 & -1.169029 & -0.039857 & $0 \mathrm{~h}$ \\
\hline 7 & $\mathrm{c}$ & 0 & -0.489886 & -2.541615 & -0.165826 & $0 \mathrm{~h}$ \\
\hline 8 & $\mathrm{~h}$ & 0 & 0.531806 & -2.904151 & -0.232123 & $0 \mathrm{~h}$ \\
\hline 9 & $\mathrm{c}$ & 0 & 2.171765 & -0.897073 & 0.957205 & $0 \mathrm{~m} \mathrm{~h} 2$ \\
\hline 10 & $\mathrm{~h}$ & 0 & 1.395901 & -1.593478 & 1.281837 & $0 \mathrm{~m}$ \\
\hline 11 & $\mathrm{c}$ & 0 & 2.174266 & 0.895515 & -0.957242 & $0 \mathrm{~m} \mathrm{~h} 2$ \\
\hline 12 & $\mathrm{~h}$ & 0 & 1.398373 & 1.590072 & -1.285582 & $0 \mathrm{~m}$ \\
\hline 13 & $\mathrm{c}$ & 0 & -1.545355 & 3.449801 & 0.227523 & $0 \mathrm{~m} \mathrm{~h} 4$ \\
\hline 14 & $\mathrm{~h}$ & 0 & -1.332237 & 4.511735 & 0.318957 & $0 \mathrm{~m}$ \\
\hline 15 & $\mathrm{c}$ & 0 & -2.125430 & 0.729676 & 0.026719 & $0 \mathrm{~m} \mathrm{~h} 3$ \\
\hline 16 & $\mathrm{c}$ & 0 & -2.125809 & -0.729149 & -0.026796 & $0 \mathrm{~m} \mathrm{~h} 6$ \\
\hline 17 & $\mathrm{c}$ & 0 & -1.546443 & -3.449324 & -0.230092 & $0 \mathrm{~m} \mathrm{~h} 7$ \\
\hline 18 & $\mathrm{~h}$ & 0 & -1.333685 & -4.511225 & -0.322739 & $0 \mathrm{~m}$ \\
\hline 19 & $\mathrm{c}$ & 0 & 3.309346 & -1.740125 & 0.364685 & $0 \mathrm{~m}$ \\
\hline 20 & $\mathrm{~h}$ & 0 & 2.974257 & -2.308627 & -0.508444 & $0 \mathrm{~m}$ \\
\hline 21 & $\mathrm{~h}$ & 0 & 3.657922 & -2.452704 & 1.120969 & $0 \mathrm{~m}$ \\
\hline 22 & $\mathrm{~h}$ & 0 & 4.171939 & -1.134322 & 0.069748 & $0 \mathrm{~m}$ \\
\hline 23 & $\mathrm{c}$ & 0 & 2.622432 & -0.153780 & 2.232166 & $0 \mathrm{~m}$ \\
\hline 24 & $\mathrm{~h}$ & 0 & 2.909277 & -0.882142 & 3.000576 & $0 \mathrm{~m}$ \\
\hline 25 & $\mathrm{~h}$ & 0 & 3.482318 & 0.498520 & 2.056593 & $0 \mathrm{~m}$ \\
\hline 26 & $\mathrm{~h}$ & 0 & 1.802407 & 0.452811 & 2.629273 & $0 \mathrm{~m}$ \\
\hline 27 & $\mathrm{c}$ & 0 & 3.307189 & 1.741159 & -0.359427 & $0 \mathrm{~m}$ \\
\hline 28 & $\mathrm{~h}$ & 0 & 4.169463 & 1.137167 & -0.059799 & $0 \mathrm{~m}$ \\
\hline 29 & $\mathrm{~h}$ & 0 & 2.966594 & 2.309072 & 0.511953 & $0 \mathrm{~m}$ \\
\hline 30 & $\mathrm{~h}$ & 0 & 3.658318 & 2.454291 & -1.114009 & $0 \mathrm{~m}$ \\
\hline 31 & $\mathrm{c}$ & 0 & 2.632608 & 0.152970 & -2.229856 & $0 \mathrm{~m}$ \\
\hline 32 & $\mathrm{~h}$ & 0 & 2.922050 & 0.881718 & -2.996910 & $0 \mathrm{~m}$ \\
\hline 33 & $\mathrm{~h}$ & 0 & 1.815674 & -0.455198 & -2.630909 & $0 \mathrm{~m}$ \\
\hline
\end{tabular}




$\begin{array}{lllrrrr}34 & \mathrm{~h} & 0 & 3.492670 & -0.497795 & -2.049645 & 0 \mathrm{~m} \\ 35 & \mathrm{c} & 0 & -2.876992 & 3.011615 & 0.186735 & 0 \mathrm{~m} \\ 36 & \mathrm{~h} & 0 & -3.685595 & 3.735606 & 0.238768 & 0 \mathrm{~m} \\ 37 & \mathrm{c} & 0 & -3.169609 & 1.646976 & 0.097812 & 0 \mathrm{~m} \\ 38 & \mathrm{~h} & 0 & -4.203241 & 1.309011 & 0.095064 & 0 \mathrm{~m} \\ 39 & \mathrm{c} & 0 & -3.170302 & -1.646208 & -0.096838 & 0 \mathrm{~m} \\ 40 & \mathrm{~h} & 0 & -4.203879 & -1.308091 & -0.092531 & 0 \mathrm{~m} \\ 41 & \mathrm{c} & 0 & -2.878003 & -3.010851 & -0.186875 & 0 \mathrm{~m} \\ 42 & \mathrm{~h} & 0 & -3.686804 & -3.734678 & -0.238147 & 0 \mathrm{~m}\end{array}$

\section{No. 33}<smiles>N#CC(c1ccccc1)c1ccccc1</smiles>

neutral molecule

Charge $=0$ Multiplicity $=1$ for low level calculation on real system.

Charge $=0$ Multiplicity $=1$ for high level calculation on model system .

Charge $=0$ Multiplicity $=1$ for low level calculation on model system .

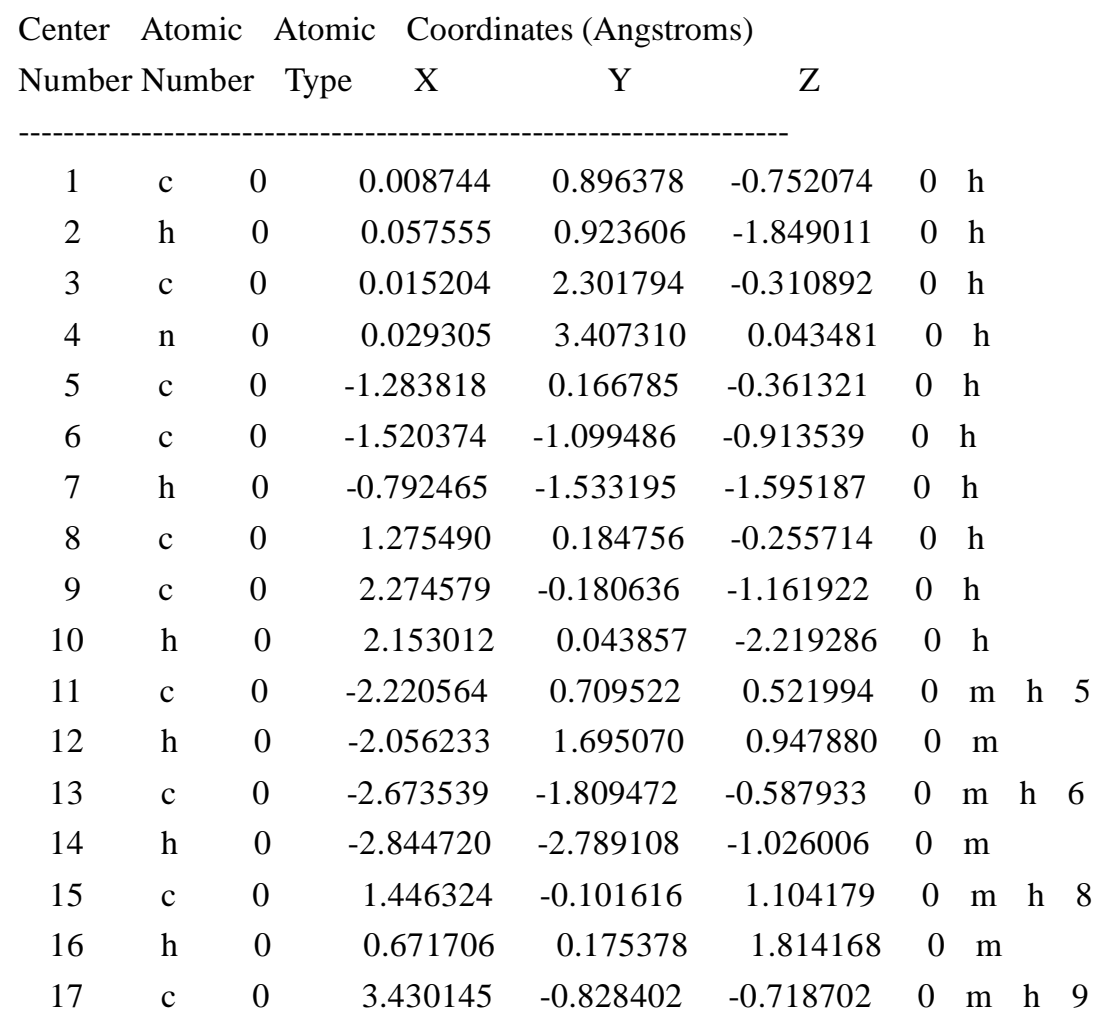




$\begin{array}{lllrcccc}18 & \mathrm{~h} & 0 & 4.198444 & -1.109183 & -1.433940 & 0 & \mathrm{~m} \\ 19 & \mathrm{c} & 0 & -3.376399 & -0.004903 & 0.850349 & 0 & \mathrm{~m} \\ 20 & \mathrm{~h} & 0 & -4.096692 & 0.430233 & 1.537787 & 0 & \mathrm{~m} \\ 21 & \mathrm{c} & 0 & -3.606630 & -1.263451 & 0.297829 & 0 & \mathrm{~m} \\ 22 & \mathrm{~h} & 0 & -4.506728 & -1.816458 & 0.551945 & 0 & \mathrm{~m} \\ 23 & \mathrm{c} & 0 & 3.595642 & -1.110372 & 0.636800 & 0 & \mathrm{~m} \\ 24 & \mathrm{~h} & 0 & 4.494134 & -1.612985 & 0.984127 & 0 & \mathrm{~m} \\ 25 & \mathrm{c} & 0 & 2.600715 & -0.743947 & 1.547299 & 0 & \mathrm{~m} \\ 26 & \mathrm{~h} & 0 & 2.723731 & -0.960090 & 2.605036 & 0 & \mathrm{~m}\end{array}$

radical

Charge $=0$ Multiplicity $=2$ for low level calculation on real system.

Charge $=0$ Multiplicity $=2$ for high level calculation on model system.

Charge $=0$ Multiplicity $=2$ for low level calculation on model system.

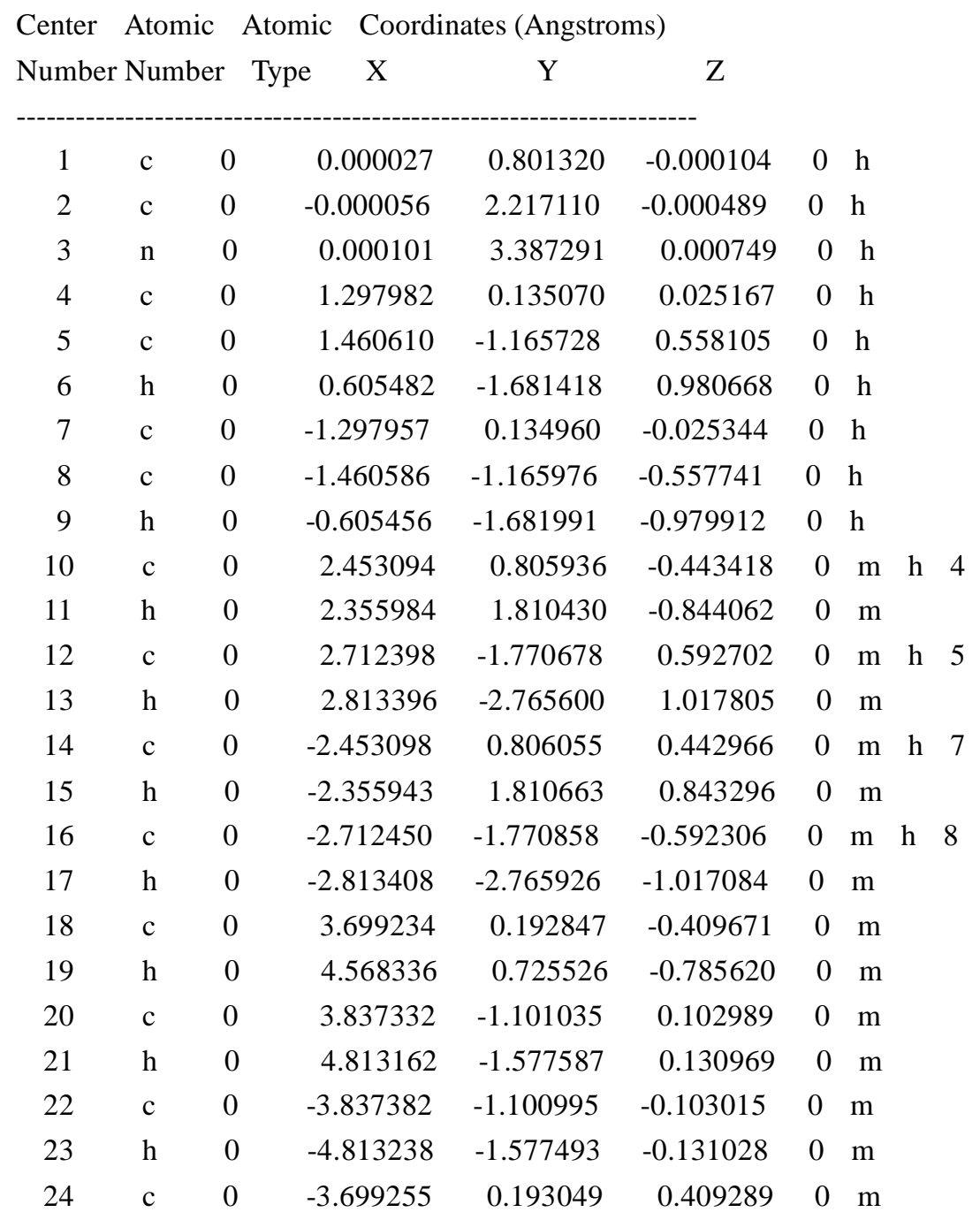




$\begin{array}{llllllll}25 & \mathrm{~h} & 0 & -4.568376 & 0.725902 & 0.784949 & 0 & \mathrm{~m}\end{array}$

\section{No. 34}<smiles>O=C1OCCCC1c1ccc2ccccc2c1</smiles>

neutral molecule

Charge $=0$ Multiplicity $=1$ for low level calculation on real system.

Charge $=0$ Multiplicity $=1$ for high level calculation on model system .

Charge $=0$ Multiplicity $=1$ for low level calculation on model system.

\begin{tabular}{|c|c|c|c|c|c|c|}
\hline Center & Atomic & & Coordi & ates (Angstro & & \\
\hline Number & Numbe & & ype & $\mathrm{Y}$ & Z & \\
\hline 1 & $\mathrm{c}$ & 0 & 1.906464 & 0.920414 & 0.395587 & $0 \mathrm{~h}$ \\
\hline 2 & $\mathrm{~h}$ & 0 & 2.119261 & 1.753036 & 1.074344 & $0 \mathrm{~h}$ \\
\hline 3 & $\mathrm{c}$ & 0 & 0.386215 & 0.804607 & 0.281535 & $0 \mathrm{~h}$ \\
\hline 4 & $\mathrm{c}$ & 0 & -0.372852 & 2.007811 & 0.233120 & $0 \mathrm{~h}$ \\
\hline 5 & $\mathrm{~h}$ & 0 & 0.144447 & 2.961234 & 0.314989 & $0 \mathrm{~h}$ \\
\hline 6 & $\mathrm{c}$ & 0 & 2.564381 & 1.202276 & -0.976385 & $0 \mathrm{~h}$ \\
\hline 7 & $\mathrm{~h}$ & 0 & 2.008961 & 1.989590 & -1.497636 & $0 \mathrm{~h}$ \\
\hline 8 & $\mathrm{~h}$ & 0 & 3.584789 & 1.578702 & -0.818469 & $0 \mathrm{~h}$ \\
\hline 9 & $\mathrm{c}$ & 0 & 2.493887 & -0.311270 & 1.092787 & $0 \mathrm{~h}$ \\
\hline 10 & o & 0 & 2.477478 & -0.446298 & 2.291435 & $0 \mathrm{~h}$ \\
\hline 11 & o & 0 & 2.968450 & -1.330854 & 0.322619 & $0 \mathrm{~h}$ \\
\hline 12 & $\mathrm{c}$ & 0 & -0.277912 & -0.402246 & 0.192664 & $0 \mathrm{~m} \mathrm{~h} 3$ \\
\hline 13 & $\mathrm{~h}$ & 0 & 0.273051 & -1.338266 & 0.240956 & $0 \mathrm{~m}$ \\
\hline 14 & $\mathrm{c}$ & 0 & -1.739946 & 1.983166 & 0.094848 & $0 \mathrm{~m} \mathrm{~h} 4$ \\
\hline 15 & $\mathrm{~h}$ & 0 & -2.303468 & 2.912928 & 0.065827 & $0 \mathrm{~m}$ \\
\hline 16 & $\mathrm{c}$ & 0 & 2.616000 & -0.082572 & -1.801117 & $0 \mathrm{~m} \mathrm{~h} 6$ \\
\hline 17 & $\mathrm{~h}$ & 0 & 1.600474 & -0.443819 & -2.000634 & $0 \mathrm{~m}$ \\
\hline 18 & $\mathrm{~h}$ & 0 & 3.098635 & 0.087117 & -2.771133 & $0 \mathrm{~m}$ \\
\hline 19 & $\mathrm{c}$ & 0 & 3.409938 & -1.133667 & -1.040391 & $0 \mathrm{~m} \mathrm{~h} 11$ \\
\hline 20 & $\mathrm{~h}$ & 0 & 4.471761 & -0.856003 & -1.000078 & $0 \mathrm{~m}$ \\
\hline 21 & $\mathrm{~h}$ & 0 & 3.336798 & -2.120854 & -1.504034 & $0 \mathrm{~m}$ \\
\hline 22 & $\mathrm{c}$ & 0 & -1.691085 & -0.465623 & 0.053139 & $0 \mathrm{~m}$ \\
\hline 23 & $\mathrm{c}$ & 0 & -2.384535 & -1.702614 & -0.034232 & $0 \mathrm{~m}$ \\
\hline 24 & $\mathrm{~h}$ & 0 & -1.813402 & -2.626928 & 0.011512 & $0 \mathrm{~m}$ \\
\hline 25 & $\mathrm{c}$ & 0 & -3.754349 & -1.734610 & -0.170800 & $0 \mathrm{~m}$ \\
\hline
\end{tabular}




$\begin{array}{lllllll}26 & \mathrm{~h} & 0 & -4.272605 & -2.687521 & -0.235433 & 0 \mathrm{~m} \\ 27 & \mathrm{c} & 0 & -4.496577 & -0.529293 & -0.225292 & 0 \mathrm{~m} \\ 28 & \mathrm{~h} & 0 & -5.577240 & -0.568131 & -0.332188 & 0 \mathrm{~m} \\ 29 & \mathrm{c} & 0 & -3.853824 & 0.685569 & -0.141358 & 0 \mathrm{~m} \\ 30 & \mathrm{~h} & 0 & -4.420255 & 1.613204 & -0.180416 & 0 \mathrm{~m} \\ 31 & \mathrm{c} & 0 & -2.442244 & 0.751874 & -0.000778 & 0 \mathrm{~m}\end{array}$

radical

Charge $=0$ Multiplicity $=2$ for low level calculation on real system.

Charge $=0$ Multiplicity $=2$ for high level calculation on model system.

Charge $=0$ Multiplicity $=2$ for low level calculation on model system.

\begin{tabular}{|c|c|c|c|c|c|c|}
\hline Center & Atomic & Atomic & Coordina & es (Angstrom & & \\
\hline Number & Numbe & r Type & $\mathrm{X}$ & $\mathrm{Y}$ & $\mathrm{Z}$ & \\
\hline 1 & $\mathrm{c}$ & 0 & 1.705005 & 0.197575 & -0.113168 & $0 \mathrm{~h}$ \\
\hline 2 & $\mathrm{c}$ & 0 & 0.278106 & 0.377977 & -0.083607 & $0 \mathrm{~h}$ \\
\hline 3 & $\mathrm{c}$ & 0 & -0.274735 & 1.712010 & -0.056904 & $0 \mathrm{~h}$ \\
\hline 4 & $\mathrm{~h}$ & 0 & 0.389088 & 2.568035 & -0.073202 & $0 \mathrm{~h}$ \\
\hline 5 & $\mathrm{c}$ & 0 & 2.603600 & 1.419100 & -0.134684 & $0 \mathrm{~h}$ \\
\hline 6 & $\mathrm{~h}$ & 0 & 2.452099 & 1.997186 & 0.790924 & $0 \mathrm{~h}$ \\
\hline 7 & $\mathrm{~h}$ & 0 & 2.304738 & 2.088119 & -0.952732 & $0 \mathrm{~h}$ \\
\hline 8 & $\mathrm{c}$ & 0 & 2.318034 & -1.147143 & -0.120657 & $0 \mathrm{~h}$ \\
\hline 9 & o & 0 & 1.740240 & -2.183197 & -0.404924 & $0 \mathrm{~h}$ \\
\hline 10 & o & 0 & 3.646933 & -1.259283 & 0.190450 & $0 \mathrm{~h}$ \\
\hline 11 & $\mathrm{c}$ & 0 & -0.643586 & -0.688119 & -0.050555 & $0 \mathrm{~m} \mathrm{~h} 2$ \\
\hline 12 & $\mathrm{~h}$ & 0 & -0.270047 & -1.702718 & -0.076717 & $0 \mathrm{~m}$ \\
\hline 13 & $\mathrm{c}$ & 0 & -1.622323 & 1.937195 & -0.006491 & $0 \mathrm{~m} \mathrm{~h} 3$ \\
\hline 14 & $\mathrm{~h}$ & 0 & -2.000643 & 2.956847 & 0.012465 & $0 \mathrm{~m}$ \\
\hline 15 & $\mathrm{c}$ & 0 & 4.088404 & 1.071927 & -0.264838 & $0 \mathrm{~m} \mathrm{~h} 5$ \\
\hline 16 & $\mathrm{~h}$ & 0 & 4.709873 & 1.932249 & 0.008611 & $0 \mathrm{~m}$ \\
\hline 17 & $\mathrm{~h}$ & 0 & 4.336442 & 0.801408 & -1.298501 & $0 \mathrm{~m}$ \\
\hline 18 & $\mathrm{c}$ & 0 & 4.388134 & -0.113999 & 0.630982 & $0 \mathrm{~m} \mathrm{~h} 10$ \\
\hline 19 & $\mathrm{~h}$ & 0 & 4.135255 & 0.106929 & 1.678169 & $0 \mathrm{~m}$ \\
\hline 20 & $\mathrm{~h}$ & 0 & 5.436496 & -0.420168 & 0.590066 & $0 \mathrm{~m}$ \\
\hline 21 & $\mathrm{c}$ & 0 & -2.039135 & -0.474397 & 0.000472 & $0 \mathrm{~m}$ \\
\hline 22 & $\mathrm{c}$ & 0 & -2.955241 & -1.563423 & 0.030741 & $0 \mathrm{~m}$ \\
\hline 23 & $\mathrm{~h}$ & 0 & -2.560881 & -2.576268 & 0.012337 & $0 \mathrm{~m}$ \\
\hline 24 & $\mathrm{c}$ & 0 & -4.313776 & -1.340067 & 0.081261 & $0 \mathrm{~m}$ \\
\hline 25 & $\mathrm{~h}$ & 0 & -5.003536 & -2.179238 & 0.103762 & $0 \mathrm{~m}$ \\
\hline 26 & $\mathrm{c}$ & 0 & -4.818950 & -0.018311 & 0.103587 & $0 \mathrm{~m}$ \\
\hline 27 & $\mathrm{~h}$ & 0 & -5.892312 & 0.146205 & 0.143205 & $0 \mathrm{~m}$ \\
\hline
\end{tabular}




$\begin{array}{lllllll}28 & \mathrm{c} & 0 & -3.955419 & 1.059704 & 0.074920 & 0 \mathrm{~m} \\ 29 & \mathrm{~h} & 0 & -4.342896 & 2.075778 & 0.091837 & 0 \mathrm{~m} \\ 30 & \mathrm{c} & 0 & -2.556628 & 0.860886 & 0.023203 & 0 \mathrm{~m}\end{array}$

No. 35

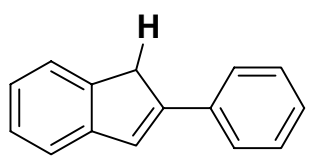

neutral molecule

Charge $=0$ Multiplicity $=1$ for low level calculation on real system.

Charge $=0$ Multiplicity $=1$ for high level calculation on model system .

Charge $=0$ Multiplicity $=1$ for low level calculation on model system.

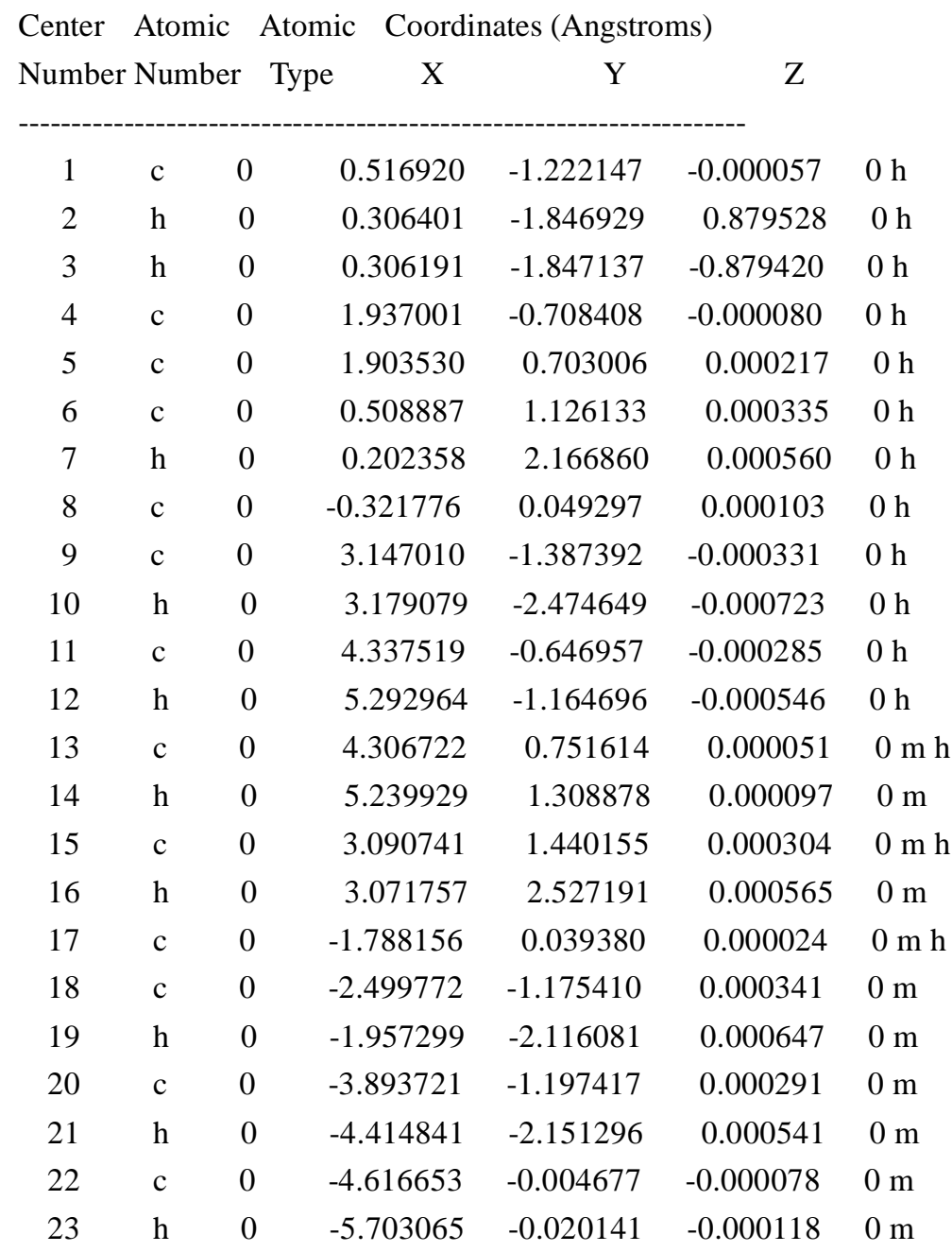




$\begin{array}{lllllll}24 & \mathrm{c} & 0 & -3.927776 & 1.212041 & -0.000418 & 0 \mathrm{~m} \\ 25 & \mathrm{~h} & 0 & -4.478498 & 2.149170 & -0.000723 & 0 \mathrm{~m} \\ 26 & \mathrm{c} & 0 & -2.537399 & 1.233846 & -0.000368 & 0 \mathrm{~m} \\ 27 & \mathrm{~h} & 0 & -2.023431 & 2.190447 & -0.000702 & 0 \mathrm{~m}\end{array}$

radical

Charge $=0$ Multiplicity $=2$ for low level calculation on real system.

Charge $=0$ Multiplicity $=2$ for high level calculation on model system.

Charge $=0$ Multiplicity $=2$ for low level calculation on model system.

\begin{tabular}{|c|c|c|c|c|c|c|}
\hline Center & Atomic & & Atomic & ates (Angstro & & \\
\hline Number & Number & & Type & $\mathrm{Y}$ & $\mathrm{Z}$ & \\
\hline 1 & $\mathrm{c}$ & 0 & 0.525680 & 1.120703 & 0.204376 & $0 \mathrm{~h}$ \\
\hline 2 & $\mathrm{~h}$ & 0 & 0.188923 & 2.128697 & 0.418498 & $0 \mathrm{~h}$ \\
\hline 3 & $\mathrm{c}$ & 0 & 1.910116 & 0.703625 & 0.128827 & $0 \mathrm{~h}$ \\
\hline 4 & $\mathrm{c}$ & 0 & 1.909973 & -0.703539 & -0.128762 & $0 \mathrm{~h}$ \\
\hline 5 & $\mathrm{c}$ & 0 & 0.525360 & -1.120143 & -0.204643 & $0 \mathrm{~h}$ \\
\hline 6 & $\mathrm{~h}$ & 0 & 0.188102 & -2.128082 & -0.418303 & $0 \mathrm{~h}$ \\
\hline 7 & $\mathrm{c}$ & 0 & -0.307835 & 0.000331 & -0.000110 & $0 \mathrm{~h}$ \\
\hline 8 & $\mathrm{c}$ & 0 & 3.109132 & 1.394192 & 0.258165 & $0 \mathrm{~h}$ \\
\hline 9 & $\mathrm{~h}$ & 0 & 3.120681 & 2.463469 & 0.455269 & $0 \mathrm{~h}$ \\
\hline 10 & $\mathrm{c}$ & 0 & 4.318119 & 0.685365 & 0.127488 & $0 \mathrm{~h}$ \\
\hline 11 & $\mathrm{~h}$ & 0 & 5.261890 & 1.214629 & 0.225864 & $0 \mathrm{~h}$ \\
\hline 12 & $\mathrm{c}$ & 0 & 4.317962 & -0.685801 & -0.127396 & $0 \mathrm{~m} \mathrm{~h}$ \\
\hline 13 & $\mathrm{~h}$ & 0 & 5.261592 & -1.215325 & -0.225718 & $0 \mathrm{~m}$ \\
\hline 14 & $\mathrm{c}$ & 0 & 3.108772 & -1.394349 & -0.258219 & $0 \mathrm{~m} \mathrm{~h} 4$ \\
\hline 15 & $\mathrm{~h}$ & 0 & 3.120169 & -2.463633 & -0.455277 & $0 \mathrm{~m}$ \\
\hline 16 & $\mathrm{c}$ & 0 & -1.780277 & 0.000216 & -0.000092 & $0 \mathrm{~m} \mathrm{~h}$ \\
\hline 17 & $\mathrm{c}$ & 0 & -2.505364 & 1.161581 & -0.321631 & $0 \mathrm{~m}$ \\
\hline 18 & $\mathrm{~h}$ & 0 & -1.969862 & 2.066034 & -0.597120 & $0 \mathrm{~m}$ \\
\hline 19 & $\mathrm{c}$ & 0 & -3.898703 & 1.162291 & -0.318663 & $0 \mathrm{~m}$ \\
\hline 20 & $\mathrm{~h}$ & 0 & -4.435742 & 2.072083 & -0.574542 & $0 \mathrm{~m}$ \\
\hline 21 & $\mathrm{c}$ & 0 & -4.603404 & -0.000327 & 0.000075 & $0 \mathrm{~m}$ \\
\hline 22 & $\mathrm{~h}$ & 0 & -5.690012 & -0.000509 & 0.000131 & $0 \mathrm{~m}$ \\
\hline 23 & $\mathrm{c}$ & 0 & -3.898280 & -1.162641 & 0.318811 & $0 \mathrm{~m}$ \\
\hline 24 & $\mathrm{~h}$ & 0 & -4.434905 & -2.072638 & 0.574829 & $0 \mathrm{~m}$ \\
\hline 25 & $\mathrm{c}$ & 0 & -2.504888 & -1.161367 & 0.321624 & $0 \mathrm{~m}$ \\
\hline 26 & $\mathrm{~h}$ & 0 & -1.969007 & -2.065552 & 0.597273 & $0 \mathrm{~m}$ \\
\hline
\end{tabular}




\section{No. 36}<smiles>c1ccc(C(c2ccccc2)c2ccccc2)cc1</smiles>

neutral molecule Charge $=0$ Multiplicity $=1$ for low level calculation on real system.

Charge $=0$ Multiplicity $=1$ for high level calculation on model system .

Charge $=0$ Multiplicity $=1$ for low level calculation on model system.

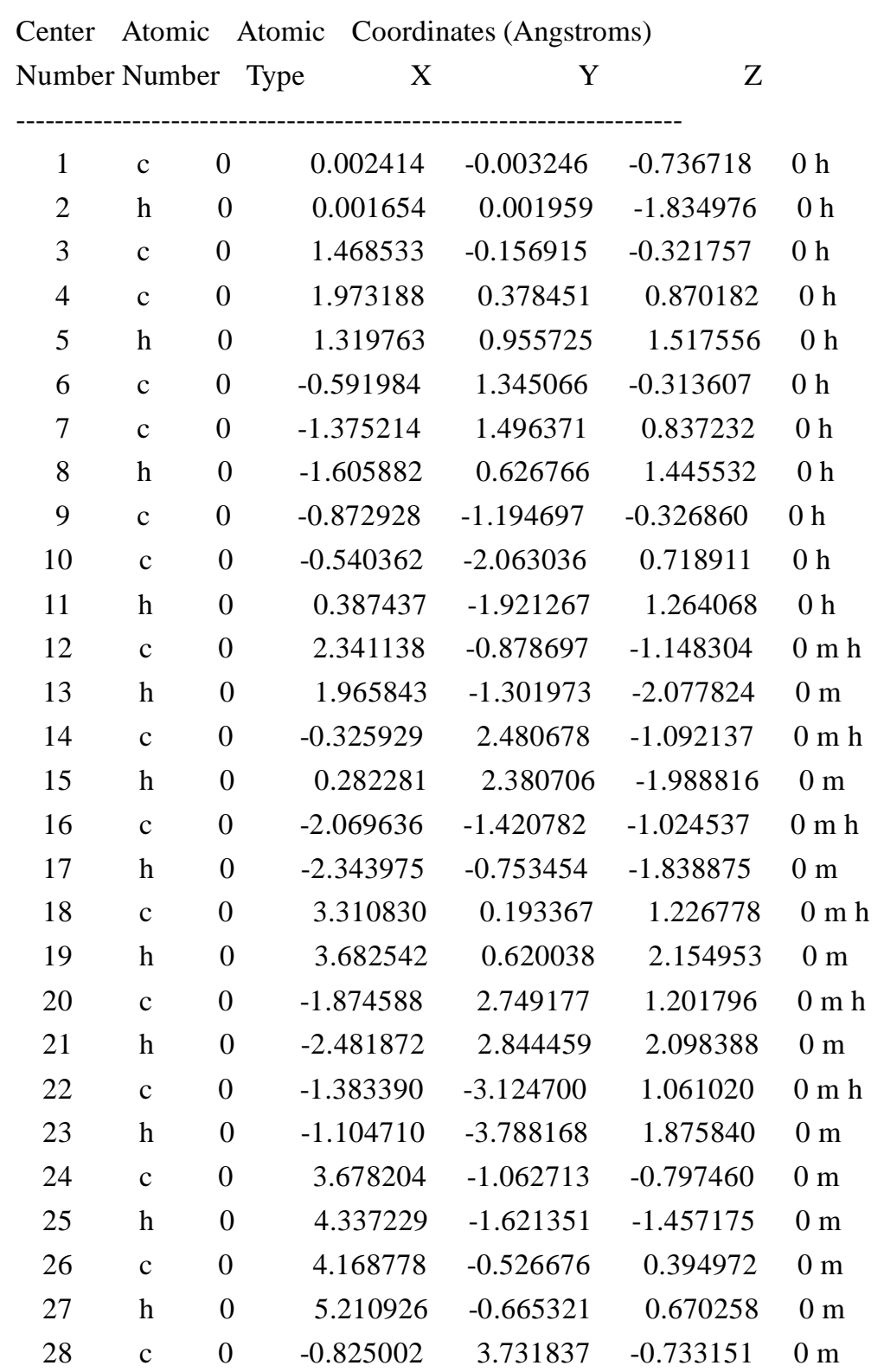




$\begin{array}{lllllll}29 & \mathrm{~h} & 0 & -0.609932 & 4.597311 & -1.354734 & 0 \mathrm{~m} \\ 30 & \mathrm{c} & 0 & -1.602860 & 3.870869 & 0.418583 & 0 \mathrm{~m} \\ 31 & \mathrm{~h} & 0 & -1.996104 & 4.844194 & 0.699769 & 0 \mathrm{~m} \\ 32 & \mathrm{c} & 0 & -2.910614 & -2.479198 & -0.687715 & 0 \mathrm{~m} \\ 33 & \mathrm{~h} & 0 & -3.830441 & -2.636489 & -1.245274 & 0 \mathrm{~m} \\ 34 & \mathrm{c} & 0 & -2.569444 & -3.337961 & 0.360564 & 0 \mathrm{~m} \\ 35 & \mathrm{~h} & 0 & -3.221553 & -4.166315 & 0.624566 & 0 \mathrm{~m}\end{array}$

radical

Charge $=0$ Multiplicity $=2$ for low level calculation on real system.

Charge $=0$ Multiplicity $=2$ for high level calculation on model system.

Charge $=0$ Multiplicity $=2$ for low level calculation on model system.

\begin{tabular}{|c|c|c|c|c|c|c|}
\hline Center & Atomic & & Coordin & ates (Angstro & ms) & \\
\hline Number & Numbe & & Type & $\mathrm{Y}$ & $\mathrm{Z}$ & \\
\hline 1 & $\mathrm{c}$ & 0 & 0.000633 & -0.001613 & -0.000124 & $0 \mathrm{~h}$ \\
\hline 2 & c & 0 & 1.393094 & -0.455414 & -0.000907 & $0 \mathrm{~h}$ \\
\hline 3 & $\mathrm{c}$ & 0 & 1.783844 & -1.638962 & 0.671157 & $0 \mathrm{~h}$ \\
\hline 4 & $\mathrm{~h}$ & 0 & 1.036476 & -2.208830 & 1.213998 & $0 \mathrm{~h}$ \\
\hline 5 & $\mathrm{c}$ & 0 & -1.089637 & -0.978974 & 0.000945 & $0 \mathrm{~h}$ \\
\hline 6 & $\mathrm{c}$ & 0 & -2.308058 & -0.723421 & 0.675383 & $0 \mathrm{~h}$ \\
\hline 7 & $\mathrm{~h}$ & 0 & -2.423244 & 0.206831 & 1.222534 & $0 \mathrm{~h}$ \\
\hline 8 & $\mathrm{c}$ & 0 & -0.302091 & 1.431651 & -0.000343 & $0 \mathrm{~h}$ \\
\hline 9 & $\mathrm{c}$ & 0 & 0.521870 & 2.359552 & 0.680994 & $0 \mathrm{~h}$ \\
\hline 10 & $\mathrm{~h}$ & 0 & 1.383961 & 1.995081 & 1.230422 & $0 \mathrm{~h}$ \\
\hline 11 & $\mathrm{c}$ & 0 & 2.405623 & 0.270778 & -0.673208 & $0 \mathrm{~m} \mathrm{~h}$ \\
\hline 12 & $\mathrm{~h}$ & 0 & 2.136954 & 1.171658 & -1.215549 & $0 \mathrm{~m}$ \\
\hline 13 & $\mathrm{c}$ & 0 & -0.970905 & -2.218702 & -0.672795 & $0 \mathrm{~m} \mathrm{~h}$ \\
\hline 14 & $\mathrm{~h}$ & 0 & -0.057889 & -2.437992 & -1.217144 & $0 \mathrm{~m}$ \\
\hline 15 & $\mathrm{c}$ & 0 & -1.431011 & 1.947296 & -0.681587 & $0 \mathrm{~m} \mathrm{~h}$ \\
\hline 16 & $\mathrm{~h}$ & 0 & -2.071782 & 1.266086 & -1.232810 & $0 \mathrm{~m}$ \\
\hline 17 & $\mathrm{c}$ & 0 & 3.107810 & -2.065798 & 0.672825 & $0 \mathrm{~m} \mathrm{~h}$ \\
\hline 18 & $\mathrm{~h}$ & 0 & 3.377626 & -2.971353 & 1.210333 & $0 \mathrm{~m}$ \\
\hline 19 & $\mathrm{c}$ & 0 & -3.342800 & -1.653211 & 0.676320 & $0 \mathrm{~m} \mathrm{~h}$ \\
\hline 20 & $\mathrm{~h}$ & 0 & -4.260577 & -1.432151 & 1.215368 & $0 \mathrm{~m}$ \\
\hline 21 & $\mathrm{c}$ & 0 & 0.230652 & 3.719913 & 0.682882 & $0 \mathrm{~m} \mathrm{~h}$ \\
\hline 22 & $\mathrm{~h}$ & 0 & 0.875747 & 4.404674 & 1.227594 & $0 \mathrm{~m}$ \\
\hline 23 & $\mathrm{c}$ & 0 & 3.727391 & -0.163438 & -0.674626 & $0 \mathrm{~m}$ \\
\hline 24 & $\mathrm{~h}$ & 0 & 4.478486 & 0.410037 & -1.211971 & $0 \mathrm{~m}$ \\
\hline 25 & $\mathrm{c}$ & 0 & 4.089546 & -1.333417 & -0.000966 & $0 \mathrm{~m}$ \\
\hline 26 & $\mathrm{~h}$ & 0 & 5.122749 & -1.669794 & -0.000594 & $0 \mathrm{~m}$ \\
\hline
\end{tabular}




$\begin{array}{lllrrrr}27 & \mathrm{c} & 0 & -2.010310 & -3.143304 & -0.673843 & 0 \mathrm{~m} \\ 28 & \mathrm{~h} & 0 & -1.892611 & -4.080313 & -1.212272 & 0 \mathrm{~m} \\ 29 & \mathrm{c} & 0 & -3.203399 & -2.869141 & 0.000956 & 0 \mathrm{~m} \\ 30 & \mathrm{~h} & 0 & -4.013458 & -3.593325 & 0.000591 & 0 \mathrm{~m} \\ 31 & \mathrm{c} & 0 & -1.714274 & 3.309387 & -0.683411 & 0 \mathrm{~m} \\ 32 & \mathrm{~h} & 0 & -2.580939 & 3.675111 & -1.228265 & 0 \mathrm{~m} \\ 33 & \mathrm{c} & 0 & -0.887848 & 4.206004 & -0.000045 & 0 \mathrm{~m} \\ 34 & \mathrm{~h} & 0 & -1.112283 & 5.269154 & 0.000120 & 0 \mathrm{~m}\end{array}$

No. 37

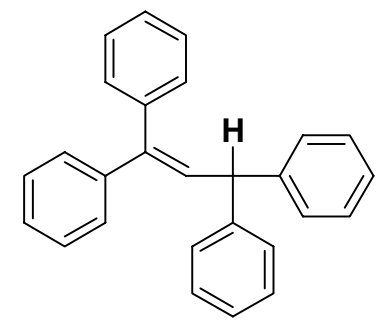

neutral molecule

Charge $=0$ Multiplicity $=1$ for low level calculation on real system.

Charge $=0$ Multiplicity $=1$ for high level calculation on model system .

Charge $=0$ Multiplicity $=1$ for low level calculation on model system .

\begin{tabular}{|c|c|c|c|c|c|c|}
\hline Center & Atomic & & tomic & ates (Angstro & & \\
\hline Number & Numbe & & ype & Y & Z & \\
\hline 1 & $\mathrm{c}$ & 0 & -1.225966 & -0.049504 & 0.181138 & $0 \mathrm{~h}$ \\
\hline 2 & $\mathrm{~h}$ & 0 & -0.984226 & -0.680085 & 1.044365 & $0 \mathrm{~h}$ \\
\hline 3 & $\mathrm{c}$ & 0 & 0.074833 & 0.420757 & -0.434725 & $0 \mathrm{~h}$ \\
\hline 4 & $\mathrm{~h}$ & 0 & -0.036932 & 1.202087 & -1.185217 & $0 \mathrm{~h}$ \\
\hline 5 & c & 0 & 1.330785 & 0.041271 & -0.113294 & $0 \mathrm{~h}$ \\
\hline 6 & $\mathrm{c}$ & 0 & -2.094786 & -0.895039 & -0.759325 & $0 \mathrm{~h}$ \\
\hline 7 & $\mathrm{c}$ & 0 & -1.677274 & -1.260653 & -2.043973 & $0 \mathrm{~h}$ \\
\hline 8 & $\mathrm{~h}$ & 0 & -0.710831 & -0.925364 & -2.409468 & $0 \mathrm{~h}$ \\
\hline 9 & $\mathrm{c}$ & 0 & -1.968965 & 1.179339 & 0.718891 & $0 \mathrm{~h}$ \\
\hline 10 & $\mathrm{c}$ & 0 & -2.703659 & 2.018406 & -0.130712 & $0 \mathrm{~h}$ \\
\hline 11 & $\mathrm{~h}$ & 0 & -2.794989 & 1.765924 & -1.184116 & $0 \mathrm{~h}$ \\
\hline 12 & $\mathrm{c}$ & 0 & 2.494564 & 0.741190 & -0.732609 & $0 \mathrm{~m} \mathrm{~h}$ \\
\hline 13 & $\mathrm{c}$ & 0 & 1.631151 & -1.062009 & 0.847677 & $0 \mathrm{~m} \mathrm{~h}$ \\
\hline 14 & $\mathrm{c}$ & 0 & -2.484072 & -2.059508 & -2.860881 & $0 \mathrm{~m} \mathrm{~h}$ \\
\hline 15 & $\mathrm{~h}$ & 0 & -2.139203 & -2.328734 & -3.856047 & $0 \mathrm{~m}$ \\
\hline
\end{tabular}




\begin{tabular}{lllrrrl}
16 & $\mathrm{c}$ & 0 & -3.343490 & -1.354624 & -0.310465 & $0 \mathrm{~m} \mathrm{~h}$ \\
17 & $\mathrm{~h}$ & 0 & -3.687479 & -1.076950 & 0.682985 & $0 \mathrm{~m}$ \\
18 & $\mathrm{c}$ & 0 & -3.332265 & 3.160627 & 0.364840 & $0 \mathrm{~m} \mathrm{~h}$ \\
19 & $\mathrm{~h}$ & 0 & -3.900265 & 3.797647 & -0.308470 & $0 \mathrm{~m}$ \\
20 & $\mathrm{c}$ & 0 & -1.878902 & 1.514460 & 2.075555 & $0 \mathrm{~m} \mathrm{~h}$ \\
21 & $\mathrm{~h}$ & 0 & -1.306345 & 0.876784 & 2.745477 & $0 \mathrm{~m}$ \\
22 & $\mathrm{c}$ & 0 & 2.476090 & 2.129731 & -0.951395 & $0 \mathrm{~m}$ \\
23 & $\mathrm{~h}$ & 0 & 1.619733 & 2.707831 & -0.615993 & $0 \mathrm{~m}$ \\
24 & $\mathrm{c}$ & 0 & 3.550443 & 2.774104 & -1.563558 & $0 \mathrm{~m}$ \\
25 & $\mathrm{~h}$ & 0 & 3.515914 & 3.849861 & -1.715785 & $0 \mathrm{~m}$ \\
26 & $\mathrm{c}$ & 0 & 4.671851 & 2.045627 & -1.964210 & $0 \mathrm{~m}$ \\
27 & $\mathrm{~h}$ & 0 & 5.511240 & 2.548151 & -2.437450 & $0 \mathrm{~m}$ \\
28 & $\mathrm{c}$ & 0 & 4.711088 & 0.667616 & -1.741170 & $0 \mathrm{~m}$ \\
29 & $\mathrm{~h}$ & 0 & 5.579958 & 0.090587 & -2.047312 & $0 \mathrm{~m}$ \\
30 & $\mathrm{c}$ & 0 & 3.638082 & 0.024014 & -1.127039 & $0 \mathrm{~m}$ \\
31 & $\mathrm{~h}$ & 0 & 3.677269 & -1.048300 & -0.959235 & $0 \mathrm{~m}$ \\
32 & $\mathrm{c}$ & 0 & 2.543073 & -0.856856 & 1.898337 & $0 \mathrm{~m}$ \\
33 & $\mathrm{~h}$ & 0 & 3.026513 & 0.110739 & 2.000120 & $0 \mathrm{~m}$ \\
34 & $\mathrm{c}$ & 0 & 2.825162 & -1.872877 & 2.809388 & $0 \mathrm{~m}$ \\
35 & $\mathrm{~h}$ & 0 & 3.525470 & -1.689779 & 3.620302 & $0 \mathrm{~m}$ \\
36 & $\mathrm{c}$ & 0 & 2.213314 & -3.122409 & 2.681203 & $0 \mathrm{~m}$ \\
37 & $\mathrm{~h}$ & 0 & 2.437869 & -3.916292 & 3.388635 & $0 \mathrm{~m}$ \\
38 & $\mathrm{c}$ & 0 & 1.318505 & -3.346138 & 1.634393 & $0 \mathrm{~m}$ \\
39 & $\mathrm{~h}$ & 0 & 0.845763 & -4.317931 & 1.518174 & $0 \mathrm{~m}$ \\
40 & $\mathrm{c}$ & 0 & 1.031311 & -2.326446 & 0.725252 & $0 \mathrm{~m}$ \\
41 & $\mathrm{~h}$ & 0 & 0.345481 & -2.512885 & -0.096248 & $0 \mathrm{~m}$ \\
42 & $\mathrm{c}$ & 0 & -3.720759 & -2.509016 & -2.402959 & $0 \mathrm{~m}$ \\
43 & $\mathrm{~h}$ & 0 & -4.348071 & -3.130727 & -3.036271 & $0 \mathrm{~m}$ \\
44 & $\mathrm{c}$ & 0 & -4.147272 & -2.153788 & -1.119856 & $0 \mathrm{~m}$ \\
45 & $\mathrm{~h}$ & 0 & -5.109009 & -2.500061 & -0.749760 & $0 \mathrm{~m}$ \\
46 & $\mathrm{c}$ & 0 & -2.510684 & 2.654559 & 2.575562 & $0 \mathrm{~m}$ \\
47 & $\mathrm{~h}$ & 0 & -2.433713 & 2.893708 & 3.633173 & $0 \mathrm{~m}$ \\
48 & $\mathrm{c}$ & 0 & -3.239040 & 3.482746 & 1.720730 & $0 \mathrm{~m}$ \\
--------------------------------------------------------------- & \\
49 & $\mathrm{~h}$ & 0 & -3.732865 & 4.370307 & 2.107382 & $0 \mathrm{~m}$ \\
\hline
\end{tabular}

radical

Charge $=0$ Multiplicity $=2$ for low level calculation on real system.

Charge $=0$ Multiplicity $=2$ for high level calculation on model system.

Charge $=0$ Multiplicity $=2$ for low level calculation on model system.

Center Atomic Atomic Coordinates (Angstroms)

$\begin{array}{llllll}\text { Number Number } & \text { Type } & \text { X } & \text { Y } & \text { Z }\end{array}$ 


\begin{tabular}{|c|c|c|c|c|c|c|}
\hline 1 & $\mathrm{c}$ & 0 & -1.281849 & 0.420576 & -0.087151 & $0 \mathrm{~h}$ \\
\hline 2 & $\mathrm{c}$ & 0 & 0.000033 & 0.993867 & -0.000068 & $0 \mathrm{~h}$ \\
\hline 3 & $\mathrm{~h}$ & 0 & 0.000096 & 2.082506 & 0.000034 & $0 \mathrm{~h}$ \\
\hline 4 & $\mathrm{c}$ & 0 & 1.281904 & 0.420493 & 0.086912 & $0 \mathrm{~h}$ \\
\hline 5 & $\mathrm{c}$ & 0 & -2.444825 & 1.288235 & 0.181050 & $0 \mathrm{~h}$ \\
\hline 6 & $\mathrm{c}$ & 0 & -2.365586 & 2.341661 & 1.118582 & $0 \mathrm{~h}$ \\
\hline 7 & $\mathrm{~h}$ & 0 & -1.452783 & 2.473349 & 1.691944 & $0 \mathrm{~h}$ \\
\hline 8 & $\mathrm{c}$ & 0 & -1.528662 & -0.969341 & -0.530728 & $0 \mathrm{~h}$ \\
\hline 9 & $\mathrm{c}$ & 0 & -2.509235 & -1.767096 & 0.091386 & $0 \mathrm{~h}$ \\
\hline 10 & $\mathrm{~h}$ & 0 & -3.069935 & -1.360631 & 0.928076 & $0 \mathrm{~h}$ \\
\hline 11 & $\mathrm{c}$ & 0 & 1.528662 & -0.969425 & 0.530613 & $0 \mathrm{~m} \mathrm{~h}$ \\
\hline 12 & $\mathrm{c}$ & 0 & 2.444908 & 1.288149 & -0.181225 & $0 \mathrm{~m} \mathrm{~h}$ \\
\hline 13 & $\mathrm{c}$ & 0 & -3.447086 & 3.188039 & 1.348349 & $0 \mathrm{~m} \mathrm{~h}$ \\
\hline 14 & $\mathrm{~h}$ & 0 & -3.358580 & 3.984083 & 2.083305 & $0 \mathrm{~m}$ \\
\hline 15 & $\mathrm{c}$ & 0 & -3.667711 & 1.118775 & -0.505199 & $0 \mathrm{~m} \mathrm{~h}$ \\
\hline 16 & $\mathrm{~h}$ & 0 & -3.757522 & 0.325438 & -1.240041 & $0 \mathrm{~m}$ \\
\hline 17 & $\mathrm{c}$ & 0 & -2.756379 & -3.068603 & -0.339611 & $0 \mathrm{~m} \mathrm{~h}$ \\
\hline 18 & $\mathrm{~h}$ & 0 & -3.509613 & -3.668417 & 0.164911 & $0 \mathrm{~m}$ \\
\hline 19 & $\mathrm{c}$ & 0 & -0.818904 & -1.522619 & -1.613333 & $0 \mathrm{~m} \mathrm{~h}$ \\
\hline 20 & $\mathrm{~h}$ & 0 & -0.073806 & -0.916468 & -2.118883 & $0 \mathrm{~m}$ \\
\hline 21 & $\mathrm{c}$ & 0 & 0.819053 & -1.522448 & 1.613454 & $0 \mathrm{~m}$ \\
\hline 22 & $\mathrm{~h}$ & 0 & 0.074101 & -0.916145 & 2.119029 & $0 \mathrm{~m}$ \\
\hline 23 & $\mathrm{c}$ & 0 & 1.072762 & -2.820070 & 2.050141 & $0 \mathrm{~m}$ \\
\hline 24 & $\mathrm{~h}$ & 0 & 0.517079 & -3.221367 & 2.893672 & $0 \mathrm{~m}$ \\
\hline 25 & $\mathrm{c}$ & 0 & 2.039881 & -3.601698 & 1.413553 & $0 \mathrm{~m}$ \\
\hline 26 & $\mathrm{~h}$ & 0 & 2.235743 & -4.615103 & 1.753697 & $0 \mathrm{~m}$ \\
\hline 27 & $\mathrm{c}$ & 0 & 2.756051 & -3.068889 & 0.339541 & $0 \mathrm{~m}$ \\
\hline 28 & $\mathrm{~h}$ & 0 & 3.509057 & -3.668896 & -0.165088 & $0 \mathrm{~m}$ \\
\hline 29 & $\mathrm{c}$ & 0 & 2.509025 & -1.767374 & -0.091562 & $0 \mathrm{~m}$ \\
\hline 30 & $\mathrm{~h}$ & 0 & 3.069538 & -1.361176 & -0.928496 & $0 \mathrm{~m}$ \\
\hline 31 & $\mathrm{c}$ & 0 & 3.667790 & 1.118626 & 0.505133 & $0 \mathrm{~m}$ \\
\hline 32 & $\mathrm{~h}$ & 0 & 3.757548 & 0.325062 & 1.239717 & $0 \mathrm{~m}$ \\
\hline 33 & $\mathrm{c}$ & 0 & 4.747050 & 1.966872 & 0.274718 & $0 \mathrm{~m}$ \\
\hline 34 & $\mathrm{~h}$ & 0 & 5.671431 & 1.819464 & 0.827412 & $0 \mathrm{~m}$ \\
\hline 35 & $\mathrm{c}$ & 0 & 4.644696 & 3.007545 & -0.652328 & $0 \mathrm{~m}$ \\
\hline 36 & $\mathrm{~h}$ & 0 & 5.489726 & 3.666185 & -0.833787 & $0 \mathrm{~m}$ \\
\hline 37 & $\mathrm{c}$ & 0 & 3.447154 & 3.188131 & -1.348224 & $0 \mathrm{~m}$ \\
\hline 38 & $\mathrm{~h}$ & 0 & 3.358657 & 3.984266 & -2.083082 & $0 \mathrm{~m}$ \\
\hline 39 & $\mathrm{c}$ & 0 & 2.365680 & 2.341672 & -1.118636 & $0 \mathrm{~m}$ \\
\hline 40 & $\mathrm{~h}$ & 0 & 1.452913 & 2.473294 & -1.692062 & $0 \mathrm{~m}$ \\
\hline 41 & $\mathrm{c}$ & 0 & -4.644675 & 3.007511 & 0.652495 & $0 \mathrm{~m}$ \\
\hline 42 & $\mathrm{~h}$ & 0 & -5.489694 & 3.666115 & 0.834130 & $0 \mathrm{~m}$ \\
\hline 43 & $\mathrm{c}$ & 0 & -4.746996 & 1.966981 & -0.274692 & $0 \mathrm{~m}$ \\
\hline
\end{tabular}




$\begin{array}{llllrrl}44 & \mathrm{~h} & 0 & -5.671380 & 1.819589 & -0.827381 & 0 \mathrm{~m} \\ 45 & \mathrm{c} & 0 & -2.040036 & -3.601673 & -1.413380 & 0 \mathrm{~m} \\ 46 & \mathrm{~h} & 0 & -2.235951 & -4.615095 & -1.753443 & 0 \mathrm{~m} \\ 47 & \mathrm{c} & 0 & -1.072669 & -2.820274 & -2.049865 & 0 \mathrm{~m} \\ 48 & \mathrm{~h} & 0 & -0.516848 & -3.221782 & -2.893206 & 0 \mathrm{~m}\end{array}$

No. 38

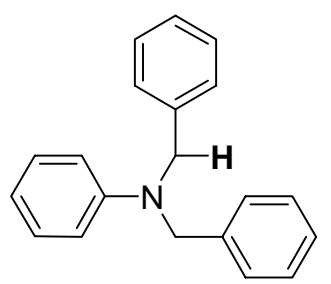

neutral molecule

Charge $=0$ Multiplicity $=1$ for low level calculation on real system.

Charge $=0$ Multiplicity $=1$ for high level calculation on model system.

Charge $=0$ Multiplicity $=1$ for low level calculation on model system .

\begin{tabular}{|c|c|c|c|c|c|c|c|}
\hline Center & Atomic & & Atomic & Coordi & lates (Angstr & & \\
\hline Number & Numbe & & Type & X & $\mathrm{Y}$ & $\mathrm{Z}$ & \\
\hline 1 & $\mathrm{c}$ & 0 & & 0.205918 & 0.583561 & -1.212887 & $0 \mathrm{~h}$ \\
\hline 2 & $\mathrm{~h}$ & 0 & & 0.639951 & 0.385418 & -2.209569 & $0 \mathrm{~h}$ \\
\hline 3 & $\mathrm{~h}$ & 0 & & 0.779616 & 1.027891 & -1.397262 & $0 \mathrm{~h}$ \\
\hline 4 & $\mathrm{c}$ & 0 & & 1.055790 & 1.607705 & -0.480531 & $0 \mathrm{~h}$ \\
\hline 5 & $\mathrm{c}$ & 0 & & 1.925469 & 2.435168 & -1.200622 & $0 \mathrm{~h}$ \\
\hline 6 & $\mathrm{~h}$ & 0 & & 2.027625 & 2.298319 & -2.275698 & $0 \mathrm{~h}$ \\
\hline 7 & $\mathrm{n}$ & 0 & & 0.047350 & -0.654057 & -0.476376 & $0 \mathrm{~h}$ \\
\hline 8 & $\mathrm{c}$ & 0 & & 1.047679 & -1.506467 & -0.185846 & $0 \mathrm{~h}$ \\
\hline 9 & $\mathrm{c}$ & 0 & & 0.872155 & -2.584095 & 0.706431 & $0 \mathrm{~h}$ \\
\hline 10 & $\mathrm{~h}$ & 0 & & 0.095556 & -2.741974 & 1.174260 & $0 \mathrm{~h}$ \\
\hline 11 & $\mathrm{c}$ & 0 & & 1.305491 & -1.296423 & -0.887412 & $0 \mathrm{~h}$ \\
\hline 12 & $\mathrm{~h}$ & 0 & & 1.286404 & -2.331218 & -0.537562 & $0 \mathrm{~h}$ \\
\hline 13 & $\mathrm{~h}$ & 0 & & 1.366209 & -1.352635 & -1.988452 & $0 \mathrm{~h}$ \\
\hline 14 & $\mathrm{c}$ & 0 & & 0.940500 & 1.794571 & 0.901973 & $0 \mathrm{~m} \mathrm{~h}$ \\
\hline 15 & $\mathrm{~h}$ & 0 & & 0.272732 & 1.152140 & 1.467345 & $0 \mathrm{~m}$ \\
\hline 16 & $\mathrm{c}$ & 0 & & 2.662400 & 3.431226 & -0.557873 & $0 \mathrm{~m} \mathrm{~h}$ \\
\hline 17 & $\mathrm{~h}$ & 0 & & 3.334086 & 4.062754 & -1.133734 & $0 \mathrm{~m}$ \\
\hline 18 & $\mathrm{c}$ & 0 & & 2.330670 & -1.301207 & -0.721976 & $0 \mathrm{~m} \mathrm{~h}$ \\
\hline 19 & $\mathrm{~h}$ & 0 & & 2.517613 & -0.474746 & -1.396377 & $0 \mathrm{~m}$ \\
\hline
\end{tabular}




$\begin{array}{lllrrrc}20 & \mathrm{c} & 0 & 1.927683 & -3.433066 & 1.022669 & 0 \mathrm{~m} \mathrm{~h} \\ 21 & \mathrm{~h} & 0 & 1.758309 & -4.254731 & 1.714196 & 0 \mathrm{~m} \\ 22 & \mathrm{c} & 0 & -2.547120 & -0.603658 & -0.353821 & 0 \mathrm{~m} \mathrm{~h} \\ 23 & \mathrm{c} & 0 & 1.679702 & 2.785362 & 1.547952 & 0 \mathrm{~m} \\ 24 & \mathrm{~h} & 0 & 1.582262 & 2.914723 & 2.622905 & 0 \mathrm{~m} \\ 25 & \mathrm{c} & 0 & 2.542475 & 3.608453 & 0.820914 & 0 \mathrm{~m} \\ 26 & \mathrm{~h} & 0 & 3.119074 & 4.379049 & 1.325757 & 0 \mathrm{~m} \\ 27 & \mathrm{c} & 0 & 3.387845 & -2.149645 & -0.386432 & 0 \mathrm{~m} \\ 28 & \mathrm{~h} & 0 & 4.368680 & -1.963155 & -0.816784 & 0 \mathrm{~m} \\ 29 & \mathrm{c} & 0 & 3.197732 & -3.225123 & 0.478162 & 0 \mathrm{~m} \\ 30 & \mathrm{~h} & 0 & 4.022989 & -3.883799 & 0.732936 & 0 \mathrm{~m} \\ 31 & \mathrm{c} & 0 & -2.653195 & -0.267785 & 1.002922 & 0 \mathrm{~m} \\ 32 & \mathrm{~h} & 0 & -1.815006 & -0.467451 & 1.665149 & 0 \mathrm{~m} \\ 33 & \mathrm{c} & 0 & -3.813840 & 0.322381 & 1.500577 & 0 \mathrm{~m} \\ 34 & \mathrm{~h} & 0 & -3.880707 & 0.576997 & 2.555227 & 0 \mathrm{~m} \\ 35 & \mathrm{c} & 0 & -4.889170 & 0.587323 & 0.647901 & 0 \mathrm{~m} \\ 36 & \mathrm{~h} & 0 & -5.792961 & 1.049513 & 1.035945 & 0 \mathrm{~m} \\ 37 & \mathrm{c} & 0 & -4.793098 & 0.259883 & -0.704299 & 0 \mathrm{~m} \\ 38 & \mathrm{~h} & 0 & -5.621172 & 0.467732 & -1.376954 & 0 \mathrm{~m} \\ 39 & \mathrm{c} & 0 & -3.627196 & -0.329265 & -1.199569 & 0 \mathrm{~m} \\ 40 & \mathrm{~h} & 0 & -3.555693 & -0.579817 & -2.256096 & 0 \mathrm{~m}\end{array}$

radical

Charge $=0$ Multiplicity $=2$ for low level calculation on real system.

Charge $=0$ Multiplicity $=2$ for high level calculation on model system.

Charge $=0$ Multiplicity $=2$ for low level calculation on model system.

\begin{tabular}{cccccccc}
$\begin{array}{c}\text { Center } \\
\text { Number }\end{array}$ & Atomic & Atomic & Coordinates (Angstroms) & \\
number & Type & $\mathrm{X}$ & $\mathrm{Y}$ & $\mathrm{Z}$ & \\
\hline 1 & $\mathrm{c}$ & 0 & -1.003526 & -0.864976 & 0.548272 & $0 \mathrm{~h}$ \\
2 & $\mathrm{~h}$ & 0 & -0.725978 & -1.188336 & 1.546231 & $0 \mathrm{~h}$ \\
3 & $\mathrm{c}$ & 0 & -2.386536 & -0.657643 & 0.276391 & $0 \mathrm{~h}$ \\
4 & $\mathrm{c}$ & 0 & -3.276323 & -0.477441 & 1.374032 & $0 \mathrm{~h}$ \\
5 & $\mathrm{~h}$ & 0 & -2.870424 & -0.493831 & 2.383182 & $0 \mathrm{~h}$ \\
6 & $\mathrm{n}$ & 0 & 0.027575 & -0.806930 & -0.381727 & $0 \mathrm{~h}$ \\
7 & $\mathrm{c}$ & 0 & 1.279200 & -1.418149 & -0.045498 & $0 \mathrm{~h}$ \\
8 & $\mathrm{c}$ & 0 & 1.927327 & -2.195751 & -1.014490 & $0 \mathrm{~h}$ \\
9 & $\mathrm{~h}$ & 0 & 1.454615 & -2.336286 & -1.982563 & $0 \mathrm{~h}$ \\
10 & $\mathrm{c}$ & 0 & 0.127360 & 0.348866 & -1.318108 & $0 \mathrm{~h}$ \\
11 & $\mathrm{~h}$ & 0 & 0.684473 & 0.017073 & -2.199141 & $0 \mathrm{~h}$ \\
12 & $\mathrm{~h}$ & 0 & -0.882828 & 0.600428 & -1.641691 & $0 \mathrm{~h}$
\end{tabular}




$\begin{array}{rrrrrrl}13 & \mathrm{c} & 0 & -2.960383 & -0.650081 & -1.026516 & 0 \mathrm{~m} \mathrm{~h} \\ 14 & \mathrm{~h} & 0 & -2.337027 & -0.874012 & -1.886239 & 0 \mathrm{~m} \\ 15 & \mathrm{c} & 0 & -4.632911 & -0.270347 & 1.179569 & 0 \mathrm{~m} \mathrm{~h} \\ 16 & \mathrm{~h} & 0 & -5.282011 & -0.131046 & 2.040705 & 0 \mathrm{~m} \\ 17 & \mathrm{c} & 0 & 1.876111 & -1.257007 & 1.211958 & 0 \mathrm{~m} \mathrm{~h} \\ 18 & \mathrm{~h} & 0 & 1.398596 & -0.629374 & 1.957843 & 0 \mathrm{~m} \\ 19 & \mathrm{c} & 0 & 3.149781 & -2.803133 & -0.730525 & 0 \mathrm{~m} \mathrm{~h} \\ 20 & \mathrm{~h} & 0 & 3.637138 & -3.408912 & -1.489909 & 0 \mathrm{~m} \\ 21 & \mathrm{c} & 0 & 0.792257 & 1.566324 & -0.705693 & 0 \mathrm{~m} \mathrm{~h} \\ 22 & \mathrm{c} & 0 & -4.322232 & -0.438653 & -1.208339 & 0 \mathrm{~m} \\ 23 & \mathrm{~h} & 0 & -4.731314 & -0.449417 & -2.215924 & 0 \mathrm{~m} \\ 24 & \mathrm{c} & 0 & -5.171096 & -0.236166 & -0.114971 & 0 \mathrm{~m} \\ 25 & \mathrm{~h} & 0 & -6.233824 & -0.069782 & -0.265585 & 0 \mathrm{~m} \\ 26 & \mathrm{c} & 0 & 3.091396 & -1.880393 & 1.497005 & 0 \mathrm{~m} \\ 27 & \mathrm{~h} & 0 & 3.543379 & -1.747150 & 2.476391 & 0 \mathrm{~m} \\ 28 & \mathrm{c} & 0 & 3.734365 & -2.652841 & 0.528827 & 0 \mathrm{~m} \\ 29 & \mathrm{~h} & 0 & 4.682372 & -3.133713 & 0.753265 & 0 \mathrm{~m} \\ 30 & \mathrm{c} & 0 & 2.147279 & 1.837070 & -0.933194 & 0 \mathrm{~m} \\ 31 & \mathrm{~h} & 0 & 2.725898 & 1.172178 & -1.570228 & 0 \mathrm{~m} \\ 32 & \mathrm{c} & 0 & 2.760681 & 2.948179 & -0.352101 & 0 \mathrm{~m} \\ 33 & \mathrm{~h} & 0 & 3.812416 & 3.145935 & -0.542355 & 0 \mathrm{~m} \\ 34 & \mathrm{c} & 0 & 2.024124 & 3.803104 & 0.469119 & 0 \mathrm{~m} \\ 35 & \mathrm{~h} & 0 & 2.499060 & 4.670440 & 0.920177 & 0 \mathrm{~m} \\ 36 & \mathrm{c} & 0 & 0.671916 & 3.542299 & 0.703783 & 0 \mathrm{~m} \\ 37 & \mathrm{~h} & 0 & 0.090529 & 4.208364 & 1.336173 & 0 \mathrm{~m} \\ 38 & \mathrm{c} & 0 & 0.060491 & 2.433050 & 0.119303 & 0 \mathrm{~m} \\ 39 & \mathrm{~h} & 0 & -0.993781 & 2.238097 & 0.298814 & 0 \mathrm{~m} \\ ----------------------------------------------------------- & \end{array}$

No. 39<smiles>C=CCCCCCC</smiles>

neutral molecule

Charge $=0$ Multiplicity $=1$ for low level calculation on real system.

Charge $=0$ Multiplicity $=1$ for high level calculation on model system.

Charge $=0$ Multiplicity $=1$ for low level calculation on model system.

$\begin{array}{lllll}\text { Center } & \text { Atomic } & \text { Atomic } & \text { Coordinates (Angstroms) } & \\ \text { Number Number } & \text { Type } & \mathrm{X} & \mathrm{Y} & \mathrm{Z}\end{array}$ 


\begin{tabular}{|c|c|c|c|c|c|c|c|c|}
\hline 1 & $\mathrm{c}$ & 0 & -7.221767 & 0.518003 & -0.132745 & 0 & & \\
\hline 2 & $\mathrm{~h}$ & 0 & -7.177491 & 1.237437 & -0.965418 & 0 & $\mathrm{~h}$ & \\
\hline 3 & $\mathrm{~h}$ & 0 & -7.256436 & 1.111470 & 0.790665 & 0 & $\mathrm{~h}$ & \\
\hline 4 & $\mathrm{c}$ & 0 & -8.474663 & -0.306083 & -0.252943 & 0 & $\mathrm{~h}$ & \\
\hline 5 & $\mathrm{~h}$ & 0 & -8.564045 & -0.899842 & -1.165089 & 0 & $\mathrm{~h}$ & \\
\hline 6 & $\mathrm{c}$ & 0 & -9.446842 & -0.372360 & 0.657459 & 0 & $\mathrm{~h}$ & \\
\hline 7 & $\mathrm{~h}$ & 0 & -10.325926 & -0.995278 & 0.514128 & 0 & $\mathrm{~h}$ & \\
\hline 8 & $\mathrm{~h}$ & 0 & -9.402465 & 0.198535 & 1.583236 & 0 & $\mathrm{~h}$ & \\
\hline 9 & $\mathrm{c}$ & 0 & -5.937877 & -0.333712 & -0.154438 & 0 & $\mathrm{~h}$ & \\
\hline 10 & $\mathrm{~h}$ & 0 & -5.962828 & -1.038960 & 0.687690 & 0 & $\mathrm{~h}$ & \\
\hline 11 & $\mathrm{~h}$ & 0 & -5.926210 & -0.948378 & -1.066557 & 0 & $\mathrm{~h}$ & \\
\hline 12 & $\mathrm{c}$ & 0 & -4.654335 & 0.503689 & -0.089901 & 0 & $\mathrm{~h}$ & \\
\hline 13 & $\mathrm{~h}$ & 0 & -4.669064 & 1.122463 & 0.819638 & 0 & $\mathrm{~h}$ & \\
\hline 14 & $\mathrm{~h}$ & 0 & -4.637669 & 1.207850 & -0.935160 & 0 & $\mathrm{~h}$ & \\
\hline 15 & $\mathrm{c}$ & 0 & -3.373517 & -0.340688 & -0.107703 & 0 & $\mathrm{~m}$ & h 12 \\
\hline 16 & $\mathrm{~h}$ & 0 & -3.389570 & -1.041898 & 0.739782 & 0 & $\mathrm{~m}$ & \\
\hline 17 & $\mathrm{~h}$ & 0 & -3.361911 & -0.962893 & -1.015010 & 0 & $\mathrm{~m}$ & \\
\hline 18 & $\mathrm{c}$ & 0 & -2.087274 & 0.493803 & -0.050263 & 0 & $\mathrm{~m}$ & \\
\hline 19 & $\mathrm{~h}$ & 0 & -2.098862 & 1.117401 & 0.856088 & 0 & $\mathrm{~m}$ & \\
\hline 20 & $\mathrm{~h}$ & 0 & -2.070703 & 1.193767 & -0.898939 & 0 & $\mathrm{~m}$ & \\
\hline 21 & $\mathrm{c}$ & 0 & -0.807493 & -0.352245 & -0.066706 & 0 & $\mathrm{~m}$ & \\
\hline 22 & $\mathrm{~h}$ & 0 & -0.822868 & -1.049877 & 0.783892 & 0 & $\mathrm{~m}$ & \\
\hline 23 & $\mathrm{~h}$ & 0 & -0.798450 & -0.978594 & -0.971224 & 0 & $\mathrm{~m}$ & \\
\hline 24 & $\mathrm{c}$ & 0 & 0.480264 & 0.480195 & -0.015177 & 0 & $\mathrm{~m}$ & \\
\hline 25 & $\mathrm{~h}$ & 0 & 0.471763 & 1.106967 & 0.889045 & 0 & $\mathrm{~m}$ & \\
\hline 26 & $\mathrm{~h}$ & 0 & 0.495948 & 1.177297 & -0.866237 & 0 & $\mathrm{~m}$ & \\
\hline 27 & c & 0 & 1.758851 & -0.367660 & -0.031710 & 0 & $\mathrm{~m}$ & \\
\hline 28 & $\mathrm{~h}$ & 0 & 1.743740 & -1.063581 & 0.820320 & 0 & $\mathrm{~m}$ & \\
\hline 29 & $\mathrm{~h}$ & 0 & 1.765737 & -0.995876 & -0.934961 & 0 & $\mathrm{~m}$ & \\
\hline 30 & c & 0 & 3.047839 & 0.463064 & 0.016452 & 0 & $\mathrm{~m}$ & \\
\hline 31 & $\mathrm{~h}$ & 0 & 3.041786 & 1.090849 & 0.920000 & 0 & $\mathrm{~m}$ & \\
\hline 32 & $\mathrm{~h}$ & 0 & 3.062896 & 1.159246 & -0.835373 & 0 & $\mathrm{~m}$ & \\
\hline 33 & $\mathrm{c}$ & 0 & 4.325357 & -0.386366 & -0.001457 & 0 & $\mathrm{~m}$ & \\
\hline 34 & $\mathrm{~h}$ & 0 & 4.310276 & -1.082345 & 0.850529 & 0 & $\mathrm{~m}$ & \\
\hline 35 & $\mathrm{~h}$ & 0 & 4.330619 & -1.014497 & -0.904775 & 0 & $\mathrm{~m}$ & \\
\hline 36 & $\mathrm{c}$ & 0 & 5.615366 & 0.442819 & 0.045649 & 0 & $\mathrm{~m}$ & \\
\hline 37 & $\mathrm{~h}$ & 0 & 5.610967 & 1.070008 & 0.949658 & 0 & $\mathrm{~m}$ & \\
\hline 38 & $\mathrm{~h}$ & 0 & 5.630200 & 1.139593 & -0.805730 & 0 & $\mathrm{~m}$ & \\
\hline 39 & $\mathrm{c}$ & 0 & 6.892095 & -0.407444 & 0.025808 & 0 & $\mathrm{~m}$ & \\
\hline 40 & $\mathrm{~h}$ & 0 & 6.877636 & -1.105183 & 0.876547 & 0 & $\mathrm{~m}$ & \\
\hline 41 & $\mathrm{~h}$ & 0 & 6.897714 & -1.034022 & -0.878751 & 0 & $\mathrm{~m}$ & \\
\hline 42 & $\mathrm{c}$ & 0 & 8.183017 & 0.420142 & 0.074481 & 0 & $\mathrm{~m}$ & \\
\hline 43 & $\mathrm{~h}$ & 0 & 8.178313 & 1.044592 & 0.979320 & 0 & $\mathrm{~m}$ & \\
\hline 44 & $\mathrm{~h}$ & 0 & 8.197874 & 1.117776 & -0.775050 & 0 & $\mathrm{~m}$ & \\
\hline
\end{tabular}




$\begin{array}{lllrrrrc}45 & \mathrm{c} & 0 & 9.452272 & -0.437780 & 0.052691 & 0 & \mathrm{~m} \\ 46 & \mathrm{~h} & 0 & 9.481831 & -1.123219 & 0.908761 & 0 & \mathrm{~m} \\ 47 & \mathrm{~h} & 0 & 9.503468 & -1.046389 & -0.858655 & 0 & \mathrm{~m} \\ 48 & \mathrm{~h} & 0 & 10.355971 & 0.181321 & 0.090648 & 0 & \mathrm{~m}\end{array}$

radical

Charge $=0$ Multiplicity $=2$ for low level calculation on real system.

Charge $=0$ Multiplicity $=2$ for high level calculation on model system.

Charge $=0$ Multiplicity $=2$ for low level calculation on model system.

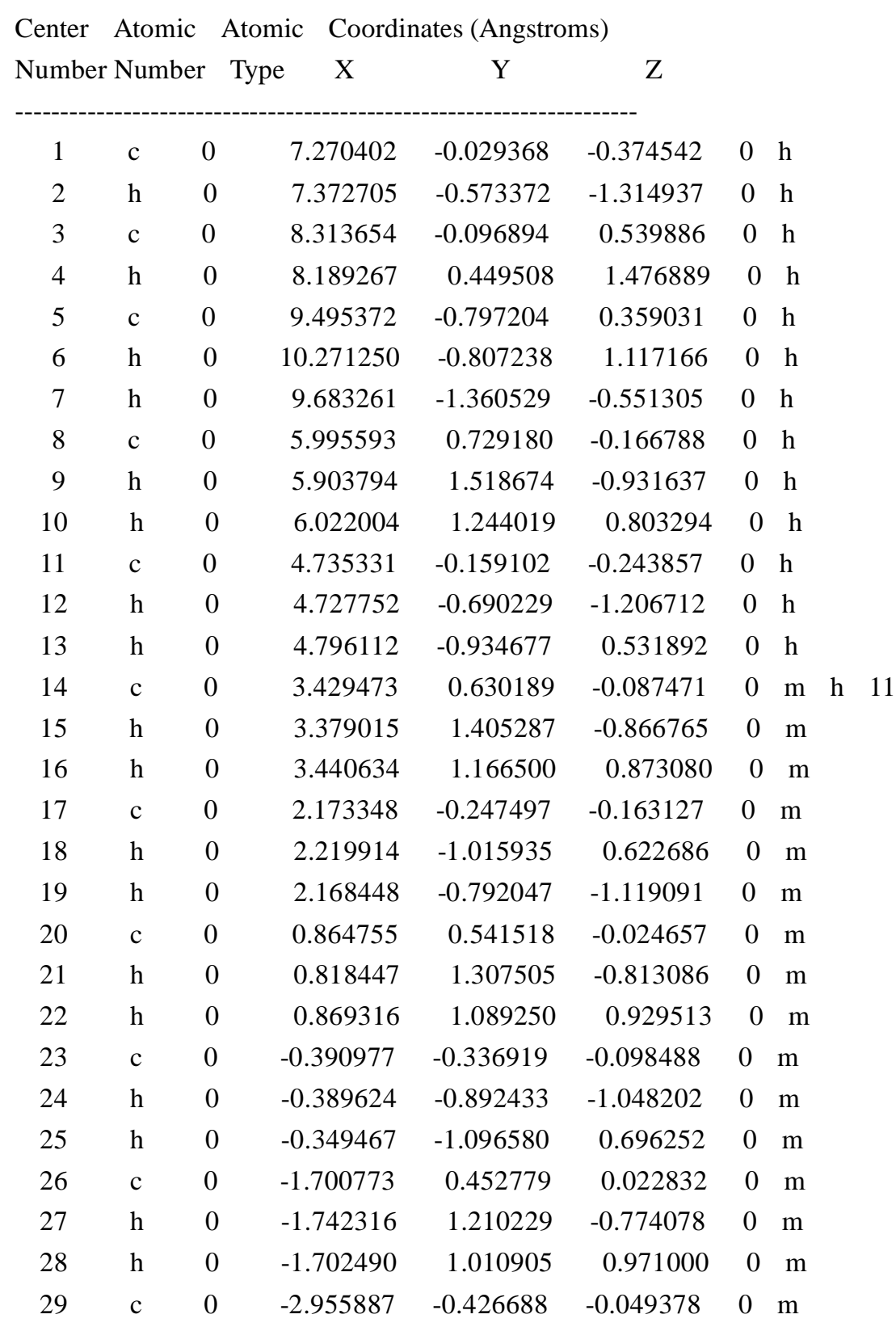




$\begin{array}{lllrrrrc}30 & \mathrm{~h} & 0 & -2.950118 & -0.990056 & -0.994448 & 0 & \mathrm{~m} \\ 31 & \mathrm{~h} & 0 & -2.917634 & -1.179792 & 0.751765 & 0 & \mathrm{~m} \\ 32 & \mathrm{c} & 0 & -4.266620 & 0.363205 & 0.060177 & 0 & \mathrm{~m} \\ 33 & \mathrm{~h} & 0 & -4.305084 & 1.114923 & -0.742286 & 0 & \mathrm{~m} \\ 34 & \mathrm{~h} & 0 & -4.272599 & 0.928147 & 1.004290 & 0 & \mathrm{~m} \\ 35 & \mathrm{c} & 0 & -5.520995 & -0.517321 & -0.010951 & 0 & \mathrm{~m} \\ 36 & \mathrm{~h} & 0 & -5.513213 & -1.084371 & -0.953843 & 0 & \mathrm{~m} \\ 37 & \mathrm{~h} & 0 & -5.483796 & -1.267313 & 0.793211 & 0 & \mathrm{~m} \\ 38 & \mathrm{c} & 0 & -6.832530 & 0.271929 & 0.093637 & 0 & \mathrm{~m} \\ 39 & \mathrm{~h} & 0 & -6.870865 & 1.021379 & -0.711152 & 0 & \mathrm{~m} \\ 40 & \mathrm{~h} & 0 & -6.841098 & 0.839878 & 1.036095 & 0 & \mathrm{~m} \\ 41 & \mathrm{c} & 0 & -8.086346 & -0.609065 & 0.023142 & 0 & \mathrm{~m} \\ 42 & \mathrm{~h} & 0 & -8.077520 & -1.175766 & -0.918914 & 0 & \mathrm{~m} \\ 43 & \mathrm{~h} & 0 & -8.048200 & -1.357510 & 0.827453 & 0 & \mathrm{~m} \\ 44 & \mathrm{c} & 0 & -9.391813 & 0.185911 & 0.128076 & 0 & \mathrm{~m} \\ 45 & \mathrm{~h} & 0 & -9.475963 & 0.919744 & -0.683187 & 0 & \mathrm{~m} \\ 46 & \mathrm{~h} & 0 & -9.446554 & 0.735428 & 1.076096 & 0 & \mathrm{~m} \\ 47 & \mathrm{~h} & 0 & -10.267305 & -0.471447 & 0.073830 & 0 & \mathrm{~m}\end{array}$

\section{No. 40}

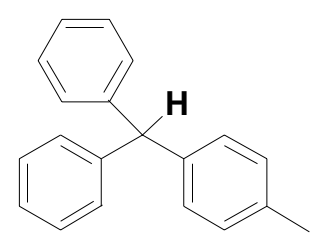

neutral molecule

Charge $=0$ Multiplicity $=1$ for low level calculation on real system.

Charge $=0$ Multiplicity $=1$ for high level calculation on model system .

Charge $=0$ Multiplicity $=1$ for low level calculation on model system.

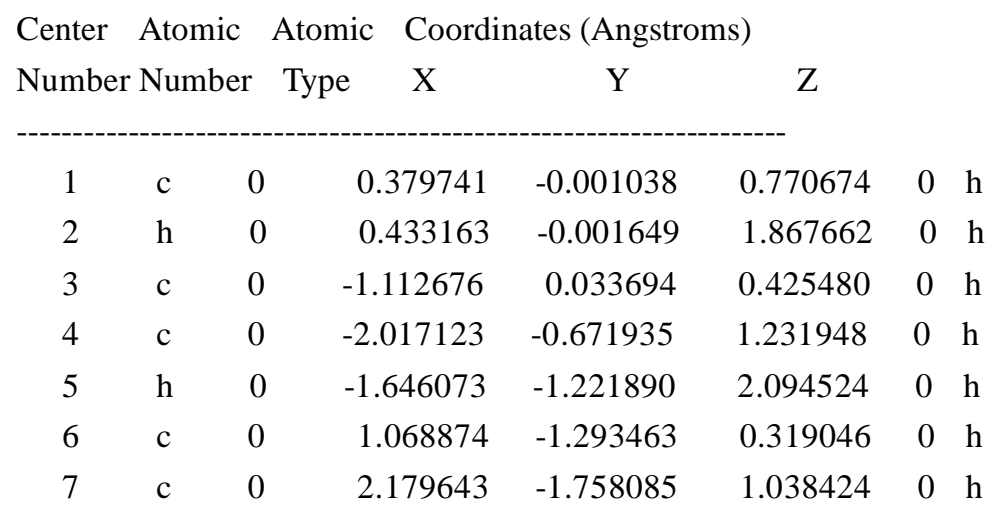




\begin{tabular}{|c|c|c|c|c|c|c|c|c|c|}
\hline 8 & $\mathrm{~h}$ & 0 & 2.520687 & -1.201097 & 1.908666 & 0 & & & \\
\hline 9 & $\mathrm{c}$ & 0 & 1.125517 & 1.258603 & 0.317626 & 0 & $\mathrm{~h}$ & & \\
\hline 10 & $\mathrm{c}$ & 0 & 1.056582 & 2.413009 & 1.110963 & 0 & $\mathrm{~h}$ & & \\
\hline 11 & $\mathrm{~h}$ & 0 & 0.489914 & 2.387466 & 2.039517 & 0 & $\mathrm{~h}$ & & \\
\hline 12 & $\mathrm{c}$ & 0 & -1.623490 & 0.722495 & -0.679647 & 0 & $\mathrm{~m}$ & $\mathrm{~h}$ & 3 \\
\hline 13 & $\mathrm{~h}$ & 0 & -0.952555 & 1.289424 & -1.318166 & 0 & $\mathrm{~m}$ & & \\
\hline 14 & $\mathrm{c}$ & 0 & -3.379267 & -0.689417 & 0.943741 & 0 & $\mathrm{~m}$ & $\mathrm{~h}$ & 4 \\
\hline 15 & $\mathrm{~h}$ & 0 & -4.055449 & -1.245839 & 1.589714 & 0 & $\mathrm{~m}$ & & \\
\hline 16 & $\mathrm{c}$ & 0 & 0.647898 & -2.023834 & -0.798640 & 0 & $\mathrm{~m}$ & $\mathrm{~h}$ & 6 \\
\hline 17 & $\mathrm{~h}$ & 0 & -0.219970 & -1.690677 & -1.359750 & 0 & $\mathrm{~m}$ & & \\
\hline 18 & $\mathrm{c}$ & 0 & 2.852445 & -2.916695 & 0.654006 & 0 & $\mathrm{~m}$ & $\mathrm{~h}$ & 7 \\
\hline 19 & $\mathrm{~h}$ & 0 & 3.707760 & -3.260792 & 1.229976 & 0 & $\mathrm{~m}$ & & \\
\hline 20 & $\mathrm{c}$ & 0 & 1.859196 & 1.314288 & -0.873714 & 0 & $\mathrm{~m}$ & $\mathrm{~h}$ & 9 \\
\hline 21 & $\mathrm{~h}$ & 0 & 1.941099 & 0.427823 & -1.495693 & 0 & $\mathrm{~m}$ & & \\
\hline 22 & $\mathrm{c}$ & 0 & 1.698707 & 3.589416 & 0.727347 & 0 & $\mathrm{~m}$ & $\mathrm{~h}$ & 10 \\
\hline 23 & $\mathrm{~h}$ & 0 & 1.635521 & 4.470188 & 1.361357 & 0 & $\mathrm{~m}$ & & \\
\hline 24 & $\mathrm{c}$ & 0 & -2.989776 & 0.700873 & -0.968732 & 0 & $\mathrm{~m}$ & & \\
\hline 25 & $\mathrm{~h}$ & 0 & -3.357743 & 1.244862 & -1.836310 & 0 & $\mathrm{~m}$ & & \\
\hline 26 & $\mathrm{c}$ & 0 & -3.893595 & 0.000278 & -0.163207 & 0 & $\mathrm{~m}$ & & \\
\hline 27 & $\mathrm{c}$ & 0 & -5.375959 & 0.009223 & -0.456125 & 0 & $\mathrm{~m}$ & & \\
\hline 28 & $\mathrm{~h}$ & 0 & -5.892685 & 0.788624 & 0.120161 & 0 & $\mathrm{~m}$ & & \\
\hline 29 & $\mathrm{~h}$ & 0 & -5.573661 & 0.204007 & -1.515517 & 0 & $\mathrm{~m}$ & & \\
\hline 30 & $\mathrm{~h}$ & 0 & -5.842657 & -0.947109 & -0.195070 & 0 & $\mathrm{~m}$ & & \\
\hline 31 & $\mathrm{c}$ & 0 & 1.322287 & -3.183637 & -1.189426 & 0 & $\mathrm{~m}$ & & \\
\hline 32 & $\mathrm{~h}$ & 0 & 0.977811 & -3.736896 & -2.059505 & 0 & $\mathrm{~m}$ & & \\
\hline 33 & $\mathrm{c}$ & 0 & 2.425408 & -3.635196 & -0.465272 & 0 & $\mathrm{~m}$ & & \\
\hline 34 & $\mathrm{~h}$ & 0 & 2.945750 & -4.540597 & -0.766323 & 0 & $\mathrm{~m}$ & & \\
\hline 35 & $\mathrm{c}$ & 0 & 2.500613 & 2.492754 & -1.263561 & 0 & $\mathrm{~m}$ & & \\
\hline 36 & $\mathrm{~h}$ & 0 & 3.066588 & 2.513452 & -2.191476 & 0 & $\mathrm{~m}$ & & \\
\hline 37 & $\mathrm{c}$ & 0 & 2.424036 & 3.634017 & -0.465506 & 0 & $\mathrm{~m}$ & & \\
\hline 38 & $\mathrm{~h}$ & 0 & 2.928137 & 4.548619 & -0.766322 & 0 & $\mathrm{~m}$ & & \\
\hline
\end{tabular}

radical

Charge $=0$ Multiplicity $=2$ for low level calculation on real system.

Charge $=0$ Multiplicity $=2$ for high level calculation on model system.

Charge $=0$ Multiplicity $=2$ for low level calculation on model system.

Center Atomic Atomic Coordinates (Angstroms)

Number Number Type $\quad \mathrm{X} \quad \mathrm{Y} \quad \mathrm{Z}$

$\begin{array}{rrrrrrrr}1 & \mathrm{c} & 0 & -0.352632 & 0.000216 & 0.000237 & 0 & \mathrm{~h} \\ 2 & \mathrm{c} & 0 & 1.110256 & -0.003000 & 0.001988 & 0 & \mathrm{~h}\end{array}$




\begin{tabular}{|c|c|c|c|c|c|c|c|c|}
\hline 3 & $\mathrm{c}$ & 0 & 1.852458 & -1.014631 & -0.654065 & 0 & $\mathrm{~h}$ & \\
\hline 4 & $\mathrm{~h}$ & 0 & 1.322438 & -1.796962 & -1.187918 & 0 & $\mathrm{~h}$ & \\
\hline 5 & $\mathrm{c}$ & 0 & -1.089499 & -1.266106 & -0.000567 & 0 & $\mathrm{~h}$ & \\
\hline 6 & $\mathrm{c}$ & 0 & -2.323803 & -1.400791 & -0.680925 & 0 & $\mathrm{~h}$ & \\
\hline 7 & $\mathrm{~h}$ & 0 & -2.719851 & -0.550893 & -1.227542 & 0 & $\mathrm{~h}$ & \\
\hline 8 & $\mathrm{c}$ & 0 & -1.083329 & 1.270064 & -0.000140 & 0 & $\mathrm{~h}$ & \\
\hline 9 & $\mathrm{c}$ & 0 & -0.583446 & 2.409857 & -0.675155 & 0 & $\mathrm{~h}$ & \\
\hline 10 & $\mathrm{~h}$ & 0 & 0.351153 & 2.331700 & -1.221404 & 0 & $\mathrm{~h}$ & \\
\hline 11 & $\mathrm{c}$ & 0 & 1.854969 & 1.002686 & 0.662893 & 0 & $\mathrm{~m}$ & $\mathrm{~h} 2$ \\
\hline 12 & $\mathrm{~h}$ & 0 & 1.327345 & 1.783594 & 1.201228 & 0 & $\mathrm{~m}$ & \\
\hline 13 & $\mathrm{c}$ & 0 & 3.241787 & -1.013043 & -0.650024 & 0 & $\mathrm{~m}$ & $\mathrm{~h}$ \\
\hline 14 & $\mathrm{~h}$ & 0 & 3.774879 & -1.802922 & -1.175461 & 0 & $\mathrm{~m}$ & \\
\hline 15 & $\mathrm{c}$ & 0 & -0.597833 & -2.407296 & 0.677990 & 0 & $\mathrm{~m}$ & h 5 \\
\hline 16 & $\mathrm{~h}$ & 0 & 0.334877 & -2.332844 & 1.228013 & 0 & $\mathrm{~m}$ & \\
\hline 17 & $\mathrm{c}$ & 0 & -3.019903 & -2.605335 & -0.685316 & 0 & $\mathrm{~m}$ & $\mathrm{~h}$ \\
\hline 18 & $\mathrm{~h}$ & 0 & -3.958201 & -2.679337 & -1.229303 & 0 & $\mathrm{~m}$ & \\
\hline 19 & $\mathrm{c}$ & 0 & -2.319677 & 1.409798 & 0.675450 & 0 & $\mathrm{~m}$ & $\mathrm{~h}$ \\
\hline 20 & $\mathrm{~h}$ & 0 & -2.721967 & 0.561121 & 1.219394 & 0 & $\mathrm{~m}$ & \\
\hline 21 & $\mathrm{c}$ & 0 & -1.280333 & 3.613981 & -0.675022 & 0 & $\mathrm{~m}$ & h 9 \\
\hline 22 & $\mathrm{~h}$ & 0 & -0.875721 & 4.466501 & -1.214791 & 0 & $\mathrm{~m}$ & \\
\hline 23 & $\mathrm{c}$ & 0 & 3.245008 & 0.991903 & 0.666446 & 0 & $\mathrm{~m}$ & \\
\hline 24 & $\mathrm{~h}$ & 0 & 3.780477 & 1.773779 & 1.201131 & 0 & $\mathrm{~m}$ & \\
\hline 25 & $\mathrm{c}$ & 0 & 3.971112 & -0.010928 & 0.007931 & 0 & $\mathrm{~m}$ & \\
\hline 26 & $\mathrm{c}$ & 0 & 5.480421 & -0.000257 & -0.015146 & 0 & $\mathrm{~m}$ & \\
\hline 27 & $\mathrm{~h}$ & 0 & 5.890649 & 0.546223 & 0.840740 & 0 & $\mathrm{~m}$ & \\
\hline 28 & $\mathrm{~h}$ & 0 & 5.862762 & 0.484804 & -0.923951 & 0 & $\mathrm{~m}$ & \\
\hline 29 & $\mathrm{~h}$ & 0 & 5.890015 & -1.016340 & 0.003384 & 0 & $\mathrm{~m}$ & \\
\hline 30 & $\mathrm{c}$ & 0 & -1.300498 & -3.608097 & 0.676687 & 0 & $\mathrm{~m}$ & \\
\hline 31 & $\mathrm{~h}$ & 0 & -0.902122 & -4.461759 & 1.219279 & 0 & $\mathrm{~m}$ & \\
\hline 32 & $\mathrm{c}$ & 0 & -2.515298 & -3.717884 & -0.005891 & 0 & $\mathrm{~m}$ & \\
\hline 33 & $\mathrm{~h}$ & 0 & -3.061393 & -4.657277 & -0.007988 & 0 & $\mathrm{~m}$ & \\
\hline 34 & $\mathrm{c}$ & 0 & -3.010046 & 2.617642 & 0.678603 & 0 & $\mathrm{~m}$ & \\
\hline 35 & $\mathrm{~h}$ & 0 & -3.950171 & 2.695322 & 1.218911 & 0 & $\mathrm{~m}$ & \\
\hline 36 & $\mathrm{c}$ & 0 & -2.497406 & 3.728660 & 0.002762 & 0 & $\mathrm{~m}$ & \\
\hline 37 & $\mathrm{~h}$ & 0 & -3.039003 & 4.670653 & 0.003858 & 0 & $\mathrm{~m}$ & \\
\hline
\end{tabular}




\section{No. 41}

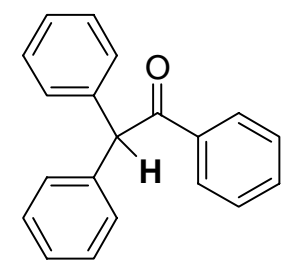

neutral molecule

Charge $=0$ Multiplicity $=1$ for low level calculation on real system.

Charge $=0$ Multiplicity $=1$ for high level calculation on model system .

Charge $=0$ Multiplicity $=1$ for low level calculation on model system.

\begin{tabular}{|c|c|c|c|c|c|c|}
\hline Center & Atomic & & Coordi & ates (Angstro & ms) & \\
\hline Number & Numbe & & Type & $\mathrm{Y}$ & Z & \\
\hline 1 & $\mathrm{c}$ & 0 & 0.384822 & -0.156860 & 0.361529 & $0 \mathrm{~h}$ \\
\hline 2 & $\mathrm{~h}$ & 0 & 0.025361 & -0.301970 & 1.385235 & $0 \mathrm{~h}$ \\
\hline 3 & $\mathrm{c}$ & 0 & -0.664973 & -0.772853 & -0.590481 & $0 \mathrm{~h}$ \\
\hline 4 & o & 0 & -0.333059 & -1.225178 & -1.675110 & $0 \mathrm{~h}$ \\
\hline 5 & $\mathrm{c}$ & 0 & 1.728932 & -0.872393 & 0.263937 & $0 \mathrm{~h}$ \\
\hline 6 & $\mathrm{c}$ & 0 & 2.563573 & -0.741268 & -0.853458 & $0 \mathrm{~h}$ \\
\hline 7 & $\mathrm{~h}$ & 0 & 2.260854 & -0.107654 & -1.679656 & $0 \mathrm{~h}$ \\
\hline 8 & $\mathrm{c}$ & 0 & 0.485726 & 1.355360 & 0.132018 & $0 \mathrm{~h}$ \\
\hline 9 & $\mathrm{c}$ & 0 & 0.746018 & 2.196139 & 1.223857 & $0 \mathrm{~h}$ \\
\hline 10 & $\mathrm{~h}$ & 0 & 0.858844 & 1.764217 & 2.215977 & $0 \mathrm{~h}$ \\
\hline 11 & $\mathrm{c}$ & 0 & -2.105211 & -0.789526 & -0.170850 & $0 \mathrm{~m} \mathrm{~h}$ \\
\hline 12 & $\mathrm{c}$ & 0 & 2.145790 & -1.692930 & 1.318921 & $0 \mathrm{~m} \mathrm{~h}$ \\
\hline 13 & $\mathrm{~h}$ & 0 & 1.508384 & -1.807481 & 2.193424 & $0 \mathrm{~m}$ \\
\hline 14 & $\mathrm{c}$ & 0 & 3.782559 & -1.415448 & -0.910292 & $0 \mathrm{~m} \mathrm{~h}$ \\
\hline 15 & $\mathrm{~h}$ & 0 & 4.417486 & -1.302309 & -1.785217 & $0 \mathrm{~m}$ \\
\hline 16 & $\mathrm{c}$ & 0 & 0.352105 & 1.930221 & -1.139051 & $0 \mathrm{~m} \mathrm{~h}$ \\
\hline 17 & $\mathrm{~h}$ & 0 & 0.140563 & 1.299211 & -1.997079 & $0 \mathrm{~m}$ \\
\hline 18 & $\mathrm{c}$ & 0 & 0.875326 & 3.573628 & 1.052041 & $0 \mathrm{~m} \mathrm{~h}$ \\
\hline 19 & $\mathrm{~h}$ & 0 & 1.079030 & 4.207213 & 1.911398 & $0 \mathrm{~m}$ \\
\hline 20 & $\mathrm{c}$ & 0 & -2.597239 & -0.076362 & 0.933539 & $0 \mathrm{~m}$ \\
\hline 21 & $\mathrm{~h}$ & 0 & -1.935243 & 0.545793 & 1.526825 & $0 \mathrm{~m}$ \\
\hline 22 & $\mathrm{c}$ & 0 & -3.952292 & -0.129169 & 1.259326 & $0 \mathrm{~m}$ \\
\hline 23 & $\mathrm{~h}$ & 0 & -4.324489 & 0.434667 & 2.110251 & $0 \mathrm{~m}$ \\
\hline 24 & $\mathrm{c}$ & 0 & -4.827410 & -0.901845 & 0.493999 & $0 \mathrm{~m}$ \\
\hline 25 & $\mathrm{~h}$ & 0 & -5.881707 & -0.946869 & 0.754049 & $0 \mathrm{~m}$ \\
\hline 26 & $\mathrm{c}$ & 0 & -4.346892 & -1.614038 & -0.608988 & $0 \mathrm{~m}$ \\
\hline 27 & $\mathrm{~h}$ & 0 & -5.026381 & -2.214549 & -1.207696 & $0 \mathrm{~m}$ \\
\hline
\end{tabular}




$\begin{array}{lllrrrl}28 & \mathrm{c} & 0 & -2.997796 & -1.552065 & -0.942450 & 0 \mathrm{~m} \\ 29 & \mathrm{~h} & 0 & -2.605201 & -2.090277 & -1.798908 & 0 \mathrm{~m} \\ 30 & \mathrm{c} & 0 & 3.366474 & -2.368366 & 1.265203 & 0 \mathrm{~m} \\ 31 & \mathrm{~h} & 0 & 3.671579 & -2.998560 & 2.096640 & 0 \mathrm{~m} \\ 32 & \mathrm{c} & 0 & 4.189596 & -2.231130 & 0.147996 & 0 \mathrm{~m} \\ 33 & \mathrm{~h} & 0 & 5.141648 & -2.753298 & 0.101735 & 0 \mathrm{~m} \\ 34 & \mathrm{c} & 0 & 0.481373 & 3.310079 & -1.311917 & 0 \mathrm{~m} \\ 35 & \mathrm{~h} & 0 & 0.372892 & 3.737513 & -2.305288 & 0 \mathrm{~m} \\ 36 & \mathrm{c} & 0 & 0.742859 & 4.136441 & -0.219159 & 0 \mathrm{~m} \\ 37 & \mathrm{~h} & 0 & 0.840809 & 5.210080 & -0.355119 & 0 \mathrm{~m}\end{array}$

radical

Charge $=0$ Multiplicity $=2$ for low level calculation on real system.

Charge $=0$ Multiplicity $=2$ for high level calculation on model system .

Charge $=0$ Multiplicity $=2$ for low level calculation on model system.

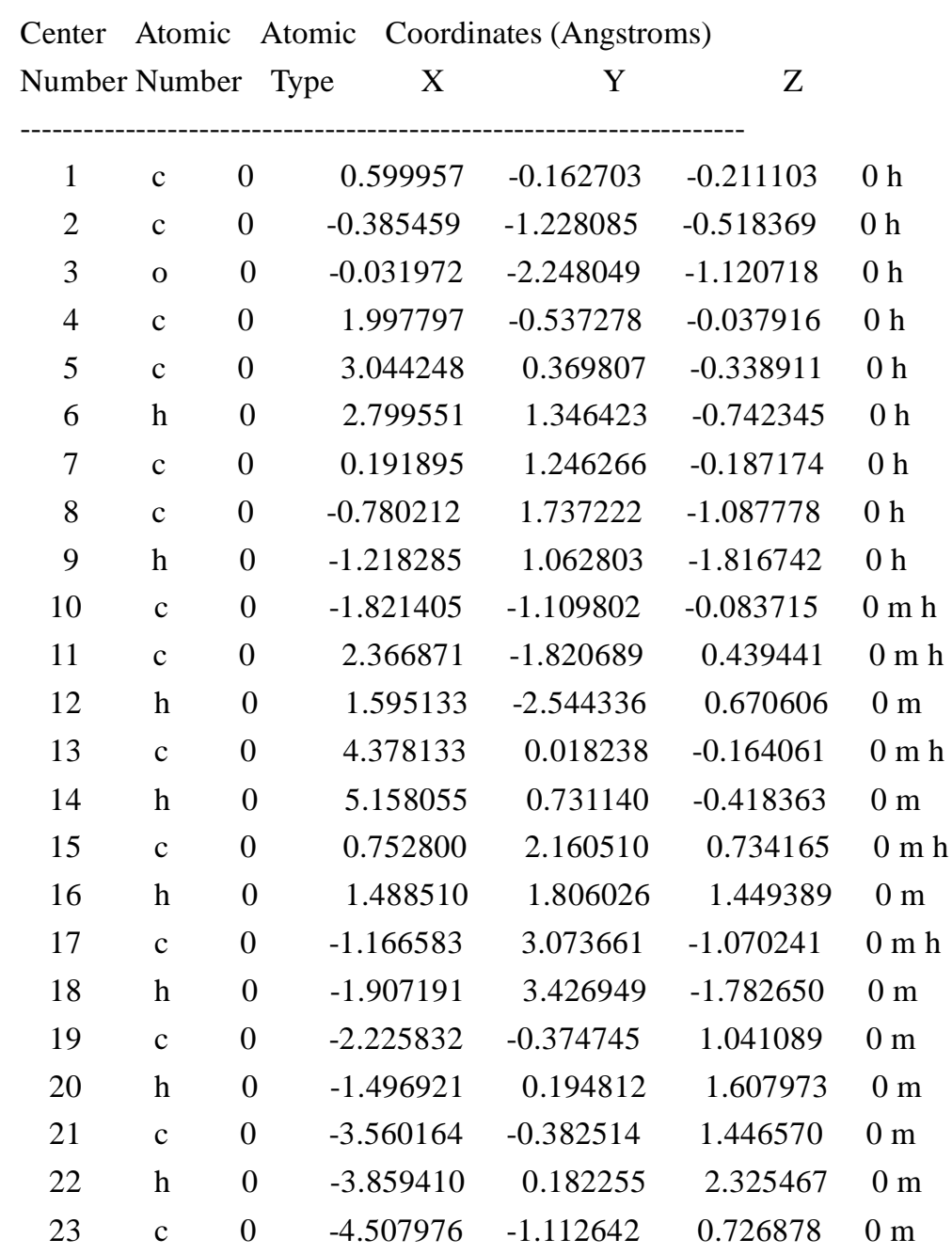




$\begin{array}{lllrrrl}24 & \mathrm{~h} & 0 & -5.549000 & -1.110349 & 1.039409 & 0 \mathrm{~m} \\ 25 & \mathrm{c} & 0 & -4.113518 & -1.851594 & -0.392086 & 0 \mathrm{~m} \\ 26 & \mathrm{~h} & 0 & -4.846901 & -2.426206 & -0.951674 & 0 \mathrm{~m} \\ 27 & \mathrm{c} & 0 & -2.778193 & -1.860176 & -0.785568 & 0 \mathrm{~m} \\ 28 & \mathrm{~h} & 0 & -2.449521 & -2.449433 & -1.635535 & 0 \mathrm{~m} \\ 29 & \mathrm{c} & 0 & 3.700694 & -2.161519 & 0.622319 & 0 \mathrm{~m} \\ 30 & \mathrm{~h} & 0 & 3.952656 & -3.148542 & 1.000775 & 0 \mathrm{~m} \\ 31 & \mathrm{c} & 0 & 4.716070 & -1.247077 & 0.322979 & 0 \mathrm{~m} \\ 32 & \mathrm{~h} & 0 & 5.758433 & -1.520747 & 0.461979 & 0 \mathrm{~m} \\ 33 & \mathrm{c} & 0 & 0.354188 & 3.493960 & 0.757176 & 0 \mathrm{~m} \\ 34 & \mathrm{~h} & 0 & 0.790854 & 4.171962 & 1.485777 & 0 \mathrm{~m} \\ 35 & \mathrm{c} & 0 & -0.604772 & 3.959191 & -0.146576 & 0 \mathrm{~m} \\ 36 & \mathrm{~h} & 0 & -0.911436 & 5.001443 & -0.131038 & 0 \mathrm{~m}\end{array}$

No. 42

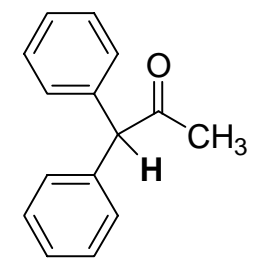

neutral molecule

Charge $=0$ Multiplicity $=1$ for low level calculation on real system.

Charge $=0$ Multiplicity $=1$ for high level calculation on model system .

Charge $=0$ Multiplicity $=1$ for low level calculation on model system.

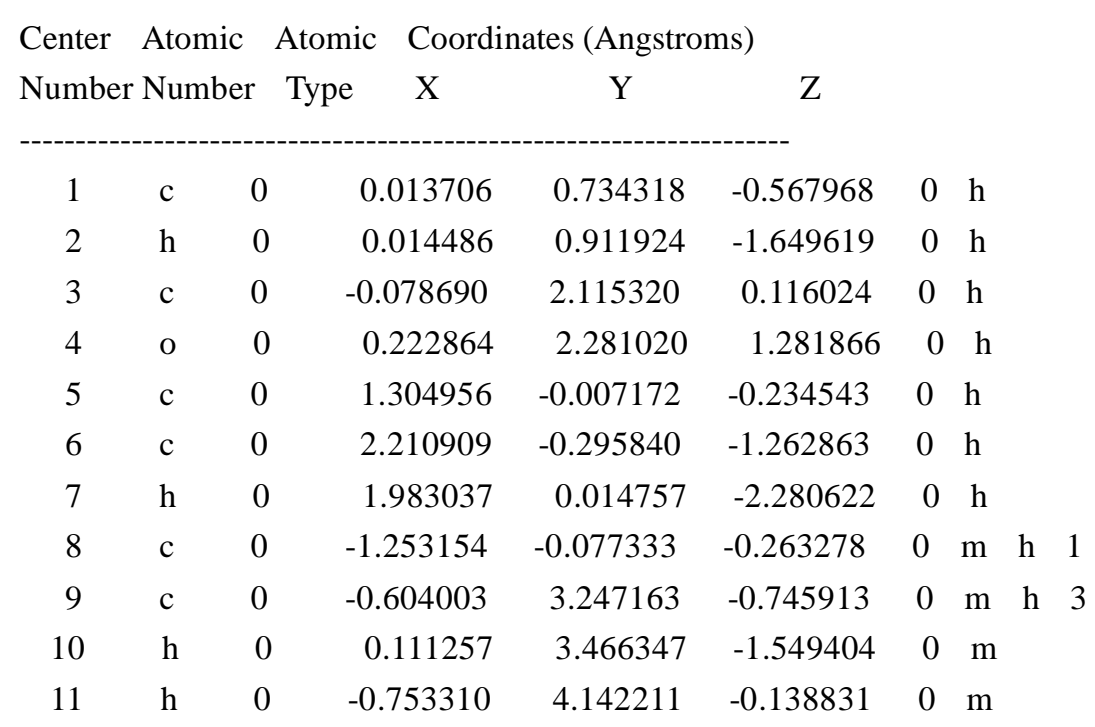




\begin{tabular}{|c|c|c|c|c|c|c|c|c|}
\hline 12 & $\mathrm{~h}$ & 0 & -1.546660 & 2.956182 & -1.225291 & 0 & $\mathrm{~m}$ & \\
\hline 13 & $\mathrm{c}$ & 0 & 1.618409 & -0.415886 & 1.069222 & 0 & $\mathrm{~m}$ & $\mathrm{~h}$ \\
\hline 14 & $\mathrm{~h}$ & 0 & 0.935473 & -0.196936 & 1.881478 & 0 & $\mathrm{~m}$ & \\
\hline 15 & $\mathrm{c}$ & 0 & 3.400955 & -0.977881 & -1.003235 & 0 & $\mathrm{~m}$ & h 6 \\
\hline 16 & $\mathrm{~h}$ & 0 & 4.087978 & -1.193413 & -1.817502 & 0 & $\mathrm{~m}$ & \\
\hline 17 & $\mathrm{c}$ & 0 & -1.845737 & -0.096861 & 1.006693 & 0 & $\mathrm{~m}$ & \\
\hline 18 & $\mathrm{~h}$ & 0 & -1.427260 & 0.506742 & 1.805778 & 0 & $\mathrm{~m}$ & \\
\hline 19 & $\mathrm{c}$ & 0 & -2.980506 & -0.873880 & 1.248010 & 0 & $\mathrm{~m}$ & \\
\hline 20 & $\mathrm{~h}$ & 0 & -3.427113 & -0.875070 & 2.238911 & 0 & $\mathrm{~m}$ & \\
\hline 21 & $\mathrm{c}$ & 0 & -3.542843 & -1.639594 & 0.226425 & 0 & $\mathrm{~m}$ & \\
\hline 22 & $\mathrm{~h}$ & 0 & -4.426926 & -2.242216 & 0.416350 & 0 & $\mathrm{~m}$ & \\
\hline 23 & $\mathrm{c}$ & 0 & -2.963002 & -1.622354 & -1.043469 & 0 & $\mathrm{~m}$ & \\
\hline 24 & $\mathrm{~h}$ & 0 & -3.393090 & -2.212003 & -1.848904 & 0 & $\mathrm{~m}$ & \\
\hline 25 & c & 0 & -1.830406 & -0.845366 & -1.284172 & 0 & $\mathrm{~m}$ & \\
\hline 26 & $\mathrm{~h}$ & 0 & -1.381975 & -0.840931 & -2.275166 & 0 & $\mathrm{~m}$ & \\
\hline 27 & $\mathrm{c}$ & 0 & 2.808458 & -1.093996 & 1.330767 & 0 & $\mathrm{~m}$ & \\
\hline 28 & $\mathrm{~h}$ & 0 & 3.036508 & -1.401851 & 2.347944 & 0 & $\mathrm{~m}$ & \\
\hline 29 & $\mathrm{c}$ & 0 & 3.703660 & -1.379433 & 0.297397 & 0 & $\mathrm{~m}$ & \\
\hline 30 & $\mathrm{~h}$ & 0 & 4.628405 & -1.911137 & 0.505359 & 0 & $\mathrm{~m}$ & \\
\hline
\end{tabular}

radical

Charge $=0$ Multiplicity $=2$ for low level calculation on real system.

Charge $=0$ Multiplicity $=2$ for high level calculation on model system.

Charge $=0$ Multiplicity $=2$ for low level calculation on model system.

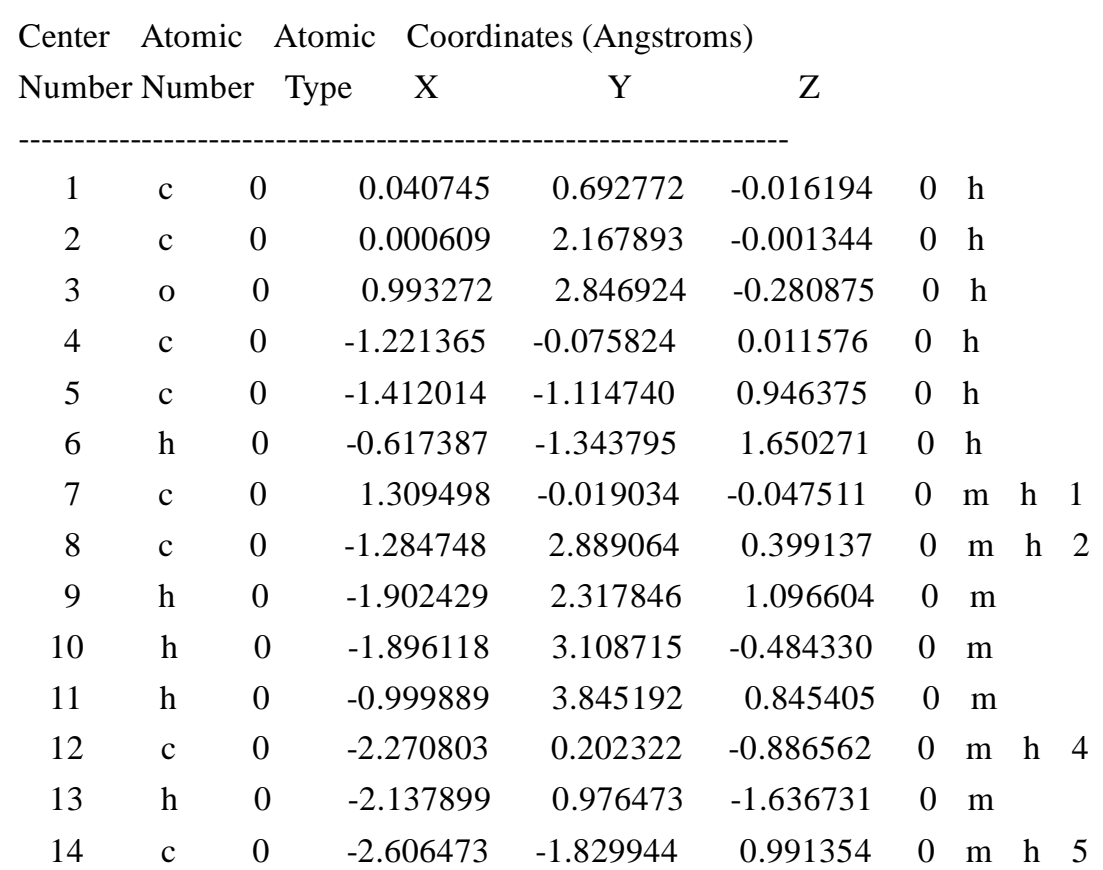




$\begin{array}{lllrrrrc}15 & \mathrm{~h} & 0 & -2.734645 & -2.616353 & 1.730451 & 0 & \mathrm{~m} \\ 16 & \mathrm{c} & 0 & 2.525364 & 0.563540 & 0.398285 & 0 & \mathrm{~m} \\ 17 & \mathrm{~h} & 0 & 2.519668 & 1.577124 & 0.774111 & 0 & \mathrm{~m} \\ 18 & \mathrm{c} & 0 & 3.715364 & -0.152530 & 0.363227 & 0 & \mathrm{~m} \\ 19 & \mathrm{~h} & 0 & 4.628506 & 0.315238 & 0.721641 & 0 & \mathrm{~m} \\ 20 & \mathrm{c} & 0 & 3.747280 & -1.463227 & -0.123578 & 0 & \mathrm{~m} \\ 21 & \mathrm{~h} & 0 & 4.683050 & -2.014936 & -0.152202 & 0 & \mathrm{~m} \\ 22 & \mathrm{c} & 0 & 2.564674 & -2.056289 & -0.575086 & 0 & \mathrm{~m} \\ 23 & \mathrm{~h} & 0 & 2.577313 & -3.069638 & -0.967632 & 0 & \mathrm{~m} \\ 24 & \mathrm{c} & 0 & 1.367960 & -1.351799 & -0.533542 & 0 & \mathrm{~m} \\ 25 & \mathrm{~h} & 0 & 0.462980 & -1.818420 & -0.906892 & 0 & \mathrm{~m} \\ 26 & \mathrm{c} & 0 & -3.459839 & -0.522951 & -0.849913 & 0 & \mathrm{~m} \\ 27 & \mathrm{~h} & 0 & -4.249085 & -0.297629 & -1.562184 & 0 & \mathrm{~m} \\ 28 & \mathrm{c} & 0 & -3.635431 & -1.538468 & 0.092716 & 0 & \mathrm{~m} \\ 29 & \mathrm{~h} & 0 & -4.565152 & -2.099915 & 0.124842 & 0 & \mathrm{~m}\end{array}$

\section{No. 43}<smiles>COc1ccc(C(c2ccc(OC)cc2)c2ccc(OC)cc2)cc1</smiles>

neutral molecule

Charge $=0$ Multiplicity $=1$ for low level calculation on real system.

Charge $=0$ Multiplicity $=1$ for high level calculation on model system .

Charge $=0$ Multiplicity $=1$ for low level calculation on model system.

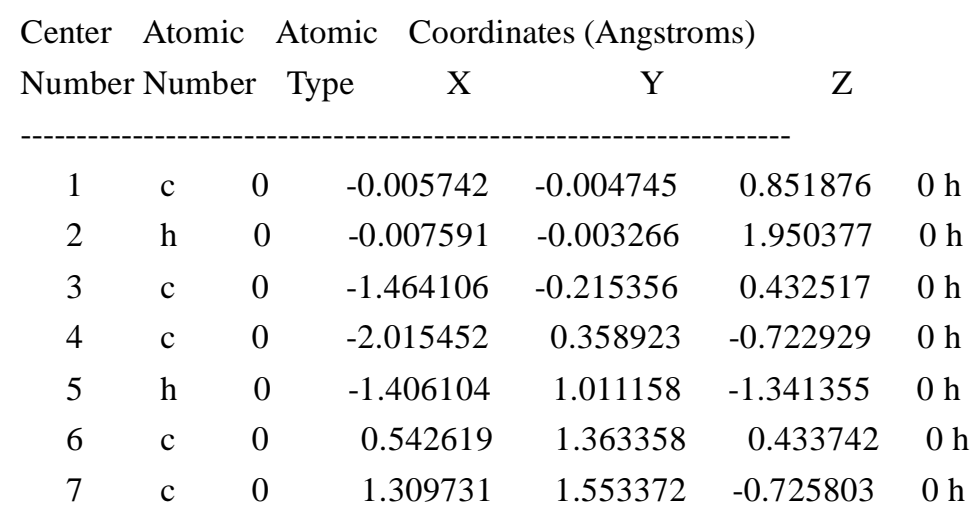




$\begin{array}{ccccccl}8 & \mathrm{~h} & 0 & 1.566442 & 0.699029 & -1.345158 & 0 \mathrm{~h} \\ 9 & \mathrm{c} & 0 & 0.909649 & -1.162047 & 0.437602 & 0 \mathrm{~h} \\ 10 & \mathrm{c} & 0 & 0.664947 & -1.964127 & -0.687066 & 0 \mathrm{~h} \\ 11 & \mathrm{~h} & 0 & -0.226324 & -1.794778 & -1.283781 & 0 \mathrm{~h} \\ 12 & \mathrm{c} & 0 & -2.294064 & -1.029576 & 1.209134 & 0 \mathrm{~m} \mathrm{~h} \\ 13 & \mathrm{~h} & 0 & -1.895373 & -1.491308 & 2.110219 & 0 \mathrm{~m} \\ 14 & \mathrm{c} & 0 & -3.336728 & 0.123202 & -1.087344 & 0 \mathrm{~m} \mathrm{~h} \\ 15 & \mathrm{~h} & 0 & -3.761170 & 0.571089 & -1.980761 & 0 \mathrm{~m} \\ 16 & \mathrm{c} & 0 & 0.257672 & 2.488673 & 1.212848 & 0 \mathrm{~m} \mathrm{~h} \\ 17 & \mathrm{~h} & 0 & -0.335955 & 2.373653 & 2.117523 & 0 \mathrm{~m} \\ 18 & \mathrm{c} & 0 & 1.763343 & 2.815619 & -1.093173 & 0 \mathrm{~m} \mathrm{~h} \\ 19 & \mathrm{~h} & 0 & 2.358545 & 2.959379 & -1.989833 & 0 \mathrm{~m} \\ 20 & \mathrm{c} & 0 & 2.058695 & -1.432444 & 1.187415 & 0 \mathrm{~m} \mathrm{~h} \\ 21 & \mathrm{~h} & 0 & 2.277260 & -0.826698 & 2.064595 & 0 \mathrm{~m} \\ 22 & \mathrm{c} & 0 & 1.535943 & -2.986411 & -1.048897 & 0 \mathrm{~m} \mathrm{~h} \\ 23 & \mathrm{~h} & 0 & 1.343045 & -3.606604 & -1.919016 & 0 \mathrm{~m} \\ 24 & \mathrm{c} & 0 & -3.624519 & -1.275717 & 0.862577 & 0 \mathrm{~m} \\ 25 & \mathrm{~h} & 0 & -4.231361 & -1.910658 & 1.498412 & 0 \mathrm{~m} \\ 26 & \mathrm{c} & 0 & -4.152998 & -0.696075 & -0.296658 & 0 \mathrm{~m} \\ 27 & \mathrm{o} & 0 & -5.437077 & -0.862005 & -0.736028 & 0 \mathrm{~m} \\ 28 & \mathrm{c} & 0 & -6.305987 & -1.677123 & 0.031656 & 0 \mathrm{~m} \\ 29 & \mathrm{~h} & 0 & -5.939293 & -2.710367 & 0.098476 & 0 \mathrm{~m} \\ 30 & \mathrm{~h} & 0 & -7.264795 & -1.669122 & -0.490417 & 0 \mathrm{~m} \\ 31 & \mathrm{~h} & 0 & -6.443558 & -1.279697 & 1.046429 & 0 \mathrm{~m} \\ 32 & \mathrm{c} & 0 & 0.707533 & 3.764060 & 0.863649 & 0 \mathrm{~m} \\ 33 & \mathrm{~h} & 0 & 0.465817 & 4.606751 & 1.501550 & 0 \mathrm{~m} \\ 34 & \mathrm{c} & 0 & 1.465447 & 3.932204 & -0.300965 & 0 \mathrm{~m} \\ 35 & \mathrm{o} & 0 & 1.961169 & 5.127295 & -0.743222 & 0 \mathrm{~m} \\ 36 & \mathrm{c} & 0 & 1.692784 & 6.287569 & 0.025072 & 0 \mathrm{~m} \\ 37 & \mathrm{~h} & 0 & 0.614560 & 6.482379 & 0.102267 & 0 \mathrm{~m} \\ 38 & \mathrm{~h} & 0 & 2.170604 & 7.114870 & -0.503335 & 0 \mathrm{~m} \\ 39 & \mathrm{~h} & 0 & 2.116237 & 6.211484 & 1.035825 & 0 \mathrm{~m} \\ 40 & \mathrm{c} & 0 & 2.943600 & -2.456530 & 0.843012 & 0 \mathrm{~m} \\ 41 & \mathrm{~h} & 0 & 3.819172 & -2.631804 & 1.458109 & 0 \mathrm{~m} \\ 43 & \mathrm{c} & 0 & 2.683149 & -3.241174 & -0.286633 & 0 \mathrm{~m} \\ 44 & \mathrm{c} & 0 & 3.472594 & -4.269567 & -0.721175 & 0 \mathrm{~m} \\ & \mathrm{~h} & 0 & 4.423636 & -4.859093 & 1.043348 & 0 \mathrm{~m} \\ & & 0 & 5.124890 & -5.399324 & -0.507064 & 0 \mathrm{~m}\end{array}$

radical

Charge $=0$ Multiplicity $=2$ for low level calculation on real system. 
Charge $=0$ Multiplicity $=2$ for high level calculation on model system .

Charge $=0$ Multiplicity $=2$ for low level calculation on model system.

\begin{tabular}{|c|c|c|c|c|c|c|}
\hline Center & Atomic & & Coordi & ates (Angstro & & \\
\hline Number & Numbe & & ype & $\mathrm{Y}$ & $\mathrm{Z}$ & \\
\hline 1 & $\mathrm{c}$ & 0 & 0.000818 & -0.001970 & -0.093207 & $0 \mathrm{~h}$ \\
\hline 2 & c & 0 & 1.409597 & -0.396452 & -0.091184 & $0 \mathrm{~h}$ \\
\hline 3 & $\mathrm{c}$ & 0 & 1.858383 & -1.559556 & 0.572988 & $0 \mathrm{~h}$ \\
\hline 4 & $\mathrm{~h}$ & 0 & 1.140836 & -2.167109 & 1.115440 & $0 \mathrm{~h}$ \\
\hline 5 & $\mathrm{c}$ & 0 & -1.046116 & -1.023758 & -0.090306 & $0 \mathrm{~h}$ \\
\hline 6 & $\mathrm{c}$ & 0 & -2.278774 & -0.826407 & 0.570637 & $0 \mathrm{~h}$ \\
\hline 7 & $\mathrm{~h}$ & 0 & -2.444954 & 0.100975 & 1.109565 & $0 \mathrm{~h}$ \\
\hline 8 & $\mathrm{c}$ & 0 & -0.360954 & 1.415823 & -0.092938 & $0 \mathrm{~h}$ \\
\hline 9 & $\mathrm{c}$ & 0 & 0.420823 & 2.384689 & 0.574333 & $0 \mathrm{~h}$ \\
\hline 10 & $\mathrm{~h}$ & 0 & 1.303614 & 2.065125 & 1.119081 & $0 \mathrm{~h}$ \\
\hline 11 & $\mathrm{c}$ & 0 & 2.400424 & 0.369499 & -0.758849 & $0 \mathrm{~m} \mathrm{~h}$ \\
\hline 12 & $\mathrm{~h}$ & 0 & 2.100627 & 1.260405 & -1.301415 & $0 \mathrm{~m}$ \\
\hline 13 & c & 0 & 3.198217 & -1.942303 & 0.580642 & $0 \mathrm{~m} \mathrm{~h}$ \\
\hline 14 & $\mathrm{~h}$ & 0 & 3.488533 & -2.837923 & 1.118749 & $0 \mathrm{~m}$ \\
\hline 15 & $\mathrm{c}$ & 0 & -0.879398 & -2.267165 & -0.754029 & $0 \mathrm{~m} \mathrm{~h}$ \\
\hline 16 & $\mathrm{~h}$ & 0 & 0.042448 & -2.456777 & -1.294524 & $0 \mathrm{~m}$ \\
\hline 17 & $\mathrm{c}$ & 0 & -3.282794 & -1.792593 & 0.578434 & $0 \mathrm{~m} \mathrm{~h}$ \\
\hline 18 & $\mathrm{~h}$ & 0 & -4.204424 & -1.591974 & 1.113405 & $0 \mathrm{~m}$ \\
\hline 19 & $\mathrm{c}$ & 0 & -1.517652 & 1.892418 & -0.763020 & $0 \mathrm{~m} \mathrm{~h}$ \\
\hline 20 & $\mathrm{~h}$ & 0 & -2.139065 & 1.188627 & -1.307549 & $0 \mathrm{~m}$ \\
\hline 21 & $\mathrm{c}$ & 0 & 0.083737 & 3.736767 & 0.582543 & $0 \mathrm{~m} \mathrm{~h}$ \\
\hline 22 & $\mathrm{~h}$ & 0 & 0.713020 & 4.434829 & 1.123523 & $0 \mathrm{~m}$ \\
\hline 23 & $\mathrm{c}$ & 0 & 3.733850 & -0.002534 & -0.761791 & $0 \mathrm{~m}$ \\
\hline 24 & $\mathrm{~h}$ & 0 & 4.478999 & 0.584849 & -1.289926 & $0 \mathrm{~m}$ \\
\hline 25 & $\mathrm{c}$ & 0 & 4.149822 & -1.163431 & -0.089685 & $0 \mathrm{~m}$ \\
\hline 26 & o & 0 & 5.485392 & -1.440785 & -0.149722 & $0 \mathrm{~m}$ \\
\hline 27 & $\mathrm{c}$ & 0 & 5.957386 & -2.608197 & 0.502494 & $0 \mathrm{~m}$ \\
\hline 28 & $\mathrm{~h}$ & 0 & 7.033295 & -2.639213 & 0.320418 & $0 \mathrm{~m}$ \\
\hline 29 & $\mathrm{~h}$ & 0 & 5.494108 & -3.515734 & 0.092916 & $0 \mathrm{~m}$ \\
\hline 30 & $\mathrm{~h}$ & 0 & 5.774250 & -2.568791 & 1.584622 & $0 \mathrm{~m}$ \\
\hline 31 & $\mathrm{c}$ & 0 & -1.870491 & -3.233588 & -0.756039 & $0 \mathrm{~m}$ \\
\hline 32 & $\mathrm{~h}$ & 0 & -1.735693 & -4.174315 & -1.281477 & $0 \mathrm{~m}$ \\
\hline 33 & $\mathrm{c}$ & 0 & -3.085209 & -3.008488 & -0.087904 & $0 \mathrm{~m}$ \\
\hline 34 & o & 0 & -3.995956 & -4.023741 & -0.149534 & $0 \mathrm{~m}$ \\
\hline 35 & $\mathrm{c}$ & 0 & -5.242631 & -3.846361 & 0.502840 & $0 \mathrm{~m}$ \\
\hline 36 & $\mathrm{~h}$ & 0 & -5.796587 & -2.992229 & 0.090937 & $0 \mathrm{~m}$ \\
\hline 37 & $\mathrm{~h}$ & 0 & -5.116659 & -3.704439 & 1.584542 & $0 \mathrm{~m}$ \\
\hline
\end{tabular}




$\begin{array}{lllrrrl}38 & \mathrm{~h} & 0 & -5.807896 & -4.762886 & 0.323595 & 0 \mathrm{~m} \\ 39 & \mathrm{c} & 0 & -1.860546 & 3.233608 & -0.765887 & 0 \mathrm{~m} \\ 40 & \mathrm{~h} & 0 & -2.739831 & 3.586657 & -1.296372 & 0 \mathrm{~m} \\ 41 & \mathrm{c} & 0 & -1.063882 & 4.173081 & -0.091263 & 0 \mathrm{~m} \\ 42 & \mathrm{o} & 0 & -1.489982 & 5.468737 & -0.152543 & 0 \mathrm{~m} \\ 43 & \mathrm{c} & 0 & -0.720792 & 6.459678 & 0.508742 & 0 \mathrm{~m} \\ 44 & \mathrm{~h} & 0 & -1.232317 & 7.406815 & 0.327140 & 0 \mathrm{~m} \\ 45 & \mathrm{~h} & 0 & 0.299276 & 6.515636 & 0.105883 & 0 \mathrm{~m} \\ 46 & \mathrm{~h} & 0 & -0.670123 & 6.277238 & 1.590539 & 0 \mathrm{~m}\end{array}$

\section{No. 44}<smiles>O=CC(=O)c1ccccc1</smiles>

neutral molecule

Charge $=0$ Multiplicity $=1$ for low level calculation on real system.

Charge $=0$ Multiplicity $=1$ for high level calculation on model system .

Charge $=0$ Multiplicity $=1$ for low level calculation on model system .

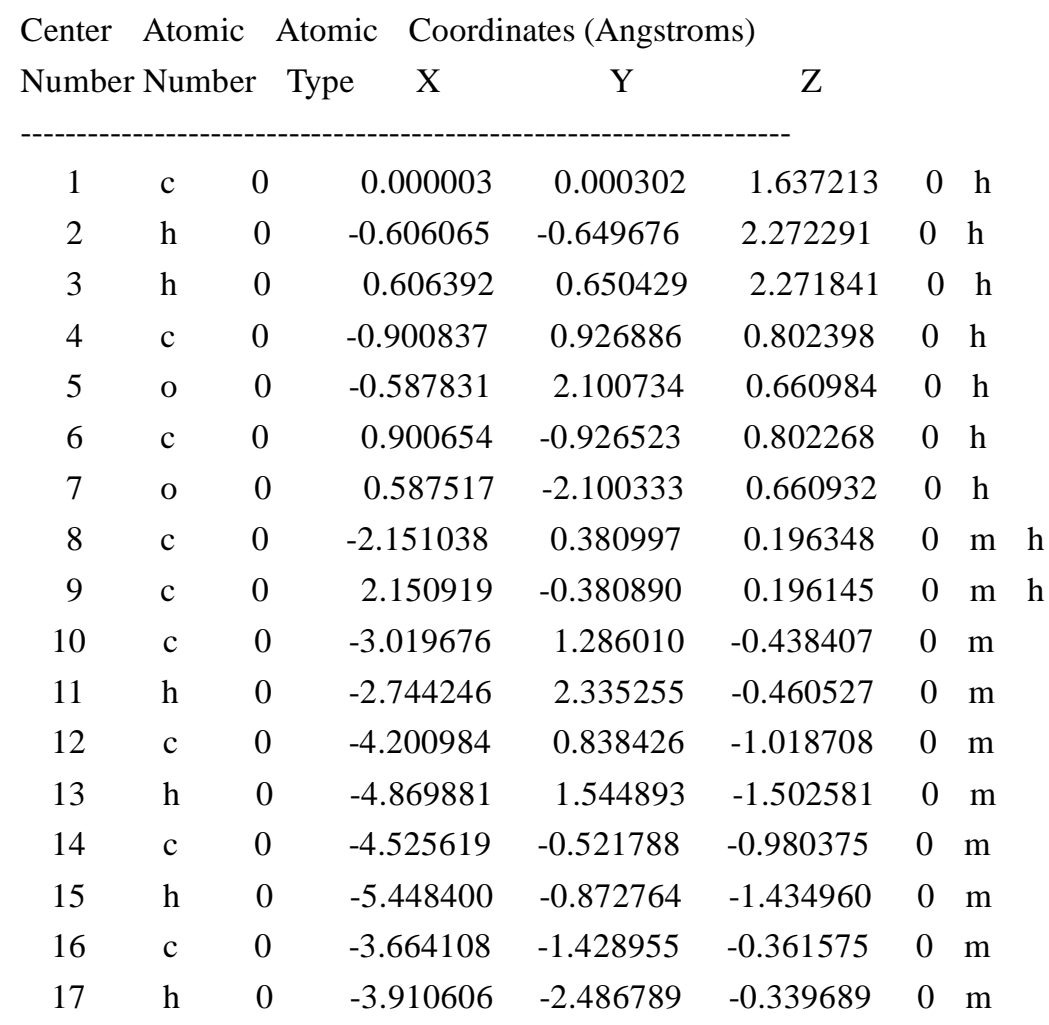




$\begin{array}{llllllll}18 & \mathrm{c} & 0 & -2.482109 & -0.983544 & 0.230445 & 0 & \mathrm{~m} \\ 19 & \mathrm{~h} & 0 & -1.808935 & -1.701937 & 0.686351 & 0 & \mathrm{~m} \\ 20 & \mathrm{c} & 0 & 3.019687 & -1.286202 & -0.437988 & 0 & \mathrm{~m} \\ 21 & \mathrm{~h} & 0 & 2.744186 & -2.335429 & -0.459799 & 0 & \mathrm{~m} \\ 22 & \mathrm{c} & 0 & 4.201165 & -0.838886 & -1.018166 & 0 & \mathrm{~m} \\ 23 & \mathrm{~h} & 0 & 4.870204 & -1.545563 & -1.501539 & 0 & \mathrm{~m} \\ 24 & \mathrm{c} & 0 & 4.525834 & 0.521316 & -0.980286 & 0 & \mathrm{~m} \\ 25 & \mathrm{~h} & 0 & 5.448797 & 0.872050 & -1.434694 & 0 & \mathrm{~m} \\ 26 & \mathrm{c} & 0 & 3.664247 & 1.428770 & -0.361990 & 0 & \mathrm{~m} \\ 27 & \mathrm{~h} & 0 & 3.910808 & 2.486593 & -0.340437 & 0 & \mathrm{~m} \\ 28 & \mathrm{c} & 0 & 2.482114 & 0.983660 & 0.229879 & 0 & \mathrm{~m} \\ 29 & \mathrm{~h} & 0 & 1.808747 & 1.702254 & 0.685200 & 0 & \mathrm{~m}\end{array}$

radical

Charge $=0$ Multiplicity $=2$ for low level calculation on real system.

Charge $=0$ Multiplicity $=2$ for high level calculation on model system .

Charge $=0$ Multiplicity $=2$ for low level calculation on model system .

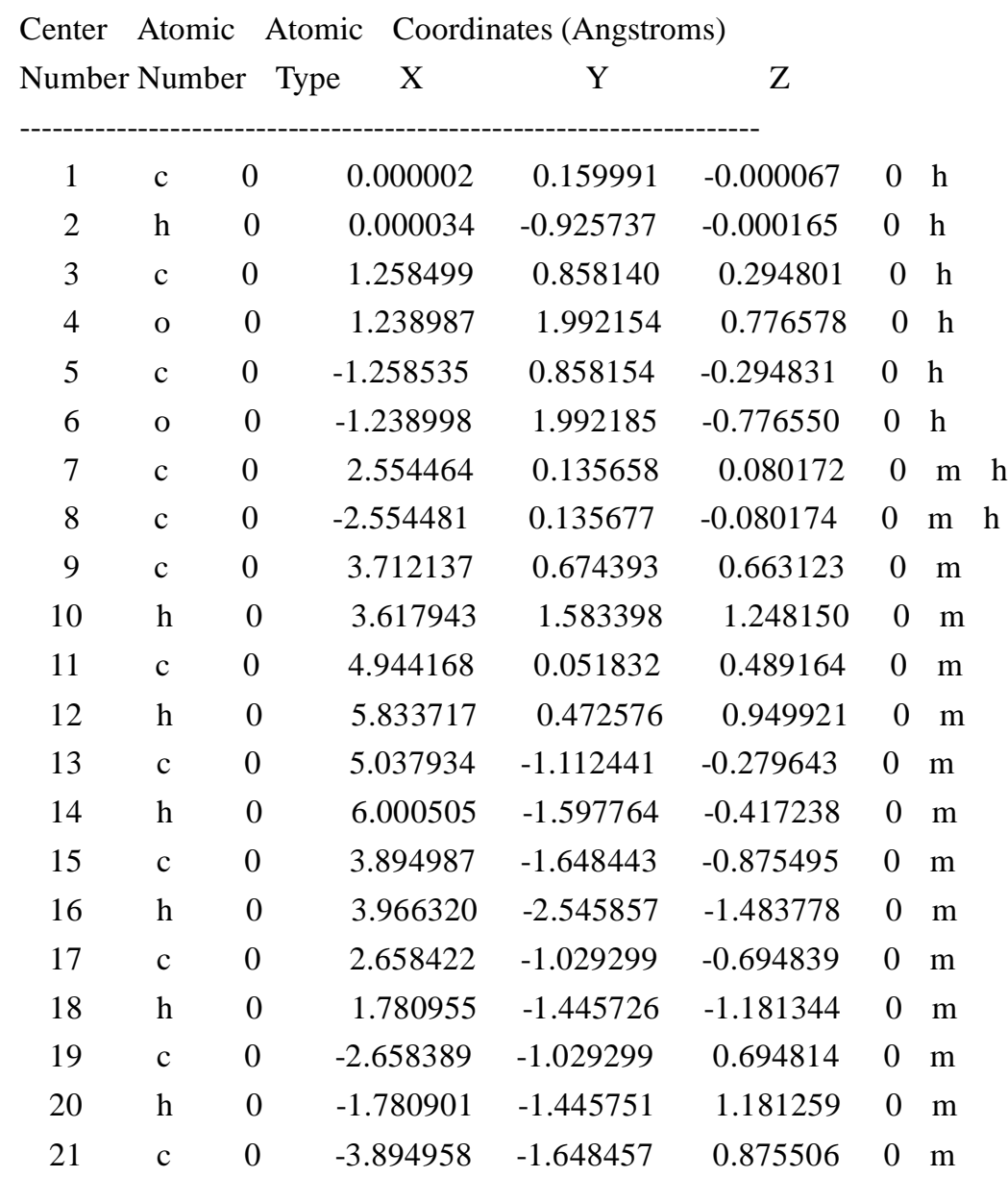




$\begin{array}{lllrrrrr}22 & \mathrm{~h} & 0 & -3.966278 & -2.545880 & 1.483777 & 0 & \mathrm{~m} \\ 23 & \mathrm{c} & 0 & -5.037919 & -1.112457 & 0.279688 & 0 & \mathrm{~m} \\ 24 & \mathrm{~h} & 0 & -6.000479 & -1.597801 & 0.417295 & 0 & \mathrm{~m} \\ 25 & \mathrm{c} & 0 & -4.944176 & 0.051816 & -0.489129 & 0 & \mathrm{~m} \\ 26 & \mathrm{~h} & 0 & -5.833746 & 0.472544 & -0.949855 & 0 & \mathrm{~m} \\ 27 & \mathrm{c} & 0 & -3.712156 & 0.674385 & -0.663109 & 0 & \mathrm{~m} \\ 28 & \mathrm{~h} & 0 & -3.617962 & 1.583392 & -1.248135 & 0 & \mathrm{~m}\end{array}$

No. 45

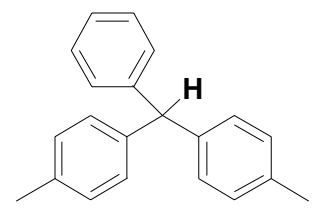

neutral molecule

Charge $=0$ Multiplicity $=1$ for low level calculation on real system.

Charge $=0$ Multiplicity $=1$ for high level calculation on model system .

Charge $=0$ Multiplicity $=1$ for low level calculation on model system .

\begin{tabular}{|c|c|c|c|c|c|c|c|c|}
\hline Center & Atomic & & Atomic Coordi & ates (Angstro & $\mathrm{ms})$ & & & \\
\hline Number & Numbe & & Type & $\mathrm{Y}$ & $\mathrm{Z}$ & & & \\
\hline 1 & $\mathrm{c}$ & 0 & 0.000984 & 0.370112 & 0.810964 & 0 & $\mathrm{~h}$ & \\
\hline 2 & $\mathrm{~h}$ & 0 & 0.000734 & 0.431704 & 1.907682 & 0 & $\mathrm{~h}$ & \\
\hline 3 & $\mathrm{c}$ & 0 & 0.022720 & 1.819571 & 0.313467 & 0 & $\mathrm{~h}$ & \\
\hline 4 & $\mathrm{c}$ & 0 & 0.703641 & 2.211855 & -0.845127 & 0 & $\mathrm{~h}$ & \\
\hline 5 & $\mathrm{~h}$ & 0 & 1.271906 & 1.477361 & -1.407892 & 0 & $\mathrm{~h}$ & \\
\hline 6 & $\mathrm{c}$ & 0 & 1.264879 & -0.410439 & 0.437291 & 0 & $\mathrm{~h}$ & \\
\hline 7 & $\mathrm{c}$ & 0 & 1.321788 & -1.290447 & -0.648090 & 0 & $\mathrm{~h}$ & \\
\hline 8 & $\mathrm{~h}$ & 0 & 0.432929 & -1.463161 & -1.247594 & 0 & $\mathrm{~h}$ & \\
\hline 9 & $\mathrm{c}$ & 0 & -1.288552 & -0.366282 & 0.434439 & 0 & $\mathrm{~h}$ & \\
\hline 10 & $\mathrm{c}$ & 0 & -2.020981 & -0.076082 & -0.721412 & 0 & $\mathrm{~h}$ & \\
\hline 11 & $\mathrm{~h}$ & 0 & -1.694986 & 0.724115 & -1.379434 & 0 & $\mathrm{~h}$ & \\
\hline 12 & $\mathrm{c}$ & 0 & -0.686060 & 2.794296 & 1.031199 & 0 & $\mathrm{~m}$ & \\
\hline 13 & $\mathrm{~h}$ & 0 & -1.222358 & 2.505000 & 1.932675 & 0 & $\mathrm{~m}$ & \\
\hline 14 & $\mathrm{c}$ & 0 & 0.672014 & 3.540461 & -1.277044 & 0 & $\mathrm{~m} \mathrm{~h}$ & 4 \\
\hline 15 & $\mathrm{~h}$ & 0 & 1.209140 & 3.824212 & -2.178616 & 0 & $\mathrm{~m}$ & \\
\hline 16 & $\mathrm{c}$ & 0 & 2.431663 & -0.227663 & 1.193967 & 0 & $\mathrm{~m} \mathrm{~h}$ & 6 \\
\hline 17 & $\mathrm{~h}$ & 0 & 2.416420 & 0.456230 & 2.040248 & 0 & $\mathrm{~m}$ & \\
\hline 18 & $\mathrm{c}$ & 0 & 2.505534 & -1.960674 & -0.966204 & 0 & $\mathrm{~m} \quad \mathrm{~h}$ & \\
\hline 19 & $\mathrm{~h}$ & 0 & 2.520289 & -2.638967 & -1.816827 & 0 & $\mathrm{~m}$ & \\
\hline
\end{tabular}




$\begin{array}{lllrrrrrrr}20 & \mathrm{c} & 0 & -1.754371 & -1.397815 & 1.262324 & 0 & \mathrm{~m} & \mathrm{~h} & 9 \\ 21 & \mathrm{~h} & 0 & -1.199947 & -1.649071 & 2.164347 & 0 & \mathrm{~m} & \\ 22 & \mathrm{c} & 0 & -3.175572 & -0.795696 & -1.037926 & 0 & \mathrm{~m} & \mathrm{~h} & 10 \\ 23 & \mathrm{~h} & 0 & -3.723783 & -0.548035 & -1.944620 & 0 & \mathrm{~m} & & \\ 24 & \mathrm{c} & 0 & -0.716895 & 4.121173 & 0.605507 & 0 & \mathrm{~m} & \\ 25 & \mathrm{~h} & 0 & -1.268781 & 4.860199 & 1.180842 & 0 & \mathrm{~m} & \\ 26 & \mathrm{c} & 0 & -0.036588 & 4.499911 & -0.554323 & 0 & \mathrm{~m} & \\ 27 & \mathrm{~h} & 0 & -0.055819 & 5.534157 & -0.887706 & 0 & \mathrm{~m} & \\ 28 & \mathrm{c} & 0 & 3.609322 & -0.899036 & 0.877234 & 0 & \mathrm{~m} & \\ 29 & \mathrm{~h} & 0 & 4.497349 & -0.737497 & 1.485154 & 0 & \mathrm{~m} & \\ 30 & \mathrm{c} & 0 & 3.668522 & -1.782481 & -0.210361 & 0 & \mathrm{~m} & \\ 31 & \mathrm{c} & 0 & 4.939427 & -2.533360 & -0.533430 & 0 & \mathrm{~m} & \\ 32 & \mathrm{~h} & 0 & 5.822461 & -1.892403 & -0.429585 & 0 & \mathrm{~m} & \\ 33 & \mathrm{~h} & 0 & 5.081331 & -3.388347 & 0.141177 & 0 & \mathrm{~m} & \\ 34 & \mathrm{~h} & 0 & 4.924542 & -2.923695 & -1.556290 & 0 & \mathrm{~m} & \\ 35 & \mathrm{c} & 0 & -2.907273 & -2.111686 & 0.946599 & 0 & \mathrm{~m} & \\ 36 & \mathrm{~h} & 0 & -3.243327 & -2.905505 & 1.610751 & 0 & \mathrm{~m} & \\ 37 & \mathrm{c} & 0 & -3.643428 & -1.822106 & -0.211029 & 0 & \mathrm{~m} & \\ 38 & \mathrm{c} & 0 & -4.912828 & -2.575144 & -0.535067 & 0 & \mathrm{~m} & \\ 39 & \mathrm{~h} & 0 & -4.833102 & -3.633370 & -0.261890 & 0 & \mathrm{~m} & \\ 40 & \mathrm{~h} & 0 & -5.772190 & -2.164739 & 0.012263 & 0 & \mathrm{~m} & \\ 41 & \mathrm{~h} & 0 & -5.150474 & -2.518987 & -1.602549 & 0 & \mathrm{~m} & \\ -------------------------------------------------------- & & & \end{array}$

radical

Charge $=0$ Multiplicity $=2$ for low level calculation on real system.

Charge $=0$ Multiplicity $=2$ for high level calculation on model system.

Charge $=0$ Multiplicity $=2$ for low level calculation on model system.

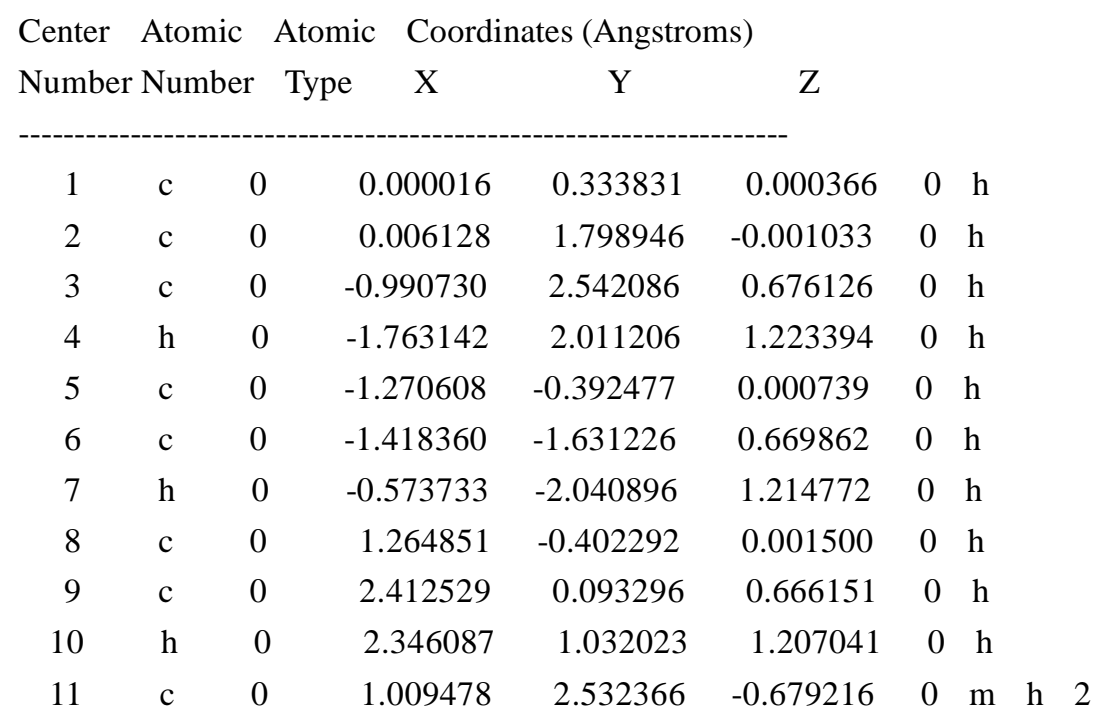




\begin{tabular}{|c|c|c|c|c|c|c|c|c|}
\hline 12 & $\mathrm{~h}$ & 0 & 1.777184 & 1.993916 & -1.225699 & 0 & $\mathrm{~m}$ & \\
\hline 13 & $\mathrm{c}$ & 0 & -0.980976 & 3.933282 & 0.676605 & 0 & $\mathrm{~m}$ & $\mathrm{~h}$ \\
\hline 14 & $\mathrm{~h}$ & 0 & -1.753405 & 4.473814 & 1.217972 & 0 & $\mathrm{~m}$ & \\
\hline 15 & $\mathrm{c}$ & 0 & -2.414618 & 0.109949 & -0.663471 & 0 & $\mathrm{~m}$ & $\mathrm{~h}$ \\
\hline 16 & $\mathrm{~h}$ & 0 & -2.343439 & 1.049137 & -1.202935 & 0 & $\mathrm{~m}$ & \\
\hline 17 & $\mathrm{c}$ & 0 & -2.628372 & -2.314317 & 0.673256 & 0 & $\mathrm{~m}$ & $\mathrm{~h}$ \\
\hline 18 & $\mathrm{~h}$ & 0 & -2.705318 & -3.254788 & 1.215316 & 0 & $\mathrm{~m}$ & \\
\hline 19 & $\mathrm{c}$ & 0 & 1.401889 & -1.646957 & -0.657553 & 0 & $\mathrm{~m}$ & $\mathrm{~h}$ \\
\hline 20 & $\mathrm{~h}$ & 0 & 0.552061 & -2.057320 & -1.193735 & 0 & $\mathrm{~m}$ & \\
\hline 21 & $\mathrm{c}$ & 0 & 3.609622 & -0.611956 & 0.669902 & 0 & $\mathrm{~m}$ & $\mathrm{~h}$ \\
\hline 22 & $\mathrm{~h}$ & 0 & 4.463354 & -0.205046 & 1.208258 & 0 & $\mathrm{~m}$ & \\
\hline 23 & $\mathrm{c}$ & 0 & 1.012131 & 3.923598 & -0.681390 & 0 & $\mathrm{~m}$ & \\
\hline 24 & $\mathrm{~h}$ & 0 & 1.789375 & 4.456578 & -1.223356 & 0 & $\mathrm{~m}$ & \\
\hline 25 & $\mathrm{c}$ & 0 & 0.018731 & 4.635347 & -0.002826 & 0 & $\mathrm{~m}$ & \\
\hline 26 & $\mathrm{~h}$ & 0 & 0.023578 & 5.721950 & -0.003451 & 0 & $\mathrm{~m}$ & \\
\hline 27 & $\mathrm{c}$ & 0 & -3.618836 & -0.584591 & -0.660379 & 0 & $\mathrm{~m}$ & \\
\hline 28 & $\mathrm{~h}$ & 0 & -4.472146 & -0.169368 & -1.192813 & 0 & $\mathrm{~m}$ & \\
\hline 29 & $\mathrm{c}$ & 0 & -3.754957 & -1.811562 & 0.004800 & 0 & $\mathrm{~m}$ & \\
\hline 30 & $\mathrm{c}$ & 0 & -5.056035 & -2.576738 & -0.018264 & 0 & $\mathrm{~m}$ & \\
\hline 31 & $\mathrm{~h}$ & 0 & -5.911655 & -1.909895 & -0.169072 & 0 & $\mathrm{~m}$ & \\
\hline 32 & $\mathrm{~h}$ & 0 & -5.215194 & -3.126413 & 0.916118 & 0 & $\mathrm{~m}$ & \\
\hline 33 & $\mathrm{~h}$ & 0 & -5.071633 & -3.314317 & -0.832649 & 0 & $\mathrm{~m}$ & \\
\hline 34 & c & 0 & 2.606096 & -2.341677 & -0.653876 & 0 & $\mathrm{~m}$ & \\
\hline 35 & $\mathrm{~h}$ & 0 & 2.672800 & -3.290587 & -1.182247 & 0 & $\mathrm{~m}$ & \\
\hline 36 & $\mathrm{c}$ & 0 & 3.737434 & -1.842119 & 0.007145 & 0 & $\mathrm{~m}$ & \\
\hline 37 & $\mathrm{c}$ & 0 & 5.051075 & -2.585463 & -0.013286 & 0 & $\mathrm{~m}$ & \\
\hline 38 & $\mathrm{~h}$ & 0 & 4.902513 & -3.659659 & -0.166606 & 0 & $\mathrm{~m}$ & \\
\hline 39 & $\mathrm{~h}$ & 0 & 5.700370 & -2.228180 & -0.824591 & 0 & $\mathrm{~m}$ & \\
\hline 40 & $\mathrm{~h}$ & 0 & 5.603416 & -2.450117 & 0.923336 & 0 & $\mathrm{~m}$ & \\
\hline
\end{tabular}

\section{No. 46}<smiles>OC(c1ccccc1)c1ccccc1</smiles>

neutral molecule

Charge $=0$ Multiplicity $=1$ for low level calculation on real system.

Charge $=0$ Multiplicity $=1$ for high level calculation on model system .

Charge $=0$ Multiplicity $=1$ for low level calculation on model system . 


\begin{tabular}{|c|c|c|c|c|c|c|c|c|}
\hline Center & Atomic & & Coordin & ates (Angstro & & & & \\
\hline Number & Numbe & & Type & $\mathrm{Y}$ & Z & & & \\
\hline 1 & $\mathrm{c}$ & 0 & -0.004757 & 0.960804 & -0.876318 & 0 & $\mathrm{~h}$ & \\
\hline 2 & $\mathrm{~h}$ & 0 & 0.030333 & 0.862692 & -1.974475 & 0 & $\mathrm{~h}$ & \\
\hline 3 & o & 0 & -0.085641 & 2.341533 & -0.521347 & 0 & h & \\
\hline 4 & $\mathrm{~h}$ & 0 & 0.779722 & 2.734780 & -0.712446 & 0 & $\mathrm{~h}$ & \\
\hline 5 & $\mathrm{c}$ & 0 & -1.273891 & 0.271023 & -0.388250 & 0 & $\mathrm{~h}$ & \\
\hline 6 & $\mathrm{c}$ & 0 & -1.502757 & -1.070306 & -0.723039 & 0 & $\mathrm{~h}$ & \\
\hline 7 & $\mathrm{~h}$ & 0 & -0.768626 & -1.609198 & -1.317988 & 0 & $\mathrm{~h}$ & \\
\hline 8 & $\mathrm{c}$ & 0 & 1.262048 & 0.324908 & -0.314632 & 0 & $\mathrm{~h}$ & \\
\hline 9 & $\mathrm{c}$ & 0 & 2.158643 & -0.348244 & -1.150004 & 0 & $\mathrm{~h}$ & \\
\hline 10 & $\mathrm{~h}$ & 0 & 1.947213 & -0.422128 & -2.214963 & 0 & $\mathrm{~h}$ & \\
\hline 11 & $\mathrm{c}$ & 0 & -2.223216 & 0.948810 & 0.381960 & 0 & $\mathrm{~m}$ & h 5 \\
\hline 12 & $\mathrm{~h}$ & 0 & -2.054667 & 1.989484 & 0.633249 & 0 & $\mathrm{~m}$ & \\
\hline 13 & $\mathrm{c}$ & 0 & -2.656237 & -1.723263 & -0.293679 & 0 & $\mathrm{~m} \quad \mathrm{l}$ & h 6 \\
\hline 14 & $\mathrm{~h}$ & 0 & -2.818104 & -2.764198 & -0.561669 & 0 & $\mathrm{~m}$ & \\
\hline 15 & $\mathrm{c}$ & 0 & 3.322851 & -0.921978 & -0.631381 & 0 & $\mathrm{~m}$ & h 9 \\
\hline 16 & $\mathrm{~h}$ & 0 & 4.009883 & -1.443294 & -1.292856 & 0 & $\mathrm{~m}$ & \\
\hline 17 & $\mathrm{c}$ & 0 & 1.554294 & 0.428000 & 1.053173 & 0 & $\mathrm{~m}$ & h 8 \\
\hline 18 & $\mathrm{~h}$ & 0 & 0.862153 & 0.955150 & 1.703848 & 0 & $\mathrm{~m}$ & \\
\hline 19 & $\mathrm{c}$ & 0 & -3.379568 & 0.293159 & 0.813495 & 0 & $\mathrm{~m}$ & \\
\hline 20 & $\mathrm{~h}$ & 0 & -4.109794 & 0.832702 & 1.411492 & 0 & $\mathrm{~m}$ & \\
\hline 21 & $\mathrm{c}$ & 0 & -3.600609 & -1.042251 & 0.479099 & 0 & $\mathrm{~m}$ & \\
\hline 22 & $\mathrm{~h}$ & 0 & -4.501023 & -1.550011 & 0.814776 & 0 & $\mathrm{~m}$ & \\
\hline 23 & $\mathrm{c}$ & 0 & 3.604004 & -0.817271 & 0.730257 & 0 & $\mathrm{~m}$ & \\
\hline 24 & $\mathrm{~h}$ & 0 & 4.509793 & -1.259712 & 1.136293 & 0 & $\mathrm{~m}$ & \\
\hline 25 & $\mathrm{c}$ & 0 & 2.716854 & -0.137725 & 1.571349 & 0 & $\mathrm{~m}$ & \\
\hline 26 & $\mathrm{~h}$ & 0 & 2.932296 & -0.052518 & 2.633329 & 0 & $\mathrm{~m}$ & \\
\hline
\end{tabular}

radical

Charge $=0$ Multiplicity $=2$ for low level calculation on real system.

Charge $=0$ Multiplicity $=2$ for high level calculation on model system.

Charge $=0$ Multiplicity $=2$ for low level calculation on model system.

\begin{tabular}{|c|c|c|c|c|c|c|}
\hline Center & Atomic & Atomi & Coordi & tes (Angstr & ns) & \\
\hline Number & Numbe & Type & $\mathrm{X}$ & $\mathrm{Y}$ & Z & \\
\hline 1 & $\mathrm{c}$ & 0 & -0.011010 & 0.910997 & -0.036179 & $0 \mathrm{~h}$ \\
\hline 2 & o & 0 & -0.045698 & 2.284952 & -0.066144 & $0 \mathrm{~h}$ \\
\hline 3 & $\mathrm{~h}$ & 0 & 0.801357 & 2.604245 & -0.418260 & 0 \\
\hline 4 & $\mathrm{c}$ & 0 & -1.306159 & 0.285068 & 0.009663 & 0 \\
\hline
\end{tabular}




\begin{tabular}{|c|c|c|c|c|c|c|c|c|}
\hline 5 & $\mathrm{c}$ & 0 & -1.495059 & -1.042572 & 0.472755 & 0 & $\mathrm{~h}$ & \\
\hline 6 & $\mathrm{~h}$ & 0 & -0.644280 & -1.611309 & 0.831665 & 0 & $\mathrm{~h}$ & \\
\hline 7 & $\mathrm{c}$ & 0 & 1.288339 & 0.275584 & -0.019088 & 0 & $\mathrm{~h}$ & \\
\hline 8 & $\mathrm{c}$ & 0 & 1.514855 & -1.016372 & -0.558097 & 0 & $\mathrm{~h}$ & \\
\hline 9 & $\mathrm{~h}$ & 0 & 0.687142 & -1.561750 & -0.998259 & 0 & $\mathrm{~h}$ & \\
\hline 10 & $\mathrm{c}$ & 0 & -2.460303 & 1.019192 & -0.368999 & 0 & $\mathrm{~m}$ & $\mathrm{~h}$ \\
\hline 11 & $\mathrm{~h}$ & 0 & -2.340587 & 2.040735 & -0.711185 & 0 & $\mathrm{~m}$ & \\
\hline 12 & $\mathrm{c}$ & 0 & -2.762693 & -1.609563 & 0.516974 & 0 & $\mathrm{~m}$ & $\mathrm{~h}$ \\
\hline 13 & $\mathrm{~h}$ & 0 & -2.878642 & -2.626946 & 0.881998 & 0 & $\mathrm{~m}$ & \\
\hline 14 & $\mathrm{c}$ & 0 & 2.789030 & -1.569442 & -0.576580 & 0 & $\mathrm{~m}$ & $\mathrm{~h}$ \\
\hline 15 & $\mathrm{~h}$ & 0 & 2.934175 & -2.557164 & -1.006634 & 0 & $\mathrm{~m}$ & \\
\hline 16 & $\mathrm{c}$ & 0 & 2.413504 & 0.978905 & 0.484701 & 0 & $\mathrm{~m}$ & $\mathrm{~h}$ \\
\hline 17 & $\mathrm{~h}$ & 0 & 2.272364 & 1.951226 & 0.950028 & 0 & $\mathrm{~m}$ & \\
\hline 18 & $\mathrm{c}$ & 0 & -3.722263 & 0.441914 & -0.317870 & 0 & $\mathrm{~m}$ & \\
\hline 19 & $\mathrm{~h}$ & 0 & -4.588291 & 1.023920 & -0.623134 & 0 & $\mathrm{~m}$ & \\
\hline 20 & $\mathrm{c}$ & 0 & -3.886245 & -0.877776 & 0.117685 & 0 & $\mathrm{~m}$ & \\
\hline 21 & $\mathrm{~h}$ & 0 & -4.875312 & -1.325671 & 0.155172 & 0 & $\mathrm{~m}$ & \\
\hline 22 & $\mathrm{c}$ & 0 & 3.885043 & -0.861301 & -0.069320 & 0 & $\mathrm{~m}$ & \\
\hline 23 & $\mathrm{~h}$ & 0 & 4.878722 & -1.299516 & -0.088032 & 0 & $\mathrm{~m}$ & \\
\hline 24 & $\mathrm{c}$ & 0 & 3.685372 & 0.416746 & 0.459099 & 0 & $\mathrm{~m}$ & \\
\hline 25 & $\mathrm{~h}$ & 0 & 4.524469 & 0.974328 & 0.867333 & 0 & $\mathrm{~m}$ & \\
\hline
\end{tabular}

No. 47<smiles>CN1c2ccccc2C(C#N)C1C#N</smiles>

neutral molecule

Charge $=0$ Multiplicity $=1$ for low level calculation on real system.

Charge $=0$ Multiplicity $=1$ for high level calculation on model system.

Charge $=0$ Multiplicity $=1$ for low level calculation on model system.

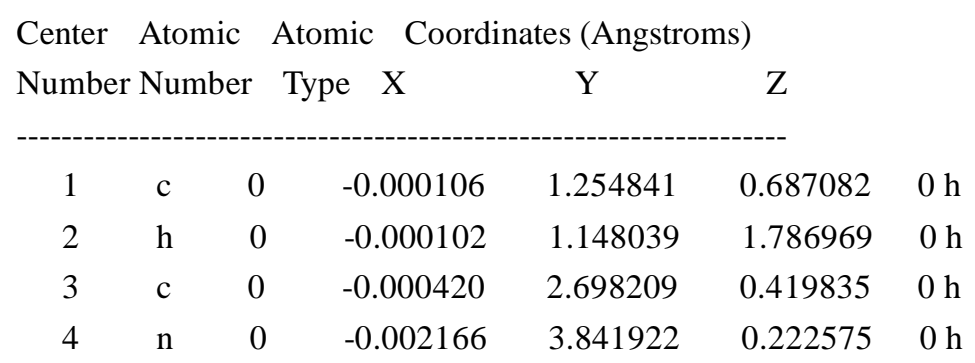




$\begin{array}{rrrrrrl}5 & \mathrm{c} & 0 & 1.250565 & 0.554029 & 0.163989 & 0 \mathrm{~h} \\ 6 & \mathrm{c} & 0 & 2.414534 & 1.221696 & -0.207497 & 0 \mathrm{~h} \\ 7 & \mathrm{~h} & 0 & 2.429782 & 2.307830 & -0.193194 & 0 \mathrm{~h} \\ 8 & \mathrm{c} & 0 & -1.250541 & 0.553644 & 0.163902 & 0 \mathrm{~h} \\ 9 & \mathrm{c} & 0 & -2.414800 & 1.220857 & -0.207604 & 0 \mathrm{~h} \\ 10 & \mathrm{~h} & 0 & -2.430478 & 2.306975 & -0.193319 & 0 \mathrm{~h} \\ 11 & \mathrm{c} & 0 & 3.557278 & 0.510833 & -0.579560 & 0 \mathrm{~m} \mathrm{~h} 6 \\ 12 & \mathrm{~h} & 0 & 4.461689 & 1.042647 & -0.858780 & 0 \mathrm{~m} \\ 13 & \mathrm{c} & 0 & 1.207510 & -0.854672 & 0.142073 & 0 \mathrm{~m} \mathrm{~h} 5 \\ 14 & \mathrm{c} & 0 & -1.206957 & -0.855044 & 0.141995 & 0 \mathrm{~m} \mathrm{~h} 8 \\ 15 & \mathrm{c} & 0 & -3.557259 & 0.509513 & -0.579635 & 0 \mathrm{~m} \mathrm{~h} 9 \\ 16 & \mathrm{~h} & 0 & -4.461891 & 1.040964 & -0.858834 & 0 \mathrm{~m} \\ 17 & \mathrm{c} & 0 & 3.523457 & -0.881973 & -0.580766 & 0 \mathrm{~m} \\ 18 & \mathrm{~h} & 0 & 4.403336 & -1.450210 & -0.870195 & 0 \mathrm{~m} \\ 19 & \mathrm{c} & 0 & 2.357967 & -1.565145 & -0.232858 & 0 \mathrm{~m} \\ 20 & \mathrm{~h} & 0 & 2.342805 & -2.647905 & -0.281274 & 0 \mathrm{~m} \\ 21 & \mathrm{n} & 0 & 0.000357 & -1.498778 & 0.486650 & 0 \mathrm{~m} \\ 22 & \mathrm{c} & 0 & 0.000477 & -2.936035 & 0.702580 & 0 \mathrm{~m} \\ 23 & \mathrm{~h} & 0 & 0.883800 & -3.210084 & 1.283935 & 0 \mathrm{~m} \\ 24 & \mathrm{~h} & 0 & 0.000622 & -3.518191 & -0.231997 & 0 \mathrm{~m} \\ 25 & \mathrm{~h} & 0 & -0.882922 & -3.210242 & 1.283757 & 0 \mathrm{~m} \\ 26 & \mathrm{c} & 0 & -2.357131 & -1.565990 & -0.232855 & 0 \mathrm{~m} \\ 27 & \mathrm{~h} & 0 & -2.341518 & -2.648756 & -0.281149 & 0 \mathrm{~m} \\ 28 & \mathrm{c} & 0 & -3.522892 & -0.883292 & -0.580747 & 0 \mathrm{~m} \\ 29 & \mathrm{~h} & 0 & -4.402551 & -1.451907 & -0.870103 & 0 \mathrm{~m} \\ ------------------------------------------------------------ & \\ & & & & & & \end{array}$

radical

Charge $=0$ Multiplicity $=2$ for low level calculation on real system.

Charge $=0$ Multiplicity $=2$ for high level calculation on model system.

Charge $=0$ Multiplicity $=2$ for low level calculation on model system.

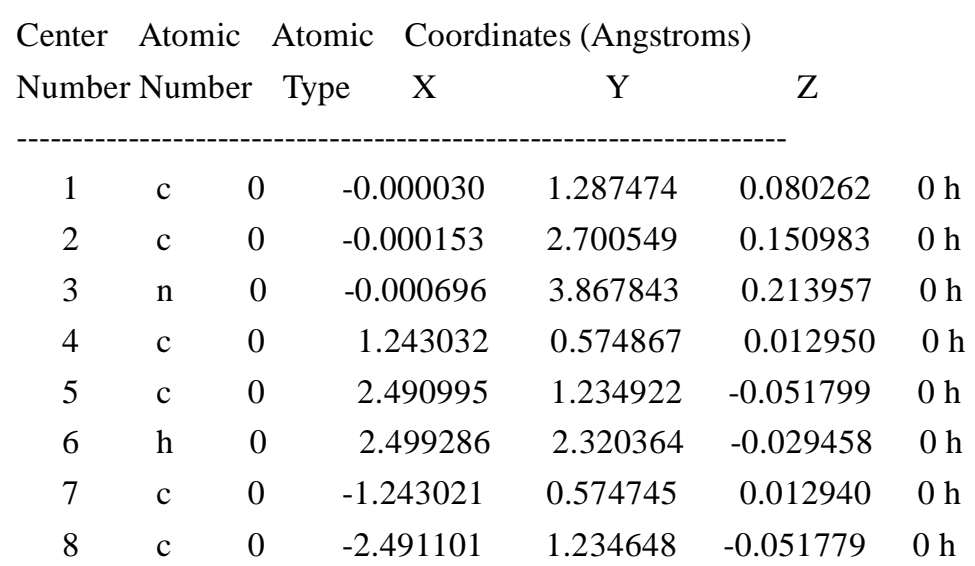




$\begin{array}{rrrrrrl}9 & \mathrm{~h} & 0 & -2.499538 & 2.320085 & -0.029407 & 0 \mathrm{~h} \\ 10 & \mathrm{c} & 0 & 3.677675 & 0.527892 & -0.149296 & 0 \mathrm{~m} \mathrm{~h} 5 \\ 11 & \mathrm{~h} & 0 & 4.623746 & 1.058524 & -0.198357 & 0 \mathrm{~m} \\ 12 & \mathrm{c} & 0 & 1.222139 & -0.851823 & 0.000356 & 0 \mathrm{~m} \mathrm{~h} 4 \\ 13 & \mathrm{c} & 0 & -1.221958 & -0.851947 & 0.000338 & 0 \mathrm{~m} \mathrm{~h} 7 \\ 14 & \mathrm{c} & 0 & -3.677688 & 0.527460 & -0.149294 & 0 \mathrm{~m} \mathrm{~h} 8 \\ 15 & \mathrm{~h} & 0 & -4.623831 & 1.057966 & -0.198331 & 0 \mathrm{~m} \\ 16 & \mathrm{c} & 0 & 3.645961 & -0.870737 & -0.200806 & 0 \mathrm{~m} \\ 17 & \mathrm{~h} & 0 & 4.567136 & -1.436824 & -0.304027 & 0 \mathrm{~m} \\ 18 & \mathrm{c} & 0 & 2.433867 & -1.551908 & -0.131260 & 0 \mathrm{~m} \\ 19 & \mathrm{~h} & 0 & 2.437483 & -2.632331 & -0.205396 & 0 \mathrm{~m} \\ 20 & \mathrm{n} & 0 & 0.000120 & -1.522687 & 0.128711 & 0 \mathrm{~m} \\ 21 & \mathrm{c} & 0 & 0.000180 & -2.955324 & 0.406383 & 0 \mathrm{~m} \\ 22 & \mathrm{~h} & 0 & 0.880035 & -3.208712 & 0.999028 & 0 \mathrm{~m} \\ 23 & \mathrm{~h} & 0 & 0.000196 & -3.561197 & -0.509477 & 0 \mathrm{~m} \\ 24 & \mathrm{~h} & 0 & -0.879643 & -3.208784 & 0.999046 & 0 \mathrm{~m} \\ 25 & \mathrm{c} & 0 & -2.433592 & -1.552194 & -0.131266 & 0 \mathrm{~m} \\ 26 & \mathrm{~h} & 0 & -2.437067 & -2.632618 & -0.205374 & 0 \mathrm{~m} \\ 27 & \mathrm{c} & 0 & -3.645785 & -0.871157 & -0.200854 & 0 \mathrm{~m} \\ 28 & \mathrm{~h} & 0 & -4.566886 & -1.437365 & -0.304068 & 0 \mathrm{~m} \\ ----------------------------------------------------------- & \end{array}$

\section{No. 48}<smiles>c1ccc(C2c3ccccc3Oc3ccccc32)cc1</smiles>

neutral molecule

Charge $=0$ Multiplicity $=1$ for low level calculation on real system.

Charge $=0$ Multiplicity $=1$ for high level calculation on model system.

Charge $=0$ Multiplicity $=1$ for low level calculation on model system.

Center Atomic Atomic Coordinates (Angstroms)

Number Number Type $\quad$ X $\quad$ Y $\quad$ Z

$\begin{array}{lllllll}1 & \mathrm{c} & 0 & -0.208773 & -0.000045 & -0.850701 & 0 \mathrm{~h} \\ 2 & \mathrm{~h} & 0 & -0.350648 & -0.000064 & -1.941374 & 0 \mathrm{~h} \\ 3 & \mathrm{c} & 0 & -1.611615 & -0.000309 & -0.234971 & 0 \mathrm{~h} \\ 4 & \mathrm{c} & 0 & -2.745519 & -0.000304 & -1.054048 & 0 \mathrm{~h} \\ 5 & \mathrm{~h} & 0 & -2.624426 & -0.000143 & -2.135571 & 0 \mathrm{~h}\end{array}$




\begin{tabular}{rrrrrrl}
6 & $\mathrm{c}$ & 0 & 0.573841 & -1.255540 & -0.487469 & $0 \mathrm{~h}$ \\
7 & $\mathrm{c}$ & 0 & 1.786948 & -1.186903 & 0.203759 & $0 \mathrm{~h}$ \\
8 & $\mathrm{c}$ & 0 & 0.573357 & 1.255749 & -0.487438 & $0 \mathrm{~h}$ \\
9 & $\mathrm{c}$ & 0 & 0.095552 & 2.526082 & -0.837934 & $0 \mathrm{~h}$ \\
10 & $\mathrm{~h}$ & 0 & -0.851317 & 2.596117 & -1.367994 & $0 \mathrm{~h}$ \\
11 & $\mathrm{c}$ & 0 & -1.787981 & -0.000539 & 1.155927 & $0 \mathrm{~m} \mathrm{~h} 3$ \\
12 & $\mathrm{~h}$ & 0 & -0.916878 & -0.000547 & 1.806305 & $0 \mathrm{~m}$ \\
13 & $\mathrm{c}$ & 0 & -4.029605 & -0.000523 & -0.501446 & $0 \mathrm{~m} \mathrm{~h} 4$ \\
14 & $\mathrm{~h}$ & 0 & -4.898189 & -0.000515 & -1.155009 & $0 \mathrm{~m}$ \\
15 & $\mathrm{c}$ & 0 & 0.096533 & -2.526049 & -0.838003 & $0 \mathrm{~m} \mathrm{~h} 6$ \\
16 & $\mathrm{~h}$ & 0 & -0.850312 & -2.596438 & -1.368056 & $0 \mathrm{~m}$ \\
17 & $\mathrm{c}$ & 0 & 2.499690 & -2.340981 & 0.545839 & $0 \mathrm{~m} \mathrm{~h} 7$ \\
18 & $\mathrm{~h}$ & 0 & 3.435709 & -2.231624 & 1.084523 & $0 \mathrm{~m}$ \\
19 & $\mathrm{o}$ & 0 & 2.364505 & 0.000431 & 0.589736 & $0 \mathrm{~m} \mathrm{~h} 7$ \\
20 & $\mathrm{c}$ & 0 & 1.786494 & 1.187541 & 0.203781 & $0 \mathrm{~m} \mathrm{~h} 8$ \\
21 & $\mathrm{c}$ & 0 & 0.793587 & 3.684931 & -0.511589 & $0 \mathrm{~m} \mathrm{~h} 9$ \\
22 & $\mathrm{~h}$ & 0 & 0.399083 & 4.655961 & -0.796392 & $0 \mathrm{~m}$ \\
23 & $\mathrm{c}$ & 0 & -3.065939 & -0.000758 & 1.710233 & $0 \mathrm{~m}$ \\
24 & $\mathrm{~h}$ & 0 & -3.184432 & -0.000935 & 2.790703 & $0 \mathrm{~m}$ \\
25 & $\mathrm{c}$ & 0 & -4.193051 & -0.000750 & 0.882696 & $0 \mathrm{~m}$ \\
26 & $\mathrm{~h}$ & 0 & -5.189500 & -0.000921 & 1.316325 & $0 \mathrm{~m}$ \\
27 & $\mathrm{c}$ & 0 & 0.795001 & -3.684625 & -0.511670 & $0 \mathrm{~m}$ \\
28 & $\mathrm{~h}$ & 0 & 0.400878 & -4.655800 & -0.796503 & $0 \mathrm{~m}$ \\
29 & $\mathrm{c}$ & 0 & 2.002609 & -3.588568 & 0.188100 & $0 \mathrm{~m}$ \\
30 & $\mathrm{~h}$ & 0 & 2.557145 & -4.484630 & 0.452644 & $0 \mathrm{~m}$ \\
31 & $\mathrm{c}$ & 0 & 2.498794 & 2.341879 & 0.545903 & $0 \mathrm{~m}$ \\
32 & $\mathrm{~h}$ & 0 & 3.434851 & 2.232862 & 1.084592 & $0 \mathrm{~m}$ \\
33 & $\mathrm{c}$ & 0 & 2.001184 & 3.589319 & 0.188216 & $0 \mathrm{~m}$ \\
34 & $\mathrm{~h}$ & 0 & 2.555355 & 4.485594 & 0.452799 & $0 \mathrm{~m}$ \\
-------------------------------------------------------------- & \\
\hline
\end{tabular}

radical

Charge $=0$ Multiplicity $=2$ for low level calculation on real system.

Charge $=0$ Multiplicity $=2$ for high level calculation on model system.

Charge $=0$ Multiplicity $=2$ for low level calculation on model system.

\begin{tabular}{|c|c|c|c|c|c|c|}
\hline Center & Atomic & Atomic & Coordi & ates (Angstro & & \\
\hline Number & Numbe & r Type & $\mathrm{X}$ & Y & Z & \\
\hline 1 & $\mathrm{c}$ & 0 & 0.186664 & -0.000270 & -0.000160 & $0 \mathrm{~h}$ \\
\hline 2 & $\mathrm{c}$ & 0 & 1.670548 & -0.001091 & -0.000213 & $0 \mathrm{~h}$ \\
\hline 3 & $\mathrm{c}$ & 0 & 2.394659 & 0.609457 & -1.040372 & $0 \mathrm{~h}$ \\
\hline 4 & $\mathrm{~h}$ & 0 & 1.853736 & 1.076743 & -1.858671 & $0 \mathrm{~h}$ \\
\hline
\end{tabular}




\begin{tabular}{rrrrrrl}
5 & $\mathrm{c}$ & 0 & -0.554453 & 1.231332 & 0.036579 & $0 \mathrm{~h}$ \\
6 & $\mathrm{c}$ & 0 & -1.973282 & 1.190137 & 0.031770 & $0 \mathrm{~h}$ \\
7 & $\mathrm{c}$ & 0 & -0.556039 & -1.230980 & -0.036736 & $0 \mathrm{~h}$ \\
8 & $\mathrm{c}$ & 0 & 0.031538 & -2.519779 & -0.116156 & $0 \mathrm{~h}$ \\
9 & $\mathrm{~h}$ & 0 & 1.112379 & -2.596278 & -0.153218 & $0 \mathrm{~h}$ \\
10 & $\mathrm{c}$ & 0 & 2.393985 & -0.612024 & 1.040223 & $0 \mathrm{~m} \mathrm{~h} 2$ \\
11 & $\mathrm{~h}$ & 0 & 1.852518 & -1.078644 & 1.858539 & $0 \mathrm{~m}$ \\
12 & $\mathrm{c}$ & 0 & 3.789317 & 0.608467 & -1.040380 & $0 \mathrm{~m} \mathrm{~h} 3$ \\
13 & $\mathrm{~h}$ & 0 & 4.327337 & 1.081016 & -1.858137 & $0 \mathrm{~m}$ \\
14 & $\mathrm{c}$ & 0 & 0.034817 & 2.519328 & 0.116127 & $0 \mathrm{~m} \mathrm{~h} 5$ \\
15 & $\mathrm{~h}$ & 0 & 1.115778 & 2.594389 & 0.152978 & $0 \mathrm{~m}$ \\
16 & $\mathrm{c}$ & 0 & -2.752631 & 2.341298 & 0.070097 & $0 \mathrm{~m} \mathrm{~h} \mathrm{6}$ \\
17 & $\mathrm{~h}$ & 0 & -3.832961 & 2.237144 & 0.059633 & $0 \mathrm{~m}$ \\
18 & $\mathrm{o}$ & 0 & -2.657368 & 0.001554 & -0.000401 & $0 \mathrm{~m} \mathrm{~h} \mathrm{6}$ \\
19 & $\mathrm{c}$ & 0 & -1.974839 & -1.187936 & -0.031853 & $0 \mathrm{~m} \mathrm{~h} \mathrm{7}$ \\
20 & $\mathrm{c}$ & 0 & -0.742409 & -3.671197 & -0.155576 & $0 \mathrm{~m} \mathrm{~h} 8$ \\
21 & $\mathrm{~h}$ & 0 & -0.256615 & -4.641094 & -0.215444 & $0 \mathrm{~m}$ \\
22 & $\mathrm{c}$ & 0 & 3.788621 & -0.612196 & 1.040469 & $0 \mathrm{~m}$ \\
23 & $\mathrm{~h}$ & 0 & 4.326140 & -1.085074 & 1.858364 & $0 \mathrm{~m}$ \\
24 & $\mathrm{c}$ & 0 & 4.492140 & -0.002198 & 0.000072 & $0 \mathrm{~m}$ \\
25 & $\mathrm{~h}$ & 0 & 5.578806 & -0.002630 & 0.000199 & $0 \mathrm{~m}$ \\
26 & $\mathrm{c}$ & 0 & -0.737563 & 3.671724 & 0.155829 & $0 \mathrm{~m}$ \\
27 & $\mathrm{~h}$ & 0 & -0.250439 & 4.640946 & 0.215839 & $0 \mathrm{~m}$ \\
28 & $\mathrm{c}$ & 0 & -2.135570 & 3.590304 & 0.127261 & $0 \mathrm{~m}$ \\
29 & $\mathrm{~h}$ & 0 & -2.741333 & 4.491105 & 0.157411 & $0 \mathrm{~m}$ \\
30 & $\mathrm{c}$ & 0 & -2.755708 & -2.338075 & -0.069878 & $0 \mathrm{~m}$ \\
31 & $\mathrm{~h}$ & 0 & -3.835900 & -2.232491 & -0.059459 & $0 \mathrm{~m}$ \\
32 & $\mathrm{c}$ & 0 & -2.140326 & -3.587911 & -0.126779 & $0 \mathrm{~m}$ \\
33 & $\mathrm{~h}$ & 0 & -2.747311 & -4.487894 & -0.156775 & $0 \mathrm{~m}$ \\
----------------------------------------------------------- & \\
\hline
\end{tabular}

\section{No. 49}<smiles>Cc1ccc(C2c3ccccc3Oc3ccccc32)cc1</smiles>

neutral molecule

Charge $=0$ Multiplicity $=1$ for low level calculation on real system.

Charge $=0$ Multiplicity $=1$ for high level calculation on model system .

Charge $=0$ Multiplicity $=1$ for low level calculation on model system. 


\begin{tabular}{|c|c|c|c|c|c|c|}
\hline Center & Atomic & & Coordi & ates (Angstro & ms) & \\
\hline Number & Numbe & & Type & $\mathrm{Y}$ & Z & \\
\hline 1 & $\mathrm{c}$ & 0 & -0.193582 & -0.000052 & 0.927870 & $0 \mathrm{~h}$ \\
\hline 2 & $\mathrm{~h}$ & 0 & -0.145503 & -0.000066 & 2.026826 & $0 \mathrm{~h}$ \\
\hline 3 & $\mathrm{c}$ & 0 & 1.256038 & -0.000261 & 0.434631 & $0 \mathrm{~h}$ \\
\hline 4 & $\mathrm{c}$ & 0 & 2.318660 & -0.000233 & 1.340751 & $0 \mathrm{~h}$ \\
\hline 5 & $\mathrm{~h}$ & 0 & 2.113468 & -0.000144 & 2.409522 & $0 \mathrm{~h}$ \\
\hline 6 & $\mathrm{c}$ & 0 & -0.943059 & -1.255537 & 0.499230 & $0 \mathrm{~h}$ \\
\hline 7 & $\mathrm{c}$ & 0 & -2.091253 & -1.186851 & -0.295096 & $0 \mathrm{~h}$ \\
\hline 8 & $\mathrm{c}$ & 0 & -0.942617 & 1.255717 & 0.499247 & $0 \mathrm{~h}$ \\
\hline 9 & $\mathrm{c}$ & 0 & -0.497651 & 2.526247 & 0.889832 & $0 \mathrm{~h}$ \\
\hline 10 & $\mathrm{~h}$ & 0 & 0.399724 & 2.596422 & 1.499887 & $0 \mathrm{~h}$ \\
\hline 11 & $\mathrm{c}$ & 0 & 1.555963 & -0.000526 & -0.935789 & $0 \mathrm{~m} \mathrm{~h} 3$ \\
\hline 12 & $\mathrm{~h}$ & 0 & 0.746629 & -0.000668 & -1.661782 & $0 \mathrm{~m}$ \\
\hline 13 & $\mathrm{c}$ & 0 & 3.644236 & -0.000403 & 0.895802 & $0 \mathrm{~m} \mathrm{~h} 4$ \\
\hline 14 & $\mathrm{~h}$ & 0 & 4.452388 & -0.000426 & 1.624050 & $0 \mathrm{~m}$ \\
\hline 15 & $\mathrm{c}$ & 0 & -0.498624 & -2.526215 & 0.889951 & $0 \mathrm{~m} \mathrm{~h} 6$ \\
\hline 16 & $\mathrm{~h}$ & 0 & 0.398665 & -2.596675 & 1.500104 & $0 \mathrm{~m}$ \\
\hline 17 & $\mathrm{c}$ & 0 & -2.771847 & -2.340695 & -0.698065 & $0 \mathrm{~m} \mathrm{~h} 7$ \\
\hline 18 & $\mathrm{~h}$ & 0 & -3.657356 & -2.231051 & -1.316251 & $0 \mathrm{~m}$ \\
\hline 19 & o & 0 & -2.632738 & 0.000393 & -0.730249 & $0 \mathrm{~m} \mathrm{~h} 7$ \\
\hline 20 & $\mathrm{c}$ & 0 & -2.090877 & 1.187424 & -0.295026 & $0 \mathrm{~m} \mathrm{~h} 8$ \\
\hline 21 & $\mathrm{c}$ & 0 & -1.165098 & 3.684952 & 0.504075 & $0 \mathrm{~m} \mathrm{~h} 9$ \\
\hline 22 & $\mathrm{~h}$ & 0 & -0.797217 & 4.656101 & 0.822258 & $0 \mathrm{~m}$ \\
\hline 23 & $\mathrm{c}$ & 0 & 2.874777 & -0.000694 & -1.376113 & $0 \mathrm{~m}$ \\
\hline 24 & $\mathrm{~h}$ & 0 & 3.080609 & -0.000966 & -2.444689 & $0 \mathrm{~m}$ \\
\hline 25 & $\mathrm{c}$ & 0 & 3.946478 & -0.000562 & -0.467987 & $0 \mathrm{~m}$ \\
\hline 26 & $\mathrm{c}$ & 0 & 5.375169 & -0.000225 & -0.959816 & $0 \mathrm{~m}$ \\
\hline 27 & $\mathrm{~h}$ & 0 & 5.587386 & 0.884166 & -1.573694 & $0 \mathrm{~m}$ \\
\hline 28 & $\mathrm{~h}$ & 0 & 6.084846 & -0.005068 & -0.126490 & $0 \mathrm{~m}$ \\
\hline 29 & $\mathrm{~h}$ & 0 & 5.584979 & -0.879273 & -1.582068 & $0 \mathrm{~m}$ \\
\hline 30 & $\mathrm{c}$ & 0 & -1.166453 & -3.684685 & 0.504175 & $0 \mathrm{~m}$ \\
\hline 31 & $\mathrm{~h}$ & 0 & -0.799015 & -4.655959 & 0.822482 & $0 \mathrm{~m}$ \\
\hline 32 & $\mathrm{c}$ & 0 & -2.308235 & -3.588422 & -0.298386 & $0 \mathrm{~m}$ \\
\hline 33 & $\mathrm{~h}$ & 0 & -2.837887 & -4.484337 & -0.610251 & $0 \mathrm{~m}$ \\
\hline 34 & $\mathrm{c}$ & 0 & -2.771124 & 2.341498 & -0.697920 & $0 \mathrm{~m}$ \\
\hline 35 & $\mathrm{~h}$ & 0 & -3.656717 & 2.232155 & -1.316037 & $0 \mathrm{~m}$ \\
\hline 36 & $\mathrm{c}$ & 0 & -2.307021 & 3.589097 & -0.298321 & $0 \mathrm{~m}$ \\
\hline 37 & $\mathrm{~h}$ & 0 & -2.836382 & 4.485203 & -0.610133 & $0 \mathrm{~m}$ \\
\hline
\end{tabular}


radical

Charge $=0$ Multiplicity $=2$ for low level calculation on real system.

Charge $=0$ Multiplicity $=2$ for high level calculation on model system .

Charge $=0$ Multiplicity $=2$ for low level calculation on model system.

Center Atomic Atomic Coordinates (Angstroms)

$\begin{array}{lllll}\text { Number Number Type } & \text { X } & \text { Y } & \text { Z }\end{array}$

\begin{tabular}{|c|c|c|c|c|c|c|}
\hline 1 & $\mathrm{c}$ & 0 & 0.163335 & -0.000813 & -0.000594 & $0 \mathrm{~h}$ \\
\hline 2 & c & 0 & -1.319817 & -0.004182 & -0.000272 & $0 \mathrm{~h}$ \\
\hline 3 & $\mathrm{c}$ & 0 & -2.047909 & -0.623511 & -1.030933 & $0 \mathrm{~h}$ \\
\hline 4 & $\mathrm{~h}$ & 0 & -1.512173 & -1.099294 & -1.847862 & $0 \mathrm{~h}$ \\
\hline 5 & $\mathrm{c}$ & 0 & 0.909227 & -1.229815 & 0.036294 & $0 \mathrm{~h}$ \\
\hline 6 & c & 0 & 2.327917 & -1.184063 & 0.030494 & $0 \mathrm{~h}$ \\
\hline 7 & $\mathrm{c}$ & 0 & 0.902677 & 1.232164 & -0.037067 & $0 \mathrm{~h}$ \\
\hline 8 & $\mathrm{c}$ & 0 & 0.311045 & 2.519111 & -0.116032 & $0 \mathrm{~h}$ \\
\hline 9 & $\mathrm{~h}$ & 0 & -0.770056 & 2.591710 & -0.152879 & $0 \mathrm{~h}$ \\
\hline 10 & $\mathrm{c}$ & 0 & -2.049796 & 0.608467 & 1.034193 & $0 \mathrm{~m} \mathrm{~h} 2$ \\
\hline 11 & $\mathrm{~h}$ & 0 & -1.514692 & 1.080762 & 1.853581 & $0 \mathrm{~m}$ \\
\hline 12 & $\mathrm{c}$ & 0 & -3.441763 & -0.627099 & -1.025984 & $0 \mathrm{~m} \mathrm{~h} 3$ \\
\hline 13 & $\mathrm{~h}$ & 0 & -3.976071 & -1.112073 & -1.840213 & $0 \mathrm{~m}$ \\
\hline 14 & $\mathrm{c}$ & 0 & 0.324626 & -2.519877 & 0.117194 & $0 \mathrm{~m} \mathrm{~h} 5$ \\
\hline 15 & $\mathrm{~h}$ & 0 & -0.756011 & -2.598301 & 0.155459 & $0 \mathrm{~m}$ \\
\hline 16 & $\mathrm{c}$ & 0 & 3.111177 & -2.332548 & 0.068648 & $0 \mathrm{~m} \mathrm{~h} 6$ \\
\hline 17 & $\mathrm{~h}$ & 0 & 4.191146 & -2.224640 & 0.057479 & $0 \mathrm{~m}$ \\
\hline 18 & $\mathrm{o}$ & 0 & 3.008269 & 0.006764 & -0.001961 & $0 \mathrm{~m} \mathrm{~h} 6$ \\
\hline 19 & $\mathrm{c}$ & 0 & 2.321570 & 1.193964 & -0.032702 & $0 \mathrm{~m} \mathrm{~h} 7$ \\
\hline 20 & $\mathrm{c}$ & 0 & 1.081066 & 3.673183 & -0.155279 & $0 \mathrm{~m} \mathrm{~h} 8$ \\
\hline 21 & $\mathrm{~h}$ & 0 & 0.591945 & 4.641465 & -0.214799 & $0 \mathrm{~m}$ \\
\hline 22 & $\mathrm{c}$ & 0 & -3.442811 & 0.600380 & 1.034483 & $0 \mathrm{~m}$ \\
\hline 23 & $\mathrm{~h}$ & 0 & -3.978025 & 1.073602 & 1.855191 & $0 \mathrm{~m}$ \\
\hline 24 & $\mathrm{c}$ & 0 & -4.166702 & -0.013800 & 0.003519 & $0 \mathrm{~m}$ \\
\hline 25 & $\mathrm{c}$ & 0 & -5.677179 & 0.007283 & -0.007344 & $0 \mathrm{~m}$ \\
\hline 26 & $\mathrm{~h}$ & 0 & -6.059699 & 0.937760 & -0.448287 & $0 \mathrm{~m}$ \\
\hline 27 & $\mathrm{~h}$ & 0 & -6.086718 & -0.061129 & 1.006497 & $0 \mathrm{~m}$ \\
\hline 28 & $\mathrm{~h}$ & 0 & -6.085744 & -0.822141 & -0.594083 & $0 \mathrm{~m}$ \\
\hline 29 & $\mathrm{c}$ & 0 & 1.100884 & -3.669736 & 0.156851 & $0 \mathrm{~m}$ \\
\hline 30 & $\mathrm{~h}$ & 0 & 0.617031 & -4.640561 & 0.217988 & $0 \mathrm{~m}$ \\
\hline 31 & $\mathrm{c}$ & 0 & 2.498535 & -3.583745 & 0.126867 & $0 \mathrm{~m}$ \\
\hline 32 & $\mathrm{~h}$ & 0 & 3.107422 & -4.482462 & 0.156924 & $0 \mathrm{~m}$ \\
\hline 33 & $\mathrm{c}$ & 0 & 3.098607 & 2.346677 & -0.070653 & $0 \mathrm{~m}$ \\
\hline 34 & $\mathrm{~h}$ & 0 & 4.179154 & 2.244585 & -0.060691 & $0 \mathrm{~m}$ \\
\hline 35 & $\mathrm{c}$ & 0 & 2.479168 & 3.594622 & -0.126984 & $0 \mathrm{~m}$ \\
\hline
\end{tabular}




\section{No. 50}<smiles>COc1ccc(C2c3ccccc3Oc3ccccc32)cc1</smiles>

neutral molecule

Charge $=0$ Multiplicity $=1$ for low level calculation on real system.

Charge $=0$ Multiplicity $=1$ for high level calculation on model system .

Charge $=0$ Multiplicity $=1$ for low level calculation on model system.

\begin{tabular}{|c|c|c|c|c|c|c|}
\hline Center & Atomic & & Atomic Coordin & ates (Angstro & & \\
\hline Number & Numbe & & Type & $\mathrm{Y}$ & $\mathrm{Z}$ & \\
\hline 1 & $\mathrm{c}$ & 0 & -0.574635 & -0.000009 & 0.999370 & $0 \mathrm{~h}$ \\
\hline 2 & $\mathrm{~h}$ & 0 & -0.627364 & -0.000028 & 2.098027 & $0 \mathrm{~h}$ \\
\hline 3 & c & 0 & 0.914176 & -0.000034 & 0.641849 & $0 \mathrm{~h}$ \\
\hline 4 & c & 0 & 1.893215 & -0.000137 & 1.644540 & $0 \mathrm{~h}$ \\
\hline 5 & $\mathrm{~h}$ & 0 & 1.589973 & -0.000215 & 2.689683 & $0 \mathrm{~h}$ \\
\hline 6 & $\mathrm{c}$ & 0 & -1.282846 & -1.255094 & 0.504637 & $0 \mathrm{~h}$ \\
\hline 7 & c & 0 & -2.338682 & -1.187115 & -0.408875 & $0 \mathrm{~h}$ \\
\hline 8 & $\mathrm{c}$ & 0 & -1.282797 & 1.255113 & 0.504661 & $0 \mathrm{~h}$ \\
\hline 9 & c & 0 & -0.887719 & 2.524933 & 0.947630 & $0 \mathrm{~h}$ \\
\hline 10 & $\mathrm{~h}$ & 0 & -0.060983 & 2.594455 & 1.650585 & $0 \mathrm{~h}$ \\
\hline 11 & $\mathrm{c}$ & 0 & 1.341883 & 0.000064 & -0.689284 & $0 \mathrm{~m} \mathrm{~h} 3$ \\
\hline 12 & $\mathrm{~h}$ & 0 & 0.605766 & 0.000144 & -1.489322 & $0 \mathrm{~m}$ \\
\hline 13 & $\mathrm{c}$ & 0 & 3.249537 & -0.000140 & 1.333645 & $0 \mathrm{~m} \mathrm{~h} 4$ \\
\hline 14 & $\mathrm{~h}$ & 0 & 4.006949 & -0.000219 & 2.111416 & $0 \mathrm{~m}$ \\
\hline 15 & $\mathrm{c}$ & 0 & -0.887828 & -2.524967 & 0.947574 & $0 \mathrm{~m} \mathrm{~h} 6$ \\
\hline 16 & $\mathrm{~h}$ & 0 & -0.061096 & -2.594553 & 1.650529 & $0 \mathrm{~m}$ \\
\hline 17 & $\mathrm{c}$ & 0 & -2.975811 & -2.341101 & -0.877388 & $0 \mathrm{~m} \mathrm{~h} 7$ \\
\hline 18 & $\mathrm{~h}$ & 0 & -3.789463 & -2.232321 & -1.587663 & $0 \mathrm{~m}$ \\
\hline 19 & o & 0 & -2.825480 & 0.000049 & -0.905364 & $0 \mathrm{~m} \mathrm{~h} 7$ \\
\hline 20 & $\mathrm{c}$ & 0 & -2.338636 & 1.187193 & -0.408852 & $0 \mathrm{~m} \mathrm{~h} 8$ \\
\hline 21 & $\mathrm{c}$ & 0 & -1.514208 & 3.683490 & 0.498250 & $0 \mathrm{~m} \mathrm{~h} 9$ \\
\hline 22 & $\mathrm{~h}$ & 0 & -1.186969 & 4.654117 & 0.859530 & $0 \mathrm{~m}$ \\
\hline 23 & $\mathrm{c}$ & 0 & 2.696766 & 0.000062 & -1.021604 & $0 \mathrm{~m}$ \\
\hline
\end{tabular}




$\begin{array}{rrrrrrr}24 & \mathrm{~h} & 0 & 2.986945 & 0.000140 & -2.066202 & 0 \mathrm{~m} \\ 25 & \mathrm{c} & 0 & 3.661179 & -0.000040 & -0.004764 & 0 \mathrm{~m} \\ 26 & \mathrm{o} & 0 & 5.011365 & -0.000072 & -0.213042 & 0 \mathrm{~m} \\ 27 & \mathrm{c} & 0 & 5.483955 & 0.000004 & -1.550208 & 0 \mathrm{~m} \\ 28 & \mathrm{~h} & 0 & 5.155288 & 0.894628 & -2.095797 & 0 \mathrm{~m} \\ 29 & \mathrm{~h} & 0 & 6.573506 & -0.000037 & -1.482695 & 0 \mathrm{~m} \\ 30 & \mathrm{~h} & 0 & 5.155228 & -0.894522 & -2.095919 & 0 \mathrm{~m} \\ 31 & \mathrm{c} & 0 & -1.514377 & -3.683478 & 0.498159 & 0 \mathrm{~m} \\ 32 & \mathrm{~h} & 0 & -1.187190 & -4.654132 & 0.859409 & 0 \mathrm{~m} \\ 33 & \mathrm{c} & 0 & -2.562275 & -3.588063 & -0.423852 & 0 \mathrm{~m} \\ 34 & \mathrm{~h} & 0 & -3.058409 & -4.484061 & -0.786517 & 0 \mathrm{~m} \\ 35 & \mathrm{c} & 0 & -2.975720 & 2.341212 & -0.877343 & 0 \mathrm{~m} \\ 36 & \mathrm{~h} & 0 & -3.789365 & 2.232478 & -1.587632 & 0 \mathrm{~m} \\ 37 & \mathrm{c} & 0 & -2.562121 & 3.588133 & -0.423772 & 0 \mathrm{~m} \\ 38 & \mathrm{~h} & 0 & -3.058221 & 4.484158 & -0.786420 & 0 \mathrm{~m} \\ ------------------------------------------------------------- & \end{array}$

radical

Charge $=0$ Multiplicity $=2$ for low level calculation on real system.

Charge $=0$ Multiplicity $=2$ for high level calculation on model system.

Charge $=0$ Multiplicity $=2$ for low level calculation on model system.

\begin{tabular}{|c|c|c|c|c|c|c|}
\hline Center & Atomic & & Coor & ates (Angs & & \\
\hline Number & Numbe & & jpe & $\mathrm{Y}$ & $\mathrm{Z}$ & \\
\hline 1 & $\mathrm{c}$ & 0 & -0.489553 & -0.010192 & 0.036141 & $0 \mathrm{~h}$ \\
\hline 2 & $\mathrm{c}$ & 0 & 0.990802 & -0.067972 & 0.094643 & $0 \mathrm{~h}$ \\
\hline 3 & $\mathrm{c}$ & 0 & 1.656276 & -0.720341 & 1.152865 & $0 \mathrm{~h}$ \\
\hline 4 & $\mathrm{~h}$ & 0 & 1.071230 & -1.176525 & 1.946627 & $0 \mathrm{~h}$ \\
\hline 5 & $\mathrm{c}$ & 0 & -1.276301 & -1.211984 & -0.038218 & $0 \mathrm{~h}$ \\
\hline 6 & $\mathrm{c}$ & 0 & -2.691602 & -1.117247 & -0.087145 & $0 \mathrm{~h}$ \\
\hline 7 & $\mathrm{c}$ & 0 & -1.187175 & 1.247418 & 0.052884 & $0 \mathrm{~h}$ \\
\hline 8 & $\mathrm{c}$ & 0 & -0.555369 & 2.512619 & 0.164425 & $0 \mathrm{~h}$ \\
\hline 9 & $\mathrm{~h}$ & 0 & 0.525141 & 2.546696 & 0.246767 & $0 \mathrm{~h}$ \\
\hline 10 & $\mathrm{c}$ & 0 & 1.785633 & 0.519949 & -0.900441 & $0 \mathrm{~m} \mathrm{~h} 2$ \\
\hline 11 & $\mathrm{~h}$ & 0 & 1.304589 & 1.020466 & -1.736354 & $0 \mathrm{~m}$ \\
\hline 12 & $\mathrm{c}$ & 0 & 3.041566 & -0.780290 & 1.210687 & $0 \mathrm{~m} \mathrm{~h} 3$ \\
\hline 13 & $\mathrm{~h}$ & 0 & 3.549543 & -1.277049 & 2.031605 & $0 \mathrm{~m}$ \\
\hline 14 & $\mathrm{c}$ & 0 & -0.733816 & -2.521129 & -0.104565 & $0 \mathrm{~m} \mathrm{~h} 5$ \\
\hline 15 & $\mathrm{~h}$ & 0 & 0.344184 & -2.636785 & -0.102219 & $0 \mathrm{~m}$ \\
\hline 16 & $\mathrm{c}$ & 0 & -3.511884 & -2.237587 & -0.163009 & $0 \mathrm{~m} \mathrm{~h} 6$ \\
\hline 17 & $\mathrm{~h}$ & 0 & -4.587115 & -2.092338 & -0.193268 & $0 \mathrm{~m}$ \\
\hline 18 & o & 0 & -3.331220 & 0.096474 & -0.074287 & $0 \mathrm{~m} \mathrm{~h} 6$ \\
\hline
\end{tabular}




$\begin{array}{lllrrrl}19 & \mathrm{c} & 0 & -2.605404 & 1.258828 & -0.008723 & 0 \mathrm{~m} \mathrm{~h} \mathrm{7} \\ 20 & \mathrm{c} & 0 & -1.285950 & 3.692733 & 0.180375 & 0 \mathrm{~m} \mathrm{~h} 8 \\ 21 & \mathrm{~h} & 0 & -0.766447 & 4.643057 & 0.266659 & 0 \mathrm{~m} \\ 22 & \mathrm{c} & 0 & 3.180786 & 0.467753 & -0.857430 & 0 \mathrm{~m} \\ 23 & \mathrm{~h} & 0 & 3.753098 & 0.929910 & -1.653898 & 0 \mathrm{~m} \\ 24 & \mathrm{c} & 0 & 3.817160 & -0.186217 & 0.203811 & 0 \mathrm{~m} \\ 25 & \mathrm{o} & 0 & 5.169028 & -0.300265 & 0.350705 & 0 \mathrm{~m} \\ 26 & \mathrm{c} & 0 & 6.003397 & 0.281078 & -0.638453 & 0 \mathrm{~m} \\ 27 & \mathrm{~h} & 0 & 5.857740 & 1.367630 & -0.702275 & 0 \mathrm{~m} \\ 28 & \mathrm{~h} & 0 & 7.028208 & 0.072791 & -0.325740 & 0 \mathrm{~m} \\ 29 & \mathrm{~h} & 0 & 5.828658 & -0.164060 & -1.626969 & 0 \mathrm{~m} \\ 30 & \mathrm{c} & 0 & -1.547054 & -3.643403 & -0.181250 & 0 \mathrm{~m} \\ 31 & \mathrm{~h} & 0 & -1.094876 & -4.630106 & -0.229411 & 0 \mathrm{~m} \\ 32 & \mathrm{c} & 0 & -2.940916 & -3.509151 & -0.205066 & 0 \mathrm{~m} \\ 33 & \mathrm{~h} & 0 & -3.578862 & -4.386017 & -0.264237 & 0 \mathrm{~m} \\ 34 & \mathrm{c} & 0 & -3.342929 & 2.437656 & 0.005653 & 0 \mathrm{~m} \\ 35 & \mathrm{~h} & 0 & -4.425110 & 2.373256 & -0.047999 & 0 \mathrm{~m} \\ 36 & \mathrm{c} & 0 & -2.683600 & 3.663141 & 0.094949 & 0 \mathrm{~m} \\ 37 & \mathrm{~h} & 0 & -3.256838 & 4.585434 & 0.106557 & 0 \mathrm{~m}\end{array}$

\section{No. 51}<smiles>CCOC(=O)C(C(=O)OCC)c1ccccc1</smiles>

neutral molecule

Charge $=0$ Multiplicity $=1$ for low level calculation on real system.

Charge $=0$ Multiplicity $=1$ for high level calculation on model system.

Charge $=0$ Multiplicity $=1$ for low level calculation on model system.

Center Atomic Atomic Coordinates (Angstroms)

$\begin{array}{lllll}\text { Number Number Type } & \text { X } & \text { Y } & \text { Z }\end{array}$

$\begin{array}{rrrrrrr}1 & \mathrm{c} & 0 & -0.006044 & 0.082009 & -0.132733 & 0 \mathrm{~h} \\ 2 & \mathrm{~h} & 0 & -0.243942 & 0.667982 & -1.021944 & 0 \mathrm{~h} \\ 3 & \mathrm{c} & 0 & 1.477704 & -0.244352 & -0.159303 & 0 \mathrm{~h} \\ 4 & \mathrm{c} & 0 & 2.085763 & -1.002786 & 0.849892 & 0 \mathrm{~h} \\ 5 & \mathrm{~h} & 0 & 1.489616 & -1.359065 & 1.680854 & 0 \mathrm{~h} \\ 6 & \mathrm{c} & 0 & -0.409981 & 0.846873 & 1.149820 & 0 \mathrm{~h} \\ 7 & \mathrm{o} & 0 & -0.237008 & 0.362944 & 2.243038 & 0 \mathrm{~h} \\ 8 & \mathrm{c} & 0 & -0.907380 & -1.162231 & -0.195194 & 0 \mathrm{~h}\end{array}$




$\begin{array}{rrrrrrl}9 & \mathrm{o} & 0 & -0.557311 & -2.311480 & -0.091878 & 0 \mathrm{~h} \\ 10 & \mathrm{c} & 0 & 3.449944 & -1.282738 & 0.788559 & 0 \mathrm{~m} \mathrm{~h} \\ 11 & \mathrm{~h} & 0 & 3.910498 & -1.871567 & 1.577267 & 0 \mathrm{~m} \\ 12 & \mathrm{c} & 0 & 2.257997 & 0.216862 & -1.226215 & 0 \mathrm{~m} \mathrm{~h} \\ 13 & \mathrm{~h} & 0 & 1.793910 & 0.793707 & -2.023956 & 0 \mathrm{~m} \\ 14 & \mathrm{o} & 0 & -0.974970 & 2.071545 & 1.064609 & 0 \mathrm{~m} \mathrm{~h} \\ 15 & \mathrm{o} & 0 & -2.187308 & -0.768615 & -0.386825 & 0 \mathrm{~m} \mathrm{~h} \\ 16 & \mathrm{c} & 0 & 4.223639 & -0.814884 & -0.276279 & 0 \mathrm{~m} \\ 17 & \mathrm{~h} & 0 & 5.286661 & -1.037004 & -0.318315 & 0 \mathrm{~m} \\ 18 & \mathrm{c} & 0 & 3.624226 & -0.063421 & -1.286449 & 0 \mathrm{~m} \\ 19 & \mathrm{~h} & 0 & 4.214641 & 0.303628 & -2.121725 & 0 \mathrm{~m} \\ 20 & \mathrm{c} & 0 & -1.331291 & 2.722567 & -0.170795 & 0 \mathrm{~m} \\ 21 & \mathrm{~h} & 0 & -1.727079 & 1.992916 & -0.883703 & 0 \mathrm{~m} \\ 22 & \mathrm{~h} & 0 & -2.156851 & 3.381650 & 0.111550 & 0 \mathrm{~m} \\ 23 & \mathrm{c} & 0 & -0.174274 & 3.533557 & -0.736877 & 0 \mathrm{~m} \\ 24 & \mathrm{~h} & 0 & 0.669261 & 2.896566 & -1.022924 & 0 \mathrm{~m} \\ 25 & \mathrm{~h} & 0 & 0.182353 & 4.252698 & 0.006984 & 0 \mathrm{~m} \\ 26 & \mathrm{~h} & 0 & -0.503400 & 4.087477 & -1.623596 & 0 \mathrm{~m} \\ 27 & \mathrm{c} & 0 & -3.179466 & -1.826176 & -0.416759 & 0 \mathrm{~m} \\ 28 & \mathrm{~h} & 0 & -2.940940 & -2.503607 & -1.242712 & 0 \mathrm{~m} \\ 29 & \mathrm{~h} & 0 & -3.106897 & -2.395804 & 0.514487 & 0 \mathrm{~m} \\ 30 & \mathrm{c} & 0 & -4.537561 & -1.172777 & -0.584774 & 0 \mathrm{~m} \\ 31 & \mathrm{~h} & 0 & -4.587302 & -0.603459 & -1.518723 & 0 \mathrm{~m} \\ 32 & \mathrm{~h} & 0 & -4.750473 & -0.494266 & 0.247345 & 0 \mathrm{~m} \\ 33 & \mathrm{~h} & 0 & -5.316928 & -1.942025 & -0.609797 & 0 \mathrm{~m} \\ ------------------------------------------------------------ & \end{array}$

radical

Charge $=0$ Multiplicity $=2$ for low level calculation on real system.

Charge $=0$ Multiplicity $=2$ for high level calculation on model system.

Charge $=0$ Multiplicity $=2$ for low level calculation on model system.

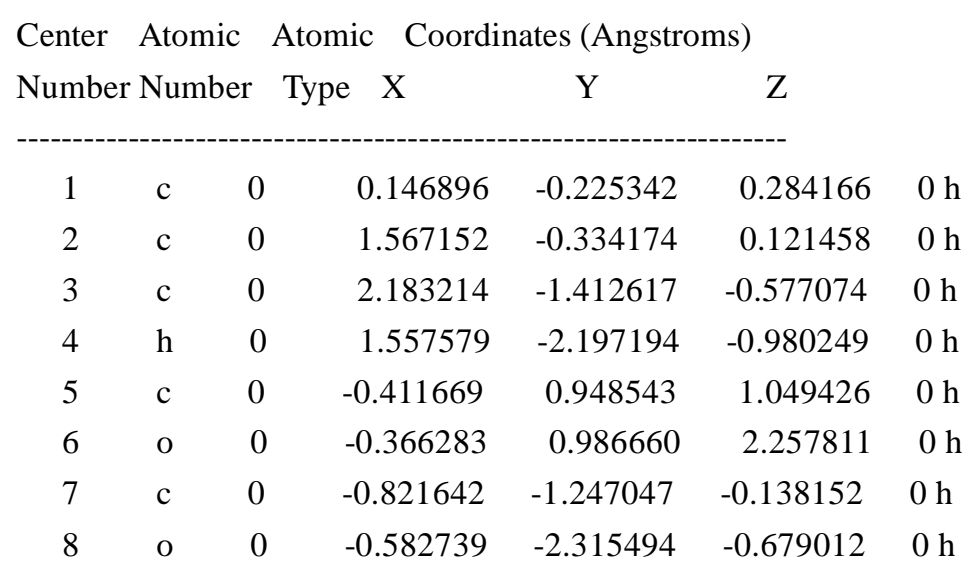




$\begin{array}{cccrrrl}9 & \mathrm{c} & 0 & 3.560707 & -1.456241 & -0.736558 & 0 \mathrm{~m} \mathrm{~h} \\ 10 & \mathrm{~h} & 0 & 4.007109 & -2.288589 & -1.273910 & 0 \mathrm{~m} \\ 11 & \mathrm{c} & 0 & 2.418696 & 0.681226 & 0.647728 & 0 \mathrm{~m} \mathrm{~h} \\ 12 & \mathrm{~h} & 0 & 1.988210 & 1.503546 & 1.209273 & 0 \mathrm{~m} \\ 13 & \mathrm{o} & 0 & -0.930816 & 1.991286 & 0.367484 & 0 \mathrm{~m} \mathrm{~h} \\ 14 & \mathrm{o} & 0 & -2.088233 & -0.845538 & 0.156216 & 0 \mathrm{~m} \mathrm{~h} \\ 15 & \mathrm{c} & 0 & 4.376331 & -0.445485 & -0.214207 & 0 \mathrm{~m} \\ 16 & \mathrm{~h} & 0 & 5.454145 & -0.489636 & -0.343579 & 0 \mathrm{~m} \\ 17 & \mathrm{c} & 0 & 3.793378 & 0.621441 & 0.480489 & 0 \mathrm{~m} \\ 18 & \mathrm{~h} & 0 & 4.418122 & 1.406480 & 0.897316 & 0 \mathrm{~m} \\ 19 & \mathrm{c} & 0 & -0.991825 & 1.983467 & -1.075929 & 0 \mathrm{~m} \\ 20 & \mathrm{~h} & 0 & -0.013606 & 1.711474 & -1.487277 & 0 \mathrm{~m} \\ 21 & \mathrm{~h} & 0 & -1.725702 & 1.236846 & -1.395453 & 0 \mathrm{~m} \\ 22 & \mathrm{c} & 0 & -1.401185 & 3.378842 & -1.512693 & 0 \mathrm{~m} \\ 23 & \mathrm{~h} & 0 & -0.665459 & 4.119660 & -1.184744 & 0 \mathrm{~m} \\ 24 & \mathrm{~h} & 0 & -1.474790 & 3.421070 & -2.604901 & 0 \mathrm{~m} \\ 25 & \mathrm{~h} & 0 & -2.373274 & 3.646824 & -1.087714 & 0 \mathrm{~m} \\ 26 & \mathrm{c} & 0 & -3.141417 & -1.787287 & -0.148027 & 0 \mathrm{~m} \\ 27 & \mathrm{~h} & 0 & -2.923520 & -2.730995 & 0.361941 & 0 \mathrm{~m} \\ 28 & \mathrm{~h} & 0 & -3.137337 & -1.985683 & -1.225060 & 0 \mathrm{~m} \\ 29 & \mathrm{c} & 0 & -4.448565 & -1.174768 & 0.317885 & 0 \mathrm{~m} \\ 30 & \mathrm{~h} & 0 & -4.649396 & -0.232363 & -0.201945 & 0 \mathrm{~m} \\ 31 & \mathrm{~h} & 0 & -5.274164 & -1.864563 & 0.111618 & 0 \mathrm{~m} \\ 32 & \mathrm{~h} & 0 & -4.423762 & -0.975545 & 1.393620 & 0 \mathrm{~m} \\ & & & & & & \end{array}$

\section{No. 52}<smiles></smiles>

neutral molecule

Charge $=0$ Multiplicity $=1$ for low level calculation on real system.

Charge $=0$ Multiplicity $=1$ for high level calculation on model system .

Charge $=0$ Multiplicity $=1$ for low level calculation on model system . 


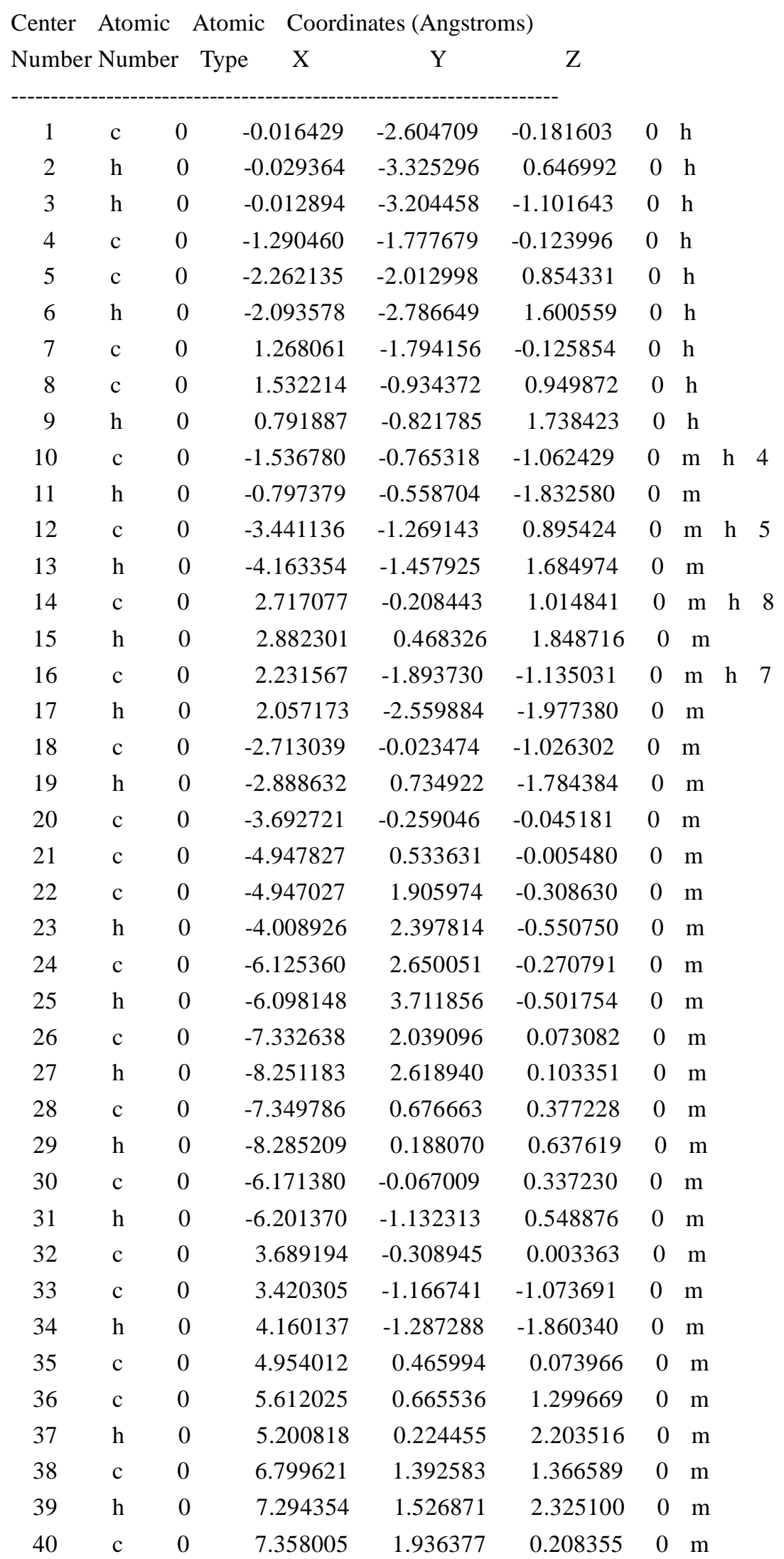




$\begin{array}{llllllll}41 & \mathrm{~h} & 0 & 8.283920 & 2.502805 & 0.260237 & 0 & \mathrm{~m} \\ 42 & \mathrm{c} & 0 & 6.716445 & 1.746585 & -1.016961 & 0 & \mathrm{~m} \\ 43 & \mathrm{~h} & 0 & 7.136991 & 2.172651 & -1.924231 & 0 & \mathrm{~m} \\ 44 & \mathrm{c} & 0 & 5.528095 & 1.020766 & -1.082650 & 0 & \mathrm{~m} \\ 45 & \mathrm{~h} & 0 & 5.023041 & 0.902633 & -2.037410 & 0 & \mathrm{~m}\end{array}$

radical

Charge $=0$ Multiplicity $=2$ for low level calculation on real system.

Charge $=0$ Multiplicity $=2$ for high level calculation on model system .

Charge $=0$ Multiplicity $=2$ for low level calculation on model system.

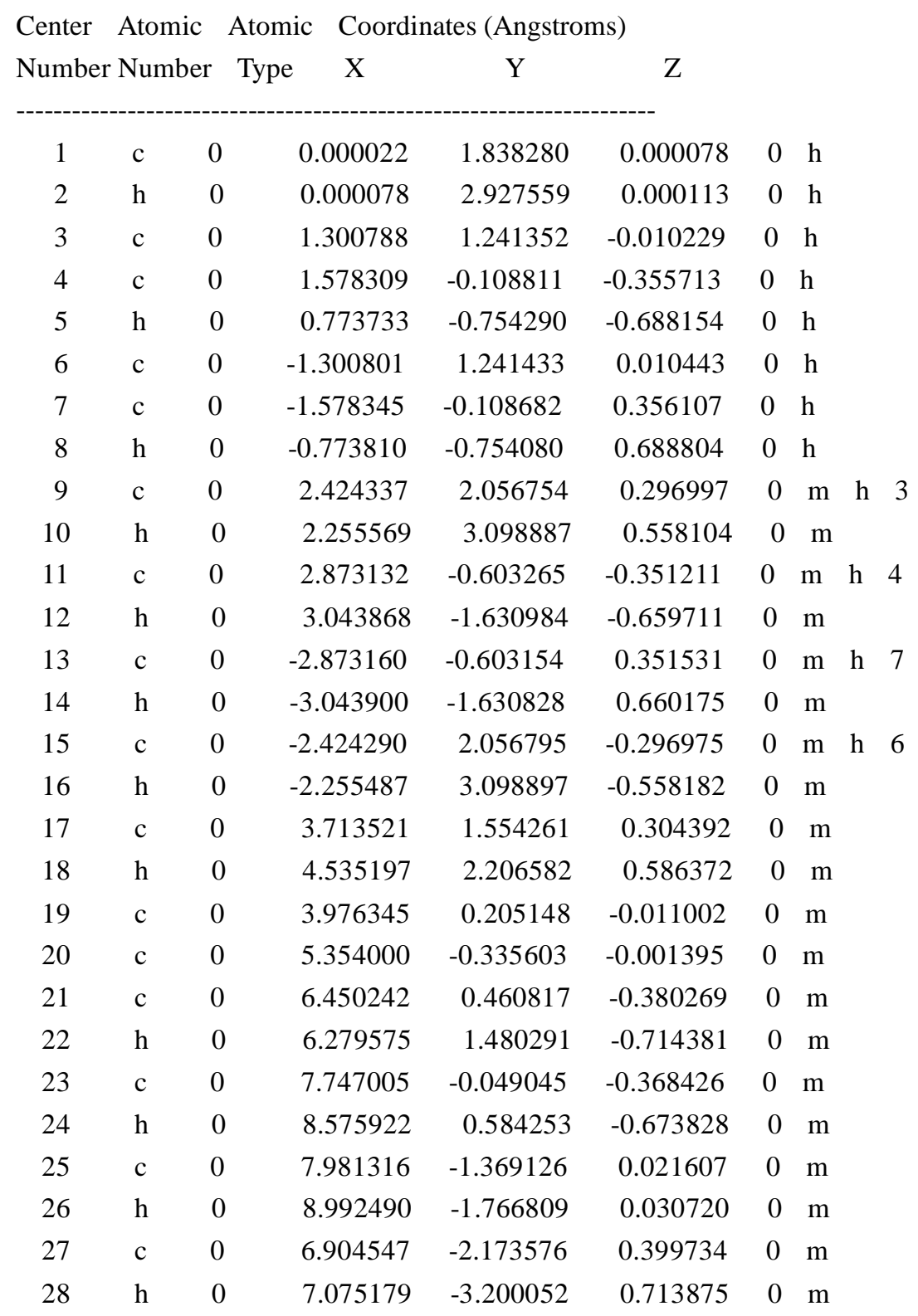




$\begin{array}{lllrrrrr}29 & \mathrm{c} & 0 & 5.607923 & -1.663312 & 0.388734 & 0 & \mathrm{~m} \\ 30 & \mathrm{~h} & 0 & 4.783069 & -2.290996 & 0.713879 & 0 & \mathrm{~m} \\ 31 & \mathrm{c} & 0 & -3.976347 & 0.205193 & 0.011066 & 0 & \mathrm{~m} \\ 32 & \mathrm{c} & 0 & -3.713486 & 1.554310 & -0.304438 & 0 & \mathrm{~m} \\ 33 & \mathrm{~h} & 0 & -4.535115 & 2.206622 & -0.586571 & 0 & \mathrm{~m} \\ 34 & \mathrm{c} & 0 & -5.354007 & -0.335601 & 0.001344 & 0 & \mathrm{~m} \\ 35 & \mathrm{c} & 0 & -5.607841 & -1.663379 & -0.388609 & 0 & \mathrm{~m} \\ 36 & \mathrm{~h} & 0 & -4.782939 & -2.291103 & -0.713550 & 0 & \mathrm{~m} \\ 37 & \mathrm{c} & 0 & -6.904474 & -2.173659 & -0.399751 & 0 & \mathrm{~m} \\ 38 & \mathrm{~h} & 0 & -7.075047 & -3.200182 & -0.713769 & 0 & \mathrm{~m} \\ 39 & \mathrm{c} & 0 & -7.981315 & -1.369177 & -0.021911 & 0 & \mathrm{~m} \\ 40 & \mathrm{~h} & 0 & -8.992482 & -1.766879 & -0.031131 & 0 & \mathrm{~m} \\ 41 & \mathrm{c} & 0 & -7.747090 & -0.049036 & 0.367973 & 0 & \mathrm{~m} \\ 42 & \mathrm{~h} & 0 & -8.576073 & 0.584282 & 0.673154 & 0 & \mathrm{~m} \\ 43 & \mathrm{c} & 0 & -6.450339 & 0.460829 & 0.379946 & 0 & \mathrm{~m} \\ 44 & \mathrm{~h} & 0 & -6.279780 & 1.480353 & 0.713955 & 0 & \mathrm{~m} \\ ------------------------------------------- & & \end{array}$

\section{No. 53}<smiles>C1=Cc2ccc3c4c(ccc(c24)C1)CCC3</smiles>

neutral molecule

Charge $=0$ Multiplicity $=1$ for low level calculation on real system.

Charge $=0$ Multiplicity $=1$ for high level calculation on model system.

Charge $=0$ Multiplicity $=1$ for low level calculation on model system.

\begin{tabular}{|c|c|c|c|c|c|c|}
\hline Center & Atomic & Atomic & Coordi & ates (Angstre & ns) & \\
\hline Number & r Number & r Type & $\mathrm{X}$ & $\mathrm{Y}$ & Z & \\
\hline 1 & $\mathrm{c}$ & 0 & 3.015805 & -1.219067 & 0.043221 & 0 \\
\hline 2 & $\mathrm{~h}$ & 0 & 3.407890 & -1.782917 & -0.818956 & 0 \\
\hline 3 & $\mathrm{~h}$ & 0 & 3.357955 & -1.790264 & 0.921569 & 0 \\
\hline 4 & $\mathrm{c}$ & 0 & 3.645045 & 0.145135 & 0.066823 & 0 \\
\hline 5 & $\mathrm{~h}$ & 0 & 4.731537 & 0.188332 & 0.100366 & $0 \quad \mathrm{l}$ \\
\hline 6 & $\mathrm{c}$ & 0 & 2.923467 & 1.274071 & 0.048097 & 0 \\
\hline 7 & $\mathrm{~h}$ & 0 & 3.418277 & 2.243207 & 0.066844 & $0 \quad \mathrm{l}$ \\
\hline 8 & $\mathrm{c}$ & 0 & 1.497361 & -1.211752 & -0.000477 & $0 \mathrm{~h}$ \\
\hline 9 & $\mathrm{c}$ & 0 & 0.794466 & -2.402644 & -0.015411 & $0 \mathrm{~h}$ \\
\hline 10 & $\mathrm{~h}$ & 0 & 1.337141 & -3.345856 & 0.008924 & 0 \\
\hline
\end{tabular}




$\begin{array}{rrrrrrrrrr}11 & \mathrm{c} & 0 & 1.464266 & 1.270056 & 0.002437 & 0 & \mathrm{~m} & \mathrm{~h} & 6 \\ 12 & \mathrm{c} & 0 & 0.771544 & 0.013643 & -0.023282 & 0 & \mathrm{~m} & \mathrm{~h} & 8 \\ 13 & \mathrm{c} & 0 & -0.612346 & -2.421476 & -0.052970 & 0 & \mathrm{~m} & \mathrm{~h} & 9 \\ 14 & \mathrm{~h} & 0 & -1.130461 & -3.378398 & -0.053388 & 0 & \mathrm{~m} & & \\ 15 & \mathrm{c} & 0 & 0.734845 & 2.444380 & -0.011623 & 0 & \mathrm{~m} & \\ 16 & \mathrm{~h} & 0 & 1.256863 & 3.398282 & 0.014791 & 0 & \mathrm{~m} & \\ 17 & \mathrm{c} & 0 & -0.675111 & 2.425169 & -0.052109 & 0 & \mathrm{~m} & \\ 18 & \mathrm{~h} & 0 & -1.218399 & 3.367895 & -0.053957 & 0 & \mathrm{~m} & \\ 19 & \mathrm{c} & 0 & -1.378813 & 1.239866 & -0.089886 & 0 & \mathrm{~m} & \\ 20 & \mathrm{c} & 0 & -0.660525 & -0.001991 & -0.071261 & 0 & \mathrm{~m} & \\ 21 & \mathrm{c} & 0 & -2.891676 & 1.227096 & -0.158452 & 0 & \mathrm{~m} & \\ 22 & \mathrm{~h} & 0 & -3.291797 & 2.133706 & 0.311582 & 0 & \mathrm{~m} & \\ 23 & \mathrm{~h} & 0 & -3.211669 & 1.262300 & -1.212172 & 0 & \mathrm{~m} & \\ 24 & \mathrm{c} & 0 & -3.482381 & -0.031298 & 0.489066 & 0 & \mathrm{~m} & \\ 25 & \mathrm{~h} & 0 & -4.572408 & -0.044523 & 0.371800 & 0 & \mathrm{~m} & & \\ 26 & \mathrm{~h} & 0 & -3.271852 & -0.023861 & 1.566628 & 0 & \mathrm{~m} & & \\ 27 & \mathrm{c} & 0 & -2.862356 & -1.281195 & -0.147020 & 0 & \mathrm{~m} & & \\ 28 & \mathrm{~h} & 0 & -3.188198 & -1.339868 & -1.197674 & 0 & \mathrm{~m} & & \\ 29 & \mathrm{~h} & 0 & -3.234793 & -2.191684 & 0.338210 & 0 & \mathrm{~m} & & \\ 30 & \mathrm{c} & 0 & -1.348606 & -1.252719 & -0.087914 & 0 & \mathrm{~m} & & \end{array}$

radical

Charge $=0$ Multiplicity $=2$ for low level calculation on real system.

Charge $=0$ Multiplicity $=2$ for high level calculation on model system.

Charge $=0$ Multiplicity $=2$ for low level calculation on model system.

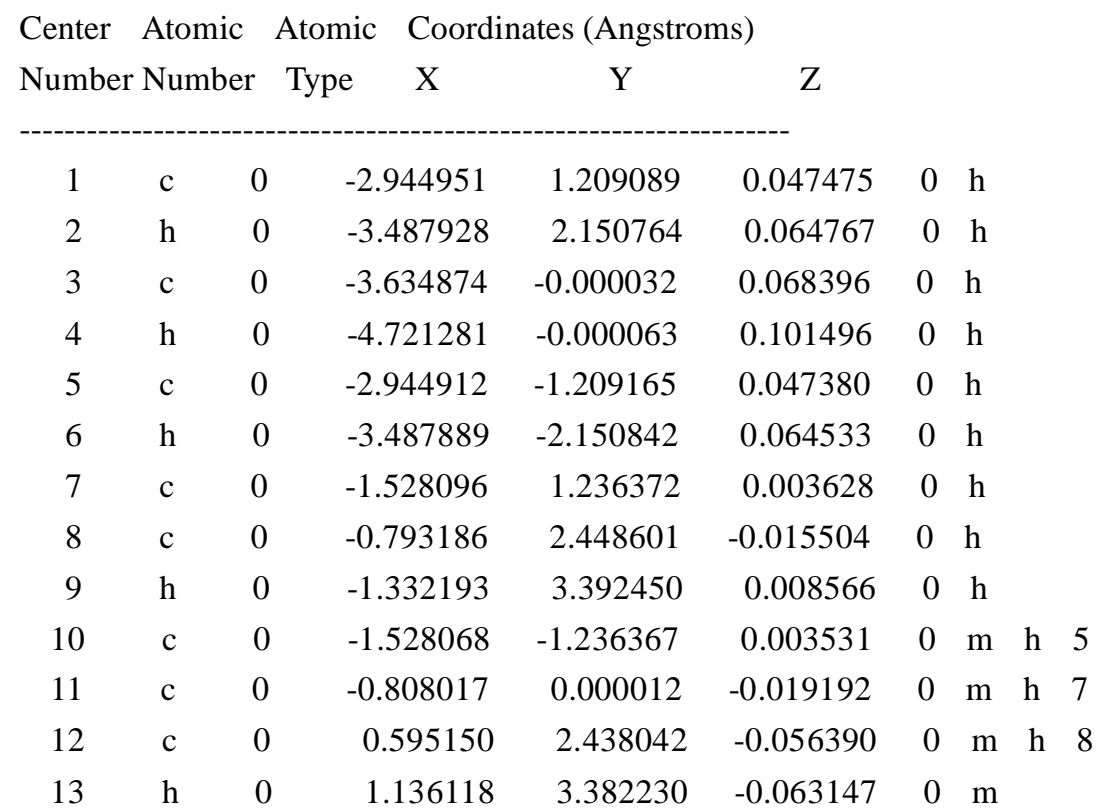




$\begin{array}{lllrrrrc}14 & \mathrm{c} & 0 & -0.793109 & -2.448587 & -0.015756 & 0 & \mathrm{~m} \\ 15 & \mathrm{~h} & 0 & -1.332121 & -3.392440 & 0.008122 & 0 & \mathrm{~m} \\ 16 & \mathrm{c} & 0 & 0.595207 & -2.438001 & -0.056447 & 0 & \mathrm{~m} \\ 17 & \mathrm{~h} & 0 & 1.136193 & -3.382179 & -0.063163 & 0 & \mathrm{~m} \\ 18 & \mathrm{c} & 0 & 1.322457 & -1.244966 & -0.087030 & 0 & \mathrm{~m} \\ 19 & \mathrm{c} & 0 & 0.623824 & -0.000002 & -0.066550 & 0 & \mathrm{~m} \\ 20 & \mathrm{c} & 0 & 2.833778 & -1.256824 & -0.150286 & 0 & \mathrm{~m} \\ 21 & \mathrm{~h} & 0 & 3.157319 & -1.313891 & -1.202529 & 0 & \mathrm{~m} \\ 22 & \mathrm{~h} & 0 & 3.219235 & -2.162711 & 0.333835 & 0 & \mathrm{~m} \\ 23 & \mathrm{c} & 0 & 3.442717 & 0.000123 & 0.484176 & 0 & \mathrm{~m} \\ 24 & \mathrm{~h} & 0 & 4.531871 & 0.000087 & 0.359418 & 0 & \mathrm{~m} \\ 25 & \mathrm{~h} & 0 & 3.239710 & 0.000396 & 1.563143 & 0 & \mathrm{~m} \\ 26 & \mathrm{c} & 0 & 2.833754 & 1.256737 & -0.150943 & 0 & \mathrm{~m} \\ 27 & \mathrm{~h} & 0 & 3.156915 & 1.312930 & -1.203354 & 0 & \mathrm{~m} \\ 28 & \mathrm{~h} & 0 & 3.219492 & 2.162935 & 0.332357 & 0 & \mathrm{~m} \\ 29 & \mathrm{c} & 0 & 1.322419 & 1.245023 & -0.087163 & 0 & \mathrm{~m}\end{array}$

No. 54

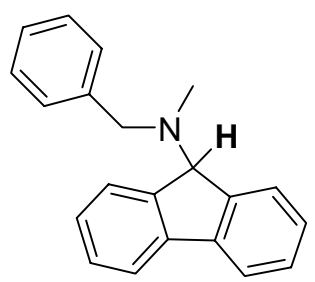

neutral molecule

Charge $=0$ Multiplicity $=1$ for low level calculation on real system.

Charge $=0$ Multiplicity $=1$ for high level calculation on model system.

Charge $=0$ Multiplicity $=1$ for low level calculation on model system.

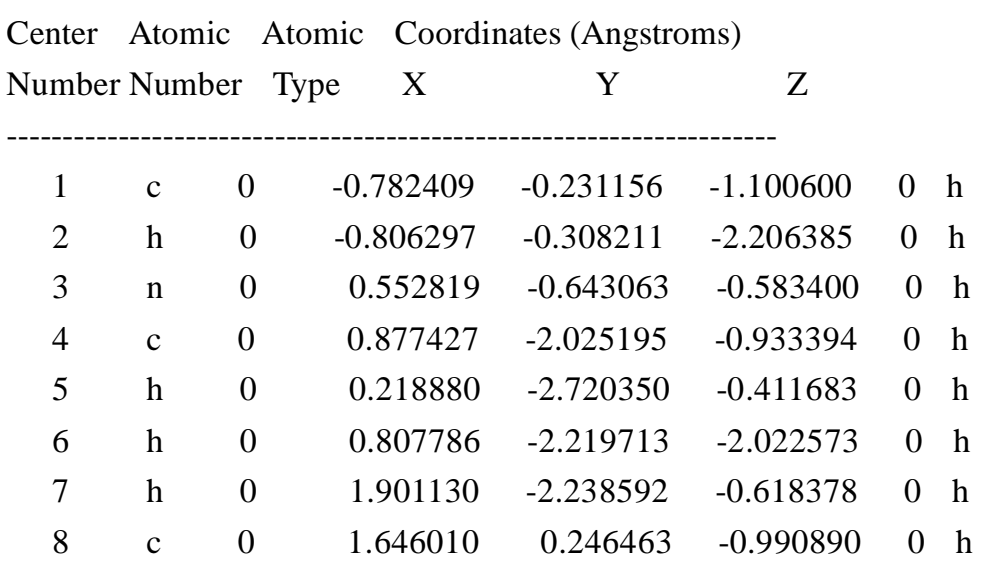




\begin{tabular}{|c|c|c|c|c|c|c|c|c|}
\hline 9 & $\mathrm{~h}$ & 0 & 1.858854 & 0.174454 & -2.077176 & 0 & & \\
\hline 10 & $\mathrm{~h}$ & 0 & 1.333065 & 1.273614 & -0.797351 & 0 & $\mathrm{~h}$ & \\
\hline 11 & $\mathrm{c}$ & 0 & -1.183070 & 1.176229 & -0.642218 & 0 & h & \\
\hline 12 & $\mathrm{c}$ & 0 & -0.738489 & 2.421554 & -1.076982 & 0 & $\mathrm{~h}$ & \\
\hline 13 & $\mathrm{~h}$ & 0 & -0.009647 & 2.508599 & -1.878001 & 0 & $\mathrm{~h}$ & \\
\hline 14 & $\mathrm{c}$ & 0 & -1.897442 & -1.075490 & -0.468579 & 0 & h & \\
\hline 15 & $\mathrm{c}$ & 0 & -2.253890 & -2.401229 & -0.693695 & 0 & h & \\
\hline 16 & $\mathrm{~h}$ & 0 & -1.734960 & -3.002673 & -1.434049 & 0 & h & \\
\hline 17 & $\mathrm{c}$ & 0 & 2.925856 & 0.005486 & -0.209502 & 0 & $\mathrm{~m}$ & h 8 \\
\hline 18 & $\mathrm{c}$ & 0 & -1.258534 & 3.583460 & -0.490830 & 0 & $\mathrm{~m}$ & h 12 \\
\hline 19 & $\mathrm{~h}$ & 0 & -0.909279 & 4.555996 & -0.826603 & 0 & $\mathrm{~m}$ & \\
\hline 20 & $\mathrm{c}$ & 0 & -2.207385 & 1.101658 & 0.323358 & 0 & $\mathrm{~m}$ & $\mathrm{~h}$ \\
\hline 21 & $\mathrm{c}$ & 0 & -2.649049 & -0.294623 & 0.432002 & 0 & $\mathrm{~m}$ & $\mathrm{~h}$ \\
\hline 22 & $\mathrm{c}$ & 0 & -3.314540 & -2.961383 & 0.031033 & 0 & $\mathrm{~m}$ & $\mathrm{~h}$ \\
\hline 23 & $\mathrm{~h}$ & 0 & -3.593973 & -3.997137 & -0.141917 & 0 & $\mathrm{~m}$ & \\
\hline 24 & $\mathrm{c}$ & 0 & 4.163546 & -0.050231 & -0.858840 & 0 & $\mathrm{~m}$ & \\
\hline 25 & $\mathrm{~h}$ & 0 & 4.202450 & 0.028286 & -1.943481 & 0 & $\mathrm{~m}$ & \\
\hline 26 & $\mathrm{c}$ & 0 & 5.346660 & -0.205110 & -0.131973 & 0 & $\mathrm{~m}$ & \\
\hline 27 & $\mathrm{~h}$ & 0 & 6.299454 & -0.246850 & -0.653720 & 0 & $\mathrm{~m}$ & \\
\hline 28 & $\mathrm{c}$ & 0 & 5.303281 & -0.314112 & 1.257837 & 0 & $\mathrm{~m}$ & \\
\hline 29 & $\mathrm{~h}$ & 0 & 6.221594 & -0.438429 & 1.825824 & 0 & $\mathrm{~m}$ & \\
\hline 30 & $\mathrm{c}$ & 0 & 4.070507 & -0.266939 & 1.915034 & 0 & $\mathrm{~m}$ & \\
\hline 31 & $\mathrm{~h}$ & 0 & 4.028144 & -0.353802 & 2.997922 & 0 & $\mathrm{~m}$ & \\
\hline 32 & $\mathrm{c}$ & 0 & 2.892154 & -0.106521 & 1.187716 & 0 & $\mathrm{~m}$ & \\
\hline 33 & $\mathrm{~h}$ & 0 & 1.930141 & -0.079833 & 1.691407 & 0 & $\mathrm{~m}$ & \\
\hline 34 & $\mathrm{c}$ & 0 & -2.228117 & 3.499554 & 0.510623 & 0 & $\mathrm{~m}$ & \\
\hline 35 & $\mathrm{~h}$ & 0 & -2.621502 & 4.407555 & 0.959888 & 0 & $\mathrm{~m}$ & \\
\hline 36 & $\mathrm{c}$ & 0 & -2.721154 & 2.255867 & 0.913792 & 0 & $\mathrm{~m}$ & \\
\hline 37 & $\mathrm{~h}$ & 0 & -3.511104 & 2.194877 & 1.657951 & 0 & $\mathrm{~m}$ & \\
\hline 38 & $\mathrm{c}$ & 0 & -3.699786 & -0.851661 & 1.160319 & 0 & $\mathrm{~m}$ & \\
\hline 39 & $\mathrm{~h}$ & 0 & -4.278252 & -0.246214 & 1.853435 & 0 & $\mathrm{~m}$ & \\
\hline 40 & $\mathrm{c}$ & 0 & -4.019621 & -2.197478 & 0.963657 & 0 & $\mathrm{~m}$ & \\
\hline 41 & $\mathrm{~h}$ & 0 & -4.837943 & -2.644988 & 1.521489 & 0 & $\mathrm{~m}$ & \\
\hline
\end{tabular}

radical

Charge $=0$ Multiplicity $=2$ for low level calculation on real system.

Charge $=0$ Multiplicity $=2$ for high level calculation on model system .

Charge $=0$ Multiplicity $=2$ for low level calculation on model system.

Center Atomic Atomic Coordinates (Angstroms)

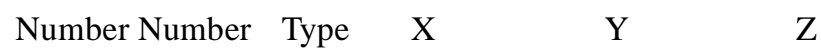




\begin{tabular}{|c|c|c|c|c|c|c|c|c|}
\hline 1 & c & 0 & -0.572441 & -0.122771 & 0.668163 & 0 & & \\
\hline 2 & $\mathrm{n}$ & 0 & 0.662762 & -0.319415 & 1.249307 & 0 & $\mathrm{~h}$ & \\
\hline 3 & $\mathrm{c}$ & 0 & 1.208391 & 0.618210 & 2.218501 & 0 & $\mathrm{~h}$ & \\
\hline 4 & $\mathrm{~h}$ & 0 & 0.392746 & 1.129267 & 2.731989 & 0 & $\mathrm{~h}$ & \\
\hline 5 & $\mathrm{~h}$ & 0 & 1.788198 & 0.058015 & 2.963484 & 0 & $\mathrm{~h}$ & \\
\hline 6 & $\mathrm{~h}$ & 0 & 1.878081 & 1.363855 & 1.768296 & 0 & $\mathrm{~h}$ & \\
\hline 7 & $\mathrm{c}$ & 0 & 1.619214 & -1.293827 & 0.713026 & 0 & $\mathrm{~h}$ & \\
\hline 8 & $\mathrm{~h}$ & 0 & 1.987251 & -1.928745 & 1.531482 & 0 & $\mathrm{~h}$ & \\
\hline 9 & $\mathrm{~h}$ & 0 & 1.083098 & -1.934586 & 0.011755 & 0 & $\mathrm{~h}$ & \\
\hline 10 & $\mathrm{c}$ & 0 & -1.465825 & -1.175559 & 0.219830 & 0 & $\mathrm{~h}$ & \\
\hline 11 & $\mathrm{c}$ & 0 & -1.397247 & -2.577482 & 0.303168 & 0 & $\mathrm{~h}$ & \\
\hline 12 & $\mathrm{~h}$ & 0 & -0.550802 & -3.073801 & 0.767931 & 0 & $\mathrm{~h}$ & \\
\hline 13 & $\mathrm{c}$ & 0 & -1.226003 & 1.146316 & 0.414251 & 0 & $\mathrm{~h}$ & \\
\hline 14 & $\mathrm{c}$ & 0 & -0.822853 & 2.482619 & 0.596864 & 0 & $\mathrm{~h}$ & \\
\hline 15 & $\mathrm{~h}$ & 0 & 0.140862 & 2.726512 & 1.030156 & 0 & $\mathrm{~h}$ & \\
\hline 16 & $\mathrm{c}$ & 0 & 2.804377 & -0.650836 & 0.005555 & 0 & $\mathrm{~m}$ & h 7 \\
\hline 17 & $\mathrm{c}$ & 0 & -2.452010 & -3.344178 & -0.190808 & 0 & $\mathrm{~m}$ & h 11 \\
\hline 18 & $\mathrm{~h}$ & 0 & -2.398148 & -4.427993 & -0.129227 & 0 & $\mathrm{~m}$ & \\
\hline 19 & $\mathrm{c}$ & 0 & -2.639399 & -0.565612 & -0.321693 & 0 & $\mathrm{~m}$ & h 10 \\
\hline 20 & $\mathrm{c}$ & 0 & -2.491152 & 0.879967 & -0.201008 & 0 & $\mathrm{~m}$ & h 13 \\
\hline 21 & $\mathrm{c}$ & 0 & -1.673581 & 3.514228 & 0.203265 & 0 & $\mathrm{~m}$ & h 14 \\
\hline 22 & $\mathrm{~h}$ & 0 & -1.362311 & 4.545437 & 0.348594 & 0 & $\mathrm{~m}$ & \\
\hline 23 & $\mathrm{c}$ & 0 & 4.111522 & -0.882102 & 0.445673 & 0 & $\mathrm{~m}$ & \\
\hline 24 & $\mathrm{~h}$ & 0 & 4.280126 & -1.520792 & 1.310395 & 0 & $\mathrm{~m}$ & \\
\hline 25 & $\mathrm{c}$ & 0 & 5.200469 & -0.307328 & -0.214394 & 0 & $\mathrm{~m}$ & \\
\hline 26 & $\mathrm{~h}$ & 0 & 6.210042 & -0.499762 & 0.139224 & 0 & $\mathrm{~m}$ & \\
\hline 27 & $\mathrm{c}$ & 0 & 4.989880 & 0.514346 & -1.321292 & 0 & $\mathrm{~m}$ & \\
\hline 28 & $\mathrm{~h}$ & 0 & 5.834256 & 0.964614 & -1.836293 & 0 & $\mathrm{~m}$ & \\
\hline 29 & $\mathrm{c}$ & 0 & 3.686619 & 0.757093 & -1.765086 & 0 & $\mathrm{~m}$ & \\
\hline 30 & $\mathrm{~h}$ & 0 & 3.515618 & 1.395850 & -2.627622 & 0 & $\mathrm{~m}$ & \\
\hline 31 & $\mathrm{c}$ & 0 & 2.602525 & 0.177832 & -1.107891 & 0 & $\mathrm{~m}$ & \\
\hline 32 & $\mathrm{~h}$ & 0 & 1.590837 & 0.370997 & -1.456180 & 0 & $\mathrm{~m}$ & \\
\hline 33 & $\mathrm{c}$ & 0 & -3.584624 & -2.737594 & -0.753381 & 0 & $\mathrm{~m}$ & \\
\hline 34 & $\mathrm{~h}$ & 0 & -4.394944 & -3.353853 & -1.133161 & 0 & $\mathrm{~m}$ & \\
\hline 35 & $\mathrm{c}$ & 0 & -3.685406 & -1.344374 & -0.808357 & 0 & $\mathrm{~m}$ & \\
\hline 36 & $\mathrm{~h}$ & 0 & -4.578084 & -0.876876 & -1.217120 & 0 & $\mathrm{~m}$ & \\
\hline 37 & $\mathrm{c}$ & 0 & -3.326726 & 1.921941 & -0.591425 & 0 & $\mathrm{~m}$ & \\
\hline 38 & $\mathrm{~h}$ & 0 & -4.284557 & 1.713948 & -1.062485 & 0 & $\mathrm{~m}$ & \\
\hline 39 & $\mathrm{c}$ & 0 & -2.919771 & 3.242642 & -0.380866 & 0 & $\mathrm{~m}$ & \\
\hline 40 & $\mathrm{~h}$ & 0 & -3.567356 & 4.062632 & -0.678947 & 0 & $\mathrm{~m}$ & \\
\hline
\end{tabular}




\section{No. 55}<smiles>C1=c2ccc3cccc4ccc(c2c43)C1</smiles>

neutral molecule

Charge $=0$ Multiplicity $=1$ for low level calculation on real system.

Charge $=0$ Multiplicity $=1$ for high level calculation on model system.

Charge $=0$ Multiplicity $=1$ for low level calculation on model system.

Center Atomic Atomic Coordinates (Angstroms)

Number Number Type $\quad \mathrm{X} \quad \mathrm{Y} \quad \mathrm{Z}$

\begin{tabular}{|c|c|c|c|c|c|c|c|c|}
\hline 1 & $\mathrm{c}$ & 0 & -3.504679 & 0.030015 & 0.379333 & 0 & $\mathrm{~h}$ & \\
\hline 2 & $\mathrm{~h}$ & 0 & -3.568912 & -0.144102 & 1.468274 & 0 & $\mathrm{~h}$ & \\
\hline 3 & $\mathrm{~h}$ & 0 & -4.539118 & 0.138707 & 0.032395 & 0 & $\mathrm{~h}$ & \\
\hline 4 & $\mathrm{c}$ & 0 & -2.723727 & 1.293357 & 0.136765 & 0 & $\mathrm{~h}$ & \\
\hline 5 & $\mathrm{~h}$ & 0 & -3.244237 & 2.247204 & 0.198284 & 0 & $\mathrm{~h}$ & \\
\hline 6 & $\mathrm{c}$ & 0 & -1.378646 & 1.274936 & -0.038389 & 0 & $\mathrm{~h}$ & \\
\hline 7 & $\mathrm{c}$ & 0 & -0.648733 & -0.007917 & -0.045844 & 0 & $\mathrm{~h}$ & \\
\hline 8 & $\mathrm{c}$ & 0 & -1.348202 & -1.214053 & -0.104163 & 0 & $\mathrm{~h}$ & \\
\hline 9 & $\mathrm{c}$ & 0 & -2.849392 & -1.177870 & -0.304711 & 0 & $\mathrm{~h}$ & \\
\hline 10 & $\mathrm{~h}$ & 0 & -3.307341 & -2.110857 & 0.043532 & 0 & $\mathrm{~h}$ & \\
\hline 11 & $\mathrm{~h}$ & 0 & -3.048312 & -1.119588 & -1.387418 & 0 & $\mathrm{~h}$ & \\
\hline 12 & $\mathrm{c}$ & 0 & -0.589216 & 2.493335 & -0.130695 & 0 & $\mathrm{~m}$ & h 6 \\
\hline 13 & $\mathrm{~h}$ & 0 & -1.122792 & 3.439806 & -0.179880 & 0 & $\mathrm{~m}$ & \\
\hline 14 & $\mathrm{c}$ & 0 & 0.775193 & -0.009594 & -0.000734 & 0 & $\mathrm{~m}$ & h \\
\hline 15 & $\mathrm{c}$ & 0 & -0.637397 & -2.431776 & -0.063759 & 0 & $\mathrm{~m}$ & h 8 \\
\hline 16 & $\mathrm{~h}$ & 0 & -1.192928 & -3.366420 & -0.101382 & 0 & $\mathrm{~m}$ & \\
\hline 17 & $\mathrm{c}$ & 0 & 0.761648 & 2.468742 & -0.112901 & 0 & $\mathrm{~m}$ & \\
\hline 18 & $\mathrm{~h}$ & 0 & 1.329075 & 3.395595 & -0.157128 & 0 & $\mathrm{~m}$ & \\
\hline 19 & $\mathrm{c}$ & 0 & 1.505850 & 1.223247 & -0.023695 & 0 & $\mathrm{~m}$ & \\
\hline 20 & $\mathrm{c}$ & 0 & 2.893043 & 1.191298 & 0.019311 & 0 & $\mathrm{~m}$ & \\
\hline 21 & $\mathrm{~h}$ & 0 & 3.448285 & 2.126124 & -0.003332 & 0 & $\mathrm{~m}$ & \\
\hline 22 & $\mathrm{c}$ & 0 & 3.590935 & -0.033958 & 0.087259 & 0 & $\mathrm{~m}$ & \\
\hline 23 & $\mathrm{~h}$ & 0 & 4.677008 & -0.028509 & 0.124493 & 0 & $\mathrm{~m}$ & \\
\hline 24 & $\mathrm{c}$ & 0 & 2.905676 & -1.228868 & 0.097056 & 0 & $\mathrm{~m}$ & \\
\hline 25 & $\mathrm{~h}$ & 0 & 3.442366 & -2.173548 & 0.140321 & 0 & $\mathrm{~m}$ & \\
\hline 26 & $\mathrm{c}$ & 0 & 1.483978 & -1.249153 & 0.043295 & 0 & $\mathrm{~m}$ & \\
\hline 27 & $\mathrm{c}$ & 0 & 0.739676 & -2.455265 & 0.021856 & 0 & $\mathrm{~m}$ & \\
\hline 28 & $\mathrm{~h}$ & 0 & 1.270867 & -3.403266 & 0.061921 & 0 & $\mathrm{~m}$ & \\
\hline
\end{tabular}


radical

Charge $=0$ Multiplicity $=2$ for low level calculation on real system.

Charge $=0$ Multiplicity $=2$ for high level calculation on model system.

Charge $=0$ Multiplicity $=2$ for low level calculation on model system.

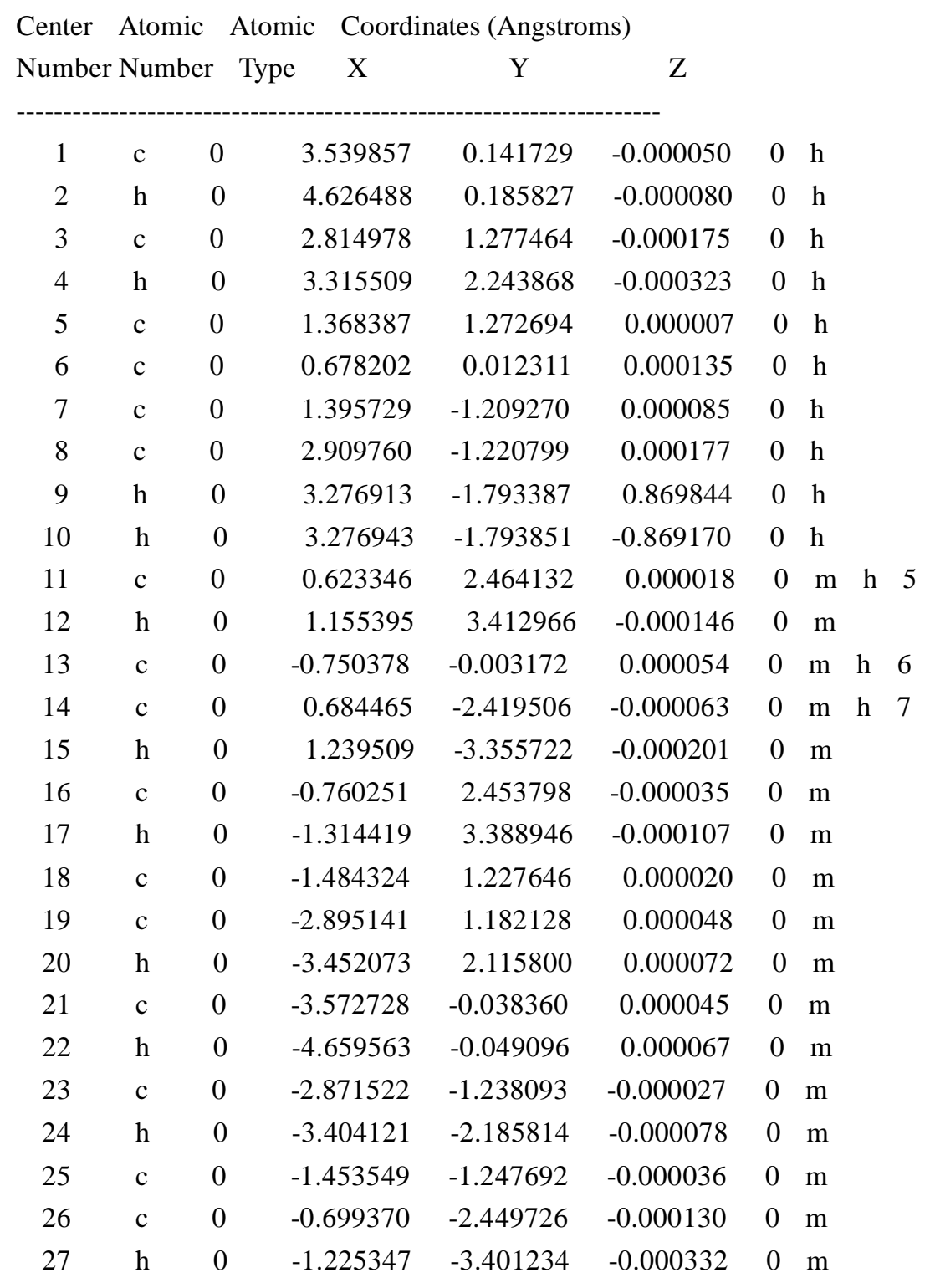




\section{No. 56}

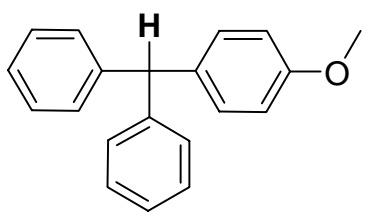

neutral molecule

Charge $=0$ Multiplicity $=1$ for low level calculation on real system.

Charge $=0$ Multiplicity $=1$ for high level calculation on model system .

Charge $=0$ Multiplicity $=1$ for low level calculation on model system .

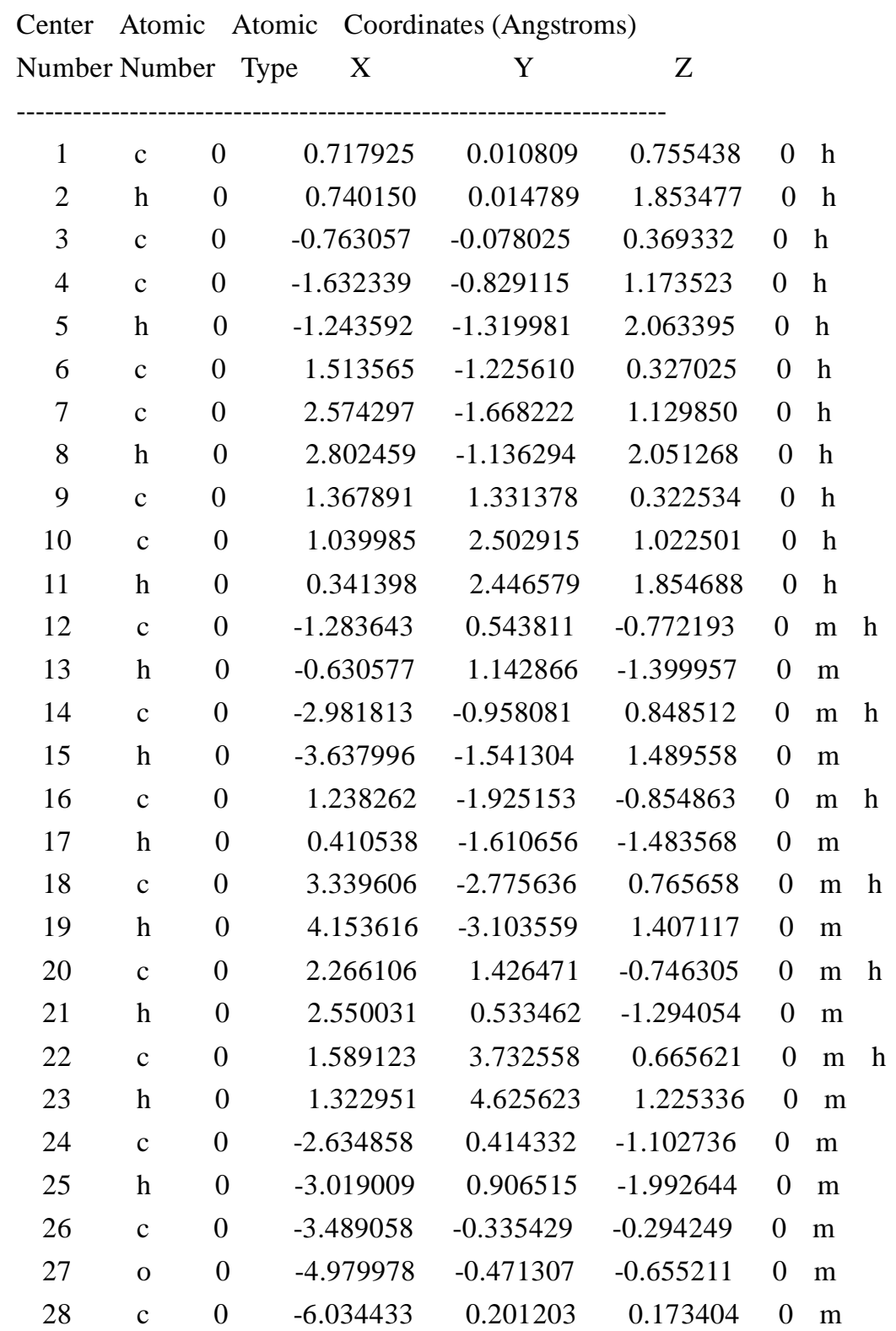




$\begin{array}{lllrrrrc}29 & \mathrm{~h} & 0 & -5.882582 & 1.307959 & 0.183628 & 0 & \mathrm{~m} \\ 30 & \mathrm{~h} & 0 & -7.052363 & -0.007444 & -0.236548 & 0 & \mathrm{~m} \\ 31 & \mathrm{~h} & 0 & -6.001290 & -0.165290 & 1.228233 & 0 & \mathrm{~m} \\ 32 & \mathrm{c} & 0 & 2.004640 & -3.032622 & -1.224835 & 0 & \mathrm{~m} \\ 33 & \mathrm{~h} & 0 & 1.771856 & -3.562904 & -2.144801 & 0 & \mathrm{~m} \\ 34 & \mathrm{c} & 0 & 3.057267 & -3.462963 & -0.416614 & 0 & \mathrm{~m} \\ 35 & \mathrm{~h} & 0 & 3.649468 & -4.328307 & -0.702194 & 0 & \mathrm{~m} \\ 36 & \mathrm{c} & 0 & 2.817067 & 2.659394 & -1.108534 & 0 & \mathrm{~m} \\ 37 & \mathrm{~h} & 0 & 3.514191 & 2.710103 & -1.941139 & 0 & \mathrm{~m} \\ 38 & \mathrm{c} & 0 & 2.482690 & 3.815609 & -0.405540 & 0 & \mathrm{~m} \\ 39 & \mathrm{~h} & 0 & 2.915231 & 4.772568 & -0.685277 & 0 & \mathrm{~m}\end{array}$

radical

Charge $=0$ Multiplicity $=2$ for low level calculation on real system.

Charge $=0$ Multiplicity $=2$ for high level calculation on model system.

Charge $=0$ Multiplicity $=2$ for low level calculation on model system.

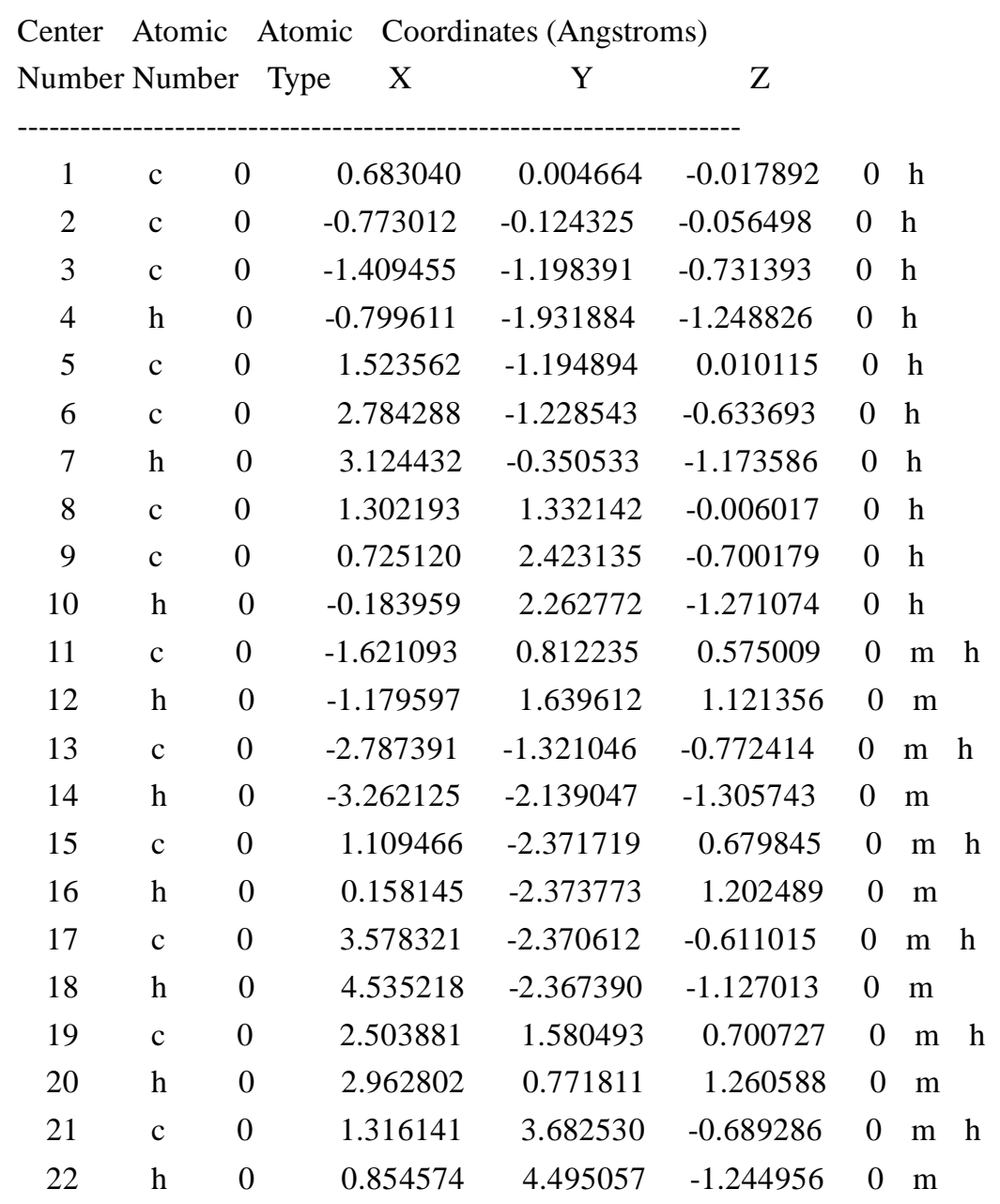




$\begin{array}{lllrrrrc}23 & \mathrm{c} & 0 & -3.008825 & 0.696521 & 0.545601 & 0 & \mathrm{~m} \\ 24 & \mathrm{~h} & 0 & -3.611426 & 1.436552 & 1.060415 & 0 & \mathrm{~m} \\ 25 & \mathrm{c} & 0 & -3.604898 & -0.375117 & -0.132046 & 0 & \mathrm{~m} \\ 26 & \mathrm{o} & 0 & -4.948523 & -0.586923 & -0.228565 & 0 & \mathrm{~m} \\ 27 & \mathrm{c} & 0 & -5.821768 & 0.343449 & 0.392135 & 0 & \mathrm{~m} \\ 28 & \mathrm{~h} & 0 & -6.833105 & -0.011581 & 0.185899 & 0 & \mathrm{~m} \\ 29 & \mathrm{~h} & 0 & -5.698809 & 1.351762 & -0.024718 & 0 & \mathrm{~m} \\ 30 & \mathrm{~h} & 0 & -5.665273 & 0.381396 & 1.478270 & 0 & \mathrm{~m} \\ 31 & \mathrm{c} & 0 & 1.909215 & -3.509951 & 0.704935 & 0 & \mathrm{~m} \\ 32 & \mathrm{~h} & 0 & 1.567228 & -4.392488 & 1.239807 & 0 & \mathrm{~m} \\ 33 & \mathrm{c} & 0 & 3.148730 & -3.519858 & 0.058965 & 0 & \mathrm{~m} \\ 34 & \mathrm{~h} & 0 & 3.771127 & -4.410357 & 0.077524 & 0 & \mathrm{~m} \\ 35 & \mathrm{c} & 0 & 3.088624 & 2.842740 & 0.713608 & 0 & \mathrm{~m} \\ 36 & \mathrm{~h} & 0 & 4.004456 & 3.002307 & 1.277256 & 0 & \mathrm{~m} \\ 37 & \mathrm{c} & 0 & 2.501137 & 3.903678 & 0.018385 & 0 & \mathrm{~m} \\ 38 & \mathrm{~h} & 0 & 2.960445 & 4.888386 & 0.027467 & 0 & \mathrm{~m}\end{array}$

\section{(2) The Cartesian Coordinates of the $10 \mathrm{~N}-\mathrm{H}$ molecules.}

\section{No.1}<smiles>c1ccc(CN(Nc2ccccc2)c2ccccc2)cc1</smiles>

neutral molecule

Charge $=0$ Multiplicity $=1$ for low level calculation on real system.

Charge $=0$ Multiplicity $=1$ for high level calculation on model system.

Charge $=0$ Multiplicity $=1$ for low level calculation on model system.

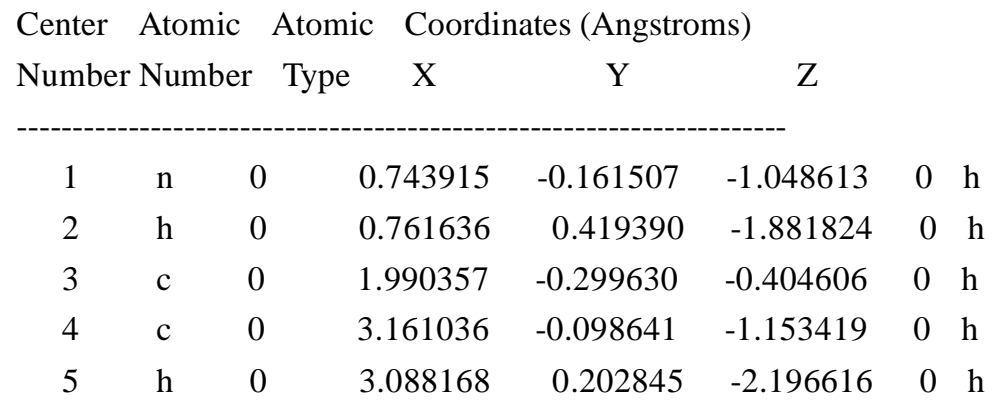




\begin{tabular}{|c|c|c|c|c|c|c|c|c|}
\hline 6 & $\mathrm{c}$ & 0 & 2.091278 & -0.691717 & 0.937431 & 0 & & \\
\hline 7 & h & 0 & 1.188751 & -0.847922 & 1.517869 & 0 & $\mathrm{~h}$ & \\
\hline 8 & $\mathrm{c}$ & 0 & 3.348456 & -0.876302 & 1.512079 & 0 & $\mathrm{~h}$ & \\
\hline 9 & $\mathrm{~h}$ & 0 & 3.411959 & -1.178737 & 2.554286 & 0 & $\mathrm{~h}$ & \\
\hline 10 & $\mathrm{n}$ & 0 & -0.409733 & 0.032692 & -0.285196 & 0 & $\mathrm{~h}$ & \\
\hline 11 & $\mathrm{c}$ & 0 & 4.409577 & -0.288340 & -0.566269 & 0 & $\mathrm{~m}$ & h 2 \\
\hline 12 & $\mathrm{~h}$ & 0 & 5.305558 & -0.125222 & -1.159761 & 0 & $\mathrm{~m}$ & \\
\hline 13 & $\mathrm{c}$ & 0 & 4.514698 & -0.678602 & 0.770740 & 0 & $\mathrm{~m}$ & $\mathrm{~h}$ \\
\hline 14 & $\mathrm{~h}$ & 0 & 5.489502 & -0.824646 & 1.226625 & 0 & $\mathrm{~m}$ & \\
\hline 15 & $\mathrm{c}$ & 0 & -0.843775 & 1.375094 & -0.114402 & 0 & $\mathrm{~m}$ & $\mathrm{~h}$ \\
\hline 16 & $\mathrm{c}$ & 0 & -1.266439 & -1.092177 & -0.145820 & 0 & $\mathrm{~m}$ & $\mathrm{~h}$ \\
\hline 17 & $\mathrm{c}$ & 0 & 0.104934 & 2.384968 & 0.125627 & 0 & $\mathrm{~m}$ & \\
\hline 18 & $\mathrm{~h}$ & 0 & 1.153347 & 2.126378 & 0.225720 & 0 & $\mathrm{~m}$ & \\
\hline 19 & $\mathrm{c}$ & 0 & -0.297960 & 3.712718 & 0.253120 & 0 & $\mathrm{~m}$ & \\
\hline 20 & $\mathrm{~h}$ & 0 & 0.451895 & 4.476308 & 0.443113 & 0 & $\mathrm{~m}$ & \\
\hline 21 & $\mathrm{c}$ & 0 & -1.645142 & 4.063696 & 0.151083 & 0 & $\mathrm{~m}$ & \\
\hline 22 & $\mathrm{~h}$ & 0 & -1.955025 & 5.099274 & 0.257042 & 0 & $\mathrm{~m}$ & \\
\hline 23 & $\mathrm{c}$ & 0 & -2.587716 & 3.063567 & -0.092713 & 0 & $\mathrm{~m}$ & \\
\hline 24 & $\mathrm{~h}$ & 0 & -3.639833 & 3.318535 & -0.191206 & 0 & $\mathrm{~m}$ & \\
\hline 25 & $\mathrm{c}$ & 0 & -2.197872 & 1.733146 & -0.235222 & 0 & $\mathrm{~m}$ & \\
\hline 26 & $\mathrm{~h}$ & 0 & -2.940713 & 0.972311 & -0.446763 & 0 & $\mathrm{~m}$ & \\
\hline 27 & $\mathrm{c}$ & 0 & -2.152300 & -1.163893 & 0.943319 & 0 & $\mathrm{~m}$ & \\
\hline 28 & $\mathrm{~h}$ & 0 & -2.189564 & -0.349042 & 1.659246 & 0 & $\mathrm{~m}$ & \\
\hline 29 & $\mathrm{c}$ & 0 & -2.971984 & -2.277723 & 1.109232 & 0 & $\mathrm{~m}$ & \\
\hline 30 & $\mathrm{~h}$ & 0 & -3.650759 & -2.314143 & 1.957452 & 0 & $\mathrm{~m}$ & \\
\hline 31 & $\mathrm{c}$ & 0 & -2.912150 & -3.346785 & 0.212406 & 0 & $\mathrm{~m}$ & \\
\hline 32 & $\mathrm{~h}$ & 0 & -3.547161 & -4.217207 & 0.349987 & 0 & $\mathrm{~m}$ & \\
\hline 33 & $\mathrm{c}$ & 0 & -2.015917 & -3.285157 & -0.855233 & 0 & $\mathrm{~m}$ & \\
\hline 34 & $\mathrm{~h}$ & 0 & -1.949699 & -4.111076 & -1.558908 & 0 & $\mathrm{~m}$ & \\
\hline 35 & $\mathrm{c}$ & 0 & -1.199431 & -2.169970 & -1.040148 & 0 & $\mathrm{~m}$ & \\
\hline 36 & $\mathrm{~h}$ & 0 & -0.495251 & -2.130851 & -1.862822 & 0 & $\mathrm{~m}$ & \\
\hline
\end{tabular}

radical

Charge $=0$ Multiplicity $=2$ for low level calculation on real system .

Charge $=0$ Multiplicity $=2$ for high level calculation on model system .

Charge $=0$ Multiplicity $=2$ for low level calculation on model system .

Center Atomic Atomic Coordinates (Angstroms)

Number Number Type $\quad X \quad$ Y $\quad$ Z

$\begin{array}{llllllll}1 & \mathrm{n} & 0 & -0.315294 & -1.237227 & -0.484456 & 0 & \mathrm{~h} \\ 2 & \mathrm{c} & 0 & -1.652996 & -1.168326 & -0.135501 & 0 & \mathrm{~h}\end{array}$




\begin{tabular}{|c|c|c|c|c|c|c|c|c|}
\hline 3 & $\mathrm{c}$ & 0 & -2.500909 & -2.028453 & -0.875586 & 0 & & \\
\hline 4 & $\mathrm{~h}$ & 0 & -2.054013 & -2.619438 & -1.669366 & 0 & $\mathrm{~h}$ & \\
\hline 5 & $\mathrm{c}$ & 0 & -2.224273 & -0.416059 & 0.920510 & 0 & $\mathrm{~h}$ & \\
\hline 6 & $\mathrm{~h}$ & 0 & -1.598825 & 0.221689 & 1.533305 & 0 & $\mathrm{~h}$ & \\
\hline 7 & $\mathrm{c}$ & 0 & -3.582852 & -0.521577 & 1.200914 & 0 & $\mathrm{~h}$ & \\
\hline 8 & $\mathrm{~h}$ & 0 & -3.999319 & 0.053011 & 2.024664 & 0 & $\mathrm{~h}$ & \\
\hline 9 & $\mathrm{n}$ & 0 & 0.520266 & -0.240536 & -0.098785 & 0 & $\mathrm{~h}$ & \\
\hline 10 & $\mathrm{c}$ & 0 & -3.858180 & -2.109776 & -0.596137 & 0 & $\mathrm{~m}$ & h 3 \\
\hline 11 & $\mathrm{~h}$ & 0 & -4.488776 & -2.770620 & -1.185389 & 0 & $\mathrm{~m}$ & \\
\hline 12 & $\mathrm{c}$ & 0 & -4.412337 & -1.354869 & 0.444183 & 0 & $\mathrm{~m}$ & $\mathrm{~h}$ \\
\hline 13 & $\mathrm{~h}$ & 0 & -5.472932 & -1.423407 & 0.669038 & 0 & $\mathrm{~m}$ & \\
\hline 14 & $\mathrm{c}$ & 0 & 1.891963 & -0.611162 & -0.032272 & 0 & $\mathrm{~m}$ & $\mathrm{~h}$ \\
\hline 15 & $\mathrm{c}$ & 0 & 0.149150 & 1.145008 & -0.103990 & 0 & $\mathrm{~m}$ & $\mathrm{~h}$ \\
\hline 16 & $\mathrm{c}$ & 0 & 2.222138 & -1.926402 & 0.333183 & 0 & $\mathrm{~m}$ & \\
\hline 17 & $\mathrm{~h}$ & 0 & 1.423731 & -2.622930 & 0.558079 & 0 & $\mathrm{~m}$ & \\
\hline 18 & $\mathrm{c}$ & 0 & 3.556582 & -2.317288 & 0.394256 & 0 & $\mathrm{~m}$ & \\
\hline 19 & $\mathrm{~h}$ & 0 & 3.797920 & -3.337561 & 0.680542 & 0 & $\mathrm{~m}$ & \\
\hline 20 & $\mathrm{c}$ & 0 & 4.579277 & -1.411122 & 0.102799 & 0 & $\mathrm{~m}$ & \\
\hline 21 & $\mathrm{~h}$ & 0 & 5.619440 & -1.719337 & 0.158020 & 0 & $\mathrm{~m}$ & \\
\hline 22 & $\mathrm{c}$ & 0 & 4.248881 & -0.104859 & -0.262197 & 0 & $\mathrm{~m}$ & \\
\hline 23 & $\mathrm{~h}$ & 0 & 5.032111 & 0.608458 & -0.504859 & 0 & $\mathrm{~m}$ & \\
\hline 24 & $\mathrm{c}$ & 0 & 2.917098 & 0.298693 & -0.337110 & 0 & $\mathrm{~m}$ & \\
\hline 25 & $\mathrm{~h}$ & 0 & 2.675622 & 1.309699 & -0.644252 & 0 & $\mathrm{~m}$ & \\
\hline 26 & $\mathrm{c}$ & 0 & 0.555952 & 1.986418 & 0.940288 & 0 & $\mathrm{~m}$ & \\
\hline 27 & $\mathrm{~h}$ & 0 & 1.149131 & 1.580738 & 1.754093 & 0 & $\mathrm{~m}$ & \\
\hline 28 & $\mathrm{c}$ & 0 & 0.193735 & 3.332331 & 0.928453 & 0 & $\mathrm{~m}$ & \\
\hline 29 & $\mathrm{~h}$ & 0 & 0.511169 & 3.978634 & 1.742235 & 0 & $\mathrm{~m}$ & \\
\hline 30 & $\mathrm{c}$ & 0 & -0.582174 & 3.845444 & -0.113875 & 0 & $\mathrm{~m}$ & \\
\hline 31 & $\mathrm{~h}$ & 0 & -0.868078 & 4.893398 & -0.117270 & 0 & $\mathrm{~m}$ & \\
\hline 32 & $\mathrm{c}$ & 0 & -0.987812 & 3.003091 & -1.151251 & 0 & $\mathrm{~m}$ & \\
\hline 33 & $\mathrm{~h}$ & 0 & -1.585989 & 3.394791 & -1.969361 & 0 & $\mathrm{~m}$ & \\
\hline 34 & $\mathrm{c}$ & 0 & -0.621418 & 1.658276 & -1.154244 & 0 & $\mathrm{~m}$ & \\
\hline 35 & $\mathrm{~h}$ & 0 & -0.926948 & 1.001004 & -1.961323 & 0 & $\mathrm{~m}$ & \\
\hline
\end{tabular}

No.2<smiles>O=[N+]([O-])c1cc([N+](=O)[O-])c(N(c2ccccc2)c2ccccc2)c([N+](=O)[O-])c1</smiles>

neutral molecule

Charge $=0$ Multiplicity $=1$ for low level calculation on real system. 
Charge $=0$ Multiplicity $=1$ for high level calculation on model system .

Charge $=0$ Multiplicity $=1$ for low level calculation on model system .

\begin{tabular}{|c|c|c|c|c|c|c|c|c|}
\hline Center & Atomic & & Atomic & lates (Angstro & ms) & & & \\
\hline Number & Numbe & & Type & $\mathrm{Y}$ & $\mathrm{Z}$ & & & \\
\hline 1 & $\mathrm{n}$ & 0 & 0.499909 & -0.663247 & 0.724467 & 0 & $\mathrm{~h}$ & \\
\hline 2 & $\mathrm{~h}$ & 0 & 0.645294 & -0.872276 & 1.711060 & 0 & & \\
\hline 3 & $\mathrm{n}$ & 0 & 1.508048 & 0.002944 & 0.022338 & 0 & $\mathrm{~h}$ & \\
\hline 4 & $\mathrm{c}$ & 0 & -0.790749 & -0.596608 & 0.314432 & 0 & $\mathrm{~h}$ & \\
\hline 5 & $\mathrm{c}$ & 0 & -1.898063 & -0.695757 & 1.219923 & 0 & $\mathrm{~h}$ & \\
\hline 6 & $\mathrm{c}$ & 0 & -1.148216 & -0.369843 & -1.047680 & 0 & $\mathrm{~h}$ & \\
\hline 7 & $\mathrm{c}$ & 0 & -2.443740 & -0.161235 & -1.463849 & 0 & $\mathrm{~h}$ & \\
\hline 8 & $\mathrm{~h}$ & 0 & -2.663158 & 0.029655 & -2.507105 & 0 & $\mathrm{~h}$ & \\
\hline 9 & $\mathrm{c}$ & 0 & 1.530545 & 1.434053 & 0.223577 & 0 & $\mathrm{~m}$ & $\mathrm{~h}$ \\
\hline 10 & $\mathrm{c}$ & 0 & 2.730745 & -0.737540 & -0.062033 & 0 & $\mathrm{~m}$ & h 3 \\
\hline 11 & $\mathrm{n}$ & 0 & -0.145374 & -0.479995 & -2.127496 & 0 & $\mathrm{~m}$ & h 6 \\
\hline 12 & $\mathrm{n}$ & 0 & -1.711380 & -0.925835 & 2.648775 & 0 & $\mathrm{~m}$ & h 5 \\
\hline 13 & $\mathrm{c}$ & 0 & -3.215766 & -0.525461 & 0.798354 & 0 & $\mathrm{~m}$ & h 5 \\
\hline 14 & $\mathrm{~h}$ & 0 & -4.020045 & -0.592169 & 1.518507 & 0 & $\mathrm{~m}$ & \\
\hline 15 & $\mathrm{c}$ & 0 & -3.478925 & -0.242363 & -0.527978 & 0 & $\mathrm{~m}$ & h 7 \\
\hline 16 & $\mathrm{c}$ & 0 & 1.255044 & 2.004117 & 1.471490 & 0 & $\mathrm{~m}$ & \\
\hline 17 & $\mathrm{~h}$ & 0 & 1.025371 & 1.372801 & 2.325013 & 0 & $m$ & \\
\hline 18 & $\mathrm{c}$ & 0 & 1.263680 & 3.391849 & 1.615465 & 0 & $\mathrm{~m}$ & \\
\hline 19 & $\mathrm{~h}$ & 0 & 1.044036 & 3.829980 & 2.585041 & 0 & $m$ & \\
\hline 20 & $\mathrm{c}$ & 0 & 1.562895 & 4.211762 & 0.526102 & 0 & $\mathrm{~m}$ & \\
\hline 21 & $\mathrm{~h}$ & 0 & 1.571282 & 5.291603 & 0.642958 & 0 & $m$ & \\
\hline 22 & $\mathrm{c}$ & 0 & 1.842024 & 3.638143 & -0.716132 & 0 & $\mathrm{~m}$ & \\
\hline 23 & $\mathrm{~h}$ & 0 & 2.062598 & 4.269357 & -1.572215 & 0 & $\mathrm{~m}$ & \\
\hline 24 & $\mathrm{c}$ & 0 & 1.815464 & 2.252825 & -0.874426 & 0 & $\mathrm{~m}$ & \\
\hline 25 & $\mathrm{~h}$ & 0 & 2.001175 & 1.799581 & -1.842448 & 0 & $\mathrm{~m}$ & \\
\hline 26 & $\mathrm{c}$ & 0 & 3.973503 & -0.088716 & -0.041479 & 0 & $\mathrm{~m}$ & \\
\hline 27 & $\mathrm{~h}$ & 0 & 4.027805 & 0.988723 & 0.058057 & 0 & $\mathrm{~m}$ & \\
\hline 28 & $\mathrm{c}$ & 0 & 5.147740 & -0.833951 & -0.145165 & 0 & $\mathrm{~m}$ & \\
\hline 29 & $\mathrm{~h}$ & 0 & 6.102861 & -0.316051 & -0.127141 & 0 & $\mathrm{~m}$ & \\
\hline 30 & $\mathrm{c}$ & 0 & 5.105926 & -2.223619 & -0.255661 & 0 & $\mathrm{~m}$ & \\
\hline 31 & $\mathrm{~h}$ & 0 & 6.024601 & -2.797801 & -0.331097 & 0 & $\mathrm{~m}$ & \\
\hline 32 & $\mathrm{c}$ & 0 & 3.866926 & -2.865935 & -0.267519 & 0 & $\mathrm{~m}$ & \\
\hline 33 & $\mathrm{~h}$ & 0 & 3.812977 & -3.947479 & -0.356611 & 0 & $\mathrm{~m}$ & \\
\hline 34 & $\mathrm{c}$ & 0 & 2.684549 & -2.135383 & -0.175386 & 0 & $\mathrm{~m}$ & \\
\hline 35 & $\mathrm{~h}$ & 0 & 1.726855 & -2.640010 & -0.206917 & 0 & $\mathrm{~m}$ & \\
\hline 36 & $\mathrm{o}$ & 0 & -0.064939 & 0.454011 & -2.920085 & 0 & $\mathrm{~m}$ & \\
\hline 37 & $\mathrm{o}$ & 0 & 0.459350 & -1.543925 & -2.204764 & 0 & $\mathrm{~m}$ & \\
\hline
\end{tabular}




$\begin{array}{lllrrrrc}38 & \mathrm{o} & 0 & -0.565206 & -1.171679 & 3.068357 & 0 & \mathrm{~m} \\ 39 & \mathrm{o} & 0 & -2.693596 & -0.866654 & 3.380692 & 0 & \mathrm{~m} \\ 40 & \mathrm{n} & 0 & -4.860232 & -0.040385 & -0.964569 & 0 & \mathrm{~m} \\ 41 & \mathrm{o} & 0 & -5.042204 & 0.221978 & -2.153484 & 0 & \mathrm{~m} \\ 42 & \mathrm{o} & 0 & -5.745899 & -0.144771 & -0.117457 & 0 & \mathrm{~m}\end{array}$

radical

Charge $=0$ Multiplicity $=2$ for low level calculation on real system.

Charge $=0$ Multiplicity $=2$ for high level calculation on model system .

Charge $=0$ Multiplicity $=2$ for low level calculation on model system.

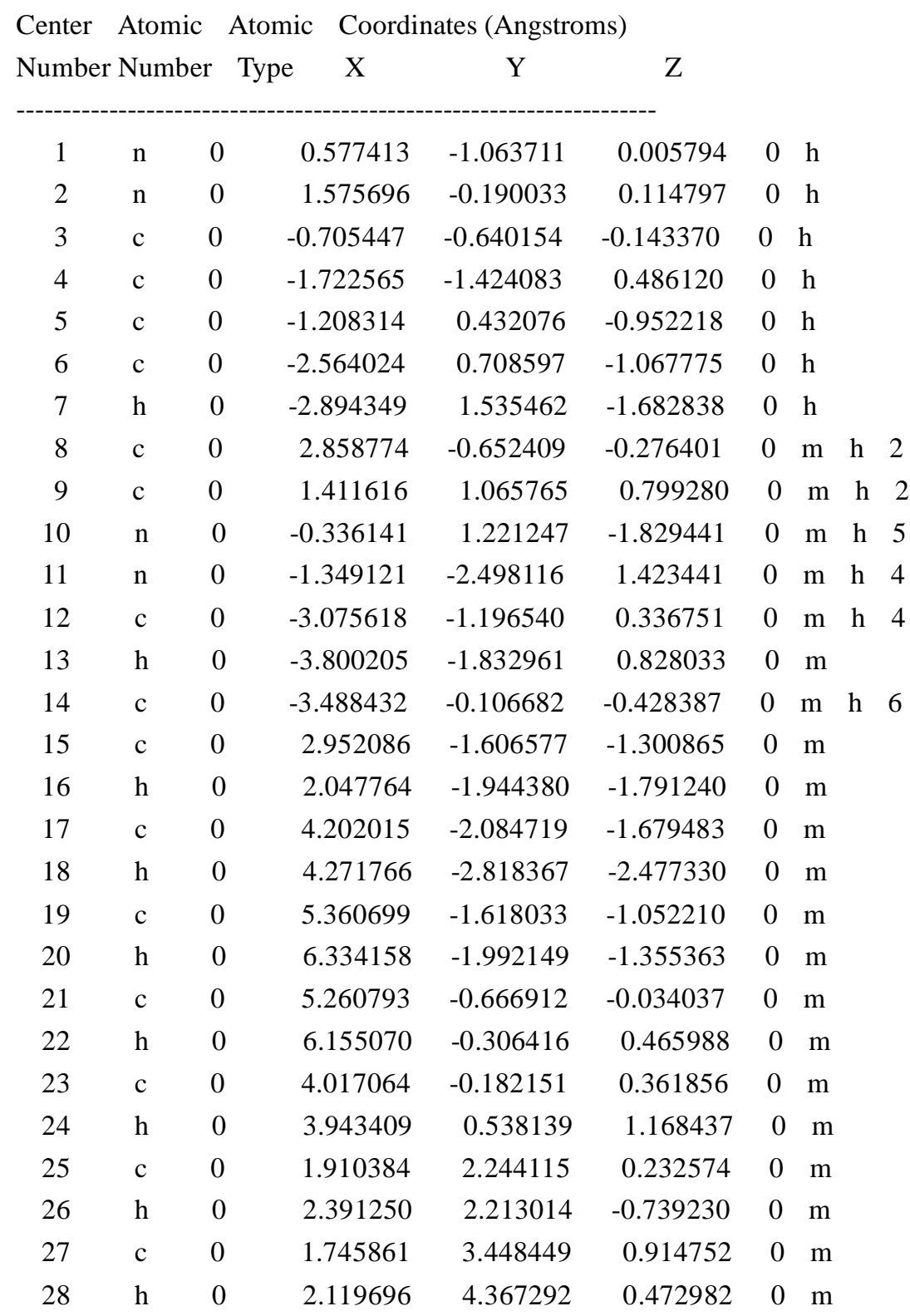




$\begin{array}{lllrrrrc}29 & \mathrm{c} & 0 & 1.089840 & 3.477106 & 2.147754 & 0 & \mathrm{~m} \\ 30 & \mathrm{~h} & 0 & 0.961863 & 4.419246 & 2.672750 & 0 & \mathrm{~m} \\ 31 & \mathrm{c} & 0 & 0.595778 & 2.294508 & 2.703918 & 0 & \mathrm{~m} \\ 32 & \mathrm{~h} & 0 & 0.092628 & 2.312329 & 3.666298 & 0 & \mathrm{~m} \\ 33 & \mathrm{c} & 0 & 0.755302 & 1.081626 & 2.036408 & 0 & \mathrm{~m} \\ 34 & \mathrm{~h} & 0 & 0.392163 & 0.151186 & 2.461858 & 0 & \mathrm{~m} \\ 35 & \mathrm{o} & 0 & 0.679564 & 0.678673 & -2.268813 & 0 & \mathrm{~m} \\ 36 & \mathrm{o} & 0 & -0.696135 & 2.363615 & -2.110053 & 0 & \mathrm{~m} \\ 37 & \mathrm{o} & 0 & -0.413395 & -2.293273 & 2.194782 & 0 & \mathrm{~m} \\ 38 & \mathrm{o} & 0 & -2.037192 & -3.515597 & 1.403072 & 0 & \mathrm{~m} \\ 39 & \mathrm{n} & 0 & -4.915592 & 0.174618 & -0.569458 & 0 & \mathrm{~m} \\ 40 & \mathrm{o} & 0 & -5.239265 & 1.145992 & -1.253070 & 0 & \mathrm{~m} \\ 41 & \mathrm{o} & 0 & -5.700561 & -0.578700 & 0.007298 & 0 & \mathrm{~m}\end{array}$

No.3

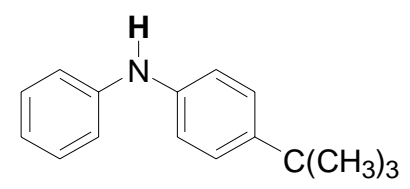

neutral molecule

Charge $=0$ Multiplicity $=1$ for low level calculation on real system.

Charge $=0$ Multiplicity $=1$ for high level calculation on model system .

Charge $=0$ Multiplicity $=1$ for low level calculation on model system .

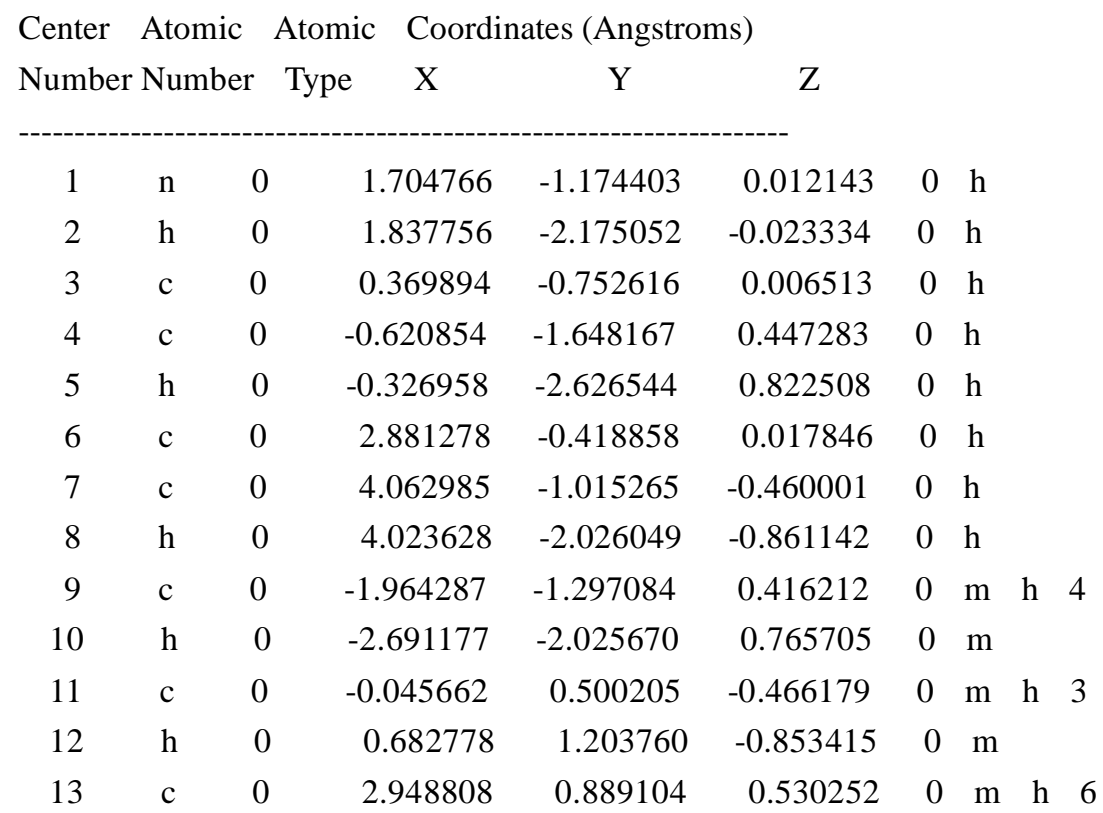




$\begin{array}{lllrrrlll}14 & \mathrm{~h} & 0 & 2.061256 & 1.353681 & 0.944368 & 0 & \mathrm{~m} \\ 15 & \mathrm{c} & 0 & 5.270497 & -0.323901 & -0.431466 & 0 & \mathrm{~m} & \mathrm{~h} \\ 16 & \mathrm{~h} & 0 & 6.168776 & -0.808496 & -0.805590 & 0 & \mathrm{~m} & \\ 17 & \mathrm{c} & 0 & 5.330191 & 0.982582 & 0.058829 & 0 & \mathrm{~m} \\ 18 & \mathrm{~h} & 0 & 6.270766 & 1.525275 & 0.070782 & 0 & \mathrm{~m} \\ 19 & \mathrm{c} & 0 & 4.161499 & 1.576565 & 0.537895 & 0 & \mathrm{~m} \\ 20 & \mathrm{~h} & 0 & 4.190587 & 2.586388 & 0.939586 & 0 & \mathrm{~m} \\ 21 & \mathrm{c} & 0 & -1.399207 & 0.841311 & -0.473770 & 0 & \mathrm{~m} \\ 22 & \mathrm{~h} & 0 & -1.666139 & 1.822931 & -0.850467 & 0 & \mathrm{~m} \\ 23 & \mathrm{c} & 0 & -2.397231 & -0.038444 & -0.036519 & 0 & \mathrm{~m} \\ 24 & \mathrm{c} & 0 & -3.895177 & 0.312087 & -0.037547 & 0 & \mathrm{~m} \\ 25 & \mathrm{c} & 0 & -4.664626 & -0.698372 & -0.921248 & 0 & \mathrm{~m} \\ 26 & \mathrm{~h} & 0 & -4.303735 & -0.667656 & -1.955675 & 0 & \mathrm{~m} \\ 27 & \mathrm{~h} & 0 & -4.550538 & -1.725549 & -0.558918 & 0 & \mathrm{~m} \\ 28 & \mathrm{~h} & 0 & -5.736615 & -0.464703 & -0.927108 & 0 & \mathrm{~m} \\ 29 & \mathrm{c} & 0 & -4.443350 & 0.245748 & 1.407962 & 0 & \mathrm{~m} & \\ 30 & \mathrm{~h} & 0 & -4.318373 & -0.750621 & 1.845587 & 0 & \mathrm{~m} \\ 31 & \mathrm{~h} & 0 & -5.514068 & 0.484681 & 1.424009 & 0 & \mathrm{~m} \\ 32 & \mathrm{~h} & 0 & -3.925173 & 0.961822 & 2.056007 & 0 & \mathrm{~m} \\ 33 & \mathrm{c} & 0 & -4.162460 & 1.726588 & -0.586309 & 0 & \mathrm{~m} \\ 34 & \mathrm{~h} & 0 & -5.238685 & 1.932928 & -0.570182 & 0 & \mathrm{~m} \\ 35 & \mathrm{~h} & 0 & -3.669444 & 2.497880 & 0.016439 & 0 & \mathrm{~m} \\ 36 & \mathrm{~h} & 0 & -3.821780 & 1.832914 & -1.622688 & 0 & \mathrm{~m} \\ -------------------------------------------------------- & & \end{array}$

radical

Charge $=0$ Multiplicity $=2$ for low level calculation on real system.

Charge $=0$ Multiplicity $=2$ for high level calculation on model system.

Charge $=0$ Multiplicity $=2$ for low level calculation on model system.

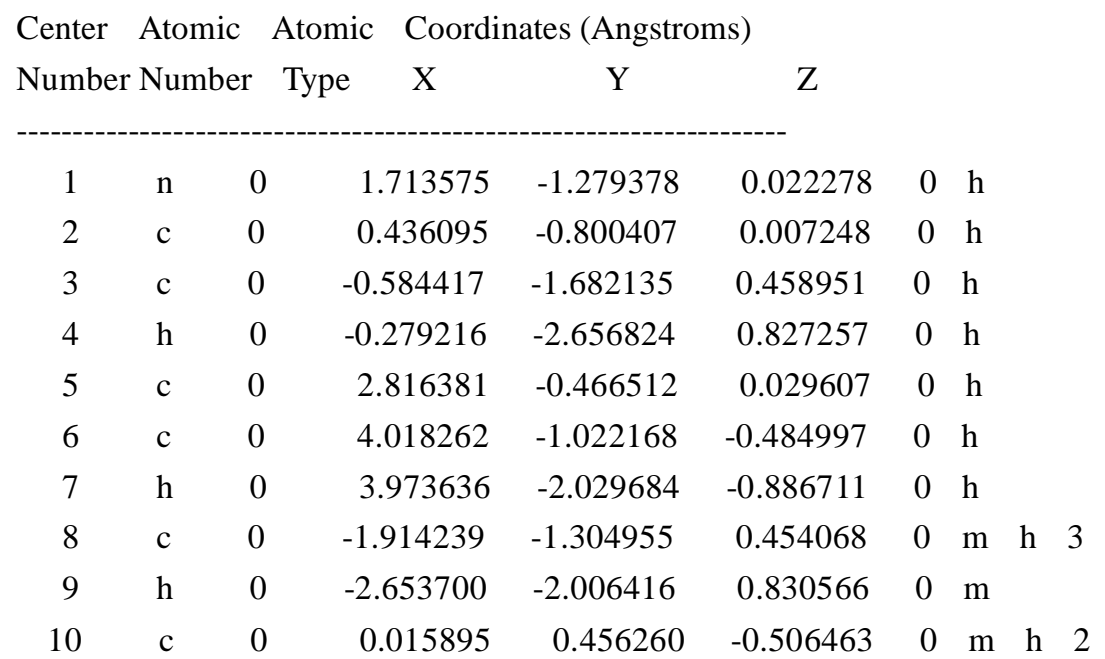




$\begin{array}{lllrrrllll}11 & \mathrm{~h} & 0 & 0.750606 & 1.130895 & -0.933144 & 0 & \mathrm{~m} & \\ 12 & \mathrm{c} & 0 & 2.878863 & 0.837422 & 0.594580 & 0 & \mathrm{~m} & \mathrm{~h} & 5 \\ 13 & \mathrm{~h} & 0 & 1.995181 & 1.255998 & 1.064361 & 0 & \mathrm{~m} & & \\ 14 & \mathrm{c} & 0 & 5.197813 & -0.292563 & -0.494567 & 0 & \mathrm{~m} & \mathrm{~h} & 6 \\ 15 & \mathrm{~h} & 0 & 6.099698 & -0.730917 & -0.913829 & 0 & \mathrm{~m} & & \\ 16 & \mathrm{c} & 0 & 5.235211 & 0.997711 & 0.050321 & 0 & \mathrm{~m} & \\ 17 & \mathrm{~h} & 0 & 6.163806 & 1.561556 & 0.058318 & 0 & \mathrm{~m} & \\ 18 & \mathrm{c} & 0 & 4.074381 & 1.546125 & 0.606893 & 0 & \mathrm{~m} & \\ 19 & \mathrm{~h} & 0 & 4.106340 & 2.533352 & 1.061060 & 0 & \mathrm{~m} & \\ 20 & \mathrm{c} & 0 & -1.329265 & 0.805564 & -0.531020 & 0 & \mathrm{~m} & \\ 21 & \mathrm{~h} & 0 & -1.598215 & 1.767933 & -0.952850 & 0 & \mathrm{~m} & \\ 22 & \mathrm{c} & 0 & -2.331215 & -0.049385 & -0.040409 & 0 & \mathrm{~m} & \\ 23 & \mathrm{c} & 0 & -3.822950 & 0.318935 & -0.041595 & 0 & \mathrm{~m} & \\ 24 & \mathrm{c} & 0 & -4.609730 & -0.712260 & -0.886470 & 0 & \mathrm{~m} & \\ 25 & \mathrm{~h} & 0 & -4.260549 & -0.716243 & -1.925211 & 0 & \mathrm{~m} & \\ 26 & \mathrm{~h} & 0 & -4.502772 & -1.728789 & -0.494153 & 0 & \mathrm{~m} & & \\ 27 & \mathrm{~h} & 0 & -5.678630 & -0.466052 & -0.887298 & 0 & \mathrm{~m} & & \\ 28 & \mathrm{c} & 0 & -4.355466 & 0.301049 & 1.412051 & 0 & \mathrm{~m} & & \\ 29 & \mathrm{~h} & 0 & -4.238232 & -0.682632 & 1.878746 & 0 & \mathrm{~m} & & \\ 30 & \mathrm{~h} & 0 & -5.422920 & 0.552723 & 1.428479 & 0 & \mathrm{~m} & & \\ 31 & \mathrm{~h} & 0 & -3.824559 & 1.031394 & 2.033226 & 0 & \mathrm{~m} & \\ 32 & \mathrm{c} & 0 & -4.082249 & 1.718955 & -0.629723 & 0 & \mathrm{~m} & & \\ 33 & \mathrm{~h} & 0 & -5.155779 & 1.936730 & -0.603738 & 0 & \mathrm{~m} & & \\ 34 & \mathrm{~h} & 0 & -3.572674 & 2.502823 & -0.057860 & 0 & \mathrm{~m} & \\ 35 & \mathrm{~h} & 0 & -3.757277 & 1.789973 & -1.674015 & 0 & \mathrm{~m} & & \\ ----------------------------------------------------------- & & & & \end{array}$

No.4

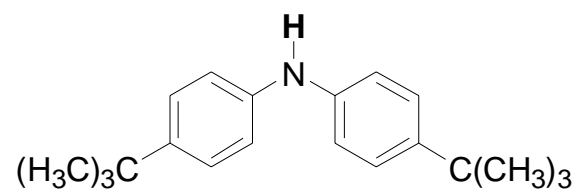

neutral molecule

Charge $=0$ Multiplicity $=1$ for low level calculation on real system.

Charge $=0$ Multiplicity $=1$ for high level calculation on model system.

Charge $=0$ Multiplicity $=1$ for low level calculation on model system.

\begin{tabular}{|c|c|c|c|c|c|c|}
\hline Center & Atomic & Atomic & Coordi & tes (Angstro & ms) & \\
\hline Number & Number & r Type & $\mathrm{X}$ & $\mathrm{Y}$ & $\mathrm{Z}$ & \\
\hline 1 & $\mathrm{n}$ & 0 & .000004 & 1.589099 & -0.000049 & 0 \\
\hline
\end{tabular}




\begin{tabular}{|c|c|c|c|c|c|c|c|c|}
\hline 2 & $\mathrm{~h}$ & 0 & 0.000021 & 2.599148 & 0.000032 & 0 & $\mathrm{~h}$ & \\
\hline 3 & $\mathrm{c}$ & 0 & 1.267112 & 0.996900 & -0.002199 & 0 & $\mathrm{~h}$ & \\
\hline 4 & $\mathrm{c}$ & 0 & 2.364001 & 1.753971 & 0.447858 & 0 & $\mathrm{~h}$ & \\
\hline 5 & $\mathrm{~h}$ & 0 & 2.197440 & 2.761007 & 0.825722 & 0 & $\mathrm{~h}$ & \\
\hline 6 & $\mathrm{c}$ & 0 & -1.267140 & 0.996942 & 0.002157 & 0 & $\mathrm{~h}$ & \\
\hline 7 & $\mathrm{c}$ & 0 & -2.364037 & 1.754028 & -0.447844 & 0 & $\mathrm{~h}$ & \\
\hline 8 & $\mathrm{~h}$ & 0 & -2.197482 & 2.761074 & -0.825689 & 0 & $\mathrm{~h}$ & \\
\hline 9 & $\mathrm{c}$ & 0 & 3.650426 & 1.231487 & 0.422820 & 0 & $\mathrm{~m}$ & $\mathrm{~h}$ \\
\hline 10 & $\mathrm{~h}$ & 0 & 4.463423 & 1.858045 & 0.780229 & 0 & $\mathrm{~m}$ & \\
\hline 11 & $\mathrm{c}$ & 0 & 1.519285 & -0.297263 & -0.479662 & 0 & $\mathrm{~m}$ & $\mathrm{~h}$ \\
\hline 12 & $\mathrm{~h}$ & 0 & 0.708125 & -0.898258 & -0.874783 & 0 & $\mathrm{~m}$ & \\
\hline 13 & $\mathrm{c}$ & 0 & -1.519291 & -0.297221 & 0.479551 & 0 & $\mathrm{~m}$ & h 6 \\
\hline 14 & $\mathrm{~h}$ & 0 & -0.708116 & -0.898239 & 0.874605 & 0 & $\mathrm{~m}$ & \\
\hline 15 & c & 0 & -3.650460 & 1.231545 & -0.422794 & 0 & $\mathrm{~m}$ & h 7 \\
\hline 16 & $\mathrm{~h}$ & 0 & -4.463451 & 1.858129 & -0.780163 & 0 & $\mathrm{~m}$ & \\
\hline 17 & $\mathrm{c}$ & 0 & -3.918656 & -0.071021 & 0.033502 & 0 & $\mathrm{~m}$ & \\
\hline 18 & $\mathrm{c}$ & 0 & -5.358145 & -0.614415 & 0.026579 & 0 & $\mathrm{~m}$ & \\
\hline 19 & $\mathrm{c}$ & 0 & -6.255511 & 0.281278 & 0.913580 & 0 & $\mathrm{~m}$ & \\
\hline 20 & $\mathrm{~h}$ & 0 & -5.900445 & 0.285909 & 1.950467 & 0 & $\mathrm{~m}$ & \\
\hline 21 & $\mathrm{~h}$ & 0 & -6.269810 & 1.318365 & 0.561745 & 0 & $\mathrm{~m}$ & \\
\hline 22 & $\mathrm{~h}$ & 0 & -7.289408 & -0.086017 & 0.908909 & 0 & $\mathrm{~m}$ & \\
\hline 23 & $\mathrm{c}$ & 0 & -5.906262 & -0.609984 & -1.420513 & 0 & $\mathrm{~m}$ & \\
\hline 24 & $\mathrm{~h}$ & 0 & -5.910499 & 0.397078 & -1.851023 & 0 & $\mathrm{~m}$ & \\
\hline 25 & $\mathrm{~h}$ & 0 & -6.936822 & -0.985838 & -1.442351 & 0 & $\mathrm{~m}$ & \\
\hline 26 & $\mathrm{~h}$ & 0 & -5.297261 & -1.247750 & -2.071345 & 0 & $\mathrm{~m}$ & \\
\hline 27 & $\mathrm{c}$ & 0 & -5.439983 & -2.055408 & 0.564423 & 0 & $\mathrm{~m}$ & \\
\hline 28 & $\mathrm{~h}$ & 0 & -6.479466 & -2.401675 & 0.538489 & 0 & $\mathrm{~m}$ & \\
\hline 29 & $\mathrm{~h}$ & 0 & -4.845137 & -2.750147 & -0.039511 & 0 & $\mathrm{~m}$ & \\
\hline 30 & $\mathrm{~h}$ & 0 & -5.095355 & -2.123359 & 1.602663 & 0 & $\mathrm{~m}$ & \\
\hline 31 & c & 0 & -2.817277 & -0.810987 & 0.481598 & 0 & $\mathrm{~m}$ & \\
\hline 32 & $\mathrm{~h}$ & 0 & -2.956691 & -1.817363 & 0.861809 & 0 & $\mathrm{~m}$ & \\
\hline 33 & $\mathrm{c}$ & 0 & 2.817289 & -0.811003 & -0.481696 & 0 & $\mathrm{~m}$ & \\
\hline 34 & $\mathrm{~h}$ & 0 & 2.956739 & -1.817348 & -0.861967 & 0 & $\mathrm{~m}$ & \\
\hline 35 & $\mathrm{c}$ & 0 & 3.918631 & -0.071058 & -0.033515 & 0 & $\mathrm{~m}$ & \\
\hline 36 & $\mathrm{c}$ & 0 & 5.358145 & -0.614411 & -0.026555 & 0 & $\mathrm{~m}$ & \\
\hline 37 & $\mathrm{c}$ & 0 & 5.440168 & -2.055350 & -0.564518 & 0 & $\mathrm{~m}$ & \\
\hline 38 & $\mathrm{~h}$ & 0 & 5.095587 & -2.123253 & -1.602776 & 0 & $\mathrm{~m}$ & \\
\hline 39 & $\mathrm{~h}$ & 0 & 4.845399 & -2.750224 & 0.039334 & 0 & $\mathrm{~m}$ & \\
\hline 40 & $\mathrm{~h}$ & 0 & 6.479693 & -2.401494 & -0.538591 & 0 & $\mathrm{~m}$ & \\
\hline 41 & $\mathrm{c}$ & 0 & 5.906138 & -0.610057 & 1.420594 & 0 & $\mathrm{~m}$ & \\
\hline 42 & $\mathrm{~h}$ & 0 & 5.297020 & -1.247778 & 2.071361 & 0 & $\mathrm{~m}$ & \\
\hline 43 & $\mathrm{~h}$ & 0 & 6.936661 & -0.986012 & 1.442497 & 0 & $\mathrm{~m}$ & \\
\hline 44 & $\mathrm{~h}$ & 0 & 5.910442 & 0.397005 & 1.851101 & 0 & $\mathrm{~m}$ & \\
\hline 45 & $\mathrm{c}$ & 0 & 6.255520 & 0.281435 & -0.913374 & 0 & $\mathrm{~m}$ & \\
\hline
\end{tabular}




$\begin{array}{llllrrrr}46 & \mathrm{~h} & 0 & 7.289487 & -0.085658 & -0.908473 & 0 & \mathrm{~m} \\ 47 & \mathrm{~h} & 0 & 6.269540 & 1.318531 & -0.561556 & 0 & \mathrm{~m} \\ 48 & \mathrm{~h} & 0 & 5.900676 & 0.285976 & -1.950339 & 0 & \mathrm{~m}\end{array}$

radical

Charge $=0$ Multiplicity $=2$ for low level calculation on real system.

Charge $=0$ Multiplicity $=2$ for high level calculation on model system .

Charge $=0$ Multiplicity $=2$ for low level calculation on model system.

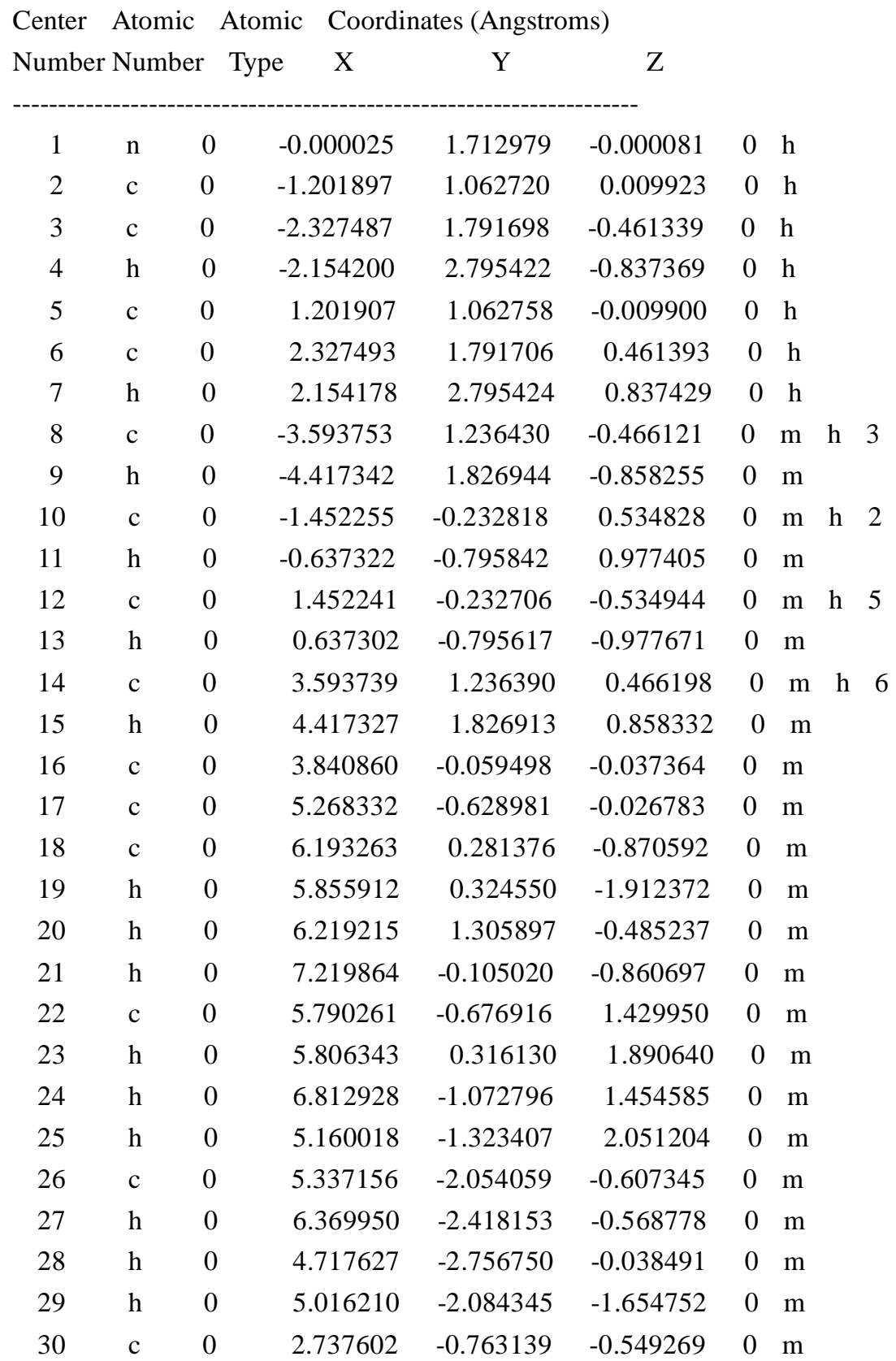




$\begin{array}{lllrrrrc}31 & \mathrm{~h} & 0 & 2.876857 & -1.749134 & -0.979395 & 0 & \mathrm{~m} \\ 32 & \mathrm{c} & 0 & -2.737635 & -0.763189 & 0.549151 & 0 & \mathrm{~m} \\ 33 & \mathrm{~h} & 0 & -2.876958 & -1.749224 & 0.979168 & 0 & \mathrm{~m} \\ 34 & \mathrm{c} & 0 & -3.840881 & -0.059467 & 0.037405 & 0 & \mathrm{~m} \\ 35 & \mathrm{c} & 0 & -5.268340 & -0.628965 & 0.026813 & 0 & \mathrm{~m} \\ 36 & \mathrm{c} & 0 & -5.336891 & -2.054346 & 0.606660 & 0 & \mathrm{~m} \\ 37 & \mathrm{~h} & 0 & -5.015646 & -2.085147 & 1.653963 & 0 & \mathrm{~m} \\ 38 & \mathrm{~h} & 0 & -4.717456 & -2.756695 & 0.037281 & 0 & \mathrm{~m} \\ 39 & \mathrm{~h} & 0 & -6.369661 & -2.418505 & 0.568182 & 0 & \mathrm{~m} \\ 40 & \mathrm{c} & 0 & -5.790544 & -0.676190 & -1.429851 & 0 & \mathrm{~m} \\ 41 & \mathrm{~h} & 0 & -5.160296 & -1.322209 & -2.051587 & 0 & \mathrm{~m} \\ 42 & \mathrm{~h} & 0 & -6.813135 & -1.072259 & -1.454490 & 0 & \mathrm{~m} \\ 43 & \mathrm{~h} & 0 & -5.806910 & 0.317112 & -1.889985 & 0 & \mathrm{~m} \\ 44 & \mathrm{c} & 0 & -6.193167 & 0.280903 & 0.871279 & 0 & \mathrm{~m} \\ 45 & \mathrm{~h} & 0 & -7.219769 & -0.105488 & 0.861290 & 0 & \mathrm{~m} \\ 46 & \mathrm{~h} & 0 & -6.219193 & 1.305655 & 0.486557 & 0 & \mathrm{~m} \\ 47 & \mathrm{~h} & 0 & -5.855692 & 0.323451 & 1.913044 & 0 & \mathrm{~m}\end{array}$

\section{No.5}

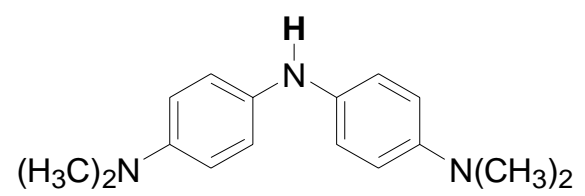

neutral molecule

Charge $=0$ Multiplicity $=1$ for low level calculation on real system.

Charge $=0$ Multiplicity $=1$ for high level calculation on model system.

Charge $=0$ Multiplicity $=1$ for low level calculation on model system.

Center Atomic Atomic Coordinates (Angstroms)

Number Number Type $\quad \mathrm{X} \quad \mathrm{Y} \quad \mathrm{Z}$

\begin{tabular}{|c|c|c|c|c|c|c|c|}
\hline 1 & $\mathrm{n}$ & 0 & -0.007715 & 1.512443 & -0.113846 & 0 & $\mathrm{~h}$ \\
\hline 2 & $\mathrm{~h}$ & 0 & -0.019414 & 2.518122 & -0.023654 & 0 & $\mathrm{~h}$ \\
\hline 3 & $\mathrm{c}$ & 0 & -1.265771 & 0.897969 & -0.096231 & 0 & $\mathrm{~h}$ \\
\hline 4 & $\mathrm{c}$ & 0 & -2.390047 & 1.637087 & -0.491790 & 0 & $\mathrm{~h}$ \\
\hline 5 & $\mathrm{~h}$ & 0 & -2.260064 & 2.662795 & -0.831864 & 0 & $\mathrm{~h}$ \\
\hline 6 & $\mathrm{c}$ & 0 & 1.261796 & 0.917453 & -0.044223 & 0 & $\mathrm{~h}$ \\
\hline 7 & $\mathrm{c}$ & 0 & 1.562757 & -0.313380 & -0.647108 & 0 & $\mathrm{~h}$ \\
\hline 8 & $\mathrm{~h}$ & 0 & 0.791591 & -0.850283 & -1.189631 & 0 & $\mathrm{~h}$ \\
\hline 9 & $\mathrm{c}$ & 0 & -3.672024 & 1.092109 & -0.448720 & 0 & $\mathrm{~m}$ \\
\hline
\end{tabular}




$\begin{array}{lllrrrlllll}10 & \mathrm{~h} & 0 & -4.507214 & 1.719095 & -0.740752 & 0 & \mathrm{~m} & & \\ 11 & \mathrm{c} & 0 & -1.476762 & -0.425536 & 0.332122 & 0 & \mathrm{~m} & \mathrm{~h} & 3 \\ 12 & \mathrm{~h} & 0 & -0.637832 & -1.026222 & 0.665563 & 0 & \mathrm{~m} & & \\ 13 & \mathrm{c} & 0 & 2.312727 & 1.598012 & 0.587312 & 0 & \mathrm{~m} & \mathrm{~h} & 6 \\ 14 & \mathrm{~h} & 0 & 2.118181 & 2.559956 & 1.057909 & 0 & \mathrm{~m} & \\ 15 & \mathrm{c} & 0 & 2.845655 & -0.849961 & -0.590041 & 0 & \mathrm{~m} & \mathrm{~h} & 7 \\ 16 & \mathrm{~h} & 0 & 3.017613 & -1.802062 & -1.078068 & 0 & \mathrm{~m} & \\ 17 & \mathrm{c} & 0 & 3.602727 & 1.078658 & 0.623087 & 0 & \mathrm{~m} & \\ 18 & \mathrm{~h} & 0 & 4.373476 & 1.660549 & 1.114276 & 0 & \mathrm{~m} & \\ 19 & \mathrm{c} & 0 & 3.904506 & -0.177664 & 0.056491 & 0 & \mathrm{~m} & \\ 20 & \mathrm{n} & 0 & 5.186692 & -0.736001 & 0.145171 & 0 & \mathrm{~m} & \\ 21 & \mathrm{c} & 0 & 6.288468 & 0.125583 & 0.536877 & 0 & \mathrm{~m} & \\ 22 & \mathrm{~h} & 0 & 6.129251 & 0.527392 & 1.543968 & 0 & \mathrm{~m} & \\ 23 & \mathrm{~h} & 0 & 6.441776 & 0.977146 & -0.148436 & 0 & \mathrm{~m} & \\ 24 & \mathrm{~h} & 0 & 7.208536 & -0.462834 & 0.563801 & 0 & \mathrm{~m} & \\ 25 & \mathrm{c} & 0 & 5.509966 & -1.862169 & -0.713603 & 0 & \mathrm{~m} & \\ 26 & \mathrm{~h} & 0 & 5.427478 & -1.629914 & -1.789443 & 0 & \mathrm{~m} & \\ 27 & \mathrm{~h} & 0 & 6.534215 & -2.182824 & -0.509426 & 0 & \mathrm{~m} & \\ 28 & \mathrm{~h} & 0 & 4.853479 & -2.713519 & -0.501252 & 0 & \mathrm{~m} & \\ 29 & \mathrm{c} & 0 & -2.751993 & -0.976847 & 0.337396 & 0 & \mathrm{~m} & \\ 30 & \mathrm{~h} & 0 & -2.866235 & -2.013853 & 0.639156 & 0 & \mathrm{~m} & \\ 31 & \mathrm{c} & 0 & -3.886495 & -0.236237 & -0.044330 & 0 & \mathrm{~m} & \\ 32 & \mathrm{n} & 0 & -5.166851 & -0.848288 & -0.053894 & 0 & \mathrm{~m} & \\ 33 & \mathrm{c} & 0 & -5.587560 & -1.502483 & 1.181703 & 0 & \mathrm{~m} & \\ 34 & \mathrm{~h} & 0 & -4.785988 & -2.123992 & 1.583796 & 0 & \mathrm{~m} & \\ 35 & \mathrm{~h} & 0 & -6.444181 & -2.153012 & 0.972952 & 0 & \mathrm{~m} & \\ 36 & \mathrm{~h} & 0 & -5.885009 & -0.781207 & 1.964989 & 0 & \mathrm{~m} & \\ 37 & \mathrm{c} & 0 & -6.247888 & -0.106024 & -0.675852 & 0 & \mathrm{~m} & & \\ 38 & \mathrm{~h} & 0 & -7.115935 & -0.766036 & -0.777567 & 0 & \mathrm{~m} & \\ 39 & \mathrm{~h} & 0 & -6.567766 & 0.783329 & -0.100070 & 0 & \mathrm{~m} & \\ 40 & \mathrm{~h} & 0 & -5.951207 & 0.220878 & -1.676806 & 0 & \mathrm{~m} & \\ ------------------------------------------------------------- & & & \end{array}$

radical

Charge $=0$ Multiplicity $=2$ for low level calculation on real system.

Charge $=0$ Multiplicity $=2$ for high level calculation on model system.

Charge $=0$ Multiplicity $=2$ for low level calculation on model system.

Center Atomic Atomic Coordinates (Angstroms)

Number Number Type $\quad \mathrm{X} \quad \mathrm{Y} \quad \mathrm{Z}$

$\begin{array}{rrrrrrrr}1 & \mathrm{n} & 0 & -0.000008 & 1.604107 & -0.000036 & 0 & \mathrm{~h} \\ 2 & \mathrm{c} & 0 & 1.200948 & 0.957073 & 0.003613 & 0 & \mathrm{~h}\end{array}$




\begin{tabular}{|c|c|c|c|c|c|c|c|c|}
\hline 3 & c & 0 & 2.330877 & 1.699132 & 0.443707 & 0 & $\mathrm{~h}$ & \\
\hline 4 & $\mathrm{~h}$ & 0 & 2.157077 & 2.716322 & 0.782040 & 0 & $\mathrm{~h}$ & \\
\hline 5 & $\mathrm{c}$ & 0 & -1.200953 & 0.957088 & -0.003715 & 0 & $\mathrm{~h}$ & \\
\hline 6 & $\mathrm{c}$ & 0 & -1.469639 & -0.352117 & 0.485130 & 0 & $\mathrm{~h}$ & \\
\hline 7 & $\mathrm{~h}$ & 0 & -0.663524 & -0.932631 & 0.921282 & 0 & $\mathrm{~h}$ & \\
\hline 8 & $\mathrm{c}$ & 0 & 3.604632 & 1.165430 & 0.468844 & 0 & $\mathrm{~m}$ & $\mathrm{~h}$ \\
\hline 9 & $\mathrm{~h}$ & 0 & 4.418728 & 1.782302 & 0.831014 & 0 & $\mathrm{~m}$ & \\
\hline 10 & $\mathrm{c}$ & 0 & 1.469682 & -0.352020 & -0.485442 & 0 & $\mathrm{~m}$ & $\mathrm{~h}$ \\
\hline 11 & $\mathrm{~h}$ & 0 & 0.663619 & -0.932444 & -0.921810 & 0 & $\mathrm{~m}$ & \\
\hline 12 & $\mathrm{c}$ & 0 & -2.330940 & 1.699229 & -0.443566 & 0 & $\mathrm{~m}$ & $\mathrm{~h}$ \\
\hline 13 & $\mathrm{~h}$ & 0 & -2.157157 & 2.716476 & -0.781744 & 0 & $\mathrm{~m}$ & \\
\hline 14 & $\mathrm{c}$ & 0 & -2.749476 & -0.882084 & 0.490038 & 0 & $\mathrm{~m}$ & $\mathrm{~h}$ \\
\hline 15 & $\mathrm{~h}$ & 0 & -2.893895 & -1.871136 & 0.909532 & 0 & $\mathrm{~m}$ & \\
\hline 16 & $\mathrm{c}$ & 0 & -3.604693 & 1.165535 & -0.468655 & 0 & $\mathrm{~m}$ & \\
\hline 17 & $\mathrm{~h}$ & 0 & -4.418827 & 1.782505 & -0.830575 & 0 & $\mathrm{~m}$ & \\
\hline 18 & $\mathrm{c}$ & 0 & -3.855339 & -0.155658 & -0.016343 & 0 & $\mathrm{~m}$ & \\
\hline 19 & $\mathrm{n}$ & 0 & -5.128532 & -0.707420 & -0.061018 & 0 & $\mathrm{~m}$ & \\
\hline 20 & $\mathrm{c}$ & 0 & -6.267315 & 0.143064 & -0.364052 & 0 & $\mathrm{~m}$ & \\
\hline 21 & $\mathrm{~h}$ & 0 & -6.409578 & 0.946523 & 0.376247 & 0 & $\mathrm{~m}$ & \\
\hline 22 & $\mathrm{~h}$ & 0 & -6.158112 & 0.607751 & -1.350994 & 0 & $\mathrm{~m}$ & \\
\hline 23 & $\mathrm{~h}$ & 0 & -7.172692 & -0.466837 & -0.385791 & 0 & $\mathrm{~m}$ & \\
\hline 24 & $\mathrm{c}$ & 0 & -5.380023 & -1.983353 & 0.586122 & 0 & $\mathrm{~m}$ & \\
\hline 25 & $\mathrm{~h}$ & 0 & -6.412992 & -2.282328 & 0.396837 & 0 & $\mathrm{~m}$ & \\
\hline 26 & $\mathrm{~h}$ & 0 & -4.729059 & -2.763905 & 0.175646 & 0 & $\mathrm{~m}$ & \\
\hline 27 & $\mathrm{~h}$ & 0 & -5.224890 & -1.949424 & 1.676738 & 0 & $\mathrm{~m}$ & \\
\hline 28 & $\mathrm{c}$ & 0 & 2.749523 & -0.881997 & -0.490289 & 0 & $\mathrm{~m}$ & \\
\hline 29 & $\mathrm{~h}$ & 0 & 2.893957 & -1.870957 & -0.909992 & 0 & $\mathrm{~m}$ & \\
\hline 30 & $\mathrm{c}$ & 0 & 3.855326 & -0.155691 & 0.016335 & 0 & $\mathrm{~m}$ & \\
\hline 31 & $\mathrm{n}$ & 0 & 5.128531 & -0.707415 & 0.061121 & 0 & $\mathrm{~m}$ & \\
\hline 32 & $\mathrm{c}$ & 0 & 5.380131 & -1.983403 & -0.585854 & 0 & $\mathrm{~m}$ & \\
\hline 33 & $\mathrm{~h}$ & 0 & 5.225436 & -1.949529 & -1.676537 & 0 & $\mathrm{~m}$ & \\
\hline 34 & $\mathrm{~h}$ & 0 & 4.728902 & -2.763831 & -0.175575 & 0 & $\mathrm{~m}$ & \\
\hline 35 & $\mathrm{~h}$ & 0 & 6.412976 & -2.282514 & -0.396101 & 0 & $\mathrm{~m}$ & \\
\hline 36 & $\mathrm{c}$ & 0 & 6.267256 & 0.143224 & 0.363991 & 0 & $\mathrm{~m}$ & \\
\hline 37 & $\mathrm{~h}$ & 0 & 7.172773 & -0.466484 & 0.385070 & 0 & $\mathrm{~m}$ & \\
\hline 38 & $\mathrm{~h}$ & 0 & 6.158378 & 0.607539 & 1.351154 & 0 & $\mathrm{~m}$ & \\
\hline 39 & $\mathrm{~h}$ & 0 & 6.408969 & 0.946972 & -0.376091 & 0 & $\mathrm{~m}$ & \\
\hline
\end{tabular}




\section{No.6}

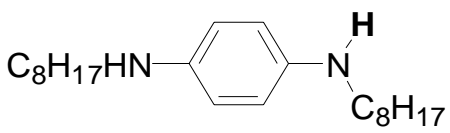

neutral molecule

Charge $=0$ Multiplicity $=1$ for low level calculation on real system.

Charge $=0$ Multiplicity $=1$ for high level calculation on model system .

Charge $=0$ Multiplicity $=1$ for low level calculation on model system .

$\begin{array}{lcccc}\text { Center Atomic } & \text { Atomic } & \text { Coordinates (Angstroms) } & \\ \text { Number Number } & \text { Type } & \text { X } & \text { Y } & \text { Z }\end{array}$

\begin{tabular}{|c|c|c|c|c|c|c|c|c|}
\hline 1 & $\mathrm{n}$ & 0 & -3.104088 & -3.182892 & -0.145930 & 0 & h & \\
\hline 2 & $\mathrm{~h}$ & 0 & -3.307099 & -4.172913 & -0.125789 & 0 & $\mathrm{~h}$ & \\
\hline 3 & $\mathrm{c}$ & 0 & -1.728945 & -2.915174 & -0.080092 & 0 & h & \\
\hline 4 & $\mathrm{c}$ & 0 & -0.808589 & -3.920956 & -0.435121 & 0 & h & \\
\hline 5 & $\mathrm{~h}$ & 0 & -1.178852 & -4.899616 & -0.736214 & 0 & $\mathrm{~h}$ & \\
\hline 6 & c & 0 & 0.558914 & -3.692625 & -0.407537 & 0 & $\mathrm{~h}$ & \\
\hline 7 & $\mathrm{~h}$ & 0 & 1.239393 & -4.499524 & -0.673871 & 0 & $\mathrm{~h}$ & \\
\hline 8 & $\mathrm{c}$ & 0 & -1.205623 & -1.669858 & 0.293760 & 0 & $\mathrm{~h}$ & \\
\hline 9 & $\mathrm{~h}$ & 0 & -1.870156 & -0.855826 & 0.563869 & 0 & $\mathrm{~h}$ & \\
\hline 10 & $\mathrm{c}$ & 0 & -4.081952 & -2.368777 & 0.567995 & 0 & $\mathrm{~h}$ & \\
\hline 11 & $\mathrm{~h}$ & 0 & -3.645008 & -1.958094 & 1.492933 & 0 & $\mathrm{~h}$ & \\
\hline 12 & $\mathrm{~h}$ & 0 & -4.899890 & -3.029777 & 0.880558 & 0 & $\mathrm{~h}$ & \\
\hline 13 & $\mathrm{c}$ & 0 & 1.086472 & -2.442702 & -0.031000 & 0 & $\mathrm{~m} \quad \mathrm{~h}$ & 6 \\
\hline 14 & $\mathrm{c}$ & 0 & 0.173207 & -1.437254 & 0.312918 & 0 & $\mathrm{~m} \mathrm{~h}$ & 8 \\
\hline 15 & $\mathrm{~h}$ & 0 & 0.526633 & -0.453316 & 0.603164 & 0 & $\mathrm{~m}$ & \\
\hline 16 & $\mathrm{c}$ & 0 & -4.667666 & -1.230261 & -0.281805 & 0 & $\mathrm{~m} \mathrm{~h}$ & 10 \\
\hline 17 & $\mathrm{~h}$ & 0 & -3.853510 & -0.592584 & -0.650345 & 0 & $\mathrm{~m}$ & \\
\hline 18 & $\mathrm{~h}$ & 0 & -5.137340 & -1.668378 & -1.172576 & 0 & $\mathrm{~m}$ & \\
\hline 19 & $\mathrm{n}$ & 0 & 2.480243 & -2.267244 & 0.027341 & 0 & $\mathrm{~m}$ & \\
\hline 20 & $\mathrm{~h}$ & 0 & 2.969077 & -2.875712 & -0.620341 & 0 & $\mathrm{~m}$ & \\
\hline 21 & $\mathrm{c}$ & 0 & 3.049730 & -0.928222 & 0.061650 & 0 & $\mathrm{~m}$ & \\
\hline 22 & $\mathrm{~h}$ & 0 & 2.660247 & -0.289151 & -0.752993 & 0 & $\mathrm{~m}$ & \\
\hline 23 & $\mathrm{~h}$ & 0 & 2.752246 & -0.447170 & 1.002979 & 0 & $\mathrm{~m}$ & \\
\hline 24 & $\mathrm{c}$ & 0 & 4.577192 & -0.981984 & -0.008289 & 0 & $\mathrm{~m}$ & \\
\hline 25 & $\mathrm{~h}$ & 0 & 4.947359 & -1.611818 & 0.811599 & 0 & $\mathrm{~m}$ & \\
\hline 26 & $\mathrm{~h}$ & 0 & 4.880372 & -1.476960 & -0.943891 & 0 & $\mathrm{~m}$ & \\
\hline 27 & $\mathrm{c}$ & 0 & 5.225573 & 0.405869 & 0.060825 & 0 & $\mathrm{~m}$ & \\
\hline 28 & $\mathrm{~h}$ & 0 & 4.836377 & 1.030835 & -0.756288 & 0 & $\mathrm{~m}$ & \\
\hline 29 & $\mathrm{~h}$ & 0 & 4.921673 & 0.901698 & 0.994365 & 0 & $\mathrm{~m}$ & \\
\hline 30 & $\mathrm{c}$ & 0 & 6.757480 & 0.369461 & -0.016224 & 0 & $\mathrm{~m}$ & \\
\hline
\end{tabular}




$\begin{array}{lllrrrrc}31 & \mathrm{~h} & 0 & 7.148070 & -0.250736 & 0.804022 & 0 & \mathrm{~m} \\ 32 & \mathrm{~h} & 0 & 7.062951 & -0.131067 & -0.947110 & 0 & \mathrm{~m} \\ 33 & \mathrm{c} & 0 & 7.402746 & 1.759743 & 0.046581 & 0 & \mathrm{~m} \\ 34 & \mathrm{~h} & 0 & 7.011013 & 2.378223 & -0.774749 & 0 & \mathrm{~m} \\ 35 & \mathrm{~h} & 0 & 7.094608 & 2.260951 & 0.976262 & 0 & \mathrm{~m} \\ 36 & \mathrm{c} & 0 & 8.934691 & 1.730323 & -0.028593 & 0 & \mathrm{~m} \\ 37 & \mathrm{~h} & 0 & 9.327910 & 1.115754 & 0.795089 & 0 & \mathrm{~m} \\ 38 & \mathrm{~h} & 0 & 9.244019 & 1.226115 & -0.956458 & 0 & \mathrm{~m} \\ 39 & \mathrm{c} & 0 & 9.578137 & 3.121793 & 0.028098 & 0 & \mathrm{~m} \\ 40 & \mathrm{~h} & 0 & 9.186654 & 3.735022 & -0.796090 & 0 & \mathrm{~m} \\ 41 & \mathrm{~h} & 0 & 9.268603 & 3.626048 & 0.954620 & 0 & \mathrm{~m} \\ 42 & \mathrm{c} & 0 & 11.108004 & 3.083569 & -0.046100 & 0 & \mathrm{~m} \\ 43 & \mathrm{~h} & 0 & 11.530823 & 2.507789 & 0.786543 & 0 & \mathrm{~m} \\ 44 & \mathrm{~h} & 0 & 11.447671 & 2.613835 & -0.977557 & 0 & \mathrm{~m} \\ 45 & \mathrm{~h} & 0 & 11.537204 & 4.091262 & -0.005711 & 0 & \mathrm{~m} \\ 46 & \mathrm{c} & 0 & -5.685333 & -0.382041 & 0.489929 & 0 & \mathrm{~m} \\ 47 & \mathrm{~h} & 0 & -5.201879 & 0.050375 & 1.378982 & 0 & \mathrm{~m} \\ 48 & \mathrm{~h} & 0 & -6.488314 & -1.031940 & 0.870248 & 0 & \mathrm{~m} \\ 49 & \mathrm{c} & 0 & -6.305334 & 0.746016 & -0.345540 & 0 & \mathrm{~m} \\ 50 & \mathrm{~h} & 0 & -5.505322 & 1.397710 & -0.726785 & 0 & \mathrm{~m} \\ 51 & \mathrm{~h} & 0 & -6.792458 & 0.314403 & -1.232406 & 0 & \mathrm{~m} \\ 52 & \mathrm{c} & 0 & -7.322484 & 1.591914 & 0.430778 & 0 & \mathrm{~m} \\ 53 & \mathrm{~h} & 0 & -6.833007 & 2.026862 & 1.315042 & 0 & \mathrm{~m} \\ 54 & \mathrm{~h} & 0 & -8.118092 & 0.937298 & 0.817100 & 0 & \mathrm{~m} \\ 55 & \mathrm{c} & 0 & -7.952975 & 2.715585 & -0.402168 & 0 & \mathrm{~m} \\ 56 & \mathrm{~h} & 0 & -7.159191 & 3.372655 & -0.788102 & 0 & \mathrm{~m} \\ 57 & \mathrm{~h} & 0 & -8.442732 & 2.281569 & -1.286836 & 0 & \mathrm{~m} \\ 58 & \mathrm{c} & 0 & -8.971582 & 3.558224 & 0.375782 & 0 & \mathrm{~m} \\ 59 & \mathrm{~h} & 0 & -8.482136 & 3.993853 & 1.258598 & 0 & \mathrm{~m} \\ 60 & \mathrm{~h} & 0 & -9.764291 & 2.901618 & 0.762010 & 0 & \mathrm{~m} \\ 61 & \mathrm{c} & 0 & -9.599065 & 4.676302 & -0.463225 & 0 & \mathrm{~m} \\ 62 & \mathrm{~h} & 0 & -8.834941 & 5.371302 & -0.832899 & 0 & \mathrm{~m} \\ & \mathrm{~h} & 0 & -10.323011 & 5.257344 & 0.119620 & 0 & \mathrm{~m} \\ & 0 & -10.124351 & 4.269338 & -1.336206 & 0 & \mathrm{~m} \\ & & & & & \end{array}$

radical

Charge $=0$ Multiplicity $=2$ for low level calculation on real system.

Charge $=0$ Multiplicity $=2$ for high level calculation on model system.

Charge $=0$ Multiplicity $=2$ for low level calculation on model system.

Center Atomic Atomic Coordinates (Angstroms)

Number Number Type $\quad \mathrm{X} \quad \mathrm{Y} \quad \mathrm{Z}$ 


\begin{tabular}{|c|c|c|c|c|c|c|c|c|}
\hline 1 & $\mathrm{n}$ & 0 & 2.686466 & 0.899947 & -0.049021 & 0 & $\mathrm{~h}$ & \\
\hline 2 & $\mathrm{c}$ & 0 & 1.432600 & 0.426347 & -0.063894 & 0 & $\mathrm{~h}$ & \\
\hline 3 & $\mathrm{c}$ & 0 & 0.372818 & 1.389166 & -0.106560 & 0 & $\mathrm{~h}$ & \\
\hline 4 & $\mathrm{~h}$ & 0 & 0.663719 & 2.435270 & -0.120172 & 0 & $\mathrm{~h}$ & \\
\hline 5 & $\mathrm{c}$ & 0 & -0.957475 & 1.028413 & -0.125428 & 0 & $\mathrm{~h}$ & \\
\hline 6 & $\mathrm{~h}$ & 0 & -1.721574 & 1.798313 & -0.153663 & 0 & $\mathrm{~h}$ & \\
\hline 7 & $\mathrm{c}$ & 0 & 1.029989 & -0.952451 & -0.039760 & 0 & $\mathrm{~h}$ & \\
\hline 8 & $\mathrm{~h}$ & 0 & 1.784629 & -1.731362 & -0.004668 & 0 & $\mathrm{~h}$ & \\
\hline 9 & $\mathrm{c}$ & 0 & 3.780661 & -0.050261 & -0.012909 & 0 & $\mathrm{~h}$ & \\
\hline 10 & $\mathrm{~h}$ & 0 & 3.710458 & -0.702892 & 0.876781 & 0 & $\mathrm{~h}$ & \\
\hline 11 & $\mathrm{~h}$ & 0 & 3.742276 & -0.730159 & -0.883900 & 0 & $\mathrm{~h}$ & \\
\hline 12 & $\mathrm{c}$ & 0 & -1.330040 & -0.338719 & -0.104178 & 0 & $\mathrm{~m}$ & h 5 \\
\hline 13 & $\mathrm{c}$ & 0 & -0.299792 & -1.312084 & -0.059010 & 0 & $\mathrm{~m}$ & \\
\hline 14 & $\mathrm{~h}$ & 0 & -0.575630 & -2.365580 & -0.044726 & 0 & $\mathrm{~m}$ & \\
\hline 15 & $\mathrm{c}$ & 0 & 5.126249 & 0.680724 & -0.000251 & 0 & $\mathrm{~m}$ & h 9 \\
\hline 16 & $\mathrm{~h}$ & 0 & 5.174159 & 1.334995 & -0.881009 & 0 & $\mathrm{~m}$ & \\
\hline 17 & $\mathrm{~h}$ & 0 & 5.154322 & 1.344277 & 0.874478 & 0 & $\mathrm{~m}$ & \\
\hline 18 & $\mathrm{n}$ & 0 & -2.646062 & -0.743575 & -0.154646 & 0 & $\mathrm{~m}$ & \\
\hline 19 & $\mathrm{~h}$ & 0 & -2.816024 & -1.709712 & 0.091848 & 0 & $\mathrm{~m}$ & \\
\hline 20 & $\mathrm{c}$ & 0 & -3.778995 & 0.153695 & 0.007758 & 0 & $\mathrm{~m}$ & \\
\hline 21 & $\mathrm{~h}$ & 0 & -3.754139 & 0.905297 & -0.792619 & 0 & $\mathrm{~m}$ & \\
\hline 22 & $\mathrm{~h}$ & 0 & -3.717920 & 0.703977 & 0.962021 & 0 & $\mathrm{~m}$ & \\
\hline 23 & $\mathrm{c}$ & 0 & -5.098091 & -0.618074 & -0.063228 & 0 & $\mathrm{~m}$ & \\
\hline 24 & $\mathrm{~h}$ & 0 & -5.131604 & -1.178972 & -1.007269 & 0 & $\mathrm{~m}$ & \\
\hline 25 & $\mathrm{~h}$ & 0 & -5.125759 & -1.362134 & 0.747099 & 0 & $\mathrm{~m}$ & \\
\hline 26 & $\mathrm{c}$ & 0 & -6.326728 & 0.293723 & 0.042175 & 0 & $\mathrm{~m}$ & \\
\hline 27 & $\mathrm{~h}$ & 0 & -6.303026 & 1.028003 & -0.775943 & 0 & $\mathrm{~m}$ & \\
\hline 28 & $\mathrm{~h}$ & 0 & -6.272655 & 0.873497 & 0.975043 & 0 & $\mathrm{~m}$ & \\
\hline 29 & $\mathrm{c}$ & 0 & -7.655430 & -0.472032 & -0.002604 & 0 & $\mathrm{~m}$ & \\
\hline 30 & $\mathrm{~h}$ & 0 & -7.685340 & -1.196192 & 0.824886 & 0 & $\mathrm{~m}$ & \\
\hline 31 & $\mathrm{~h}$ & 0 & -7.701476 & -1.065360 & -0.927707 & 0 & $\mathrm{~m}$ & \\
\hline 32 & $\mathrm{c}$ & 0 & -8.886521 & 0.440009 & 0.076087 & 0 & $\mathrm{~m}$ & \\
\hline 33 & $\mathrm{~h}$ & 0 & -8.857607 & 1.159840 & -0.755295 & 0 & $\mathrm{~m}$ & \\
\hline 34 & $\mathrm{~h}$ & 0 & -8.836662 & 1.038994 & 0.997550 & 0 & $\mathrm{~m}$ & \\
\hline 35 & $\mathrm{c}$ & 0 & -10.217014 & -0.322659 & 0.039258 & 0 & $\mathrm{~m}$ & \\
\hline 36 & $\mathrm{~h}$ & 0 & -10.249561 & -1.038224 & 0.874452 & 0 & $\mathrm{~m}$ & \\
\hline 37 & $\mathrm{~h}$ & 0 & -10.264801 & -0.926840 & -0.879115 & 0 & $\mathrm{~m}$ & \\
\hline 38 & $\mathrm{c}$ & 0 & -11.448398 & 0.589868 & 0.107699 & 0 & $\mathrm{~m}$ & \\
\hline 39 & $\mathrm{~h}$ & 0 & -11.416484 & 1.303320 & -0.728004 & 0 & $\mathrm{~m}$ & \\
\hline 40 & $\mathrm{~h}$ & 0 & -11.400265 & 1.194401 & 1.024654 & 0 & $\mathrm{~m}$ & \\
\hline 41 & $\mathrm{c}$ & 0 & -12.773104 & -0.179011 & 0.072102 & 0 & $\mathrm{~m}$ & \\
\hline 42 & $\mathrm{~h}$ & 0 & -13.631750 & 0.500077 & 0.123762 & 0 & $\mathrm{~m}$ & \\
\hline 43 & $\mathrm{~h}$ & 0 & -12.849267 & -0.876892 & 0.915096 & 0 & $\mathrm{~m}$ & \\
\hline
\end{tabular}




$\begin{array}{rrrrrrrc}44 & \mathrm{~h} & 0 & -12.866950 & -0.764813 & -0.850664 & 0 & \mathrm{~m} \\ 45 & \mathrm{c} & 0 & 6.332545 & -0.264577 & 0.017643 & 0 & \mathrm{~m} \\ 46 & \mathrm{~h} & 0 & 6.269923 & -0.924644 & 0.896333 & 0 & \mathrm{~m} \\ 47 & \mathrm{~h} & 0 & 6.292187 & -0.927093 & -0.860473 & 0 & \mathrm{~m} \\ 48 & \mathrm{c} & 0 & 7.680329 & 0.468427 & 0.033612 & 0 & \mathrm{~m} \\ 49 & \mathrm{~h} & 0 & 7.740602 & 1.133433 & -0.840659 & 0 & \mathrm{~m} \\ 50 & \mathrm{~h} & 0 & 7.725064 & 1.124419 & 0.915583 & 0 & \mathrm{~m} \\ 51 & \mathrm{c} & 0 & 8.892353 & -0.471913 & 0.038913 & 0 & \mathrm{~m} \\ 52 & \mathrm{~h} & 0 & 8.831216 & -1.139145 & 0.911804 & 0 & \mathrm{~m} \\ 53 & \mathrm{~h} & 0 & 8.848280 & -1.125837 & -0.844992 & 0 & \mathrm{~m} \\ 54 & \mathrm{c} & 0 & 10.239052 & 0.262569 & 0.057556 & 0 & \mathrm{~m} \\ 55 & \mathrm{~h} & 0 & 10.299635 & 0.932845 & -0.813035 & 0 & \mathrm{~m} \\ 56 & \mathrm{~h} & 0 & 10.285988 & 0.913501 & 0.943522 & 0 & \mathrm{~m} \\ 57 & \mathrm{c} & 0 & 11.452007 & -0.676627 & 0.056424 & 0 & \mathrm{~m} \\ 58 & \mathrm{~h} & 0 & 11.391557 & -1.347163 & 0.925639 & 0 & \mathrm{~m} \\ 59 & \mathrm{~h} & 0 & 11.406476 & -1.325270 & -0.830130 & 0 & \mathrm{~m} \\ 60 & \mathrm{c} & 0 & 12.792449 & 0.065241 & 0.077164 & 0 & \mathrm{~m} \\ 61 & \mathrm{~h} & 0 & 13.637618 & -0.632799 & 0.074946 & 0 & \mathrm{~m} \\ 62 & \mathrm{~h} & 0 & 12.897670 & 0.718304 & -0.798073 & 0 & \mathrm{~m} \\ 63 & \mathrm{~h} & 0 & 12.883092 & 0.695077 & 0.970860 & 0 & \mathrm{~m} \\ ---------------------------------------------------------- & & \end{array}$

No.7

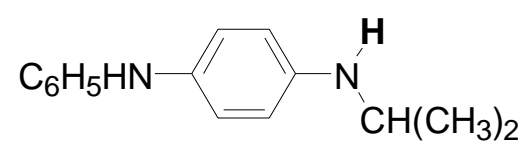

neutral molecule

Charge $=0$ Multiplicity $=1$ for low level calculation on real system.

Charge $=0$ Multiplicity $=1$ for high level calculation on model system.

Charge $=0$ Multiplicity $=1$ for low level calculation on model system.

\begin{tabular}{|c|c|c|c|c|c|c|}
\hline Center & Atomic & Atomi & Coordi & ates (Angstr & & \\
\hline Number & Numbe & ar Type & $\mathrm{X}$ & $\mathrm{Y}$ & $\mathrm{Z}$ & \\
\hline 1 & $\mathrm{n}$ & 0 & 3.564952 & -0.680158 & -0.514346 & $0 \mathrm{~h}$ \\
\hline 2 & $\mathrm{~h}$ & 0 & 3.570195 & -1.697448 & -0.552634 & $0 \mathrm{~h}$ \\
\hline 3 & $\mathrm{c}$ & 0 & 4.543566 & -0.268245 & 0.522652 & $0 \mathrm{~h}$ \\
\hline 4 & $\mathrm{~h}$ & 0 & 4.144124 & -0.478991 & 1.531980 & $0 \mathrm{~h}$ \\
\hline 5 & $\mathrm{c}$ & 0 & 2.214754 & -0.240598 & -0.361716 & $0 \mathrm{~h}$ \\
\hline 6 & $\mathrm{c}$ & 0 & 1.230930 & -1.064044 & 0.203335 & $0 \mathrm{~h}$ \\
\hline 7 & $\mathrm{~h}$ & 0 & 1.511001 & -2.045355 & 0.582212 & $0 \mathrm{~h}$ \\
\hline
\end{tabular}




$\begin{array}{rrrrrrllll}8 & \mathrm{c} & 0 & 1.817154 & 1.023090 & -0.823951 & 0 & \mathrm{~h} & & \\ 9 & \mathrm{~h} & 0 & 2.548802 & 1.662191 & -1.307607 & 0 & \mathrm{~h} & & \\ 10 & \mathrm{c} & 0 & 0.496206 & 1.443667 & -0.715867 & 0 & \mathrm{~h} & \\ 11 & \mathrm{~h} & 0 & 0.209703 & 2.420085 & -1.101062 & 0 & \mathrm{~h} & & \\ 12 & \mathrm{c} & 0 & 5.813764 & -1.100082 & 0.324875 & 0 & \mathrm{~m} & \mathrm{~h} & 3 \\ 13 & \mathrm{~h} & 0 & 5.608055 & -2.174061 & 0.421040 & 0 & \mathrm{~m} & & \\ 14 & \mathrm{~h} & 0 & 6.568576 & -0.838549 & 1.073950 & 0 & \mathrm{~m} & \\ 15 & \mathrm{~h} & 0 & 6.235281 & -0.922683 & -0.671096 & 0 & \mathrm{~m} & & \\ 16 & \mathrm{c} & 0 & 4.853115 & 1.228408 & 0.445409 & 0 & \mathrm{~m} & \mathrm{~h} & 3 \\ 17 & \mathrm{~h} & 0 & 5.604910 & 1.494451 & 1.197448 & 0 & \mathrm{~m} & \\ 18 & \mathrm{~h} & 0 & 5.244139 & 1.487946 & -0.545411 & 0 & \mathrm{~m} & & \\ 19 & \mathrm{~h} & 0 & 3.963506 & 1.835533 & 0.636060 & 0 & \mathrm{~m} & \\ 20 & \mathrm{c} & 0 & -0.097024 & -0.654612 & 0.310766 & 0 & \mathrm{~m} & \mathrm{~h} & 6 \\ 21 & \mathrm{~h} & 0 & -0.822613 & -1.306827 & 0.783912 & 0 & \mathrm{~m} & \\ 22 & \mathrm{c} & 0 & -0.487052 & 0.612871 & -0.150121 & 0 & \mathrm{~m} & \mathrm{~h} & 10 \\ 23 & \mathrm{n} & 0 & -1.794480 & 1.104122 & -0.027650 & 0 & \mathrm{~m} & \\ 24 & \mathrm{~h} & 0 & -1.873081 & 2.109999 & 0.016753 & 0 & \mathrm{~m} & & \\ 25 & \mathrm{c} & 0 & -3.006117 & 0.410320 & 0.029590 & 0 & \mathrm{~m} & & \\ 26 & \mathrm{c} & 0 & -3.169839 & -0.892087 & -0.475912 & 0 & \mathrm{~m} & & \\ 27 & \mathrm{~h} & 0 & -2.331876 & -1.402179 & -0.937274 & 0 & \mathrm{~m} & & \\ 28 & \mathrm{c} & 0 & -4.415080 & -1.515678 & -0.413926 & 0 & \mathrm{~m} & & \\ 29 & \mathrm{~h} & 0 & -4.518963 & -2.521991 & -0.812178 & 0 & \mathrm{~m} & & \\ 30 & \mathrm{c} & 0 & -5.522639 & -0.863678 & 0.130427 & 0 & \mathrm{~m} & & \\ 31 & \mathrm{~h} & 0 & -6.488963 & -1.357431 & 0.172371 & 0 & \mathrm{~m} & & \\ 32 & \mathrm{c} & 0 & -5.367676 & 0.436898 & 0.615833 & 0 & \mathrm{~m} & & \\ 33 & \mathrm{~h} & 0 & -6.216939 & 0.966296 & 1.040377 & 0 & \mathrm{~m} & & \\ 34 & \mathrm{c} & 0 & -4.126848 & 1.065309 & 0.574245 & 0 & \mathrm{~m} & & \\ 35 & \mathrm{~h} & 0 & -4.012449 & 2.072033 & 0.971300 & 0 & \mathrm{~m} & & \end{array}$

radical

Charge $=0$ Multiplicity $=2$ for low level calculation on real system.

Charge $=0$ Multiplicity $=2$ for high level calculation on model system.

Charge $=0$ Multiplicity $=2$ for low level calculation on model system.

\begin{tabular}{|c|c|c|c|c|c|c|}
\hline Center & Atomic & Atomic & Coordi & ates (Angstro & & \\
\hline Number & Numbe & r Type & $\mathrm{X}$ & $\mathrm{Y}$ & Z & \\
\hline 1 & $\mathrm{n}$ & 0 & 3.664890 & 0.456690 & -0.227307 & 0 \\
\hline 2 & $\mathrm{c}$ & 0 & 4.347675 & -0.806789 & 0.000330 & 0 \\
\hline 3 & $\mathrm{~h}$ & 0 & 3.726592 & -1.675718 & -0.274796 & 0 \\
\hline 4 & $\mathrm{c}$ & 0 & 2.331357 & 0.525808 & -0.139462 & 0 \\
\hline 5 & $\mathrm{c}$ & 0 & 1.737587 & 1.810108 & -0.390182 & 0 \\
\hline
\end{tabular}




\begin{tabular}{|c|c|c|c|c|c|c|c|c|}
\hline 6 & $\mathrm{~h}$ & 0 & 2.414028 & 2.623535 & -0.633431 & 0 & & \\
\hline 7 & $\mathrm{c}$ & 0 & 1.416418 & -0.531044 & 0.193266 & 0 & $\mathrm{~h}$ & \\
\hline 8 & $\mathrm{~h}$ & 0 & 1.798218 & -1.517056 & 0.434495 & 0 & $\mathrm{~h}$ & \\
\hline 9 & $\mathrm{c}$ & 0 & 0.051867 & -0.324592 & 0.250589 & 0 & $\mathrm{~h}$ & \\
\hline 10 & $\mathrm{~h}$ & 0 & -0.599532 & -1.139196 & 0.547157 & 0 & $\mathrm{~h}$ & \\
\hline 11 & $\mathrm{c}$ & 0 & 5.609000 & -0.835443 & -0.873444 & 0 & $\mathrm{~m}$ & $\mathrm{~h}$ \\
\hline 12 & $\mathrm{~h}$ & 0 & 5.346868 & -0.752064 & -1.933467 & 0 & $\mathrm{~m}$ & \\
\hline 13 & $\mathrm{~h}$ & 0 & 6.262797 & 0.006685 & -0.621345 & 0 & $\mathrm{~m}$ & \\
\hline 14 & $\mathrm{~h}$ & 0 & 6.164846 & -1.769277 & -0.726947 & 0 & $\mathrm{~m}$ & \\
\hline 15 & $\mathrm{c}$ & 0 & 4.707605 & -0.928545 & 1.494604 & 0 & $\mathrm{~m}$ & $\mathrm{~h}$ \\
\hline 16 & $\mathrm{~h}$ & 0 & 5.314225 & -0.070936 & 1.805699 & 0 & $\mathrm{~m}$ & \\
\hline 17 & $\mathrm{~h}$ & 0 & 5.279845 & -1.845314 & 1.679789 & 0 & $\mathrm{~m}$ & \\
\hline 18 & $\mathrm{~h}$ & 0 & 3.807371 & -0.950592 & 2.118355 & 0 & $\mathrm{~m}$ & \\
\hline 19 & $\mathrm{c}$ & 0 & 0.379052 & 2.009366 & -0.344333 & 0 & $\mathrm{~m}$ & $\mathrm{~h}$ \\
\hline 20 & $\mathrm{~h}$ & 0 & -0.034900 & 2.992813 & -0.559559 & 0 & $\mathrm{~m}$ & \\
\hline 21 & $\mathrm{c}$ & 0 & -0.503759 & 0.944340 & -0.032712 & 0 & $\mathrm{~m}$ & $\mathrm{~h}$ \\
\hline 22 & $\mathrm{n}$ & 0 & -1.863577 & 1.215646 & 0.033337 & 0 & $\mathrm{~m}$ & \\
\hline 23 & $\mathrm{~h}$ & 0 & -2.100231 & 2.193579 & 0.133263 & 0 & $\mathrm{~m}$ & \\
\hline 24 & $\mathrm{c}$ & 0 & -2.960458 & 0.341356 & 0.004102 & 0 & $\mathrm{~m}$ & \\
\hline 25 & $\mathrm{c}$ & 0 & -4.153057 & 0.746612 & 0.628693 & 0 & $\mathrm{~m}$ & \\
\hline 26 & $\mathrm{~h}$ & 0 & -4.181670 & 1.697750 & 1.155946 & 0 & $\mathrm{~m}$ & \\
\hline 27 & $\mathrm{c}$ & 0 & -5.284534 & -0.062838 & 0.585836 & 0 & $\mathrm{~m}$ & \\
\hline 28 & $\mathrm{~h}$ & 0 & -6.196111 & 0.270320 & 1.074781 & 0 & $\mathrm{~m}$ & \\
\hline 29 & $\mathrm{c}$ & 0 & -5.248210 & -1.297347 & -0.066592 & 0 & $\mathrm{~m}$ & \\
\hline 30 & $\mathrm{~h}$ & 0 & -6.128531 & -1.932682 & -0.091573 & 0 & $\mathrm{~m}$ & \\
\hline 31 & $\mathrm{c}$ & 0 & -4.067337 & -1.699639 & -0.692841 & 0 & $\mathrm{~m}$ & \\
\hline 32 & $\mathrm{~h}$ & 0 & -4.028503 & -2.648388 & -1.221670 & 0 & $\mathrm{~m}$ & \\
\hline 33 & $\mathrm{c}$ & 0 & -2.932598 & -0.890237 & -0.672214 & 0 & $\mathrm{~m}$ & \\
\hline 34 & $\mathrm{~h}$ & 0 & -2.038150 & -1.196507 & -1.202743 & 0 & $\mathrm{~m}$ & \\
\hline
\end{tabular}

\section{No.8}

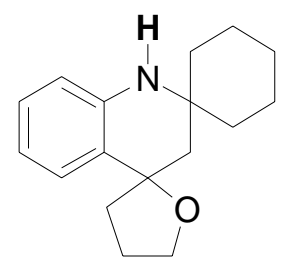

neutral molecule

Charge $=0$ Multiplicity $=1$ for low level calculation on real system.

Charge $=0$ Multiplicity $=1$ for high level calculation on model system .

Charge $=0$ Multiplicity $=1$ for low level calculation on model system. 


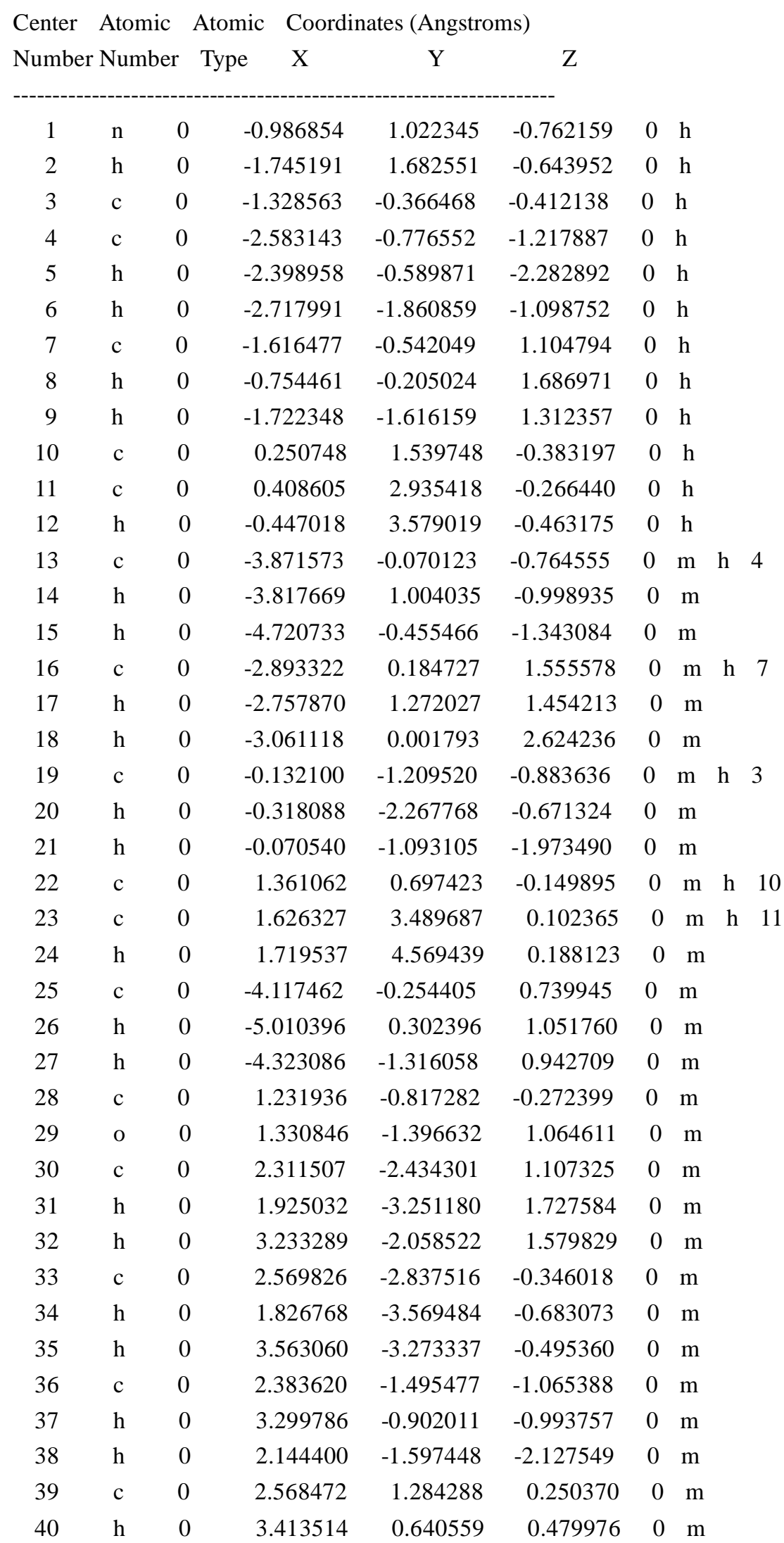




$\begin{array}{llllllll}41 & \mathrm{c} & 0 & 2.719049 & 2.661907 & 0.377460 & 0 & \mathrm{~m} \\ 42 & \mathrm{~h} & 0 & 3.670223 & 3.084076 & 0.688088 & 0 & \mathrm{~m}\end{array}$

radical

Charge $=0$ Multiplicity $=2$ for low level calculation on real system .

Charge $=0$ Multiplicity $=2$ for high level calculation on model system .

Charge $=0$ Multiplicity $=2$ for low level calculation on model system .

\begin{tabular}{|c|c|c|c|c|c|c|c|c|}
\hline Center & Atomic & & Atomic & ates (Angstro & $\mathrm{ns})$ & & & \\
\hline Number & Numbe & & Type & $\mathrm{Y}$ & $\mathrm{Z}$ & & & \\
\hline 1 & $\mathrm{n}$ & 0 & -1.034143 & 1.086284 & -0.108957 & 0 & $\mathrm{~h}$ & \\
\hline 2 & $\mathrm{c}$ & 0 & -1.404495 & -0.292124 & -0.397301 & 0 & $\mathrm{~h}$ & \\
\hline 3 & $\mathrm{c}$ & 0 & -2.601845 & -0.245158 & -1.388583 & 0 & $\mathrm{~h}$ & \\
\hline 4 & $\mathrm{~h}$ & 0 & -2.281571 & 0.267683 & -2.304839 & 0 & $\mathrm{~h}$ & \\
\hline 5 & $\mathrm{~h}$ & 0 & -2.842067 & -1.280851 & -1.671938 & 0 & $\mathrm{~h}$ & \\
\hline 6 & $\mathrm{c}$ & 0 & -1.897266 & -0.938151 & 0.937510 & 0 & $\mathrm{~h}$ & \\
\hline 7 & $\mathrm{~h}$ & 0 & -1.078616 & -0.932645 & 1.661174 & 0 & $\mathrm{~h}$ & \\
\hline 8 & $\mathrm{~h}$ & 0 & -2.120024 & -1.993500 & 0.722073 & 0 & $\mathrm{~h}$ & \\
\hline 9 & $\mathrm{c}$ & 0 & 0.260585 & 1.436188 & -0.043825 & 0 & $\mathrm{~h}$ & \\
\hline 10 & $\mathrm{c}$ & 0 & 0.520454 & 2.822260 & 0.205723 & 0 & $\mathrm{~h}$ & \\
\hline 11 & $\mathrm{~h}$ & 0 & -0.345279 & 3.465454 & 0.328809 & 0 & $\mathrm{~h}$ & \\
\hline 12 & $\mathrm{c}$ & 0 & -3.850066 & 0.434505 & -0.812223 & 0 & $\mathrm{~m}$ & h 3 \\
\hline 13 & $\mathrm{~h}$ & 0 & -3.624056 & 1.491348 & -0.628958 & 0 & $\mathrm{~m}$ & \\
\hline 14 & $\mathrm{~h}$ & 0 & -4.659664 & 0.400983 & -1.553156 & 0 & $\mathrm{~m}$ & \\
\hline 15 & $\mathrm{c}$ & 0 & -3.147648 & -0.259948 & 1.514887 & 0 & $\mathrm{~m}$ & $\mathrm{~h}$ \\
\hline 16 & $\mathrm{~h}$ & 0 & -2.897727 & 0.764627 & 1.815659 & 0 & $\mathrm{~m}$ & \\
\hline 17 & $\mathrm{~h}$ & 0 & -3.457933 & -0.792888 & 2.423402 & 0 & $\mathrm{~m}$ & \\
\hline 18 & $\mathrm{c}$ & 0 & -0.265844 & -1.112616 & -1.043060 & 0 & $\mathrm{~m}$ & h 2 \\
\hline 19 & $\mathrm{~h}$ & 0 & -0.173347 & -0.805018 & -2.094419 & 0 & $\mathrm{~m}$ & \\
\hline 20 & $\mathrm{~h}$ & 0 & -0.519709 & -2.179042 & -1.040089 & 0 & $\mathrm{~m}$ & \\
\hline 21 & $\mathrm{c}$ & 0 & 1.386155 & 0.549573 & -0.212440 & 0 & $\mathrm{~m}$ & h 9 \\
\hline 22 & $\mathrm{c}$ & 0 & 1.808964 & 3.314977 & 0.277330 & 0 & $\mathrm{~m}$ & h 10 \\
\hline 23 & $\mathrm{~h}$ & 0 & 1.981867 & 4.371364 & 0.464468 & 0 & $\mathrm{~m}$ & \\
\hline 24 & $\mathrm{c}$ & 0 & -4.297269 & -0.226824 & 0.498613 & 0 & $\mathrm{~m}$ & \\
\hline 25 & $\mathrm{~h}$ & 0 & -5.162194 & 0.304043 & 0.917119 & 0 & $\mathrm{~m}$ & \\
\hline 26 & $\mathrm{~h}$ & 0 & -4.630871 & -1.255794 & 0.293165 & 0 & $\mathrm{~m}$ & \\
\hline 27 & $\mathrm{c}$ & 0 & 1.110268 & -0.937954 & -0.400447 & 0 & $\mathrm{~m}$ & \\
\hline 28 & o & 0 & 1.086049 & -1.592582 & 0.892361 & 0 & $\mathrm{~m}$ & \\
\hline 29 & $\mathrm{c}$ & 0 & 2.423532 & -1.925309 & 1.247728 & 0 & $\mathrm{~m}$ & \\
\hline 30 & $\mathrm{~h}$ & 0 & 2.920997 & -1.068855 & 1.726877 & 0 & $\mathrm{~m}$ & \\
\hline 31 & $\mathrm{~h}$ & 0 & 2.370528 & -2.739625 & 1.976179 & 0 & $\mathrm{~m}$ & \\
\hline
\end{tabular}




$\begin{array}{llllllll}32 & \mathrm{c} & 0 & 3.130799 & -2.313253 & -0.069867 & 0 & \mathrm{~m} \\ 33 & \mathrm{~h} & 0 & 3.194644 & -3.400110 & -0.177255 & 0 & \mathrm{~m} \\ 34 & \mathrm{~h} & 0 & 4.153650 & -1.923709 & -0.106648 & 0 & \mathrm{~m} \\ 35 & \mathrm{c} & 0 & 2.220615 & -1.706299 & -1.167584 & 0 & \mathrm{~m} \\ 36 & \mathrm{~h} & 0 & 1.751752 & -2.503009 & -1.753518 & 0 & \mathrm{~m} \\ 37 & \mathrm{~h} & 0 & 2.758845 & -1.063920 & -1.869150 & 0 & \mathrm{~m} \\ 38 & \mathrm{c} & 0 & 2.669869 & 1.081644 & -0.141396 & 0 & \mathrm{~m} \\ 39 & \mathrm{~h} & 0 & 3.530355 & 0.434469 & -0.285244 & 0 & \mathrm{~m} \\ 40 & \mathrm{c} & 0 & 2.894618 & 2.444207 & 0.100449 & 0 & \mathrm{~m} \\ 41 & \mathrm{~h} & 0 & 3.912491 & 2.821352 & 0.147025 & 0 & \mathrm{~m}\end{array}$

\section{No.9}<smiles>O=[N+]([O-])c1ccc(N(c2ccccc2)c2ccccc2)c([N+](=O)[O-])c1</smiles>

neutral molecule

Charge $=0$ Multiplicity $=1$ for low level calculation on real system.

Charge $=0$ Multiplicity $=1$ for high level calculation on model system.

Charge $=0$ Multiplicity $=1$ for low level calculation on model system.

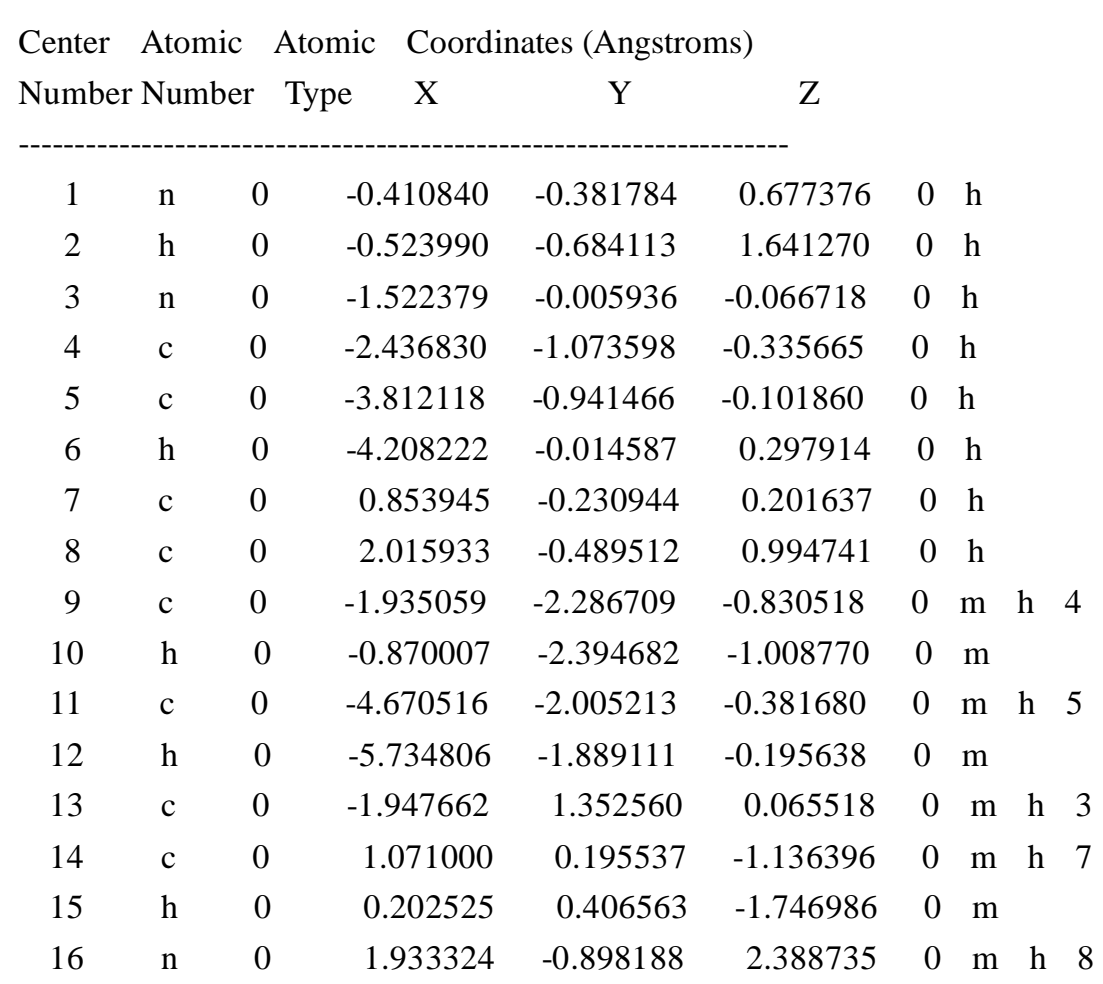




\begin{tabular}{|c|c|c|c|c|c|c|c|}
\hline 17 & $\mathrm{c}$ & 0 & 3.299529 & -0.345794 & 0.466098 & 0 & $\mathrm{~m} \mathrm{~h}$ \\
\hline 18 & $\mathrm{~h}$ & 0 & 4.160132 & -0.550725 & 1.088110 & 0 & $\mathrm{~m}$ \\
\hline 19 & $\mathrm{c}$ & 0 & -2.799880 & -3.346726 & -1.090607 & 0 & $\mathrm{~m}$ \\
\hline 20 & $\mathrm{~h}$ & 0 & -2.396550 & -4.280332 & -1.473130 & 0 & $\mathrm{~m}$ \\
\hline 21 & $\mathrm{c}$ & 0 & -4.173232 & -3.211825 & -0.874168 & 0 & $\mathrm{~m}$ \\
\hline 22 & $\mathrm{~h}$ & 0 & -4.845740 & -4.038244 & -1.084299 & 0 & $\mathrm{~m}$ \\
\hline 23 & $\mathrm{c}$ & 0 & -2.728939 & 1.922508 & -0.952105 & 0 & $\mathrm{~m}$ \\
\hline 24 & $\mathrm{~h}$ & 0 & -3.018736 & 1.318793 & -1.806502 & 0 & $\mathrm{~m}$ \\
\hline 25 & $\mathrm{c}$ & 0 & -3.126856 & 3.253869 & -0.863531 & 0 & $\mathrm{~m}$ \\
\hline 26 & $\mathrm{~h}$ & 0 & -3.732598 & 3.681149 & -1.658042 & 0 & $\mathrm{~m}$ \\
\hline 27 & $\mathrm{c}$ & 0 & -2.736274 & 4.041959 & 0.222358 & 0 & $\mathrm{~m}$ \\
\hline 28 & $\mathrm{~h}$ & 0 & -3.040153 & 5.082777 & 0.282554 & 0 & $\mathrm{~m}$ \\
\hline 29 & $\mathrm{c}$ & 0 & -1.945078 & 3.479014 & 1.222883 & 0 & $\mathrm{~m}$ \\
\hline 30 & $\mathrm{~h}$ & 0 & -1.630478 & 4.078813 & 2.072519 & 0 & $\mathrm{~m}$ \\
\hline 31 & $\mathrm{c}$ & 0 & -1.553869 & 2.140907 & 1.153629 & 0 & $\mathrm{~m}$ \\
\hline 32 & $\mathrm{~h}$ & 0 & -0.939228 & 1.715574 & 1.939943 & 0 & $\mathrm{~m}$ \\
\hline 33 & $\mathrm{c}$ & 0 & 2.339732 & 0.336061 & -1.651935 & 0 & $\mathrm{~m}$ \\
\hline 34 & $\mathrm{~h}$ & 0 & 2.494035 & 0.656082 & -2.675259 & 0 & $\mathrm{~m}$ \\
\hline 35 & $\mathrm{c}$ & 0 & 3.455207 & 0.061159 & -0.844779 & 0 & $\mathrm{~m}$ \\
\hline 36 & $\mathrm{n}$ & 0 & 4.800044 & 0.209835 & -1.394035 & 0 & $\mathrm{~m}$ \\
\hline 37 & o & 0 & 4.895916 & 0.583629 & -2.564974 & 0 & $\mathrm{~m}$ \\
\hline 38 & o & 0 & 5.753956 & -0.046726 & -0.658811 & 0 & $\mathrm{~m}$ \\
\hline 39 & o & 0 & 2.976444 & -1.088277 & 3.007213 & 0 & $\mathrm{~m}$ \\
\hline 40 & o & 0 & 0.807254 & -1.031895 & 2.904960 & 0 & $\mathrm{~m}$ \\
\hline
\end{tabular}

radical

Charge $=0$ Multiplicity $=2$ for low level calculation on real system.

Charge $=0$ Multiplicity $=2$ for high level calculation on model system.

Charge $=0$ Multiplicity $=2$ for low level calculation on model system.

Center Atomic Atomic Coordinates (Angstroms)

Number Number Type $\quad \mathrm{X} \quad \mathrm{Y} \quad$ Z

\begin{tabular}{|c|c|c|c|c|c|c|c|}
\hline 1 & $\mathrm{n}$ & 0 & 0.538596 & -0.930876 & 0.110151 & & $\mathrm{~h}$ \\
\hline 2 & $\mathrm{n}$ & 0 & 1.547975 & -0.072579 & -0.106716 & 0 & $\mathrm{~h}$ \\
\hline 3 & c & 0 & 2.802339 & -0.665480 & -0.424513 & 0 & $\mathrm{~h}$ \\
\hline 4 & $\mathrm{c}$ & 0 & 4.010073 & -0.071595 & -0.027048 & & $\mathrm{~h}$ \\
\hline 5 & $\mathrm{~h}$ & 0 & 4.004974 & 0.847766 & 0.546562 & 0 & $\mathrm{~h}$ \\
\hline 6 & $\mathrm{c}$ & 0 & -0.741517 & -0.499611 & -0.118710 & & $\mathrm{~h}$ \\
\hline 7 & $\mathrm{c}$ & 0 & -1.810383 & -1.099082 & 0.604464 & & $\mathrm{~h}$ \\
\hline 8 & $\mathrm{c}$ & 0 & 2.816782 & -1.875620 & -1.135516 & 0 & $\mathrm{~m} \mathrm{~h}$ \\
\hline 9 & $\mathrm{~h}$ & 0 & 1.876999 & -2.328150 & -1.428416 & 0 & $\mathrm{~m}$ \\
\hline
\end{tabular}




$\begin{array}{lllrrrrrrr}10 & \mathrm{c} & 0 & 5.218024 & -0.683994 & -0.353393 & 0 & \mathrm{~m} & \mathrm{~h} & 4 \\ 11 & \mathrm{~h} & 0 & 6.148811 & -0.221624 & -0.037297 & 0 & \mathrm{~m} & & \\ 12 & \mathrm{c} & 0 & 1.459236 & 1.307405 & 0.290733 & 0 & \mathrm{~m} & \mathrm{~h} & 2 \\ 13 & \mathrm{c} & 0 & -1.105847 & 0.391668 & -1.164174 & 0 & \mathrm{~m} & \mathrm{~h} & 6 \\ 14 & \mathrm{~h} & 0 & -0.320522 & 0.852431 & -1.751109 & 0 & \mathrm{~m} & & \\ 15 & \mathrm{n} & 0 & -1.542745 & -1.930519 & 1.783384 & 0 & \mathrm{~m} & \mathrm{~h} & 7 \\ 16 & \mathrm{c} & 0 & -3.141412 & -0.880467 & 0.283467 & 0 & \mathrm{~m} & \mathrm{~h} & 7 \\ 17 & \mathrm{~h} & 0 & -3.928323 & -1.365950 & 0.845261 & 0 & \mathrm{~m} & \\ 18 & \mathrm{c} & 0 & 4.031495 & -2.478720 & -1.446334 & 0 & \mathrm{~m} & \\ 19 & \mathrm{~h} & 0 & 4.034479 & -3.414684 & -1.997695 & 0 & \mathrm{~m} & \\ 20 & \mathrm{c} & 0 & 5.237626 & -1.886506 & -1.062771 & 0 & \mathrm{~m} & \\ 21 & \mathrm{~h} & 0 & 6.183118 & -2.359179 & -1.311926 & 0 & \mathrm{~m} & \\ 22 & \mathrm{c} & 0 & 0.856508 & 1.627152 & 1.513263 & 0 & \mathrm{~m} & \\ 23 & \mathrm{~h} & 0 & 0.481044 & 0.829973 & 2.147664 & 0 & \mathrm{~m} & \\ 24 & \mathrm{c} & 0 & 0.754891 & 2.963925 & 1.894610 & 0 & \mathrm{~m} & \\ 25 & \mathrm{~h} & 0 & 0.291093 & 3.213404 & 2.844574 & 0 & \mathrm{~m} & \\ 26 & \mathrm{c} & 0 & 1.254626 & 3.975345 & 1.070890 & 0 & \mathrm{~m} & & \\ 27 & \mathrm{~h} & 0 & 1.173673 & 5.014969 & 1.374500 & 0 & \mathrm{~m} & \\ 28 & \mathrm{c} & 0 & 1.860158 & 3.646548 & -0.144465 & 0 & \mathrm{~m} & & \\ 29 & \mathrm{~h} & 0 & 2.246411 & 4.428761 & -0.791669 & 0 & \mathrm{~m} & \\ 30 & \mathrm{c} & 0 & 1.963207 & 2.314107 & -0.541154 & 0 & \mathrm{~m} & \\ 31 & \mathrm{~h} & 0 & 2.425772 & 2.051947 & -1.487768 & 0 & \mathrm{~m} & & \\ 32 & \mathrm{c} & 0 & -2.429044 & 0.633381 & -1.479879 & 0 & \mathrm{~m} & & \\ 33 & \mathrm{~h} & 0 & -2.700252 & 1.300484 & -2.289125 & 0 & \mathrm{~m} & \\ 34 & \mathrm{c} & 0 & -3.441802 & -0.008042 & -0.756116 & 0 & \mathrm{~m} & & \\ 35 & \mathrm{n} & 0 & -4.839533 & 0.247290 & -1.092166 & 0 & \mathrm{~m} & & \\ 36 & \mathrm{o} & 0 & -5.069264 & 1.029230 & -2.017759 & 0 & \mathrm{~m} & & \\ 37 & \mathrm{o} & 0 & -5.704103 & -0.331971 & -0.432761 & 0 & \mathrm{~m} & & \\ 38 & \mathrm{o} & 0 & -2.348766 & -2.824531 & 2.038751 & 0 & \mathrm{~m} & & \\ 39 & \mathrm{o} & 0 & -0.555003 & -1.660958 & 2.468489 & 0 & \mathrm{~m} & & \\ & & & & & & & & \end{array}$

\section{No.10}

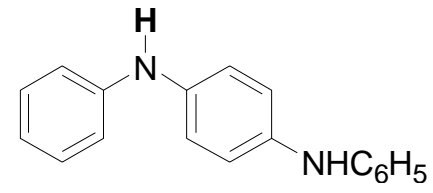

neutral molecule

Charge $=0$ Multiplicity $=1$ for low level calculation on real system.

Charge $=0$ Multiplicity $=1$ for high level calculation on model system .

Charge $=0$ Multiplicity $=1$ for low level calculation on model system. 


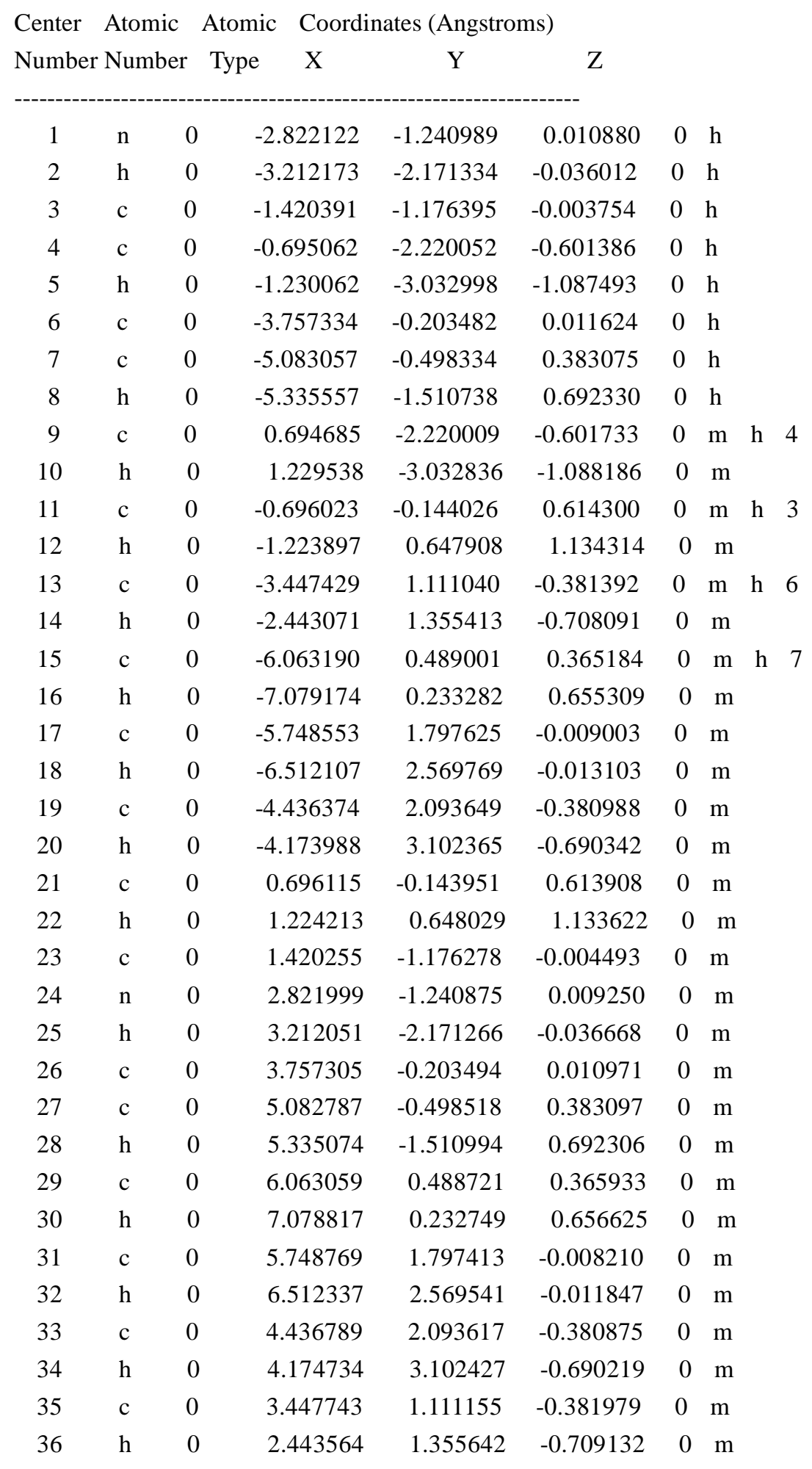

radical

Charge $=0$ Multiplicity $=2$ for low level calculation on real system. 
Charge $=0$ Multiplicity $=2$ for high level calculation on model system .

Charge $=0$ Multiplicity $=2$ for low level calculation on model system.

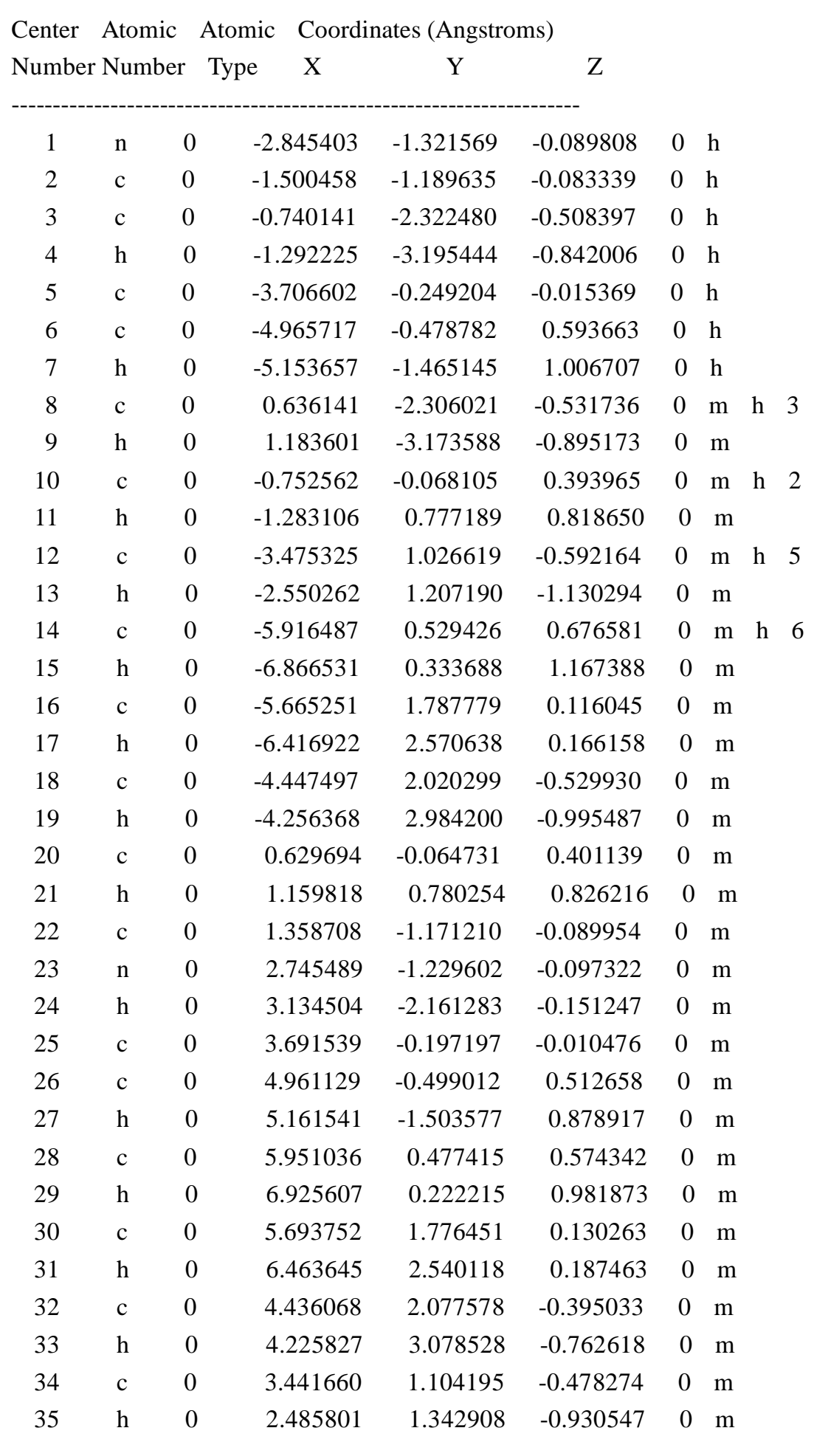




\title{
(3) The Cartesian Coordinates of Deoxynucleoside
}

\author{
dA
}

s1

neutral molecule

Charge $=0$ Multiplicity $=1$ for low level calculation on real system.

Charge $=0$ Multiplicity $=1$ for high level calculation on model system.

Charge $=0$ Multiplicity $=1$ for low level calculation on model system.

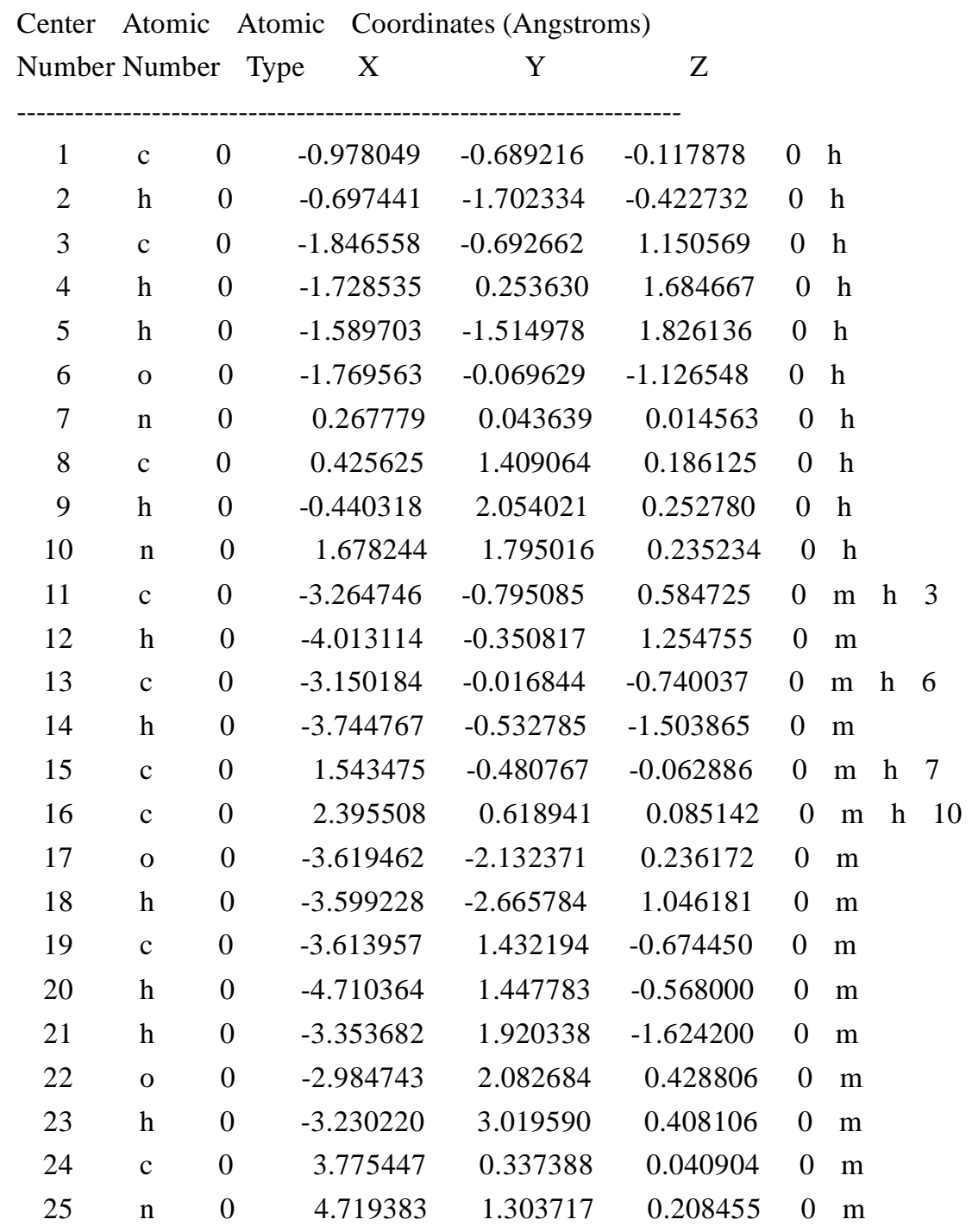




$\begin{array}{lllrrrrr}26 & \mathrm{~h} & 0 & 4.433402 & 2.268289 & 0.124136 & 0 & \mathrm{~m} \\ 27 & \mathrm{~h} & 0 & 5.668715 & 1.065463 & -0.040452 & 0 & \mathrm{~m} \\ 28 & \mathrm{n} & 0 & 4.176831 & -0.933162 & -0.144009 & 0 & \mathrm{~m} \\ 29 & \mathrm{c} & 0 & 3.236742 & -1.884646 & -0.266662 & 0 & \mathrm{~m} \\ 30 & \mathrm{~h} & 0 & 3.614816 & -2.894596 & -0.412857 & 0 & \mathrm{~m} \\ 31 & \mathrm{n} & 0 & 1.906443 & -1.758281 & -0.235014 & 0 & \mathrm{~m}\end{array}$

radical

Charge $=0$ Multiplicity $=2$ for low level calculation on real system.

Charge $=0$ Multiplicity $=2$ for high level calculation on model system.

Charge $=0$ Multiplicity $=2$ for low level calculation on model system.

\begin{tabular}{|c|c|c|c|c|c|c|c|c|}
\hline Center & Atomic & & Atomic Coordi & ates (Angstro & & & & \\
\hline Number & Numbe & & Type & $\mathrm{Y}$ & $\mathrm{Z}$ & & & \\
\hline 1 & $\mathrm{c}$ & 0 & 1.009581 & 0.126170 & -0.512221 & 0 & $\mathrm{~h}$ & \\
\hline 2 & $\mathrm{c}$ & 0 & 1.583648 & 1.172013 & 0.392829 & 0 & $\mathrm{~h}$ & \\
\hline 3 & $\mathrm{~h}$ & 0 & 1.444110 & 0.901605 & 1.450061 & 0 & $\mathrm{~h}$ & \\
\hline 4 & $\mathrm{~h}$ & 0 & 1.141974 & 2.161014 & 0.239939 & 0 & $\mathrm{~h}$ & \\
\hline 5 & o & 0 & 1.946782 & -0.810992 & -0.861993 & 0 & $\mathrm{~h}$ & \\
\hline 6 & $\mathrm{n}$ & 0 & -0.280966 & -0.388169 & -0.398257 & 0 & $\mathrm{~h}$ & \\
\hline 7 & $\mathrm{c}$ & 0 & -0.709252 & -1.680372 & -0.695958 & 0 & $\mathrm{~h}$ & \\
\hline 8 & $\mathrm{~h}$ & 0 & 0.003543 & -2.431772 & -1.000618 & 0 & $\mathrm{~h}$ & \\
\hline 9 & $\mathrm{n}$ & 0 & -2.005079 & -1.854321 & -0.570153 & 0 & $\mathrm{~h}$ & \\
\hline 10 & $\mathrm{c}$ & 0 & 3.075368 & 1.102902 & 0.020504 & 0 & $\mathrm{~m}$ & $\mathrm{~h}$ \\
\hline 11 & $\mathrm{~h}$ & 0 & 3.718104 & 1.328004 & 0.881729 & 0 & $\mathrm{~m}$ & \\
\hline 12 & $\mathrm{c}$ & 0 & 3.257241 & -0.355157 & -0.446528 & 0 & $\mathrm{~m}$ & h 5 \\
\hline 13 & $\mathrm{~h}$ & 0 & 3.908332 & -0.379055 & -1.325748 & 0 & $\mathrm{~m}$ & \\
\hline 14 & $\mathrm{c}$ & 0 & -1.432737 & 0.314955 & -0.061099 & 0 & $\mathrm{~m}$ & h 6 \\
\hline 15 & $\mathrm{c}$ & 0 & -2.470857 & -0.617000 & -0.169833 & 0 & & h 9 \\
\hline 16 & o & 0 & 3.410498 & 1.924063 & -1.093804 & 0 & $\mathrm{~m}$ & \\
\hline 17 & $\mathrm{~h}$ & 0 & 3.134987 & 2.830946 & -0.888213 & 0 & $\mathrm{~m}$ & \\
\hline 18 & $\mathrm{c}$ & 0 & 3.807563 & -1.299955 & 0.609717 & 0 & $\mathrm{~m}$ & \\
\hline 19 & $\mathrm{~h}$ & 0 & 4.872073 & -1.058773 & 0.766532 & 0 & $\mathrm{~m}$ & \\
\hline 20 & $\mathrm{~h}$ & 0 & 3.746287 & -2.328299 & 0.224374 & 0 & $\mathrm{~m}$ & \\
\hline 21 & o & 0 & 3.055304 & -1.142459 & 1.804746 & 0 & $\mathrm{~m}$ & \\
\hline 22 & $\mathrm{~h}$ & 0 & 3.321471 & -1.834157 & 2.427955 & 0 & $\mathrm{~m}$ & \\
\hline 23 & $\mathrm{c}$ & 0 & -3.763011 & -0.134442 & 0.122808 & 0 & $\mathrm{~m}$ & \\
\hline 24 & $\mathrm{n}$ & 0 & -4.858241 & -0.940440 & 0.094162 & 0 & $\mathrm{~m}$ & \\
\hline 25 & $\mathrm{~h}$ & 0 & -4.779638 & -1.837482 & -0.362212 & 0 & $\mathrm{~m}$ & \\
\hline 26 & $\mathrm{~h}$ & 0 & -5.762483 & -0.491474 & 0.114646 & 0 & $\mathrm{~m}$ & \\
\hline 27 & $\mathrm{n}$ & 0 & -3.916922 & 1.156178 & 0.467027 & 0 & $\mathrm{~m}$ & \\
\hline
\end{tabular}




$\begin{array}{llllllll}28 & \mathrm{c} & 0 & -2.821033 & 1.931400 & 0.527940 & 0 & \mathrm{~m} \\ 29 & \mathrm{~h} & 0 & -3.000004 & 2.966701 & 0.811394 & 0 & \mathrm{~m} \\ 30 & \mathrm{n} & 0 & -1.548577 & 1.604575 & 0.284309 & 0 & \mathrm{~m}\end{array}$

\section{s2}

neutral molecule

Charge $=0$ Multiplicity $=1$ for low level calculation on real system .

Charge $=0$ Multiplicity $=1$ for high level calculation on model system .

Charge $=0$ Multiplicity $=1$ for low level calculation on model system .

$\begin{array}{lllcc}\text { Center } & \text { Atomic } & \text { Atomic } & \text { Coordinates (Angstroms) } & \\ \text { Number Number } & \text { Type } & \text { X } & \text { Y } & \text { Z }\end{array}$

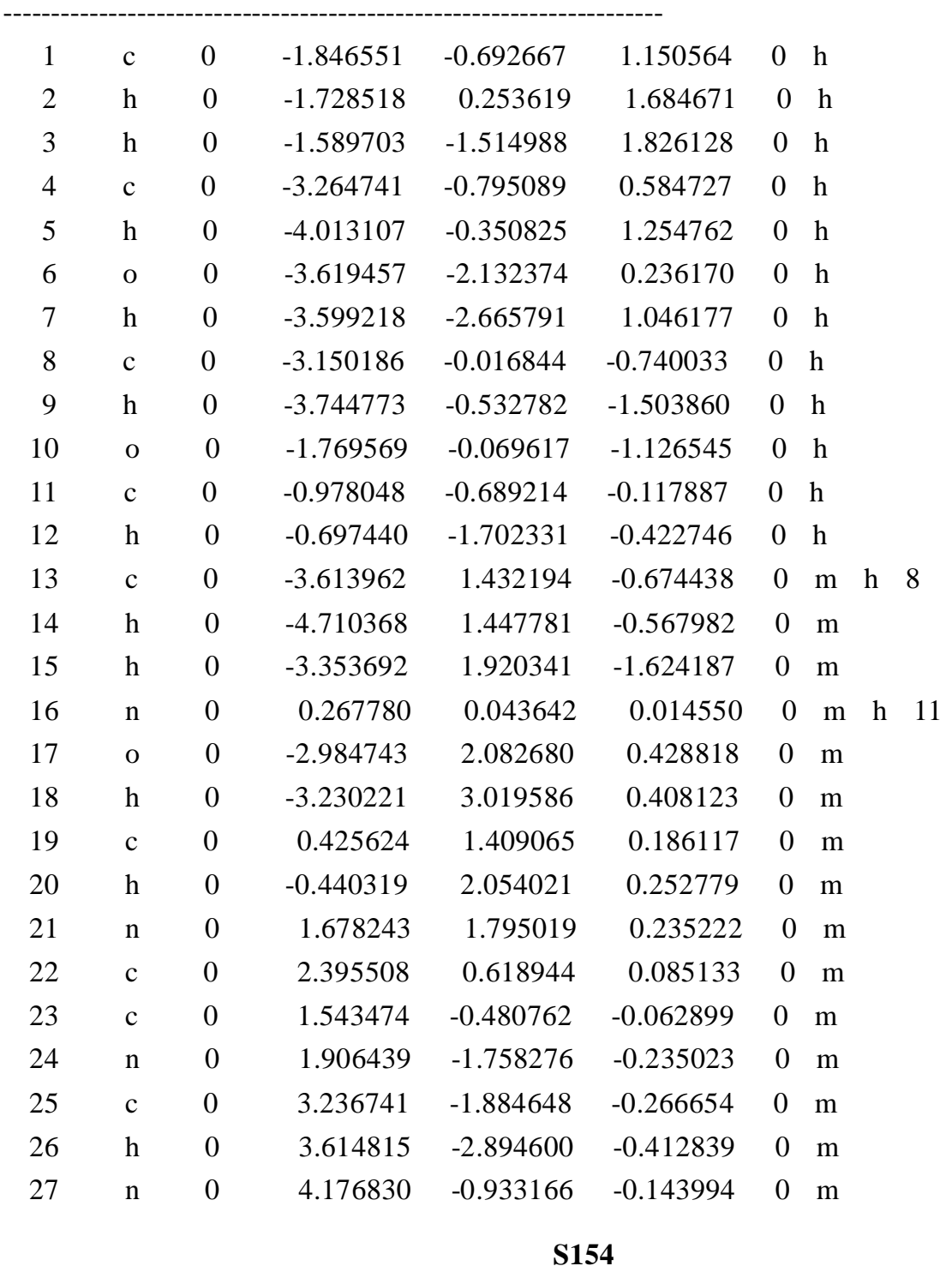




$\begin{array}{lllrrrrr}28 & \mathrm{c} & 0 & 3.775447 & 0.337387 & 0.040906 & 0 & \mathrm{~m} \\ 29 & \mathrm{n} & 0 & 4.719384 & 1.303714 & 0.208462 & 0 & \mathrm{~m} \\ 30 & \mathrm{~h} & 0 & 4.433407 & 2.268286 & 0.124139 & 0 & \mathrm{~m} \\ 31 & \mathrm{~h} & 0 & 5.668718 & 1.065456 & -0.040437 & 0 & \mathrm{~m}\end{array}$

radical

Charge $=0$ Multiplicity $=2$ for low level calculation on real system.

Charge $=0$ Multiplicity $=2$ for high level calculation on model system.

Charge $=0$ Multiplicity $=2$ for low level calculation on model system.

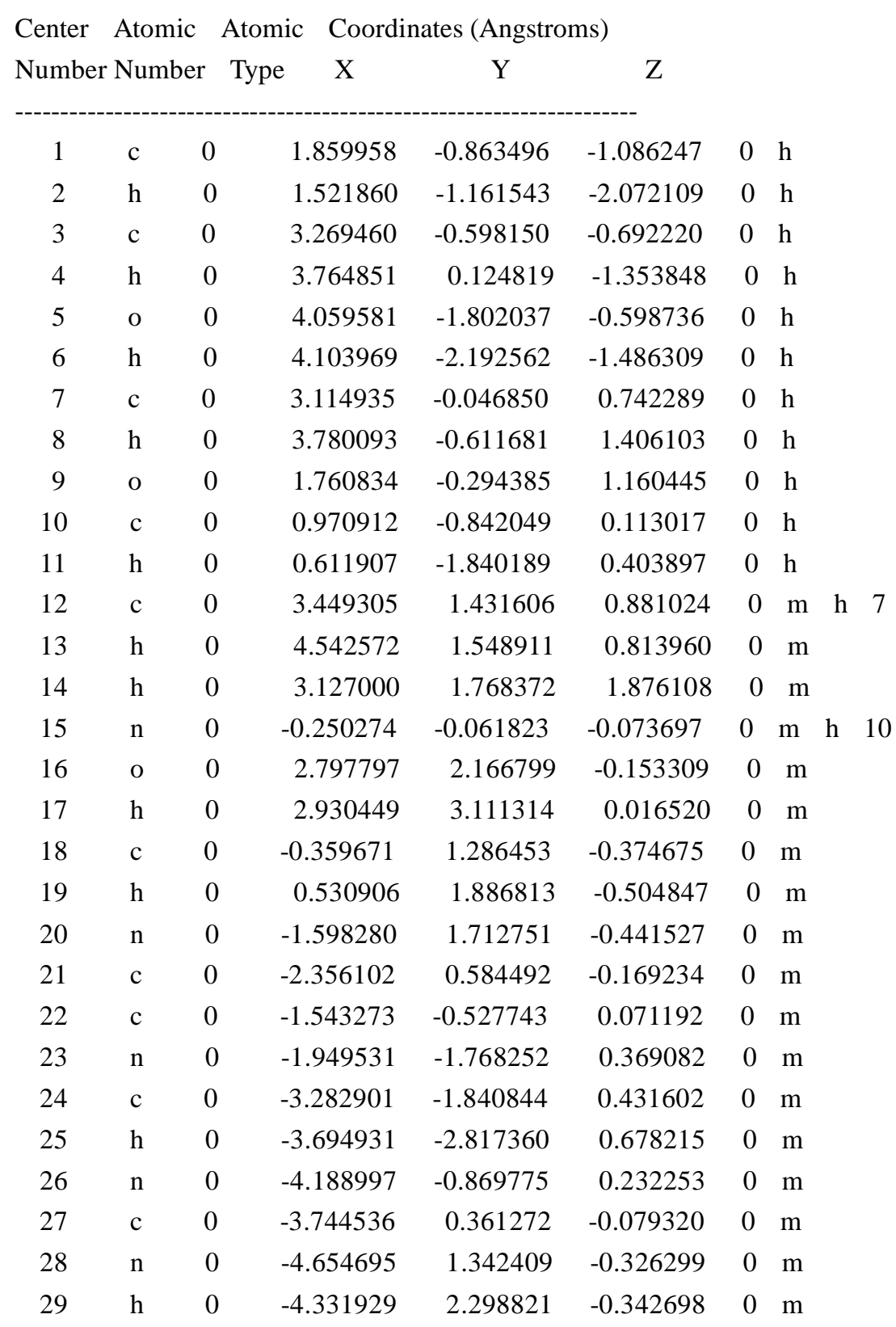




\section{s3}

neutral molecule

Charge $=0$ Multiplicity $=1$ for low level calculation on real system.

Charge $=0$ Multiplicity $=1$ for high level calculation on model system .

Charge $=0$ Multiplicity $=1$ for low level calculation on model system.

Center Atomic Atomic Coordinates (Angstroms)

Number Number Type $\quad X \quad$ Y $\quad$ Z

\begin{tabular}{|c|c|c|c|c|c|c|c|c|}
\hline 1 & $\mathrm{c}$ & 0 & -3.264748 & -0.795089 & 0.584723 & 0 & $\mathrm{~h}$ & \\
\hline 2 & $\mathrm{~h}$ & 0 & -4.013108 & -0.350823 & 1.254763 & 0 & $\mathrm{~h}$ & \\
\hline 3 & o & 0 & -3.619463 & -2.132375 & 0.236164 & 0 & $\mathrm{~h}$ & \\
\hline 4 & $\mathrm{~h}$ & 0 & -3.599222 & -2.665792 & 1.046171 & 0 & $\mathrm{~h}$ & \\
\hline 5 & $\mathrm{c}$ & 0 & -3.150186 & -0.016843 & -0.740036 & 0 & h & \\
\hline 6 & $\mathrm{~h}$ & 0 & -3.744768 & -0.532781 & -1.503867 & 0 & h & \\
\hline 7 & o & 0 & -1.769566 & -0.069617 & -1.126541 & 0 & $\mathrm{~h}$ & \\
\hline 8 & $\mathrm{c}$ & 0 & -0.978047 & -0.689214 & -0.117880 & 0 & h & \\
\hline 9 & $\mathrm{~h}$ & 0 & -0.697438 & -1.702333 & -0.422730 & 0 & h & \\
\hline 10 & $\mathrm{c}$ & 0 & -1.846548 & -0.692667 & 1.150572 & 0 & $\mathrm{~h}$ & \\
\hline 11 & $\mathrm{~h}$ & 0 & -1.728517 & 0.253621 & 1.684677 & 0 & $\mathrm{~h}$ & \\
\hline 12 & $\mathrm{~h}$ & 0 & -1.589691 & -1.514988 & 1.826132 & 0 & h & \\
\hline 13 & $\mathrm{c}$ & 0 & -3.613961 & 1.432194 & -0.674444 & 0 & $\mathrm{~m}$ & h \\
\hline 14 & $\mathrm{~h}$ & 0 & -4.710367 & 1.447782 & -0.567994 & 0 & $\mathrm{~m}$ & \\
\hline 15 & $\mathrm{~h}$ & 0 & -3.353685 & 1.920342 & -1.624191 & 0 & $\mathrm{~m}$ & \\
\hline 16 & $\mathrm{n}$ & 0 & 0.267781 & 0.043642 & 0.014555 & 0 & $\mathrm{~m}$ & $\mathrm{~h}$ \\
\hline 17 & o & 0 & -2.984748 & 2.082680 & 0.428816 & 0 & $\mathrm{~m}$ & \\
\hline 18 & $\mathrm{~h}$ & 0 & -3.230226 & 3.019586 & 0.408120 & 0 & $\mathrm{~m}$ & \\
\hline 19 & $\mathrm{c}$ & 0 & 0.425626 & 1.409066 & 0.186122 & 0 & $\mathrm{~m}$ & \\
\hline 20 & $\mathrm{~h}$ & 0 & -0.440317 & 2.054022 & 0.252785 & 0 & $\mathrm{~m}$ & \\
\hline 21 & $\mathrm{n}$ & 0 & 1.678245 & 1.795019 & 0.235224 & 0 & $\mathrm{~m}$ & \\
\hline 22 & $\mathrm{c}$ & 0 & 2.395510 & 0.618944 & 0.085133 & 0 & $\mathrm{~m}$ & \\
\hline 23 & $\mathrm{c}$ & 0 & 1.543474 & -0.480762 & -0.062897 & 0 & $\mathrm{~m}$ & \\
\hline 24 & $\mathrm{n}$ & 0 & 1.906439 & -1.758276 & -0.235022 & 0 & $\mathrm{~m}$ & \\
\hline 25 & $\mathrm{c}$ & 0 & 3.236741 & -1.884649 & -0.266656 & 0 & $\mathrm{~m}$ & \\
\hline 26 & $\mathrm{~h}$ & 0 & 3.614815 & -2.894600 & -0.412842 & 0 & $\mathrm{~m}$ & \\
\hline 27 & $\mathrm{n}$ & 0 & 4.176830 & -0.933166 & -0.143998 & 0 & $\mathrm{~m}$ & \\
\hline 28 & $\mathrm{c}$ & 0 & 3.775448 & 0.337387 & 0.040904 & 0 & $\mathrm{~m}$ & \\
\hline 29 & $\mathrm{n}$ & 0 & 4.719386 & 1.303713 & 0.208458 & 0 & $\mathrm{~m}$ & \\
\hline
\end{tabular}




$\begin{array}{llllllll}30 & \mathrm{~h} & 0 & 4.433409 & 2.268286 & 0.124136 & 0 & \mathrm{~m} \\ 31 & \mathrm{~h} & 0 & 5.668719 & 1.065456 & -0.040443 & 0 & \mathrm{~m}\end{array}$

radical

Charge $=0$ Multiplicity $=2$ for low level calculation on real system.

Charge $=0$ Multiplicity $=2$ for high level calculation on model system .

Charge $=0$ Multiplicity $=2$ for low level calculation on model system .

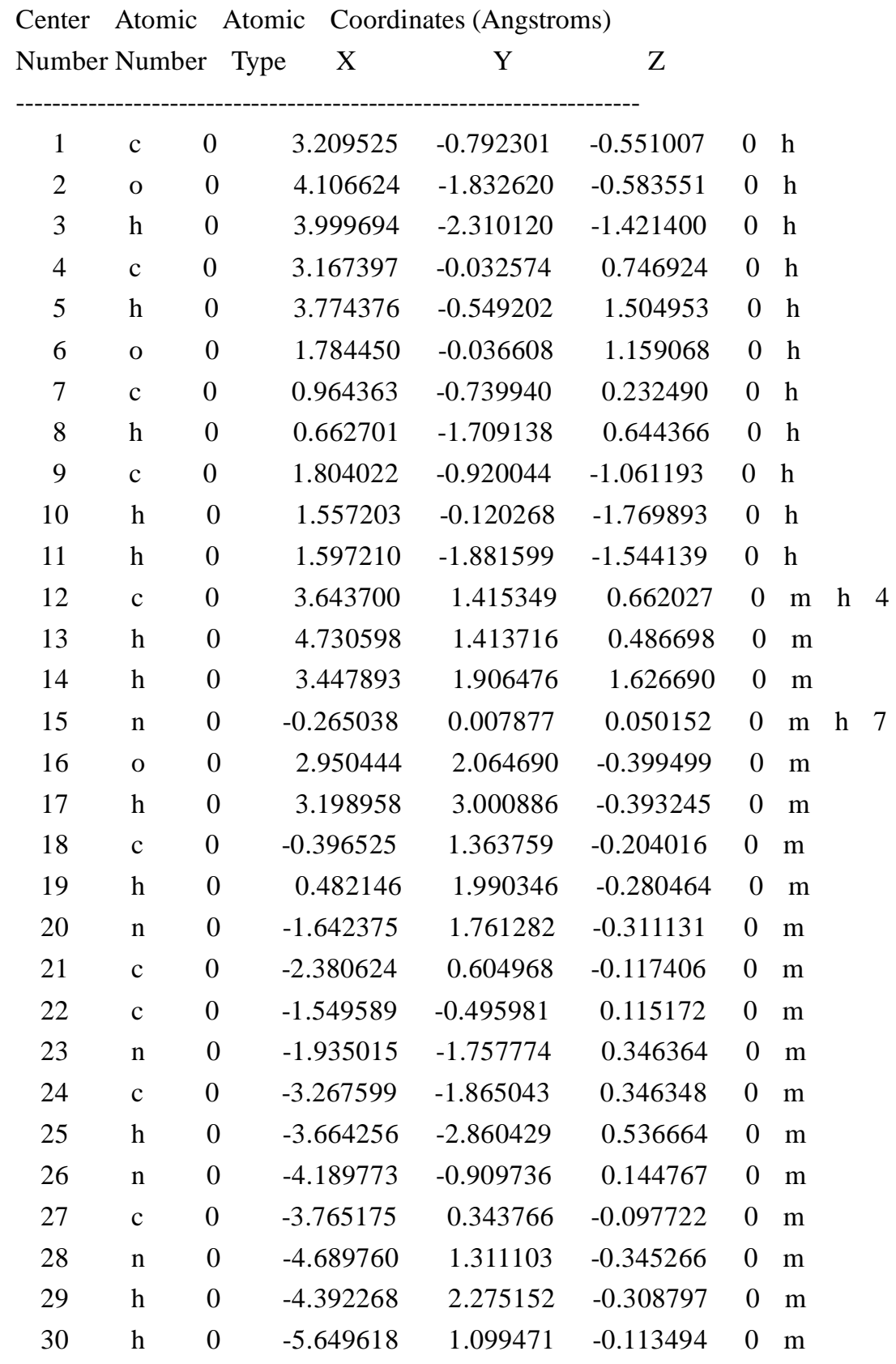




\section{s4}

neutral molecule

Charge $=0$ Multiplicity $=1$ for low level calculation on real system.

Charge $=0$ Multiplicity $=1$ for high level calculation on model system.

Charge $=0$ Multiplicity $=1$ for low level calculation on model system .

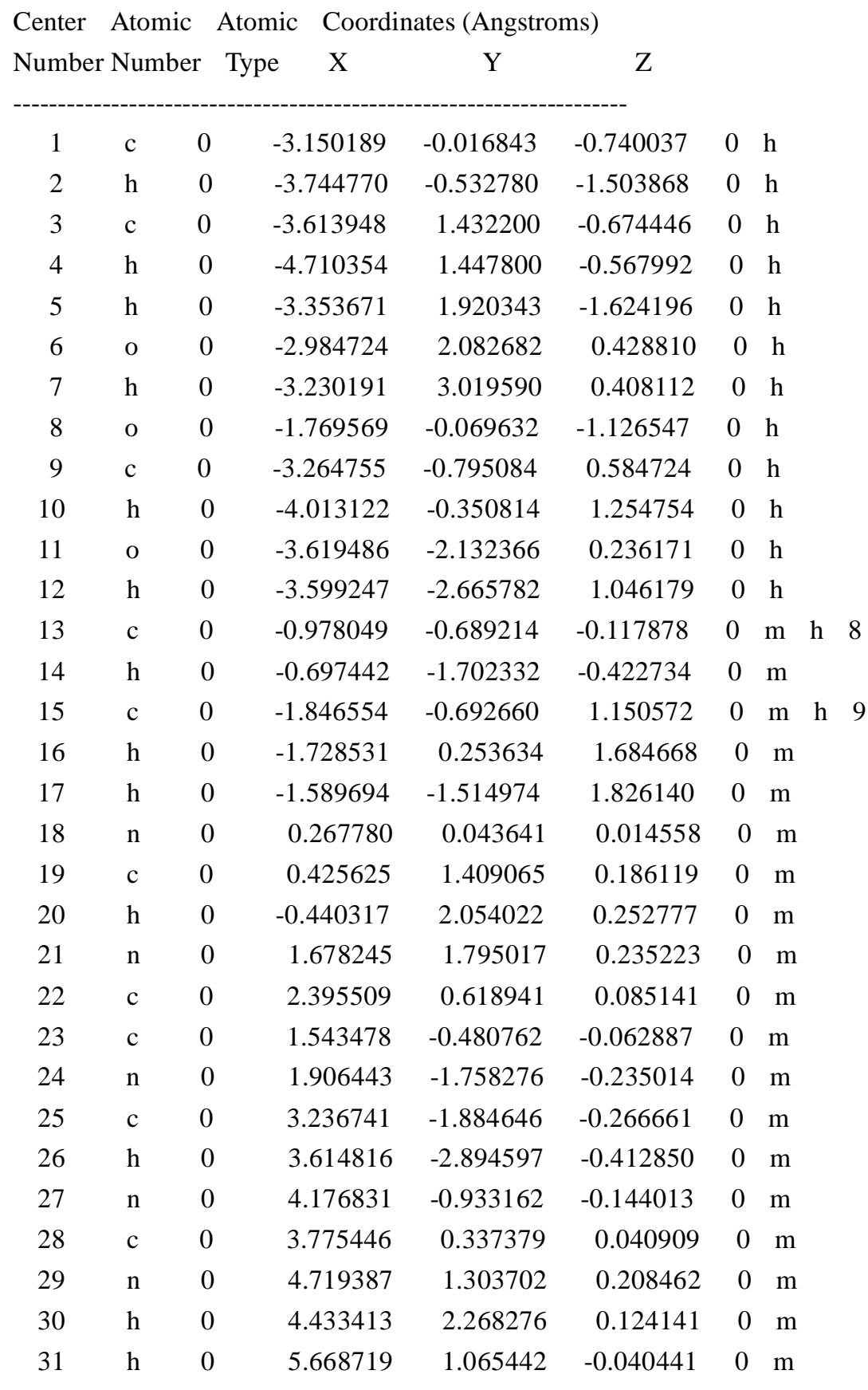


radical

Charge $=0$ Multiplicity $=2$ for low level calculation on real system.

Charge $=0$ Multiplicity $=2$ for high level calculation on model system.

Charge $=0$ Multiplicity $=2$ for low level calculation on model system.

Center Atomic Atomic Coordinates (Angstroms)

Number Number Type X $\quad$ Y $\quad$ Z

$\begin{array}{rrrrrrrrr}1 & \mathrm{c} & 0 & -3.088469 & 0.009401 & -0.535660 & 0 & \mathrm{~h} & \\ 2 & \mathrm{c} & 0 & -3.896862 & 1.223598 & -0.792030 & 0 & \mathrm{~h} & \\ 3 & \mathrm{~h} & 0 & -4.963216 & 0.983939 & -0.672679 & 0 & \mathrm{~h} & \\ 4 & \mathrm{~h} & 0 & -3.732763 & 1.565372 & -1.823736 & 0 & \mathrm{~h} & \\ 5 & \mathrm{o} & 0 & -3.492984 & 2.241531 & 0.150729 & 0 & \mathrm{~h} & \\ 6 & \mathrm{~h} & 0 & -3.972991 & 3.056286 & -0.071224 & 0 & \mathrm{~h} & \\ 7 & \mathrm{o} & 0 & -1.794239 & 0.064080 & -0.994840 & 0 & \mathrm{~h} & \\ 8 & \mathrm{c} & 0 & -3.197729 & -0.893096 & 0.655653 & 0 & \mathrm{~h} & \\ 9 & \mathrm{~h} & 0 & -3.881446 & -0.486467 & 1.413313 & 0 & \mathrm{~h} & \\ 10 & \mathrm{o} & 0 & -3.648349 & -2.181717 & 0.211890 & 0 & \mathrm{~h} & \\ 11 & \mathrm{~h} & 0 & -3.731670 & -2.745652 & 0.998745 & 0 & \mathrm{~h} & \\ 12 & \mathrm{c} & 0 & -0.931720 & -0.711554 & -0.142747 & 0 & \mathrm{~m} & \mathrm{~h} \\ 13 & \mathrm{~h} & 0 & -0.672807 & -1.652928 & -0.634148 & 0 & \mathrm{~m} & \\ 14 & \mathrm{c} & 0 & -1.738677 & -0.921384 & 1.149857 & 0 & \mathrm{~m} & \mathrm{~h} \\ 15 & \mathrm{~h} & 0 & -1.558385 & -0.082832 & 1.831018 & 0 & \mathrm{~m} & \\ 16 & \mathrm{~h} & 0 & -1.465923 & -1.850554 & 1.658379 & 0 & \mathrm{~m} & \\ 17 & \mathrm{n} & 0 & 0.296874 & 0.030869 & 0.021929 & 0 & \mathrm{~m} & \\ 18 & \mathrm{c} & 0 & 0.426146 & 1.380488 & 0.315736 & 0 & \mathrm{~m} & \\ 19 & \mathrm{~h} & 0 & -0.450460 & 2.005556 & 0.428813 & 0 & \mathrm{~m} & \\ 20 & \mathrm{n} & 0 & 1.670476 & 1.784603 & 0.391236 & 0 & \mathrm{~m} & \\ 21 & \mathrm{c} & 0 & 2.411061 & 0.641919 & 0.133658 & 0 & \mathrm{~m} & \\ 22 & \mathrm{c} & 0 & 1.582751 & -0.458315 & -0.108433 & 0 & \mathrm{~m} & \\ 23 & \mathrm{n} & 0 & 1.967320 & -1.707412 & -0.396810 & 0 & \mathrm{~m} & \\ 24 & \mathrm{c} & 0 & 3.300317 & -1.802814 & -0.447147 & 0 & \mathrm{~m} & \\ 25 & \mathrm{~h} & 0 & 3.697473 & -2.787246 & -0.686237 & 0 & \mathrm{~m} & \\ 26 & \mathrm{n} & 0 & 4.220973 & -0.846885 & -0.243969 & 0 & \mathrm{~m} & \\ 27 & \mathrm{c} & 0 & 3.796833 & 0.394260 & 0.056884 & 0 & \mathrm{~m} & \\ 28 & \mathrm{n} & 0 & 4.721365 & 1.359404 & 0.303531 & 0 & \mathrm{~m} & \\ 29 & \mathrm{~h} & 0 & 4.418513 & 2.322076 & 0.324622 & 0 & \mathrm{~m} & \\ 30 & \mathrm{~h} & 0 & 5.677288 & 1.162224 & 0.044849 & 0 & \mathrm{~m} & \\ ----------------------------------------------- & & & \end{array}$




\section{s5}

neutral molecule

Charge $=0$ Multiplicity $=1$ for low level calculation on real system.

Charge $=0$ Multiplicity $=1$ for high level calculation on model system .

Charge $=0$ Multiplicity $=1$ for low level calculation on model system .

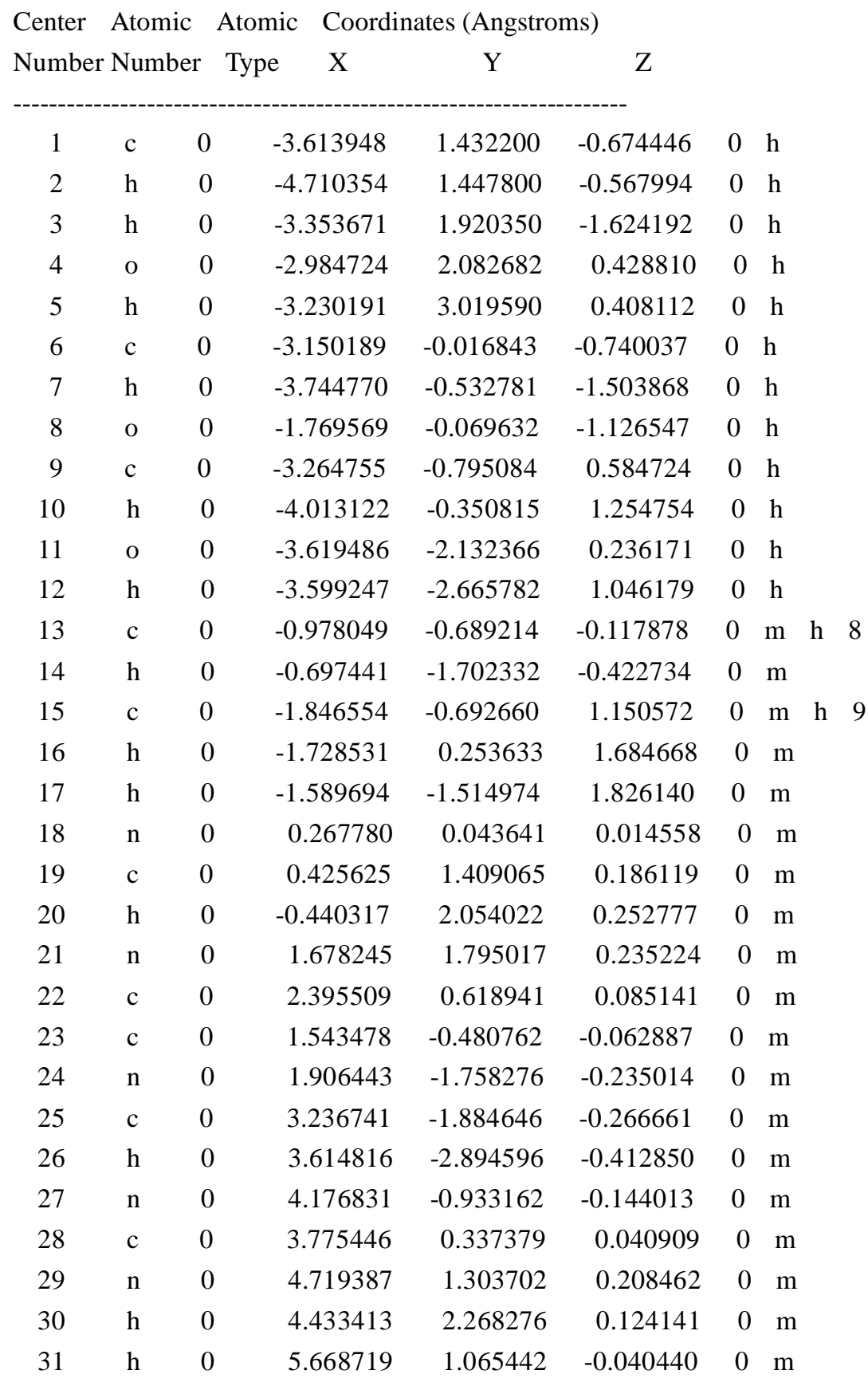


radical

Charge $=0$ Multiplicity $=2$ for low level calculation on real system.

Charge $=0$ Multiplicity $=2$ for high level calculation on model system.

Charge $=0$ Multiplicity $=2$ for low level calculation on model system.

Center Atomic Atomic Coordinates (Angstroms)

Number Number Type X $\quad$ Y $\quad$ Z

$\begin{array}{rrrrrrrrr}1 & \mathrm{c} & 0 & -3.690363 & 1.429290 & -0.544049 & 0 & \mathrm{~h} & \\ 2 & \mathrm{~h} & 0 & -4.716465 & 1.698247 & -0.785649 & 0 & \mathrm{~h} & \\ 3 & \mathrm{o} & 0 & -3.051230 & 2.170013 & 0.422337 & 0 & \mathrm{~h} & \\ 4 & \mathrm{~h} & 0 & -3.427855 & 3.062656 & 0.442833 & 0 & \mathrm{~h} & \\ 5 & \mathrm{c} & 0 & -3.216192 & 0.029823 & -0.699328 & 0 & \mathrm{~h} & \\ 6 & \mathrm{~h} & 0 & -3.826159 & -0.461157 & -1.465725 & 0 & \mathrm{~h} & \\ 7 & \mathrm{o} & 0 & -1.833910 & 0.011362 & -1.130828 & 0 & \mathrm{~h} & \\ 8 & \mathrm{c} & 0 & -3.256960 & -0.843740 & 0.584670 & 0 & \mathrm{~h} & \\ 9 & \mathrm{~h} & 0 & -4.012782 & -0.483172 & 1.295443 & 0 & \mathrm{~h} & \\ 10 & \mathrm{o} & 0 & -3.550040 & -2.171264 & 0.156621 & 0 & \mathrm{~h} & \\ 11 & \mathrm{~h} & 0 & -3.480961 & -2.756451 & 0.927514 & 0 & \mathrm{~h} & \\ 12 & \mathrm{c} & 0 & -1.009145 & -0.648438 & -0.180623 & 0 & \mathrm{~m} & \mathrm{~h} \\ 13 & \mathrm{~h} & 0 & -0.740577 & -1.650950 & -0.529357 & 0 & \mathrm{~m} & \\ 14 & \mathrm{c} & 0 & -1.831056 & -0.704847 & 1.116133 & 0 & \mathrm{~m} & \mathrm{~h} \\ 15 & \mathrm{~h} & 0 & -1.723825 & 0.235596 & 1.665436 & 0 & \mathrm{~m} & \\ 16 & \mathrm{~h} & 0 & -1.526477 & -1.529507 & 1.768911 & 0 & \mathrm{~m} & \\ 17 & \mathrm{n} & 0 & 0.242064 & 0.080867 & -0.063474 & 0 & \mathrm{~m} & \\ 18 & \mathrm{c} & 0 & 0.413241 & 1.448315 & 0.068901 & 0 & \mathrm{~m} & \\ 19 & \mathrm{~h} & 0 & -0.442923 & 2.108463 & 0.089425 & 0 & \mathrm{~m} & \\ 20 & \mathrm{n} & 0 & 1.669415 & 1.820309 & 0.140422 & 0 & \mathrm{~m} & \\ 21 & \mathrm{c} & 0 & 2.374419 & 0.631302 & 0.049985 & 0 & \mathrm{~m} & \\ 22 & \mathrm{c} & 0 & 1.511744 & -0.461943 & -0.084628 & 0 & \mathrm{~m} & \\ 23 & \mathrm{n} & 0 & 1.861460 & -1.749767 & -0.200908 & 0 & \mathrm{~m} & \\ 24 & \mathrm{c} & 0 & 3.190222 & -1.894191 & -0.189565 & 0 & \mathrm{~m} & \\ 25 & \mathrm{~h} & 0 & 3.558381 & -2.913444 & -0.289206 & 0 & \mathrm{~m} & \\ 26 & \mathrm{n} & 0 & 4.139607 & -0.951090 & -0.073052 & 0 & \mathrm{~m} & \\ 27 & \mathrm{c} & 0 & 3.751062 & 0.330343 & 0.055397 & 0 & \mathrm{~m} & \\ 28 & \mathrm{n} & 0 & 4.703217 & 1.289561 & 0.217216 & 0 & \mathrm{~m} & \\ 29 & \mathrm{~h} & 0 & 4.433268 & 2.253936 & 0.088934 & 0 & \mathrm{~m} & \\ 30 & \mathrm{~h} & 0 & 5.655644 & 1.030256 & 0.003618 & 0 & \mathrm{~m} & \end{array}$




\section{b2}

neutral molecule

Charge $=0$ Multiplicity $=1$ for low level calculation on real system.

Charge $=0$ Multiplicity $=1$ for high level calculation on model system.

Charge $=0$ Multiplicity $=1$ for low level calculation on model system .

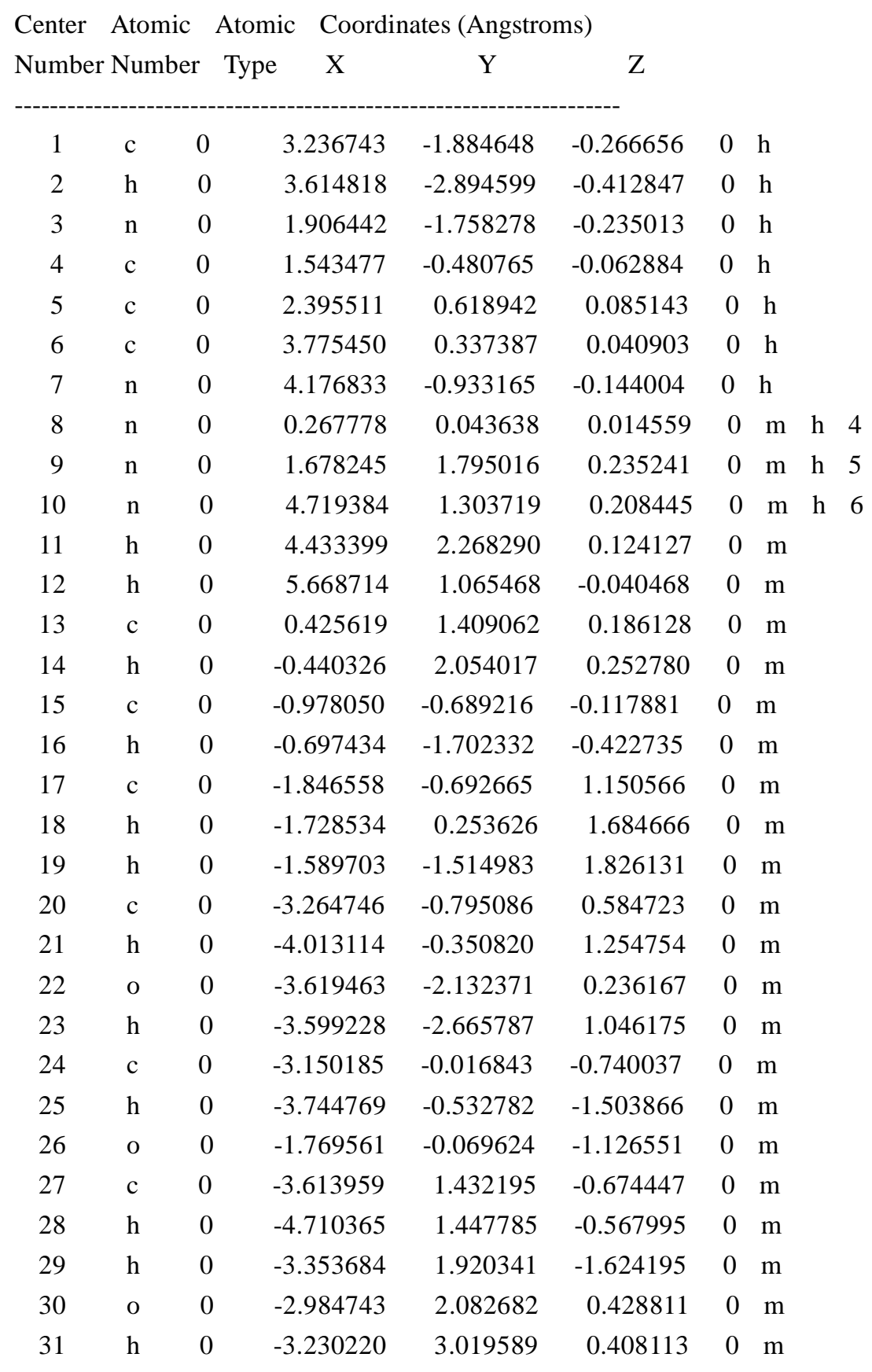


radical

Charge $=0$ Multiplicity $=2$ for low level calculation on real system.

Charge $=0$ Multiplicity $=2$ for high level calculation on model system.

Charge $=0$ Multiplicity $=2$ for low level calculation on model system.

Center Atomic Atomic Coordinates (Angstroms)

Number Number Type X $\quad$ Y $\quad$ Z

\begin{tabular}{|c|c|c|c|c|c|c|c|c|}
\hline 1 & c & 0 & 3.229702 & -1.904101 & -0.273239 & 0 & $\mathrm{~h}$ & \\
\hline 2 & $\mathrm{n}$ & 0 & 1.921666 & -1.816822 & -0.250596 & 0 & $\mathrm{~h}$ & \\
\hline 3 & $\mathrm{c}$ & 0 & 1.558636 & -0.528608 & -0.073878 & 0 & $\mathrm{~h}$ & \\
\hline 4 & $\mathrm{c}$ & 0 & 2.430856 & 0.557333 & 0.078310 & 0 & $\mathrm{~h}$ & \\
\hline 5 & $\mathrm{c}$ & 0 & 3.811623 & 0.270990 & 0.034359 & 0 & $\mathrm{~h}$ & \\
\hline 6 & $\mathrm{n}$ & 0 & 4.188824 & -1.016483 & -0.154841 & 0 & $\mathrm{~h}$ & \\
\hline 7 & $\mathrm{n}$ & 0 & 0.293953 & 0.011421 & 0.005717 & 0 & $\mathrm{~m}$ & h 3 \\
\hline 8 & $\mathrm{n}$ & 0 & 1.728298 & 1.742305 & 0.234844 & 0 & $\mathrm{~m}$ & h 4 \\
\hline 9 & $\mathrm{n}$ & 0 & 4.762894 & 1.221970 & 0.197802 & 0 & $\mathrm{~m}$ & h 5 \\
\hline 10 & $\mathrm{~h}$ & 0 & 4.489910 & 2.192825 & 0.156666 & 0 & $\mathrm{~m}$ & \\
\hline 11 & $\mathrm{~h}$ & 0 & 5.718091 & 0.976437 & -0.017713 & 0 & $\mathrm{~m}$ & \\
\hline 12 & $\mathrm{c}$ & 0 & 0.471035 & 1.376245 & 0.184217 & 0 & $\mathrm{~m}$ & \\
\hline 13 & $\mathrm{~h}$ & 0 & -0.386219 & 2.032114 & 0.255336 & 0 & $\mathrm{~m}$ & \\
\hline 14 & $\mathrm{c}$ & 0 & -0.963562 & -0.703298 & -0.130193 & 0 & $\mathrm{~m}$ & \\
\hline 15 & $\mathrm{~h}$ & 0 & -0.699080 & -1.717238 & -0.446762 & 0 & $\mathrm{~m}$ & \\
\hline 16 & $\mathrm{c}$ & 0 & -1.826832 & -0.707173 & 1.141743 & 0 & $\mathrm{~m}$ & \\
\hline 17 & $\mathrm{~h}$ & 0 & -1.691091 & 0.230910 & 1.686044 & 0 & $\mathrm{~m}$ & \\
\hline 18 & $\mathrm{~h}$ & 0 & -1.581261 & -1.541482 & 1.806580 & 0 & $\mathrm{~m}$ & \\
\hline 19 & $\mathrm{c}$ & 0 & -3.248686 & -0.779633 & 0.579635 & 0 & $\mathrm{~m}$ & \\
\hline 20 & $\mathrm{~h}$ & 0 & -3.987145 & -0.331185 & 1.257786 & 0 & $\mathrm{~m}$ & \\
\hline 21 & o & 0 & -3.625836 & -2.106453 & 0.216208 & 0 & $\mathrm{~m}$ & \\
\hline 22 & $\mathrm{~h}$ & 0 & -3.615957 & -2.649601 & 1.019937 & 0 & $\mathrm{~m}$ & \\
\hline 23 & $\mathrm{c}$ & 0 & -3.125948 & 0.012158 & -0.736133 & 0 & $\mathrm{~m}$ & \\
\hline 24 & $\mathrm{~h}$ & 0 & -3.732016 & -0.484252 & -1.503692 & 0 & $\mathrm{~m}$ & \\
\hline 25 & o & 0 & -1.747500 & -0.061347 & -1.129197 & 0 & $\mathrm{~m}$ & \\
\hline 26 & $\mathrm{c}$ & 0 & -3.563109 & 1.468331 & -0.652462 & 0 & $\mathrm{~m}$ & \\
\hline 27 & $\mathrm{~h}$ & 0 & -4.658430 & 1.502205 & -0.539945 & 0 & $\mathrm{~m}$ & \\
\hline 28 & $\mathrm{~h}$ & 0 & -3.299152 & 1.962088 & -1.598275 & 0 & $\mathrm{~m}$ & \\
\hline 29 & o & 0 & -2.916507 & 2.095482 & 0.454310 & 0 & $\mathrm{~m}$ & \\
\hline 30 & $\mathrm{~h}$ & 0 & -3.150637 & 3.035526 & 0.448843 & 0 & $\mathrm{~m}$ & \\
\hline
\end{tabular}




\section{b6}

neutral molecule

Charge $=0$ Multiplicity $=1$ for low level calculation on real system.

Charge $=0$ Multiplicity $=1$ for high level calculation on model system.

Charge $=0$ Multiplicity $=1$ for low level calculation on model system .

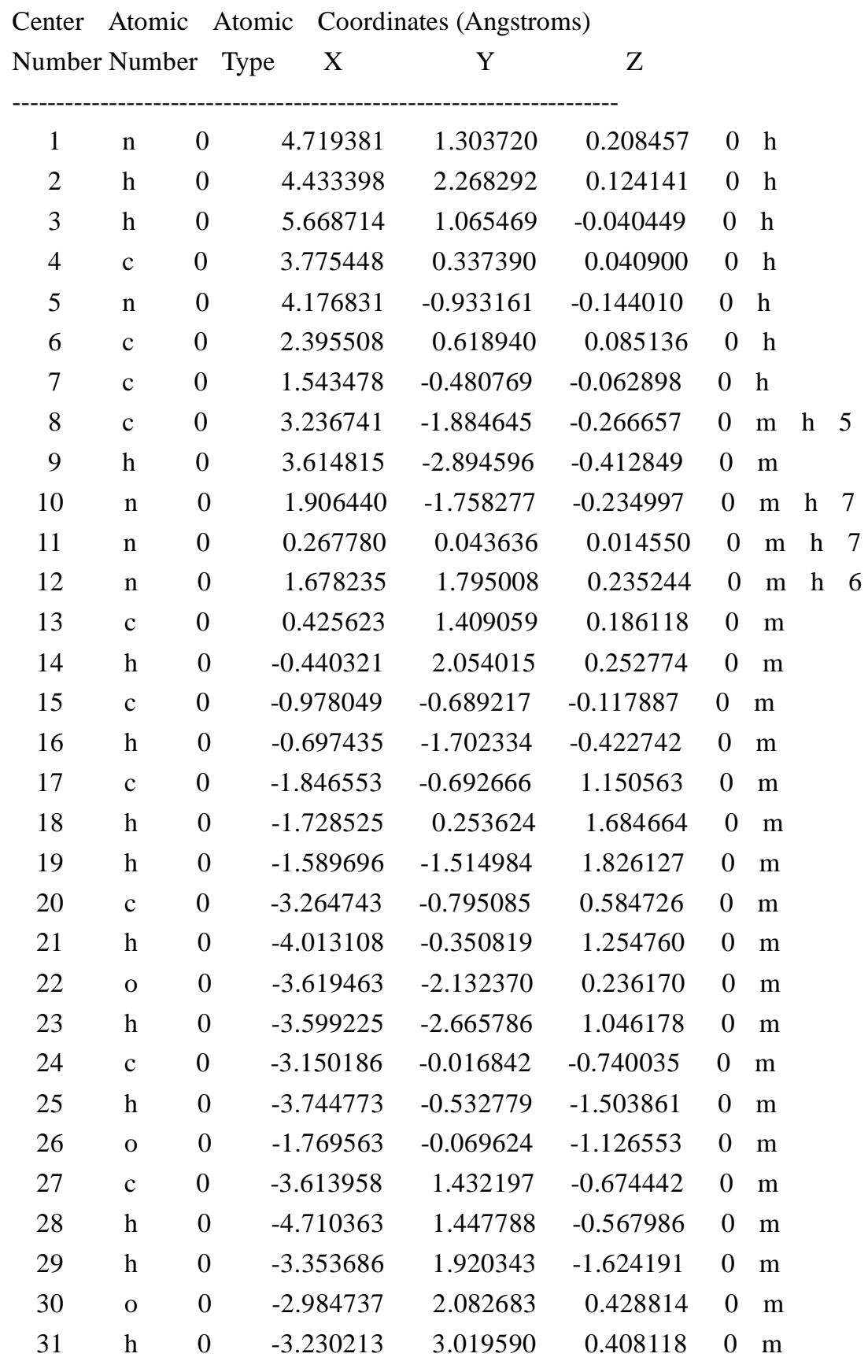


radical

Charge $=0$ Multiplicity $=2$ for low level calculation on real system.

Charge $=0$ Multiplicity $=2$ for high level calculation on model system.

Charge $=0$ Multiplicity $=2$ for low level calculation on model system.

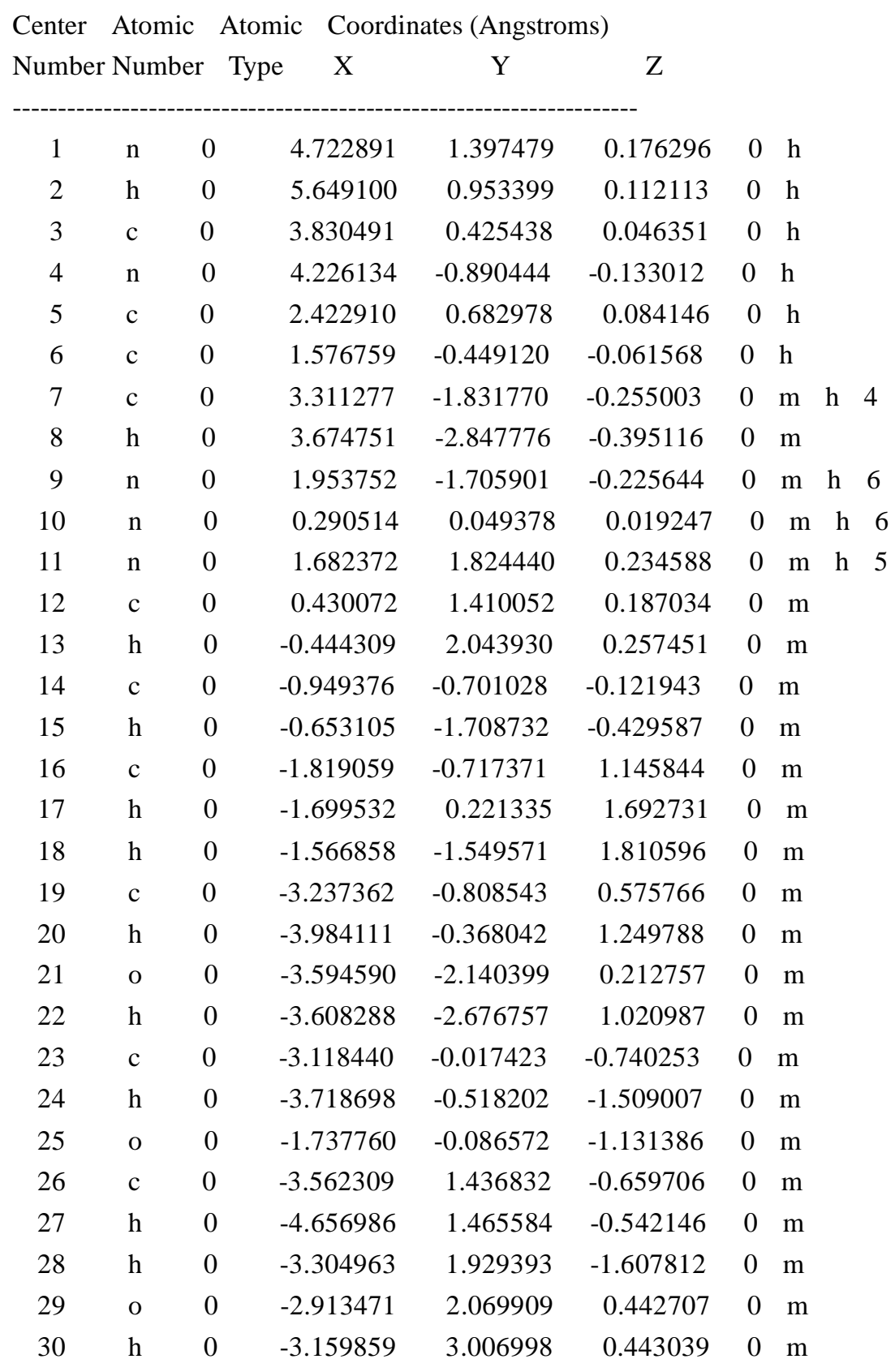




\section{b8}

neutral molecule

Charge $=0$ Multiplicity $=1$ for low level calculation on real system.

Charge $=0$ Multiplicity $=1$ for high level calculation on model system.

Charge $=0$ Multiplicity $=1$ for low level calculation on model system .

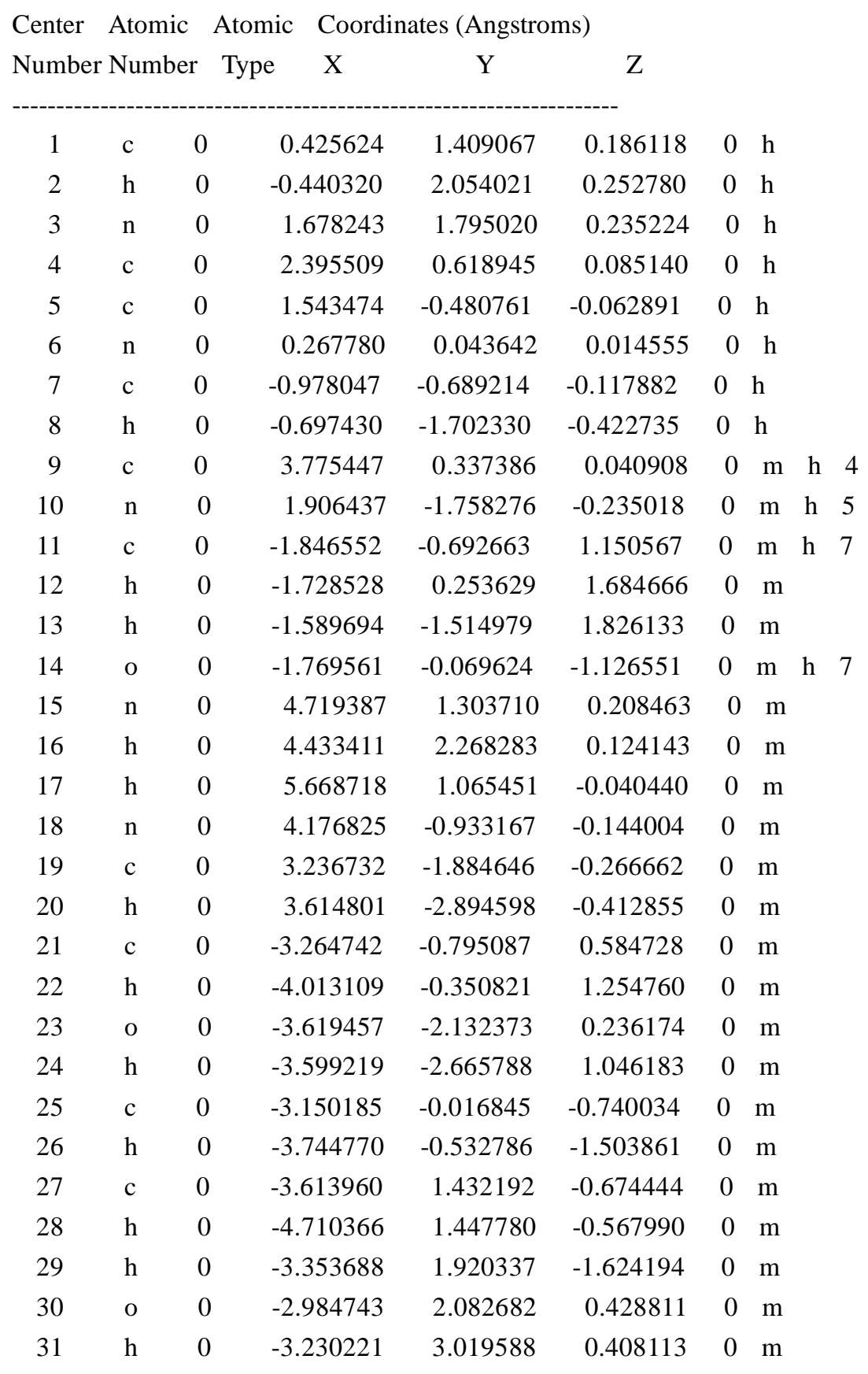


radical

Charge $=0$ Multiplicity $=2$ for low level calculation on real system.

Charge $=0$ Multiplicity $=2$ for high level calculation on model system.

Charge $=0$ Multiplicity $=2$ for low level calculation on model system.

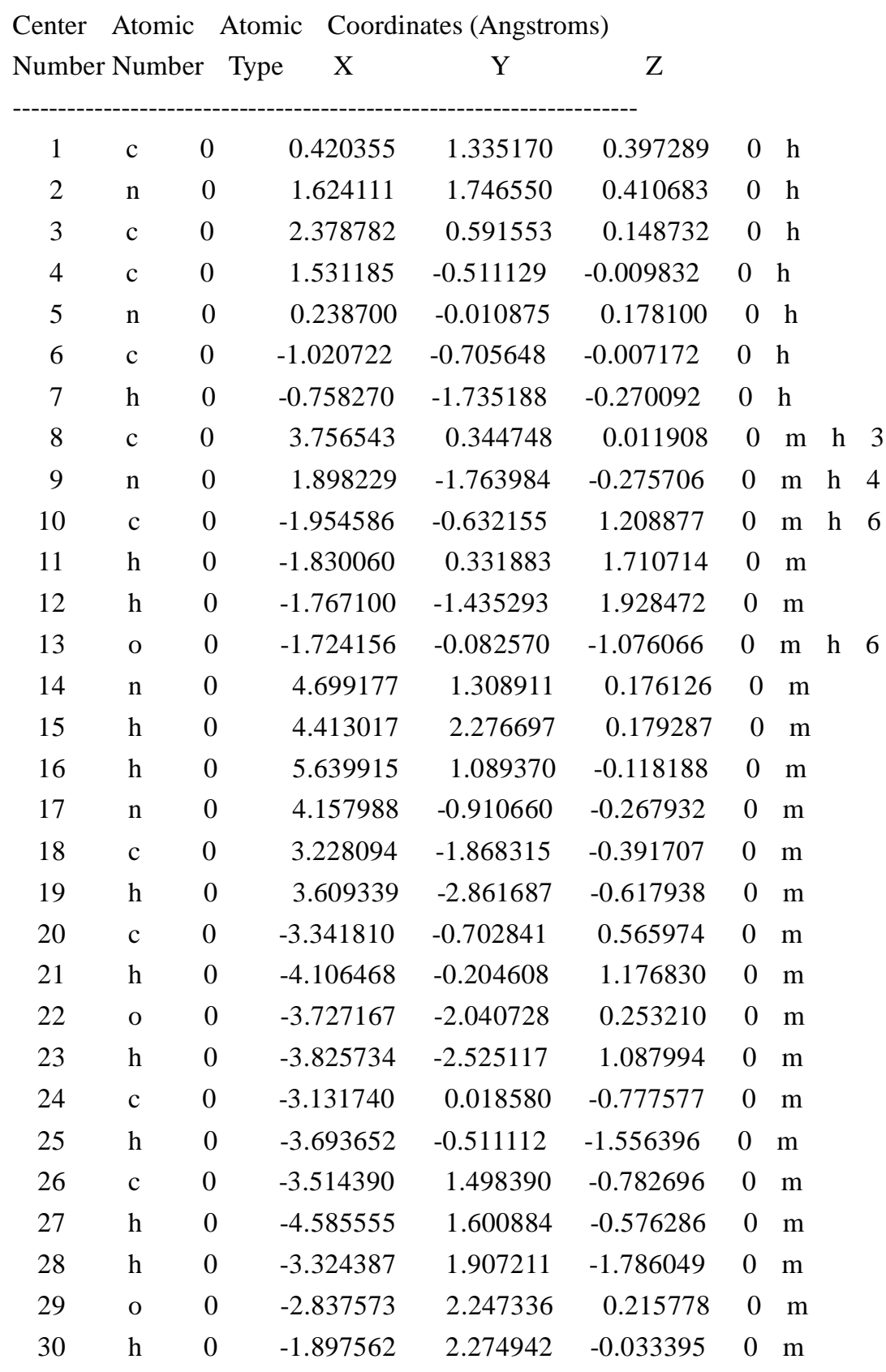




\section{dC}

\section{s1}

neutral molecule

Charge $=0$ Multiplicity $=1$ for low level calculation on real system.

Charge $=0$ Multiplicity $=1$ for high level calculation on model system .

Charge $=0$ Multiplicity $=1$ for low level calculation on model system .

Center Atomic Atomic Coordinates (Angstroms)

Number Number Type $\quad \mathrm{X} \quad \mathrm{Y} \quad \mathrm{Z}$

\begin{tabular}{|c|c|c|c|c|c|c|c|c|}
\hline 1 & $\mathrm{c}$ & 0 & -0.573268 & -0.736961 & -0.326703 & 0 & $\mathrm{~h}$ & \\
\hline 2 & $\mathrm{~h}$ & 0 & -0.510239 & -1.707549 & -0.821799 & 0 & $\mathrm{~h}$ & \\
\hline 3 & $\mathrm{c}$ & 0 & -1.347257 & -0.829668 & 0.994485 & 0 & $\mathrm{~h}$ & \\
\hline 4 & $\mathrm{~h}$ & 0 & -1.050432 & -0.008859 & 1.654330 & 0 & $\mathrm{~h}$ & \\
\hline 5 & $\mathrm{~h}$ & 0 & -1.172118 & -1.780631 & 1.506454 & 0 & $\mathrm{~h}$ & \\
\hline 6 & o & 0 & -1.291671 & 0.197169 & -1.124834 & 0 & $\mathrm{~h}$ & \\
\hline 7 & $\mathrm{n}$ & 0 & 0.809749 & -0.271986 & -0.180737 & 0 & $\mathrm{~h}$ & \\
\hline 8 & $\mathrm{c}$ & 0 & 1.833609 & -1.276480 & -0.074893 & 0 & $\mathrm{~h}$ & \\
\hline 9 & o & 0 & 1.495674 & -2.453631 & -0.054433 & 0 & $\mathrm{~h}$ & \\
\hline 10 & $\mathrm{c}$ & 0 & -2.794178 & -0.630824 & 0.541081 & 0 & $\mathrm{~m}$ & h 3 \\
\hline 11 & $\mathrm{~h}$ & 0 & -3.424119 & -0.199997 & 1.331515 & 0 & $\mathrm{~m}$ & \\
\hline 12 & $\mathrm{c}$ & 0 & -2.642316 & 0.331108 & -0.656091 & 0 & $\mathrm{~m}$ & $\mathrm{~h}$ \\
\hline 13 & $\mathrm{~h}$ & 0 & -3.324089 & 0.004176 & -1.451118 & 0 & $\mathrm{~m}$ & \\
\hline 14 & $\mathrm{c}$ & 0 & 1.098878 & 1.051417 & -0.087684 & 0 & $\mathrm{~m}$ & $\mathrm{~h}$ \\
\hline 15 & $\mathrm{~h}$ & 0 & 0.247385 & 1.721162 & -0.122776 & 0 & $\mathrm{~m}$ & \\
\hline 16 & $\mathrm{n}$ & 0 & 3.128050 & -0.837208 & -0.006442 & 0 & $\mathrm{~m}$ & $\mathrm{~h}$ \\
\hline 17 & $\mathrm{o}$ & 0 & -3.376438 & -1.824882 & 0.020007 & 0 & $\mathrm{~m}$ & \\
\hline 18 & $\mathrm{~h}$ & 0 & -3.332070 & -2.503738 & 0.711579 & 0 & $\mathrm{~m}$ & \\
\hline 19 & $\mathrm{c}$ & 0 & -2.933001 & 1.793027 & -0.351411 & 0 & $\mathrm{~m}$ & \\
\hline 20 & $\mathrm{~h}$ & 0 & -4.006809 & 1.899361 & -0.130126 & 0 & $\mathrm{~m}$ & \\
\hline 21 & $\mathrm{~h}$ & 0 & -2.708346 & 2.388200 & -1.248607 & 0 & $\mathrm{~m}$ & \\
\hline 22 & o & 0 & -2.134075 & 2.209120 & 0.754281 & 0 & $\mathrm{~m}$ & \\
\hline 23 & $\mathrm{~h}$ & 0 & -2.322321 & 3.143298 & 0.928619 & 0 & $\mathrm{~m}$ & \\
\hline 24 & $\mathrm{c}$ & 0 & 2.386157 & 1.474054 & 0.043184 & 0 & $\mathrm{~m}$ & \\
\hline 25 & $\mathrm{~h}$ & 0 & 2.627315 & 2.527109 & 0.125393 & 0 & $\mathrm{~m}$ & \\
\hline 26 & $\mathrm{c}$ & 0 & 3.393170 & 0.453719 & 0.058591 & 0 & $\mathrm{~m}$ & \\
\hline 27 & $\mathrm{n}$ & 0 & 4.708977 & 0.809823 & 0.183226 & 0 & $\mathrm{~m}$ & \\
\hline 28 & $\mathrm{~h}$ & 0 & 4.992204 & 1.744212 & -0.071973 & 0 & $\mathrm{~m}$ & \\
\hline 29 & $\mathrm{~h}$ & 0 & 5.377525 & 0.070280 & 0.012657 & 0 & $\mathrm{~m}$ & \\
\hline
\end{tabular}


radical

Charge $=0$ Multiplicity $=2$ for low level calculation on real system.

Charge $=0$ Multiplicity $=2$ for high level calculation on model system .

Charge $=0$ Multiplicity $=2$ for low level calculation on model system.

\begin{tabular}{|c|c|c|c|c|c|c|c|c|}
\hline Center & Atomic & & Atomic & ates (Angstro & & & & \\
\hline Number & Numbe & & Type & $\mathrm{Y}$ & $\mathrm{Z}$ & & & \\
\hline 1 & $\mathrm{c}$ & 0 & 0.539196 & 0.313290 & -0.317215 & 0 & $\mathrm{~h}$ & \\
\hline 2 & $\mathrm{c}$ & 0 & 1.402138 & 1.138216 & 0.588287 & 0 & & \\
\hline 3 & $\mathrm{~h}$ & 0 & 1.324671 & 0.792542 & 1.629355 & 0 & $\mathrm{~h}$ & \\
\hline 4 & $\mathrm{~h}$ & 0 & 1.140787 & 2.196948 & 0.572650 & 0 & & \\
\hline 5 & o & 0 & 1.254450 & -0.693454 & -0.909993 & 0 & $\mathrm{~h}$ & \\
\hline 6 & $\mathrm{n}$ & 0 & -0.816036 & 0.050276 & -0.188670 & 0 & $\mathrm{~h}$ & \\
\hline 7 & $\mathrm{c}$ & 0 & -1.701343 & 1.114447 & 0.325449 & 0 & $\mathrm{~h}$ & \\
\hline 8 & o & 0 & -1.186363 & 2.173647 & 0.653814 & 0 & & \\
\hline 9 & $\mathrm{c}$ & 0 & 2.806195 & 0.847752 & 0.033735 & 0 & $\mathrm{~m}$ & $\mathrm{~h}$ \\
\hline 10 & $\mathrm{~h}$ & 0 & 3.565191 & 0.851004 & 0.827433 & 0 & $\mathrm{~m}$ & \\
\hline 11 & $\mathrm{c}$ & 0 & 2.660576 & -0.547573 & -0.596893 & 0 & $\mathrm{~m}$ & h 5 \\
\hline 12 & $\mathrm{~h}$ & 0 & 3.209566 & -0.584795 & -1.542549 & 0 & $\mathrm{~m}$ & \\
\hline 13 & $\mathrm{c}$ & 0 & -1.353728 & -1.133980 & -0.620338 & 0 & $\mathrm{~m}$ & h 6 \\
\hline 14 & $\mathrm{~h}$ & 0 & -0.647356 & -1.859968 & -0.997792 & 0 & $\mathrm{~m}$ & \\
\hline 15 & $\mathrm{n}$ & 0 & -3.025351 & 0.836934 & 0.387083 & 0 & $\mathrm{~m}$ & $\mathrm{~h}$ \\
\hline 16 & $\mathrm{o}$ & 0 & 3.175649 & 1.725703 & -1.026105 & 0 & $\mathrm{~m}$ & \\
\hline 17 & $\mathrm{~h}$ & 0 & 3.032735 & 2.635137 & -0.720989 & 0 & $\mathrm{~m}$ & \\
\hline 18 & $\mathrm{c}$ & 0 & 3.107065 & -1.702285 & 0.285348 & 0 & $\mathrm{~m}$ & \\
\hline 19 & $\mathrm{~h}$ & 0 & 4.206976 & -1.672822 & 0.357041 & 0 & $\mathrm{~m}$ & \\
\hline 20 & $\mathrm{~h}$ & 0 & 2.825222 & -2.646884 & -0.203587 & 0 & $\mathrm{~m}$ & \\
\hline 21 & o & 0 & 2.495648 & -1.570883 & 1.560718 & 0 & $\mathrm{~m}$ & \\
\hline 22 & $\mathrm{~h}$ & 0 & 2.717062 & -2.351989 & 2.088636 & 0 & $\mathrm{~m}$ & \\
\hline 23 & $\mathrm{c}$ & 0 & -2.700523 & -1.361284 & -0.563801 & 0 & $\mathrm{~m}$ & \\
\hline 24 & $\mathrm{~h}$ & 0 & -3.113207 & -2.306678 & -0.895907 & 0 & $\mathrm{~m}$ & \\
\hline 25 & $\mathrm{c}$ & 0 & -3.512202 & -0.323622 & -0.029141 & 0 & $\mathrm{~m}$ & \\
\hline 26 & $\mathrm{n}$ & 0 & -4.865295 & -0.507973 & 0.114532 & 0 & $\mathrm{~m}$ & \\
\hline 27 & $\mathrm{~h}$ & 0 & -5.323187 & -1.192173 & -0.470126 & 0 & $\mathrm{~m}$ & \\
\hline 28 & $\mathrm{~h}$ & 0 & -5.390999 & 0.335154 & 0.305161 & 0 & $\mathrm{~m}$ & \\
\hline
\end{tabular}

\section{s2}

neutral molecule

Charge $=0$ Multiplicity $=1$ for low level calculation on real system . 
Charge $=0$ Multiplicity $=1$ for high level calculation on model system .

Charge $=0$ Multiplicity $=1$ for low level calculation on model system.

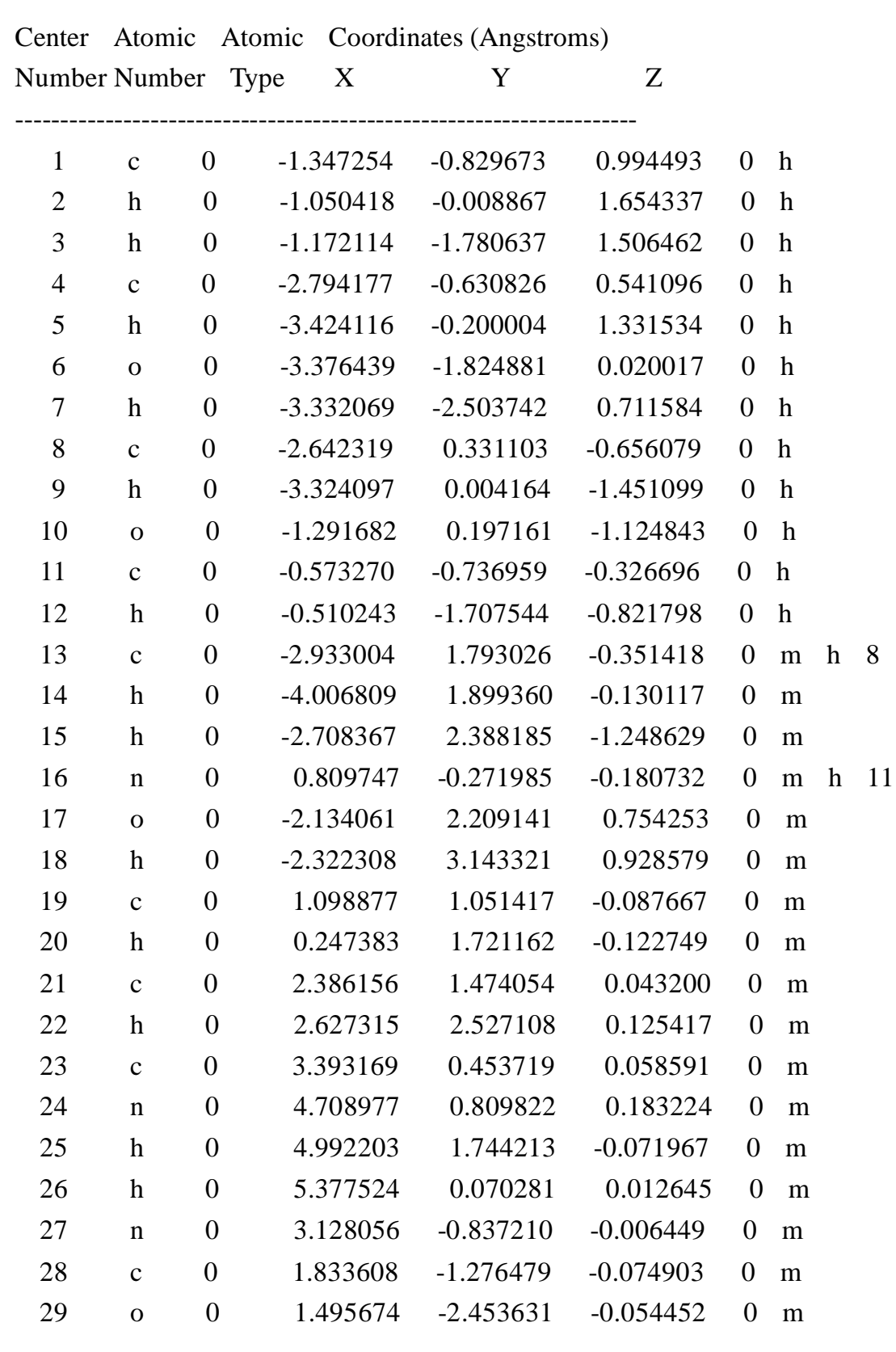

radical

Charge $=0$ Multiplicity $=2$ for low level calculation on real system.

Charge $=0$ Multiplicity $=2$ for high level calculation on model system.

Charge $=0$ Multiplicity $=2$ for low level calculation on model system. 


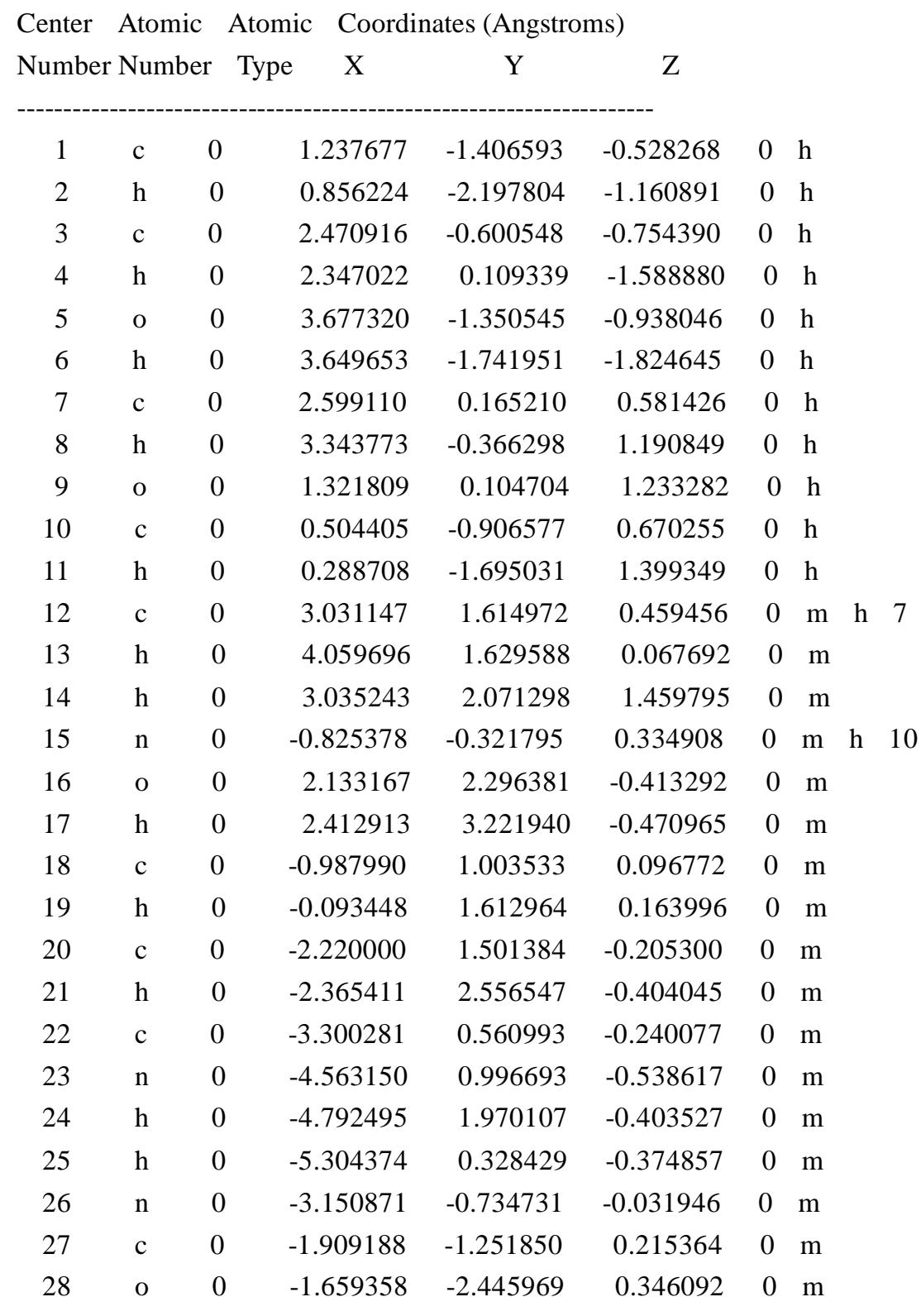

\section{s3}

neutral molecule

Charge $=0$ Multiplicity $=1$ for low level calculation on real system.

Charge $=0$ Multiplicity $=1$ for high level calculation on model system.

Charge $=0$ Multiplicity $=1$ for low level calculation on model system .

Center Atomic Atomic Coordinates (Angstroms)

Number Number Type $\quad \mathrm{X} \quad \mathrm{Y} \quad \mathrm{Z}$ 


\begin{tabular}{|c|c|c|c|c|c|c|c|}
\hline 1 & $\mathrm{c}$ & 0 & -2.794185 & -0.630818 & 0.541087 & 0 & h \\
\hline 2 & $\mathrm{~h}$ & 0 & -3.424138 & -0.199995 & 1.331513 & 0 & $\mathrm{~h}$ \\
\hline 3 & o & 0 & -3.376432 & -1.824888 & 0.020024 & 0 & $\mathrm{~h}$ \\
\hline 4 & $\mathrm{~h}$ & 0 & -3.332064 & -2.503735 & 0.711605 & 0 & $\mathrm{~h}$ \\
\hline 5 & $\mathrm{c}$ & 0 & -2.642319 & 0.331100 & -0.656096 & 0 & $\mathrm{~h}$ \\
\hline 6 & $\mathrm{~h}$ & 0 & -3.324081 & 0.004145 & -1.451123 & 0 & $\mathrm{~h}$ \\
\hline 7 & o & 0 & -1.291673 & 0.197168 & -1.124838 & 0 & $\mathrm{~h}$ \\
\hline 8 & $\mathrm{c}$ & 0 & -0.573266 & -0.736956 & -0.326704 & 0 & h \\
\hline 9 & $\mathrm{~h}$ & 0 & -0.510238 & -1.707543 & -0.821803 & 0 & $\mathrm{~h}$ \\
\hline 10 & $\mathrm{c}$ & 0 & -1.347254 & -0.829664 & 0.994484 & 0 & $\mathrm{~h}$ \\
\hline 11 & $\mathrm{~h}$ & 0 & -1.050435 & -0.008851 & 1.654327 & 0 & $\mathrm{~h}$ \\
\hline 12 & $\mathrm{~h}$ & 0 & -1.172110 & -1.780625 & 1.506456 & 0 & $\mathrm{~h}$ \\
\hline 13 & $\mathrm{c}$ & 0 & -2.933013 & 1.793019 & -0.351419 & 0 & \\
\hline 14 & $\mathrm{~h}$ & 0 & -4.006821 & 1.899346 & -0.130136 & 0 & $\mathrm{~m}$ \\
\hline 15 & $\mathrm{~h}$ & 0 & -2.708361 & 2.388190 & -1.248617 & 0 & $\mathrm{~m}$ \\
\hline 16 & $\mathrm{n}$ & 0 & 0.809752 & -0.271982 & -0.180736 & 0 & $\mathrm{~m}$ \\
\hline 17 & o & 0 & -2.134090 & 2.209119 & 0.754272 & 0 & $\mathrm{~m}$ \\
\hline 18 & $\mathrm{~h}$ & 0 & -2.322342 & 3.143296 & 0.928608 & 0 & $\mathrm{~m}$ \\
\hline 19 & $\mathrm{c}$ & 0 & 1.098882 & 1.051420 & -0.087676 & 0 & $\mathrm{~m}$ \\
\hline 20 & $\mathrm{~h}$ & 0 & 0.247389 & 1.721165 & -0.122763 & 0 & $\mathrm{~m}$ \\
\hline 21 & $\mathrm{c}$ & 0 & 2.386161 & 1.474056 & 0.043193 & 0 & $\mathrm{~m}$ \\
\hline 22 & $\mathrm{~h}$ & 0 & 2.627320 & 2.527110 & 0.125407 & 0 & $\mathrm{~m}$ \\
\hline 23 & $\mathrm{c}$ & 0 & 3.393173 & 0.453720 & 0.058593 & 0 & $\mathrm{~m}$ \\
\hline 24 & $\mathrm{n}$ & 0 & 4.708981 & 0.809823 & 0.183229 & 0 & $\mathrm{~m}$ \\
\hline 25 & $\mathrm{~h}$ & 0 & 4.992208 & 1.744213 & -0.071965 & 0 & $\mathrm{~m}$ \\
\hline 26 & $\mathrm{~h}$ & 0 & 5.377529 & 0.070281 & 0.012655 & 0 & $\mathrm{~m}$ \\
\hline 27 & $\mathrm{n}$ & 0 & 3.128059 & -0.837208 & -0.006442 & 0 & $\mathrm{~m}$ \\
\hline 28 & $\mathrm{c}$ & 0 & 1.833612 & -1.276477 & -0.074899 & 0 & $\mathrm{~m}$ \\
\hline 29 & o & 0 & 1.495677 & -2.453629 & -0.054445 & 0 & $\mathrm{~m}$ \\
\hline
\end{tabular}

radical

Charge $=0$ Multiplicity $=2$ for low level calculation on real system.

Charge $=0$ Multiplicity $=2$ for high level calculation on model system .

Charge $=0$ Multiplicity $=2$ for low level calculation on model system.

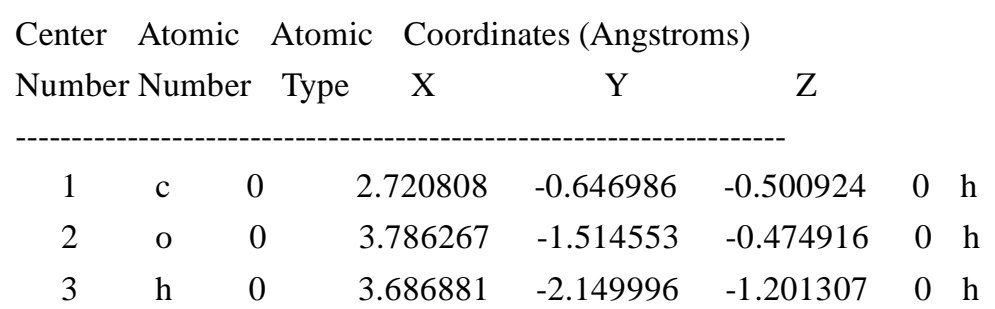




$\begin{array}{rrrrrrllll}4 & \mathrm{c} & 0 & 2.646270 & 0.295146 & 0.670828 & 0 & \mathrm{~h} & \\ 5 & \mathrm{~h} & 0 & 3.336682 & -0.038747 & 1.461061 & 0 & \mathrm{~h} & \\ 6 & \mathrm{o} & 0 & 1.289538 & 0.216783 & 1.151246 & 0 & \mathrm{~h} & \\ 7 & \mathrm{c} & 0 & 0.552628 & -0.779107 & 0.452445 & 0 & \mathrm{~h} & \\ 8 & \mathrm{~h} & 0 & 0.450999 & -1.684893 & 1.054301 & 0 & \mathrm{~h} & \\ 9 & \mathrm{c} & 0 & 1.328421 & -1.075962 & -0.855826 & 0 & \mathrm{~h} & \\ 10 & \mathrm{~h} & 0 & 0.912214 & -0.469132 & -1.670511 & 0 & \mathrm{~h} & \\ 11 & \mathrm{~h} & 0 & 1.241359 & -2.130778 & -1.136191 & 0 & \mathrm{~h} & \\ 12 & \mathrm{c} & 0 & 2.960375 & 1.755392 & 0.357372 & 0 & \mathrm{~m} & \mathrm{~h} & 4 \\ 13 & \mathrm{~h} & 0 & 4.018931 & 1.824665 & 0.063765 & 0 & \mathrm{~m} & \\ 14 & \mathrm{~h} & 0 & 2.814452 & 2.354347 & 1.269368 & 0 & \mathrm{~m} & \\ 15 & \mathrm{n} & 0 & -0.811878 & -0.287662 & 0.240818 & 0 & \mathrm{~m} & \mathrm{~h} & 7 \\ 16 & \mathrm{o} & 0 & 2.098313 & 2.191814 & -0.688885 & 0 & \mathrm{~m} & \\ 17 & \mathrm{~h} & 0 & 2.320019 & 3.111752 & -0.895966 & 0 & \mathrm{~m} & \\ 18 & \mathrm{c} & 0 & -1.067405 & 1.033309 & 0.053580 & 0 & \mathrm{~m} & \\ 19 & \mathrm{~h} & 0 & -0.202799 & 1.687226 & 0.073822 & 0 & \mathrm{~m} & \\ 20 & \mathrm{c} & 0 & -2.341880 & 1.468606 & -0.147087 & 0 & \mathrm{~m} & & \\ 21 & \mathrm{~h} & 0 & -2.558681 & 2.518367 & -0.304765 & 0 & \mathrm{~m} & \\ 22 & \mathrm{c} & 0 & -3.368835 & 0.468976 & -0.132621 & 0 & \mathrm{~m} & \\ 23 & \mathrm{n} & 0 & -4.672301 & 0.840665 & -0.322079 & 0 & \mathrm{~m} & \\ 24 & \mathrm{~h} & 0 & -4.944412 & 1.794982 & -0.138857 & 0 & \mathrm{~m} & \\ 25 & \mathrm{~h} & 0 & -5.361570 & 0.126139 & -0.129234 & 0 & \mathrm{~m} & \\ 26 & \mathrm{n} & 0 & -3.133207 & -0.820930 & 0.020436 & 0 & \mathrm{~m} & \\ 27 & \mathrm{c} & 0 & -1.851138 & -1.277148 & 0.157831 & 0 & \mathrm{~m} & \\ 28 & \mathrm{o} & 0 & -1.532597 & -2.459269 & 0.218392 & 0 & \mathrm{~m} & \\ ---------------------------------------------------------- & & & \end{array}$

\section{s4}

neutral molecule

Charge $=0$ Multiplicity $=1$ for low level calculation on real system.

Charge $=0$ Multiplicity $=1$ for high level calculation on model system .

Charge $=0$ Multiplicity $=1$ for low level calculation on model system .

\begin{tabular}{|c|c|c|c|c|c|c|}
\hline Center & Atomic & Atomic & Coord & tes (Angstr & $\mathrm{ns})$ & \\
\hline Number & Numbe & Type & $\mathrm{X}$ & $\mathrm{Y}$ & Z & \\
\hline 1 & $\mathrm{c}$ & -2 & 2.642317 & 0.331097 & -0.656089 & $0 \mathrm{~h}$ \\
\hline 2 & $\mathrm{~h}$ & -3 & 3.324087 & 0.004167 & -1.451119 & \\
\hline 3 & $\mathrm{c}$ & -2 & 2.933020 & 1.793013 & -0.351411 & \\
\hline 4 & $\mathrm{~h}$ & 0 & 4.006831 & 1.899334 & -0.130132 & $0 \mathrm{~h}$ \\
\hline 5 & $\mathrm{~h}$ & -2 & 2.708368 & 2.388187 & -1.248607 & $0 \mathrm{~h}$ \\
\hline
\end{tabular}




\begin{tabular}{|c|c|c|c|c|c|c|c|}
\hline 6 & o & 0 & -2.134105 & 2.209116 & 0.754284 & 0 & $\mathrm{~h}$ \\
\hline 7 & $\mathrm{~h}$ & 0 & -2.322363 & 3.143292 & 0.928621 & 0 & $\mathrm{~h}$ \\
\hline 8 & o & 0 & -1.291668 & 0.197173 & -1.124825 & 0 & $\mathrm{~h}$ \\
\hline 9 & $\mathrm{c}$ & 0 & -2.794187 & -0.630824 & 0.541077 & 0 & $\mathrm{~h}$ \\
\hline 10 & $\mathrm{~h}$ & 0 & -3.424132 & -0.200013 & 1.331516 & 0 & $\mathrm{~h}$ \\
\hline 11 & o & 0 & -3.376449 & -1.824873 & 0.019982 & 0 & $\mathrm{~h}$ \\
\hline 12 & $\mathrm{~h}$ & 0 & -3.332091 & -2.503738 & 0.711546 & 0 & $\mathrm{~h}$ \\
\hline 13 & $\mathrm{c}$ & 0 & -0.573261 & -0.736955 & -0.326695 & 0 & $\mathrm{~m}$ \\
\hline 14 & $\mathrm{~h}$ & 0 & -0.510227 & -1.707537 & -0.821802 & 0 & $\mathrm{~m}$ \\
\hline 15 & $\mathrm{c}$ & 0 & -1.347259 & -0.829672 & 0.994493 & 0 & $\mathrm{~m}$ \\
\hline 16 & $\mathrm{~h}$ & 0 & -1.050444 & -0.008864 & 1.654343 & 0 & $\mathrm{~m}$ \\
\hline 17 & $\mathrm{~h}$ & 0 & -1.172125 & -1.780635 & 1.506465 & 0 & $\mathrm{~m}$ \\
\hline 18 & $\mathrm{n}$ & 0 & 0.809757 & -0.271982 & -0.180734 & 0 & $\mathrm{~m}$ \\
\hline 19 & $\mathrm{c}$ & 0 & 1.098890 & 1.051421 & -0.087684 & 0 & $\mathrm{~m}$ \\
\hline 20 & $\mathrm{~h}$ & 0 & 0.247397 & 1.721167 & -0.122774 & 0 & $\mathrm{~m}$ \\
\hline 21 & $\mathrm{c}$ & 0 & 2.386170 & 1.474056 & 0.043179 & 0 & $\mathrm{~m}$ \\
\hline 22 & $\mathrm{~h}$ & 0 & 2.627330 & 2.527111 & 0.125385 & 0 & $\mathrm{~m}$ \\
\hline 23 & $\mathrm{c}$ & 0 & 3.393180 & 0.453719 & 0.058584 & 0 & $\mathrm{~m}$ \\
\hline 24 & $\mathrm{n}$ & 0 & 4.708989 & 0.809821 & 0.183214 & 0 & $\mathrm{~m}$ \\
\hline 25 & $\mathrm{~h}$ & 0 & 4.992216 & 1.744209 & -0.071987 & 0 & $\mathrm{~m}$ \\
\hline 26 & $\mathrm{~h}$ & 0 & 5.377535 & 0.070277 & 0.012644 & 0 & $\mathrm{~m}$ \\
\hline 27 & $\mathrm{n}$ & 0 & 3.128065 & -0.837201 & -0.006422 & 0 & $\mathrm{~m}$ \\
\hline 28 & $\mathrm{c}$ & 0 & 1.833616 & -1.276477 & -0.074892 & 0 & $\mathrm{~m}$ \\
\hline 29 & o & 0 & 1.495679 & -2.453628 & -0.054428 & 0 & $\mathrm{~m}$ \\
\hline
\end{tabular}

radical

Charge $=0$ Multiplicity $=2$ for low level calculation on real system.

Charge $=0$ Multiplicity $=2$ for high level calculation on model system .

Charge $=0$ Multiplicity $=2$ for low level calculation on model system .

Center Atomic Atomic Coordinates (Angstroms)

Number Number Type $\quad \mathrm{X} \quad \mathrm{Y} \quad$ Z

$\begin{array}{llllllll}1 & \mathrm{c} & 0 & -2.549396 & 0.273823 & -0.413930 & 0 & \mathrm{~h} \\ 2 & \mathrm{c} & 0 & -3.249230 & 1.570997 & -0.526749 & 0 & \mathrm{~h} \\ 3 & \mathrm{~h} & 0 & -4.314024 & 1.428090 & -0.294342 & 0 & \mathrm{~h} \\ 4 & \mathrm{~h} & 0 & -3.169112 & 1.947604 & -1.556868 & 0 & \mathrm{~h} \\ 5 & \mathrm{o} & 0 & -2.643539 & 2.505557 & 0.396006 & 0 & \mathrm{~h} \\ 6 & \mathrm{~h} & 0 & -3.056070 & 3.372238 & 0.246417 & 0 & \mathrm{~h} \\ 7 & \mathrm{o} & 0 & -1.294252 & 0.233418 & -0.970258 & 0 & \mathrm{~h} \\ 8 & \mathrm{c} & 0 & -2.706570 & -0.766001 & 0.651952 & 0 & \mathrm{~h} \\ 9 & \mathrm{~h} & 0 & -3.251805 & -0.377670 & 1.524060 & 0 & \mathrm{~h}\end{array}$




\begin{tabular}{|c|c|c|c|c|c|c|c|}
\hline 10 & o & 0 & -3.403644 & -1.888904 & 0.090408 & 0 & $\mathrm{~h}$ \\
\hline 11 & $\mathrm{~h}$ & 0 & -3.480664 & -2.559457 & 0.789546 & 0 & $\mathrm{~h}$ \\
\hline 12 & $\mathrm{c}$ & 0 & -0.505981 & -0.784304 & -0.332334 & 0 & $\mathrm{~m}$ \\
\hline 13 & $\mathrm{~h}$ & 0 & -0.434482 & -1.661597 & -0.975227 & 0 & $\mathrm{~m}$ \\
\hline 14 & $\mathrm{c}$ & 0 & -1.236898 & -1.085942 & 0.985629 & 0 & $\mathrm{~m}$ \\
\hline 15 & $\mathrm{~h}$ & 0 & -0.875393 & -0.405342 & 1.764982 & 0 & $\mathrm{~m}$ \\
\hline 16 & $\mathrm{~h}$ & 0 & -1.069400 & -2.114875 & 1.314208 & 0 & $\mathrm{~m}$ \\
\hline 17 & $\mathrm{n}$ & 0 & 0.850731 & -0.266989 & -0.173569 & 0 & $\mathrm{~m}$ \\
\hline 18 & $\mathrm{c}$ & 0 & 1.076282 & 1.051602 & 0.073896 & 0 & $\mathrm{~m}$ \\
\hline 19 & $\mathrm{~h}$ & 0 & 0.197126 & 1.685905 & 0.116969 & 0 & $\mathrm{~m}$ \\
\hline 20 & $\mathrm{c}$ & 0 & 2.343692 & 1.517789 & 0.233806 & 0 & $\mathrm{~m}$ \\
\hline 21 & $\mathrm{~h}$ & 0 & 2.535624 & 2.565217 & 0.432623 & 0 & $\mathrm{~m}$ \\
\hline 22 & $\mathrm{c}$ & 0 & 3.399723 & 0.553360 & 0.112496 & 0 & $\mathrm{~m}$ \\
\hline 23 & $\mathrm{n}$ & 0 & 4.695650 & 0.956997 & 0.264674 & 0 & $\mathrm{~m}$ \\
\hline 24 & $\mathrm{~h}$ & 0 & 4.931699 & 1.931952 & 0.157827 & 0 & $\mathrm{~m}$ \\
\hline 25 & $\mathrm{~h}$ & 0 & 5.402696 & 0.280555 & 0.009928 & 0 & $\mathrm{~m}$ \\
\hline 26 & $\mathrm{n}$ & 0 & 3.196625 & -0.731372 & -0.111946 & 0 & $\mathrm{~m}$ \\
\hline 27 & $\mathrm{c}$ & 0 & 1.925853 & -1.223574 & -0.222857 & 0 & $\mathrm{~m}$ \\
\hline 28 & o & 0 & 1.641175 & -2.406267 & -0.360618 & 0 & $\mathrm{~m}$ \\
\hline
\end{tabular}

\section{s5}

neutral molecule

Charge $=0$ Multiplicity $=1$ for low level calculation on real system.

Charge $=0$ Multiplicity $=1$ for high level calculation on model system.

Charge $=0$ Multiplicity $=1$ for low level calculation on model system .

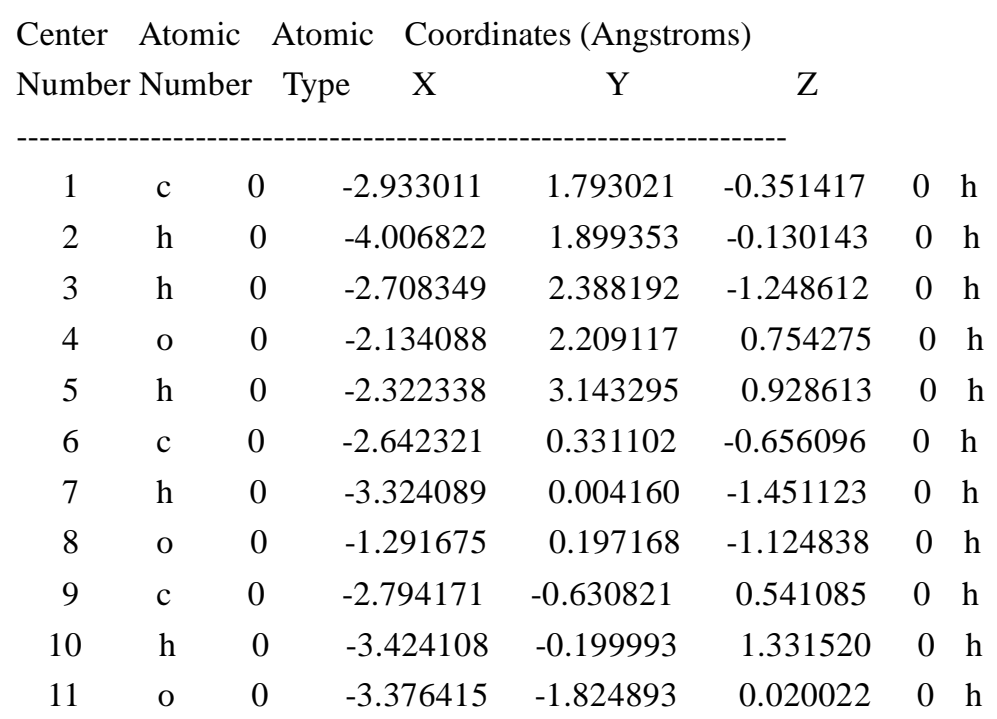




\begin{tabular}{|c|c|c|c|c|c|c|c|c|}
\hline 12 & $\mathrm{~h}$ & 0 & -3.332036 & -2.503742 & 0.711600 & 0 & $\mathrm{~h}$ & \\
\hline 13 & c & 0 & -0.573269 & -0.736957 & -0.326704 & 0 & $\mathrm{~m}$ & h $\varepsilon$ \\
\hline 14 & $\mathrm{~h}$ & 0 & -0.510243 & -1.707544 & -0.821803 & 0 & $\mathrm{~m}$ & \\
\hline 15 & $\mathrm{c}$ & 0 & -1.347258 & -0.829664 & 0.994484 & 0 & $\mathrm{~m}$ & $\mathrm{~h}$ \\
\hline 16 & $\mathrm{~h}$ & 0 & -1.050438 & -0.008853 & 1.654327 & 0 & $\mathrm{~m}$ & \\
\hline 17 & $\mathrm{~h}$ & 0 & -1.172116 & -1.780626 & 1.506455 & 0 & $\mathrm{~m}$ & \\
\hline 18 & $\mathrm{n}$ & 0 & 0.809748 & -0.271983 & -0.180737 & 0 & $\mathrm{~m}$ & \\
\hline 19 & $\mathrm{c}$ & 0 & 1.098877 & 1.051420 & -0.087677 & 0 & $\mathrm{~m}$ & \\
\hline 20 & $\mathrm{~h}$ & 0 & 0.247384 & 1.721165 & -0.122766 & 0 & $\mathrm{~m}$ & \\
\hline 21 & $\mathrm{c}$ & 0 & 2.386156 & 1.474057 & 0.043191 & 0 & $\mathrm{~m}$ & \\
\hline 22 & $\mathrm{~h}$ & 0 & 2.627315 & 2.527111 & 0.125404 & 0 & $\mathrm{~m}$ & \\
\hline 23 & $\mathrm{c}$ & 0 & 3.393169 & 0.453722 & 0.058592 & 0 & $\mathrm{~m}$ & \\
\hline 24 & $\mathrm{n}$ & 0 & 4.708976 & 0.809826 & 0.183228 & 0 & $\mathrm{~m}$ & \\
\hline 25 & $\mathrm{~h}$ & 0 & 4.992203 & 1.744216 & -0.071967 & 0 & $\mathrm{~m}$ & \\
\hline 26 & $\mathrm{~h}$ & 0 & 5.377525 & 0.070284 & 0.012655 & 0 & $\mathrm{~m}$ & \\
\hline 27 & $\mathrm{n}$ & 0 & 3.128056 & -0.837207 & -0.006442 & 0 & $\mathrm{~m}$ & \\
\hline 28 & $\mathrm{c}$ & 0 & 1.833608 & -1.276477 & -0.074898 & 0 & $\mathrm{~m}$ & \\
\hline 29 & o & 0 & 1.495674 & -2.453628 & -0.054443 & 0 & $\mathrm{~m}$ & \\
\hline
\end{tabular}

radical

Charge $=0$ Multiplicity $=2$ for low level calculation on real system.

Charge $=0$ Multiplicity $=2$ for high level calculation on model system.

Charge $=0$ Multiplicity $=2$ for low level calculation on model system.

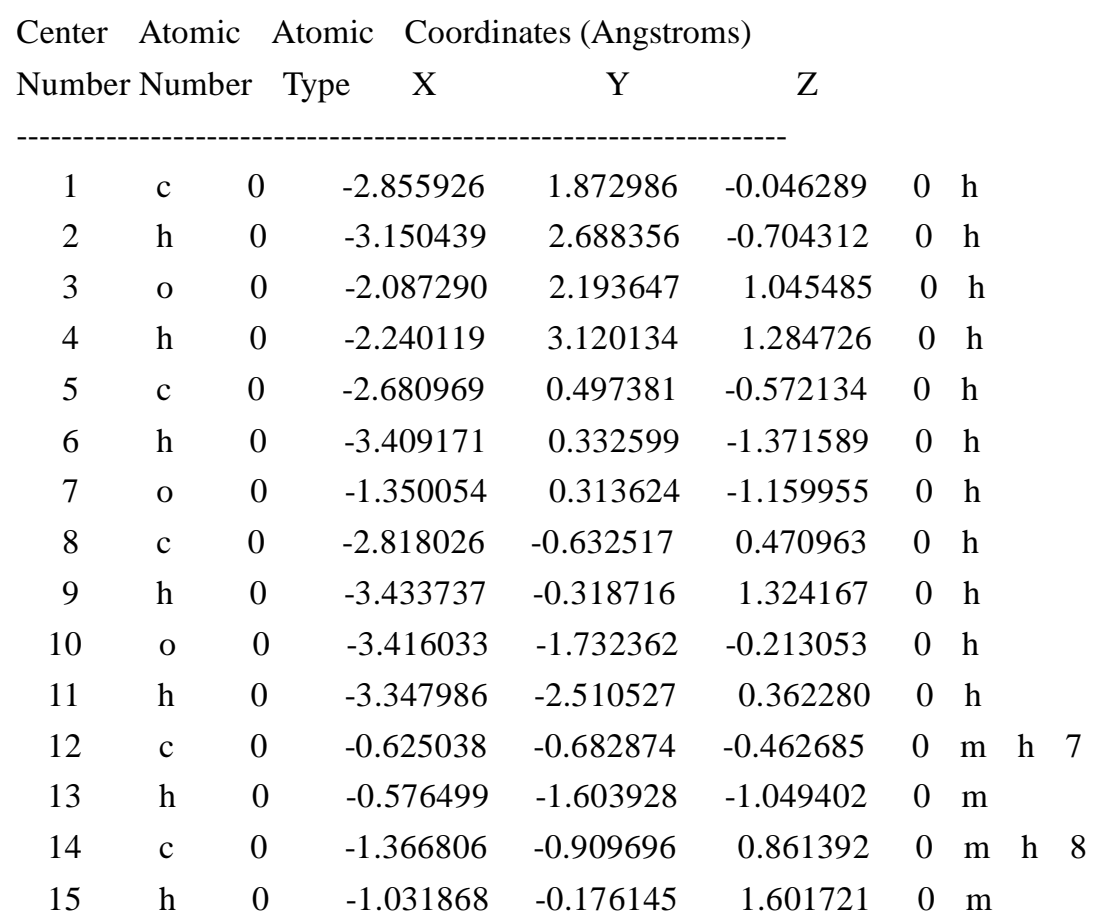




$\begin{array}{lllrrrrc}16 & \mathrm{~h} & 0 & -1.194334 & -1.913615 & 1.256919 & 0 & \mathrm{~m} \\ 17 & \mathrm{n} & 0 & 0.770446 & -0.237434 & -0.284086 & 0 & \mathrm{~m} \\ 18 & \mathrm{c} & 0 & 1.136351 & 1.060494 & -0.409159 & 0 & \mathrm{~m} \\ 19 & \mathrm{~h} & 0 & 0.336062 & 1.748495 & -0.649804 & 0 & \mathrm{~m} \\ 20 & \mathrm{c} & 0 & 2.436025 & 1.436230 & -0.245306 & 0 & \mathrm{~m} \\ 21 & \mathrm{~h} & 0 & 2.737605 & 2.472738 & -0.337295 & 0 & \mathrm{~m} \\ 22 & \mathrm{c} & 0 & 3.369717 & 0.389717 & 0.044746 & 0 & \mathrm{~m} \\ 23 & \mathrm{n} & 0 & 4.690603 & 0.697419 & 0.233882 & 0 & \mathrm{~m} \\ 24 & \mathrm{~h} & 0 & 5.054392 & 1.556135 & -0.151936 & 0 & \mathrm{~m} \\ 25 & \mathrm{~h} & 0 & 5.321285 & -0.093094 & 0.252102 & 0 & \mathrm{~m} \\ 26 & \mathrm{n} & 0 & 3.028841 & -0.879235 & 0.176693 & 0 & \mathrm{~m} \\ 27 & \mathrm{c} & 0 & 1.720342 & -1.258214 & 0.056827 & 0 & \mathrm{~m} \\ 28 & \mathrm{o} & 0 & 1.304821 & -2.401000 & 0.215881 & 0 & \mathrm{~m} \\ ----------------------------------------------------------------- & & \end{array}$

\section{b4}

neutral molecule

Charge $=0$ Multiplicity $=1$ for low level calculation on real system.

Charge $=0$ Multiplicity $=1$ for high level calculation on model system .

Charge $=0$ Multiplicity $=1$ for low level calculation on model system.

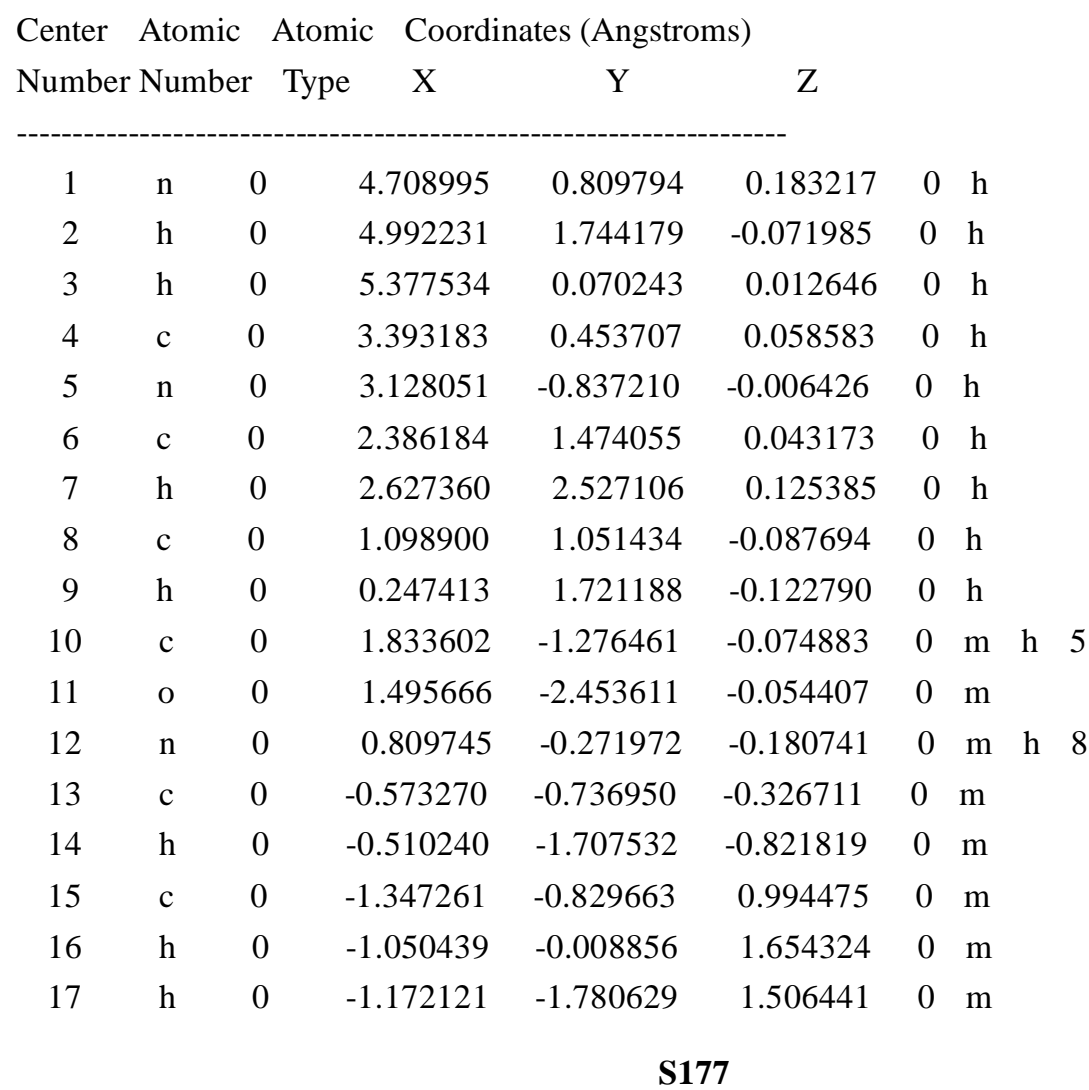




$\begin{array}{llllllll}18 & \mathrm{c} & 0 & -2.794181 & -0.630821 & 0.541070 & 0 & \mathrm{~m} \\ 19 & \mathrm{~h} & 0 & -3.424125 & -0.199999 & 1.331504 & 0 & \mathrm{~m} \\ 20 & \mathrm{o} & 0 & -3.376438 & -1.824878 & 0.019990 & 0 & \mathrm{~m} \\ 21 & \mathrm{~h} & 0 & -3.332070 & -2.503737 & 0.711559 & 0 & \mathrm{~m} \\ 22 & \mathrm{c} & 0 & -2.642321 & 0.331100 & -0.656097 & 0 & \mathrm{~m} \\ 23 & \mathrm{~h} & 0 & -3.324088 & 0.004172 & -1.451131 & 0 & \mathrm{~m} \\ 24 & \mathrm{o} & 0 & -1.291679 & 0.197183 & -1.124835 & 0 & \mathrm{~m} \\ 25 & \mathrm{c} & 0 & -2.933021 & 1.793011 & -0.351391 & 0 & \mathrm{~m} \\ 26 & \mathrm{~h} & 0 & -4.006828 & 1.899328 & -0.130096 & 0 & \mathrm{~m} \\ 27 & \mathrm{~h} & 0 & -2.708380 & 2.388200 & -1.248580 & 0 & \mathrm{~m} \\ 28 & \mathrm{o} & 0 & -2.134091 & 2.209096 & 0.754300 & 0 & \mathrm{~m} \\ 29 & \mathrm{~h} & 0 & -2.322347 & 3.143269 & 0.928656 & 0 & \mathrm{~m}\end{array}$

radical

Charge $=0$ Multiplicity $=2$ for low level calculation on real system.

Charge $=0$ Multiplicity $=2$ for high level calculation on model system .

Charge $=0$ Multiplicity $=2$ for low level calculation on model system.

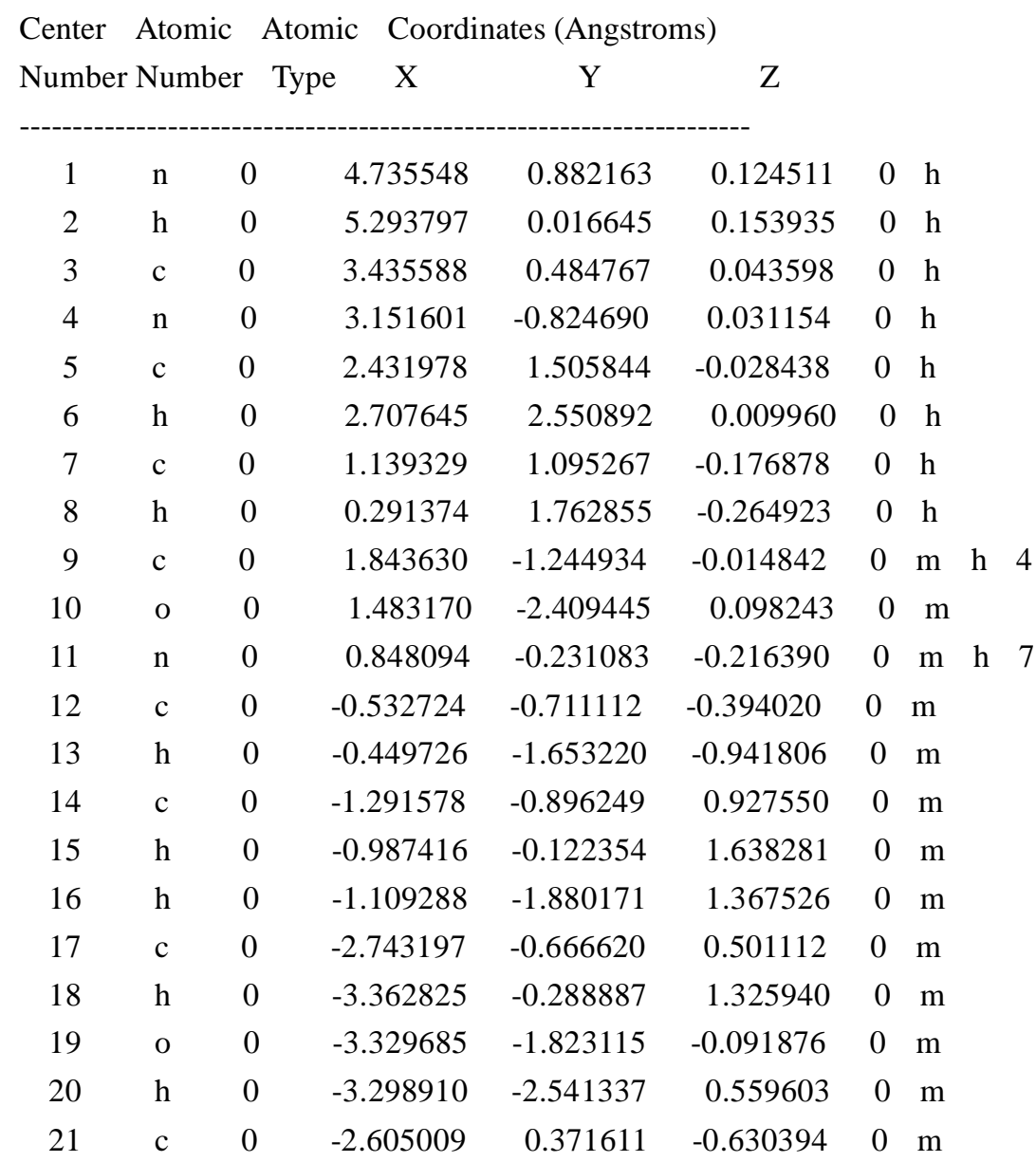




$\begin{array}{cccccccc}22 & \mathrm{~h} & 0 & -3.302876 & 0.107082 & -1.433563 & 0 & \mathrm{~m} \\ 23 & \mathrm{o} & 0 & -1.263604 & 0.246342 & -1.137820 & 0 & \mathrm{~m} \\ 24 & \mathrm{c} & 0 & -2.864940 & 1.814234 & -0.224740 & 0 & \mathrm{~m} \\ 25 & \mathrm{~h} & 0 & -3.928082 & 1.916524 & 0.044000 & 0 & \mathrm{~m} \\ 26 & \mathrm{~h} & 0 & -2.668391 & 2.462568 & -1.091185 & 0 & \mathrm{~m} \\ 27 & \mathrm{o} & 0 & -2.017163 & 2.148685 & 0.871193 & 0 & \mathrm{~m} \\ 28 & \mathrm{~h} & 0 & -2.192215 & 3.068077 & 1.121687 & 0 & \mathrm{~m}\end{array}$

\section{b5}

neutral molecule

Charge $=0$ Multiplicity $=1$ for low level calculation on real system.

Charge $=0$ Multiplicity $=1$ for high level calculation on model system .

Charge $=0$ Multiplicity $=1$ for low level calculation on model system .

Center Atomic Atomic Coordinates (Angstroms)

Number Number Type X $\quad$ Y $\quad$ Z

\begin{tabular}{|c|c|c|c|c|c|c|c|}
\hline 1 & $\mathrm{c}$ & 0 & 2.386160 & 1.474053 & 0.043180 & 0 & $\mathrm{~h}$ \\
\hline 2 & $\mathrm{~h}$ & 0 & 2.627324 & 2.527106 & 0.125396 & 0 & $\mathrm{~h}$ \\
\hline 3 & $\mathrm{c}$ & 0 & 3.393171 & 0.453717 & 0.058584 & 0 & $\mathrm{~h}$ \\
\hline 4 & $\mathrm{n}$ & 0 & 4.708979 & 0.809819 & 0.183218 & 0 & $\mathrm{~h}$ \\
\hline 5 & $\mathrm{~h}$ & 0 & 4.992207 & 1.744207 & -0.071980 & 0 & $\mathrm{~h}$ \\
\hline 6 & $\mathrm{~h}$ & 0 & 5.377526 & 0.070275 & 0.012648 & 0 & $\mathrm{~h}$ \\
\hline 7 & $\mathrm{n}$ & 0 & 3.128056 & -0.837204 & -0.006426 & 0 & $\mathrm{~h}$ \\
\hline 8 & $\mathrm{c}$ & 0 & 1.098881 & 1.051418 & -0.087687 & 0 & $\mathrm{~h}$ \\
\hline 9 & $\mathrm{~h}$ & 0 & 0.247386 & 1.721162 & -0.122779 & 0 & $\mathrm{~h}$ \\
\hline 10 & $\mathrm{n}$ & 0 & 0.809749 & -0.271984 & -0.180742 & 0 & $\mathrm{~m}$ \\
\hline 11 & c & 0 & 1.833610 & -1.276475 & -0.074887 & 0 & $\mathrm{~m}$ \\
\hline 12 & o & 0 & 1.495678 & -2.453627 & -0.054422 & 0 & $\mathrm{~m}$ \\
\hline 13 & $\mathrm{c}$ & 0 & -0.573269 & -0.736957 & -0.326708 & 0 & $\mathrm{~m}$ \\
\hline 14 & $\mathrm{~h}$ & 0 & -0.510243 & -1.707543 & -0.821809 & 0 & $\mathrm{~m}$ \\
\hline 15 & $\mathrm{c}$ & 0 & -1.347258 & -0.829659 & 0.994480 & 0 & $\mathrm{~m}$ \\
\hline 16 & $\mathrm{~h}$ & 0 & -1.050432 & -0.008848 & 1.654323 & 0 & $\mathrm{~m}$ \\
\hline 17 & $\mathrm{~h}$ & 0 & -1.172121 & -1.780621 & 1.506452 & 0 & $\mathrm{~m}$ \\
\hline 18 & $\mathrm{c}$ & 0 & -2.794178 & -0.630813 & 0.541076 & 0 & $\mathrm{~m}$ \\
\hline 19 & $\mathrm{~h}$ & 0 & -3.424119 & -0.199984 & 1.331508 & 0 & $\mathrm{~m}$ \\
\hline 20 & o & 0 & -3.376441 & -1.824872 & 0.020005 & 0 & $\mathrm{~m}$ \\
\hline 21 & $\mathrm{~h}$ & 0 & -3.332074 & -2.503727 & 0.711578 & 0 & $\mathrm{~m}$ \\
\hline 22 & $\mathrm{c}$ & 0 & -2.642316 & 0.331099 & -0.656098 & 0 & $\mathrm{~m}$ \\
\hline 23 & $\mathrm{~h}$ & 0 & -3.324085 & 0.004168 & -1.451129 & 0 & $\mathrm{~m}$ \\
\hline
\end{tabular}




$\begin{array}{llllllll}24 & \mathrm{o} & 0 & -1.291674 & 0.197173 & -1.124836 & 0 & \mathrm{~m} \\ 25 & \mathrm{c} & 0 & -2.933009 & 1.793013 & -0.351401 & 0 & \mathrm{~m} \\ 26 & \mathrm{~h} & 0 & -4.006816 & 1.899336 & -0.130105 & 0 & \mathrm{~m} \\ 27 & \mathrm{~h} & 0 & -2.708367 & 2.388195 & -1.248594 & 0 & \mathrm{~m} \\ 28 & \mathrm{o} & 0 & -2.134076 & 2.209102 & 0.754286 & 0 & \mathrm{~m} \\ 29 & \mathrm{~h} & 0 & -2.322328 & 3.143277 & 0.928636 & 0 & \mathrm{~m}\end{array}$

radical

Charge $=0$ Multiplicity $=2$ for low level calculation on real system.

Charge $=0$ Multiplicity $=2$ for high level calculation on model system.

Charge $=0$ Multiplicity $=2$ for low level calculation on model system.

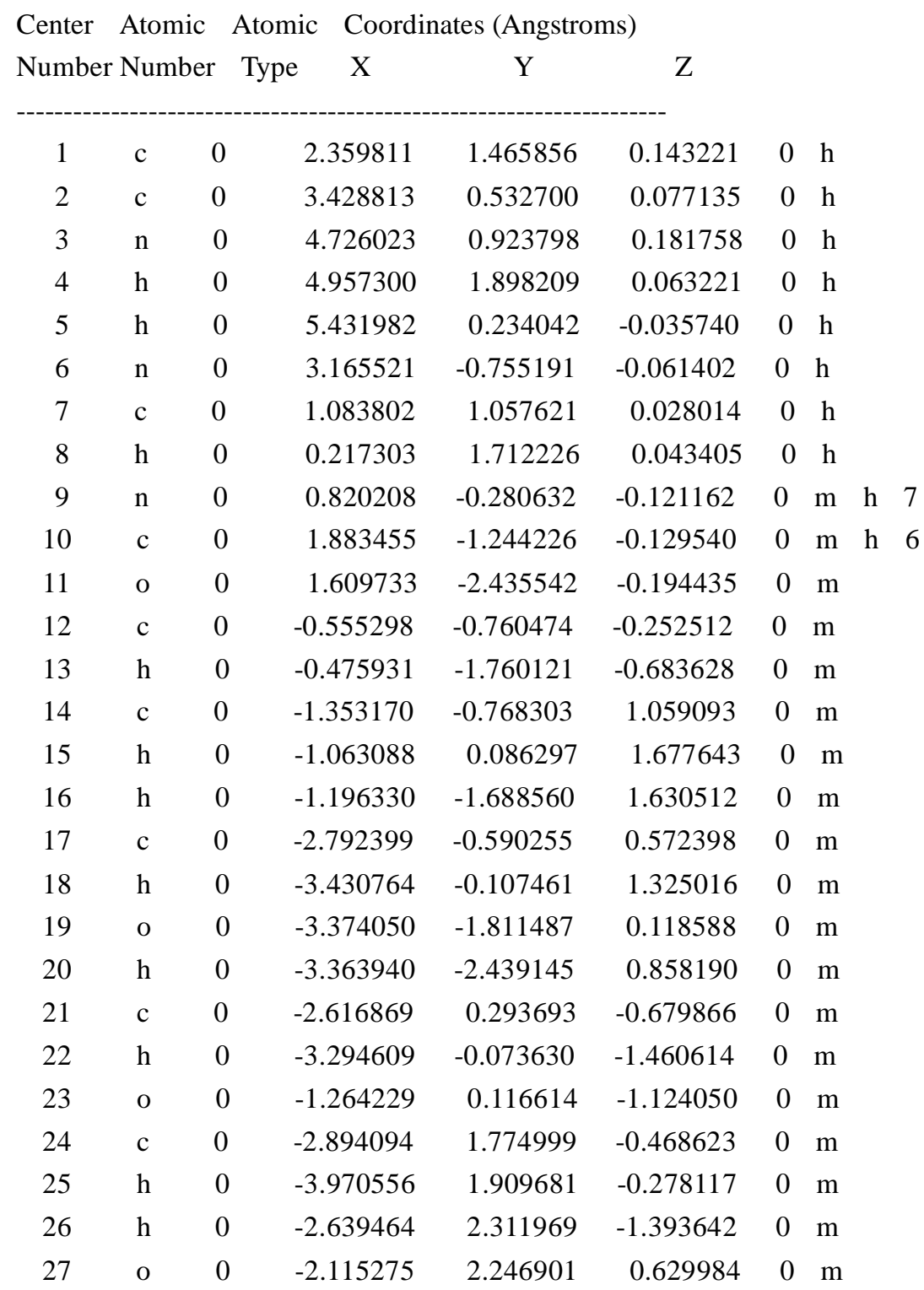




$28 \quad \mathrm{~h} \quad 0 \quad 0 \quad-2.267899 \quad 3.199104 \quad 0.722781 \quad 0 \quad \mathrm{~m}$

\section{b6}

neutral molecule

Charge $=0$ Multiplicity $=1$ for low level calculation on real system.

Charge $=0$ Multiplicity $=1$ for high level calculation on model system .

Charge $=0$ Multiplicity $=1$ for low level calculation on model system.

Center Atomic Atomic Coordinates (Angstroms)

Number Number Type $\mathrm{X} \quad \mathrm{Y} \quad \mathrm{Z}$

\begin{tabular}{|c|c|c|c|c|c|c|c|c|}
\hline 1 & $\mathrm{c}$ & 0 & 1.098881 & 1.051418 & -0.087687 & 0 & $\mathrm{~h}$ & \\
\hline 2 & $\mathrm{~h}$ & 0 & 0.247386 & 1.721162 & -0.122778 & 0 & $\mathrm{~h}$ & \\
\hline 3 & $\mathrm{c}$ & 0 & 2.386160 & 1.474053 & 0.043180 & 0 & $\mathrm{~h}$ & \\
\hline 4 & $\mathrm{~h}$ & 0 & 2.627320 & 2.527108 & 0.125390 & 0 & $\mathrm{~h}$ & \\
\hline 5 & $\mathrm{n}$ & 0 & 0.809749 & -0.271984 & -0.180742 & 0 & $\mathrm{~h}$ & \\
\hline 6 & $\mathrm{c}$ & 0 & 1.833610 & -1.276475 & -0.074887 & 0 & $\mathrm{~h}$ & \\
\hline 7 & o & 0 & 1.495678 & -2.453627 & -0.054422 & 0 & $\mathrm{~h}$ & \\
\hline 8 & $\mathrm{c}$ & 0 & -0.573269 & -0.736957 & -0.326708 & 0 & h & \\
\hline 9 & $\mathrm{~h}$ & 0 & -0.510243 & -1.707543 & -0.821809 & 0 & $\mathrm{~h}$ & \\
\hline 10 & $\mathrm{c}$ & 0 & 3.393171 & 0.453717 & 0.058585 & 0 & $\mathrm{~m}$ & h 3 \\
\hline 11 & $\mathrm{n}$ & 0 & 3.128056 & -0.837204 & -0.006426 & 0 & $\mathrm{~m}$ & h 6 \\
\hline 12 & $\mathrm{c}$ & 0 & -1.347258 & -0.829659 & 0.994480 & 0 & $\mathrm{~m}$ & 8 \\
\hline 13 & $\mathrm{~h}$ & 0 & -1.050432 & -0.008849 & 1.654323 & 0 & $\mathrm{~m}$ & \\
\hline 14 & $\mathrm{~h}$ & 0 & -1.172121 & -1.780621 & 1.506452 & 0 & $\mathrm{~m}$ & \\
\hline 15 & o & 0 & -1.291674 & 0.197173 & -1.124836 & 0 & $\mathrm{~m}$ & 8 \\
\hline 16 & $\mathrm{n}$ & 0 & 4.708979 & 0.809819 & 0.183219 & 0 & $\mathrm{~m}$ & \\
\hline 17 & $\mathrm{~h}$ & 0 & 4.992207 & 1.744208 & -0.071979 & 0 & $\mathrm{~m}$ & \\
\hline 18 & $\mathrm{~h}$ & 0 & 5.377526 & 0.070275 & 0.012648 & 0 & $\mathrm{~m}$ & \\
\hline 19 & $\mathrm{c}$ & 0 & -2.794178 & -0.630813 & 0.541076 & 0 & $\mathrm{~m}$ & \\
\hline 20 & $\mathrm{~h}$ & 0 & -3.424119 & -0.199984 & 1.331508 & 0 & $\mathrm{~m}$ & \\
\hline 21 & o & 0 & -3.376441 & -1.824872 & 0.020004 & 0 & $\mathrm{~m}$ & \\
\hline 22 & $\mathrm{~h}$ & 0 & -3.332074 & -2.503727 & 0.711578 & 0 & $\mathrm{~m}$ & \\
\hline 23 & $\mathrm{c}$ & 0 & -2.642316 & 0.331099 & -0.656098 & 0 & $\mathrm{~m}$ & \\
\hline 24 & $\mathrm{~h}$ & 0 & -3.324085 & 0.004168 & -1.451129 & 0 & $\mathrm{~m}$ & \\
\hline 25 & $\mathrm{c}$ & 0 & -2.933009 & 1.793013 & -0.351401 & 0 & $\mathrm{~m}$ & \\
\hline 26 & $\mathrm{~h}$ & 0 & -4.006816 & 1.899336 & -0.130105 & 0 & $\mathrm{~m}$ & \\
\hline 27 & $\mathrm{~h}$ & 0 & -2.708367 & 2.388195 & -1.248594 & 0 & $\mathrm{~m}$ & \\
\hline 28 & o & 0 & -2.134076 & 2.209102 & 0.754287 & 0 & $\mathrm{~m}$ & \\
\hline 29 & $\mathrm{~h}$ & 0 & -2.322328 & 3.143277 & 0.928636 & 0 & $\mathrm{~m}$ & \\
\hline
\end{tabular}


radical

Charge $=0$ Multiplicity $=2$ for low level calculation on real system.

Charge $=0$ Multiplicity $=2$ for high level calculation on model system .

Charge $=0$ Multiplicity $=2$ for low level calculation on model system .

Center Atomic Atomic Coordinates (Angstroms)

Number Number Type $\quad \mathrm{X} \quad \mathrm{Y} \quad \mathrm{Z}$

\begin{tabular}{|c|c|c|c|c|c|c|c|c|}
\hline 1 & $\mathrm{c}$ & 0 & 1.113264 & 0.971674 & -0.403082 & 0 & $\mathrm{~h}$ & \\
\hline 2 & $\mathrm{c}$ & 0 & 2.380156 & 1.425600 & -0.269540 & 0 & $\mathrm{~h}$ & \\
\hline 3 & $\mathrm{~h}$ & 0 & 2.661471 & 2.467949 & -0.356893 & 0 & $\mathrm{~h}$ & \\
\hline 4 & $\mathrm{n}$ & 0 & 0.736019 & -0.289827 & -0.276910 & 0 & $\mathrm{~h}$ & \\
\hline 5 & $\mathrm{c}$ & 0 & 1.731507 & -1.310656 & 0.071289 & 0 & $\mathrm{~h}$ & \\
\hline 6 & o & 0 & 1.321551 & -2.450203 & 0.232397 & 0 & $\mathrm{~h}$ & \\
\hline 7 & $\mathrm{c}$ & 0 & -0.653430 & -0.748722 & -0.486354 & 0 & $\mathrm{~h}$ & \\
\hline 8 & $\mathrm{~h}$ & 0 & -0.580601 & -1.668356 & -1.072105 & 0 & $\mathrm{~h}$ & \\
\hline 9 & $\mathrm{c}$ & 0 & 3.333749 & 0.386074 & 0.018073 & 0 & $\mathrm{~m} \mathrm{~h}$ & 2 \\
\hline 10 & $\mathrm{n}$ & 0 & 3.020328 & -0.890424 & 0.170154 & 0 & $\mathrm{~m}$ & 5 \\
\hline 11 & $\mathrm{c}$ & 0 & -1.419594 & -0.964872 & 0.828498 & 0 & $\mathrm{~m} h$ & 7 \\
\hline 12 & $\mathrm{~h}$ & 0 & -1.011735 & -0.310031 & 1.601852 & 0 & $\mathrm{~m}$ & \\
\hline 13 & $\mathrm{~h}$ & 0 & -1.355853 & -2.001312 & 1.169470 & 0 & $\mathrm{~m}$ & \\
\hline 14 & $\mathrm{o}$ & 0 & -1.351480 & 0.241170 & -1.215219 & 0 & $\mathrm{~m} \mathrm{~h}$ & 7 \\
\hline 15 & $\mathrm{n}$ & 0 & 4.649184 & 0.718863 & 0.186759 & 0 & $\mathrm{~m}$ & \\
\hline 16 & $\mathrm{~h}$ & 0 & 4.990554 & 1.587685 & -0.196215 & 0 & $\mathrm{~m}$ & \\
\hline 17 & $\mathrm{~h}$ & 0 & 5.298438 & -0.055959 & 0.211737 & 0 & $\mathrm{~m}$ & \\
\hline 18 & $\mathrm{c}$ & 0 & -2.840943 & -0.521731 & 0.470588 & 0 & $\mathrm{~m}$ & \\
\hline 19 & $\mathrm{~h}$ & 0 & -3.387101 & -0.136959 & 1.342890 & 0 & $\mathrm{~m}$ & \\
\hline 20 & $\mathrm{o}$ & 0 & -3.586366 & -1.545231 & -0.187695 & 0 & $\mathrm{~m}$ & \\
\hline 21 & $\mathrm{~h}$ & 0 & -3.609158 & -2.317420 & 0.398921 & 0 & $\mathrm{~m}$ & \\
\hline 22 & $\mathrm{c}$ & 0 & -2.595879 & 0.575400 & -0.578483 & 0 & $\mathrm{~m}$ & \\
\hline 23 & $\mathrm{~h}$ & 0 & -3.381866 & 0.525982 & -1.340638 & 0 & $\mathrm{~m}$ & \\
\hline 24 & $\mathrm{c}$ & 0 & -2.550008 & 1.995846 & -0.030486 & 0 & $\mathrm{~m}$ & \\
\hline 25 & $\mathrm{~h}$ & 0 & -3.569850 & 2.293185 & 0.264669 & 0 & $\mathrm{~m}$ & \\
\hline 26 & $\mathrm{~h}$ & 0 & -2.220904 & 2.661862 & -0.840443 & 0 & $\mathrm{~m}$ & \\
\hline 27 & $\mathrm{o}$ & 0 & -1.658866 & 2.048557 & 1.078397 & 0 & $\mathrm{~m}$ & \\
\hline 28 & $\mathrm{~h}$ & 0 & -1.463754 & 2.977070 & 1.270675 & 0 & $\mathrm{~m}$ & \\
\hline
\end{tabular}




\section{dG}

\section{s1}

neutral molecule

Charge $=0$ Multiplicity $=1$ for low level calculation on real system .

Charge $=0$ Multiplicity $=1$ for high level calculation on model system .

Charge $=0$ Multiplicity $=1$ for low level calculation on model system .

Center Atomic Atomic Coordinates (Angstroms)

Number Number Type $\quad X \quad$ Y $\quad$ Z

\begin{tabular}{|c|c|c|c|c|c|c|c|c|}
\hline 1 & $\mathrm{c}$ & 0 & 1.104641 & 0.606564 & -0.045105 & 0 & $\mathrm{~h}$ & \\
\hline 2 & $\mathrm{~h}$ & 0 & 0.692111 & 1.595833 & -0.266103 & 0 & $\mathrm{~h}$ & \\
\hline 3 & $\mathrm{c}$ & 0 & 2.001706 & 0.628299 & 1.203289 & 0 & $\mathrm{~h}$ & \\
\hline 4 & $\mathrm{~h}$ & 0 & 2.018460 & -0.360345 & 1.668495 & 0 & $\mathrm{~h}$ & \\
\hline 5 & $\mathrm{~h}$ & 0 & 1.663945 & 1.361713 & 1.942679 & 0 & $\mathrm{~h}$ & \\
\hline 6 & o & 0 & 1.939283 & 0.172556 & -1.114279 & 0 & $\mathrm{~h}$ & \\
\hline 7 & $\mathrm{n}$ & 0 & -0.034240 & -0.286806 & 0.056303 & 0 & $\mathrm{~h}$ & \\
\hline 8 & $\mathrm{c}$ & 0 & -0.012070 & -1.669975 & 0.183123 & 0 & $\mathrm{~h}$ & \\
\hline 9 & $\mathrm{~h}$ & 0 & 0.928586 & -2.201617 & 0.223854 & 0 & $\mathrm{~h}$ & \\
\hline 10 & $\mathrm{n}$ & 0 & -1.205438 & -2.204598 & 0.216885 & 0 & $\mathrm{~h}$ & \\
\hline 11 & $\mathrm{c}$ & 0 & 3.378835 & 0.951696 & 0.619759 & 0 & $\mathrm{~m}$ & $\mathrm{~h}$ \\
\hline 12 & $\mathrm{~h}$ & 0 & 4.196331 & 0.560810 & 1.239925 & 0 & $\mathrm{~m}$ & \\
\hline 13 & $\mathrm{c}$ & 0 & 3.325730 & 0.262668 & -0.757045 & 0 & $\mathrm{~m}$ & $\mathrm{~h}$ \\
\hline 14 & $\mathrm{~h}$ & 0 & 3.832136 & 0.901390 & -1.491038 & 0 & $\mathrm{~m}$ & \\
\hline 15 & $\mathrm{c}$ & 0 & -1.361646 & 0.065618 & -0.005601 & 0 & $\mathrm{~m}$ & $\mathrm{~h}$ \\
\hline 16 & $\mathrm{c}$ & 0 & -2.069116 & -1.131414 & 0.102755 & 0 & & h \\
\hline 17 & o & 0 & 3.550282 & 2.346223 & 0.366371 & 0 & $\mathrm{~m}$ & \\
\hline 18 & $\mathrm{~h}$ & 0 & 3.511931 & 2.808230 & 1.218496 & 0 & $\mathrm{~m}$ & \\
\hline 19 & $\mathrm{c}$ & 0 & 3.965642 & -1.118760 & -0.805676 & 0 & $\mathrm{~m}$ & \\
\hline 20 & $\mathrm{~h}$ & 0 & 5.059403 & -1.004117 & -0.737454 & 0 & $\mathrm{~m}$ & \\
\hline 21 & $\mathrm{~h}$ & 0 & 3.727455 & -1.573166 & -1.777496 & 0 & $\mathrm{~m}$ & \\
\hline 22 & o & 0 & 3.468256 & -1.910657 & 0.271376 & 0 & $\mathrm{~m}$ & \\
\hline 23 & $\mathrm{~h}$ & 0 & 3.786946 & -2.817937 & 0.154501 & 0 & $\mathrm{~m}$ & \\
\hline 24 & $\mathrm{c}$ & 0 & -3.507945 & -1.092386 & 0.091116 & 0 & $\mathrm{~m}$ & \\
\hline 25 & o & 0 & -4.341375 & -1.976353 & 0.186994 & 0 & $\mathrm{~m}$ & \\
\hline 26 & $\mathrm{n}$ & 0 & -3.948034 & 0.272648 & -0.054309 & 0 & $\mathrm{~m}$ & \\
\hline 27 & $\mathrm{~h}$ & 0 & -4.954831 & 0.378071 & 0.002020 & 0 & $\mathrm{~m}$ & \\
\hline 28 & $\mathrm{c}$ & 0 & -3.147755 & 1.379941 & -0.162899 & 0 & $\mathrm{~m}$ & \\
\hline 29 & $\mathrm{n}$ & 0 & -3.783505 & 2.604560 & -0.236603 & 0 & $\mathrm{~m}$ & \\
\hline 30 & $\mathrm{~h}$ & 0 & -4.652421 & 2.634211 & -0.756087 & 0 & $\mathrm{~m}$ & \\
\hline
\end{tabular}




$\begin{array}{llllllll}31 & \mathrm{~h} & 0 & -3.145614 & 3.350237 & -0.487260 & 0 & \mathrm{~m} \\ 32 & \mathrm{n} & 0 & -1.837946 & 1.331199 & -0.148067 & 0 & \mathrm{~m}\end{array}$

radical

Charge $=0$ Multiplicity $=2$ for low level calculation on real system.

Charge $=0$ Multiplicity $=2$ for high level calculation on model system .

Charge $=0$ Multiplicity $=2$ for low level calculation on model system .

\begin{tabular}{|c|c|c|c|c|c|c|c|c|}
\hline Center & Atomic & & Atomic & ates (Angstro & & & & \\
\hline Number & Numbe & & Type & $\mathrm{Y}$ & $\mathrm{Z}$ & & & \\
\hline 1 & c & 0 & 1.180385 & 0.026252 & -0.551562 & 0 & $\mathrm{~h}$ & \\
\hline 2 & $\mathrm{c}$ & 0 & 1.622910 & 1.165912 & 0.316481 & 0 & $\mathrm{~h}$ & \\
\hline 3 & $\mathrm{~h}$ & 0 & 1.470426 & 0.934374 & 1.380908 & 0 & $\mathrm{~h}$ & \\
\hline 4 & $\mathrm{~h}$ & 0 & 1.098577 & 2.100612 & 0.096887 & 0 & $\mathrm{~h}$ & \\
\hline 5 & o & 0 & 2.223013 & -0.811301 & -0.852727 & 0 & $\mathrm{~h}$ & \\
\hline 6 & $\mathrm{n}$ & 0 & -0.040171 & -0.637702 & -0.414663 & 0 & $\mathrm{~h}$ & \\
\hline 7 & $\mathrm{c}$ & 0 & -0.283309 & -2.004877 & -0.579632 & 0 & h & \\
\hline 8 & $\mathrm{~h}$ & 0 & 0.530343 & -2.680967 & -0.792631 & 0 & $\mathrm{~h}$ & \\
\hline 9 & $\mathrm{n}$ & 0 & -1.544993 & -2.326485 & -0.451849 & 0 & $\mathrm{~h}$ & \\
\hline 10 & $\mathrm{c}$ & 0 & 3.127552 & 1.229416 & -0.003319 & 0 & $\mathrm{~m} \mathrm{~h}$ & h 2 \\
\hline 11 & $\mathrm{~h}$ & 0 & 3.712732 & 1.546080 & 0.869968 & 0 & $\mathrm{~m}$ & \\
\hline 12 & $\mathrm{c}$ & 0 & 3.465394 & -0.218887 & -0.407054 & 0 & $\mathrm{~m} h$ & 5 \\
\hline 13 & $\mathrm{~h}$ & 0 & 4.151318 & -0.211510 & -1.259587 & 0 & $\mathrm{~m}$ & \\
\hline 14 & $\mathrm{c}$ & 0 & -1.278081 & -0.071366 & -0.170812 & 0 & $\mathrm{~m} h$ & 6 \\
\hline 15 & $\mathrm{c}$ & 0 & -2.184360 & -1.132678 & -0.193970 & 0 & $\mathrm{~m} h$ & 9 \\
\hline 16 & o & 0 & 3.423845 & 2.040626 & -1.136097 & 0 & $\mathrm{~m}$ & \\
\hline 17 & $\mathrm{~h}$ & 0 & 3.091887 & 2.933940 & -0.956663 & 0 & $\mathrm{~m}$ & \\
\hline 18 & $\mathrm{c}$ & 0 & 4.057695 & -1.068653 & 0.706240 & 0 & $\mathrm{~m}$ & \\
\hline 19 & $\mathrm{~h}$ & 0 & 5.090533 & -0.728733 & 0.890026 & 0 & $\mathrm{~m}$ & \\
\hline 20 & $\mathrm{~h}$ & 0 & 4.100232 & -2.111983 & 0.361932 & 0 & $\mathrm{~m}$ & \\
\hline 21 & o & 0 & 3.250021 & -0.932074 & 1.867441 & 0 & $\mathrm{~m}$ & \\
\hline 22 & $\mathrm{~h}$ & 0 & 3.518084 & -1.605480 & 2.509450 & 0 & $\mathrm{~m}$ & \\
\hline 23 & $\mathrm{c}$ & 0 & -3.578021 & -0.863815 & 0.048002 & 0 & $\mathrm{~m}$ & \\
\hline 24 & o & 0 & -4.544483 & -1.604264 & 0.100159 & 0 & $\mathrm{~m}$ & \\
\hline 25 & $\mathrm{n}$ & 0 & -3.763594 & 0.547962 & 0.263388 & 0 & $\mathrm{~m}$ & \\
\hline 26 & $\mathrm{~h}$ & 0 & -4.714512 & 0.795557 & 0.513049 & 0 & $\mathrm{~m}$ & \\
\hline 27 & $\mathrm{c}$ & 0 & -2.787188 & 1.510128 & 0.243150 & 0 & $\mathrm{~m}$ & \\
\hline 28 & $\mathrm{n}$ & 0 & -3.184652 & 2.804043 & 0.526879 & 0 & $\mathrm{~m}$ & \\
\hline 29 & $\mathrm{~h}$ & 0 & -4.088627 & 3.086189 & 0.167041 & 0 & $\mathrm{~m}$ & \\
\hline 30 & $\mathrm{~h}$ & 0 & -2.454719 & 3.477231 & 0.327939 & 0 & $\mathrm{~m}$ & \\
\hline 31 & $\mathrm{n}$ & 0 & -1.521347 & 1.253927 & 0.021434 & 0 & $\mathrm{~m}$ & \\
\hline
\end{tabular}


neutral molecule

Charge $=0$ Multiplicity $=1$ for low level calculation on real system.

Charge $=0$ Multiplicity $=1$ for high level calculation on model system .

Charge $=0$ Multiplicity $=1$ for low level calculation on model system.

Center Atomic Atomic Coordinates (Angstroms)

Number Number Type $\quad X \quad$ Y $\quad$ Z

\begin{tabular}{|c|c|c|c|c|c|c|c|c|}
\hline 1 & $\mathrm{c}$ & 0 & 2.001707 & 0.628296 & 1.203290 & 0 & $\mathrm{~h}$ & \\
\hline 2 & $\mathrm{~h}$ & 0 & 2.018457 & -0.360348 & 1.668497 & 0 & $\mathrm{~h}$ & \\
\hline 3 & $\mathrm{~h}$ & 0 & 1.663952 & 1.361717 & 1.942676 & 0 & $\mathrm{~h}$ & \\
\hline 4 & $\mathrm{c}$ & 0 & 3.378835 & 0.951696 & 0.619759 & 0 & $\mathrm{~h}$ & \\
\hline 5 & $\mathrm{~h}$ & 0 & 4.196332 & 0.560809 & 1.239924 & 0 & $\mathrm{~h}$ & \\
\hline 6 & o & 0 & 3.550280 & 2.346223 & 0.366374 & 0 & $\mathrm{~h}$ & \\
\hline 7 & $\mathrm{~h}$ & 0 & 3.511930 & 2.808229 & 1.218500 & 0 & $\mathrm{~h}$ & \\
\hline 8 & $\mathrm{c}$ & 0 & 3.325730 & 0.262670 & -0.757046 & 0 & h & \\
\hline 9 & $\mathrm{~h}$ & 0 & 3.832134 & 0.901393 & -1.491038 & 0 & $\mathrm{~h}$ & \\
\hline 10 & o & 0 & 1.939282 & 0.172560 & -1.114275 & 0 & $\mathrm{~h}$ & \\
\hline 11 & $\mathrm{c}$ & 0 & 1.104641 & 0.606563 & -0.045104 & 0 & $\mathrm{~h}$ & \\
\hline 12 & $\mathrm{~h}$ & 0 & 0.692110 & 1.595832 & -0.266100 & 0 & $\mathrm{~h}$ & \\
\hline 13 & $\mathrm{c}$ & 0 & 3.965648 & -1.118755 & -0.805677 & 0 & $\mathrm{~m}$ & h 8 \\
\hline 14 & $\mathrm{~h}$ & 0 & 5.059408 & -1.004108 & -0.737453 & 0 & $\mathrm{~m}$ & \\
\hline 15 & $\mathrm{~h}$ & 0 & 3.727464 & -1.573162 & -1.777497 & 0 & $\mathrm{~m}$ & \\
\hline 16 & $\mathrm{n}$ & 0 & -0.034239 & -0.286808 & 0.056304 & 0 & $\mathrm{~m}$ & h 11 \\
\hline 17 & o & 0 & 3.468263 & -1.910656 & 0.271374 & 0 & $\mathrm{~m}$ & \\
\hline 18 & $\mathrm{~h}$ & 0 & 3.786957 & -2.817934 & 0.154499 & 0 & $\mathrm{~m}$ & \\
\hline 19 & $\mathrm{c}$ & 0 & -0.012068 & -1.669978 & 0.183121 & 0 & $\mathrm{~m}$ & \\
\hline 20 & $\mathrm{~h}$ & 0 & 0.928589 & -2.201619 & 0.223850 & 0 & $\mathrm{~m}$ & \\
\hline 21 & $\mathrm{n}$ & 0 & -1.205435 & -2.204603 & 0.216883 & 0 & $\mathrm{~m}$ & \\
\hline 22 & $\mathrm{c}$ & 0 & -2.069117 & -1.131422 & 0.102746 & 0 & $\mathrm{~m}$ & \\
\hline 23 & $\mathrm{c}$ & 0 & -1.361644 & 0.065619 & -0.005598 & 0 & $\mathrm{~m}$ & \\
\hline 24 & $\mathrm{n}$ & 0 & -1.837949 & 1.331198 & -0.148070 & 0 & $\mathrm{~m}$ & \\
\hline 25 & $\mathrm{c}$ & 0 & -3.147758 & 1.379939 & -0.162895 & 0 & $\mathrm{~m}$ & \\
\hline 26 & $\mathrm{n}$ & 0 & -3.783503 & 2.604560 & -0.236606 & 0 & $\mathrm{~m}$ & \\
\hline 27 & $\mathrm{~h}$ & 0 & -4.652421 & 2.634211 & -0.756087 & 0 & $\mathrm{~m}$ & \\
\hline 28 & $\mathrm{~h}$ & 0 & -3.145609 & 3.350233 & -0.487270 & 0 & $\mathrm{~m}$ & \\
\hline 29 & $\mathrm{n}$ & 0 & -3.948046 & 0.272652 & -0.054303 & 0 & $\mathrm{~m}$ & \\
\hline 30 & $\mathrm{~h}$ & 0 & -4.954842 & 0.378082 & 0.002019 & 0 & $\mathrm{~m}$ & \\
\hline
\end{tabular}




$\begin{array}{llllllll}31 & \mathrm{c} & 0 & -3.507946 & -1.092388 & 0.091109 & 0 & \mathrm{~m} \\ 32 & \mathrm{o} & 0 & -4.341380 & -1.976349 & 0.187002 & 0 & \mathrm{~m}\end{array}$

radical

Charge $=0$ Multiplicity $=2$ for low level calculation on real system.

Charge $=0$ Multiplicity $=2$ for high level calculation on model system .

Charge $=0$ Multiplicity $=2$ for low level calculation on model system .

\begin{tabular}{|c|c|c|c|c|c|c|c|c|}
\hline Center & Atomic & & Atomic & ates (Angstro & & & & \\
\hline Number & Number & & Type & $\mathrm{Y}$ & $\mathrm{Z}$ & & & \\
\hline 1 & $\mathrm{c}$ & 0 & -2.060422 & 0.692423 & -1.213723 & 0 & $\mathrm{~h}$ & \\
\hline 2 & $\mathrm{~h}$ & 0 & -1.762272 & 0.779086 & -2.252237 & 0 & $\mathrm{~h}$ & \\
\hline 3 & $\mathrm{c}$ & 0 & -3.458288 & 0.684225 & -0.705488 & 0 & $\mathrm{~h}$ & \\
\hline 4 & $\mathrm{~h}$ & 0 & -4.095154 & -0.043283 & -1.225027 & 0 & $\mathrm{~h}$ & \\
\hline 5 & o & 0 & -4.068868 & 1.992171 & -0.743510 & 0 & $\mathrm{~h}$ & \\
\hline 6 & $\mathrm{~h}$ & 0 & -4.139092 & 2.254281 & -1.675535 & 0 & $\mathrm{~h}$ & \\
\hline 7 & $\mathrm{c}$ & 0 & -3.265719 & 0.318735 & 0.782167 & 0 & $\mathrm{~h}$ & \\
\hline 8 & $\mathrm{~h}$ & 0 & -3.829258 & 1.031133 & 1.395792 & 0 & $\mathrm{~h}$ & \\
\hline 9 & o & 0 & -1.869913 & 0.487064 & 1.089136 & 0 & $\mathrm{~h}$ & \\
\hline 10 & $\mathrm{c}$ & 0 & -1.097553 & 0.754498 & -0.074865 & 0 & h & \\
\hline 11 & $\mathrm{~h}$ & 0 & -0.615822 & 1.737127 & 0.031136 & 0 & $\mathrm{~h}$ & \\
\hline 12 & $\mathrm{c}$ & 0 & -3.726342 & -1.088263 & 1.139369 & 0 & $\mathrm{~m} \mathrm{~h}$ & h 7 \\
\hline 13 & $\mathrm{~h}$ & 0 & -4.827477 & -1.096546 & 1.179443 & 0 & $\mathrm{~m}$ & \\
\hline 14 & $\mathrm{~h}$ & 0 & -3.343553 & -1.331679 & 2.139876 & 0 & $\mathrm{~m}$ & \\
\hline 15 & $\mathrm{n}$ & 0 & 0.014636 & -0.183409 & -0.195501 & 0 & $\mathrm{~m} \mathrm{~h}$ & 10 \\
\hline 16 & o & 0 & -3.250920 & -2.009078 & 0.160259 & 0 & $\mathrm{~m}$ & \\
\hline 17 & $\mathrm{~h}$ & 0 & -3.383578 & -2.907726 & 0.496656 & 0 & $\mathrm{~m}$ & \\
\hline 18 & $\mathrm{c}$ & 0 & -0.043584 & -1.555192 & -0.410266 & 0 & $\mathrm{~m}$ & \\
\hline 19 & $\mathrm{~h}$ & 0 & -0.998791 & -2.050265 & -0.514994 & 0 & $\mathrm{~m}$ & \\
\hline 20 & $\mathrm{n}$ & 0 & 1.133915 & -2.124107 & -0.428301 & 0 & $\mathrm{~m}$ & \\
\hline 21 & $\mathrm{c}$ & 0 & 2.024008 & -1.088127 & -0.211876 & 0 & $\mathrm{~m}$ & \\
\hline 22 & $\mathrm{c}$ & 0 & 1.349275 & 0.121934 & -0.059157 & 0 & $\mathrm{~m}$ & \\
\hline 23 & $\mathrm{n}$ & 0 & 1.856119 & 1.360827 & 0.177378 & 0 & $\mathrm{~m}$ & \\
\hline 24 & $\mathrm{c}$ & 0 & 3.165198 & 1.367517 & 0.246819 & 0 & $\mathrm{~m}$ & \\
\hline 25 & $\mathrm{n}$ & 0 & 3.833048 & 2.564538 & 0.417259 & 0 & $\mathrm{~m}$ & \\
\hline 26 & $\mathrm{~h}$ & 0 & 4.680927 & 2.536965 & 0.970369 & 0 & $\mathrm{~m}$ & \\
\hline 27 & $\mathrm{~h}$ & 0 & 3.208277 & 3.315372 & 0.684836 & 0 & $\mathrm{~m}$ & \\
\hline 28 & $\mathrm{n}$ & 0 & 3.935738 & 0.242781 & 0.105880 & 0 & $\mathrm{~m}$ & \\
\hline 29 & $\mathrm{~h}$ & 0 & 4.946744 & 0.318616 & 0.097307 & 0 & $\mathrm{~m}$ & \\
\hline 30 & $\mathrm{c}$ & 0 & 3.461853 & -1.096223 & -0.139265 & 0 & $\mathrm{~m}$ & \\
\hline 31 & o & 0 & 4.271488 & -1.999737 & -0.254251 & 0 & $\mathrm{~m}$ & \\
\hline
\end{tabular}




\section{s3}

neutral molecule

Charge $=0$ Multiplicity $=1$ for low level calculation on real system.

Charge $=0$ Multiplicity $=1$ for high level calculation on model system.

Charge $=0$ Multiplicity $=1$ for low level calculation on model system.

Center Atomic Atomic Coordinates (Angstroms)

Number Number Type $\quad X \quad$ Y $\quad$ Z

\begin{tabular}{|c|c|c|c|c|c|c|c|}
\hline 1 & $\mathrm{c}$ & 0 & 3.378830 & 0.951689 & 0.619762 & 0 & $\mathrm{~h}$ \\
\hline 2 & $\mathrm{~h}$ & 0 & 4.196331 & 0.560797 & 1.239919 & 0 & $\mathrm{~h}$ \\
\hline 3 & o & 0 & 3.550270 & 2.346222 & 0.366401 & 0 & $\mathrm{~h}$ \\
\hline 4 & $\mathrm{~h}$ & 0 & 3.511915 & 2.808213 & 1.218534 & 0 & $\mathrm{~h}$ \\
\hline 5 & $\mathrm{c}$ & 0 & 3.325733 & 0.262678 & -0.757050 & 0 & $\mathrm{~h}$ \\
\hline 6 & $\mathrm{~h}$ & 0 & 3.832134 & 0.901412 & -1.491035 & 0 & $\mathrm{~h}$ \\
\hline 7 & o & 0 & 1.939286 & 0.172561 & -1.114283 & 0 & $\mathrm{~h}$ \\
\hline 8 & c & 0 & 1.104641 & 0.606558 & -0.045106 & 0 & $\mathrm{~h}$ \\
\hline 9 & $\mathrm{~h}$ & 0 & 0.692103 & 1.595827 & -0.266088 & 0 & $\mathrm{~h}$ \\
\hline 10 & $\mathrm{c}$ & 0 & 2.001707 & 0.628294 & 1.203288 & 0 & $\mathrm{~h}$ \\
\hline 11 & $\mathrm{~h}$ & 0 & 2.018469 & -0.360351 & 1.668489 & 0 & $\mathrm{~h}$ \\
\hline 12 & $\mathrm{~h}$ & 0 & 1.663940 & 1.361703 & 1.942681 & 0 & $\mathrm{~h}$ \\
\hline 13 & $\mathrm{c}$ & 0 & 3.965657 & -1.118744 & -0.805685 & 0 & \\
\hline 14 & $\mathrm{~h}$ & 0 & 5.059417 & -1.004092 & -0.737459 & 0 & $\mathrm{~m}$ \\
\hline 15 & $\mathrm{~h}$ & 0 & 3.727479 & -1.573148 & -1.777508 & 0 & $\mathrm{~m}$ \\
\hline 16 & $\mathrm{n}$ & 0 & -0.034241 & -0.286812 & 0.056304 & 0 & $\mathrm{~m}$ \\
\hline 17 & o & 0 & 3.468275 & -1.910652 & 0.271362 & 0 & $\mathrm{~m}$ \\
\hline 18 & $\mathrm{~h}$ & 0 & 3.786973 & -2.817928 & 0.154484 & 0 & $\mathrm{~m}$ \\
\hline 19 & $\mathrm{c}$ & 0 & -0.012071 & -1.669981 & 0.183127 & 0 & $\mathrm{~m}$ \\
\hline 20 & $\mathrm{~h}$ & 0 & 0.928586 & -2.201623 & 0.223858 & 0 & $\mathrm{~m}$ \\
\hline 21 & $\mathrm{n}$ & 0 & -1.205439 & -2.204604 & 0.216890 & 0 & $\mathrm{~m}$ \\
\hline 22 & $\mathrm{c}$ & 0 & -2.069119 & -1.131423 & 0.102749 & 0 & $\mathrm{~m}$ \\
\hline 23 & $\mathrm{c}$ & 0 & -1.361645 & 0.065617 & -0.005599 & 0 & $\mathrm{~m}$ \\
\hline 24 & $\mathrm{n}$ & 0 & -1.837949 & 1.331196 & -0.148075 & 0 & $\mathrm{~m}$ \\
\hline 25 & $\mathrm{c}$ & 0 & -3.147757 & 1.379939 & -0.162900 & 0 & $\mathrm{~m}$ \\
\hline 26 & $\mathrm{n}$ & 0 & -3.783501 & 2.604560 & -0.236616 & 0 & $\mathrm{~m}$ \\
\hline 27 & $\mathrm{~h}$ & 0 & -4.652419 & 2.634210 & -0.756097 & 0 & $\mathrm{~m}$ \\
\hline 28 & $\mathrm{~h}$ & 0 & -3.145607 & 3.350231 & -0.487282 & 0 & $\mathrm{~m}$ \\
\hline 29 & $\mathrm{n}$ & 0 & -3.948046 & 0.272652 & -0.054305 & 0 & $\mathrm{~m}$ \\
\hline 30 & $\mathrm{~h}$ & 0 & -4.954842 & 0.378084 & 0.002017 & 0 & $\mathrm{~m}$ \\
\hline
\end{tabular}




$\begin{array}{rrrrrrrr}31 & \mathrm{c} & 0 & -3.507948 & -1.092387 & 0.091112 & 0 & \mathrm{~m} \\ 32 & \mathrm{o} & 0 & -4.341383 & -1.976347 & 0.187008 & 0 & \mathrm{~m}\end{array}$

radical

Charge $=0$ Multiplicity $=2$ for low level calculation on real system.

Charge $=0$ Multiplicity $=2$ for high level calculation on model system .

Charge $=0$ Multiplicity $=2$ for low level calculation on model system .

\begin{tabular}{|c|c|c|c|c|c|c|c|}
\hline Center & Atomic & & Atomic & ates (Angstro & & & \\
\hline Number & Numbe & & Type & $\mathrm{Y}$ & $\mathrm{Z}$ & & \\
\hline 1 & $\mathrm{c}$ & 0 & -3.337167 & 0.937013 & -0.591594 & 0 & $\mathrm{~h}$ \\
\hline 2 & o & 0 & -4.049830 & 2.110672 & -0.663228 & 0 & $\mathrm{~h}$ \\
\hline 3 & $\mathrm{~h}$ & 0 & -3.914585 & 2.503312 & -1.540203 & 0 & $\mathrm{~h}$ \\
\hline 4 & $\mathrm{c}$ & 0 & -3.352863 & 0.273153 & 0.759238 & 0 & $\mathrm{~h}$ \\
\hline 5 & $\mathrm{~h}$ & 0 & -3.874144 & 0.911335 & 1.487116 & 0 & $\mathrm{~h}$ \\
\hline 6 & o & 0 & -1.967621 & 0.143234 & 1.148174 & 0 & $\mathrm{~h}$ \\
\hline 7 & $\mathrm{c}$ & 0 & -1.095461 & 0.650354 & 0.143507 & 0 & $\mathrm{~h}$ \\
\hline 8 & $\mathrm{~h}$ & 0 & -0.671902 & 1.611256 & 0.455039 & 0 & $\mathrm{~h}$ \\
\hline 9 & $\mathrm{c}$ & 0 & -1.947599 & 0.808331 & -1.144867 & 0 & $\mathrm{~h}$ \\
\hline 10 & $\mathrm{~h}$ & 0 & -1.845128 & -0.093125 & -1.759803 & 0 & $\mathrm{~h}$ \\
\hline 11 & $\mathrm{~h}$ & 0 & -1.626732 & 1.670905 & -1.739279 & 0 & $\mathrm{~h}$ \\
\hline 12 & $\mathrm{c}$ & 0 & -3.998472 & -1.109936 & 0.786898 & 0 & $\mathrm{~m} \quad \mathrm{~h} \quad 4$ \\
\hline 13 & $\mathrm{~h}$ & 0 & -5.084404 & -0.990117 & 0.649275 & 0 & $\mathrm{~m}$ \\
\hline 14 & $\mathrm{~h}$ & 0 & -3.820739 & -1.558713 & 1.775159 & 0 & $\mathrm{~m}$ \\
\hline 15 & $\mathrm{n}$ & 0 & 0.030367 & -0.253243 & 0.008898 & 0 & $\mathrm{~m} \mathrm{~h}$ \\
\hline 16 & o & 0 & -3.432020 & -1.902324 & -0.252060 & 0 & $\mathrm{~m}$ \\
\hline 17 & $\mathrm{~h}$ & 0 & -3.748886 & -2.811510 & -0.146252 & 0 & $\mathrm{~m}$ \\
\hline 18 & $\mathrm{c}$ & 0 & -0.010864 & -1.630081 & -0.173905 & 0 & $\mathrm{~m}$ \\
\hline 19 & $\mathrm{~h}$ & 0 & -0.959702 & -2.147338 & -0.211565 & 0 & $\mathrm{~m}$ \\
\hline 20 & $\mathrm{n}$ & 0 & 1.176469 & -2.173106 & -0.260733 & 0 & $\mathrm{~m}$ \\
\hline 21 & $\mathrm{c}$ & 0 & 2.053855 & -1.113248 & -0.129871 & 0 & $\mathrm{~m}$ \\
\hline 22 & $\mathrm{c}$ & 0 & 1.362335 & 0.085520 & 0.042592 & 0 & $\mathrm{~m}$ \\
\hline 23 & $\mathrm{n}$ & 0 & 1.854611 & 1.341404 & 0.216817 & 0 & $\mathrm{~m}$ \\
\hline 24 & $\mathrm{c}$ & 0 & 3.164891 & 1.377936 & 0.194370 & 0 & $\mathrm{~m}$ \\
\hline 25 & $\mathrm{n}$ & 0 & 3.814906 & 2.593340 & 0.291705 & 0 & $\mathrm{~m}$ \\
\hline 26 & $\mathrm{~h}$ & 0 & 4.699753 & 2.596749 & 0.784383 & 0 & $\mathrm{~m}$ \\
\hline 27 & $\mathrm{~h}$ & 0 & 3.192981 & 3.335637 & 0.588023 & 0 & $\mathrm{~m}$ \\
\hline 28 & $\mathrm{n}$ & 0 & 3.950317 & 0.268042 & 0.023154 & 0 & $\mathrm{~m}$ \\
\hline 29 & $\mathrm{~h}$ & 0 & 4.956042 & 0.366361 & -0.059274 & 0 & $\mathrm{~m}$ \\
\hline 30 & $\mathrm{c}$ & 0 & 3.492838 & -1.087264 & -0.157913 & 0 & $\mathrm{~m}$ \\
\hline 31 & o & 0 & 4.314696 & -1.974142 & -0.309414 & 0 & $\mathrm{~m}$ \\
\hline
\end{tabular}




\section{s4}

neutral molecule

Charge $=0$ Multiplicity $=1$ for low level calculation on real system.

Charge $=0$ Multiplicity $=1$ for high level calculation on model system .

Charge $=0$ Multiplicity $=1$ for low level calculation on model system .

Center Atomic Atomic Coordinates (Angstroms)

Number Number Type $\quad \mathrm{X} \quad \mathrm{Y} \quad \mathrm{Z}$

\begin{tabular}{|c|c|c|c|c|c|c|c|c|}
\hline 1 & $\mathrm{c}$ & 0 & 3.325733 & 0.262678 & -0.757046 & 0 & $\mathrm{~h}$ & \\
\hline 2 & $\mathrm{~h}$ & 0 & 3.832136 & 0.901412 & -1.491030 & 0 & $\mathrm{~h}$ & \\
\hline 3 & $\mathrm{c}$ & 0 & 3.965661 & -1.118743 & -0.805689 & 0 & $\mathrm{~h}$ & \\
\hline 4 & $\mathrm{~h}$ & 0 & 5.059420 & -1.004089 & -0.737462 & 0 & $\mathrm{~h}$ & \\
\hline 5 & $\mathrm{~h}$ & 0 & 3.727483 & -1.573142 & -1.777514 & 0 & $\mathrm{~h}$ & \\
\hline 6 & o & 0 & 3.468279 & -1.910657 & 0.271353 & 0 & $\mathrm{~h}$ & \\
\hline 7 & $\mathrm{~h}$ & 0 & 3.786980 & -2.817932 & 0.154471 & 0 & $\mathrm{~h}$ & \\
\hline 8 & o & 0 & 1.939286 & 0.172561 & -1.114280 & 0 & $\mathrm{~h}$ & \\
\hline 9 & $\mathrm{c}$ & 0 & 3.378825 & 0.951692 & 0.619764 & 0 & $\mathrm{~h}$ & \\
\hline 10 & $\mathrm{~h}$ & 0 & 4.196314 & 0.560805 & 1.239939 & 0 & $\mathrm{~h}$ & \\
\hline 11 & o & 0 & 3.550264 & 2.346224 & 0.366401 & 0 & $\mathrm{~h}$ & \\
\hline 12 & $\mathrm{~h}$ & 0 & 3.511905 & 2.808217 & 1.218533 & 0 & $\mathrm{~h}$ & \\
\hline 13 & $\mathrm{c}$ & 0 & 1.104641 & 0.606560 & -0.045105 & 0 & $\mathrm{~m}$ & h 8 \\
\hline 14 & $\mathrm{~h}$ & 0 & 0.692103 & 1.595829 & -0.266089 & 0 & $\mathrm{~m}$ & \\
\hline 15 & $\mathrm{c}$ & 0 & 2.001706 & 0.628298 & 1.203290 & 0 & $\mathrm{~m}$ & h 9 \\
\hline 16 & $\mathrm{~h}$ & 0 & 2.018467 & -0.360346 & 1.668493 & 0 & $\mathrm{~m}$ & \\
\hline 17 & $\mathrm{~h}$ & 0 & 1.663939 & 1.361708 & 1.942681 & 0 & $\mathrm{~m}$ & \\
\hline 18 & $\mathrm{n}$ & 0 & -0.034240 & -0.286811 & 0.056306 & 0 & $\mathrm{~m}$ & \\
\hline 19 & $\mathrm{c}$ & 0 & -0.012069 & -1.669980 & 0.183131 & 0 & $\mathrm{~m}$ & \\
\hline 20 & $\mathrm{~h}$ & 0 & 0.928587 & -2.201621 & 0.223863 & 0 & $\mathrm{~m}$ & \\
\hline 21 & $\mathrm{n}$ & 0 & -1.205437 & -2.204604 & 0.216894 & 0 & $\mathrm{~m}$ & \\
\hline 22 & $\mathrm{c}$ & 0 & -2.069118 & -1.131424 & 0.102751 & 0 & $\mathrm{~m}$ & \\
\hline 23 & $\mathrm{c}$ & 0 & -1.361644 & 0.065617 & -0.005599 & 0 & $\mathrm{~m}$ & \\
\hline 24 & $\mathrm{n}$ & 0 & -1.837949 & 1.331195 & -0.148076 & 0 & $\mathrm{~m}$ & \\
\hline 25 & $\mathrm{c}$ & 0 & -3.147758 & 1.379937 & -0.162903 & 0 & $\mathrm{~m}$ & \\
\hline 26 & $\mathrm{n}$ & 0 & -3.783502 & 2.604558 & -0.236620 & 0 & $\mathrm{~m}$ & \\
\hline 27 & $\mathrm{~h}$ & 0 & -4.652420 & 2.634207 & -0.756102 & 0 & $\mathrm{~m}$ & \\
\hline 28 & $\mathrm{~h}$ & 0 & -3.145608 & 3.350229 & -0.487288 & 0 & $\mathrm{~m}$ & \\
\hline 29 & $\mathrm{n}$ & 0 & -3.948046 & 0.272651 & -0.054306 & 0 & $\mathrm{~m}$ & \\
\hline 30 & $\mathrm{~h}$ & 0 & -4.954842 & 0.378082 & 0.002014 & 0 & $\mathrm{~m}$ & \\
\hline
\end{tabular}




$\begin{array}{llllllll}31 & \mathrm{c} & 0 & -3.507947 & -1.092389 & 0.091113 & 0 & \mathrm{~m} \\ 32 & \mathrm{o} & 0 & -4.341382 & -1.976349 & 0.187009 & 0 & \mathrm{~m}\end{array}$

radical

Charge $=0$ Multiplicity $=2$ for low level calculation on real system.

Charge $=0$ Multiplicity $=2$ for high level calculation on model system .

Charge $=0$ Multiplicity $=2$ for low level calculation on model system .

\begin{tabular}{|c|c|c|c|c|c|c|c|}
\hline Center & Atomic & & Atomic & ates (Angstro & & & \\
\hline Number & Numbe & & Type & Y & $\mathrm{Z}$ & & \\
\hline 1 & $\mathrm{c}$ & 0 & 3.216738 & 0.221516 & -0.529614 & 0 & $\mathrm{~h}$ \\
\hline 2 & $\mathrm{c}$ & 0 & 4.293174 & -0.519473 & -1.221848 & 0 & $\mathrm{~h}$ \\
\hline 3 & $\mathrm{~h}$ & 0 & 5.191607 & 0.114801 & -1.262790 & 0 & $\mathrm{~h}$ \\
\hline 4 & $\mathrm{~h}$ & 0 & 3.979918 & -0.737219 & -2.252813 & 0 & $\mathrm{~h}$ \\
\hline 5 & o & 0 & 4.568980 & -1.735675 & -0.497560 & 0 & $\mathrm{~h}$ \\
\hline 6 & $\mathrm{~h}$ & 0 & 5.316582 & -2.172789 & -0.937478 & 0 & $\mathrm{~h}$ \\
\hline 7 & o & 0 & 1.924503 & -0.100072 & -0.867707 & 0 & $\mathrm{~h}$ \\
\hline 8 & $\mathrm{c}$ & 0 & 3.294566 & 0.857061 & 0.823971 & 0 & $\mathrm{~h}$ \\
\hline 9 & $\mathrm{~h}$ & 0 & 4.099417 & 0.420883 & 1.431313 & 0 & $\mathrm{~h}$ \\
\hline 10 & $\mathrm{o}$ & 0 & 3.509453 & 2.269878 & 0.659737 & 0 & $\mathrm{~h}$ \\
\hline 11 & $\mathrm{~h}$ & 0 & 3.672267 & 2.640875 & 1.543045 & 0 & $\mathrm{~h}$ \\
\hline 12 & $\mathrm{c}$ & 0 & 1.037473 & 0.450393 & 0.127328 & 0 & $\mathrm{~m} h$ \\
\hline 13 & $\mathrm{~h}$ & 0 & 0.694041 & 1.435956 & -0.198552 & 0 & $\mathrm{~m}$ \\
\hline 14 & $\mathrm{c}$ & 0 & 1.899871 & 0.535514 & 1.394617 & 0 & $\mathrm{~m} h$ \\
\hline 15 & $\mathrm{~h}$ & 0 & 1.911267 & -0.437475 & 1.897779 & 0 & $\mathrm{~m}$ \\
\hline 16 & $\mathrm{~h}$ & 0 & 1.532077 & 1.289657 & 2.095665 & 0 & $\mathrm{~m}$ \\
\hline 17 & $\mathrm{n}$ & 0 & -0.128443 & -0.390071 & 0.217496 & 0 & $\mathrm{~m}$ \\
\hline 18 & $\mathrm{c}$ & 0 & -0.180763 & -1.757415 & 0.468668 & 0 & $\mathrm{~m}$ \\
\hline 19 & $\mathrm{~h}$ & 0 & 0.722673 & -2.333436 & 0.615063 & 0 & $\mathrm{~m}$ \\
\hline 20 & $\mathrm{n}$ & 0 & -1.396368 & -2.235483 & 0.464635 & 0 & $\mathrm{~m}$ \\
\hline 21 & $\mathrm{c}$ & 0 & -2.199669 & -1.142976 & 0.196093 & 0 & $\mathrm{~m}$ \\
\hline 22 & $\mathrm{c}$ & 0 & -1.433418 & 0.008637 & 0.030985 & 0 & $\mathrm{~m}$ \\
\hline 23 & $\mathrm{n}$ & 0 & -1.835646 & 1.273665 & -0.254551 & 0 & $\mathrm{~m}$ \\
\hline 24 & $\mathrm{c}$ & 0 & -3.139119 & 1.376506 & -0.364632 & 0 & $\mathrm{~m}$ \\
\hline 25 & $\mathrm{n}$ & 0 & -3.706386 & 2.613092 & -0.589546 & 0 & $\mathrm{~m}$ \\
\hline 26 & $\mathrm{~h}$ & 0 & -4.548919 & 2.637879 & -1.150257 & 0 & $\mathrm{~m}$ \\
\hline 27 & $\mathrm{~h}$ & 0 & -3.022717 & 3.309703 & -0.858951 & 0 & $\mathrm{~m}$ \\
\hline 28 & $\mathrm{n}$ & 0 & -3.995883 & 0.317390 & -0.214816 & 0 & $\mathrm{~m}$ \\
\hline 29 & $\mathrm{~h}$ & 0 & -4.998224 & 0.468173 & -0.241311 & 0 & $\mathrm{~m}$ \\
\hline 30 & $\mathrm{c}$ & 0 & -3.632144 & -1.046053 & 0.080546 & 0 & $\mathrm{~m}$ \\
\hline 31 & $\mathrm{o}$ & 0 & -4.509332 & -1.882807 & 0.195539 & 0 & $\mathrm{~m}$ \\
\hline
\end{tabular}


neutral molecule

Charge $=0$ Multiplicity $=1$ for low level calculation on real system.

Charge $=0$ Multiplicity $=1$ for high level calculation on model system.

Charge $=0$ Multiplicity $=1$ for low level calculation on model system.

Center Atomic Atomic Coordinates (Angstroms)

Number Number Type $\quad X \quad$ Y $\quad$ Z

\begin{tabular}{|c|c|c|c|c|c|c|c|c|}
\hline 1 & $\mathrm{c}$ & 0 & 3.965661 & -1.118743 & -0.805689 & 0 & $\mathrm{~h}$ & \\
\hline 2 & $\mathrm{~h}$ & 0 & 5.059420 & -1.004090 & -0.737451 & 0 & $\mathrm{~h}$ & \\
\hline 3 & $\mathrm{~h}$ & 0 & 3.727483 & -1.573134 & -1.777518 & 0 & $\mathrm{~h}$ & \\
\hline 4 & o & 0 & 3.468279 & -1.910657 & 0.271353 & 0 & $\mathrm{~h}$ & \\
\hline 5 & $\mathrm{~h}$ & 0 & 3.786979 & -2.817932 & 0.154470 & 0 & $\mathrm{~h}$ & \\
\hline 6 & $\mathrm{c}$ & 0 & 3.325733 & 0.262678 & -0.757047 & 0 & $\mathrm{~h}$ & \\
\hline 7 & $\mathrm{~h}$ & 0 & 3.832136 & 0.901412 & -1.491030 & 0 & $\mathrm{~h}$ & \\
\hline 8 & o & 0 & 1.939286 & 0.172561 & -1.114280 & 0 & $\mathrm{~h}$ & \\
\hline 9 & $\mathrm{c}$ & 0 & 3.378825 & 0.951692 & 0.619764 & 0 & $\mathrm{~h}$ & \\
\hline 10 & $\mathrm{~h}$ & 0 & 4.196314 & 0.560805 & 1.239939 & 0 & $\mathrm{~h}$ & \\
\hline 11 & o & 0 & 3.550264 & 2.346224 & 0.366401 & 0 & $\mathrm{~h}$ & \\
\hline 12 & $\mathrm{~h}$ & 0 & 3.511905 & 2.808217 & 1.218533 & 0 & $\mathrm{~h}$ & \\
\hline 13 & $\mathrm{c}$ & 0 & 1.104641 & 0.606560 & -0.045105 & 0 & $\mathrm{~m}$ & h 8 \\
\hline 14 & $\mathrm{~h}$ & 0 & 0.692103 & 1.595828 & -0.266089 & 0 & $\mathrm{~m}$ & \\
\hline 15 & $\mathrm{c}$ & 0 & 2.001706 & 0.628298 & 1.203290 & 0 & $\mathrm{~m}$ & h 9 \\
\hline 16 & $\mathrm{~h}$ & 0 & 2.018467 & -0.360347 & 1.668493 & 0 & $\mathrm{~m}$ & \\
\hline 17 & $\mathrm{~h}$ & 0 & 1.663939 & 1.361708 & 1.942681 & 0 & $\mathrm{~m}$ & \\
\hline 18 & $\mathrm{n}$ & 0 & -0.034240 & -0.286811 & 0.056306 & 0 & $\mathrm{~m}$ & \\
\hline 19 & $\mathrm{c}$ & 0 & -0.012069 & -1.669980 & 0.183131 & 0 & $\mathrm{~m}$ & \\
\hline 20 & $\mathrm{~h}$ & 0 & 0.928587 & -2.201621 & 0.223863 & 0 & $\mathrm{~m}$ & \\
\hline 21 & $\mathrm{n}$ & 0 & -1.205437 & -2.204604 & 0.216893 & 0 & $\mathrm{~m}$ & \\
\hline 22 & $\mathrm{c}$ & 0 & -2.069118 & -1.131424 & 0.102751 & 0 & $\mathrm{~m}$ & \\
\hline 23 & $\mathrm{c}$ & 0 & -1.361644 & 0.065617 & -0.005599 & 0 & $\mathrm{~m}$ & \\
\hline 24 & $\mathrm{n}$ & 0 & -1.837949 & 1.331195 & -0.148076 & 0 & $\mathrm{~m}$ & \\
\hline 25 & $\mathrm{c}$ & 0 & -3.147758 & 1.379937 & -0.162903 & 0 & $\mathrm{~m}$ & \\
\hline 26 & $\mathrm{n}$ & 0 & -3.783502 & 2.604558 & -0.236620 & 0 & $\mathrm{~m}$ & \\
\hline 27 & $\mathrm{~h}$ & 0 & -3.145608 & 3.350229 & -0.487287 & 0 & $\mathrm{~m}$ & \\
\hline 28 & $\mathrm{~h}$ & 0 & -4.652420 & 2.634207 & -0.756102 & 0 & $\mathrm{~m}$ & \\
\hline 29 & $\mathrm{n}$ & 0 & -3.948046 & 0.272651 & -0.054306 & 0 & $\mathrm{~m}$ & \\
\hline 30 & $\mathrm{~h}$ & 0 & -4.954842 & 0.378082 & 0.002014 & 0 & $\mathrm{~m}$ & \\
\hline
\end{tabular}




$\begin{array}{llllllll}31 & \mathrm{c} & 0 & -3.507947 & -1.092389 & 0.091113 & 0 & \mathrm{~m} \\ 32 & \mathrm{o} & 0 & -4.341382 & -1.976349 & 0.187009 & 0 & \mathrm{~m}\end{array}$

radical

Charge $=0$ Multiplicity $=2$ for low level calculation on real system.

Charge $=0$ Multiplicity $=2$ for high level calculation on model system .

Charge $=0$ Multiplicity $=2$ for low level calculation on model system .

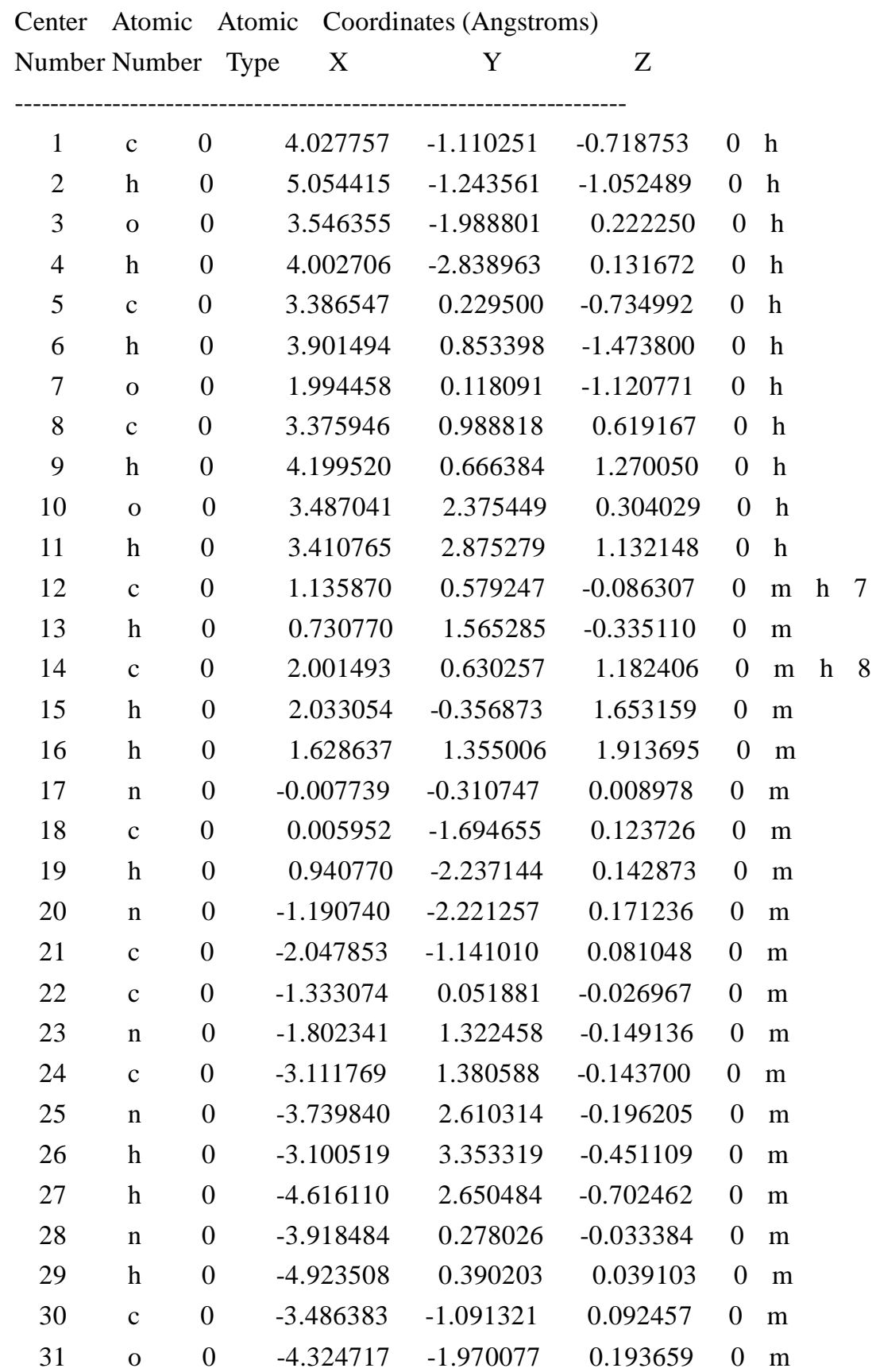




\section{b1}

neutral molecule

Charge $=0$ Multiplicity $=1$ for low level calculation on real system.

Charge $=0$ Multiplicity $=1$ for high level calculation on model system .

Charge $=0$ Multiplicity $=1$ for low level calculation on model system.

Center Atomic Atomic Coordinates (Angstroms)

Number Number Type $\quad \mathrm{X} \quad \mathrm{Y} \quad \mathrm{Z}$

\begin{tabular}{|c|c|c|c|c|c|c|c|}
\hline 1 & $\mathrm{n}$ & 0 & -3.948032 & 0.272650 & -0.054310 & 0 & $\mathrm{~h}$ \\
\hline 2 & $\mathrm{~h}$ & 0 & -4.954829 & 0.378076 & 0.002014 & 0 & $\mathrm{~h}$ \\
\hline 3 & $\mathrm{c}$ & 0 & -3.147750 & 1.379943 & -0.162896 & 0 & $\mathrm{~h}$ \\
\hline 4 & $\mathrm{n}$ & 0 & -3.783497 & 2.604563 & -0.236598 & 0 & $\mathrm{~h}$ \\
\hline 5 & $\mathrm{~h}$ & 0 & -4.652412 & 2.634218 & -0.756083 & 0 & $\mathrm{~h}$ \\
\hline 6 & $\mathrm{~h}$ & 0 & -3.145604 & 3.350239 & -0.487251 & 0 & $\mathrm{~h}$ \\
\hline 7 & $\mathrm{n}$ & 0 & -1.837931 & 1.331186 & -0.148058 & 0 & $\mathrm{~h}$ \\
\hline 8 & $\mathrm{c}$ & 0 & -3.507946 & -1.092385 & 0.091112 & 0 & $\mathrm{~h}$ \\
\hline 9 & o & 0 & -4.341385 & -1.976344 & 0.186985 & 0 & $\mathrm{~h}$ \\
\hline 10 & $\mathrm{c}$ & 0 & -1.361647 & 0.065613 & -0.005605 & 0 & $\mathrm{~m} \mathrm{l}$ \\
\hline 11 & $\mathrm{c}$ & 0 & -2.069118 & -1.131416 & 0.102754 & 0 & $\mathrm{~m} \mathrm{~h}$ \\
\hline 12 & $\mathrm{n}$ & 0 & -1.205434 & -2.204594 & 0.216893 & 0 & $\mathrm{~m}$ \\
\hline 13 & $\mathrm{c}$ & 0 & -0.012077 & -1.669973 & 0.183114 & 0 & $\mathrm{~m}$ \\
\hline 14 & $\mathrm{~h}$ & 0 & 0.928577 & -2.201618 & 0.223843 & 0 & $\mathrm{~m}$ \\
\hline 15 & $\mathrm{n}$ & 0 & -0.034242 & -0.286812 & 0.056302 & 0 & $\mathrm{~m}$ \\
\hline 16 & $\mathrm{c}$ & 0 & 1.104637 & 0.606560 & -0.045111 & 0 & $\mathrm{~m}$ \\
\hline 17 & $\mathrm{~h}$ & 0 & 0.692104 & 1.595828 & -0.266109 & 0 & $\mathrm{~m}$ \\
\hline 18 & $\mathrm{c}$ & 0 & 2.001701 & 0.628304 & 1.203285 & 0 & $\mathrm{~m}$ \\
\hline 19 & $\mathrm{~h}$ & 0 & 2.018456 & -0.360336 & 1.668497 & 0 & $\mathrm{~m}$ \\
\hline 20 & $\mathrm{~h}$ & 0 & 1.663938 & 1.361723 & 1.942670 & 0 & $\mathrm{~m}$ \\
\hline 21 & $\mathrm{c}$ & 0 & 3.378830 & 0.951701 & 0.619754 & 0 & $\mathrm{~m}$ \\
\hline 22 & $\mathrm{~h}$ & 0 & 4.196326 & 0.560821 & 1.239924 & 0 & $\mathrm{~m}$ \\
\hline 23 & $\mathrm{o}$ & 0 & 3.550273 & 2.346227 & 0.366358 & 0 & $\mathrm{~m}$ \\
\hline 24 & $\mathrm{~h}$ & 0 & 3.511921 & 2.808239 & 1.218480 & 0 & $\mathrm{~m}$ \\
\hline 25 & $\mathrm{c}$ & 0 & 3.325728 & 0.262665 & -0.757046 & 0 & $\mathrm{~m}$ \\
\hline 26 & $\mathrm{~h}$ & 0 & 3.832133 & 0.901383 & -1.491042 & 0 & $\mathrm{~m}$ \\
\hline 27 & o & 0 & 1.939293 & 0.172544 & -1.114271 & 0 & $\mathrm{~m}$ \\
\hline 28 & $\mathrm{c}$ & 0 & 3.965643 & -1.118762 & -0.805668 & 0 & $\mathrm{~m}$ \\
\hline 29 & $\mathrm{~h}$ & 0 & 5.059404 & -1.004116 & -0.737445 & 0 & $\mathrm{~m}$ \\
\hline 30 & $\mathrm{~h}$ & 0 & 3.727459 & -1.573175 & -1.777485 & 0 & $\mathrm{~m}$ \\
\hline
\end{tabular}




$\begin{array}{llllllll}31 & \mathrm{o} & 0 & 3.468258 & -1.910654 & 0.271389 & 0 & \mathrm{~m} \\ 32 & \mathrm{~h} & 0 & 3.786950 & -2.817934 & 0.154521 & 0 & \mathrm{~m}\end{array}$

radical

Charge $=0$ Multiplicity $=2$ for low level calculation on real system.

Charge $=0$ Multiplicity $=2$ for high level calculation on model system .

Charge $=0$ Multiplicity $=2$ for low level calculation on model system .

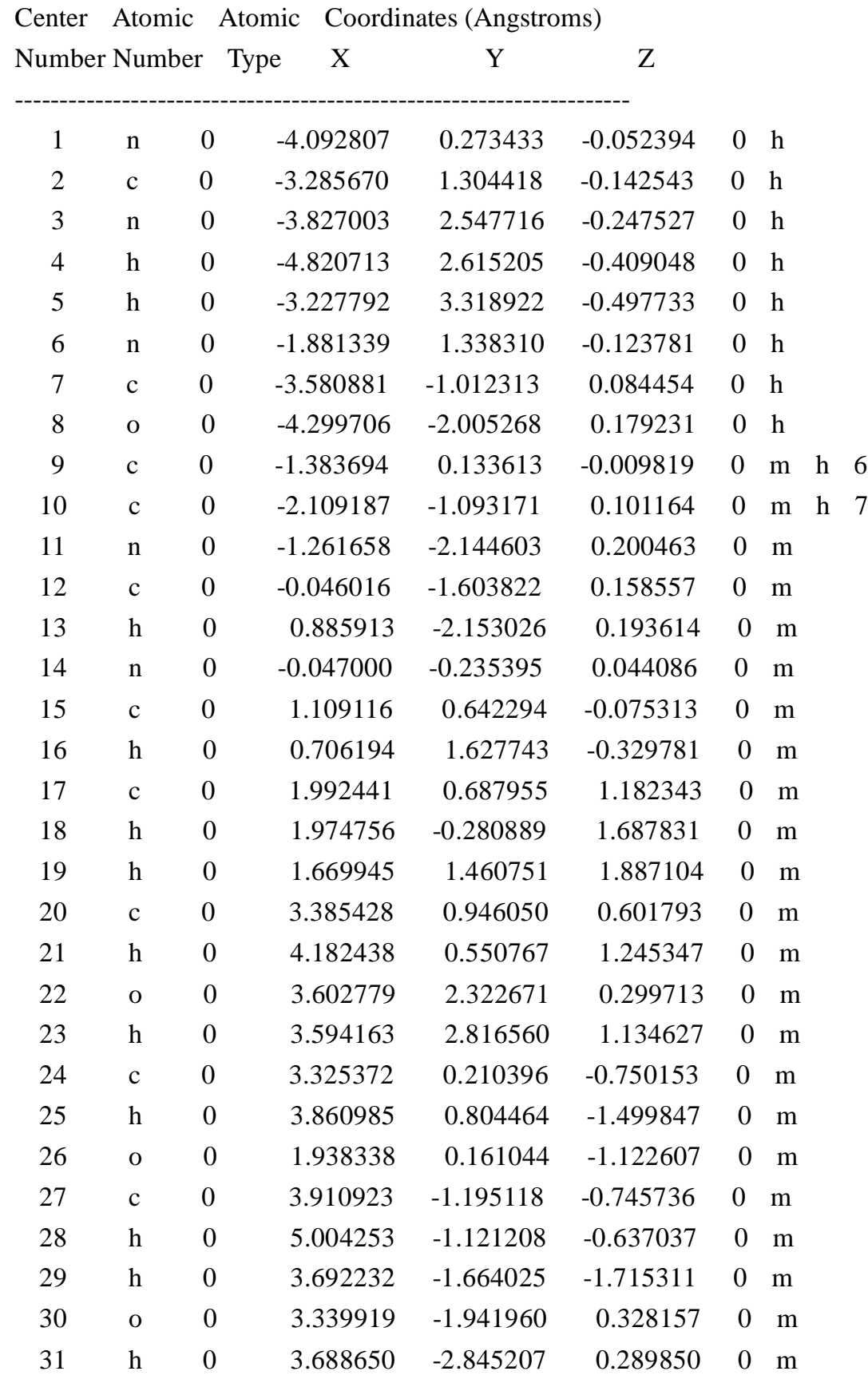




\section{b2}

neutral molecule

Charge $=0$ Multiplicity $=1$ for low level calculation on real system.

Charge $=0$ Multiplicity $=1$ for high level calculation on model system.

Charge $=0$ Multiplicity $=1$ for low level calculation on model system.

Center Atomic Atomic Coordinates (Angstroms)

Number Number Type $\quad X \quad$ Y $\quad$ Z

\begin{tabular}{|c|c|c|c|c|c|c|c|}
\hline 1 & $\mathrm{n}$ & 0 & -3.783530 & 2.604548 & -0.236615 & 0 & $\mathrm{~h}$ \\
\hline 2 & $\mathrm{~h}$ & 0 & -4.652439 & 2.634186 & -0.756111 & 0 & $\mathrm{~h}$ \\
\hline 3 & $\mathrm{~h}$ & 0 & -3.145646 & 3.350232 & -0.487266 & 0 & $\mathrm{~h}$ \\
\hline 4 & $\mathrm{c}$ & 0 & -3.147765 & 1.379938 & -0.162896 & 0 & $\mathrm{~h}$ \\
\hline 5 & $\mathrm{n}$ & 0 & -1.837945 & 1.331196 & -0.148047 & 0 & $\mathrm{~h}$ \\
\hline 6 & $\mathrm{n}$ & 0 & -3.948031 & 0.272634 & -0.054313 & 0 & $\mathrm{~h}$ \\
\hline 7 & $\mathrm{~h}$ & 0 & -4.954830 & 0.378043 & 0.001999 & 0 & $\mathrm{~h}$ \\
\hline 8 & $\mathrm{c}$ & 0 & -3.507925 & -1.092393 & 0.091125 & 0 & $\mathrm{~h}$ \\
\hline 9 & o & 0 & -4.341351 & -1.976365 & 0.186994 & 0 & $\mathrm{~h}$ \\
\hline 10 & $\mathrm{c}$ & 0 & -1.361647 & 0.065629 & -0.005594 & 0 & $\mathrm{~m}$ \\
\hline 11 & $\mathrm{c}$ & 0 & -2.069115 & -1.131406 & 0.102747 & 0 & $\mathrm{~m}$ \\
\hline 12 & $\mathrm{n}$ & 0 & -1.205415 & -2.204571 & 0.216884 & 0 & $\mathrm{~m}$ \\
\hline 13 & $\mathrm{c}$ & 0 & -0.012084 & -1.669963 & 0.183123 & 0 & $\mathrm{~m}$ \\
\hline 14 & $\mathrm{~h}$ & 0 & 0.928570 & -2.201610 & 0.223857 & 0 & $\mathrm{~m}$ \\
\hline 15 & $\mathrm{n}$ & 0 & -0.034244 & -0.286802 & 0.056307 & 0 & $\mathrm{~m}$ \\
\hline 16 & $\mathrm{c}$ & 0 & 1.104639 & 0.606567 & -0.045104 & 0 & $\mathrm{~m}$ \\
\hline 17 & $\mathrm{~h}$ & 0 & 0.692108 & 1.595837 & -0.266096 & 0 & $\mathrm{~m}$ \\
\hline 18 & $\mathrm{c}$ & 0 & 2.001707 & 0.628301 & 1.203289 & 0 & $\mathrm{~m}$ \\
\hline 19 & $\mathrm{~h}$ & 0 & 2.018459 & -0.360342 & 1.668495 & 0 & $\mathrm{~m}$ \\
\hline 20 & $\mathrm{~h}$ & 0 & 1.663948 & 1.361717 & 1.942679 & 0 & $\mathrm{~m}$ \\
\hline 21 & $\mathrm{c}$ & 0 & 3.378834 & 0.951697 & 0.619755 & 0 & $\mathrm{~m}$ \\
\hline 22 & $\mathrm{~h}$ & 0 & 4.196331 & 0.560809 & 1.239920 & 0 & $\mathrm{~m}$ \\
\hline 23 & o & 0 & 3.550282 & 2.346223 & 0.366366 & 0 & $\mathrm{~m}$ \\
\hline 24 & $\mathrm{~h}$ & 0 & 3.511934 & 2.808230 & 1.218491 & 0 & $\mathrm{~m}$ \\
\hline 25 & $\mathrm{c}$ & 0 & 3.325726 & 0.262668 & -0.757048 & 0 & $\mathrm{~m}$ \\
\hline 26 & $\mathrm{~h}$ & 0 & 3.832131 & 0.901388 & -1.491042 & 0 & $\mathrm{~m}$ \\
\hline 27 & o & 0 & 1.939289 & 0.172554 & -1.114270 & 0 & $\mathrm{~m}$ \\
\hline 28 & $\mathrm{c}$ & 0 & 3.965636 & -1.118761 & -0.805680 & 0 & $\mathrm{~m}$ \\
\hline 29 & $\mathrm{~h}$ & 0 & 5.059398 & -1.004120 & -0.737460 & 0 & $\mathrm{~m}$ \\
\hline 30 & $\mathrm{~h}$ & 0 & 3.727447 & -1.573168 & -1.777499 & 0 & $\mathrm{~m}$ \\
\hline
\end{tabular}




$\begin{array}{llllllll}31 & \mathrm{o} & 0 & 3.468251 & -1.910657 & 0.271374 & 0 & \mathrm{~m} \\ 32 & \mathrm{~h} & 0 & 3.786940 & -2.817938 & 0.154499 & 0 & \mathrm{~m}\end{array}$

radical

Charge $=0$ Multiplicity $=2$ for low level calculation on real system.

Charge $=0$ Multiplicity $=2$ for high level calculation on model system .

Charge $=0$ Multiplicity $=2$ for low level calculation on model system .

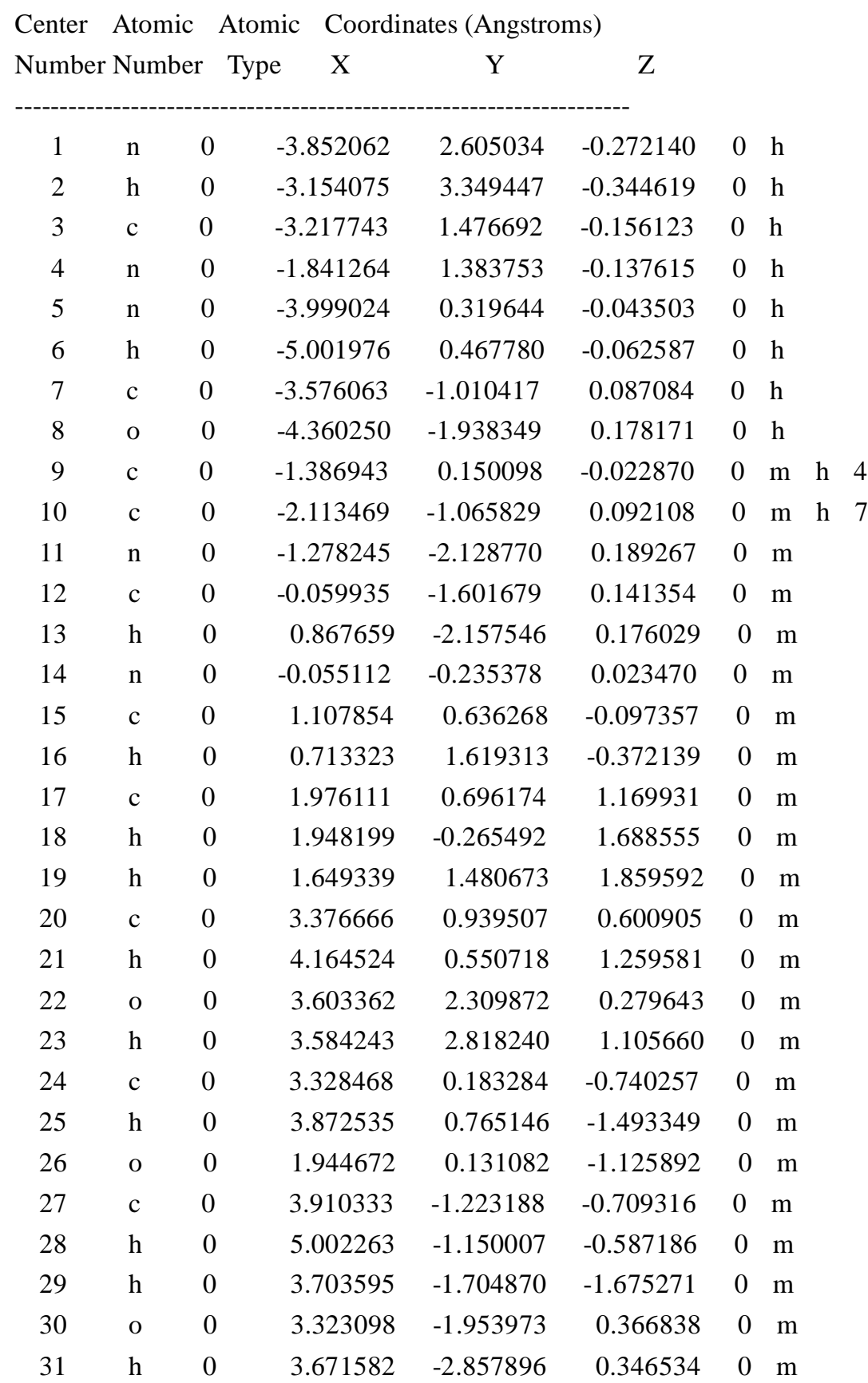




\section{b8}

neutral molecule

Charge $=0$ Multiplicity $=1$ for low level calculation on real system .

Charge $=0$ Multiplicity $=1$ for high level calculation on model system .

Charge $=0$ Multiplicity $=1$ for low level calculation on model system .

Center Atomic Atomic Coordinates (Angstroms)

Number Number Type $\quad X \quad$ Y $\quad$ Z

\begin{tabular}{|c|c|c|c|c|c|c|c|c|}
\hline 1 & $\mathrm{c}$ & 0 & -0.012075 & -1.669975 & 0.183150 & 0 & $\mathrm{~h}$ & \\
\hline 2 & $\mathrm{~h}$ & 0 & 0.928579 & -2.201620 & 0.223897 & 0 & $\mathrm{~h}$ & \\
\hline 3 & $\mathrm{n}$ & 0 & -1.205432 & -2.204595 & 0.216910 & 0 & $\mathrm{~h}$ & \\
\hline 4 & $\mathrm{c}$ & 0 & -2.069114 & -1.131417 & 0.102751 & 0 & $\mathrm{~h}$ & \\
\hline 5 & $\mathrm{c}$ & 0 & -1.361646 & 0.065617 & -0.005601 & 0 & $\mathrm{~h}$ & \\
\hline 6 & $\mathrm{n}$ & 0 & -0.034246 & -0.286807 & 0.056311 & 0 & $\mathrm{~h}$ & \\
\hline 7 & $\mathrm{c}$ & 0 & 1.104634 & 0.606563 & -0.045102 & 0 & $\mathrm{~h}$ & \\
\hline 8 & $\mathrm{~h}$ & 0 & 0.692102 & 1.595833 & -0.266092 & 0 & $\mathrm{~h}$ & \\
\hline 9 & $\mathrm{c}$ & 0 & -3.507942 & -1.092384 & 0.091103 & 0 & $\mathrm{~m}$ & h 4 \\
\hline 10 & o & 0 & -4.341377 & -1.976347 & 0.186972 & 0 & $\mathrm{~m}$ & \\
\hline 11 & $\mathrm{n}$ & 0 & -1.837947 & 1.331183 & -0.148056 & 0 & $\mathrm{~m}$ & $\mathrm{~h}$ \\
\hline 12 & $\mathrm{c}$ & 0 & 2.001705 & 0.628298 & 1.203289 & 0 & $\mathrm{~m}$ & $\mathrm{~h}$ \\
\hline 13 & $\mathrm{~h}$ & 0 & 2.018460 & -0.360346 & 1.668494 & 0 & $\mathrm{~m}$ & \\
\hline 14 & $\mathrm{~h}$ & 0 & 1.663947 & 1.361712 & 1.942680 & 0 & $\mathrm{~m}$ & \\
\hline 15 & o & 0 & 1.939283 & 0.172553 & -1.114270 & 0 & $\mathrm{~m}$ & $\mathrm{~h}$ \\
\hline 16 & $\mathrm{n}$ & 0 & -3.948025 & 0.272653 & -0.054318 & 0 & $\mathrm{~m}$ & \\
\hline 17 & $\mathrm{~h}$ & 0 & -4.954822 & 0.378080 & 0.002006 & 0 & $\mathrm{~m}$ & \\
\hline 18 & $\mathrm{c}$ & 0 & -3.147741 & 1.379944 & -0.162897 & 0 & $\mathrm{~m}$ & \\
\hline 19 & $\mathrm{n}$ & 0 & -3.783486 & 2.604566 & -0.236599 & 0 & $\mathrm{~m}$ & \\
\hline 20 & $\mathrm{~h}$ & 0 & -4.652398 & 2.634223 & -0.756088 & 0 & $\mathrm{~m}$ & \\
\hline 21 & $\mathrm{~h}$ & 0 & -3.145590 & 3.350241 & -0.487247 & 0 & $\mathrm{~m}$ & \\
\hline 22 & $\mathrm{c}$ & 0 & 3.378831 & 0.951696 & 0.619752 & 0 & $\mathrm{~m}$ & \\
\hline 23 & $\mathrm{~h}$ & 0 & 4.196330 & 0.560809 & 1.239915 & 0 & $\mathrm{~m}$ & \\
\hline 24 & o & 0 & 3.550276 & 2.346223 & 0.366365 & 0 & $\mathrm{~m}$ & \\
\hline 25 & $\mathrm{~h}$ & 0 & 3.511929 & 2.808229 & 1.218490 & 0 & $\mathrm{~m}$ & \\
\hline 26 & $\mathrm{c}$ & 0 & 3.325721 & 0.262668 & -0.757051 & 0 & $\mathrm{~m}$ & \\
\hline 27 & $\mathrm{~h}$ & 0 & 3.832123 & 0.901390 & -1.491046 & 0 & $\mathrm{~m}$ & \\
\hline 28 & $\mathrm{c}$ & 0 & 3.965633 & -1.118760 & -0.805686 & 0 & $\mathrm{~m}$ & \\
\hline 29 & $\mathrm{~h}$ & 0 & 5.059394 & -1.004117 & -0.737468 & 0 & $\mathrm{~m}$ & \\
\hline 30 & $\mathrm{~h}$ & 0 & 3.727442 & -1.573166 & -1.777504 & 0 & $\mathrm{~m}$ & \\
\hline
\end{tabular}




$\begin{array}{llllllll}31 & \mathrm{o} & 0 & 3.468252 & -1.910658 & 0.271369 & 0 & \mathrm{~m} \\ 32 & \mathrm{~h} & 0 & 3.786941 & -2.817937 & 0.154492 & 0 & \mathrm{~m}\end{array}$

radical

Charge $=0$ Multiplicity $=2$ for low level calculation on real system.

Charge $=0$ Multiplicity $=2$ for high level calculation on model system .

Charge $=0$ Multiplicity $=2$ for low level calculation on model system .

\begin{tabular}{|c|c|c|c|c|c|c|c|c|}
\hline Center & Atomic & & Atomic & lates (Angstro & & & & \\
\hline Number & Numbe & & Type & Y & $\mathrm{Z}$ & & & \\
\hline 1 & $\mathrm{c}$ & 0 & 0.009047 & -1.596168 & 0.367212 & 0 & $\mathrm{~h}$ & \\
\hline 2 & $\mathrm{n}$ & 0 & -1.132026 & -2.147596 & 0.364671 & 0 & $\mathrm{~h}$ & \\
\hline 3 & $\mathrm{c}$ & 0 & -2.036723 & -1.098856 & 0.172374 & 0 & $\mathrm{~h}$ & \\
\hline 4 & $\mathrm{c}$ & 0 & -1.341056 & 0.106216 & 0.082374 & 0 & $\mathrm{~h}$ & \\
\hline 5 & $\mathrm{n}$ & 0 & 0.001242 & -0.221088 & 0.236160 & 0 & $\mathrm{~h}$ & \\
\hline 6 & $\mathrm{c}$ & 0 & 1.153894 & 0.648225 & 0.085374 & 0 & $\mathrm{~h}$ & \\
\hline 7 & $\mathrm{~h}$ & 0 & 0.752436 & 1.656286 & -0.054997 & 0 & $\mathrm{~h}$ & \\
\hline 8 & $\mathrm{c}$ & 0 & -3.470493 & -1.095936 & 0.076295 & 0 & $\mathrm{~m} \mathrm{~h}$ & 3 \\
\hline 9 & o & 0 & -4.287454 & -1.996328 & 0.138084 & 0 & $\mathrm{~m}$ & \\
\hline 10 & $\mathrm{n}$ & 0 & -1.828666 & 1.352713 & -0.114480 & 0 & $\mathrm{~m} \mathrm{~h}$ & 4 \\
\hline 11 & $\mathrm{c}$ & 0 & 2.138597 & 0.567287 & 1.261285 & 0 & $\mathrm{~m} \mathrm{l}$ & h 6 \\
\hline 12 & $\mathrm{~h}$ & 0 & 2.132002 & -0.443351 & 1.678854 & 0 & $\mathrm{~m}$ & \\
\hline 13 & $\mathrm{~h}$ & 0 & 1.898690 & 1.282145 & 2.054745 & 0 & $\mathrm{~m}$ & \\
\hline 14 & o & 0 & 1.881380 & 0.246224 & -1.068874 & 0 & $\mathrm{~m}$ & 6 \\
\hline 15 & $\mathrm{n}$ & 0 & -3.923727 & 0.259106 & -0.121190 & 0 & $\mathrm{~m}$ & \\
\hline 16 & $\mathrm{~h}$ & 0 & -4.934209 & 0.344410 & -0.135008 & 0 & $\mathrm{~m}$ & \\
\hline 17 & $\mathrm{c}$ & 0 & -3.138744 & 1.379019 & -0.207984 & 0 & $\mathrm{~m}$ & \\
\hline 18 & $\mathrm{n}$ & 0 & -3.782901 & 2.587450 & -0.346239 & 0 & $\mathrm{~m}$ & \\
\hline 19 & $\mathrm{~h}$ & 0 & -4.645746 & 2.599239 & -0.874864 & 0 & $\mathrm{~m}$ & \\
\hline 20 & $\mathrm{~h}$ & 0 & -3.152299 & 3.345267 & -0.575980 & 0 & $\mathrm{~m}$ & \\
\hline 21 & $\mathrm{c}$ & 0 & 3.486752 & 0.838064 & 0.589453 & 0 & $\mathrm{~m}$ & \\
\hline 22 & $\mathrm{~h}$ & 0 & 4.319880 & 0.370042 & 1.130473 & 0 & $\mathrm{~m}$ & \\
\hline 23 & o & 0 & 3.723176 & 2.232027 & 0.391063 & 0 & $\mathrm{~m}$ & \\
\hline 24 & $\mathrm{~h}$ & 0 & 3.818204 & 2.643345 & 1.264500 & 0 & $\mathrm{~m}$ & \\
\hline 25 & $\mathrm{c}$ & 0 & 3.299025 & 0.220260 & -0.806657 & 0 & $\mathrm{~m}$ & \\
\hline 26 & $\mathrm{~h}$ & 0 & 3.798409 & 0.854756 & -1.548889 & 0 & $\mathrm{~m}$ & \\
\hline 27 & $\mathrm{c}$ & 0 & 3.797109 & -1.218367 & -0.946755 & 0 & $\mathrm{~m}$ & \\
\hline 28 & $\mathrm{~h}$ & 0 & 4.883709 & -1.242010 & -0.805494 & 0 & $\mathrm{~m}$ & \\
\hline 29 & $\mathrm{~h}$ & 0 & 3.583828 & -1.564115 & -1.968763 & 0 & $\mathrm{~m}$ & \\
\hline 30 & o & 0 & 3.247373 & -2.098106 & 0.022198 & 0 & $\mathrm{~m}$ & \\
\hline 31 & $\mathrm{~h}$ & 0 & 2.307390 & -2.229108 & -0.194626 & 0 & $\mathrm{~m}$ & \\
\hline
\end{tabular}




\section{dT}

\section{s1}

neutral molecule

Charge $=0$ Multiplicity $=1$ for low level calculation on real system.

Charge $=0$ Multiplicity $=1$ for high level calculation on model system .

Charge $=0$ Multiplicity $=1$ for low level calculation on model system .

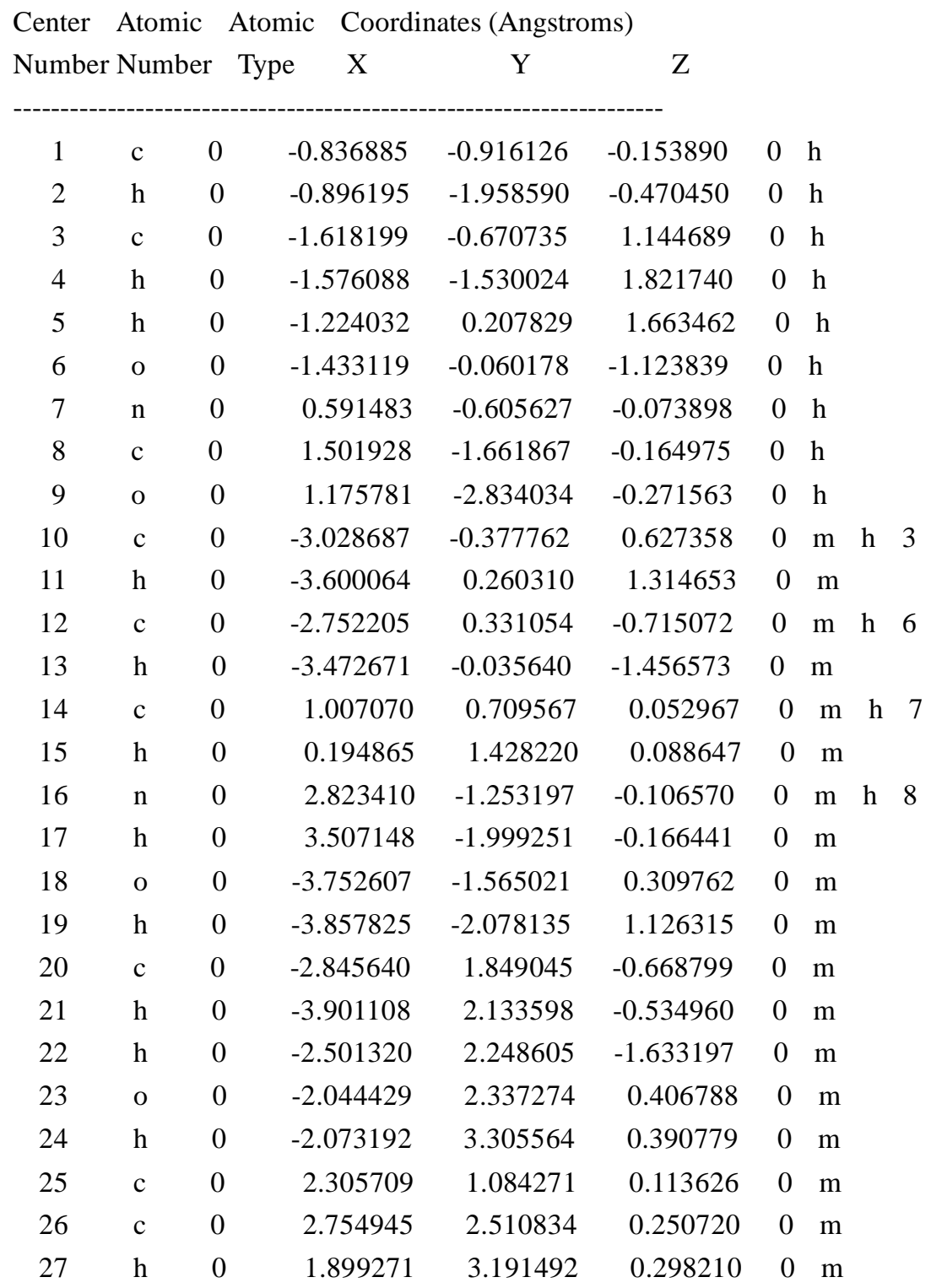




$\begin{array}{llllllll}28 & \mathrm{~h} & 0 & 3.388809 & 2.805002 & -0.593780 & 0 & \mathrm{~m} \\ 29 & \mathrm{~h} & 0 & 3.359558 & 2.646124 & 1.155053 & 0 & \mathrm{~m} \\ 30 & \mathrm{c} & 0 & 3.335445 & 0.049441 & 0.031381 & 0 & \mathrm{~m} \\ 31 & \mathrm{o} & 0 & 4.542837 & 0.239499 & 0.072825 & 0 & \mathrm{~m}\end{array}$

radical

Charge $=0$ Multiplicity $=2$ for low level calculation on real system.

Charge $=0$ Multiplicity $=2$ for high level calculation on model system.

Charge $=0$ Multiplicity $=2$ for low level calculation on model system .

Center Atomic Atomic Coordinates (Angstroms)

Number Number Type $\quad \mathrm{X} \quad \mathrm{Y} \quad \mathrm{Z}$

\begin{tabular}{|c|c|c|c|c|c|c|c|c|}
\hline 1 & $\mathrm{c}$ & 0 & -0.809363 & -0.497512 & -0.382448 & 0 & & \\
\hline 2 & $\mathrm{c}$ & 0 & -1.747251 & -1.141264 & 0.599533 & 0 & $\mathrm{~h}$ & \\
\hline 3 & $\mathrm{~h}$ & 0 & -1.667339 & -2.230625 & 0.608746 & 0 & $\mathrm{~h}$ & \\
\hline 4 & $\mathrm{~h}$ & 0 & -1.557044 & -0.776163 & 1.619631 & 0 & $\mathrm{~h}$ & \\
\hline 5 & o & 0 & -1.374925 & 0.618415 & -0.943085 & 0 & $\mathrm{~h}$ & \\
\hline 6 & $\mathrm{n}$ & 0 & 0.583132 & -0.363936 & -0.164163 & 0 & $\mathrm{~h}$ & \\
\hline 7 & $\mathrm{c}$ & 0 & 1.296736 & -1.531665 & 0.169066 & 0 & $\mathrm{~h}$ & \\
\hline 8 & o & 0 & 0.764384 & -2.617838 & 0.331785 & 0 & $\mathrm{~h}$ & \\
\hline 9 & $\mathrm{c}$ & 0 & -3.112741 & -0.637808 & 0.105170 & 0 & $\mathrm{~m}$ & h 2 \\
\hline 10 & $\mathrm{~h}$ & 0 & -3.817728 & -0.497524 & 0.935675 & 0 & $\mathrm{~m}$ & \\
\hline 11 & $\mathrm{c}$ & 0 & -2.772600 & 0.702458 & -0.572535 & 0 & $\mathrm{~m}$ & $\mathrm{~h}$ \\
\hline 12 & $\mathrm{~h}$ & 0 & -3.350404 & 0.802972 & -1.496111 & 0 & $\mathrm{~m}$ & \\
\hline 13 & $\mathrm{c}$ & 0 & 1.242886 & 0.842991 & -0.362605 & 0 & $\mathrm{~m}$ & $\mathrm{~h} \quad \mathrm{C}$ \\
\hline 14 & $\mathrm{~h}$ & 0 & 0.583983 & 1.661208 & -0.618957 & 0 & $\mathrm{~m}$ & \\
\hline 15 & $\mathrm{n}$ & 0 & 2.656120 & -1.329931 & 0.302505 & 0 & $\mathrm{~m}$ & $\mathrm{~h}$ \\
\hline 16 & $\mathrm{~h}$ & 0 & 3.187801 & -2.158910 & 0.543456 & 0 & $\mathrm{~m}$ & \\
\hline 17 & o & 0 & -3.678557 & -1.462682 & -0.908158 & 0 & $\mathrm{~m}$ & \\
\hline 18 & $\mathrm{~h}$ & 0 & -3.698761 & -2.373383 & -0.575379 & 0 & $\mathrm{~m}$ & \\
\hline 19 & $\mathrm{c}$ & 0 & -2.990394 & 1.934000 & 0.291170 & 0 & $\mathrm{~m}$ & \\
\hline 20 & $\mathrm{~h}$ & 0 & -4.075493 & 2.066148 & 0.433389 & 0 & $\mathrm{~m}$ & \\
\hline 21 & $\mathrm{~h}$ & 0 & -2.610326 & 2.814522 & -0.248196 & 0 & $\mathrm{~m}$ & \\
\hline 22 & o & 0 & -2.317114 & 1.749748 & 1.529075 & 0 & $\mathrm{~m}$ & \\
\hline 23 & $\mathrm{~h}$ & 0 & -2.420165 & 2.555812 & 2.056007 & 0 & $\mathrm{~m}$ & \\
\hline 24 & $\mathrm{c}$ & 0 & 2.584815 & 1.000868 & -0.253839 & 0 & $\mathrm{~m}$ & \\
\hline 25 & $\mathrm{c}$ & 0 & 3.280467 & 2.313519 & -0.475356 & 0 & $\mathrm{~m}$ & \\
\hline 26 & $\mathrm{~h}$ & 0 & 2.567623 & 3.100158 & -0.741352 & 0 & $\mathrm{~m}$ & \\
\hline 27 & $\mathrm{~h}$ & 0 & 4.023563 & 2.231168 & -1.276960 & 0 & $\mathrm{~m}$ & \\
\hline 28 & $\mathrm{~h}$ & 0 & 3.825610 & 2.624866 & 0.423287 & 0 & $\mathrm{~m}$ & \\
\hline 29 & $\mathrm{c}$ & 0 & 3.401189 & -0.151576 & 0.106259 & 0 & $\mathrm{~m}$ & \\
\hline
\end{tabular}




\section{s2}

neutral molecule

Charge $=0$ Multiplicity $=1$ for low level calculation on real system.

Charge $=0$ Multiplicity $=1$ for high level calculation on model system .

Charge $=0$ Multiplicity $=1$ for low level calculation on model system.

Center Atomic Atomic Coordinates (Angstroms)

Number Number Type $\quad X \quad$ Y $\quad$ Z

\begin{tabular}{|c|c|c|c|c|c|c|c|c|}
\hline 1 & $\mathrm{c}$ & 0 & -1.618197 & -0.670731 & 1.144689 & 0 & $\mathrm{~h}$ & \\
\hline 2 & $\mathrm{~h}$ & 0 & -1.224032 & 0.207830 & 1.663468 & 0 & h & \\
\hline 3 & $\mathrm{~h}$ & 0 & -1.576101 & -1.530021 & 1.821740 & 0 & h & \\
\hline 4 & $\mathrm{c}$ & 0 & -3.028686 & -0.377759 & 0.627358 & 0 & $\mathrm{~h}$ & \\
\hline 5 & $\mathrm{~h}$ & 0 & -3.600062 & 0.260315 & 1.314652 & 0 & $\mathrm{~h}$ & \\
\hline 6 & o & 0 & -3.752605 & -1.565017 & 0.309762 & 0 & $\mathrm{~h}$ & \\
\hline 7 & $\mathrm{~h}$ & 0 & -3.857824 & -2.078130 & 1.126316 & 0 & h & \\
\hline 8 & $\mathrm{c}$ & 0 & -2.752205 & 0.331049 & -0.715077 & 0 & h & \\
\hline 9 & $\mathrm{~h}$ & 0 & -3.472666 & -0.035647 & -1.456583 & 0 & h & \\
\hline 10 & o & 0 & -1.433117 & -0.060176 & -1.123841 & 0 & h & \\
\hline 11 & $\mathrm{c}$ & 0 & -0.836883 & -0.916125 & -0.153890 & 0 & h & \\
\hline 12 & $\mathrm{~h}$ & 0 & -0.896194 & -1.958588 & -0.470449 & 0 & $\mathrm{~h}$ & \\
\hline 13 & $\mathrm{c}$ & 0 & -2.845643 & 1.849039 & -0.668801 & 0 & $\mathrm{~m}$ & h \\
\hline 14 & $\mathrm{~h}$ & 0 & -3.901113 & 2.133590 & -0.534969 & 0 & $\mathrm{~m}$ & \\
\hline 15 & $\mathrm{~h}$ & 0 & -2.501316 & 2.248602 & -1.633194 & 0 & $\mathrm{~m}$ & \\
\hline 16 & $\mathrm{n}$ & 0 & 0.591484 & -0.605626 & -0.073897 & 0 & $\mathrm{~m}$ & h \\
\hline 17 & o & 0 & -2.044441 & 2.337267 & 0.406794 & 0 & $\mathrm{~m}$ & \\
\hline 18 & $\mathrm{~h}$ & 0 & -2.073205 & 3.305557 & 0.390787 & 0 & $\mathrm{~m}$ & \\
\hline 19 & $\mathrm{c}$ & 0 & 1.007073 & 0.709568 & 0.052966 & 0 & $\mathrm{~m}$ & \\
\hline 20 & $\mathrm{~h}$ & 0 & 0.194868 & 1.428221 & 0.088645 & 0 & $\mathrm{~m}$ & \\
\hline 21 & $\mathrm{c}$ & 0 & 2.305711 & 1.084271 & 0.113625 & 0 & $\mathrm{~m}$ & \\
\hline 22 & $\mathrm{c}$ & 0 & 2.754948 & 2.510834 & 0.250717 & 0 & $\mathrm{~m}$ & \\
\hline 23 & $\mathrm{~h}$ & 0 & 1.899274 & 3.191493 & 0.298206 & 0 & $\mathrm{~m}$ & \\
\hline 24 & $\mathrm{~h}$ & 0 & 3.388813 & 2.805001 & -0.593782 & 0 & $\mathrm{~m}$ & \\
\hline 25 & $\mathrm{~h}$ & 0 & 3.359562 & 2.646125 & 1.155051 & 0 & $\mathrm{~m}$ & \\
\hline 26 & $\mathrm{c}$ & 0 & 3.335447 & 0.049441 & 0.031381 & 0 & $\mathrm{~m}$ & \\
\hline 27 & o & 0 & 4.542840 & 0.239499 & 0.072825 & 0 & $\mathrm{~m}$ & \\
\hline 28 & $\mathrm{n}$ & 0 & 2.823404 & -1.253194 & -0.106569 & 0 & $\mathrm{~m}$ & \\
\hline 29 & $\mathrm{~h}$ & 0 & 3.507138 & -1.999253 & -0.166437 & 0 & $\mathrm{~m}$ & \\
\hline
\end{tabular}




$\begin{array}{llllllll}30 & \mathrm{c} & 0 & 1.501930 & -1.661866 & -0.164974 & 0 & \mathrm{~m} \\ 31 & \mathrm{o} & 0 & 1.175781 & -2.834034 & -0.271560 & 0 & \mathrm{~m}\end{array}$

radical

Charge $=0$ Multiplicity $=2$ for low level calculation on real system.

Charge $=0$ Multiplicity $=2$ for high level calculation on model system .

Charge $=0$ Multiplicity $=2$ for low level calculation on model system .

\begin{tabular}{|c|c|c|c|c|c|c|c|c|}
\hline Center & Atomic & & Coordin & ates (Angstro & & & & \\
\hline Number & Numbe & & Type & $\mathrm{Y}$ & $\mathrm{Z}$ & & & \\
\hline 1 & $\mathrm{c}$ & 0 & -1.596854 & -0.887539 & 1.088602 & 0 & $\mathrm{~h}$ & \\
\hline 2 & $\mathrm{~h}$ & 0 & -1.307387 & -1.294240 & 2.051116 & 0 & $\mathrm{~h}$ & \\
\hline 3 & $\mathrm{c}$ & 0 & -2.902997 & -0.245430 & 0.779280 & 0 & $\mathrm{~h}$ & \\
\hline 4 & $\mathrm{~h}$ & 0 & -3.105405 & 0.622108 & 1.422164 & 0 & $\mathrm{~h}$ & \\
\hline 5 & o & 0 & -4.014941 & -1.161055 & 0.829086 & 0 & $\mathrm{~h}$ & \\
\hline 6 & $\mathrm{~h}$ & 0 & -4.134986 & -1.428305 & 1.754369 & 0 & $\mathrm{~h}$ & \\
\hline 7 & $\mathrm{c}$ & 0 & -2.728531 & 0.174728 & -0.700097 & 0 & $\mathrm{~h}$ & \\
\hline 8 & $\mathrm{~h}$ & 0 & -3.520234 & -0.309378 & -1.285417 & 0 & $\mathrm{~h}$ & \\
\hline 9 & o & 0 & -1.457316 & -0.319895 & -1.153618 & 0 & $\mathrm{~h}$ & \\
\hline 10 & $\mathrm{c}$ & 0 & -0.807164 & -1.101003 & -0.159021 & 0 & $\mathrm{~h}$ & \\
\hline 11 & $\mathrm{~h}$ & 0 & -0.761201 & -2.153343 & -0.465372 & 0 & $\mathrm{~h}$ & \\
\hline 12 & $\mathrm{c}$ & 0 & -2.804693 & 1.674724 & -0.938960 & 0 & $\mathrm{~m} \mathrm{~h}$ & 7 \\
\hline 13 & $\mathrm{~h}$ & 0 & -3.851205 & 1.995132 & -0.817773 & 0 & $\mathrm{~m}$ & \\
\hline 14 & $\mathrm{~h}$ & 0 & -2.497841 & 1.879087 & -1.973903 & 0 & $\mathrm{~m}$ & \\
\hline 15 & $\mathrm{n}$ & 0 & 0.603472 & -0.691001 & -0.054228 & 0 & $\mathrm{~m}$ & 10 \\
\hline 16 & o & 0 & -1.957866 & 2.343841 & -0.005324 & 0 & $\mathrm{~m}$ & \\
\hline 17 & $\mathrm{~h}$ & 0 & -1.915423 & 3.279913 & -0.250819 & 0 & $\mathrm{~m}$ & \\
\hline 18 & $\mathrm{c}$ & 0 & 0.917366 & 0.640351 & 0.165580 & 0 & $\mathrm{~m}$ & \\
\hline 19 & $\mathrm{~h}$ & 0 & 0.053324 & 1.292639 & 0.247513 & 0 & $\mathrm{~m}$ & \\
\hline 20 & $\mathrm{c}$ & 0 & 2.184857 & 1.106723 & 0.244077 & 0 & $\mathrm{~m}$ & \\
\hline 21 & $\mathrm{c}$ & 0 & 2.526873 & 2.549905 & 0.481046 & 0 & $\mathrm{~m}$ & \\
\hline 22 & $\mathrm{~h}$ & 0 & 1.622438 & 3.156568 & 0.588528 & 0 & $\mathrm{~m}$ & \\
\hline 23 & $\mathrm{~h}$ & 0 & 3.122691 & 2.952837 & -0.346217 & 0 & $\mathrm{~m}$ & \\
\hline 24 & $\mathrm{~h}$ & 0 & 3.134072 & 2.665180 & 1.386347 & 0 & $\mathrm{~m}$ & \\
\hline 25 & $\mathrm{c}$ & 0 & 3.288935 & 0.162345 & 0.078402 & 0 & $\mathrm{~m}$ & \\
\hline 26 & o & 0 & 4.478892 & 0.439519 & 0.127191 & 0 & $\mathrm{~m}$ & \\
\hline 27 & $\mathrm{n}$ & 0 & 2.875991 & -1.161819 & -0.158510 & 0 & $\mathrm{~m}$ & \\
\hline 28 & $\mathrm{~h}$ & 0 & 3.614032 & -1.844821 & -0.287293 & 0 & $\mathrm{~m}$ & \\
\hline 29 & $\mathrm{c}$ & 0 & 1.588914 & -1.662599 & -0.246835 & 0 & $\mathrm{~m}$ & \\
\hline 30 & o & 0 & 1.350063 & -2.842018 & -0.458150 & 0 & $\mathrm{~m}$ & \\
\hline
\end{tabular}




\section{s3}

neutral molecule

Charge $=0$ Multiplicity $=1$ for low level calculation on real system.

Charge $=0$ Multiplicity $=1$ for high level calculation on model system.

Charge $=0$ Multiplicity $=1$ for low level calculation on model system .

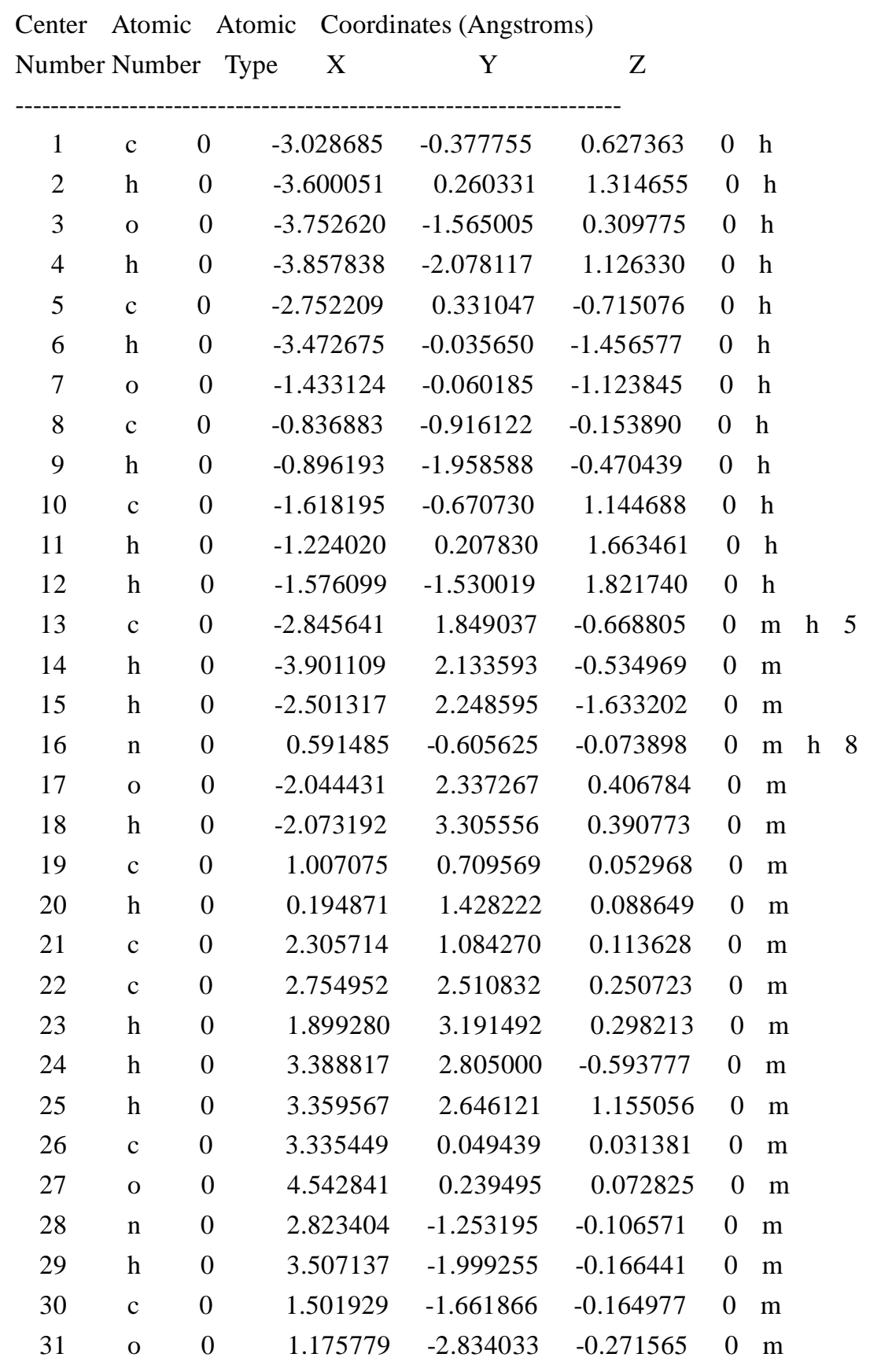


radical

Charge $=0$ Multiplicity $=2$ for low level calculation on real system.

Charge $=0$ Multiplicity $=2$ for high level calculation on model system.

Charge $=0$ Multiplicity $=2$ for low level calculation on model system.

Center Atomic Atomic Coordinates (Angstroms)

Number Number Type X $\quad$ Y $\quad$ Z

\begin{tabular}{|c|c|c|c|c|c|c|c|c|}
\hline 1 & $\mathrm{c}$ & 0 & -2.937819 & -0.426922 & 0.616483 & 0 & h & \\
\hline 2 & o & 0 & -4.093144 & -1.152339 & 0.771850 & 0 & $\mathrm{~h}$ & \\
\hline 3 & $\mathrm{~h}$ & 0 & -4.079573 & -1.580618 & 1.642240 & 0 & $\mathrm{~h}$ & \\
\hline 4 & $\mathrm{c}$ & 0 & -2.768117 & 0.217007 & -0.733379 & 0 & $\mathrm{~h}$ & \\
\hline 5 & $\mathrm{~h}$ & 0 & -3.492622 & -0.208373 & -1.445120 & 0 & $\mathrm{~h}$ & \\
\hline 6 & o & 0 & -1.428661 & -0.106239 & -1.155330 & 0 & $\mathrm{~h}$ & \\
\hline 7 & $\mathrm{c}$ & 0 & -0.803707 & -1.004845 & -0.244924 & 0 & $\mathrm{~h}$ & \\
\hline 8 & $\mathrm{~h}$ & 0 & -0.811290 & -2.023695 & -0.637209 & 0 & $\mathrm{~h}$ & \\
\hline 9 & $\mathrm{c}$ & 0 & -1.597260 & -0.911772 & 1.083544 & 0 & $\mathrm{~h}$ & \\
\hline 10 & $\mathrm{~h}$ & 0 & -1.113630 & -0.189492 & 1.754302 & 0 & $\mathrm{~h}$ & \\
\hline 11 & $\mathrm{~h}$ & 0 & -1.633773 & -1.880037 & 1.595469 & 0 & $\mathrm{~h}$ & \\
\hline 12 & $\mathrm{c}$ & 0 & -2.924323 & 1.735268 & -0.750015 & 0 & $\mathrm{~m}$ & h 4 \\
\hline 13 & $\mathrm{~h}$ & 0 & -3.980844 & 1.976951 & -0.557989 & 0 & $\mathrm{~m}$ & \\
\hline 14 & $\mathrm{~h}$ & 0 & -2.659162 & 2.106404 & -1.751024 & 0 & $\mathrm{~m}$ & \\
\hline 15 & $\mathrm{n}$ & 0 & 0.607740 & -0.635212 & -0.136571 & 0 & $\mathrm{~m}$ & $\mathrm{~h}$ \\
\hline 16 & o & 0 & -2.076755 & 2.293022 & 0.250434 & 0 & $\mathrm{~m}$ & \\
\hline 17 & $\mathrm{~h}$ & 0 & -2.122178 & 3.257999 & 0.178551 & 0 & $\mathrm{~m}$ & \\
\hline 18 & $\mathrm{c}$ & 0 & 0.962142 & 0.689821 & 0.060455 & 0 & $\mathrm{~m}$ & \\
\hline 19 & $\mathrm{~h}$ & 0 & 0.118490 & 1.372113 & 0.096548 & 0 & $\mathrm{~m}$ & \\
\hline 20 & $\mathrm{c}$ & 0 & 2.242625 & 1.112057 & 0.176996 & 0 & $\mathrm{~m}$ & \\
\hline 21 & $\mathrm{c}$ & 0 & 2.628606 & 2.548218 & 0.386926 & 0 & $\mathrm{~m}$ & \\
\hline 22 & $\mathrm{~h}$ & 0 & 1.744205 & 3.190306 & 0.442017 & 0 & $\mathrm{~m}$ & \\
\hline 23 & $\mathrm{~h}$ & 0 & 3.269613 & 2.905026 & -0.427575 & 0 & $\mathrm{~m}$ & \\
\hline 24 & $\mathrm{~h}$ & 0 & 3.205422 & 2.667503 & 1.311405 & 0 & $\mathrm{~m}$ & \\
\hline 25 & $\mathrm{c}$ & 0 & 3.316197 & 0.123999 & 0.082753 & 0 & $\mathrm{~m}$ & \\
\hline 26 & o & 0 & 4.513202 & 0.359564 & 0.167848 & 0 & $\mathrm{~m}$ & \\
\hline 27 & $\mathrm{n}$ & 0 & 2.862973 & -1.192048 & -0.123921 & 0 & $\mathrm{~m}$ & \\
\hline 28 & $\mathrm{~h}$ & 0 & 3.578864 & -1.906682 & -0.190923 & 0 & $\mathrm{~m}$ & \\
\hline 29 & $\mathrm{c}$ & 0 & 1.562193 & -1.650551 & -0.234655 & 0 & $\mathrm{~m}$ & \\
\hline 30 & o & 0 & 1.285141 & -2.830289 & -0.391346 & 0 & $\mathrm{~m}$ & \\
\hline
\end{tabular}




\section{s4}

neutral molecule

Charge $=0$ Multiplicity $=1$ for low level calculation on real system.

Charge $=0$ Multiplicity $=1$ for high level calculation on model system.

Charge $=0$ Multiplicity $=1$ for low level calculation on model system .

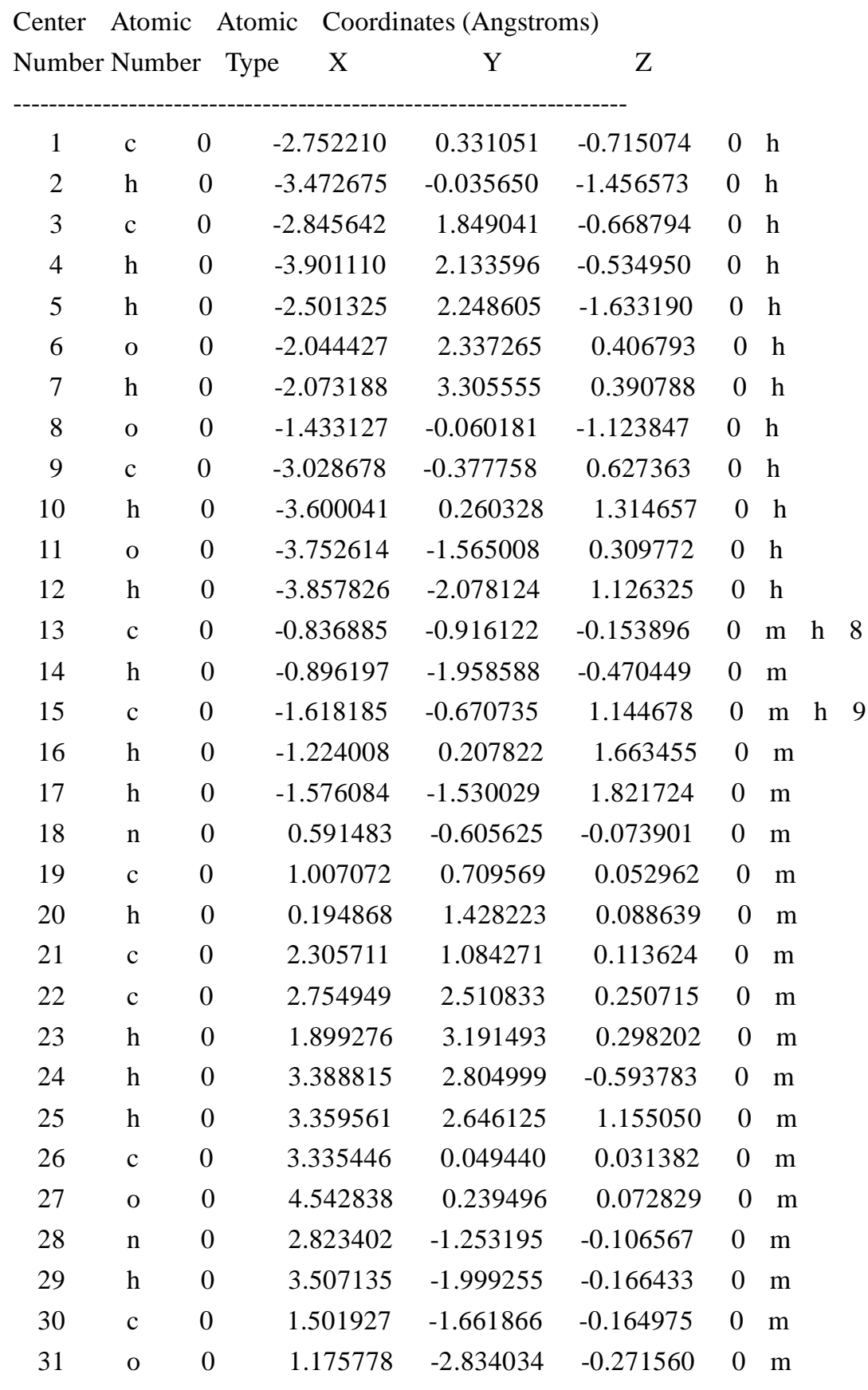


radical

Charge $=0$ Multiplicity $=2$ for low level calculation on real system.

Charge $=0$ Multiplicity $=2$ for high level calculation on model system.

Charge $=0$ Multiplicity $=2$ for low level calculation on model system.

Center Atomic Atomic Coordinates (Angstroms)

Number Number Type X $\quad$ Y $\quad$ Z

$\begin{array}{rrrrrrrrr}1 & \mathrm{c} & 0 & -2.676271 & 0.288133 & -0.502855 & 0 & \mathrm{~h} & \\ 2 & \mathrm{c} & 0 & -3.150157 & 1.659086 & -0.797681 & 0 & \mathrm{~h} & \\ 3 & \mathrm{~h} & 0 & -4.231323 & 1.720772 & -0.609703 & 0 & \mathrm{~h} & \\ 4 & \mathrm{~h} & 0 & -2.968598 & 1.893660 & -1.855922 & 0 & \mathrm{~h} & \\ 5 & \mathrm{o} & 0 & -2.431111 & 2.578971 & 0.055637 & 0 & \mathrm{~h} & \\ 6 & \mathrm{~h} & 0 & -2.683841 & 3.480669 & -0.202615 & 0 & \mathrm{~h} & \\ 7 & \mathrm{o} & 0 & -1.444926 & -0.027867 & -1.025363 & 0 & \mathrm{~h} & \\ 8 & \mathrm{c} & 0 & -2.953223 & -0.503523 & 0.739494 & 0 & \mathrm{~h} & \\ 9 & \mathrm{~h} & 0 & -3.448777 & 0.105378 & 1.508148 & 0 & \mathrm{~h} & \\ 10 & \mathrm{o} & 0 & -3.769370 & -1.629960 & 0.387957 & 0 & \mathrm{~h} & \\ 11 & \mathrm{~h} & 0 & -3.953177 & -2.121252 & 1.205839 & 0 & \mathrm{~h} & \\ 12 & \mathrm{c} & 0 & -0.779251 & -0.983473 & -0.180594 & 0 & \mathrm{~m} & \mathrm{~h} \\ 13 & \mathrm{~h} & 0 & -0.819525 & -1.972698 & -0.635913 & 0 & \mathrm{~m} & \\ 14 & \mathrm{c} & 0 & -1.529528 & -0.917547 & 1.159128 & 0 & \mathrm{~m} & \mathrm{~h} \\ 15 & \mathrm{~h} & 0 & -1.086893 & -0.140875 & 1.792807 & 0 & \mathrm{~m} & \\ 16 & \mathrm{~h} & 0 & -1.490344 & -1.869966 & 1.695426 & 0 & \mathrm{~m} & \\ 17 & \mathrm{n} & 0 & 0.629094 & -0.611178 & -0.113598 & 0 & \mathrm{~m} & \\ 18 & \mathrm{c} & 0 & 0.987232 & 0.709773 & 0.114684 & 0 & \mathrm{~m} & \\ 19 & \mathrm{~h} & 0 & 0.148628 & 1.394828 & 0.188107 & 0 & \mathrm{~m} & \\ 20 & \mathrm{c} & 0 & 2.268546 & 1.132067 & 0.203962 & 0 & \mathrm{~m} & \\ 21 & \mathrm{c} & 0 & 2.658696 & 2.562335 & 0.442253 & 0 & \mathrm{~m} & \\ 22 & \mathrm{~h} & 0 & 1.776126 & 3.201446 & 0.540820 & 0 & \mathrm{~m} & \\ 23 & \mathrm{~h} & 0 & 3.275415 & 2.941278 & -0.380762 & 0 & \mathrm{~m} & \\ 24 & \mathrm{~h} & 0 & 3.262508 & 2.655574 & 1.352228 & 0 & \mathrm{~m} & \\ 25 & \mathrm{c} & 0 & 3.340680 & 0.147961 & 0.045534 & 0 & \mathrm{~m} & \\ 26 & \mathrm{o} & 0 & 4.538046 & 0.384500 & 0.105702 & 0 & \mathrm{~m} & \\ 27 & \mathrm{n} & 0 & 2.884483 & -1.160824 & -0.198406 & 0 & \mathrm{~m} & \\ 28 & \mathrm{~h} & 0 & 3.600044 & -1.869441 & -0.315917 & 0 & \mathrm{~m} & \\ 29 & \mathrm{c} & 0 & 1.582468 & -1.618881 & -0.288819 & 0 & \mathrm{~m} & \\ 30 & \mathrm{o} & 0 & 1.298557 & -2.789514 & -0.487577 & 0 & \mathrm{~m} & \\ -------------------------------------------------------- & & & & \end{array}$




\section{s5}

neutral molecule

Charge $=0$ Multiplicity $=1$ for low level calculation on real system.

Charge $=0$ Multiplicity $=1$ for high level calculation on model system .

Charge $=0$ Multiplicity $=1$ for low level calculation on model system .

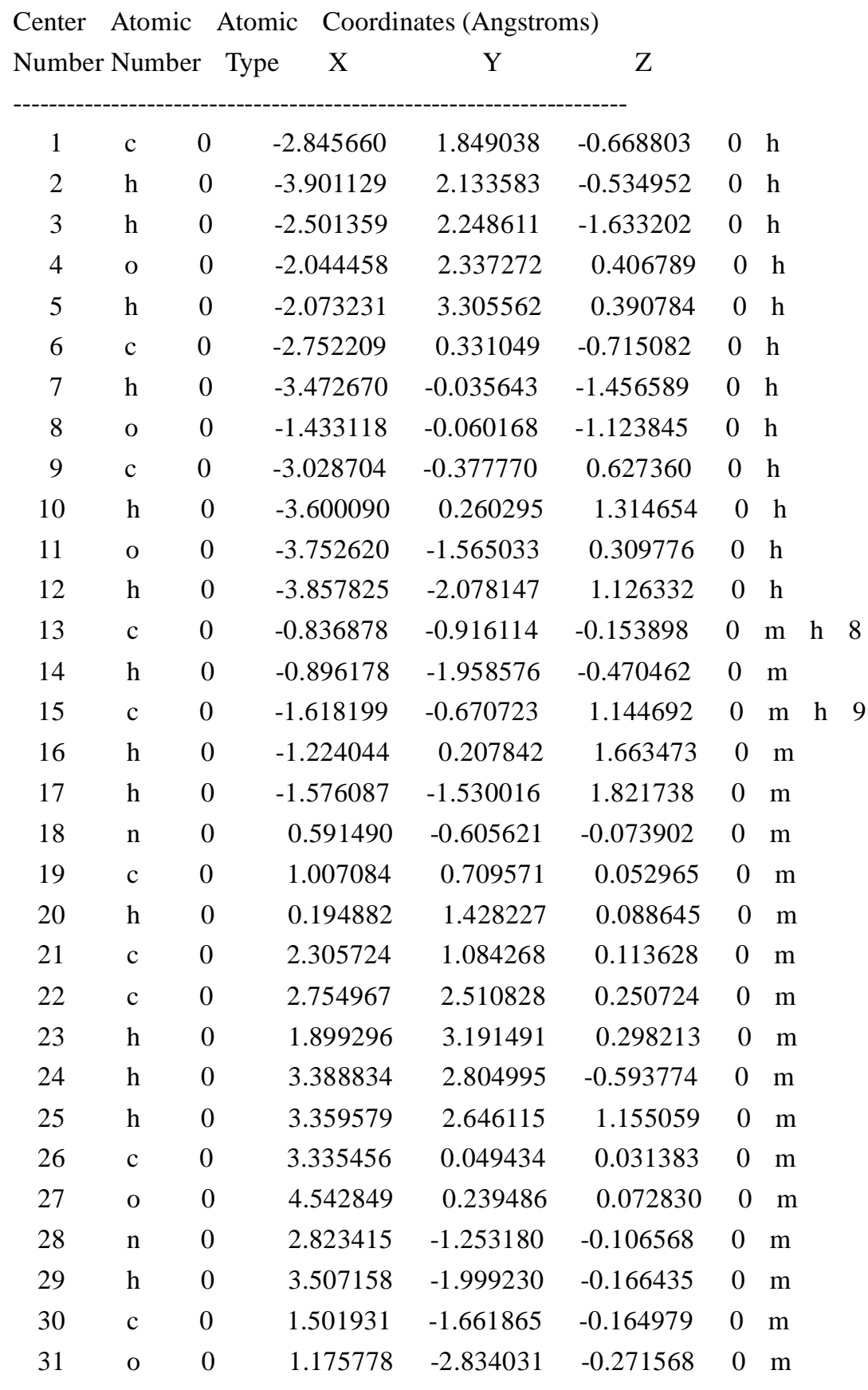


radical

Charge $=0$ Multiplicity $=2$ for low level calculation on real system.

Charge $=0$ Multiplicity $=2$ for high level calculation on model system.

Charge $=0$ Multiplicity $=2$ for low level calculation on model system.

Center Atomic Atomic Coordinates (Angstroms)

Number Number Type X $\quad$ Y $\quad$ Z

\begin{tabular}{|c|c|c|c|c|c|c|c|}
\hline 1 & $\mathrm{c}$ & 0 & -2.871149 & 1.877071 & -0.593177 & 0 & h \\
\hline 2 & $\mathrm{~h}$ & 0 & -3.048400 & 2.489821 & -1.474554 & 0 & $\mathrm{~h}$ \\
\hline 3 & o & 0 & -2.130448 & 2.437828 & 0.420626 & 0 & $\mathrm{~h}$ \\
\hline 4 & $\mathrm{~h}$ & 0 & -2.192499 & 3.403531 & 0.366859 & 0 & $\mathrm{~h}$ \\
\hline 5 & $\mathrm{c}$ & 0 & -2.788776 & 0.399495 & -0.700297 & 0 & $\mathrm{~h}$ \\
\hline 6 & $\mathrm{~h}$ & 0 & -3.506845 & 0.061793 & -1.453891 & 0 & $\mathrm{~h}$ \\
\hline 7 & o & 0 & -1.458703 & -0.021442 & -1.137351 & 0 & $\mathrm{~h}$ \\
\hline 8 & $\mathrm{c}$ & 0 & -3.053980 & -0.371100 & 0.614095 & 0 & $\mathrm{~h}$ \\
\hline 9 & $\mathrm{~h}$ & 0 & -3.644026 & 0.227666 & 1.319706 & 0 & $\mathrm{~h}$ \\
\hline 10 & o & 0 & -3.749667 & -1.558962 & 0.243283 & 0 & $\mathrm{~h}$ \\
\hline 11 & $\mathrm{~h}$ & 0 & -3.853356 & -2.104871 & 1.038515 & 0 & $\mathrm{~h}$ \\
\hline 12 & $\mathrm{c}$ & 0 & -0.867760 & -0.891088 & -0.181151 & 0 & $\mathrm{~m}$ \\
\hline 13 & $\mathrm{~h}$ & 0 & -0.933318 & -1.930054 & -0.508335 & 0 & $\mathrm{~m}$ \\
\hline 14 & $\mathrm{c}$ & 0 & -1.641600 & -0.661322 & 1.124418 & 0 & $\mathrm{~m}$ \\
\hline 15 & $\mathrm{~h}$ & 0 & -1.246913 & 0.213914 & 1.650782 & 0 & $\mathrm{~m}$ \\
\hline 16 & $\mathrm{~h}$ & 0 & -1.591121 & -1.524942 & 1.795519 & 0 & $\mathrm{~m}$ \\
\hline 17 & $\mathrm{n}$ & 0 & 0.566165 & -0.592102 & -0.094905 & 0 & $\mathrm{~m}$ \\
\hline 18 & $\mathrm{c}$ & 0 & 0.996015 & 0.718717 & 0.012638 & 0 & $\mathrm{~m}$ \\
\hline 19 & $\mathrm{~h}$ & 0 & 0.193358 & 1.447563 & 0.020876 & 0 & $\mathrm{~m}$ \\
\hline 20 & $\mathrm{c}$ & 0 & 2.297369 & 1.081751 & 0.085810 & 0 & $\mathrm{~m}$ \\
\hline 21 & $\mathrm{c}$ & 0 & 2.760472 & 2.505690 & 0.201092 & 0 & $\mathrm{~m}$ \\
\hline 22 & $\mathrm{~h}$ & 0 & 1.912141 & 3.196919 & 0.221690 & 0 & $\mathrm{~m}$ \\
\hline 23 & $\mathrm{~h}$ & 0 & 3.410208 & 2.775633 & -0.639393 & 0 & $\mathrm{~m}$ \\
\hline 24 & $\mathrm{~h}$ & 0 & 3.352934 & 2.652592 & 1.111627 & 0 & $\mathrm{~m}$ \\
\hline 25 & $\mathrm{c}$ & 0 & 3.316314 & 0.033988 & 0.039249 & 0 & $\mathrm{~m}$ \\
\hline 26 & o & 0 & 4.524992 & 0.211988 & 0.094747 & 0 & $\mathrm{~m}$ \\
\hline 27 & $\mathrm{n}$ & 0 & 2.791160 & -1.264950 & -0.080065 & 0 & $\mathrm{~m}$ \\
\hline 28 & $\mathrm{~h}$ & 0 & 3.466710 & -2.020115 & -0.112894 & 0 & $\mathrm{~m}$ \\
\hline 29 & $\mathrm{c}$ & 0 & 1.465460 & -1.659920 & -0.146211 & 0 & $\mathrm{~m}$ \\
\hline 30 & o & 0 & 1.127034 & -2.830633 & -0.227618 & 0 & $\mathrm{~m}$ \\
\hline
\end{tabular}




\section{b3}

neutral molecule

Charge $=0$ Multiplicity $=1$ for low level calculation on real system.

Charge $=0$ Multiplicity $=1$ for high level calculation on model system.

Charge $=0$ Multiplicity $=1$ for low level calculation on model system .

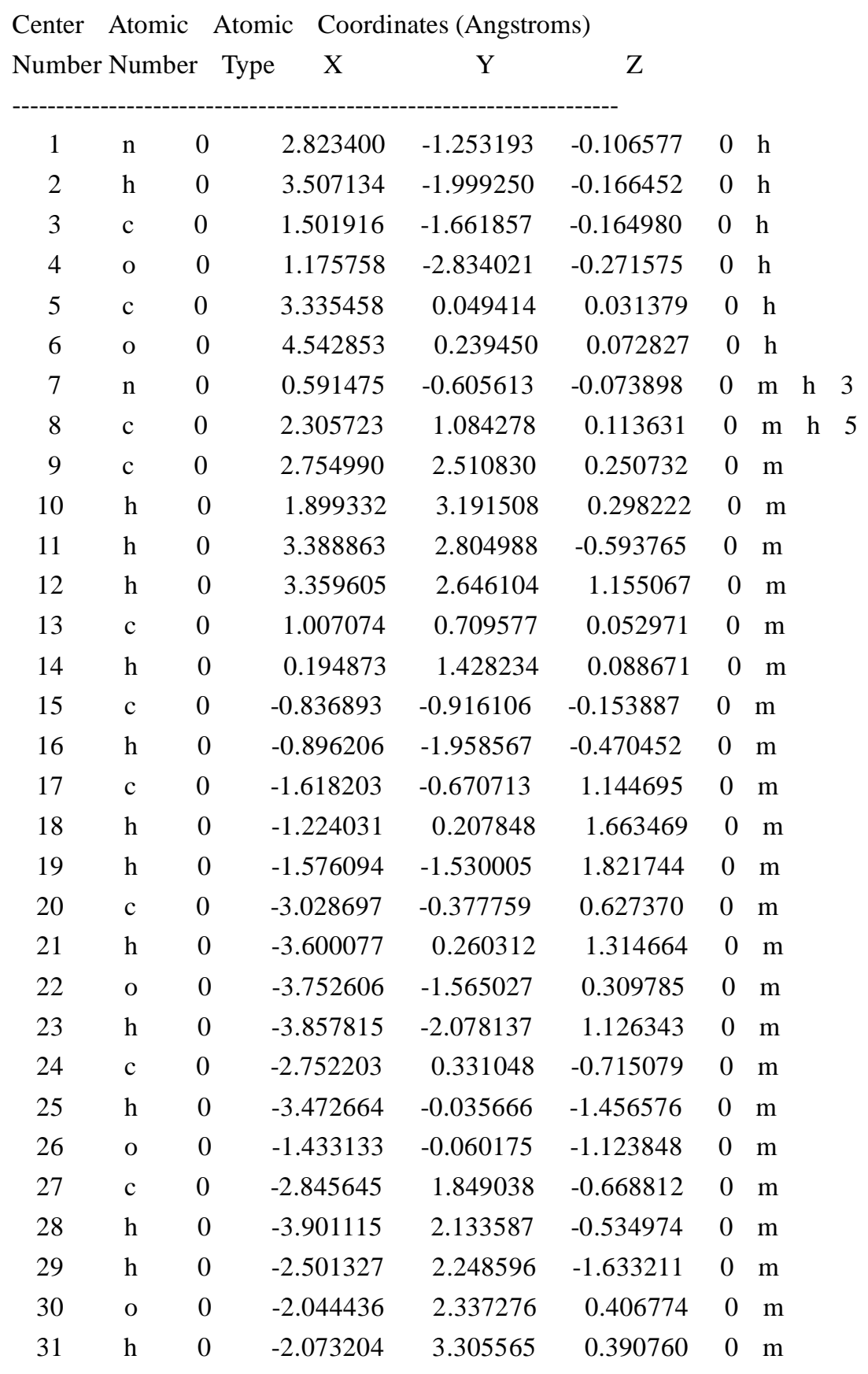


radical

Charge $=0$ Multiplicity $=2$ for low level calculation on real system.

Charge $=0$ Multiplicity $=2$ for high level calculation on model system.

Charge $=0$ Multiplicity $=2$ for low level calculation on model system.

Center Atomic Atomic Coordinates (Angstroms)

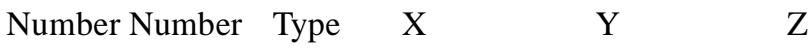

\begin{tabular}{|c|c|c|c|c|c|c|c|}
\hline 1 & $\mathrm{n}$ & 0 & 2.867589 & -1.226029 & -0.415571 & 0 & $\mathrm{~h}$ \\
\hline 2 & $\mathrm{c}$ & 0 & 1.549705 & -1.612954 & -0.344645 & 0 & $\mathrm{~h}$ \\
\hline 3 & o & 0 & 1.244553 & -2.802382 & -0.387845 & 0 & $\mathrm{~h}$ \\
\hline 4 & $\mathrm{c}$ & 0 & 3.342291 & -0.045714 & 0.180009 & 0 & $\mathrm{~h}$ \\
\hline 5 & o & 0 & 4.470463 & 0.111934 & 0.609215 & 0 & $\mathrm{~h}$ \\
\hline 6 & $\mathrm{n}$ & 0 & 0.599008 & -0.591293 & -0.215462 & 0 & $\mathrm{~m} \mathrm{~h}$ \\
\hline 7 & $\mathrm{c}$ & 0 & 2.343797 & 1.039492 & 0.038771 & 0 & $\begin{array}{lll}\mathrm{m} & \mathrm{h} & 4\end{array}$ \\
\hline 8 & $\mathrm{c}$ & 0 & 2.847067 & 2.451706 & 0.107039 & 0 & $\mathrm{~m}$ \\
\hline 9 & $\mathrm{~h}$ & 0 & 2.020290 & 3.167309 & 0.057394 & 0 & $\mathrm{~m}$ \\
\hline 10 & $\mathrm{~h}$ & 0 & 3.536044 & 2.664935 & -0.719540 & 0 & $\mathrm{~m}$ \\
\hline 11 & $\mathrm{~h}$ & 0 & 3.409977 & 2.611884 & 1.032788 & 0 & $\mathrm{~m}$ \\
\hline 12 & $\mathrm{c}$ & 0 & 1.031402 & 0.709897 & -0.099423 & 0 & $\mathrm{~m}$ \\
\hline 13 & $\mathrm{~h}$ & 0 & 0.241536 & 1.455100 & -0.106752 & 0 & $\mathrm{~m}$ \\
\hline 14 & $\mathrm{c}$ & 0 & -0.825784 & -0.921198 & -0.178418 & 0 & $\mathrm{~m}$ \\
\hline 15 & $\mathrm{~h}$ & 0 & -0.893663 & -1.975198 & -0.455480 & 0 & $\mathrm{~m}$ \\
\hline 16 & $\mathrm{c}$ & 0 & -1.517026 & -0.639433 & 1.163048 & 0 & $\mathrm{~m}$ \\
\hline 17 & $\mathrm{~h}$ & 0 & -1.092563 & 0.255551 & 1.627018 & 0 & $\mathrm{~m}$ \\
\hline 18 & $\mathrm{~h}$ & 0 & -1.424831 & -1.477676 & 1.860924 & 0 & $\mathrm{~m}$ \\
\hline 19 & $\mathrm{c}$ & 0 & -2.961605 & -0.366347 & 0.734606 & 0 & $\mathrm{~m}$ \\
\hline 20 & $\mathrm{~h}$ & 0 & -3.487180 & 0.290718 & 1.440271 & 0 & $\mathrm{~m}$ \\
\hline 21 & o & 0 & -3.698259 & -1.565021 & 0.503656 & 0 & $\mathrm{~m}$ \\
\hline 22 & $\mathrm{~h}$ & 0 & -3.757971 & -2.046962 & 1.343513 & 0 & $\mathrm{~m}$ \\
\hline 23 & $\mathrm{c}$ & 0 & -2.782468 & 0.301374 & -0.644272 & 0 & $\mathrm{~m}$ \\
\hline 24 & $\mathrm{~h}$ & 0 & -3.552861 & -0.086729 & -1.321597 & 0 & $\mathrm{~m}$ \\
\hline 25 & o & 0 & -1.494856 & -0.105212 & -1.133899 & 0 & $\mathrm{~m}$ \\
\hline 26 & $\mathrm{c}$ & 0 & -2.871152 & 1.820181 & -0.639115 & 0 & $\mathrm{~m}$ \\
\hline 27 & $\mathrm{~h}$ & 0 & -3.910660 & 2.111445 & -0.422348 & 0 & $\mathrm{~m}$ \\
\hline 28 & $\mathrm{~h}$ & 0 & -2.613002 & 2.188414 & -1.642152 & 0 & $\mathrm{~m}$ \\
\hline 29 & o & 0 & -1.977760 & 2.339861 & 0.345681 & 0 & $\mathrm{~m}$ \\
\hline 30 & $\mathrm{~h}$ & 0 & -2.031793 & 3.307009 & 0.323126 & 0 & $\mathrm{~m}$ \\
\hline
\end{tabular}




\section{b5}

neutral molecule

Charge $=0$ Multiplicity $=1$ for low level calculation on real system.

Charge $=0$ Multiplicity $=1$ for high level calculation on model system.

Charge $=0$ Multiplicity $=1$ for low level calculation on model system .

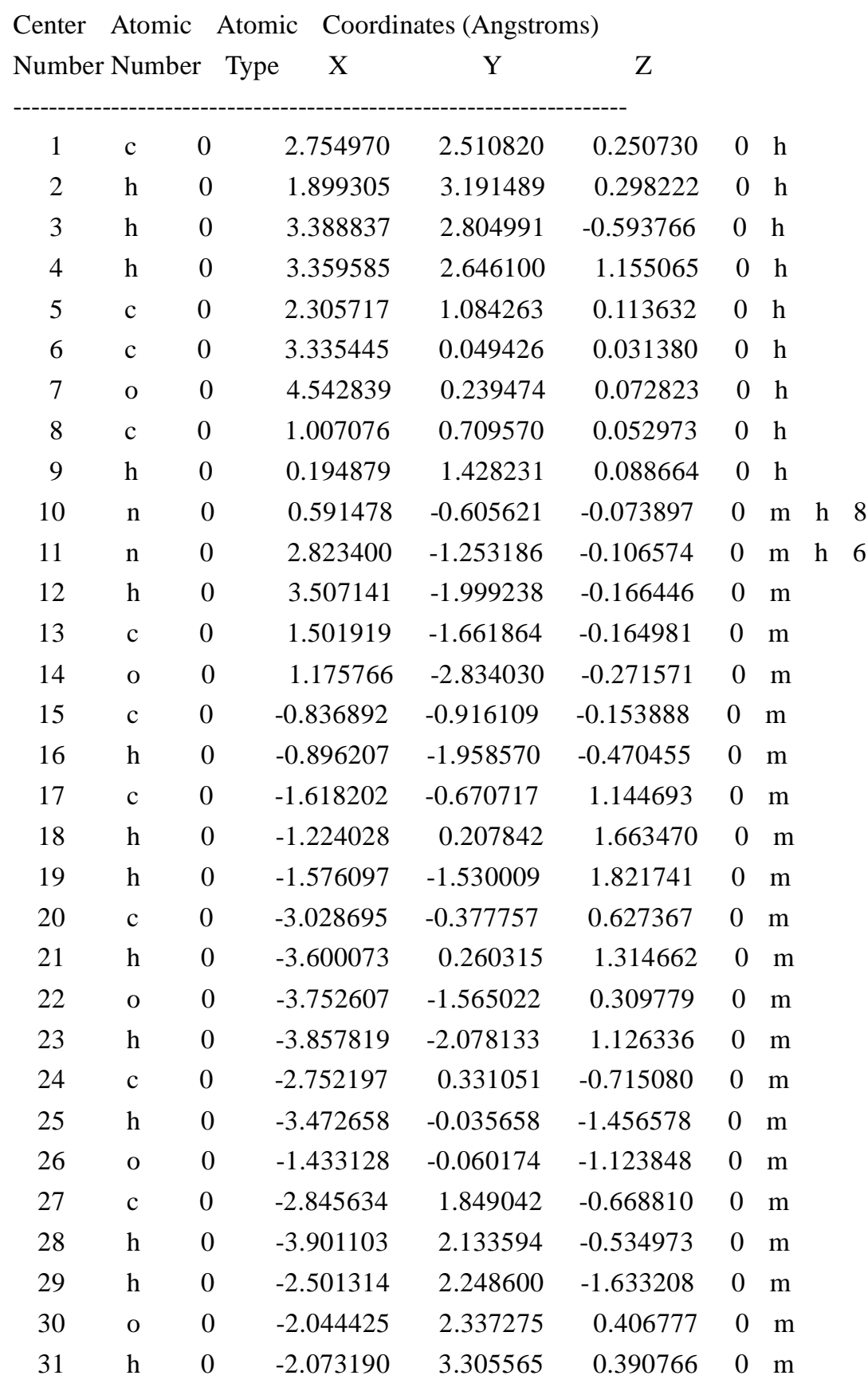


radical

Charge $=0$ Multiplicity $=2$ for low level calculation on real system.

Charge $=0$ Multiplicity $=2$ for high level calculation on model system.

Charge $=0$ Multiplicity $=2$ for low level calculation on model system.

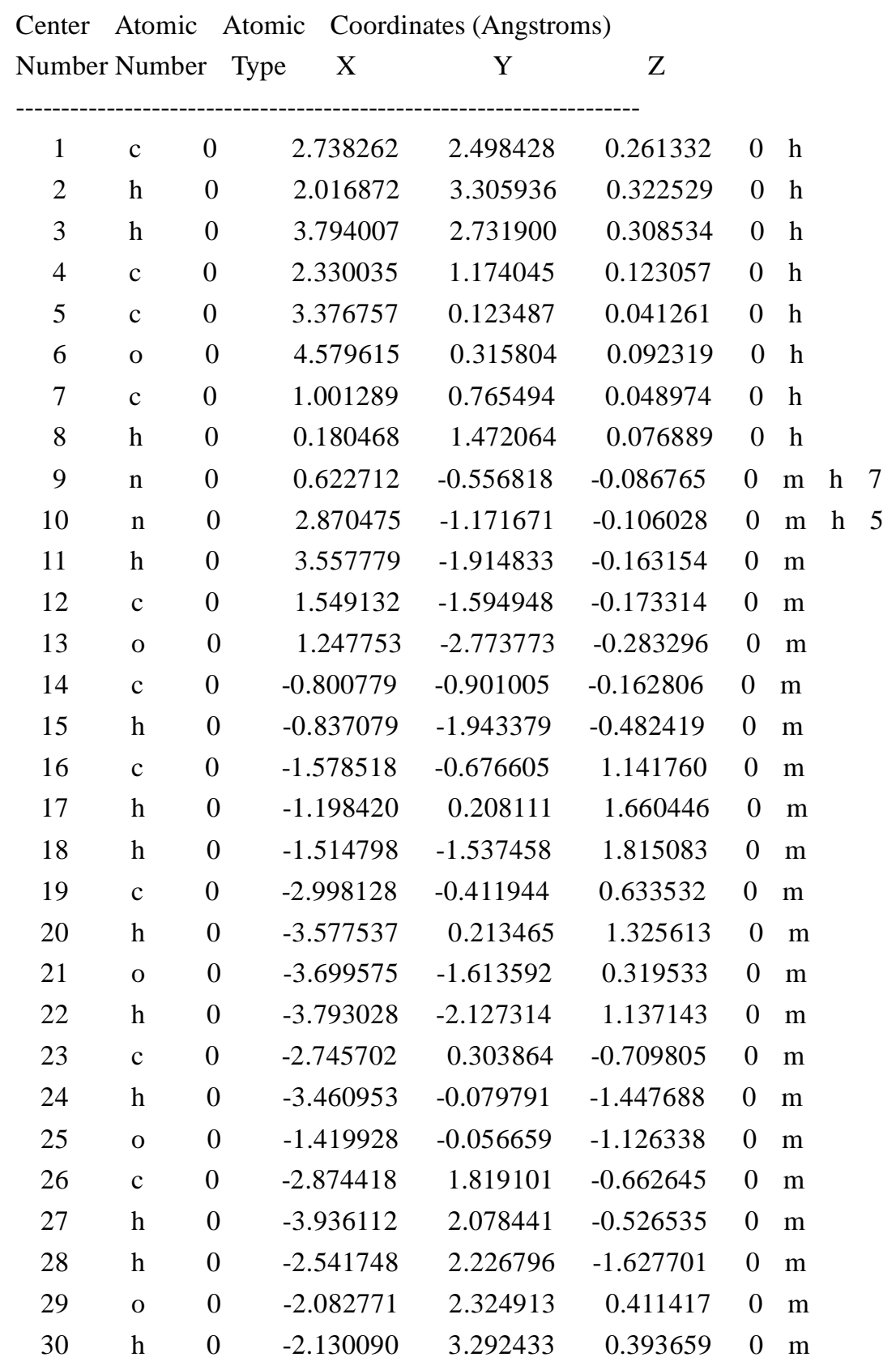




\section{b6}

neutral molecule

Charge $=0$ Multiplicity $=1$ for low level calculation on real system.

Charge $=0$ Multiplicity $=1$ for high level calculation on model system.

Charge $=0$ Multiplicity $=1$ for low level calculation on model system .

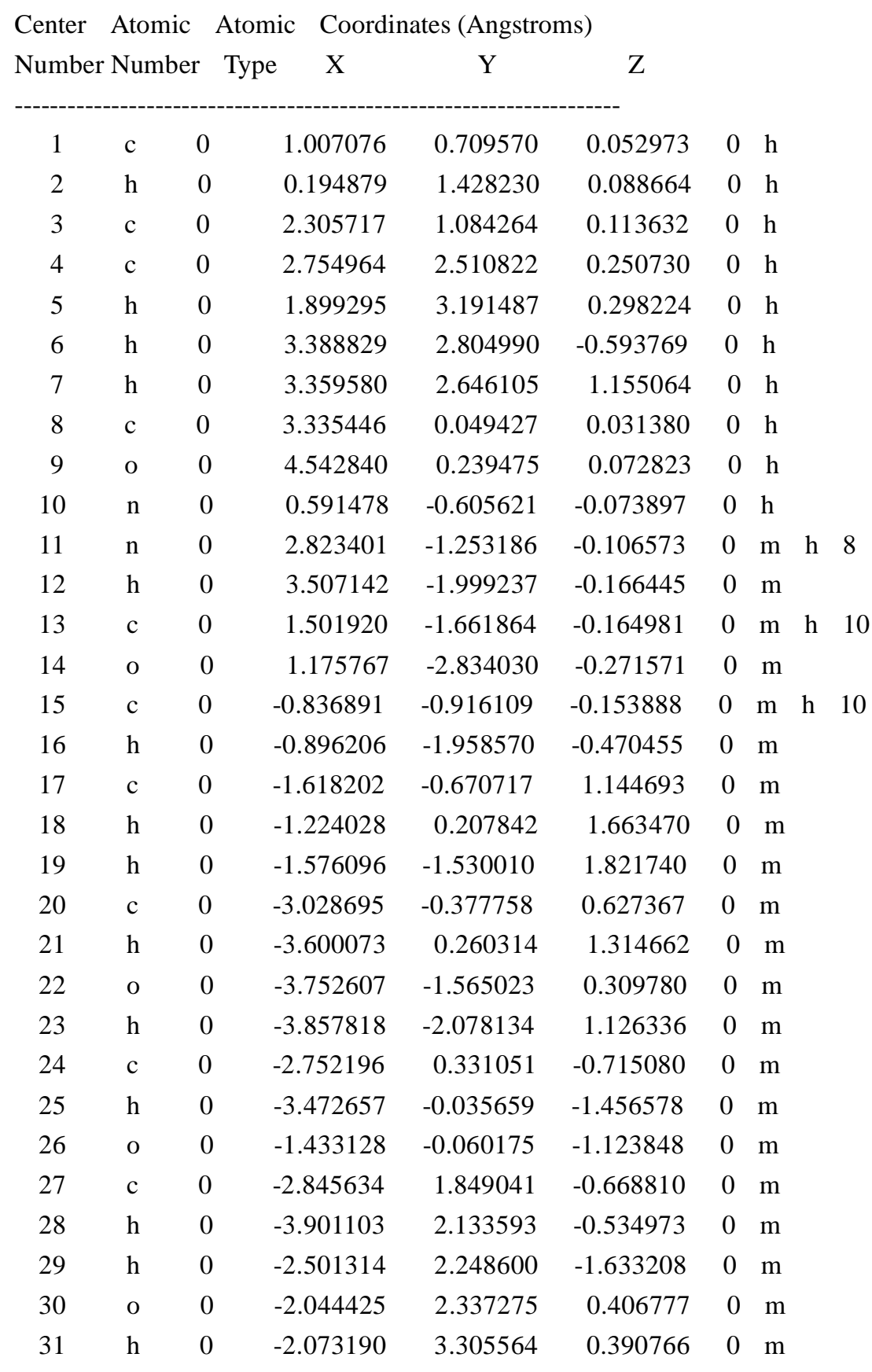


radical

Charge $=0$ Multiplicity $=2$ for low level calculation on real system.

Charge $=0$ Multiplicity $=2$ for high level calculation on model system.

Charge $=0$ Multiplicity $=2$ for low level calculation on model system.

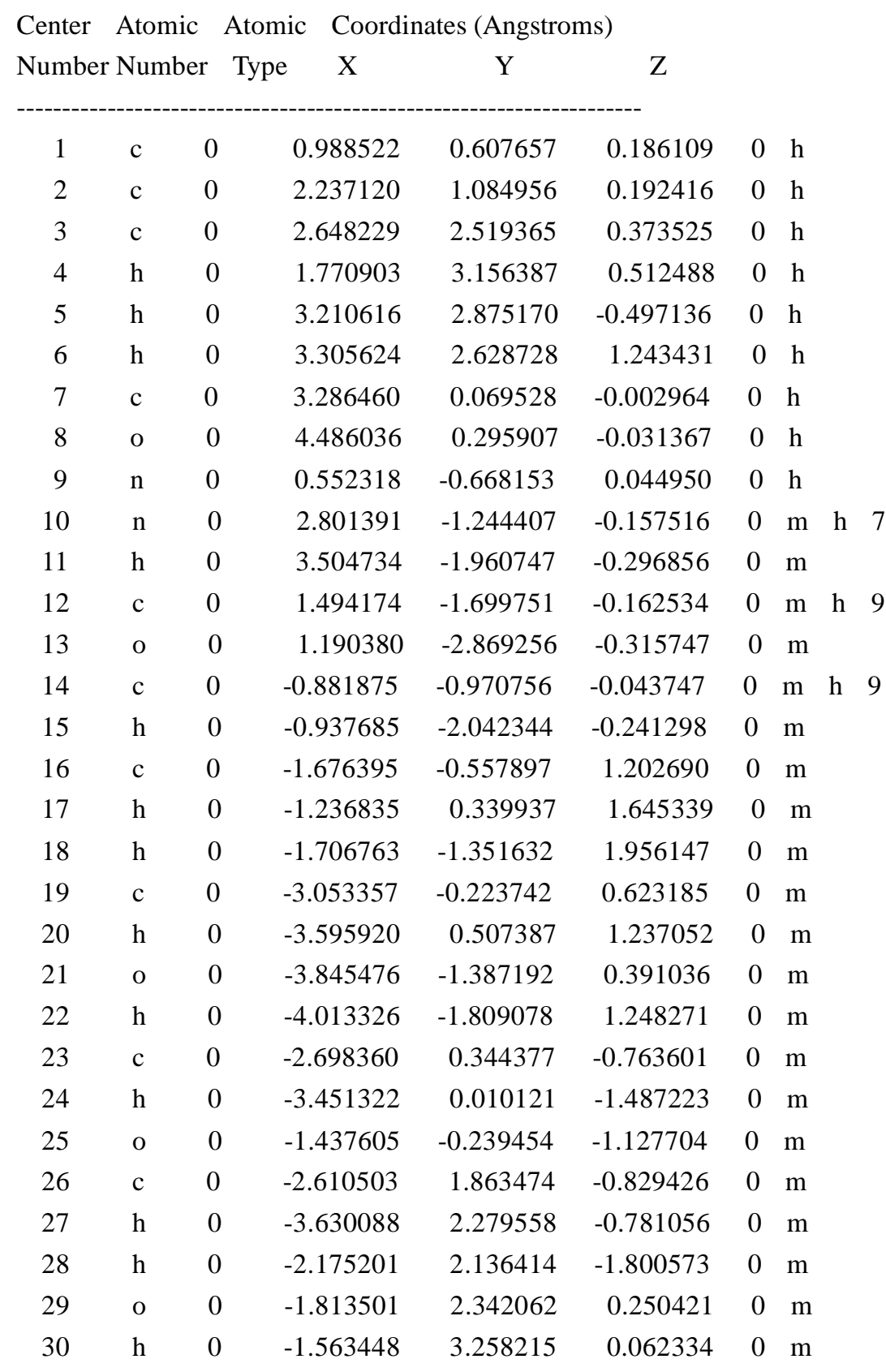




\section{(4) The Cartesian Coordinates of Nucleoside}

$\mathbf{r A}$

s1

neutral molecule

Charge $=0$ Multiplicity $=1$ for low level calculation on real system.

Charge $=0$ Multiplicity $=1$ for high level calculation on model system .

Charge $=0$ Multiplicity $=1$ for low level calculation on model system .

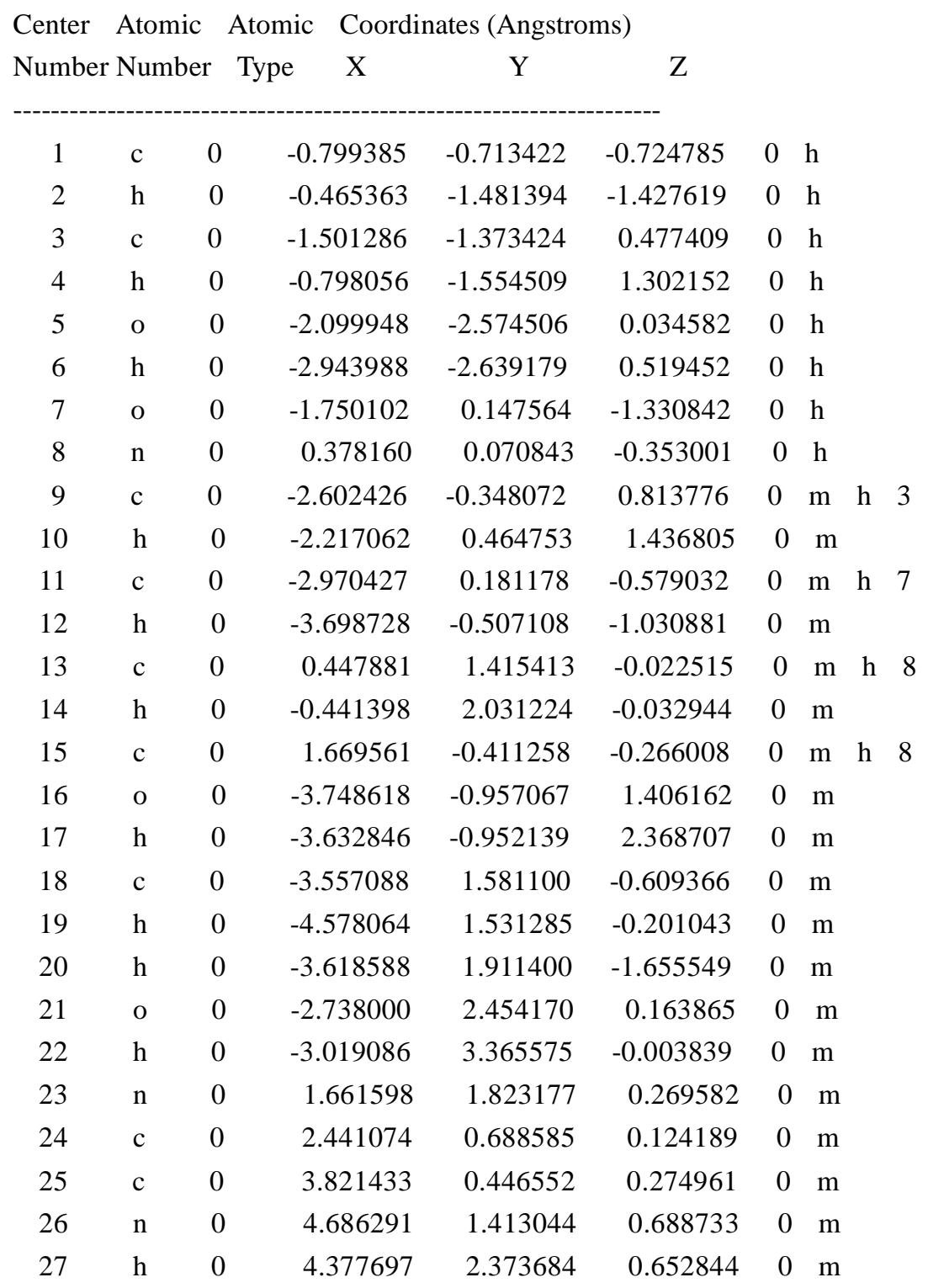




$\begin{array}{lllrrrrc}28 & \mathrm{~h} & 0 & 5.672020 & 1.225877 & 0.573300 & 0 & \mathrm{~m} \\ 29 & \mathrm{n} & 0 & 4.298211 & -0.787294 & 0.031659 & 0 & \mathrm{~m} \\ 30 & \mathrm{c} & 0 & 3.429428 & -1.743903 & -0.336147 & 0 & \mathrm{~m} \\ 31 & \mathrm{~h} & 0 & 3.867409 & -2.722805 & -0.520805 & 0 & \mathrm{~m} \\ 32 & \mathrm{n} & 0 & 2.106712 & -1.654692 & -0.506346 & 0 & \mathrm{~m}\end{array}$

radical

Charge $=0$ Multiplicity $=2$ for low level calculation on real system.

Charge $=0$ Multiplicity $=2$ for high level calculation on model system .

Charge $=0$ Multiplicity $=2$ for low level calculation on model system.

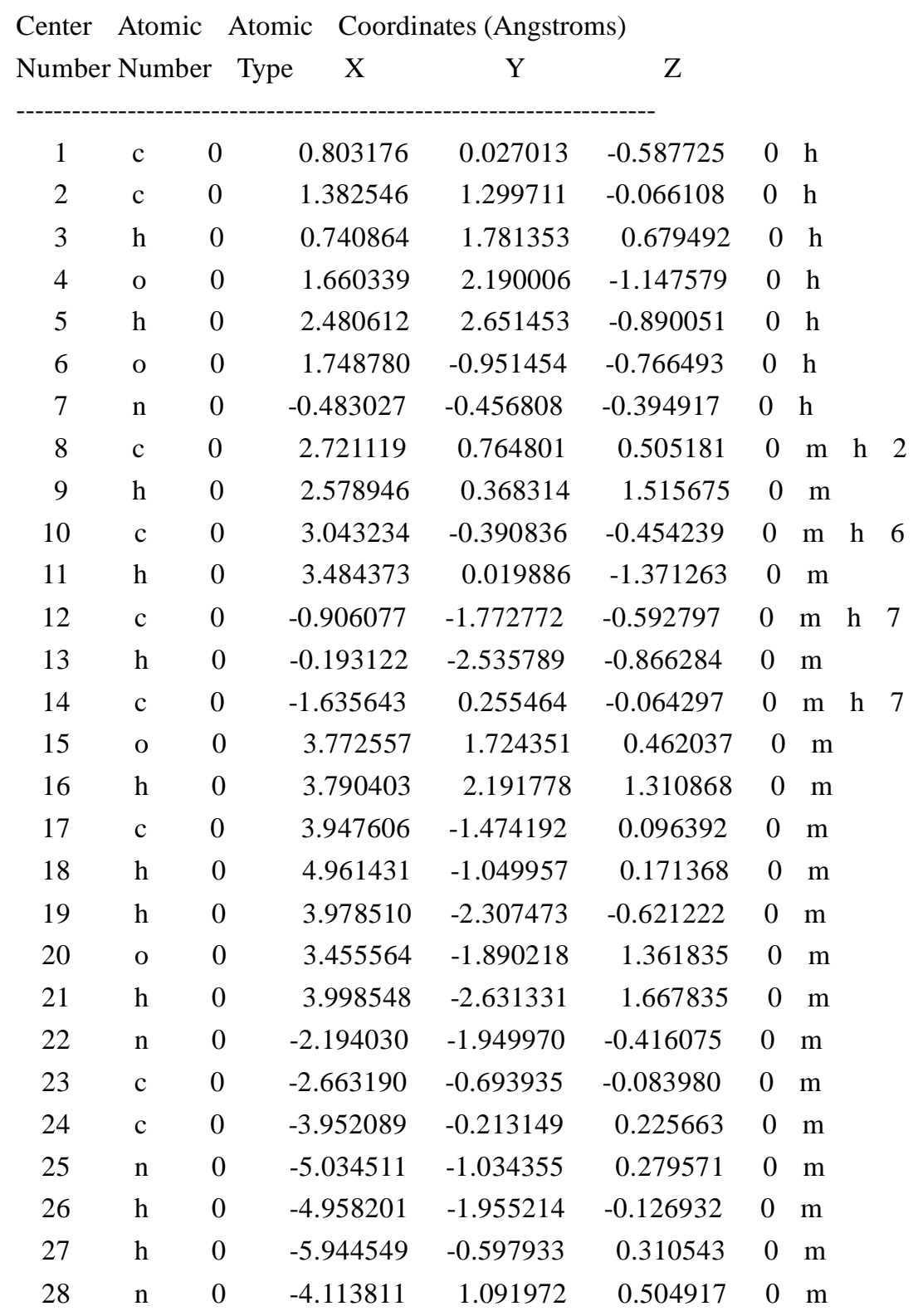




$\begin{array}{llllllll}29 & \mathrm{c} & 0 & -3.026940 & 1.881662 & 0.492143 & 0 & \mathrm{~m} \\ 30 & \mathrm{~h} & 0 & -3.208865 & 2.927581 & 0.730452 & 0 & \mathrm{~m} \\ 31 & \mathrm{n} & 0 & -1.758810 & 1.556768 & 0.225036 & 0 & \mathrm{~m}\end{array}$

\section{s2}

neutral molecule

Charge $=0$ Multiplicity $=1$ for low level calculation on real system.

Charge $=0$ Multiplicity $=1$ for high level calculation on model system.

Charge $=0$ Multiplicity $=1$ for low level calculation on model system.

Center Atomic Atomic Coordinates (Angstroms)

Number Number Type $\quad \mathrm{X} \quad \mathrm{Y} \quad \mathrm{Z}$

\begin{tabular}{|c|c|c|c|c|c|c|c|c|}
\hline 1 & $\mathrm{c}$ & 0 & -1.501287 & -1.373420 & 0.477410 & 0 & $\mathrm{~h}$ & \\
\hline 2 & $\mathrm{~h}$ & 0 & -0.798061 & -1.554502 & 1.302158 & 0 & $\mathrm{~h}$ & \\
\hline 3 & o & 0 & -2.099952 & -2.574504 & 0.034592 & 0 & $\mathrm{~h}$ & \\
\hline 4 & $\mathrm{~h}$ & 0 & -2.943992 & -2.639170 & 0.519461 & 0 & $\mathrm{~h}$ & \\
\hline 5 & $\mathrm{c}$ & 0 & -2.602428 & -0.348067 & 0.813770 & 0 & $\mathrm{~h}$ & \\
\hline 6 & $\mathrm{~h}$ & 0 & -2.217067 & 0.464760 & 1.436797 & 0 & $\mathrm{~h}$ & \\
\hline 7 & o & 0 & -3.748620 & -0.957056 & 1.406164 & 0 & $\mathrm{~h}$ & \\
\hline 8 & $\mathrm{~h}$ & 0 & -3.632848 & -0.952113 & 2.368709 & 0 & $\mathrm{~h}$ & \\
\hline 9 & $\mathrm{c}$ & 0 & -0.799381 & -0.713421 & -0.724784 & 0 & $\mathrm{~h}$ & \\
\hline 10 & $\mathrm{~h}$ & 0 & -0.465359 & -1.481396 & -1.427615 & 0 & $\mathrm{~h}$ & \\
\hline 11 & $\mathrm{c}$ & 0 & -2.970430 & 0.181169 & -0.579044 & 0 & $\mathrm{~m}$ & h 5 \\
\hline 12 & $\mathrm{~h}$ & 0 & -3.698728 & -0.507125 & -1.030885 & 0 & $\mathrm{~m}$ & \\
\hline 13 & o & 0 & -1.750096 & 0.147565 & -1.330846 & 0 & $\mathrm{~m}$ & h 9 \\
\hline 14 & $\mathrm{n}$ & 0 & 0.378164 & 0.070843 & -0.352999 & 0 & $\mathrm{~m}$ & h 9 \\
\hline 15 & $\mathrm{c}$ & 0 & -3.557097 & 1.581089 & -0.609375 & 0 & $\mathrm{~m}$ & \\
\hline 16 & $\mathrm{~h}$ & 0 & -4.578076 & 1.531267 & -0.201061 & 0 & $\mathrm{~m}$ & \\
\hline 17 & $\mathrm{~h}$ & 0 & -3.618589 & 1.911394 & -1.655557 & 0 & $\mathrm{~m}$ & \\
\hline 18 & o & 0 & -2.738020 & 2.454157 & 0.163868 & 0 & $\mathrm{~m}$ & \\
\hline 19 & $\mathrm{~h}$ & 0 & -3.019108 & 3.365562 & -0.003833 & 0 & $\mathrm{~m}$ & \\
\hline 20 & $\mathrm{c}$ & 0 & 0.447884 & 1.415411 & -0.022507 & 0 & $\mathrm{~m}$ & \\
\hline 21 & $\mathrm{~h}$ & 0 & -0.441396 & 2.031222 & -0.032933 & 0 & $\mathrm{~m}$ & \\
\hline 22 & $\mathrm{n}$ & 0 & 1.661601 & 1.823175 & 0.269588 & 0 & $\mathrm{~m}$ & \\
\hline 23 & $\mathrm{c}$ & 0 & 2.441076 & 0.688583 & 0.124191 & 0 & $\mathrm{~m}$ & \\
\hline 24 & $\mathrm{c}$ & 0 & 3.821437 & 0.446554 & 0.274958 & 0 & $\mathrm{~m}$ & \\
\hline 25 & $\mathrm{n}$ & 0 & 4.686294 & 1.413050 & 0.688724 & 0 & $\mathrm{~m}$ & \\
\hline 26 & $\mathrm{~h}$ & 0 & 4.377697 & 2.373688 & 0.652834 & 0 & $\mathrm{~m}$ & \\
\hline 27 & $\mathrm{~h}$ & 0 & 5.672022 & 1.225884 & 0.573288 & 0 & $\mathrm{~m}$ & \\
\hline
\end{tabular}




$\begin{array}{llllllll}28 & \mathrm{n} & 0 & 4.298217 & -0.787292 & 0.031659 & 0 & \mathrm{~m} \\ 29 & \mathrm{c} & 0 & 3.429434 & -1.743904 & -0.336142 & 0 & \mathrm{~m} \\ 30 & \mathrm{~h} & 0 & 3.867417 & -2.722805 & -0.520798 & 0 & \mathrm{~m} \\ 31 & \mathrm{n} & 0 & 2.106719 & -1.654690 & -0.506344 & 0 & \mathrm{~m} \\ 32 & \mathrm{c} & 0 & 1.669565 & -0.411257 & -0.266010 & 0 & \mathrm{~m}\end{array}$

radical

Charge $=0$ Multiplicity $=2$ for low level calculation on real system.

Charge $=0$ Multiplicity $=2$ for high level calculation on model system .

Charge $=0$ Multiplicity $=2$ for low level calculation on model system.

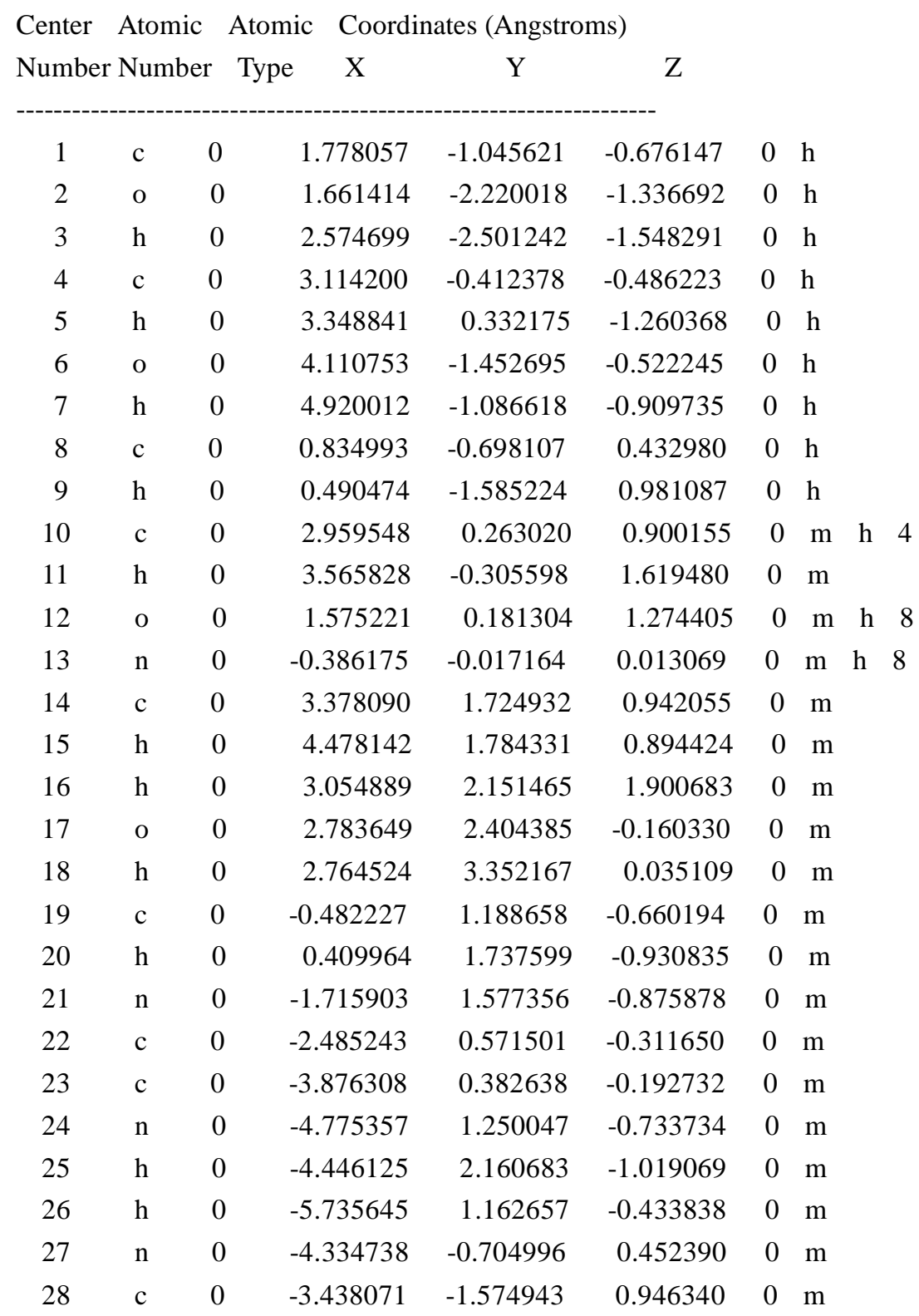




$\begin{array}{llllllll}29 & \mathrm{~h} & 0 & -3.861063 & -2.436942 & 1.458491 & 0 & \mathrm{~m} \\ 30 & \mathrm{n} & 0 & -2.103660 & -1.522810 & 0.896045 & 0 & \mathrm{~m} \\ 31 & \mathrm{c} & 0 & -1.683373 & -0.424081 & 0.256833 & 0 & \mathrm{~m}\end{array}$

\section{s3}

neutral molecule

Charge $=0$ Multiplicity $=1$ for low level calculation on real system.

Charge $=0$ Multiplicity $=1$ for high level calculation on model system.

Charge $=0$ Multiplicity $=1$ for low level calculation on model system.

$\begin{array}{lcccc}\text { Center Atomic } & \text { Atomic } & \text { Coordinates (Angstroms) } & \\ \text { Number Number } & \text { Type } & \text { X } & \text { Y } & \text { Z }\end{array}$

\begin{tabular}{|c|c|c|c|c|c|c|c|c|}
\hline 1 & $\mathrm{c}$ & 0 & -2.602428 & -0.348070 & 0.813778 & 0 & $\mathrm{~h}$ & \\
\hline 2 & $\mathrm{~h}$ & 0 & -2.217065 & 0.464756 & 1.436805 & 0 & $\mathrm{~h}$ & \\
\hline 3 & o & 0 & -3.748618 & -0.957067 & 1.406167 & 0 & $\mathrm{~h}$ & \\
\hline 4 & $\mathrm{~h}$ & 0 & -3.632844 & -0.952136 & 2.368712 & 0 & $\mathrm{~h}$ & \\
\hline 5 & $\mathrm{c}$ & 0 & -2.970432 & 0.181175 & -0.579031 & 0 & $\mathrm{~h}$ & \\
\hline 6 & $\mathrm{~h}$ & 0 & -3.698732 & -0.507117 & -1.030876 & 0 & $\mathrm{~h}$ & \\
\hline 7 & $\mathrm{c}$ & 0 & -1.501288 & -1.373422 & 0.477410 & 0 & $\mathrm{~h}$ & \\
\hline 8 & $\mathrm{~h}$ & 0 & -0.798060 & -1.554499 & 1.302157 & 0 & $\mathrm{~h}$ & \\
\hline 9 & o & 0 & -2.099954 & -2.574503 & 0.034586 & 0 & $\mathrm{~h}$ & \\
\hline 10 & $\mathrm{~h}$ & 0 & -2.943994 & -2.639171 & 0.519456 & 0 & $\mathrm{~h}$ & \\
\hline 11 & $\mathrm{c}$ & 0 & -3.557091 & 1.581098 & -0.609377 & 0 & $\mathrm{~m}$ & \\
\hline 12 & $\mathrm{~h}$ & 0 & -4.578068 & 1.531287 & -0.201058 & 0 & $\mathrm{~m}$ & \\
\hline 13 & $\mathrm{~h}$ & 0 & -3.618585 & 1.911390 & -1.655563 & 0 & $\mathrm{~m}$ & \\
\hline 14 & o & 0 & -1.750107 & 0.147561 & -1.330841 & 0 & $\mathrm{~m}$ & h 5 \\
\hline 15 & $\mathrm{c}$ & 0 & -0.799385 & -0.713416 & -0.724782 & 0 & $\mathrm{~m}$ & h 7 \\
\hline 16 & $\mathrm{~h}$ & 0 & -0.465364 & -1.481387 & -1.427618 & 0 & $\mathrm{~m}$ & \\
\hline 17 & o & 0 & -2.738002 & 2.454171 & 0.163849 & 0 & $\mathrm{~m}$ & \\
\hline 18 & $\mathrm{~h}$ & 0 & -3.019085 & 3.365575 & -0.003863 & 0 & $\mathrm{~m}$ & \\
\hline 19 & $\mathrm{n}$ & 0 & 0.378162 & 0.070845 & -0.352994 & 0 & $\mathrm{~m}$ & \\
\hline 20 & $\mathrm{c}$ & 0 & 0.447883 & 1.415411 & -0.022495 & 0 & $\mathrm{~m}$ & \\
\hline 21 & $\mathrm{~h}$ & 0 & -0.441396 & 2.031222 & -0.032915 & 0 & $\mathrm{~m}$ & \\
\hline 22 & $\mathrm{n}$ & 0 & 1.661601 & 1.823173 & 0.269601 & 0 & $\mathrm{~m}$ & \\
\hline 23 & $\mathrm{c}$ & 0 & 2.441075 & 0.688581 & 0.124195 & 0 & $\mathrm{~m}$ & \\
\hline 24 & $\mathrm{c}$ & 0 & 3.821436 & 0.446551 & 0.274958 & 0 & $\mathrm{~m}$ & \\
\hline 25 & $\mathrm{n}$ & 0 & 4.686295 & 1.413043 & 0.688728 & 0 & $\mathrm{~m}$ & \\
\hline 26 & $\mathrm{~h}$ & 0 & 4.377699 & 2.373682 & 0.652844 & 0 & $\mathrm{~m}$ & \\
\hline 27 & $\mathrm{~h}$ & 0 & 5.672023 & 1.225878 & 0.573289 & 0 & $\mathrm{~m}$ & \\
\hline
\end{tabular}




$\begin{array}{llllllll}28 & \mathrm{n} & 0 & 4.298215 & -0.787294 & 0.031650 & 0 & \mathrm{~m} \\ 29 & \mathrm{c} & 0 & 3.429431 & -1.743904 & -0.336154 & 0 & \mathrm{~m} \\ 30 & \mathrm{~h} & 0 & 3.867414 & -2.722804 & -0.520817 & 0 & \mathrm{~m} \\ 31 & \mathrm{n} & 0 & 2.106716 & -1.654688 & -0.506353 & 0 & \mathrm{~m} \\ 32 & \mathrm{c} & 0 & 1.669563 & -0.411257 & -0.266011 & 0 & \mathrm{~m}\end{array}$

radical

Charge $=0$ Multiplicity $=2$ for low level calculation on real system.

Charge $=0$ Multiplicity $=2$ for high level calculation on model system .

Charge $=0$ Multiplicity $=2$ for low level calculation on model system.

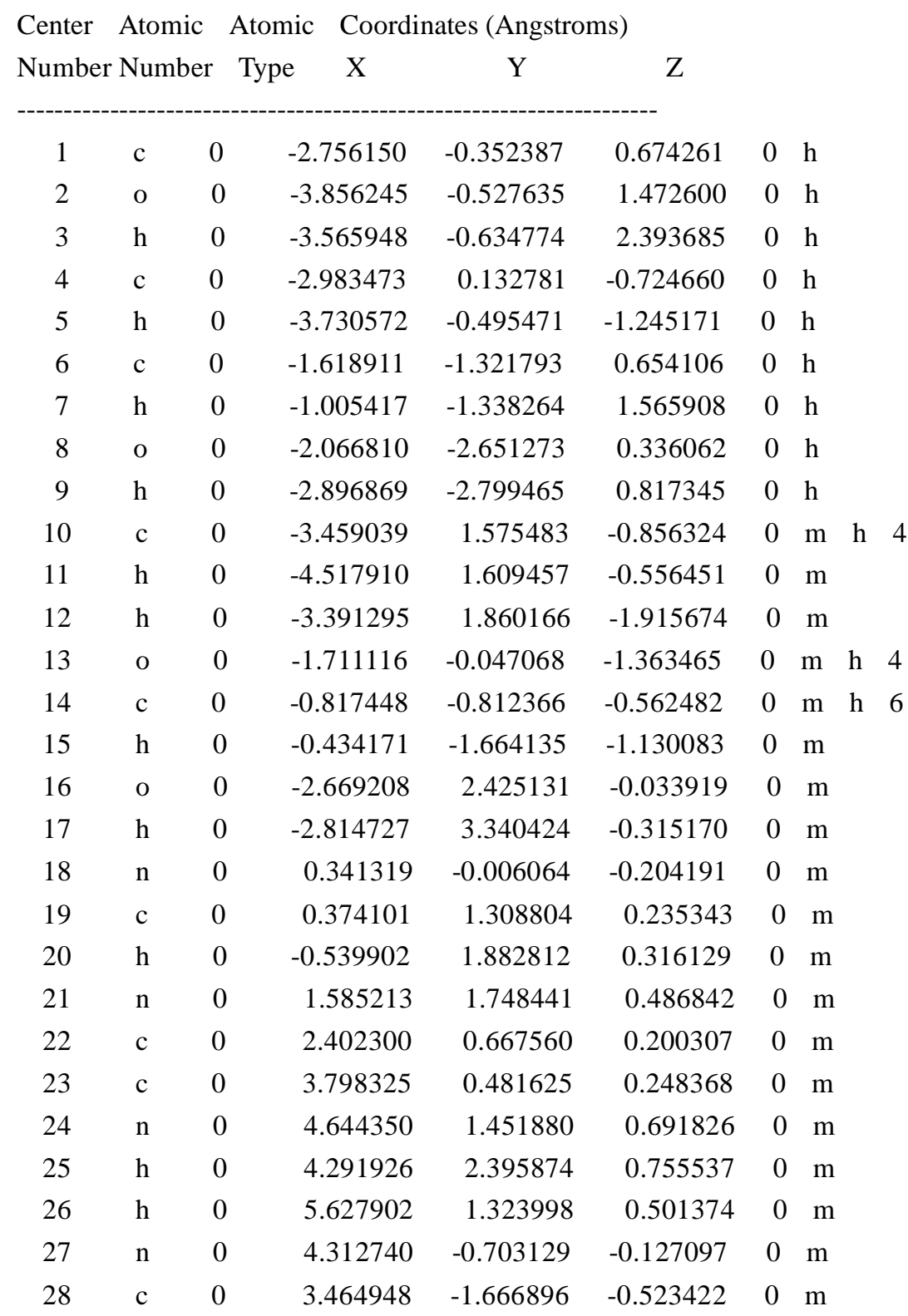




$\begin{array}{llllllll}29 & \mathrm{~h} & 0 & 3.932182 & -2.604886 & -0.816744 & 0 & \mathrm{~m} \\ 30 & \mathrm{n} & 0 & 2.131504 & -1.627621 & -0.606838 & 0 & \mathrm{~m} \\ 31 & \mathrm{c} & 0 & 1.656341 & -0.431732 & -0.236283 & 0 & \mathrm{~m}\end{array}$

\section{s4}

neutral molecule

Charge $=0$ Multiplicity $=1$ for low level calculation on real system.

Charge $=0$ Multiplicity $=1$ for high level calculation on model system .

Charge $=0$ Multiplicity $=1$ for low level calculation on model system .

$\begin{array}{lcccc}\text { Center Atomic } & \text { Atomic } & \text { Coordinates (Angstroms) } & \\ \text { Number Number } & \text { Type } & \text { X } & \text { Y } & \text { Z }\end{array}$

\begin{tabular}{|c|c|c|c|c|c|c|c|c|}
\hline 1 & $\mathrm{c}$ & 0 & -2.970427 & 0.181173 & -0.579029 & 0 & $\mathrm{~h}$ & \\
\hline 2 & $\mathrm{~h}$ & 0 & -3.698738 & -0.507111 & -1.030866 & 0 & h & \\
\hline 3 & $\mathrm{c}$ & 0 & -3.557087 & 1.581096 & -0.609366 & 0 & $\mathrm{~h}$ & \\
\hline 4 & $\mathrm{~h}$ & 0 & -4.578061 & 1.531283 & -0.201039 & 0 & $\mathrm{~h}$ & \\
\hline 5 & $\mathrm{~h}$ & 0 & -3.618590 & 1.911392 & -1.655550 & 0 & $\mathrm{~h}$ & \\
\hline 6 & o & 0 & -1.750105 & 0.147555 & -1.330844 & 0 & $\mathrm{~h}$ & \\
\hline 7 & $\mathrm{c}$ & 0 & -2.602412 & -0.348076 & 0.813776 & 0 & $\mathrm{~h}$ & \\
\hline 8 & $\mathrm{~h}$ & 0 & -2.217048 & 0.464749 & 1.436805 & 0 & $\mathrm{~h}$ & \\
\hline 9 & o & 0 & -3.748602 & -0.957055 & 1.406182 & 0 & $\mathrm{~h}$ & \\
\hline 10 & $\mathrm{~h}$ & 0 & -3.632822 & -0.952110 & 2.368726 & 0 & $\mathrm{~h}$ & \\
\hline 11 & o & 0 & -2.737995 & 2.454167 & 0.163859 & 0 & $\mathrm{~m}$ & $\mathrm{~h}$ \\
\hline 12 & $\mathrm{~h}$ & 0 & -3.019079 & 3.365572 & -0.003847 & 0 & $\mathrm{~m}$ & \\
\hline 13 & $\mathrm{c}$ & 0 & -0.799387 & -0.713430 & -0.724788 & 0 & $\mathrm{~m} \mathrm{l}$ & h \\
\hline 14 & $\mathrm{~h}$ & 0 & -0.465376 & -1.481410 & -1.427618 & 0 & $\mathrm{~m}$ & \\
\hline 15 & $\mathrm{c}$ & 0 & -1.501279 & -1.373420 & 0.477398 & 0 & $\mathrm{~m} \quad 1$ & h \\
\hline 16 & $\mathrm{~h}$ & 0 & -0.798045 & -1.554499 & 1.302139 & 0 & $\mathrm{~m}$ & \\
\hline 17 & o & 0 & -2.099947 & -2.574500 & 0.034575 & 0 & $\mathrm{~m}$ & \\
\hline 18 & $\mathrm{~h}$ & 0 & -2.943983 & -2.639171 & 0.519451 & 0 & $\mathrm{~m}$ & \\
\hline 19 & $\mathrm{n}$ & 0 & 0.378156 & 0.070837 & -0.353003 & 0 & $\mathrm{~m}$ & \\
\hline 20 & $\mathrm{c}$ & 0 & 0.447873 & 1.415407 & -0.022516 & 0 & $\mathrm{~m}$ & \\
\hline 21 & $\mathrm{~h}$ & 0 & -0.441407 & 2.031216 & -0.032945 & 0 & $\mathrm{~m}$ & \\
\hline 22 & $\mathrm{n}$ & 0 & 1.661589 & 1.823174 & 0.269580 & 0 & $\mathrm{~m}$ & \\
\hline 23 & $\mathrm{c}$ & 0 & 2.441066 & 0.688583 & 0.124188 & 0 & $\mathrm{~m}$ & \\
\hline 24 & $\mathrm{c}$ & 0 & 3.821427 & 0.446558 & 0.274959 & 0 & $\mathrm{~m}$ & \\
\hline 25 & $\mathrm{n}$ & 0 & 4.686282 & 1.413056 & 0.688723 & 0 & $\mathrm{~m}$ & \\
\hline 26 & $\mathrm{~h}$ & 0 & 4.377684 & 2.373694 & 0.652829 & 0 & $\mathrm{~m}$ & \\
\hline 27 & $\mathrm{~h}$ & 0 & 5.672011 & 1.225892 & 0.573289 & 0 & $\mathrm{~m}$ & \\
\hline
\end{tabular}




$\begin{array}{llllllll}28 & \mathrm{n} & 0 & 4.298210 & -0.787289 & 0.031664 & 0 & \mathrm{~m} \\ 29 & \mathrm{c} & 0 & 3.429430 & -1.743904 & -0.336134 & 0 & \mathrm{~m} \\ 30 & \mathrm{~h} & 0 & 3.867415 & -2.722805 & -0.520786 & 0 & \mathrm{~m} \\ 31 & \mathrm{n} & 0 & 2.106715 & -1.654693 & -0.506339 & 0 & \mathrm{~m} \\ 32 & \mathrm{c} & 0 & 1.669558 & -0.411260 & -0.266010 & 0 & \mathrm{~m}\end{array}$

radical

Charge $=0$ Multiplicity $=2$ for low level calculation on real system.

Charge $=0$ Multiplicity $=2$ for high level calculation on model system .

Charge $=0$ Multiplicity $=2$ for low level calculation on model system.

\begin{tabular}{|c|c|c|c|c|c|c|c|c|}
\hline Center & Atomic & & Atomic & ates (Angstro & & & & \\
\hline Number & Numbe & & Type & $\mathrm{Y}$ & $\mathrm{Z}$ & & & \\
\hline 1 & $\mathrm{c}$ & 0 & -2.819637 & 0.298304 & -0.427330 & 0 & $\mathrm{~h}$ & \\
\hline 2 & $\mathrm{c}$ & 0 & -3.593008 & 1.549103 & -0.585692 & 0 & $\mathrm{~h}$ & \\
\hline 3 & $\mathrm{~h}$ & 0 & -4.571063 & 1.427668 & -0.099843 & 0 & $\mathrm{~h}$ & \\
\hline 4 & $\mathrm{~h}$ & 0 & -3.754139 & 1.748927 & -1.653898 & 0 & $\mathrm{~h}$ & \\
\hline 5 & o & 0 & -1.791944 & 0.099220 & -1.320393 & 0 & $\mathrm{~h}$ & \\
\hline 6 & $\mathrm{c}$ & 0 & -2.562398 & -0.410800 & 0.863600 & 0 & $\mathrm{~h}$ & \\
\hline 7 & $\mathrm{~h}$ & 0 & -2.180933 & 0.270325 & 1.640066 & 0 & $\mathrm{~h}$ & \\
\hline 8 & o & 0 & -3.767179 & -1.036764 & 1.325406 & 0 & $\mathrm{~h}$ & \\
\hline 9 & $\mathrm{~h}$ & 0 & -3.680604 & -1.181414 & 2.281972 & 0 & $\mathrm{~h}$ & \\
\hline 10 & o & 0 & -2.852994 & 2.625474 & 0.032265 & 0 & $\mathrm{~m}$ & $\mathrm{~h}$ \\
\hline 11 & $\mathrm{~h}$ & 0 & -3.323892 & 3.455541 & -0.146612 & 0 & $\mathrm{~m}$ & \\
\hline 12 & $\mathrm{c}$ & 0 & -0.805720 & -0.761420 & -0.747162 & 0 & $\mathrm{~m} \mathrm{l}$ & h 5 \\
\hline 13 & $\mathrm{~h}$ & 0 & -0.478654 & -1.490960 & -1.489174 & 0 & $\mathrm{~m}$ & \\
\hline 14 & $\mathrm{c}$ & 0 & -1.490273 & -1.455928 & 0.446781 & 0 & $\mathrm{~m} 1$ & h 6 \\
\hline 15 & $\mathrm{~h}$ & 0 & -0.762690 & -1.676519 & 1.238797 & 0 & $\mathrm{~m}$ & \\
\hline 16 & o & 0 & -2.119697 & -2.629879 & -0.007899 & 0 & $\mathrm{~m}$ & \\
\hline 17 & $\mathrm{~h}$ & 0 & -3.033231 & -2.588108 & 0.335608 & 0 & $\mathrm{~m}$ & \\
\hline 18 & $\mathrm{n}$ & 0 & 0.345789 & 0.050155 & -0.368519 & 0 & $\mathrm{~m}$ & \\
\hline 19 & $\mathrm{c}$ & 0 & 0.387468 & 1.393289 & -0.014532 & 0 & $\mathrm{~m}$ & \\
\hline 20 & $\mathrm{~h}$ & 0 & -0.507019 & 2.003590 & -0.025234 & 0 & $\mathrm{~m}$ & \\
\hline 21 & $\mathrm{n}$ & 0 & 1.591701 & 1.815132 & 0.291308 & 0 & $\mathrm{~m}$ & \\
\hline 22 & $\mathrm{c}$ & 0 & 2.393208 & 0.697869 & 0.133453 & 0 & $\mathrm{~m}$ & \\
\hline 23 & $\mathrm{c}$ & 0 & 3.777262 & 0.478315 & 0.290227 & 0 & $\mathrm{~m}$ & \\
\hline 24 & $\mathrm{n}$ & 0 & 4.621480 & 1.451992 & 0.723454 & 0 & $\mathrm{~m}$ & \\
\hline 25 & $\mathrm{~h}$ & 0 & 4.296277 & 2.407604 & 0.715788 & 0 & $\mathrm{~m}$ & \\
\hline 26 & $\mathrm{~h}$ & 0 & 5.612115 & 1.282020 & 0.626200 & 0 & $\mathrm{~m}$ & \\
\hline 27 & $\mathrm{n}$ & 0 & 4.276720 & -0.743456 & 0.030186 & 0 & $\mathrm{~m}$ & \\
\hline 28 & $\mathrm{c}$ & 0 & 3.428442 & -1.709226 & -0.359169 & 0 & $\mathrm{~m}$ & \\
\hline
\end{tabular}




$\begin{array}{llllllll}29 & \mathrm{~h} & 0 & 3.884883 & -2.677040 & -0.556438 & 0 & \mathrm{~m} \\ 30 & \mathrm{n} & 0 & 2.105014 & -1.640615 & -0.537261 & 0 & \mathrm{~m} \\ 31 & \mathrm{c} & 0 & 1.646079 & -0.409257 & -0.279583 & 0 & \mathrm{~m}\end{array}$

\section{s5}

neutral molecule

Charge $=0$ Multiplicity $=1$ for low level calculation on real system.

Charge $=0$ Multiplicity $=1$ for high level calculation on model system.

Charge $=0$ Multiplicity $=1$ for low level calculation on model system.

$\begin{array}{lcccc}\text { Center Atomic } & \text { Atomic } & \text { Coordinates (Angstroms) } & \\ \text { Number Number } & \text { Type } & \text { X } & \text { Y } & \text { Z }\end{array}$

\begin{tabular}{|c|c|c|c|c|c|c|c|}
\hline 1 & $\mathrm{c}$ & 0 & -3.557090 & 1.581097 & -0.609373 & 0 & $\mathrm{~h}$ \\
\hline 2 & $\mathrm{~h}$ & 0 & -4.578063 & 1.531286 & -0.201044 & 0 & $\mathrm{~h}$ \\
\hline 3 & $\mathrm{~h}$ & 0 & -3.618589 & 1.911385 & -1.655560 & 0 & $\mathrm{~h}$ \\
\hline 4 & o & 0 & -2.738005 & 2.454170 & 0.163857 & 0 & $\mathrm{~h}$ \\
\hline 5 & $\mathrm{~h}$ & 0 & -3.019091 & 3.365574 & -0.003850 & 0 & $\mathrm{~h}$ \\
\hline 6 & $\mathrm{c}$ & 0 & -2.970427 & 0.181176 & -0.579034 & 0 & $\mathrm{~h}$ \\
\hline 7 & $\mathrm{~h}$ & 0 & -3.698734 & -0.507110 & -1.030875 & 0 & h \\
\hline 8 & o & 0 & -1.750101 & 0.147561 & -1.330842 & 0 & $\mathrm{~h}$ \\
\hline 9 & $\mathrm{c}$ & 0 & -2.602418 & -0.348073 & 0.813772 & 0 & $\mathrm{~h}$ \\
\hline 10 & $\mathrm{~h}$ & 0 & -2.217055 & 0.464752 & 1.436802 & 0 & $\mathrm{~h}$ \\
\hline 11 & $\mathrm{c}$ & 0 & -0.799385 & -0.713425 & -0.724783 & 0 & $\mathrm{~m}$ \\
\hline 12 & $\mathrm{~h}$ & 0 & -0.465369 & -1.481402 & -1.427613 & 0 & $\mathrm{~m}$ \\
\hline 13 & $\mathrm{c}$ & 0 & -1.501289 & -1.373426 & 0.477411 & 0 & $\mathrm{~m}$ \\
\hline 14 & $\mathrm{~h}$ & 0 & -0.798060 & -1.554509 & 1.302156 & 0 & $\mathrm{~m}$ \\
\hline 15 & o & 0 & -3.748612 & -0.957057 & 1.406168 & 0 & $\mathrm{~m}$ \\
\hline 16 & $\mathrm{~h}$ & 0 & -3.632838 & -0.952116 & 2.368713 & 0 & $\mathrm{~m}$ \\
\hline 17 & o & 0 & -2.099950 & -2.574508 & 0.034584 & 0 & $\mathrm{~m}$ \\
\hline 18 & $\mathrm{~h}$ & 0 & -2.943990 & -2.639180 & 0.519452 & 0 & $\mathrm{~m}$ \\
\hline 19 & $\mathrm{n}$ & 0 & 0.378159 & 0.070841 & -0.352998 & 0 & $\mathrm{~m}$ \\
\hline 20 & $\mathrm{c}$ & 0 & 0.447878 & 1.415408 & -0.022504 & 0 & $\mathrm{~m}$ \\
\hline 21 & $\mathrm{~h}$ & 0 & -0.441402 & 2.031218 & -0.032928 & 0 & $\mathrm{~m}$ \\
\hline 22 & $\mathrm{n}$ & 0 & 1.661595 & 1.823174 & 0.269591 & 0 & $\mathrm{~m}$ \\
\hline 23 & $\mathrm{c}$ & 0 & 2.441071 & 0.688583 & 0.124191 & 0 & $\mathrm{~m}$ \\
\hline 24 & $\mathrm{c}$ & 0 & 3.821432 & 0.446555 & 0.274958 & 0 & $\mathrm{~m}$ \\
\hline 25 & $\mathrm{n}$ & 0 & 4.686288 & 1.413051 & 0.688725 & 0 & $\mathrm{~m}$ \\
\hline 26 & $\mathrm{~h}$ & 0 & 4.377691 & 2.373690 & 0.652836 & 0 & $\mathrm{~m}$ \\
\hline 27 & $\mathrm{~h}$ & 0 & 5.672017 & 1.225887 & 0.573287 & 0 & $\mathrm{~m}$ \\
\hline
\end{tabular}




$\begin{array}{llllllll}28 & \mathrm{n} & 0 & 4.298214 & -0.787290 & 0.031656 & 0 & \mathrm{~m} \\ 29 & \mathrm{c} & 0 & 3.429432 & -1.743902 & -0.336145 & 0 & \mathrm{~m} \\ 30 & \mathrm{~h} & 0 & 3.867417 & -2.722803 & -0.520803 & 0 & \mathrm{~m} \\ 31 & \mathrm{n} & 0 & 2.106717 & -1.654690 & -0.506346 & 0 & \mathrm{~m} \\ 32 & \mathrm{c} & 0 & 1.669561 & -0.411258 & -0.266010 & 0 & \mathrm{~m}\end{array}$

radical

Charge $=0$ Multiplicity $=2$ for low level calculation on real system.

Charge $=0$ Multiplicity $=2$ for high level calculation on model system .

Charge $=0$ Multiplicity $=2$ for low level calculation on model system.

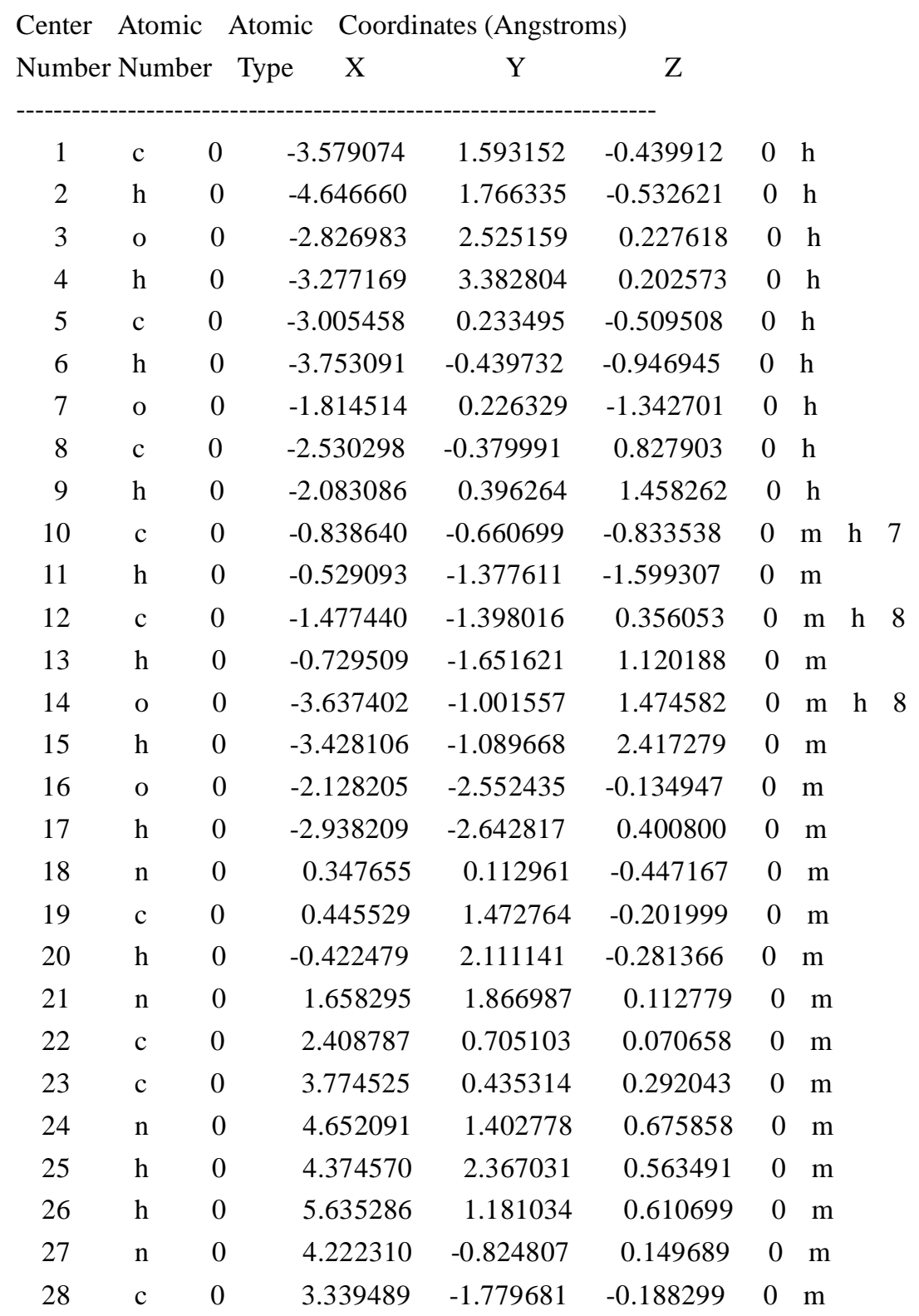




$\begin{array}{llllllll}29 & \mathrm{~h} & 0 & 3.754216 & -2.780698 & -0.289100 & 0 & \mathrm{~m} \\ 30 & \mathrm{n} & 0 & 2.027772 & -1.665421 & -0.418166 & 0 & \mathrm{~m} \\ 31 & \mathrm{c} & 0 & 1.619796 & -0.396427 & -0.278620 & 0 & \mathrm{~m}\end{array}$

\section{b2}

neutral molecule

Charge $=0$ Multiplicity $=1$ for low level calculation on real system.

Charge $=0$ Multiplicity $=1$ for high level calculation on model system .

Charge $=0$ Multiplicity $=1$ for low level calculation on model system .

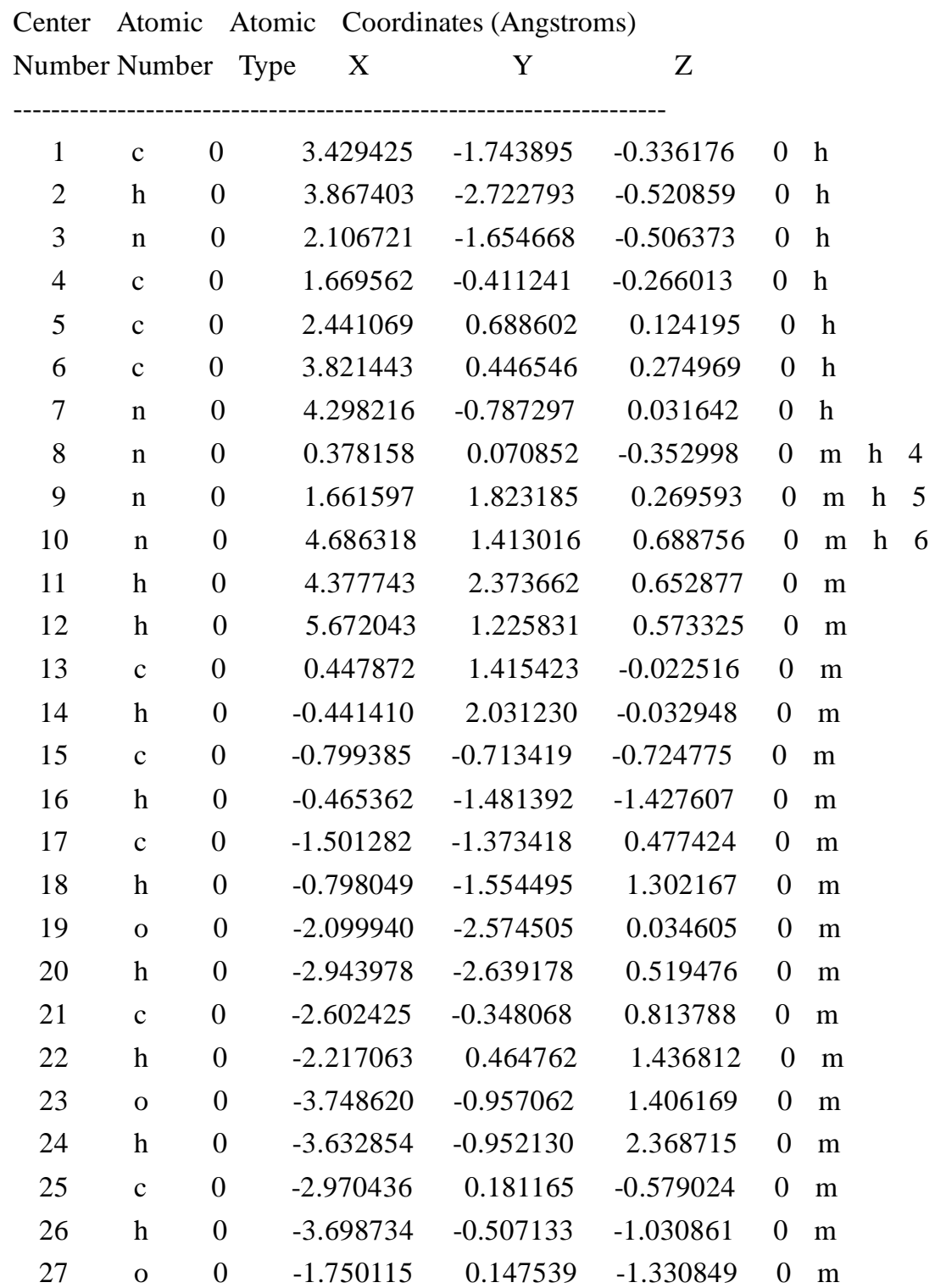




$\begin{array}{llllllll}28 & \mathrm{c} & 0 & -3.557099 & 1.581086 & -0.609379 & 0 & \mathrm{~m} \\ 29 & \mathrm{~h} & 0 & -4.578075 & 1.531275 & -0.201056 & 0 & \mathrm{~m} \\ 30 & \mathrm{~h} & 0 & -3.618599 & 1.911370 & -1.655567 & 0 & \mathrm{~m} \\ 31 & \mathrm{o} & 0 & -2.738013 & 2.454167 & 0.163839 & 0 & \mathrm{~m} \\ 32 & \mathrm{~h} & 0 & -3.019099 & 3.365570 & -0.003878 & 0 & \mathrm{~m}\end{array}$

radical

Charge $=0$ Multiplicity $=2$ for low level calculation on real system.

Charge $=0$ Multiplicity $=2$ for high level calculation on model system .

Charge $=0$ Multiplicity $=2$ for low level calculation on model system.

\begin{tabular}{|c|c|c|c|c|c|c|c|c|}
\hline Center & Atomic & & Atomic Coordi & ates (Angstro & $\mathrm{ms})$ & & & \\
\hline Number & Numbe & & Type & $\mathrm{Y}$ & $\mathrm{Z}$ & & & \\
\hline 1 & $\mathrm{c}$ & 0 & 3.414321 & -1.771170 & -0.331716 & 0 & $\mathrm{~h}$ & \\
\hline 2 & $\mathrm{n}$ & 0 & 2.117352 & -1.715014 & -0.514619 & 0 & $\mathrm{~h}$ & \\
\hline 3 & $\mathrm{c}$ & 0 & 1.683429 & -0.457908 & -0.278489 & 0 & $\mathrm{~h}$ & \\
\hline 4 & $\mathrm{c}$ & 0 & 2.478728 & 0.627367 & 0.112427 & 0 & $\mathrm{~h}$ & \\
\hline 5 & $\mathrm{c}$ & 0 & 3.858544 & 0.375705 & 0.268154 & 0 & $\mathrm{~h}$ & \\
\hline 6 & $\mathrm{n}$ & 0 & 4.308265 & -0.878559 & 0.024715 & 0 & $\mathrm{~h}$ & \\
\hline 7 & $\mathrm{n}$ & 0 & 0.404358 & 0.045303 & -0.366855 & 0 & $\mathrm{~m}$ & h 3 \\
\hline 8 & $\mathrm{n}$ & 0 & 1.717411 & 1.775153 & 0.257208 & 0 & $\mathrm{~m}$ & h 4 \\
\hline 9 & $\mathrm{n}$ & 0 & 4.734867 & 1.326855 & 0.673646 & 0 & $\mathrm{~m}$ & $\mathrm{~h}$ \\
\hline 10 & $\mathrm{~h}$ & 0 & 4.436001 & 2.291040 & 0.671438 & 0 & $\mathrm{~m}$ & \\
\hline 11 & $\mathrm{~h}$ & 0 & 5.720683 & 1.125289 & 0.590729 & 0 & $\mathrm{~m}$ & \\
\hline 12 & $\mathrm{c}$ & 0 & 0.497498 & 1.390864 & -0.035362 & 0 & $\mathrm{~m}$ & \\
\hline 13 & $\mathrm{~h}$ & 0 & -0.381583 & 2.020953 & -0.044587 & 0 & $\mathrm{~m}$ & \\
\hline 14 & $\mathrm{c}$ & 0 & -0.788307 & -0.716781 & -0.742796 & 0 & $\mathrm{~m}$ & \\
\hline 15 & $\mathrm{~h}$ & 0 & -0.471451 & -1.479413 & -1.459433 & 0 & $\mathrm{~m}$ & \\
\hline 16 & $\mathrm{c}$ & 0 & -1.491994 & -1.382138 & 0.455213 & 0 & $\mathrm{~m}$ & \\
\hline 17 & $\mathrm{~h}$ & 0 & -0.786162 & -1.587498 & 1.272054 & 0 & $\mathrm{~m}$ & \\
\hline 18 & o & 0 & -2.113937 & -2.565455 & -0.001932 & 0 & $\mathrm{~m}$ & \\
\hline 19 & $\mathrm{~h}$ & 0 & -2.955046 & -2.624789 & 0.488660 & 0 & $\mathrm{~m}$ & \\
\hline 20 & $\mathrm{c}$ & 0 & -2.573027 & -0.342850 & 0.814361 & 0 & $\mathrm{~m}$ & \\
\hline 21 & $\mathrm{~h}$ & 0 & -2.169489 & 0.454623 & 1.445767 & 0 & $\mathrm{~m}$ & \\
\hline 22 & o & 0 & -3.724835 & -0.940864 & 1.406272 & 0 & $\mathrm{~m}$ & \\
\hline 23 & $\mathrm{~h}$ & 0 & -3.601998 & -0.952987 & 2.367897 & 0 & $\mathrm{~m}$ & \\
\hline 24 & $\mathrm{c}$ & 0 & -2.942250 & 0.211721 & -0.568244 & 0 & $\mathrm{~m}$ & \\
\hline 25 & $\mathrm{~h}$ & 0 & -3.684561 & -0.458365 & -1.024275 & 0 & $\mathrm{~m}$ & \\
\hline 26 & o & 0 & -1.727570 & 0.167869 & -1.329753 & 0 & $\mathrm{~m}$ & \\
\hline 27 & $\mathrm{c}$ & 0 & -3.505503 & 1.621366 & -0.575733 & 0 & $\mathrm{~m}$ & \\
\hline 28 & $\mathrm{~h}$ & 0 & -4.522586 & 1.583220 & -0.156706 & 0 & $\mathrm{~m}$ & \\
\hline
\end{tabular}




$\begin{array}{llllllll}29 & \mathrm{~h} & 0 & -3.572934 & 1.965435 & -1.617114 & 0 & \mathrm{~m} \\ 30 & \mathrm{o} & 0 & -2.663287 & 2.471072 & 0.198683 & 0 & \mathrm{~m} \\ 31 & \mathrm{~h} & 0 & -2.938235 & 3.388297 & 0.053854 & 0 & \mathrm{~m}\end{array}$

\section{b6}

neutral molecule

Charge $=0$ Multiplicity $=1$ for low level calculation on real system.

Charge $=0$ Multiplicity $=1$ for high level calculation on model system.

Charge $=0$ Multiplicity $=1$ for low level calculation on model system.

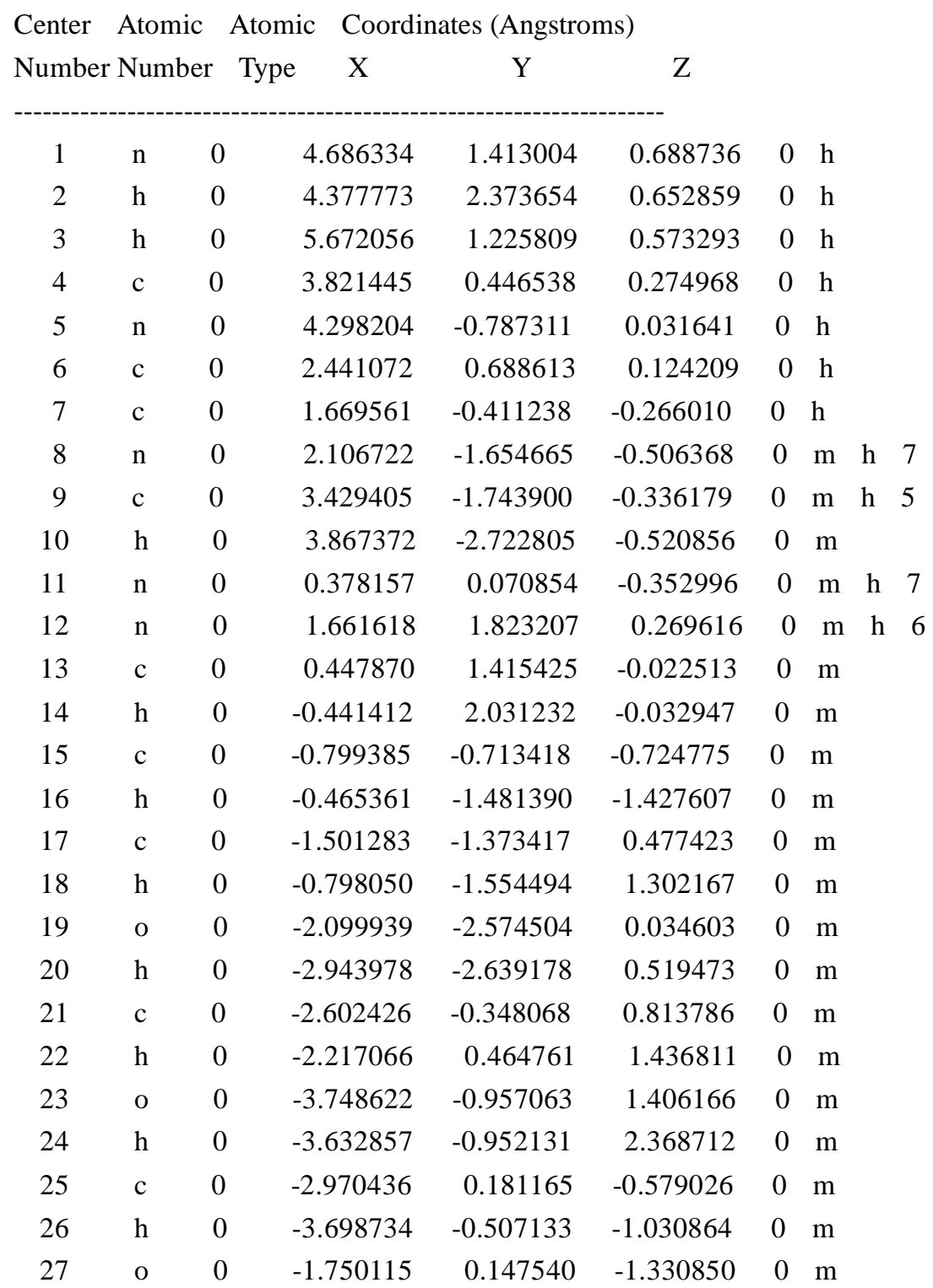




$\begin{array}{llllllll}28 & \mathrm{c} & 0 & -3.557101 & 1.581085 & -0.609381 & 0 & \mathrm{~m} \\ 29 & \mathrm{~h} & 0 & -4.578077 & 1.531274 & -0.201059 & 0 & \mathrm{~m} \\ 30 & \mathrm{~h} & 0 & -3.618599 & 1.911370 & -1.655569 & 0 & \mathrm{~m} \\ 31 & \mathrm{o} & 0 & -2.738016 & 2.454167 & 0.163839 & 0 & \mathrm{~m} \\ 32 & \mathrm{~h} & 0 & -3.019103 & 3.365569 & -0.003879 & 0 & \mathrm{~m}\end{array}$

radical

Charge $=0$ Multiplicity $=2$ for low level calculation on real system.

Charge $=0$ Multiplicity $=2$ for high level calculation on model system .

Charge $=0$ Multiplicity $=2$ for low level calculation on model system.

\begin{tabular}{|c|c|c|c|c|c|c|c|c|}
\hline Center & Atomic & & Atomic & ates (Angstro & $\mathrm{ms})$ & & & \\
\hline Number & Numbe & & Type & $\mathrm{Y}$ & $\mathrm{Z}$ & & & \\
\hline 1 & $\mathrm{n}$ & 0 & 4.697585 & 1.497732 & 0.676270 & 0 & $\mathrm{~h}$ & \\
\hline 2 & $\mathrm{~h}$ & 0 & 5.636688 & 1.079172 & 0.721512 & 0 & $\mathrm{~h}$ & \\
\hline 3 & $\mathrm{c}$ & 0 & 3.872508 & 0.529757 & 0.303471 & 0 & $\mathrm{~h}$ & \\
\hline 4 & $\mathrm{n}$ & 0 & 4.337078 & -0.753366 & 0.060829 & 0 & $\mathrm{~h}$ & \\
\hline 5 & $\mathrm{c}$ & 0 & 2.468566 & 0.756861 & 0.134091 & 0 & $\mathrm{~h}$ & \\
\hline 6 & $\mathrm{c}$ & 0 & 1.698567 & -0.370518 & -0.259728 & 0 & $\mathrm{~h}$ & \\
\hline 7 & $\mathrm{n}$ & 0 & 2.140191 & -1.596320 & -0.487561 & 0 & $\mathrm{~m}$ & h 6 \\
\hline 8 & $\mathrm{c}$ & 0 & 3.488356 & -1.693480 & -0.304902 & 0 & $\mathrm{~m}$ & h 4 \\
\hline 9 & $\mathrm{~h}$ & 0 & 3.905374 & -2.682171 & -0.485188 & 0 & $\mathrm{~m}$ & \\
\hline 10 & $\mathrm{n}$ & 0 & 0.400090 & 0.093036 & -0.352470 & 0 & $\mathrm{~m}$ & $\mathrm{~h}$ \\
\hline 11 & $\mathrm{n}$ & 0 & 1.673539 & 1.860704 & 0.269931 & 0 & $\mathrm{~m}$ & $\mathrm{~h}$ \\
\hline 12 & $\mathrm{c}$ & 0 & 0.459075 & 1.431857 & -0.028310 & 0 & $\mathrm{~m}$ & \\
\hline 13 & $\mathrm{~h}$ & 0 & -0.435482 & 2.041238 & -0.037632 & 0 & $\mathrm{~m}$ & \\
\hline 14 & $\mathrm{c}$ & 0 & -0.771826 & -0.700564 & -0.740370 & 0 & $\mathrm{~m}$ & \\
\hline 15 & $\mathrm{~h}$ & 0 & -0.426760 & -1.451450 & -1.456141 & 0 & $\mathrm{~m}$ & \\
\hline 16 & $\mathrm{c}$ & 0 & -1.464894 & -1.386869 & 0.451811 & 0 & $\mathrm{~m}$ & \\
\hline 17 & $\mathrm{~h}$ & 0 & -0.759827 & -1.574763 & 1.273765 & 0 & $\mathrm{~m}$ & \\
\hline 18 & o & 0 & -2.049615 & -2.584891 & -0.013090 & 0 & $\mathrm{~m}$ & \\
\hline 19 & $\mathrm{~h}$ & 0 & -2.896449 & -2.665667 & 0.464841 & 0 & $\mathrm{~m}$ & \\
\hline 20 & $\mathrm{c}$ & 0 & -2.575838 & -0.377185 & 0.807251 & 0 & $\mathrm{~m}$ & \\
\hline 21 & $\mathrm{~h}$ & 0 & -2.199556 & 0.422679 & 1.452254 & 0 & $\mathrm{~m}$ & \\
\hline 22 & o & 0 & -3.718111 & -1.010793 & 1.378602 & 0 & $\mathrm{~m}$ & \\
\hline 23 & $\mathrm{~h}$ & 0 & -3.614459 & -1.018823 & 2.342548 & 0 & $\mathrm{~m}$ & \\
\hline 24 & $\mathrm{c}$ & 0 & -2.944829 & 0.184076 & -0.573223 & 0 & $\mathrm{~m}$ & \\
\hline 25 & $\mathrm{~h}$ & 0 & -3.676123 & -0.490387 & -1.039600 & 0 & $\mathrm{~m}$ & \\
\hline 26 & o & 0 & -1.725653 & 0.161003 & -1.331261 & 0 & $\mathrm{~m}$ & \\
\hline 27 & $\mathrm{c}$ & 0 & -3.521677 & 1.588301 & -0.570275 & 0 & $\mathrm{~m}$ & \\
\hline 28 & $\mathrm{~h}$ & 0 & -4.537277 & 1.538074 & -0.149310 & 0 & $\mathrm{~m}$ & \\
\hline
\end{tabular}




$\begin{array}{llllllll}29 & \mathrm{~h} & 0 & -3.594006 & 1.940214 & -1.608570 & 0 & \mathrm{~m} \\ 30 & \mathrm{o} & 0 & -2.683791 & 2.437475 & 0.209399 & 0 & \mathrm{~m} \\ 31 & \mathrm{~h} & 0 & -2.972189 & 3.353630 & 0.084443 & 0 & \mathrm{~m}\end{array}$

\section{b8}

neutral molecule

Charge $=0$ Multiplicity $=1$ for low level calculation on real system.

Charge $=0$ Multiplicity $=1$ for high level calculation on model system.

Charge $=0$ Multiplicity $=1$ for low level calculation on model system.

\begin{tabular}{|c|c|c|c|c|c|c|c|c|}
\hline Center & Atomic & & Atomic & ates (Angstro & ms) & & & \\
\hline Number & Numbe & & Type & $\mathrm{Y}$ & Z & & & \\
\hline 1 & $\mathrm{c}$ & 0 & 0.447879 & 1.415414 & -0.022497 & 0 & $\mathrm{~h}$ & \\
\hline 2 & $\mathrm{~h}$ & 0 & -0.441405 & 2.031218 & -0.032926 & 0 & $\mathrm{~h}$ & \\
\hline 3 & $\mathrm{n}$ & 0 & 1.661605 & 1.823182 & 0.269599 & & $\mathrm{~h}$ & \\
\hline 4 & $\mathrm{c}$ & 0 & 2.441075 & 0.688598 & 0.124197 & 0 & $\mathrm{~h}$ & \\
\hline 5 & $\mathrm{c}$ & 0 & 1.669566 & -0.411246 & -0.266002 & & $\mathrm{~h}$ & \\
\hline 6 & $\mathrm{n}$ & 0 & 0.378159 & 0.070847 & -0.352993 & & $\mathrm{~h}$ & \\
\hline 7 & $\mathrm{c}$ & 0 & -0.799386 & -0.713417 & -0.724778 & & $\mathrm{~h}$ & \\
\hline 8 & $\mathrm{~h}$ & 0 & -0.465364 & -1.481387 & -1.427614 & & $\mathrm{~h}$ & \\
\hline 9 & $\mathrm{c}$ & 0 & 3.821452 & 0.446544 & 0.274957 & 0 & $\mathrm{~m}$ & $\mathrm{~h}$ \\
\hline 10 & $\mathrm{n}$ & 0 & 2.106701 & -1.654671 & -0.506355 & 0 & $\mathrm{~m}$ & h 5 \\
\hline 11 & $\mathrm{c}$ & 0 & -1.501288 & -1.373421 & 0.477415 & 0 & $\mathrm{~m}$ & h 7 \\
\hline 12 & $\mathrm{~h}$ & 0 & -0.798058 & -1.554506 & 1.302158 & 0 & $\mathrm{~m}$ & \\
\hline 13 & o & 0 & -1.750111 & 0.147549 & -1.330849 & 0 & $\mathrm{~m} \quad 1$ & h 7 \\
\hline 14 & $\mathrm{n}$ & 0 & 4.686326 & 1.413023 & 0.688723 & & $\mathrm{~m}$ & \\
\hline 15 & $\mathrm{~h}$ & 0 & 4.377745 & 2.373667 & 0.652841 & & $\mathrm{~m}$ & \\
\hline 16 & $\mathrm{~h}$ & 0 & 5.672051 & 1.225842 & 0.573280 & 0 & $\mathrm{~m}$ & \\
\hline 17 & $\mathrm{n}$ & 0 & 4.298211 & -0.787309 & 0.031647 & 0 & $\mathrm{~m}$ & \\
\hline 18 & $\mathrm{c}$ & 0 & 3.429411 & -1.743905 & -0.336154 & 0 & $\mathrm{~m}$ & \\
\hline 19 & $\mathrm{~h}$ & 0 & 3.867378 & -2.722812 & -0.520818 & 0 & $\mathrm{~m}$ & \\
\hline 20 & o & 0 & -2.099950 & -2.574502 & 0.034587 & 0 & $\mathrm{~m}$ & \\
\hline 21 & $\mathrm{~h}$ & 0 & -2.943990 & -2.639175 & 0.519456 & 0 & $\mathrm{~m}$ & \\
\hline 22 & $\mathrm{c}$ & 0 & -2.602427 & -0.348068 & 0.813783 & 0 & $\mathrm{~m}$ & \\
\hline 23 & $\mathrm{~h}$ & 0 & -2.217064 & 0.464756 & 1.436813 & 0 & $\mathrm{~m}$ & \\
\hline 24 & o & 0 & -3.748626 & -0.957061 & 1.406157 & 0 & $\mathrm{~m}$ & \\
\hline 25 & $\mathrm{~h}$ & 0 & -3.632862 & -0.952136 & 2.368704 & 0 & $\mathrm{~m}$ & \\
\hline 26 & $\mathrm{c}$ & 0 & -2.970433 & 0.181175 & -0.579027 & 0 & $\mathrm{~m}$ & \\
\hline 27 & $\mathrm{~h}$ & 0 & -3.698734 & -0.507117 & -1.030869 & 0 & $\mathrm{~m}$ & \\
\hline
\end{tabular}




$\begin{array}{llllllll}28 & \mathrm{c} & 0 & -3.557091 & 1.581098 & -0.609374 & 0 & \mathrm{~m} \\ 29 & \mathrm{~h} & 0 & -4.578067 & 1.531289 & -0.201054 & 0 & \mathrm{~m} \\ 30 & \mathrm{~h} & 0 & -3.618586 & 1.911389 & -1.655560 & 0 & \mathrm{~m} \\ 31 & \mathrm{o} & 0 & -2.738002 & 2.454172 & 0.163852 & 0 & \mathrm{~m} \\ 32 & \mathrm{~h} & 0 & -3.019085 & 3.365576 & -0.003860 & 0 & \mathrm{~m}\end{array}$

radical

Charge $=0$ Multiplicity $=2$ for low level calculation on real system.

Charge $=0$ Multiplicity $=2$ for high level calculation on model system .

Charge $=0$ Multiplicity $=2$ for low level calculation on model system.

\begin{tabular}{|c|c|c|c|c|c|c|c|c|}
\hline Center & Atomic & & Atomic & ates (Angstro & & & & \\
\hline Number & Numbe & & Type & $\mathrm{Y}$ & $\mathrm{Z}$ & & & \\
\hline 1 & $\mathrm{c}$ & 0 & 0.427395 & 1.350417 & -0.021886 & & $\mathrm{~h}$ & \\
\hline 2 & $\mathrm{n}$ & 0 & 1.592299 & 1.785981 & 0.257266 & 0 & $\mathrm{~h}$ & \\
\hline 3 & $\mathrm{c}$ & 0 & 2.413734 & 0.656840 & 0.124721 & 0 & $\mathrm{~h}$ & \\
\hline 4 & $\mathrm{c}$ & 0 & 1.648814 & -0.456149 & -0.244975 & 0 & $\mathrm{~h}$ & \\
\hline 5 & $\mathrm{n}$ & 0 & 0.336895 & 0.015296 & -0.333387 & 0 & $\mathrm{~h}$ & \\
\hline 6 & $\mathrm{c}$ & 0 & -0.857500 & -0.733455 & -0.728679 & & $\mathrm{~h}$ & \\
\hline 7 & $\mathrm{~h}$ & 0 & -0.538513 & -1.500041 & -1.439793 & 0 & $\mathrm{~h}$ & \\
\hline 8 & $\mathrm{c}$ & 0 & 3.795646 & 0.440055 & 0.275258 & 0 & $\mathrm{~m}$ & h 3 \\
\hline 9 & $\mathrm{n}$ & 0 & 2.095803 & -1.692071 & -0.470100 & 0 & $\mathrm{~m}$ & \\
\hline 10 & $\mathrm{c}$ & 0 & -1.593810 & -1.376034 & 0.459821 & 0 & $\mathrm{~m}$ & h 6 \\
\hline 11 & $\mathrm{~h}$ & 0 & -0.902149 & -1.602464 & 1.283653 & 0 & $\mathrm{~m}$ & \\
\hline 12 & o & 0 & -1.761490 & 0.173242 & -1.333159 & 0 & $\mathrm{~m}$ & h 6 \\
\hline 13 & $\mathrm{n}$ & 0 & 4.656179 & 1.414770 & 0.668377 & 0 & $\mathrm{~m}$ & \\
\hline 14 & $\mathrm{~h}$ & 0 & 4.350153 & 2.376134 & 0.642898 & 0 & $\mathrm{~m}$ & \\
\hline 15 & $\mathrm{~h}$ & 0 & 5.643567 & 1.224127 & 0.576121 & 0 & $\mathrm{~m}$ & \\
\hline 16 & $\mathrm{n}$ & 0 & 4.279328 & -0.796178 & 0.046050 & 0 & $\mathrm{~m}$ & \\
\hline 17 & $\mathrm{c}$ & 0 & 3.422368 & -1.766205 & -0.304726 & 0 & $\mathrm{~m}$ & \\
\hline 18 & $\mathrm{~h}$ & 0 & 3.869379 & -2.742653 & -0.477908 & 0 & $\mathrm{~m}$ & \\
\hline 19 & o & 0 & -2.245447 & -2.539879 & -0.002876 & 0 & $\mathrm{~m}$ & \\
\hline 20 & $\mathrm{~h}$ & 0 & -3.102574 & -2.560315 & 0.463251 & 0 & $\mathrm{~m}$ & \\
\hline 21 & $\mathrm{c}$ & 0 & -2.644304 & -0.301269 & 0.808296 & 0 & $\mathrm{~m}$ & \\
\hline 22 & $\mathrm{~h}$ & 0 & -2.223983 & 0.478768 & 1.451645 & 0 & $\mathrm{~m}$ & \\
\hline 23 & o & 0 & -3.823665 & -0.868025 & 1.375751 & 0 & $\mathrm{~m}$ & \\
\hline 24 & $\mathrm{~h}$ & 0 & -3.730561 & -0.866681 & 2.340681 & 0 & $\mathrm{~m}$ & \\
\hline 25 & $\mathrm{c}$ & 0 & -2.975685 & 0.290611 & -0.566301 & 0 & $\mathrm{~m}$ & \\
\hline 26 & $\mathrm{~h}$ & 0 & -3.763911 & -0.311620 & -1.037764 & 0 & $\mathrm{~m}$ & \\
\hline 27 & $\mathrm{c}$ & 0 & -3.389225 & 1.758373 & -0.545279 & 0 & $\mathrm{~m}$ & \\
\hline 28 & $\mathrm{~h}$ & 0 & -4.338062 & 1.846244 & -0.005566 & 0 & $\mathrm{~m}$ & \\
\hline
\end{tabular}




$\begin{array}{cccccccc}29 & \mathrm{~h} & 0 & -3.551942 & 2.099311 & -1.578476 & 0 & \mathrm{~m} \\ 30 & \mathrm{o} & 0 & -2.458426 & 2.581415 & 0.135213 & 0 & \mathrm{~m} \\ 31 & \mathrm{~h} & 0 & -1.607302 & 2.511480 & -0.333102 & 0 & \mathrm{~m}\end{array}$

\section{rC}

\section{s1}

neutral molecule

Charge $=0$ Multiplicity $=1$ for low level calculation on real system.

Charge $=0$ Multiplicity $=1$ for high level calculation on model system.

Charge $=0$ Multiplicity $=1$ for low level calculation on model system .

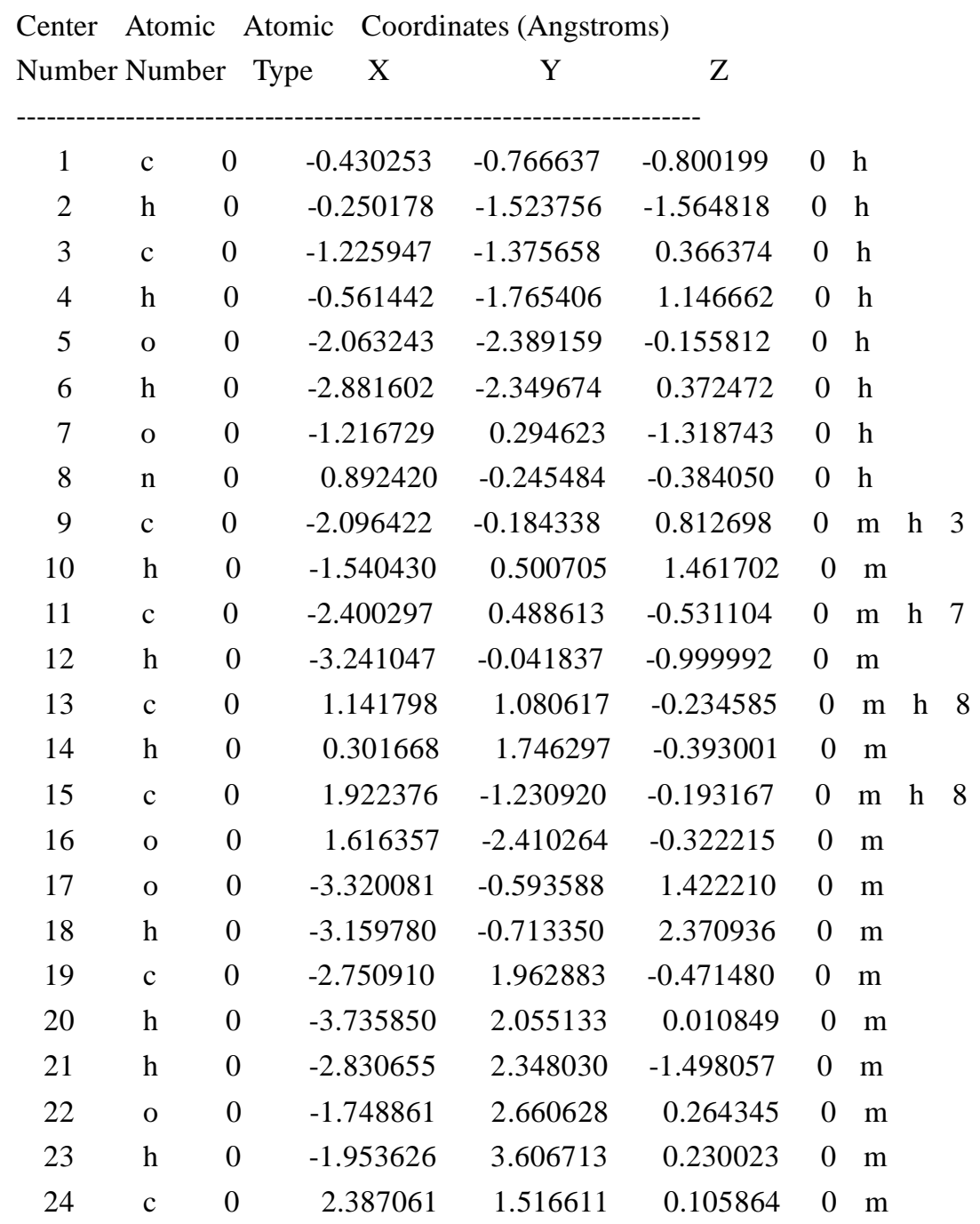




$\begin{array}{llllllll}25 & \mathrm{~h} & 0 & 2.591698 & 2.571893 & 0.241735 & 0 & \mathrm{~m} \\ 26 & \mathrm{c} & 0 & 3.397179 & 0.514699 & 0.268236 & 0 & \mathrm{~m} \\ 27 & \mathrm{n} & 0 & 4.664083 & 0.885670 & 0.631478 & 0 & \mathrm{~m} \\ 28 & \mathrm{~h} & 0 & 4.967212 & 1.832631 & 0.458246 & 0 & \mathrm{~m} \\ 29 & \mathrm{~h} & 0 & 5.367504 & 0.163394 & 0.550514 & 0 & \mathrm{~m} \\ 30 & \mathrm{n} & 0 & 3.174849 & -0.777888 & 0.118089 & 0 & \mathrm{~m}\end{array}$

radical

Charge $=0$ Multiplicity $=2$ for low level calculation on real system.

Charge $=0$ Multiplicity $=2$ for high level calculation on model system .

Charge $=0$ Multiplicity $=2$ for low level calculation on model system .

\begin{tabular}{|c|c|c|c|c|c|c|c|c|}
\hline Center & Atomic & & Atomic & ates (Angstro & ms) & & & \\
\hline Number & Numbe & & Type & $\mathrm{Y}$ & Z & & & \\
\hline 1 & $\mathrm{c}$ & 0 & 0.334135 & 0.040933 & 0.126385 & 0 & $\mathrm{~h}$ & \\
\hline 2 & $\mathrm{c}$ & 0 & 1.118274 & 1.307173 & 0.009036 & 0 & $\mathrm{~h}$ & \\
\hline 3 & $\mathrm{~h}$ & 0 & 0.837244 & 2.046170 & 0.760550 & 0 & $\mathrm{~h}$ & \\
\hline 4 & o & 0 & 0.983826 & 1.844035 & -1.314438 & 0 & $\mathrm{~h}$ & \\
\hline 5 & $\mathrm{~h}$ & 0 & 1.859745 & 2.220712 & -1.523348 & 0 & $\mathrm{~h}$ & \\
\hline 6 & o & 0 & 1.066566 & -1.040846 & -0.297220 & 0 & $\mathrm{~h}$ & \\
\hline 7 & $\mathrm{n}$ & 0 & -1.042711 & -0.112144 & 0.014112 & 0 & $\mathrm{~h}$ & \\
\hline 8 & $\mathrm{c}$ & 0 & 2.543803 & 0.737163 & 0.177262 & 0 & $\mathrm{~m}$ & h 2 \\
\hline 9 & $\mathrm{~h}$ & 0 & 2.785189 & 0.594691 & 1.235004 & 0 & $\mathrm{~m}$ & \\
\hline 10 & $\mathrm{c}$ & 0 & 2.432041 & -0.625872 & -0.521287 & 0 & $\mathrm{~m} \quad 1$ & h 6 \\
\hline 11 & $\mathrm{~h}$ & 0 & 2.573409 & -0.480293 & -1.599742 & 0 & $\mathrm{~m}$ & \\
\hline 12 & $\mathrm{c}$ & 0 & -1.586556 & -1.183191 & -0.641778 & 0 & $\mathrm{~m} \quad \mathrm{r}$ & h 7 \\
\hline 13 & $\mathrm{~h}$ & 0 & -0.879344 & -1.859852 & -1.102178 & 0 & $\mathrm{~m}$ & \\
\hline 14 & $\mathrm{c}$ & 0 & -1.913683 & 0.847873 & 0.711061 & 0 & $\mathrm{~m}$ & h 7 \\
\hline 15 & o & 0 & -1.382597 & 1.742112 & 1.350205 & 0 & $\mathrm{~m}$ & \\
\hline 16 & o & 0 & 3.535119 & 1.517480 & -0.486671 & 0 & $\mathrm{~m}$ & \\
\hline 17 & $\mathrm{~h}$ & 0 & 3.859676 & 2.184243 & 0.137804 & 0 & $\mathrm{~m}$ & \\
\hline 18 & $\mathrm{c}$ & 0 & 3.385590 & -1.699052 & -0.034567 & 0 & $\mathrm{~m}$ & \\
\hline 19 & $\mathrm{~h}$ & 0 & 4.393627 & -1.429988 & -0.388971 & 0 & $\mathrm{~m}$ & \\
\hline 20 & $\mathrm{~h}$ & 0 & 3.105969 & -2.656627 & -0.498936 & 0 & $\mathrm{~m}$ & \\
\hline 21 & o & 0 & 3.325388 & -1.766074 & 1.381672 & 0 & $\mathrm{~m}$ & \\
\hline 22 & $\mathrm{~h}$ & 0 & 3.867484 & -2.513816 & 1.672622 & 0 & $\mathrm{~m}$ & \\
\hline 23 & $\mathrm{c}$ & 0 & -2.937091 & -1.361515 & -0.700655 & 0 & $\mathrm{~m}$ & \\
\hline 24 & $\mathrm{~h}$ & 0 & -3.360602 & -2.217412 & -1.212645 & 0 & $\mathrm{~m}$ & \\
\hline 25 & $\mathrm{c}$ & 0 & -3.748733 & -0.374713 & -0.065339 & 0 & $\mathrm{~m}$ & \\
\hline 26 & $\mathrm{n}$ & 0 & -5.112960 & -0.502350 & -0.076132 & 0 & $\mathrm{~m}$ & \\
\hline 27 & $\mathrm{~h}$ & 0 & -5.546075 & -1.061327 & -0.796473 & 0 & $\mathrm{~m}$ & \\
\hline
\end{tabular}




$\begin{array}{llllllll}28 & \mathrm{~h} & 0 & -5.625057 & 0.314914 & 0.228733 & 0 & \mathrm{~m} \\ 29 & \mathrm{n} & 0 & -3.253523 & 0.664797 & 0.584661 & 0 & \mathrm{~m}\end{array}$

s2

neutral molecule

Charge $=0$ Multiplicity $=1$ for low level calculation on real system.

Charge $=0$ Multiplicity $=1$ for high level calculation on model system .

Charge $=0$ Multiplicity $=1$ for low level calculation on model system .

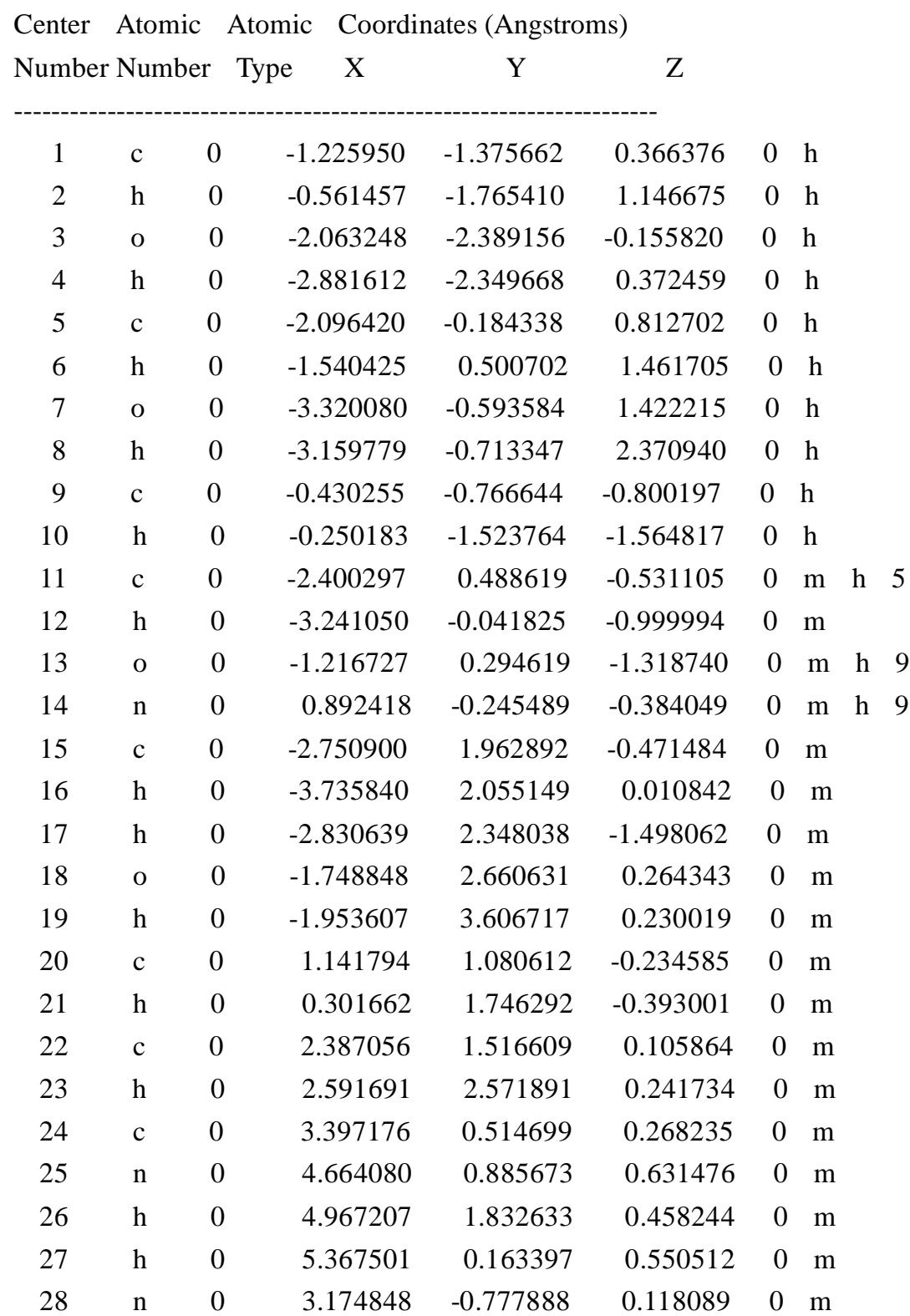




$\begin{array}{llllllll}29 & \text { c } & 0 & 1.922376 & -1.230923 & -0.193166 & 0 & \mathrm{~m} \\ 30 & \text { o } & 0 & 1.616358 & -2.410268 & -0.322213 & 0 & \mathrm{~m}\end{array}$

radical

Charge $=0$ Multiplicity $=2$ for low level calculation on real system.

Charge $=0$ Multiplicity $=2$ for high level calculation on model system .

Charge $=0$ Multiplicity $=2$ for low level calculation on model system .

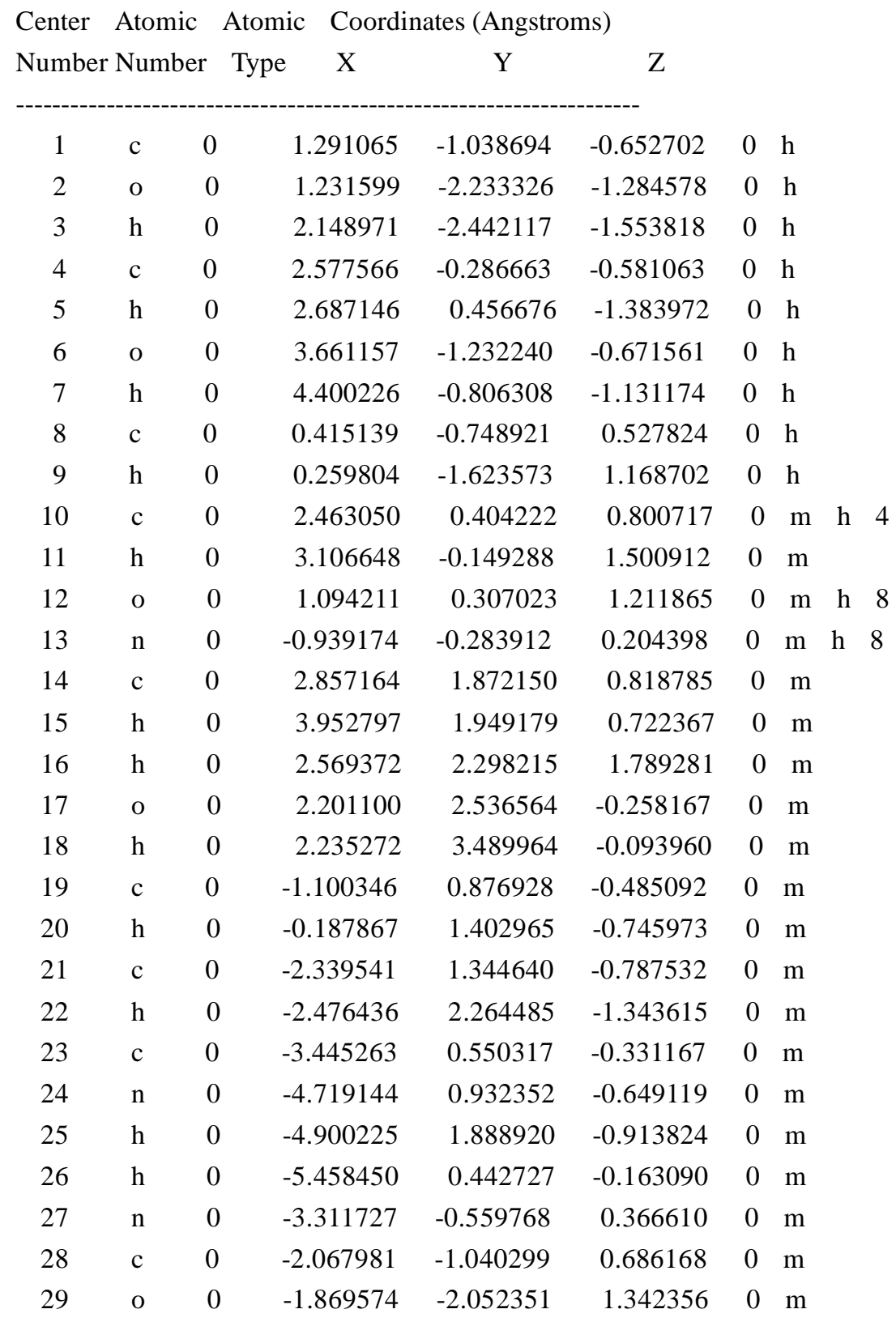




\section{s3}

neutral molecule

Charge $=0$ Multiplicity $=1$ for low level calculation on real system.

Charge $=0$ Multiplicity $=1$ for high level calculation on model system.

Charge $=0$ Multiplicity $=1$ for low level calculation on model system.

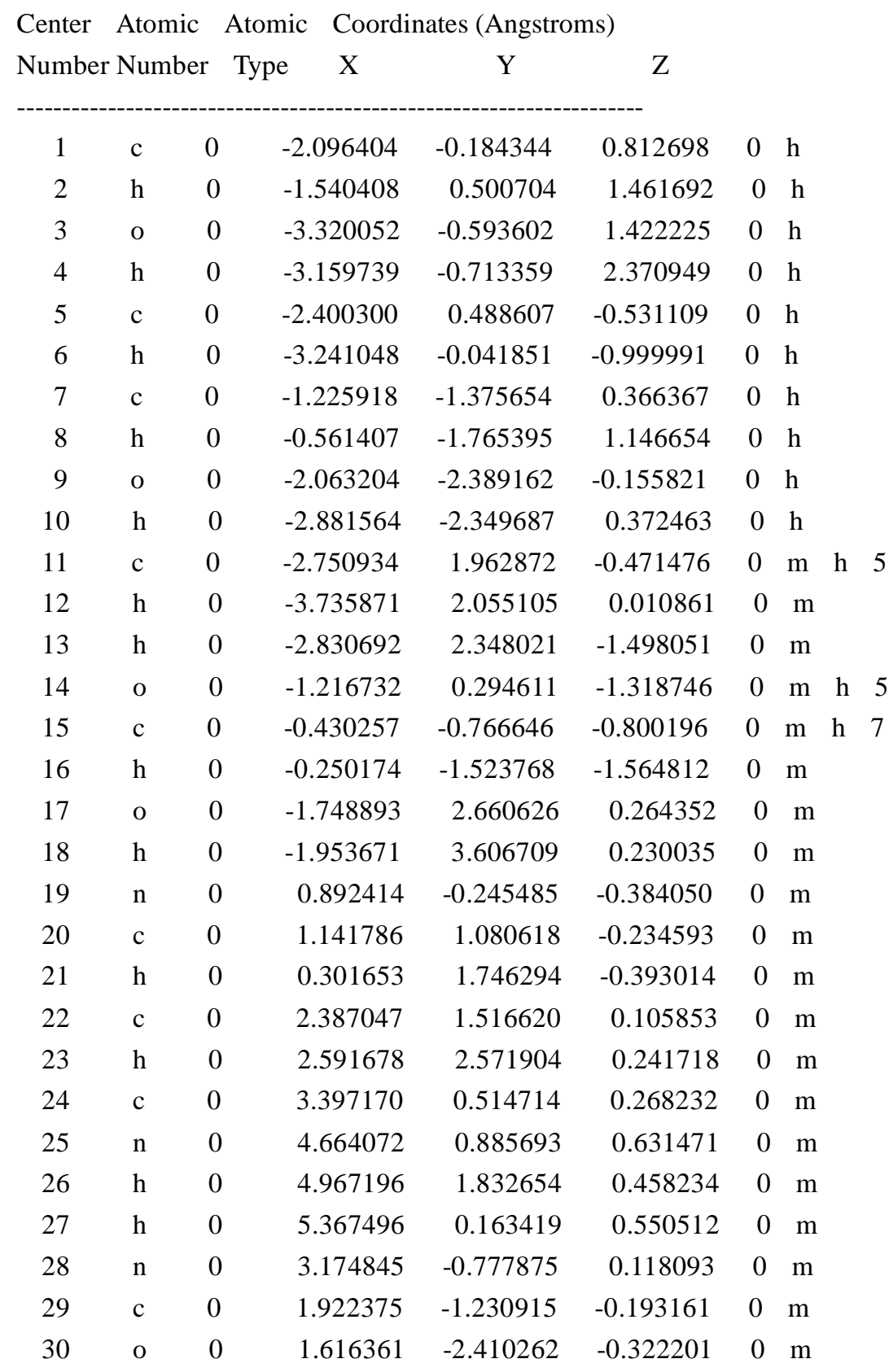


radical

Charge $=0$ Multiplicity $=2$ for low level calculation on real system.

Charge $=0$ Multiplicity $=2$ for high level calculation on model system .

Charge $=0$ Multiplicity $=2$ for low level calculation on model system.

Center Atomic Atomic Coordinates (Angstroms)

Number Number Type $\mathrm{X} \quad \mathrm{Y} \quad \mathrm{Z}$

\begin{tabular}{|c|c|c|c|c|c|c|c|}
\hline 1 & $\mathrm{c}$ & 0 & -2.152719 & -0.235499 & 0.721235 & 0 & $\mathrm{~h}$ \\
\hline 2 & o & 0 & -3.174297 & -0.329469 & 1.631014 & 0 & $\mathrm{~h}$ \\
\hline 3 & $\mathrm{~h}$ & 0 & -2.817137 & -0.666558 & 2.469185 & 0 & $\mathrm{~h}$ \\
\hline 4 & c & 0 & -2.442005 & 0.468571 & -0.569345 & 0 & h \\
\hline 5 & $\mathrm{~h}$ & 0 & -3.303627 & 0.001444 & -1.082286 & 0 & $\mathrm{~h}$ \\
\hline 6 & $\mathrm{c}$ & 0 & -1.246333 & -1.383793 & 0.402098 & 0 & $\mathrm{~h}$ \\
\hline 7 & $\mathrm{~h}$ & 0 & -0.579918 & -1.706903 & 1.210569 & 0 & $\mathrm{~h}$ \\
\hline 8 & o & 0 & -1.997740 & -2.498924 & -0.109085 & 0 & $\mathrm{~h}$ \\
\hline 9 & $\mathrm{~h}$ & 0 & -2.725779 & -2.664278 & 0.511049 & 0 & $\mathrm{~h}$ \\
\hline 10 & $\mathrm{c}$ & 0 & -2.746103 & 1.956969 & -0.485581 & 0 & $\mathrm{~m} \quad \mathrm{~h} \quad 4$ \\
\hline 11 & $\mathrm{~h}$ & 0 & -3.728929 & 2.067073 & -0.002075 & 0 & $\mathrm{~m}$ \\
\hline 12 & $\mathrm{~h}$ & 0 & -2.820027 & 2.352017 & -1.509489 & 0 & $\mathrm{~m}$ \\
\hline 13 & $\mathrm{o}$ & 0 & -1.262528 & 0.224997 & -1.356296 & 0 & $\mathrm{~m} \quad \mathrm{~h} \quad 4$ \\
\hline 14 & $\mathrm{c}$ & 0 & -0.460734 & -0.799699 & -0.785704 & 0 & $\mathrm{~m} h \quad \mathrm{~h}$ \\
\hline 15 & $\mathrm{~h}$ & 0 & -0.268000 & -1.584136 & -1.518760 & 0 & $\mathrm{~m}$ \\
\hline 16 & $\mathrm{o}$ & 0 & -1.729955 & 2.621552 & 0.255120 & 0 & $\mathrm{~m}$ \\
\hline 17 & $\mathrm{~h}$ & 0 & -1.909522 & 3.573073 & 0.220285 & 0 & $\mathrm{~m}$ \\
\hline 18 & $\mathrm{n}$ & 0 & 0.852291 & -0.253724 & -0.383995 & 0 & $\mathrm{~m}$ \\
\hline 19 & $\mathrm{c}$ & 0 & 1.072393 & 1.075335 & -0.219898 & 0 & $\mathrm{~m}$ \\
\hline 20 & $\mathrm{~h}$ & 0 & 0.216774 & 1.724848 & -0.360282 & 0 & $\mathrm{~m}$ \\
\hline 21 & $\mathrm{c}$ & 0 & 2.311782 & 1.532677 & 0.114588 & 0 & $\mathrm{~m}$ \\
\hline 22 & $\mathrm{~h}$ & 0 & 2.495757 & 2.590350 & 0.261143 & 0 & $\mathrm{~m}$ \\
\hline 23 & $\mathrm{c}$ & 0 & 3.343549 & 0.551053 & 0.258671 & 0 & $\mathrm{~m}$ \\
\hline 24 & $\mathrm{n}$ & 0 & 4.608347 & 0.945448 & 0.604674 & 0 & $\mathrm{~m}$ \\
\hline 25 & $\mathrm{~h}$ & 0 & 4.890310 & 1.898530 & 0.429482 & 0 & $\mathrm{~m}$ \\
\hline 26 & $\mathrm{~h}$ & 0 & 5.324287 & 0.237126 & 0.511484 & 0 & $\mathrm{~m}$ \\
\hline 27 & $\mathrm{n}$ & 0 & 3.144805 & -0.745830 & 0.108434 & 0 & $\mathrm{~m}$ \\
\hline 28 & $\mathrm{c}$ & 0 & 1.896828 & -1.220964 & -0.184317 & 0 & $\mathrm{~m}$ \\
\hline 29 & o & 0 & 1.605490 & -2.407123 & -0.290075 & 0 & $\mathrm{~m}$ \\
\hline
\end{tabular}

s4

neutral molecule 
Charge $=0$ Multiplicity $=1$ for low level calculation on real system .

Charge $=0$ Multiplicity $=1$ for high level calculation on model system .

Charge $=0$ Multiplicity $=1$ for low level calculation on model system .

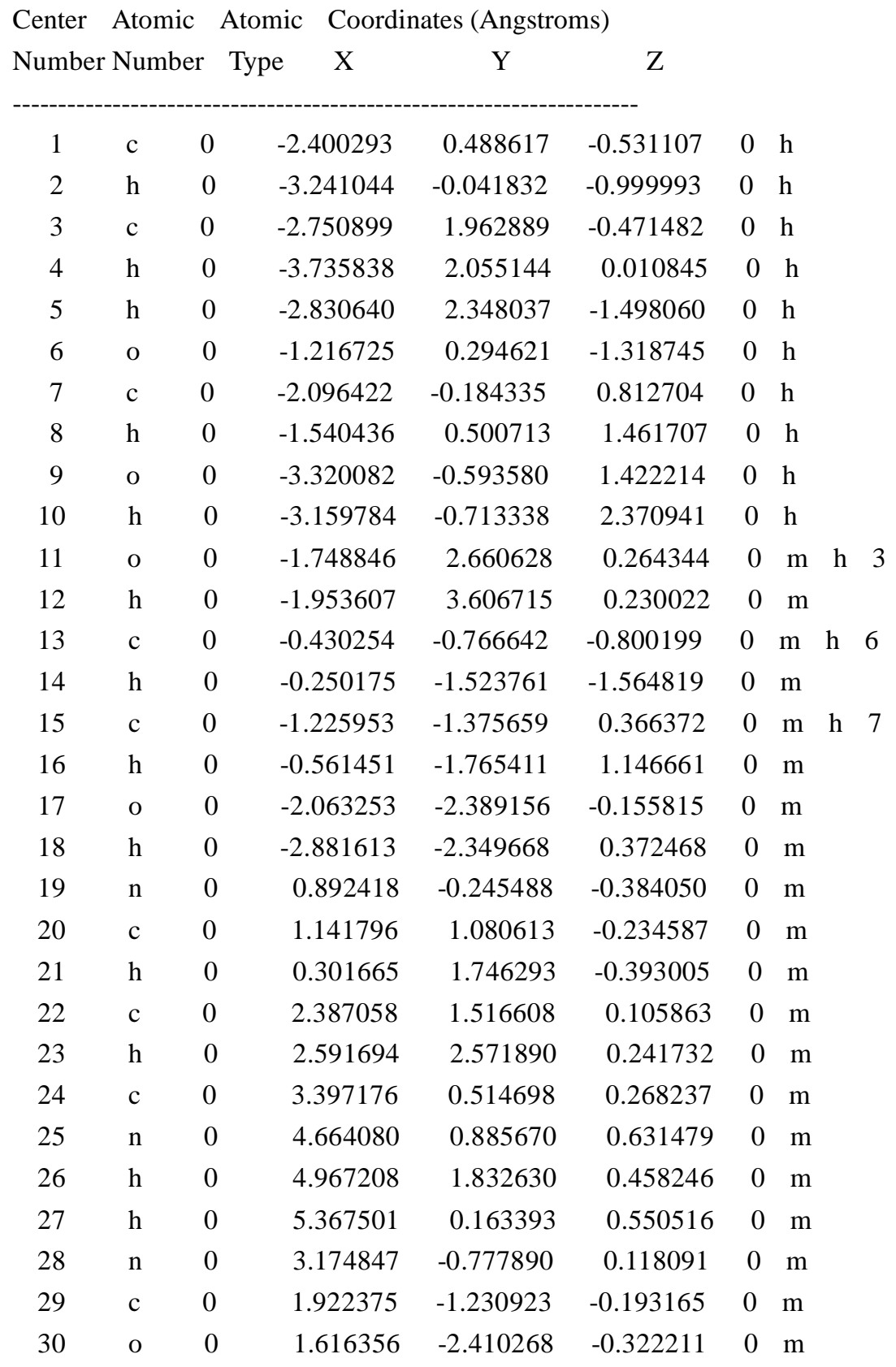

radical

Charge $=0$ Multiplicity $=2$ for low level calculation on real system.

Charge $=0$ Multiplicity $=2$ for high level calculation on model system .

Charge $=0$ Multiplicity $=2$ for low level calculation on model system. 


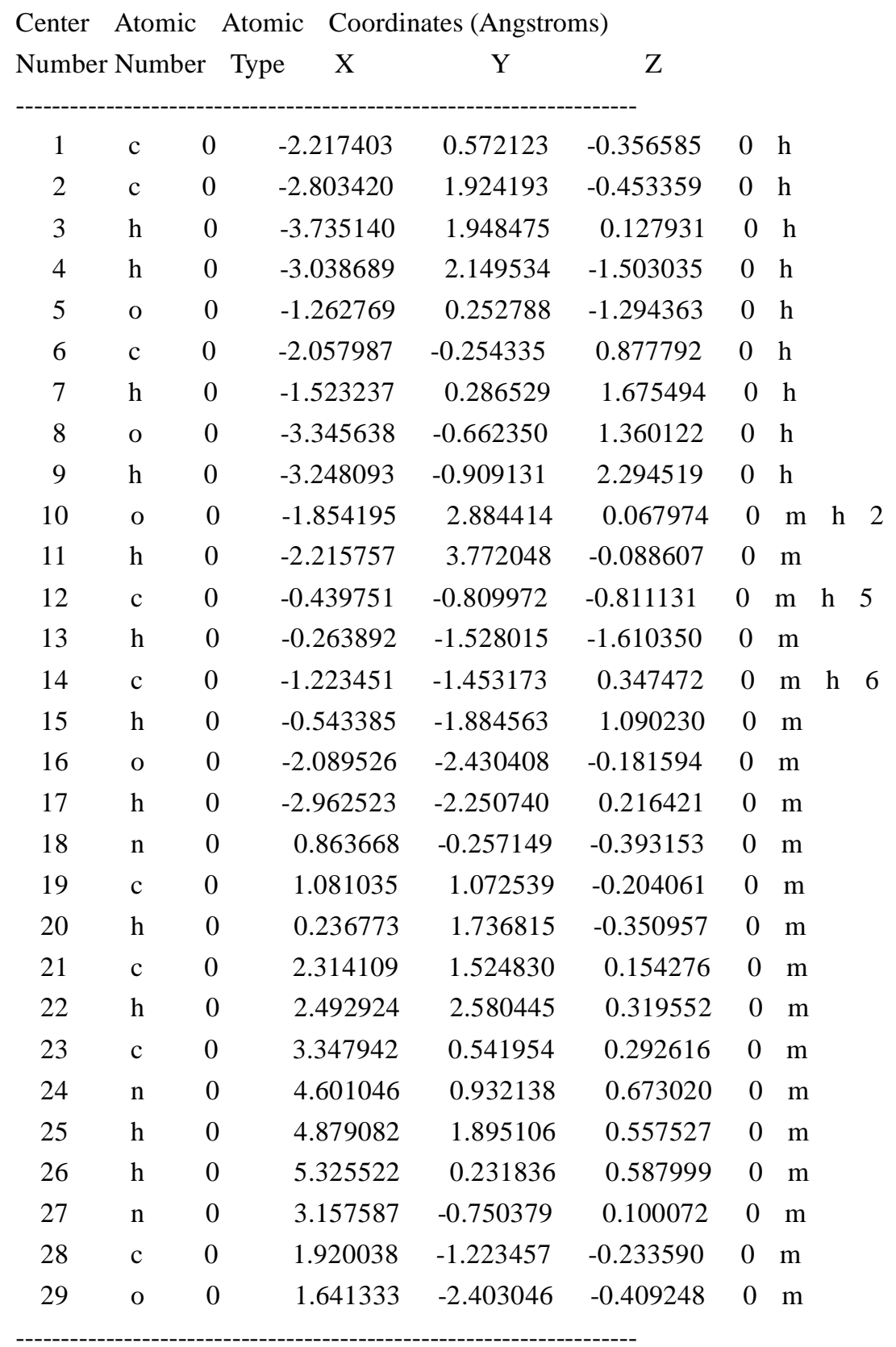

\section{s5}

neutral molecule

Charge $=0$ Multiplicity $=1$ for low level calculation on real system .

Charge $=0$ Multiplicity $=1$ for high level calculation on model system .

Charge $=0$ Multiplicity $=1$ for low level calculation on model system . 


\begin{tabular}{|c|c|c|c|c|c|c|c|c|c|}
\hline Center & Atomi & & Atomic & Coordi & lates (Angstro & & & & \\
\hline Number & Numb & & Type & $X$ & Y & $\mathrm{Z}$ & & & \\
\hline 1 & $\mathrm{c}$ & 0 & & 2.750900 & 1.962890 & -0.471482 & 0 & $\mathrm{~h}$ & \\
\hline 2 & $\mathrm{~h}$ & 0 & & 3.735841 & 2.055143 & 0.010843 & 0 & $\mathrm{~h}$ & \\
\hline 3 & $\mathrm{~h}$ & 0 & & 2.830631 & 2.348032 & -1.498063 & 0 & $\mathrm{~h}$ & \\
\hline 4 & o & 0 & & 1.748848 & 2.660629 & 0.264345 & 0 & $\mathrm{~h}$ & \\
\hline 5 & $\mathrm{~h}$ & 0 & & 1.953608 & 3.606715 & 0.230023 & 0 & $\mathrm{~h}$ & \\
\hline 6 & $\mathrm{c}$ & 0 & & 2.400294 & 0.488618 & -0.531107 & 0 & $\mathrm{~h}$ & \\
\hline 7 & $\mathrm{~h}$ & 0 & & 3.241045 & -0.041832 & -0.999993 & 0 & $\mathrm{~h}$ & \\
\hline 8 & o & 0 & & 1.216725 & 0.294622 & -1.318745 & 0 & $\mathrm{~h}$ & \\
\hline 9 & $\mathrm{c}$ & 0 & & 2.096423 & -0.184334 & 0.812704 & 0 & $\mathrm{~h}$ & \\
\hline 10 & $\mathrm{~h}$ & 0 & & 1.540437 & 0.500714 & 1.461707 & 0 & $\mathrm{~h}$ & \\
\hline 11 & $\mathrm{c}$ & 0 & & 0.430254 & -0.766642 & -0.800200 & 0 & $\mathrm{~m}$ & h 8 \\
\hline 12 & $\mathrm{~h}$ & 0 & & 0.250174 & -1.523760 & -1.564819 & 0 & $\mathrm{~m}$ & \\
\hline 13 & $\mathrm{c}$ & 0 & & 1.225952 & -1.375660 & 0.366372 & 0 & $\mathrm{~m}$ & h 9 \\
\hline 14 & $\mathrm{~h}$ & 0 & & 0.561450 & -1.765411 & 1.146661 & 0 & $\mathrm{~m}$ & \\
\hline 15 & o & 0 & & 3.320083 & -0.593581 & 1.422215 & 0 & $\mathrm{~m}$ & $\mathrm{~h}$ \\
\hline 16 & $\mathrm{~h}$ & 0 & & 3.159782 & -0.713348 & 2.370940 & 0 & $\mathrm{~m}$ & \\
\hline 17 & o & 0 & & 2.063252 & -2.389157 & -0.155815 & 0 & $\mathrm{~m}$ & \\
\hline 18 & $\mathrm{~h}$ & 0 & & 2.881613 & -2.349668 & 0.372468 & 0 & $\mathrm{~m}$ & \\
\hline 19 & $\mathrm{n}$ & 0 & & 0.892418 & -0.245488 & -0.384050 & 0 & $\mathrm{~m}$ & \\
\hline 20 & $\mathrm{c}$ & 0 & & 1.141796 & 1.080613 & -0.234587 & 0 & $\mathrm{~m}$ & \\
\hline 21 & $\mathrm{~h}$ & 0 & & 0.301665 & 1.746293 & -0.393005 & 0 & $\mathrm{~m}$ & \\
\hline 22 & $\mathrm{c}$ & 0 & & 2.387058 & 1.516608 & 0.105863 & 0 & $\mathrm{~m}$ & \\
\hline 23 & $\mathrm{~h}$ & 0 & & 2.591693 & 2.571891 & 0.241733 & 0 & $\mathrm{~m}$ & \\
\hline 24 & $\mathrm{c}$ & 0 & & 3.397177 & 0.514698 & 0.268237 & 0 & $\mathrm{~m}$ & \\
\hline 25 & $\mathrm{n}$ & 0 & & 4.664080 & 0.885671 & 0.631479 & 0 & $\mathrm{~m}$ & \\
\hline 26 & $\mathrm{~h}$ & 0 & & 4.967208 & 1.832631 & 0.458246 & 0 & $\mathrm{~m}$ & \\
\hline 27 & $\mathrm{~h}$ & 0 & & 5.367501 & 0.163394 & 0.550517 & 0 & $\mathrm{~m}$ & \\
\hline 28 & $\mathrm{n}$ & 0 & & 3.174847 & -0.777889 & 0.118091 & 0 & $\mathrm{~m}$ & \\
\hline 29 & $\mathrm{c}$ & 0 & & 1.922376 & -1.230924 & -0.193165 & 0 & $\mathrm{~m}$ & \\
\hline 30 & o & 0 & & 1.616358 & -2.410268 & -0.322211 & 0 & $\mathrm{~m}$ & \\
\hline
\end{tabular}

radical

Charge $=0$ Multiplicity $=2$ for low level calculation on real system.

Charge $=0$ Multiplicity $=2$ for high level calculation on model system.

Charge $=0$ Multiplicity $=2$ for low level calculation on model system.

Center Atomic Atomic Coordinates (Angstroms)

Number Number Type $\quad \mathrm{X} \quad \mathrm{Y} \quad \mathrm{Z}$ 


\begin{tabular}{|c|c|c|c|c|c|c|c|}
\hline 1 & $\mathrm{c}$ & 0 & -2.745564 & 1.976839 & -0.439987 & 0 & $\mathrm{~h}$ \\
\hline 2 & $\mathrm{~h}$ & 0 & -3.774476 & 2.322186 & -0.478861 & 0 & $\mathrm{~h}$ \\
\hline 3 & o & 0 & -1.827589 & 2.762543 & 0.212180 & 0 & $\mathrm{~h}$ \\
\hline 4 & $\mathrm{~h}$ & 0 & -2.140098 & 3.679644 & 0.219289 & 0 & $\mathrm{~h}$ \\
\hline 5 & $\mathrm{c}$ & 0 & -2.420282 & 0.537347 & -0.518005 & 0 & $\mathrm{~h}$ \\
\hline 6 & $\mathrm{~h}$ & 0 & -3.267747 & 0.013997 & -0.977953 & 0 & $\mathrm{~h}$ \\
\hline 7 & o & 0 & -1.234462 & 0.325142 & -1.328974 & 0 & $\mathrm{~h}$ \\
\hline 8 & $\mathrm{c}$ & 0 & -2.088182 & -0.172311 & 0.814099 & 0 & $\mathrm{~h}$ \\
\hline 9 & $\mathrm{~h}$ & 0 & -1.505217 & 0.495675 & 1.458860 & 0 & $\mathrm{~h}$ \\
\hline 10 & $\mathrm{c}$ & 0 & -0.456081 & -0.745675 & -0.828448 & 0 & $\mathrm{~m}$ \\
\hline 11 & $\mathrm{~h}$ & 0 & -0.280276 & -1.491935 & -1.605331 & 0 & $\mathrm{~m}$ \\
\hline 12 & $\mathrm{c}$ & 0 & -1.247981 & -1.367367 & 0.332596 & 0 & $\mathrm{~m}$ \\
\hline 13 & $\mathrm{~h}$ & 0 & -0.582353 & -1.787106 & 1.096105 & 0 & $\mathrm{~m}$ \\
\hline 14 & $\mathrm{o}$ & 0 & -3.302273 & -0.570111 & 1.445868 & 0 & $\mathrm{~m}$ \\
\hline 15 & $\mathrm{~h}$ & 0 & -3.113795 & -0.738879 & 2.382035 & 0 & $\mathrm{~m}$ \\
\hline 16 & o & 0 & -2.109193 & -2.353742 & -0.203035 & 0 & $\mathrm{~m}$ \\
\hline 17 & $\mathrm{~h}$ & 0 & -2.919423 & -2.314074 & 0.337435 & 0 & $\mathrm{~m}$ \\
\hline 18 & $\mathrm{n}$ & 0 & 0.872106 & -0.236701 & -0.401846 & 0 & $\mathrm{~m}$ \\
\hline 19 & $\mathrm{c}$ & 0 & 1.146036 & 1.088385 & -0.302513 & 0 & $\mathrm{~m}$ \\
\hline 20 & $\mathrm{~h}$ & 0 & 0.324113 & 1.760557 & -0.514326 & 0 & $\mathrm{~m}$ \\
\hline 21 & $\mathrm{c}$ & 0 & 2.391733 & 1.516979 & 0.045467 & 0 & $\mathrm{~m}$ \\
\hline 22 & $\mathrm{~h}$ & 0 & 2.613686 & 2.573253 & 0.139668 & 0 & $\mathrm{~m}$ \\
\hline 23 & $\mathrm{c}$ & 0 & 3.378646 & 0.503983 & 0.270394 & 0 & $\mathrm{~m}$ \\
\hline 24 & $\mathrm{n}$ & 0 & 4.642503 & 0.865492 & 0.653151 & 0 & $\mathrm{~m}$ \\
\hline 25 & $\mathrm{~h}$ & 0 & 4.967169 & 1.800289 & 0.454574 & 0 & $\mathrm{~m}$ \\
\hline 26 & $\mathrm{~h}$ & 0 & 5.334488 & 0.128782 & 0.614691 & 0 & $\mathrm{~m}$ \\
\hline 27 & $\mathrm{n}$ & 0 & 3.136131 & -0.788925 & 0.159979 & 0 & $\mathrm{~m}$ \\
\hline 28 & $\mathrm{c}$ & 0 & 1.884242 & -1.231372 & -0.169373 & 0 & $\mathrm{~m}$ \\
\hline 29 & o & 0 & 1.565186 & -2.409118 & -0.279858 & 0 & $\mathrm{~m}$ \\
\hline
\end{tabular}

\section{b4}

neutral molecule

Charge $=0$ Multiplicity $=1$ for low level calculation on real system.

Charge $=0$ Multiplicity $=1$ for high level calculation on model system.

Charge $=0$ Multiplicity $=1$ for low level calculation on model system .

Center Atomic Atomic Coordinates (Angstroms)

Number Number Type $\quad \mathrm{X} \quad \mathrm{Y} \quad \mathrm{Z}$ 


\begin{tabular}{|c|c|c|c|c|c|c|c|c|}
\hline 1 & $\mathrm{n}$ & 0 & 4.664087 & 0.885675 & 0.631479 & 0 & $\mathrm{~h}$ & \\
\hline 2 & $\mathrm{~h}$ & 0 & 4.967215 & 1.832634 & 0.458245 & 0 & $\mathrm{~h}$ & \\
\hline 3 & $\mathrm{~h}$ & 0 & 5.367504 & 0.163394 & 0.550524 & 0 & $\mathrm{~h}$ & \\
\hline 4 & $\mathrm{c}$ & 0 & 3.397182 & 0.514705 & 0.268237 & 0 & $\mathrm{~h}$ & \\
\hline 5 & $\mathrm{n}$ & 0 & 3.174859 & -0.777883 & 0.118088 & 0 & $\mathrm{~h}$ & \\
\hline 6 & $\mathrm{c}$ & 0 & 2.387067 & 1.516619 & 0.105862 & 0 & $\mathrm{~h}$ & \\
\hline 7 & $\mathrm{~h}$ & 0 & 2.591701 & 2.571901 & 0.241732 & 0 & $\mathrm{~h}$ & \\
\hline 8 & $\mathrm{c}$ & 0 & 1.141803 & 1.080627 & -0.234587 & 0 & $\mathrm{~h}$ & \\
\hline 9 & $\mathrm{~h}$ & 0 & 0.301679 & 1.746313 & -0.393014 & 0 & $\mathrm{~h}$ & \\
\hline 10 & $\mathrm{c}$ & 0 & 1.922389 & -1.230922 & -0.193167 & 0 & $\mathrm{~m}$ & $\mathrm{~h}$ \\
\hline 11 & o & 0 & 1.616372 & -2.410267 & -0.322213 & 0 & $\mathrm{~m}$ & \\
\hline 12 & $\mathrm{n}$ & 0 & 0.892417 & -0.245479 & -0.384051 & 0 & $\mathrm{~m}$ & h \\
\hline 13 & $\mathrm{c}$ & 0 & -0.430254 & -0.766636 & -0.800200 & 0 & $\mathrm{~m}$ & \\
\hline 14 & $\mathrm{~h}$ & 0 & -0.250170 & -1.523748 & -1.564825 & 0 & $\mathrm{~m}$ & \\
\hline 15 & $\mathrm{c}$ & 0 & -1.225945 & -1.375661 & 0.366372 & 0 & $\mathrm{~m}$ & \\
\hline 16 & $\mathrm{~h}$ & 0 & -0.561438 & -1.765407 & 1.146661 & 0 & $\mathrm{~m}$ & \\
\hline 17 & o & 0 & -2.063237 & -2.389164 & -0.155814 & 0 & $\mathrm{~m}$ & \\
\hline 18 & $\mathrm{~h}$ & 0 & -2.881597 & -2.349683 & 0.372471 & 0 & $\mathrm{~m}$ & \\
\hline 19 & $\mathrm{c}$ & 0 & -2.096425 & -0.184344 & 0.812698 & 0 & $\mathrm{~m}$ & \\
\hline 20 & $\mathrm{~h}$ & 0 & -1.540435 & 0.500701 & 1.461702 & 0 & $\mathrm{~m}$ & \\
\hline 21 & o & 0 & -3.320082 & -0.593599 & 1.422209 & 0 & $\mathrm{~m}$ & \\
\hline 22 & $\mathrm{~h}$ & 0 & -3.159781 & -0.713362 & 2.370935 & 0 & $\mathrm{~m}$ & \\
\hline 23 & $\mathrm{c}$ & 0 & -2.400306 & 0.488611 & -0.531109 & 0 & $\mathrm{~m}$ & \\
\hline 24 & h & 0 & -3.241054 & -0.041839 & -0.999999 & 0 & $\mathrm{~m}$ & \\
\hline 25 & o & 0 & -1.216728 & 0.294626 & -1.318742 & 0 & $\mathrm{~m}$ & \\
\hline 26 & $\mathrm{c}$ & 0 & -2.750928 & 1.962879 & -0.471476 & 0 & $\mathrm{~m}$ & \\
\hline 27 & h & 0 & -3.735868 & 2.055119 & 0.010853 & 0 & $\mathrm{~m}$ & \\
\hline 28 & h & 0 & -2.830675 & 2.348031 & -1.498052 & 0 & $\mathrm{~m}$ & \\
\hline 29 & o & 0 & -1.748883 & 2.660626 & 0.264352 & 0 & $\mathrm{~m}$ & \\
\hline 30 & $\mathrm{~h}$ & 0 & -1.953654 & 3.606710 & 0.230036 & 0 & $\mathrm{~m}$ & \\
\hline
\end{tabular}

radical

Charge $=0$ Multiplicity $=2$ for low level calculation on real system.

Charge $=0$ Multiplicity $=2$ for high level calculation on model system.

Charge $=0$ Multiplicity $=2$ for low level calculation on model system.

\begin{tabular}{ccccccccc} 
Center & Atomic & Atomic & \multicolumn{2}{l}{ Coordinates (Angstroms) } \\
Number Number & Type & X & Y & Z & & \\
- & & & & & \\
1 & $\mathrm{n}$ & 0 & 4.685238 & 1.028921 & 0.606661 & 0 & $\mathrm{~h}$ \\
2 & $\mathrm{~h}$ & 0 & 5.280023 & 0.188383 & 0.644905 & 0 & $\mathrm{~h}$ \\
3 & $\mathrm{c}$ & 0 & 3.435564 & 0.586617 & 0.289467 & 0 & $\mathrm{~h}$
\end{tabular}




\begin{tabular}{|c|c|c|c|c|c|c|c|c|}
\hline 4 & $\mathrm{n}$ & 0 & 3.233345 & -0.725368 & 0.112980 & 0 & & \\
\hline 5 & $\mathrm{c}$ & 0 & 2.397627 & 1.564574 & 0.156820 & 0 & $\mathrm{~h}$ & \\
\hline 6 & $\mathrm{~h}$ & 0 & 2.608296 & 2.613911 & 0.312342 & 0 & $\mathrm{~h}$ & \\
\hline 7 & $\mathrm{c}$ & 0 & 1.156567 & 1.115589 & -0.192612 & 0 & $\mathrm{~h}$ & \\
\hline 8 & $\mathrm{~h}$ & 0 & 0.299361 & 1.762268 & -0.339265 & 0 & $\mathrm{~h}$ & \\
\hline 9 & $\mathrm{c}$ & 0 & 1.976135 & -1.194692 & -0.188844 & 0 & $\mathrm{~m}$ & h 4 \\
\hline 10 & o & 0 & 1.687416 & -2.377325 & -0.312055 & 0 & $\mathrm{~m}$ & \\
\hline 11 & $\mathrm{n}$ & 0 & 0.940859 & -0.214740 & -0.368870 & 0 & $\mathrm{~m}$ & h 7 \\
\hline 12 & $\mathrm{c}$ & 0 & -0.375672 & -0.753690 & -0.794078 & 0 & $\mathrm{~m}$ & \\
\hline 13 & $\mathrm{~h}$ & 0 & -0.171844 & -1.509824 & -1.554683 & 0 & $\mathrm{~m}$ & \\
\hline 14 & $\mathrm{c}$ & 0 & -1.167544 & -1.378267 & 0.368976 & 0 & $\mathrm{~m}$ & \\
\hline 15 & $\mathrm{~h}$ & 0 & -0.503303 & -1.749414 & 1.158899 & 0 & $\mathrm{~m}$ & \\
\hline 16 & o & 0 & -1.972087 & -2.411406 & -0.160896 & 0 & $\mathrm{~m}$ & \\
\hline 17 & $\mathrm{~h}$ & 0 & -2.800557 & -2.388193 & 0.352865 & 0 & $\mathrm{~m}$ & \\
\hline 18 & $\mathrm{c}$ & 0 & -2.070425 & -0.205144 & 0.803270 & 0 & $\mathrm{~m}$ & \\
\hline 19 & $\mathrm{~h}$ & 0 & -1.540625 & 0.487857 & 1.465556 & 0 & $\mathrm{~m}$ & \\
\hline 20 & o & 0 & -3.294262 & -0.644918 & 1.387765 & 0 & $\mathrm{~m}$ & \\
\hline 21 & $\mathrm{~h}$ & 0 & -3.157002 & -0.747574 & 2.342102 & 0 & $\mathrm{~m}$ & \\
\hline 22 & $\mathrm{c}$ & 0 & -2.366436 & 0.469265 & -0.541881 & 0 & $\mathrm{~m}$ & \\
\hline 23 & $\mathrm{~h}$ & 0 & -3.193327 & -0.069046 & -1.025038 & 0 & $\mathrm{~m}$ & \\
\hline 24 & o & 0 & -1.169272 & 0.292828 & -1.317161 & 0 & $\mathrm{~m}$ & \\
\hline 25 & $\mathrm{c}$ & 0 & -2.730473 & 1.940094 & -0.479871 & 0 & $\mathrm{~m}$ & \\
\hline 26 & $\mathrm{~h}$ & 0 & -3.720439 & 2.022605 & -0.006548 & 0 & $\mathrm{~m}$ & \\
\hline 27 & $\mathrm{~h}$ & 0 & -2.802927 & 2.329591 & -1.505093 & 0 & $\mathrm{~m}$ & \\
\hline 28 & o & 0 & -1.739554 & 2.639105 & 0.269378 & 0 & $\mathrm{~m}$ & \\
\hline 29 & $\mathrm{~h}$ & 0 & -1.943732 & 3.585401 & 0.234840 & 0 & $\mathrm{~m}$ & \\
\hline
\end{tabular}

\section{b5}

neutral molecule

Charge $=0$ Multiplicity $=1$ for low level calculation on real system.

Charge $=0$ Multiplicity $=1$ for high level calculation on model system.

Charge $=0$ Multiplicity $=1$ for low level calculation on model system.

\begin{tabular}{ccccccccc} 
Center & Atomic & Atomic & \multicolumn{3}{l}{ Coordinates (Angstroms) } \\
Number Number & Type & X & Y & Z & & \\
- & & & & & & \\
1 & $\mathrm{c}$ & 0 & 2.387047 & 1.516618 & 0.105870 & 0 & $\mathrm{~h}$ \\
2 & $\mathrm{~h}$ & 0 & 2.591672 & 2.571901 & 0.241749 & 0 & $\mathrm{~h}$ \\
3 & $\mathrm{c}$ & 0 & 3.397171 & 0.514712 & 0.268241 & 0 & $\mathrm{~h}$ \\
4 & $\mathrm{n}$ & 0 & 4.664071 & 0.885689 & 0.631492 & 0 & $\mathrm{~h}$
\end{tabular}




\begin{tabular}{|c|c|c|c|c|c|c|c|c|}
\hline 5 & $\mathrm{~h}$ & 0 & 4.967194 & 1.832653 & 0.458267 & 0 & $\mathrm{~h}$ & \\
\hline 6 & $\mathrm{~h}$ & 0 & 5.367496 & 0.163417 & 0.550528 & 0 & $\mathrm{~h}$ & \\
\hline 7 & $\mathrm{n}$ & 0 & 3.174850 & -0.777876 & 0.118086 & 0 & $\mathrm{~h}$ & \\
\hline 8 & $\mathrm{c}$ & 0 & 1.141789 & 1.080618 & -0.234589 & 0 & $\mathrm{~h}$ & \\
\hline 9 & $\mathrm{~h}$ & 0 & 0.301660 & 1.746298 & -0.393014 & 0 & $\mathrm{~h}$ & \\
\hline 10 & $\mathrm{n}$ & 0 & 0.892420 & -0.245484 & -0.384062 & 0 & $\mathrm{~m}$ & h 8 \\
\hline 11 & $\mathrm{c}$ & 0 & 1.922397 & -1.230922 & -0.193183 & 0 & $\mathrm{~m}$ & $\mathrm{~h}$ \\
\hline 12 & o & 0 & 1.616386 & -2.410269 & -0.322228 & 0 & $\mathrm{~m}$ & \\
\hline 13 & $\mathrm{c}$ & 0 & -0.430253 & -0.766644 & -0.800200 & 0 & $\mathrm{~m}$ & \\
\hline 14 & $\mathrm{~h}$ & 0 & -0.250174 & -1.523762 & -1.564820 & 0 & $\mathrm{~m}$ & \\
\hline 15 & $\mathrm{c}$ & 0 & -1.225939 & -1.375659 & 0.366381 & 0 & $\mathrm{~m}$ & \\
\hline 16 & $\mathrm{~h}$ & 0 & -0.561428 & -1.765400 & 1.146668 & 0 & $\mathrm{~m}$ & \\
\hline 17 & o & 0 & -2.063234 & -2.389167 & -0.155794 & 0 & $\mathrm{~m}$ & \\
\hline 18 & $\mathrm{~h}$ & 0 & -2.881591 & -2.349681 & 0.372495 & 0 & $\mathrm{~m}$ & \\
\hline 19 & $\mathrm{c}$ & 0 & -2.096416 & -0.184339 & 0.812702 & 0 & $\mathrm{~m}$ & \\
\hline 20 & $\mathrm{~h}$ & 0 & -1.540423 & 0.500710 & 1.461698 & 0 & $\mathrm{~m}$ & \\
\hline 21 & o & 0 & -3.320069 & -0.593590 & 1.422223 & 0 & $\mathrm{~m}$ & \\
\hline 22 & $\mathrm{~h}$ & 0 & -3.159764 & -0.713345 & 2.370949 & 0 & $\mathrm{~m}$ & \\
\hline 23 & $\mathrm{c}$ & 0 & -2.400303 & 0.488606 & -0.531109 & 0 & $\mathrm{~m}$ & \\
\hline 24 & $\mathrm{~h}$ & 0 & -3.241055 & -0.041847 & -0.999990 & 0 & $\mathrm{~m}$ & \\
\hline 25 & o & 0 & -1.216730 & 0.294614 & -1.318747 & 0 & $\mathrm{~m}$ & \\
\hline 26 & $\mathrm{c}$ & 0 & -2.750925 & 1.962874 & -0.471485 & 0 & $\mathrm{~m}$ & \\
\hline 27 & $\mathrm{~h}$ & 0 & -3.735863 & 2.055119 & 0.010849 & 0 & $\mathrm{~m}$ & \\
\hline 28 & $\mathrm{~h}$ & 0 & -2.830678 & 2.348019 & -1.498063 & 0 & $\mathrm{~m}$ & \\
\hline 29 & o & 0 & -1.748876 & 2.660626 & 0.264333 & 0 & $\mathrm{~m}$ & \\
\hline 30 & $\mathrm{~h}$ & 0 & -1.953647 & 3.606710 & 0.230010 & 0 & $\mathrm{~m}$ & \\
\hline
\end{tabular}

radical

Charge $=0$ Multiplicity $=2$ for low level calculation on real system.

Charge $=0$ Multiplicity $=2$ for high level calculation on model system.

Charge $=0$ Multiplicity $=2$ for low level calculation on model system.

\begin{tabular}{|c|c|c|c|c|c|c|}
\hline Center & Atomic & Atomi & Coordil & ates (Angstro & & \\
\hline Number & Numbe & r Type & $\mathrm{X}$ & $\mathrm{Y}$ & Z & \\
\hline 1 & $\mathrm{c}$ & 0 & 2.383392 & 1.516306 & 0.132890 & 0 \\
\hline 2 & $\mathrm{c}$ & 0 & 3.441404 & 0.582746 & 0.280007 & $0 \mathrm{~h}$ \\
\hline 3 & $\mathrm{n}$ & 0 & 4.697650 & 0.971144 & 0.628263 & 0 \\
\hline 4 & $\mathrm{~h}$ & 0 & 4.952834 & 1.942253 & 0.532100 & 0 \\
\hline 5 & $\mathrm{~h}$ & 0 & 5.428128 & 0.277857 & 0.541276 & 0 \\
\hline 6 & $\mathrm{n}$ & 0 & 3.204679 & -0.706872 & 0.105160 & 0 \\
\hline 7 & $\mathrm{c}$ & 0 & 1.143718 & 1.114748 & -0.205613 & 0 \\
\hline
\end{tabular}




\begin{tabular}{|c|c|c|c|c|c|c|c|}
\hline 8 & $\mathrm{~h}$ & 0 & 0.296287 & 1.777554 & -0.348363 & 0 & $\mathrm{~h}$ \\
\hline 9 & $\mathrm{n}$ & 0 & 0.906437 & -0.224915 & -0.376103 & 0 & $\mathrm{~m}$ \\
\hline 10 & $\mathrm{c}$ & 0 & 1.955007 & -1.184687 & -0.203752 & 0 & $\mathrm{~m}$ \\
\hline 11 & o & 0 & 1.684166 & -2.371996 & -0.340172 & 0 & $\mathrm{~m}$ \\
\hline 12 & $\mathrm{c}$ & 0 & -0.409799 & -0.760251 & -0.790605 & 0 & $\mathrm{~m}$ \\
\hline 13 & $\mathrm{~h}$ & 0 & -0.219804 & -1.520988 & -1.549809 & 0 & $\mathrm{~m}$ \\
\hline 14 & $\mathrm{c}$ & 0 & -1.204929 & -1.368957 & 0.376861 & 0 & $\mathrm{~m}$ \\
\hline 15 & $\mathrm{~h}$ & 0 & -0.539385 & -1.746527 & 1.162744 & 0 & $\mathrm{~m}$ \\
\hline 16 & o & 0 & -2.030205 & -2.394121 & -0.140813 & 0 & $\mathrm{~m}$ \\
\hline 17 & $\mathrm{~h}$ & 0 & -2.853370 & -2.354847 & 0.380165 & 0 & $\mathrm{~m}$ \\
\hline 18 & $\mathrm{c}$ & 0 & -2.087957 & -0.182636 & 0.812399 & 0 & $\mathrm{~m}$ \\
\hline 19 & $\mathrm{~h}$ & 0 & -1.541827 & 0.509248 & 1.462351 & 0 & $\mathrm{~m}$ \\
\hline 20 & o & 0 & -3.312033 & -0.600032 & 1.415383 & 0 & $\mathrm{~m}$ \\
\hline 21 & $\mathrm{~h}$ & 0 & -3.159289 & -0.708016 & 2.366748 & 0 & $\mathrm{~m}$ \\
\hline 22 & $\mathrm{c}$ & 0 & -2.389250 & 0.483077 & -0.535947 & 0 & $\mathrm{~m}$ \\
\hline 23 & $\mathrm{~h}$ & 0 & -3.225756 & -0.052586 & -1.006397 & 0 & $\mathrm{~m}$ \\
\hline 24 & o & 0 & -1.201382 & 0.292309 & -1.317996 & 0 & $\mathrm{~m}$ \\
\hline 25 & $\mathrm{c}$ & 0 & -2.743598 & 1.956939 & -0.483378 & 0 & $\mathrm{~m}$ \\
\hline 26 & $\mathrm{~h}$ & 0 & -3.731456 & 2.050441 & -0.007299 & 0 & $\mathrm{~m}$ \\
\hline 27 & $\mathrm{~h}$ & 0 & -2.817052 & 2.338322 & -1.511692 & 0 & $\mathrm{~m}$ \\
\hline 28 & o & 0 & -1.746196 & 2.656703 & 0.256417 & 0 & $\mathrm{~m}$ \\
\hline 29 & $\mathrm{~h}$ & 0 & -1.933402 & 3.605173 & 0.197208 & 0 & $\mathrm{~m}$ \\
\hline
\end{tabular}

\section{b6}

neutral molecule

Charge $=0$ Multiplicity $=1$ for low level calculation on real system.

Charge $=0$ Multiplicity $=1$ for high level calculation on model system.

Charge $=0$ Multiplicity $=1$ for low level calculation on model system.

Center Atomic Atomic Coordinates (Angstroms)

Number Number Type $\mathrm{X} \quad \mathrm{Y} \quad \mathrm{Z}$

$\begin{array}{llllllllll}1 & \mathrm{c} & 0 & 1.141789 & 1.080618 & -0.234589 & 0 & \mathrm{~h} & \\ 2 & \mathrm{~h} & 0 & 0.301659 & 1.746295 & -0.393022 & 0 & \mathrm{~h} & \\ 3 & \mathrm{n} & 0 & 0.892420 & -0.245484 & -0.384062 & 0 & \mathrm{~h} & \\ 4 & \mathrm{c} & 0 & 1.922397 & -1.230922 & -0.193183 & 0 & \mathrm{~h} & \\ 5 & \mathrm{o} & 0 & 1.616386 & -2.410269 & -0.322228 & 0 & \mathrm{~h} & \\ 6 & \mathrm{c} & 0 & 2.387047 & 1.516618 & 0.105870 & 0 & \mathrm{~h} & \\ 7 & \mathrm{~h} & 0 & 2.591676 & 2.571900 & 0.241748 & 0 & \mathrm{~h} & & \\ 8 & \mathrm{c} & 0 & 3.397171 & 0.514712 & 0.268241 & 0 & \mathrm{~m} & \mathrm{~h} & 6\end{array}$




\begin{tabular}{|c|c|c|c|c|c|c|c|c|}
\hline 9 & $\mathrm{n}$ & 0 & 4.664071 & 0.885689 & 0.631492 & 0 & $\mathrm{~m}$ & \\
\hline 10 & $\mathrm{~h}$ & 0 & 4.967194 & 1.832653 & 0.458267 & 0 & $\mathrm{~m}$ & \\
\hline 11 & $\mathrm{~h}$ & 0 & 5.367496 & 0.163417 & 0.550528 & 0 & $\mathrm{~m}$ & \\
\hline 12 & $\mathrm{n}$ & 0 & 3.174850 & -0.777876 & 0.118086 & 0 & $\mathrm{~m}$ & h \\
\hline 13 & $\mathrm{c}$ & 0 & -0.430253 & -0.766644 & -0.800200 & 0 & $\mathrm{~m} \mathrm{~h}$ & 3 \\
\hline 14 & $\mathrm{~h}$ & 0 & -0.250174 & -1.523762 & -1.564821 & 0 & $\mathrm{~m}$ & \\
\hline 15 & $\mathrm{c}$ & 0 & -1.225939 & -1.375659 & 0.366381 & 0 & $\mathrm{~m}$ & \\
\hline 16 & $\mathrm{~h}$ & 0 & -0.561428 & -1.765400 & 1.146668 & 0 & $\mathrm{~m}$ & \\
\hline 17 & o & 0 & -2.063234 & -2.389167 & -0.155794 & 0 & $\mathrm{~m}$ & \\
\hline 18 & $\mathrm{~h}$ & 0 & -2.881591 & -2.349681 & 0.372495 & 0 & $\mathrm{~m}$ & \\
\hline 19 & $\mathrm{c}$ & 0 & -2.096416 & -0.184339 & 0.812702 & 0 & $\mathrm{~m}$ & \\
\hline 20 & $\mathrm{~h}$ & 0 & -1.540423 & 0.500710 & 1.461698 & 0 & $\mathrm{~m}$ & \\
\hline 21 & o & 0 & -3.320070 & -0.593590 & 1.422223 & 0 & $\mathrm{~m}$ & \\
\hline 22 & $\mathrm{~h}$ & 0 & -3.159764 & -0.713345 & 2.370949 & 0 & $\mathrm{~m}$ & \\
\hline 23 & $\mathrm{c}$ & 0 & -2.400304 & 0.488606 & -0.531109 & 0 & $\mathrm{~m}$ & \\
\hline 24 & $\mathrm{~h}$ & 0 & -3.241055 & -0.041847 & -0.999990 & 0 & $\mathrm{~m}$ & \\
\hline 25 & o & 0 & -1.216730 & 0.294615 & -1.318747 & 0 & $\mathrm{~m}$ & \\
\hline 26 & $\mathrm{c}$ & 0 & -2.750925 & 1.962874 & -0.471485 & 0 & $\mathrm{~m}$ & \\
\hline 27 & $\mathrm{~h}$ & 0 & -3.735863 & 2.055119 & 0.010849 & 0 & $\mathrm{~m}$ & \\
\hline 28 & $\mathrm{~h}$ & 0 & -2.830678 & 2.348019 & -1.498063 & 0 & $\mathrm{~m}$ & \\
\hline 29 & o & 0 & -1.748876 & 2.660626 & 0.264333 & 0 & $\mathrm{~m}$ & \\
\hline 30 & $\mathrm{~h}$ & 0 & -1.953647 & 3.606710 & 0.230011 & 0 & $\mathrm{~m}$ & \\
\hline
\end{tabular}

radical

Charge $=0$ Multiplicity $=2$ for low level calculation on real system.

Charge $=0$ Multiplicity $=2$ for high level calculation on model system.

Charge $=0$ Multiplicity $=2$ for low level calculation on model system.

Center Atomic Atomic Coordinates (Angstroms)

Number Number Type $\quad X \quad$ Y $\quad$ Z

\begin{tabular}{|c|c|c|c|c|c|c|c|}
\hline 1 & $\mathrm{c}$ & 0 & 1.124992 & 0.988619 & -0.147724 & 0 & $\mathrm{~h}$ \\
\hline 2 & $\mathrm{n}$ & 0 & 0.835002 & -0.289487 & -0.328198 & 0 & h \\
\hline 3 & $\mathrm{c}$ & 0 & 1.908698 & -1.296832 & -0.175503 & 0 & $\mathrm{~h}$ \\
\hline 4 & o & 0 & 1.585039 & -2.462522 & -0.320112 & 0 & $\mathrm{~h}$ \\
\hline 5 & c & 0 & 2.340625 & 1.485456 & 0.165626 & 0 & $\mathrm{~h}$ \\
\hline 6 & $\mathrm{~h}$ & 0 & 2.535289 & 2.532587 & 0.362165 & 0 & $\mathrm{~h}$ \\
\hline 7 & c & 0 & 3.366800 & 0.475202 & 0.257320 & 0 & $\mathrm{~m} \mathrm{~h}$ \\
\hline 8 & $\mathrm{n}$ & 0 & 4.637709 & 0.851021 & 0.571750 & 0 & $\mathrm{~m}$ \\
\hline 9 & $\mathrm{~h}$ & 0 & 4.920593 & 1.813664 & 0.470861 & 0 & $\mathrm{~m}$ \\
\hline 10 & $\mathrm{~h}$ & 0 & 5.352482 & 0.140818 & 0.492560 & 0 & $\mathrm{~m}$ \\
\hline 11 & $\mathrm{n}$ & 0 & 3.150137 & -0.818994 & 0.093443 & 0 & h 3 \\
\hline
\end{tabular}




\begin{tabular}{|c|c|c|c|c|c|c|c|}
\hline 12 & $\mathrm{c}$ & 0 & -0.491243 & -0.777446 & -0.781780 & 0 & $\mathrm{~m} h$ \\
\hline 13 & $\mathrm{~h}$ & 0 & -0.308780 & -1.542645 & -1.537573 & 0 & $\mathrm{~m}$ \\
\hline 14 & $\mathrm{c}$ & 0 & -1.329612 & -1.342842 & 0.374235 & 0 & $\mathrm{~m}$ \\
\hline 15 & $\mathrm{~h}$ & 0 & -0.693216 & -1.760282 & 1.164962 & 0 & $\mathrm{~m}$ \\
\hline 16 & o & 0 & -2.205412 & -2.317318 & -0.154150 & 0 & $\mathrm{~m}$ \\
\hline 17 & $\mathrm{~h}$ & 0 & -3.040807 & -2.210078 & 0.337883 & 0 & $\mathrm{~m}$ \\
\hline 18 & $\mathrm{c}$ & 0 & -2.144359 & -0.106070 & 0.805683 & 0 & $\mathrm{~m}$ \\
\hline 19 & $\mathrm{~h}$ & 0 & -1.566937 & 0.546529 & 1.469798 & 0 & $\mathrm{~m}$ \\
\hline 20 & o & 0 & -3.399684 & -0.458231 & 1.384051 & 0 & $\mathrm{~m}$ \\
\hline 21 & $\mathrm{~h}$ & 0 & -3.275156 & -0.566882 & 2.339403 & 0 & $\mathrm{~m}$ \\
\hline 22 & $\mathrm{c}$ & 0 & -2.385355 & 0.600987 & -0.531993 & 0 & $\mathrm{~m}$ \\
\hline 23 & $\mathrm{~h}$ & 0 & -3.270542 & 0.163267 & -1.012601 & 0 & $\mathrm{~m}$ \\
\hline 24 & o & 0 & -1.217356 & 0.309620 & -1.325060 & 0 & $\mathrm{~m}$ \\
\hline 25 & $\mathrm{c}$ & 0 & -2.540000 & 2.114579 & -0.447646 & 0 & $\mathrm{~m}$ \\
\hline 26 & $\mathrm{~h}$ & 0 & -3.419413 & 2.346205 & 0.162441 & 0 & $\mathrm{~m}$ \\
\hline 27 & $\mathrm{~h}$ & 0 & -2.710941 & 2.515389 & -1.458136 & 0 & $\mathrm{~m}$ \\
\hline 28 & o & 0 & -1.430349 & 2.739459 & 0.175367 & 0 & $\mathrm{~m}$ \\
\hline 29 & $\mathrm{~h}$ & 0 & -0.643684 & 2.485684 & -0.340805 & 0 & $\mathrm{~m}$ \\
\hline
\end{tabular}

\section{rG}

\section{s1}

neutral molecule

Charge $=0$ Multiplicity $=1$ for low level calculation on real system.

Charge $=0$ Multiplicity $=1$ for high level calculation on model system .

Charge $=0$ Multiplicity $=1$ for low level calculation on model system .

\begin{tabular}{|c|c|c|c|c|c|c|c|}
\hline $\begin{array}{l}\text { Center } \\
\text { Number }\end{array}$ & $\begin{array}{l}\text { Atomic } \\
\text { Number }\end{array}$ & $\begin{array}{c}\text { Atomic } \\
\text { Type }\end{array}$ & $\begin{array}{l}\text { c Coordi } \\
\mathrm{X}\end{array}$ & $\begin{array}{c}\text { ates (Angstro } \\
\text { Y }\end{array}$ & ms) & & \\
\hline 1 & $\mathrm{c}$ & 0 & 0.904804 & 0.670054 & -0.621434 & 0 & $\mathrm{~h}$ \\
\hline 2 & $\mathrm{~h}$ & 0 & 0.449926 & 1.441293 & -1.248586 & 0 & $\mathrm{~h}$ \\
\hline 3 & c & 0 & 1.572093 & 1.324948 & 0.604477 & 0 & $\mathrm{~h}$ \\
\hline 4 & $\mathrm{~h}$ & 0 & 0.895979 & 1.335250 & 1.470776 & 0 & $\mathrm{~h}$ \\
\hline 5 & o & 0 & 1.970871 & 2.632936 & 0.245082 & 0 & $\mathrm{~h}$ \\
\hline 6 & $\mathrm{~h}$ & 0 & 2.827215 & 2.774659 & 0.691011 & 0 & $\mathrm{~h}$ \\
\hline 7 & o & 0 & 1.928028 & -0.015305 & -1.326681 & 0 & $\mathrm{~h}$ \\
\hline 8 & $\mathrm{n}$ & 0 & 0.149838 & -0.281401 & -0.278877 & 0 & $\mathrm{~h}$ \\
\hline 9 & $\mathrm{c}$ & 0 & 2.822640 & 0.446296 & 0.807695 & 0 & \\
\hline
\end{tabular}




\begin{tabular}{|c|c|c|c|c|c|c|c|c|}
\hline 10 & $\mathrm{~h}$ & 0 & 2.590209 & -0.453220 & 1.385219 & 0 & $\mathrm{~m}$ & \\
\hline 11 & $\mathrm{c}$ & 0 & 3.180725 & 0.070383 & -0.636358 & 0 & $\mathrm{~m}$ & $\mathrm{~h}$ \\
\hline 12 & $\mathrm{~h}$ & 0 & 3.784187 & 0.881762 & -1.067979 & 0 & $\mathrm{~m}$ & \\
\hline 13 & $\mathrm{c}$ & 0 & -0.026434 & -1.632208 & 0.025614 & 0 & $\mathrm{~m}$ & h \\
\hline 14 & $\mathrm{~h}$ & 0 & 0.938131 & -2.120130 & -0.005486 & 0 & $\mathrm{~m}$ & \\
\hline 15 & $\mathrm{c}$ & 0 & -1.489469 & 0.008439 & -0.180923 & 0 & $\mathrm{~m}$ & h \\
\hline 16 & o & 0 & 3.904117 & 1.172242 & 1.390925 & 0 & $\mathrm{~m}$ & \\
\hline 17 & $\mathrm{~h}$ & 0 & 3.859467 & 1.064782 & 2.353323 & 0 & $\mathrm{~m}$ & \\
\hline 18 & $\mathrm{c}$ & 0 & 3.945854 & -1.231784 & -0.796148 & 0 & $\mathrm{~m}$ & \\
\hline 19 & $\mathrm{~h}$ & 0 & 4.977503 & -1.067236 & -0.448965 & 0 & $\mathrm{~m}$ & \\
\hline 20 & $\mathrm{~h}$ & 0 & 3.977296 & -1.487660 & -1.864043 & 0 & $\mathrm{~m}$ & \\
\hline 21 & o & 0 & 3.306769 & -2.251666 & -0.034288 & 0 & $\mathrm{~m}$ & \\
\hline 22 & $\mathrm{~h}$ & 0 & 3.643127 & -3.111358 & -0.326267 & 0 & $\mathrm{~m}$ & \\
\hline 23 & $\mathrm{n}$ & 0 & -1.172218 & -2.201544 & 0.305877 & 0 & $\mathrm{~m}$ & \\
\hline 24 & $\mathrm{c}$ & 0 & -2.103809 & -1.189735 & 0.182880 & 0 & $\mathrm{~m}$ & \\
\hline 25 & $\mathrm{c}$ & 0 & -3.530836 & -1.206723 & 0.375277 & 0 & $\mathrm{~m}$ & \\
\hline 26 & o & 0 & -4.290566 & -2.100549 & 0.705789 & 0 & $\mathrm{~m}$ & \\
\hline 27 & $\mathrm{n}$ & 0 & -4.063627 & 0.107984 & 0.121087 & 0 & $\mathrm{~m}$ & \\
\hline 28 & $\mathrm{~h}$ & 0 & -5.056476 & 0.182831 & 0.312536 & 0 & $\mathrm{~m}$ & \\
\hline 29 & $\mathrm{c}$ & 0 & -3.351599 & 1.220275 & -0.247422 & 0 & $\mathrm{~m}$ & \\
\hline 30 & $\mathrm{n}$ & 0 & -4.060696 & 2.397518 & -0.385296 & 0 & $\mathrm{~m}$ & \\
\hline 31 & $\mathrm{~h}$ & 0 & -4.991073 & 2.324851 & -0.778680 & 0 & $\mathrm{~m}$ & \\
\hline 32 & $\mathrm{~h}$ & 0 & -3.504567 & 3.129826 & -0.809948 & 0 & $\mathrm{~m}$ & \\
\hline 33 & $\mathrm{n}$ & 0 & -2.051977 & 1.223646 & -0.418716 & 0 & $\mathrm{~m}$ & \\
\hline
\end{tabular}

radical

Charge $=0$ Multiplicity $=2$ for low level calculation on real system.

Charge $=0$ Multiplicity $=2$ for high level calculation on model system.

Charge $=0$ Multiplicity $=2$ for low level calculation on model system.

Center Atomic Atomic Coordinates (Angstroms)

Number Number Type $\quad \mathrm{X} \quad \mathrm{Y} \quad$ Z

\begin{tabular}{|c|c|c|c|c|c|c|c|}
\hline 1 & $\mathrm{c}$ & 0 & 0.969814 & -0.008777 & -0.592393 & & h \\
\hline 2 & $\mathrm{c}$ & 0 & 1.408664 & 1.266207 & 0.058779 & 0 & $\mathrm{~h}$ \\
\hline 3 & $\mathrm{~h}$ & 0 & 0.730929 & 1.577819 & 0.862604 & 0 & $\mathrm{~h}$ \\
\hline 4 & o & 0 & 1.554358 & 2.301202 & -0.909781 & 0 & $\mathrm{~h}$ \\
\hline 5 & $\mathrm{~h}$ & 0 & 2.346246 & 2.798084 & -0.629212 & 0 & $\mathrm{~h}$ \\
\hline 6 & o & 0 & 2.025941 & -0.840686 & -0.868568 & 0 & $\mathrm{~h}$ \\
\hline 7 & $\mathrm{n}$ & 0 & -0.221161 & -0.687133 & -0.348684 & & h \\
\hline 8 & $\mathrm{c}$ & 0 & 2.813019 & 0.841895 & 0.564484 & 0 & $\mathrm{~m} \mathrm{~h} 2$ \\
\hline 9 & $\mathrm{~h}$ & 0 & 2.738547 & 0.336623 & 1.532589 & 0 & $\mathrm{~m}$ \\
\hline
\end{tabular}




$\begin{array}{lllrrrrrrr}10 & \mathrm{c} & 0 & 3.250439 & -0.169420 & -0.505319 & 0 & \mathrm{~m} & \mathrm{~h} & 6 \\ 11 & \mathrm{~h} & 0 & 3.631985 & 0.375873 & -1.378036 & 0 & \mathrm{~m} & & \\ 12 & \mathrm{c} & 0 & -0.414652 & -2.074534 & -0.366402 & 0 & \mathrm{~m} & \mathrm{~h} & 7 \\ 13 & \mathrm{~h} & 0 & 0.422618 & -2.738596 & -0.516552 & 0 & \mathrm{~m} & & \\ 14 & \mathrm{c} & 0 & -1.478819 & -0.143614 & -0.142895 & 0 & \mathrm{~m} & \mathrm{~h} & 7 \\ 15 & \mathrm{o} & 0 & 3.742518 & 1.920799 & 0.606422 & 0 & \mathrm{~m} & \\ 16 & \mathrm{~h} & 0 & 3.730226 & 2.297571 & 1.499266 & 0 & \mathrm{~m} & \\ 17 & \mathrm{c} & 0 & 4.276311 & -1.193806 & -0.064274 & 0 & \mathrm{~m} & \\ 18 & \mathrm{~h} & 0 & 5.239094 & -0.671357 & 0.052641 & 0 & \mathrm{~m} & \\ 19 & \mathrm{~h} & 0 & 4.389297 & -1.944266 & -0.860355 & 0 & \mathrm{~m} & \\ 20 & \mathrm{o} & 0 & 3.844553 & -1.782271 & 1.153930 & 0 & \mathrm{~m} & \\ 21 & \mathrm{~h} & 0 & 4.448501 & -2.507377 & 1.370952 & 0 & \mathrm{~m} & \\ 22 & \mathrm{n} & 0 & -1.660668 & -2.424796 & -0.184900 & 0 & \mathrm{~m} & \\ 23 & \mathrm{c} & 0 & -2.342615 & -1.233815 & -0.041620 & 0 & \mathrm{~m} & \\ 24 & \mathrm{c} & 0 & -3.742979 & -0.991402 & 0.192559 & 0 & \mathrm{~m} & \\ 25 & \mathrm{o} & 0 & -4.679631 & -1.758012 & 0.333813 & 0 & \mathrm{~m} & & \\ 26 & \mathrm{n} & 0 & -3.979124 & 0.427196 & 0.264349 & 0 & \mathrm{~m} & & \\ 27 & \mathrm{~h} & 0 & -4.937501 & 0.664525 & 0.494921 & 0 & \mathrm{~m} & \\ 28 & \mathrm{c} & 0 & -3.037784 & 1.416271 & 0.136654 & 0 & \mathrm{~m} & \\ 29 & \mathrm{n} & 0 & -3.471963 & 2.718226 & 0.294472 & 0 & \mathrm{~m} & \\ 30 & \mathrm{~h} & 0 & -4.392707 & 2.935145 & -0.067694 & 0 & \mathrm{~m} & \\ 31 & \mathrm{~h} & 0 & -2.767031 & 3.387955 & 0.009678 & 0 & \mathrm{~m} & \\ 32 & \mathrm{n} & 0 & -1.765726 & 1.181609 & -0.073063 & 0 & \mathrm{~m} & & \\ --------------------------------------------------------- & & & & \end{array}$

\section{s2}

neutral molecule

Charge $=0$ Multiplicity $=1$ for low level calculation on real system.

Charge $=0$ Multiplicity $=1$ for high level calculation on model system.

Charge $=0$ Multiplicity $=1$ for low level calculation on model system.

\begin{tabular}{|c|c|c|c|c|c|c|}
\hline Center & Atomic & Atom & Coordi & ates (Angstro & & \\
\hline Number & Number & r Type & $\mathrm{X}$ & $\mathrm{Y}$ & Z & \\
\hline 1 & $\mathrm{c}$ & 0 & 1.572104 & 1.324951 & 0.604479 & $0 \mathrm{~h}$ \\
\hline 2 & $\mathrm{~h}$ & 0 & 0.895997 & 1.335250 & 1.470784 & $0 \mathrm{~h}$ \\
\hline 3 & o & 0 & 1.970882 & 2.632938 & 0.245081 & $0 \mathrm{~h}$ \\
\hline 4 & $\mathrm{~h}$ & 0 & 2.827230 & 2.774660 & 0.691004 & 0 \\
\hline 5 & $\mathrm{c}$ & 0 & 2.822647 & 0.446294 & 0.807694 & 0 \\
\hline 6 & $\mathrm{~h}$ & 0 & 2.590214 & -0.453222 & 1.385218 & 0 \\
\hline 7 & o & 0 & 3.904129 & 1.172235 & 1.390922 & 0 \\
\hline
\end{tabular}




\begin{tabular}{|c|c|c|c|c|c|c|c|c|}
\hline 8 & $\mathrm{~h}$ & 0 & 3.859480 & 1.064774 & 2.353320 & 0 & $\mathrm{~h}$ & \\
\hline 9 & $\mathrm{c}$ & 0 & 0.904809 & 0.670061 & -0.621432 & 0 & $\mathrm{~h}$ & \\
\hline 10 & $\mathrm{~h}$ & 0 & 0.449934 & 1.441302 & -1.248582 & 0 & $\mathrm{~h}$ & \\
\hline 11 & $\mathrm{c}$ & 0 & 3.180728 & 0.070379 & -0.636360 & 0 & $\mathrm{~m}$ & $\mathrm{~h}$ \\
\hline 12 & $\mathrm{~h}$ & 0 & 3.784193 & 0.881756 & -1.067982 & 0 & $\mathrm{~m}$ & \\
\hline 13 & o & 0 & 1.928029 & -0.015303 & -1.326680 & 0 & $\mathrm{~m}$ & h 9 \\
\hline 14 & $\mathrm{n}$ & 0 & -0.149836 & -0.281390 & -0.278873 & 0 & $\mathrm{~m}$ & h 9 \\
\hline 15 & $\mathrm{c}$ & 0 & 3.945851 & -1.231791 & -0.796152 & 0 & $\mathrm{~m}$ & \\
\hline 16 & $\mathrm{~h}$ & 0 & 4.977501 & -1.067247 & -0.448971 & 0 & $\mathrm{~m}$ & \\
\hline 17 & $\mathrm{~h}$ & 0 & 3.977289 & -1.487666 & -1.864047 & 0 & $\mathrm{~m}$ & \\
\hline 18 & o & 0 & 3.306762 & -2.251671 & -0.034291 & 0 & $\mathrm{~m}$ & \\
\hline 19 & $\mathrm{~h}$ & 0 & 3.643116 & -3.111363 & -0.326271 & 0 & $\mathrm{~m}$ & \\
\hline 20 & $\mathrm{c}$ & 0 & -0.026438 & -1.632197 & 0.025618 & 0 & $\mathrm{~m}$ & \\
\hline 21 & $\mathrm{~h}$ & 0 & 0.938125 & -2.120124 & -0.005484 & 0 & $\mathrm{~m}$ & \\
\hline 22 & $\mathrm{n}$ & 0 & -1.172224 & -2.201531 & 0.305873 & 0 & $\mathrm{~m}$ & \\
\hline 23 & $\mathrm{c}$ & 0 & -2.103802 & -1.189727 & 0.182879 & 0 & $\mathrm{~m}$ & \\
\hline 24 & c & 0 & -3.530828 & -1.206728 & 0.375277 & 0 & $\mathrm{~m}$ & \\
\hline 25 & o & 0 & -4.290551 & -2.100560 & 0.705791 & 0 & $\mathrm{~m}$ & \\
\hline 26 & $\mathrm{n}$ & 0 & -4.063631 & 0.107974 & 0.121086 & 0 & $\mathrm{~m}$ & \\
\hline 27 & $\mathrm{~h}$ & 0 & -5.056481 & 0.182813 & 0.312537 & 0 & $\mathrm{~m}$ & \\
\hline 28 & $\mathrm{c}$ & 0 & -3.351613 & 1.220271 & -0.247424 & 0 & $\mathrm{~m}$ & \\
\hline 29 & $\mathrm{n}$ & 0 & -4.060721 & 2.397508 & -0.385297 & 0 & $\mathrm{~m}$ & \\
\hline 30 & $\mathrm{~h}$ & 0 & -4.991098 & 2.324832 & -0.778681 & 0 & $\mathrm{~m}$ & \\
\hline 31 & $\mathrm{~h}$ & 0 & -3.504598 & 3.129821 & -0.809951 & 0 & $\mathrm{~m}$ & \\
\hline 32 & $\mathrm{n}$ & 0 & -2.051991 & 1.223655 & -0.418701 & 0 & $\mathrm{~m}$ & \\
\hline 33 & $\mathrm{c}$ & 0 & -1.489472 & 0.008451 & -0.180927 & 0 & $\mathrm{~m}$ & \\
\hline
\end{tabular}

radical

Charge $=0$ Multiplicity $=2$ for low level calculation on real system.

Charge $=0$ Multiplicity $=2$ for high level calculation on model system.

Charge $=0$ Multiplicity $=2$ for low level calculation on model system.

\begin{tabular}{|c|c|c|c|c|c|c|}
\hline Center & Atomic & Atomi & Coordi & ates (Angstro & ns) & \\
\hline Number & Numbe & ar Type & $\mathrm{X}$ & $\mathrm{Y}$ & Z & \\
\hline 1 & $\mathrm{c}$ & 0 & 2.050923 & -0.484666 & -1.188241 & \\
\hline 2 & o & 0 & 2.041747 & -1.311999 & -2.259973 & $0 \mathrm{~h}$ \\
\hline 3 & $\mathrm{~h}$ & 0 & 2.982800 & -1.508308 & -2.451248 & \\
\hline 4 & $\mathrm{c}$ & 0 & 3.355827 & -0.164237 & -0.511804 & \\
\hline 5 & $\mathrm{~h}$ & 0 & 3.683189 & 0.871066 & -0.684519 & \\
\hline 6 & o & 0 & 4.353008 & -1.079770 & -0.983989 & 0 \\
\hline 7 & $\mathrm{~h}$ & 0 & 5.187592 & -0.600557 & -1.089848 & 0 \\
\hline
\end{tabular}




$\begin{array}{rrrrrrrrrr}8 & \mathrm{c} & 0 & 0.955240 & -0.587041 & -0.164123 & 0 & \mathrm{~h} & & \\ 9 & \mathrm{~h} & 0 & 0.589112 & -1.616145 & -0.045540 & 0 & \mathrm{~h} & & \\ 10 & \mathrm{c} & 0 & 2.991900 & -0.354293 & 0.977088 & 0 & \mathrm{~m} & \mathrm{~h} & 4 \\ 11 & \mathrm{~h} & 0 & 3.234551 & -1.390880 & 1.257944 & 0 & \mathrm{~m} & & \\ 12 & \mathrm{o} & 0 & 1.573916 & -0.126573 & 1.048444 & 0 & \mathrm{~m} & \mathrm{~h} & 8 \\ 13 & \mathrm{n} & 0 & -0.212744 & 0.225216 & -0.426740 & 0 & \mathrm{~m} & \mathrm{~h} & 8 \\ 14 & \mathrm{c} & 0 & 3.641735 & 0.630588 & 1.940911 & 0 & \mathrm{~m} & & \\ 15 & \mathrm{~h} & 0 & 4.731363 & 0.524715 & 1.895369 & 0 & \mathrm{~m} & \\ 16 & \mathrm{~h} & 0 & 3.323001 & 0.396596 & 2.968050 & 0 & \mathrm{~m} & \\ 17 & \mathrm{o} & 0 & 3.340733 & 1.970454 & 1.593800 & 0 & \mathrm{~m} & \\ 18 & \mathrm{~h} & 0 & 2.370086 & 2.026604 & 1.574766 & 0 & \mathrm{~m} & \\ 19 & \mathrm{c} & 0 & -0.252488 & 1.519008 & -0.933162 & 0 & \mathrm{~m} & \\ 20 & \mathrm{~h} & 0 & 0.649225 & 2.009788 & -1.273141 & 0 & \mathrm{~m} & \\ 21 & \mathrm{n} & 0 & -1.455297 & 2.026158 & -0.964320 & 0 & \mathrm{~m} & \\ 22 & \mathrm{c} & 0 & -2.264029 & 1.030698 & -0.446678 & 0 & \mathrm{~m} & \\ 23 & \mathrm{c} & 0 & -3.690308 & 1.001368 & -0.247057 & 0 & \mathrm{~m} & & \\ 24 & \mathrm{o} & 0 & -4.553711 & 1.829885 & -0.474160 & 0 & \mathrm{~m} & & \\ 25 & \mathrm{n} & 0 & -4.066822 & -0.270683 & 0.315196 & 0 & \mathrm{~m} & & \\ 26 & \mathrm{~h} & 0 & -5.069522 & -0.384965 & 0.413327 & 0 & \mathrm{~m} & & \\ 27 & \mathrm{c} & 0 & -3.225209 & -1.308563 & 0.621260 & 0 & \mathrm{~m} & & \\ 28 & \mathrm{n} & 0 & -3.807432 & -2.465073 & 1.096395 & 0 & \mathrm{~m} & & \\ 29 & \mathrm{~h} & 0 & -4.619605 & -2.360548 & 1.691641 & 0 & \mathrm{~m} & & \\ 30 & \mathrm{~h} & 0 & -3.125712 & -3.121495 & 1.456245 & 0 & \mathrm{~m} & & \\ 31 & \mathrm{n} & 0 & -1.926835 & -1.265700 & 0.439358 & 0 & \mathrm{~m} & & \\ 32 & \mathrm{c} & 0 & -1.513211 & -0.091407 & -0.102399 & 0 & \mathrm{~m} & & \\ ----------------------------------------------------------~ & & & & \end{array}$

\section{s3}

neutral molecule

Charge $=0$ Multiplicity $=1$ for low level calculation on real system.

Charge $=0$ Multiplicity $=1$ for high level calculation on model system.

Charge $=0$ Multiplicity $=1$ for low level calculation on model system .

\begin{tabular}{|c|c|c|c|c|c|c|}
\hline Center & Atomic & Atomic & Coordi & ates (Angstro & & \\
\hline Number & Number & r Type & $\mathrm{X}$ & $\mathrm{Y}$ & Z & \\
\hline 1 & $\mathrm{c}$ & 0 & 2.822644 & 0.446288 & 0.807698 & 0 \\
\hline 2 & $\mathrm{~h}$ & 0 & 2.590208 & -0.453226 & 1.385222 & 0 \\
\hline 3 & o & 0 & 3.904127 & 1.172227 & 1.390927 & 0 \\
\hline 4 & $\mathrm{~h}$ & 0 & 3.859477 & 1.064768 & 2.353326 & 0 \\
\hline 5 & $\mathrm{c}$ & 0 & 3.180730 & 0.070381 & -0.636356 & 0 \\
\hline
\end{tabular}




\begin{tabular}{|c|c|c|c|c|c|c|c|c|}
\hline 6 & $\mathrm{~h}$ & 0 & 3.784193 & 0.881762 & -1.067973 & 0 & & \\
\hline 7 & $\mathrm{c}$ & 0 & 1.572104 & 1.324949 & 0.604481 & 0 & $\mathrm{~h}$ & \\
\hline 8 & $\mathrm{~h}$ & 0 & 0.895991 & 1.335251 & 1.470781 & 0 & $\mathrm{~h}$ & \\
\hline 9 & o & 0 & 1.970887 & 2.632935 & 0.245082 & 0 & $\mathrm{~h}$ & \\
\hline 10 & $\mathrm{~h}$ & 0 & 2.827234 & 2.774654 & 0.691007 & 0 & $\mathrm{~h}$ & \\
\hline 11 & $\mathrm{c}$ & 0 & 3.945851 & -1.231789 & -0.796152 & 0 & $\mathrm{~m}$ & $\mathrm{~h}$ \\
\hline 12 & $\mathrm{~h}$ & 0 & 4.977501 & -1.067250 & -0.448966 & 0 & $\mathrm{~m}$ & \\
\hline 13 & $\mathrm{~h}$ & 0 & 3.977293 & -1.487659 & -1.864048 & 0 & $\mathrm{~m}$ & \\
\hline 14 & o & 0 & 1.928035 & -0.015288 & -1.326685 & 0 & $\mathrm{~m}$ & $\mathrm{~h}$ \\
\hline 15 & $\mathrm{c}$ & 0 & 0.904810 & 0.670060 & -0.621431 & 0 & $\mathrm{~m}$ & $\mathrm{~h}$ \\
\hline 16 & $\mathrm{~h}$ & 0 & 0.449935 & 1.441301 & -1.248581 & 0 & $\mathrm{~m}$ & \\
\hline 17 & o & 0 & 3.306757 & -2.251672 & -0.034300 & 0 & $\mathrm{~m}$ & \\
\hline 18 & $\mathrm{~h}$ & 0 & 3.643111 & -3.111364 & -0.326283 & 0 & $\mathrm{~m}$ & \\
\hline 19 & $\mathrm{n}$ & 0 & -0.149837 & -0.281391 & -0.278872 & 0 & $\mathrm{~m}$ & \\
\hline 20 & $\mathrm{c}$ & 0 & -0.026439 & -1.632198 & 0.025618 & 0 & $\mathrm{~m}$ & \\
\hline 21 & $\mathrm{~h}$ & 0 & 0.938124 & -2.120125 & -0.005484 & 0 & $\mathrm{~m}$ & \\
\hline 22 & $\mathrm{n}$ & 0 & -1.172225 & -2.201531 & 0.305874 & 0 & $\mathrm{~m}$ & \\
\hline 23 & $\mathrm{c}$ & 0 & -2.103803 & -1.189728 & 0.182879 & 0 & $\mathrm{~m}$ & \\
\hline 24 & $\mathrm{c}$ & 0 & -3.530829 & -1.206728 & 0.375277 & 0 & $\mathrm{~m}$ & \\
\hline 25 & o & 0 & -4.290552 & -2.100560 & 0.705790 & 0 & $\mathrm{~m}$ & \\
\hline 26 & $\mathrm{n}$ & 0 & -4.063632 & 0.107975 & 0.121087 & 0 & $\mathrm{~m}$ & \\
\hline 27 & $\mathrm{~h}$ & 0 & -5.056481 & 0.182814 & 0.312537 & 0 & $\mathrm{~m}$ & \\
\hline 28 & $\mathrm{c}$ & 0 & -3.351613 & 1.220272 & -0.247424 & 0 & $\mathrm{~m}$ & \\
\hline 29 & $\mathrm{n}$ & 0 & -4.060719 & 2.397509 & -0.385296 & 0 & $\mathrm{~m}$ & \\
\hline 30 & $\mathrm{~h}$ & 0 & -4.991097 & 2.324834 & -0.778680 & 0 & $\mathrm{~m}$ & \\
\hline 31 & $\mathrm{~h}$ & 0 & -3.504596 & 3.129821 & -0.809950 & 0 & $\mathrm{~m}$ & \\
\hline 32 & $\mathrm{n}$ & 0 & -2.051991 & 1.223655 & -0.418700 & 0 & $\mathrm{~m}$ & \\
\hline 33 & c & 0 & -1.489473 & 0.008452 & -0.180935 & 0 & $\mathrm{~m}$ & \\
\hline
\end{tabular}

radical

Charge $=0$ Multiplicity $=2$ for low level calculation on real system.

Charge $=0$ Multiplicity $=2$ for high level calculation on model system .

Charge $=0$ Multiplicity $=2$ for low level calculation on model system.

\begin{tabular}{|c|c|c|c|c|c|c|}
\hline Center & Atomic & Atomic & c Coordi & tes (Angstro & & \\
\hline Number & Numbe & r Type & $\mathrm{X}$ & $\mathrm{Y}$ & Z & \\
\hline 1 & $\mathrm{c}$ & 0 & 2.967083 & 0.466486 & 0.663918 & 0 \\
\hline 2 & o & 0 & 4.074132 & 0.718456 & 1.431323 & $0 \mathrm{~h}$ \\
\hline 3 & $\mathrm{~h}$ & 0 & 3.824405 & 0.682459 & 2.369761 & 0 \\
\hline 4 & $\mathrm{c}$ & 0 & 3.180503 & 0.134227 & -0.780725 & 0 \\
\hline 5 & $\mathrm{~h}$ & 0 & 3.816145 & 0.894122 & -1.273220 & 0 \\
\hline
\end{tabular}




\begin{tabular}{|c|c|c|c|c|c|c|c|c|}
\hline 6 & c & 0 & 1.711609 & 1.266834 & 0.784845 & 0 & & \\
\hline 7 & $\mathrm{~h}$ & 0 & 1.149154 & 1.109496 & 1.715909 & 0 & $\mathrm{~h}$ & \\
\hline 8 & o & 0 & 1.960968 & 2.671790 & 0.592826 & 0 & $\mathrm{~h}$ & \\
\hline 9 & $\mathrm{~h}$ & 0 & 2.795159 & 2.879277 & 1.044250 & 0 & $\mathrm{~h}$ & \\
\hline 10 & $\mathrm{c}$ & 0 & 3.823138 & -1.221380 & -1.056985 & 0 & $\mathrm{~m}$ & h 4 \\
\hline 11 & $\mathrm{~h}$ & 0 & 4.895078 & -1.141244 & -0.817968 & 0 & $\mathrm{~m}$ & \\
\hline 12 & $\mathrm{~h}$ & 0 & 3.724922 & -1.430090 & -2.131331 & 0 & $\mathrm{~m}$ & \\
\hline 13 & o & 0 & 1.864697 & 0.202431 & -1.349090 & 0 & $\mathrm{~m}$ & h 4 \\
\hline 14 & $\mathrm{c}$ & 0 & 0.922945 & 0.772932 & -0.446688 & 0 & $\mathrm{~m}$ & h 6 \\
\hline 15 & $\mathrm{~h}$ & 0 & 0.416042 & 1.618993 & -0.916966 & 0 & $\mathrm{~m}$ & \\
\hline 16 & o & 0 & 3.199549 & -2.224722 & -0.266956 & 0 & $\mathrm{~m}$ & \\
\hline 17 & $\mathrm{~h}$ & 0 & 3.382650 & -3.087326 & -0.667112 & 0 & $\mathrm{~m}$ & \\
\hline 18 & $\mathrm{n}$ & 0 & -0.111416 & -0.200392 & -0.128183 & 0 & $\mathrm{~m}$ & \\
\hline 19 & $\mathrm{c}$ & 0 & 0.042145 & -1.533228 & 0.236586 & 0 & $\mathrm{~m}$ & \\
\hline 20 & $\mathrm{~h}$ & 0 & 1.024859 & -1.984210 & 0.269854 & 0 & $\mathrm{~m}$ & \\
\hline 21 & $\mathrm{n}$ & 0 & -1.096803 & -2.133859 & 0.473619 & 0 & $\mathrm{~m}$ & \\
\hline 22 & $\mathrm{c}$ & 0 & -2.055823 & -1.162465 & 0.259316 & 0 & $\mathrm{~m}$ & \\
\hline 23 & $\mathrm{c}$ & 0 & -3.489611 & -1.226515 & 0.374752 & 0 & $\mathrm{~m}$ & \\
\hline 24 & o & 0 & -4.234113 & -2.135788 & 0.697957 & 0 & $\mathrm{~m}$ & \\
\hline 25 & $\mathrm{n}$ & 0 & -4.054455 & 0.056801 & 0.041474 & 0 & $\mathrm{~m}$ & \\
\hline 26 & $\mathrm{~h}$ & 0 & -5.058321 & 0.101261 & 0.176475 & 0 & $\mathrm{~m}$ & \\
\hline 27 & $\mathrm{c}$ & 0 & -3.363083 & 1.180606 & -0.330551 & 0 & $\mathrm{~m}$ & \\
\hline 28 & $\mathrm{n}$ & 0 & -4.104836 & 2.324986 & -0.551172 & 0 & $\mathrm{~m}$ & \\
\hline 29 & $\mathrm{~h}$ & 0 & -5.008669 & 2.202618 & -0.991348 & 0 & $\mathrm{~m}$ & \\
\hline 30 & $\mathrm{~h}$ & 0 & -3.552631 & 3.061185 & -0.974153 & 0 & $\mathrm{~m}$ & \\
\hline 31 & $\mathrm{n}$ & 0 & -2.057125 & 1.225856 & -0.432108 & 0 & $\mathrm{~m}$ & \\
\hline 32 & $\mathrm{c}$ & 0 & -1.465276 & 0.042898 & -0.117477 & 0 & $\mathrm{~m}$ & \\
\hline
\end{tabular}

\section{s4}

neutral molecule

Charge $=0$ Multiplicity $=1$ for low level calculation on real system.

Charge $=0$ Multiplicity $=1$ for high level calculation on model system .

Charge $=0$ Multiplicity $=1$ for low level calculation on model system .

Center Atomic Atomic Coordinates (Angstroms)

Number Number Type $\quad \mathrm{X} \quad \mathrm{Y} \quad$ Z

$\begin{array}{llllllll}1 & \mathrm{c} & 0 & 3.180734 & 0.070379 & -0.636356 & 0 & \mathrm{~h} \\ 2 & \mathrm{~h} & 0 & 3.784210 & 0.881747 & -1.067978 & 0 & \mathrm{~h} \\ 3 & \mathrm{c} & 0 & 3.945844 & -1.231799 & -0.796146 & 0 & \mathrm{~h}\end{array}$




\begin{tabular}{|c|c|c|c|c|c|c|c|c|}
\hline 4 & $\mathrm{~h}$ & 0 & 4.977494 & -1.067267 & -0.448958 & 0 & & \\
\hline 5 & $\mathrm{~h}$ & 0 & 3.977286 & -1.487672 & -1.864042 & 0 & $\mathrm{~h}$ & \\
\hline 6 & o & 0 & 1.928036 & -0.015295 & -1.326680 & 0 & h & \\
\hline 7 & $\mathrm{c}$ & 0 & 2.822648 & 0.446294 & 0.807696 & 0 & $\mathrm{~h}$ & \\
\hline 8 & $\mathrm{~h}$ & 0 & 2.590214 & -0.453220 & 1.385223 & 0 & $\mathrm{~h}$ & \\
\hline 9 & o & 0 & 3.904133 & 1.172230 & 1.390924 & 0 & $\mathrm{~h}$ & \\
\hline 10 & $\mathrm{~h}$ & 0 & 3.859480 & 1.064774 & 2.353323 & 0 & $\mathrm{~h}$ & \\
\hline 11 & o & 0 & 3.306739 & -2.251674 & -0.034292 & 0 & $\mathrm{~m}$ & h 3 \\
\hline 12 & $\mathrm{~h}$ & 0 & 3.643086 & -3.111369 & -0.326272 & 0 & $\mathrm{~m}$ & \\
\hline 13 & $\mathrm{c}$ & 0 & 0.904810 & 0.670063 & -0.621435 & 0 & $\mathrm{~m}$ & h \\
\hline 14 & $\mathrm{~h}$ & 0 & 0.449929 & 1.441304 & -1.248580 & 0 & $\mathrm{~m}$ & \\
\hline 15 & $\mathrm{c}$ & 0 & 1.572106 & 1.324952 & 0.604476 & 0 & $\mathrm{~m}$ & $\mathrm{~h}$ \\
\hline 16 & $\mathrm{~h}$ & 0 & 0.895993 & 1.335254 & 1.470776 & 0 & $\mathrm{~m}$ & \\
\hline 17 & o & 0 & 1.970886 & 2.632937 & 0.245075 & 0 & $\mathrm{~m}$ & \\
\hline 18 & $\mathrm{~h}$ & 0 & 2.827233 & 2.774659 & 0.691000 & 0 & $\mathrm{~m}$ & \\
\hline 19 & $\mathrm{n}$ & 0 & -0.149836 & -0.281388 & -0.278875 & 0 & $\mathrm{~m}$ & \\
\hline 20 & $\mathrm{c}$ & 0 & -0.026437 & -1.632196 & 0.025614 & 0 & $\mathrm{~m}$ & \\
\hline 21 & $\mathrm{~h}$ & 0 & 0.938126 & -2.120123 & -0.005490 & 0 & $\mathrm{~m}$ & \\
\hline 22 & $\mathrm{n}$ & 0 & -1.172223 & -2.201530 & 0.305870 & 0 & $\mathrm{~m}$ & \\
\hline 23 & $\mathrm{c}$ & 0 & -2.103801 & -1.189727 & 0.182878 & 0 & $\mathrm{~m}$ & \\
\hline 24 & $\mathrm{c}$ & 0 & -3.530827 & -1.206728 & 0.375277 & 0 & $\mathrm{~m}$ & \\
\hline 25 & o & 0 & -4.290549 & -2.100560 & 0.705791 & 0 & $\mathrm{~m}$ & \\
\hline 26 & $\mathrm{n}$ & 0 & -4.063630 & 0.107975 & 0.121089 & 0 & $\mathrm{~m}$ & \\
\hline 27 & $\mathrm{~h}$ & 0 & -5.056479 & 0.182813 & 0.312541 & 0 & $\mathrm{~m}$ & \\
\hline 28 & $\mathrm{c}$ & 0 & -3.351612 & 1.220273 & -0.247421 & 0 & $\mathrm{~m}$ & \\
\hline 29 & $\mathrm{n}$ & 0 & -4.060720 & 2.397509 & -0.385292 & 0 & $\mathrm{~m}$ & \\
\hline 30 & $\mathrm{~h}$ & 0 & -4.991098 & 2.324834 & -0.778675 & 0 & $\mathrm{~m}$ & \\
\hline 31 & $\mathrm{~h}$ & 0 & -3.504598 & 3.129822 & -0.809945 & 0 & $\mathrm{~m}$ & \\
\hline 32 & $\mathrm{n}$ & 0 & -2.051991 & 1.223657 & -0.418699 & 0 & $\mathrm{~m}$ & \\
\hline 33 & $\mathrm{c}$ & 0 & -1.489472 & 0.008454 & -0.180936 & 0 & $\mathrm{~m}$ & \\
\hline
\end{tabular}

radical

Charge $=0$ Multiplicity $=2$ for low level calculation on real system.

Charge $=0$ Multiplicity $=2$ for high level calculation on model system.

Charge $=0$ Multiplicity $=2$ for low level calculation on model system.

\begin{tabular}{|c|c|c|c|c|c|c|}
\hline Center & Atomic & Atomi & Coord & ates (Angstr & & \\
\hline Number & Numbe & Type & $X$ & $\mathrm{Y}$ & $\mathrm{Z}$ & \\
\hline 1 & $\mathrm{c}$ & 0 & 3.061042 & -0.068073 & -0.485231 & 0 \\
\hline 2 & $\mathrm{c}$ & 0 & 3.986633 & -1.192019 & -0.750043 & 0 \\
\hline 3 & $\mathrm{~h}$ & 0 & 4.969255 & -0.955324 & -0.318474 & 0 \\
\hline
\end{tabular}




\begin{tabular}{|c|c|c|c|c|c|c|c|c|}
\hline 4 & $\mathrm{~h}$ & 0 & 4.102304 & -1.323527 & -1.834556 & 0 & & \\
\hline 5 & o & 0 & 1.972736 & 0.035082 & -1.320531 & 0 & $\mathrm{~h}$ & \\
\hline 6 & $\mathrm{c}$ & 0 & 2.770989 & 0.517031 & 0.859641 & 0 & $\mathrm{~h}$ & \\
\hline 7 & $\mathrm{~h}$ & 0 & 2.532567 & -0.258510 & 1.603531 & 0 & $\mathrm{~h}$ & \\
\hline 8 & o & 0 & 3.894624 & 1.285598 & 1.312635 & 0 & $\mathrm{~h}$ & \\
\hline 9 & $\mathrm{~h}$ & 0 & 3.846882 & 1.341239 & 2.281101 & 0 & $\mathrm{~h}$ & \\
\hline 10 & o & 0 & 3.441853 & -2.382307 & -0.141031 & 0 & $\mathrm{~m}$ & h 2 \\
\hline 11 & $\mathrm{~h}$ & 0 & 4.000664 & -3.134615 & -0.394794 & 0 & $\mathrm{~m}$ & \\
\hline 12 & $\mathrm{c}$ & 0 & 0.905651 & 0.716548 & -0.656845 & 0 & $\mathrm{~m}$ & h 5 \\
\hline 13 & $\mathrm{~h}$ & 0 & 0.459313 & 1.444552 & -1.335195 & 0 & $\mathrm{~m}$ & \\
\hline 14 & $\mathrm{c}$ & 0 & 1.540047 & 1.417950 & 0.560937 & 0 & $\mathrm{~m}$ & h 6 \\
\hline 15 & $\mathrm{~h}$ & 0 & 0.827432 & 1.471624 & 1.394516 & 0 & $\mathrm{~m}$ & \\
\hline 16 & o & 0 & 1.969670 & 2.702555 & 0.176083 & 0 & $\mathrm{~m}$ & \\
\hline 17 & $\mathrm{~h}$ & 0 & 2.895435 & 2.770646 & 0.481432 & 0 & $\mathrm{~m}$ & \\
\hline 18 & $\mathrm{n}$ & 0 & -0.115645 & -0.261766 & -0.304924 & 0 & $\mathrm{~m}$ & \\
\hline 19 & $\mathrm{c}$ & 0 & 0.037912 & -1.608631 & 0.017446 & 0 & $\mathrm{~m}$ & \\
\hline 20 & $\mathrm{~h}$ & 0 & 1.007346 & -2.088738 & -0.019301 & 0 & $\mathrm{~m}$ & \\
\hline 21 & $\mathrm{n}$ & 0 & -1.094945 & -2.192384 & 0.315569 & 0 & $\mathrm{~m}$ & \\
\hline 22 & $\mathrm{c}$ & 0 & -2.046768 & -1.200257 & 0.189671 & 0 & $\mathrm{~m}$ & \\
\hline 23 & $\mathrm{c}$ & 0 & -3.471565 & -1.240216 & 0.398032 & 0 & $\mathrm{~m}$ & \\
\hline 24 & o & 0 & -4.211044 & -2.142902 & 0.747522 & 0 & $\mathrm{~m}$ & \\
\hline 25 & $n$ & 0 & -4.030126 & 0.062225 & 0.133707 & 0 & $\mathrm{~m}$ & \\
\hline 26 & $\mathrm{~h}$ & 0 & -5.022433 & 0.120904 & 0.333763 & 0 & $\mathrm{~m}$ & \\
\hline 27 & $\mathrm{c}$ & 0 & -3.342727 & 1.182669 & -0.255677 & 0 & $\mathrm{~m}$ & \\
\hline 28 & $\mathrm{n}$ & 0 & -4.071968 & 2.344918 & -0.399148 & 0 & $\mathrm{~m}$ & \\
\hline 29 & $\mathrm{~h}$ & 0 & -5.011047 & 2.255133 & -0.766810 & 0 & $\mathrm{~m}$ & \\
\hline 30 & $\mathrm{~h}$ & 0 & -3.536905 & 3.083807 & -0.838791 & 0 & $\mathrm{~m}$ & \\
\hline 31 & $\mathrm{n}$ & 0 & -2.044445 & 1.206233 & -0.441881 & 0 & $\mathrm{~m}$ & \\
\hline 32 & $\mathrm{c}$ & 0 & -1.460151 & 0.004001 & -0.195783 & 0 & $\mathrm{~m}$ & \\
\hline
\end{tabular}

s5

neutral molecule

Charge $=0$ Multiplicity $=1$ for low level calculation on real system.

Charge $=0$ Multiplicity $=1$ for high level calculation on model system .

Charge $=0$ Multiplicity $=1$ for low level calculation on model system.

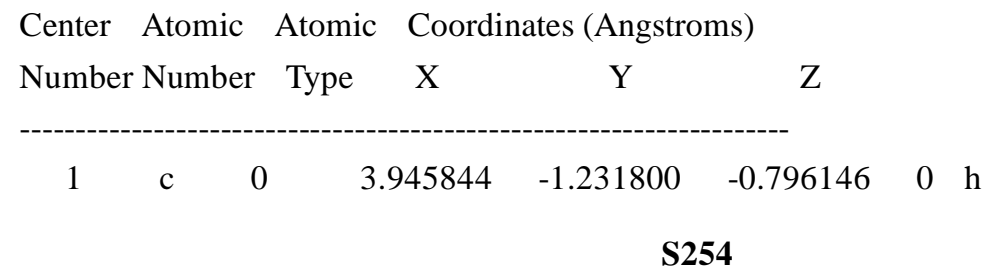




\begin{tabular}{|c|c|c|c|c|c|c|c|c|}
\hline 2 & $\mathrm{~h}$ & 0 & 4.977495 & -1.067271 & -0.448960 & 0 & & \\
\hline 3 & $\mathrm{~h}$ & 0 & 3.977287 & -1.487684 & -1.864038 & 0 & $\mathrm{~h}$ & \\
\hline 4 & o & 0 & 3.306739 & -2.251675 & -0.034291 & 0 & $\mathrm{~h}$ & \\
\hline 5 & $\mathrm{~h}$ & 0 & 3.643085 & -3.111370 & -0.326270 & 0 & $\mathrm{~h}$ & \\
\hline 6 & $\mathrm{c}$ & 0 & 3.180734 & 0.070378 & -0.636357 & 0 & $\mathrm{~h}$ & \\
\hline 7 & $\mathrm{~h}$ & 0 & 3.784211 & 0.881746 & -1.067978 & 0 & $\mathrm{~h}$ & \\
\hline 8 & o & 0 & 1.928036 & -0.015296 & -1.326680 & 0 & $\mathrm{~h}$ & \\
\hline 9 & $\mathrm{c}$ & 0 & 2.822648 & 0.446293 & 0.807696 & 0 & $\mathrm{~h}$ & \\
\hline 10 & $\mathrm{~h}$ & 0 & 2.590214 & -0.453220 & 1.385223 & 0 & $\mathrm{~h}$ & \\
\hline 11 & $\mathrm{c}$ & 0 & 0.904810 & 0.670063 & -0.621435 & 0 & $\mathrm{~m}$ & h 8 \\
\hline 12 & $\mathrm{~h}$ & 0 & 0.449930 & 1.441303 & -1.248581 & 0 & $\mathrm{~m}$ & \\
\hline 13 & $\mathrm{c}$ & 0 & 1.572106 & 1.324952 & 0.604475 & 0 & $\mathrm{~m}$ & $\mathrm{~h}$ \\
\hline 14 & $\mathrm{~h}$ & 0 & 0.895991 & 1.335252 & 1.470774 & 0 & $\mathrm{~m}$ & \\
\hline 15 & o & 0 & 3.904129 & 1.172235 & 1.390924 & 0 & $\mathrm{~m}$ & $\mathrm{~h}$ \\
\hline 16 & $\mathrm{~h}$ & 0 & 3.859478 & 1.064777 & 2.353323 & 0 & $\mathrm{~m}$ & \\
\hline 17 & o & 0 & 1.970887 & 2.632937 & 0.245075 & 0 & $\mathrm{~m}$ & \\
\hline 18 & $\mathrm{~h}$ & 0 & 2.827233 & 2.774659 & 0.691000 & 0 & $\mathrm{~m}$ & \\
\hline 19 & $\mathrm{n}$ & 0 & -0.149835 & -0.281389 & -0.278875 & 0 & $\mathrm{~m}$ & \\
\hline 20 & $\mathrm{c}$ & 0 & -0.026436 & -1.632196 & 0.025614 & 0 & $\mathrm{~m}$ & \\
\hline 21 & $\mathrm{~h}$ & 0 & 0.938127 & -2.120123 & -0.005490 & 0 & $\mathrm{~m}$ & \\
\hline 22 & $\mathrm{n}$ & 0 & -1.172222 & -2.201530 & 0.305871 & 0 & $\mathrm{~m}$ & \\
\hline 23 & $\mathrm{c}$ & 0 & -2.103801 & -1.189727 & 0.182878 & 0 & $\mathrm{~m}$ & \\
\hline 24 & $\mathrm{c}$ & 0 & -3.530827 & -1.206727 & 0.375278 & 0 & $\mathrm{~m}$ & \\
\hline 25 & o & 0 & -4.290549 & -2.100560 & 0.705792 & 0 & $\mathrm{~m}$ & \\
\hline 26 & $\mathrm{n}$ & 0 & -4.063630 & 0.107975 & 0.121089 & 0 & $\mathrm{~m}$ & \\
\hline 27 & $\mathrm{~h}$ & 0 & -5.056479 & 0.182814 & 0.312541 & 0 & $\mathrm{~m}$ & \\
\hline 28 & $\mathrm{c}$ & 0 & -3.351612 & 1.220272 & -0.247422 & 0 & $\mathrm{~m}$ & \\
\hline 29 & $\mathrm{n}$ & 0 & -4.060720 & 2.397509 & -0.385293 & 0 & $\mathrm{~m}$ & \\
\hline 30 & $\mathrm{~h}$ & 0 & -4.991097 & 2.324834 & -0.778675 & 0 & $\mathrm{~m}$ & \\
\hline 31 & $\mathrm{~h}$ & 0 & -3.504597 & 3.129822 & -0.809947 & 0 & $\mathrm{~m}$ & \\
\hline 32 & $\mathrm{n}$ & 0 & -2.051990 & 1.223656 & -0.418700 & 0 & $\mathrm{~m}$ & \\
\hline 33 & $\mathrm{c}$ & 0 & -1.489471 & 0.008454 & -0.180936 & 0 & $\mathrm{~m}$ & \\
\hline
\end{tabular}

radical

Charge $=0$ Multiplicity $=2$ for low level calculation on real system.

Charge $=0$ Multiplicity $=2$ for high level calculation on model system.

Charge $=0$ Multiplicity $=2$ for low level calculation on model system.

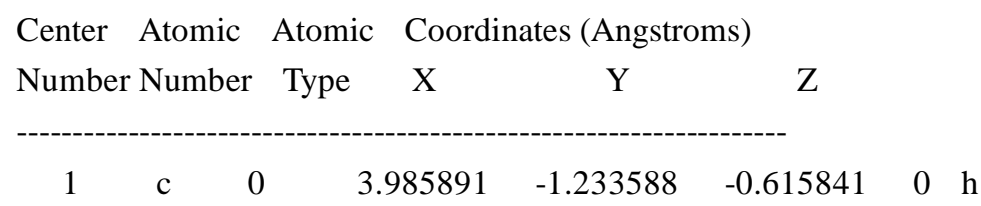




\begin{tabular}{|c|c|c|c|c|c|c|c|c|}
\hline 2 & $\mathrm{~h}$ & 0 & 5.054543 & -1.256101 & -0.803918 & 0 & h & \\
\hline 3 & o & 0 & 3.418765 & -2.293993 & 0.040793 & 0 & $\mathrm{~h}$ & \\
\hline 4 & $\mathrm{~h}$ & 0 & 3.965189 & -3.084576 & -0.082808 & 0 & $\mathrm{~h}$ & \\
\hline 5 & $\mathrm{c}$ & 0 & 3.226201 & 0.032372 & -0.569134 & 0 & $\mathrm{~h}$ & \\
\hline 6 & $\mathrm{~h}$ & 0 & 3.849301 & 0.831872 & -0.989183 & 0 & $\mathrm{~h}$ & \\
\hline 7 & o & 0 & 2.005592 & -0.079894 & -1.350372 & 0 & $\mathrm{~h}$ & \\
\hline 8 & $\mathrm{c}$ & 0 & 2.737480 & 0.483520 & 0.826586 & 0 & $\mathrm{~h}$ & \\
\hline 9 & $\mathrm{~h}$ & 0 & 2.442430 & -0.388836 & 1.419121 & 0 & $\mathrm{~h}$ & \\
\hline 10 & $\mathrm{c}$ & 0 & 0.940805 & 0.627004 & -0.745582 & 0 & $\mathrm{~m}$ & h 7 \\
\hline 11 & $\mathrm{~h}$ & 0 & 0.508092 & 1.349255 & -1.443508 & 0 & $\mathrm{~m}$ & \\
\hline 12 & $\mathrm{c}$ & 0 & 1.523112 & 1.360802 & 0.475973 & 0 & $\mathrm{~m}$ & h \\
\hline 13 & $\mathrm{~h}$ & 0 & 0.785354 & 1.437502 & 1.287193 & 0 & $\mathrm{~m}$ & \\
\hline 14 & o & 0 & 3.773178 & 1.222935 & 1.468473 & 0 & $\mathrm{~m}$ & h 8 \\
\hline 15 & $\mathrm{~h}$ & 0 & 3.618962 & 1.194618 & 2.425246 & 0 & $\mathrm{~m}$ & \\
\hline 16 & o & 0 & 1.968072 & 2.636695 & 0.060497 & 0 & $\mathrm{~m}$ & \\
\hline 17 & $\mathrm{~h}$ & 0 & 2.786906 & 2.802681 & 0.564319 & 0 & $\mathrm{~m}$ & \\
\hline 18 & $\mathrm{n}$ & 0 & -0.117229 & -0.323207 & -0.388399 & 0 & $\mathrm{~m}$ & \\
\hline 19 & $\mathrm{c}$ & 0 & -0.005016 & -1.683264 & -0.128403 & 0 & $\mathrm{~m}$ & \\
\hline 20 & $\mathrm{~h}$ & 0 & 0.947966 & -2.186626 & -0.203636 & 0 & $\mathrm{~m}$ & \\
\hline 21 & $\mathrm{n}$ & 0 & -1.148045 & -2.244092 & 0.180546 & 0 & $\mathrm{~m}$ & \\
\hline 22 & $\mathrm{c}$ & 0 & -2.066696 & -1.215337 & 0.123071 & 0 & $\mathrm{~m}$ & \\
\hline 23 & $\mathrm{c}$ & 0 & -3.484856 & -1.214660 & 0.373168 & 0 & $\mathrm{~m}$ & \\
\hline 24 & o & 0 & -4.246582 & -2.105508 & 0.707091 & 0 & $\mathrm{~m}$ & \\
\hline 25 & $\mathrm{n}$ & 0 & -4.003800 & 0.116303 & 0.182088 & 0 & $\mathrm{~m}$ & \\
\hline 26 & $\mathrm{~h}$ & 0 & -4.986667 & 0.201267 & 0.416119 & 0 & $\mathrm{~m}$ & \\
\hline 27 & $\mathrm{c}$ & 0 & -3.287184 & 1.228443 & -0.178269 & 0 & $\mathrm{~m}$ & \\
\hline 28 & $\mathrm{n}$ & 0 & -3.979478 & 2.421819 & -0.246186 & 0 & $\mathrm{~m}$ & \\
\hline 29 & $\mathrm{~h}$ & 0 & -4.926526 & 2.378609 & -0.602444 & 0 & $\mathrm{~m}$ & \\
\hline 30 & $\mathrm{~h}$ & 0 & -3.427945 & 3.158138 & -0.670004 & 0 & $\mathrm{~m}$ & \\
\hline 31 & $\mathrm{n}$ & 0 & -1.995813 & 1.215852 & -0.403215 & 0 & $\mathrm{~m}$ & \\
\hline 32 & $\mathrm{c}$ & 0 & -1.446279 & -0.016361 & -0.228598 & 0 & $\mathrm{~m}$ & \\
\hline
\end{tabular}

\section{b1}

neutral molecule

Charge $=0$ Multiplicity $=1$ for low level calculation on real system.

Charge $=0$ Multiplicity $=1$ for high level calculation on model system.

Charge $=0$ Multiplicity $=1$ for low level calculation on model system.

Center Atomic Atomic Coordinates (Angstroms)

Number Number Type $\quad \mathrm{X} \quad \mathrm{Y} \quad \mathrm{Z}$ 


$\begin{array}{rrrrrrrrr}1 & \mathrm{n} & 0 & -4.063626 & 0.107988 & 0.121085 & 0 & \mathrm{~h} & \\ 2 & \mathrm{~h} & 0 & -5.056474 & 0.182839 & 0.312535 & 0 & \mathrm{~h} & \\ 3 & \mathrm{c} & 0 & -3.351595 & 1.220279 & -0.247421 & 0 & \mathrm{~h} & \\ 4 & \mathrm{n} & 0 & -4.060691 & 2.397523 & -0.385292 & 0 & \mathrm{~h} & \\ 5 & \mathrm{~h} & 0 & -4.991068 & 2.324858 & -0.778678 & 0 & \mathrm{~h} & \\ 6 & \mathrm{~h} & 0 & -3.504560 & 3.129832 & -0.809941 & 0 & \mathrm{~h} & \\ 7 & \mathrm{n} & 0 & -2.051973 & 1.223651 & -0.418694 & 0 & \mathrm{~h} & \\ 8 & \mathrm{c} & 0 & -3.530837 & -1.206720 & 0.375272 & 0 & \mathrm{~h} & \\ 9 & \mathrm{o} & 0 & -4.290562 & -2.100552 & 0.705781 & 0 & \mathrm{~h} & \\ 10 & \mathrm{c} & 0 & -2.103810 & -1.189733 & 0.182878 & 0 & \mathrm{~m} & \mathrm{~h} \\ 11 & \mathrm{c} & 0 & -1.489467 & 0.008440 & -0.180922 & 0 & \mathrm{~m} & \mathrm{~h} \\ 12 & \mathrm{n} & 0 & -0.149837 & -0.281401 & -0.278874 & 0 & \mathrm{~m} & \\ 13 & \mathrm{c} & 0 & -0.026444 & -1.632210 & 0.025616 & 0 & \mathrm{~m} & \\ 14 & \mathrm{~h} & 0 & 0.938117 & -2.120141 & -0.005489 & 0 & \mathrm{~m} & \\ 15 & \mathrm{n} & 0 & -1.172242 & -2.201546 & 0.305872 & 0 & \mathrm{~m} & \\ 16 & \mathrm{c} & 0 & 0.904806 & 0.670051 & -0.621437 & 0 & \mathrm{~m} & \\ 17 & \mathrm{~h} & 0 & 0.449918 & 1.441289 & -1.248581 & 0 & \mathrm{~m} & \\ 18 & \mathrm{c} & 0 & 1.572096 & 1.324951 & 0.604471 & 0 & \mathrm{~m} & \\ 19 & \mathrm{~h} & 0 & 0.895982 & 1.335259 & 1.470770 & 0 & \mathrm{~m} & \\ 20 & \mathrm{o} & 0 & 1.970875 & 2.632936 & 0.245068 & 0 & \mathrm{~m} & \\ 21 & \mathrm{~h} & 0 & 2.827220 & 2.774662 & 0.690995 & 0 & \mathrm{~m} & \\ 22 & \mathrm{c} & 0 & 2.822642 & 0.446299 & 0.807693 & 0 & \mathrm{~m} & \\ 23 & \mathrm{~h} & 0 & 2.590211 & -0.453214 & 1.385222 & 0 & \mathrm{~m} & \\ 24 & \mathrm{o} & 0 & 3.904120 & 1.172248 & 1.390918 & 0 & \mathrm{~m} & \\ 25 & \mathrm{~h} & 0 & 3.859470 & 1.064793 & 2.353317 & 0 & \mathrm{~m} & \\ 26 & \mathrm{c} & 0 & 3.180727 & 0.070378 & -0.636358 & 0 & \mathrm{~m} & \\ 27 & \mathrm{~h} & 0 & 3.784189 & 0.881754 & -1.067984 & 0 & \mathrm{~m} & \\ 28 & \mathrm{o} & 0 & 1.928025 & -0.015316 & -1.326683 & 0 & \mathrm{~m} & \\ 29 & \mathrm{c} & 0 & 3.945854 & -1.231791 & -0.796140 & 0 & \mathrm{~m} & \\ 30 & \mathrm{~h} & 0 & 4.977504 & -1.067242 & -0.448959 & 0 & \mathrm{~m} & \\ 31 & \mathrm{~h} & 0 & 3.977296 & -1.487673 & -1.864034 & 0 & \mathrm{~m} & \\ 32 & \mathrm{o} & 0 & 3.306768 & -2.251668 & -0.034275 & 0 & \mathrm{~m} & \\ 33 & \mathrm{~h} & 0 & 3.643126 & -3.111362 & -0.326249 & 0 & \mathrm{~m} & \\ ----------------------------------------------------------- & & & \end{array}$

radical

Charge $=0$ Multiplicity $=2$ for low level calculation on real system.

Charge $=0$ Multiplicity $=2$ for high level calculation on model system.

Charge $=0$ Multiplicity $=2$ for low level calculation on model system.

Center Atomic Atomic Coordinates (Angstroms)

Number Number Type $\quad \mathrm{X} \quad \mathrm{Y} \quad \mathrm{Z}$ 


\begin{tabular}{|c|c|c|c|c|c|c|c|}
\hline 1 & $\mathrm{n}$ & 0 & -4.166434 & 0.137410 & 0.195452 & 0 & $\mathrm{~h}$ \\
\hline 2 & $\mathrm{c}$ & 0 & -3.423786 & 1.171074 & -0.125829 & 0 & $\mathrm{~h}$ \\
\hline 3 & $\mathrm{n}$ & 0 & -4.017010 & 2.387929 & -0.245114 & 0 & $\mathrm{~h}$ \\
\hline 4 & $\mathrm{~h}$ & 0 & -5.024677 & 2.428439 & -0.221042 & 0 & $\mathrm{~h}$ \\
\hline 5 & $\mathrm{~h}$ & 0 & -3.491779 & 3.151728 & -0.641247 & 0 & $\mathrm{~h}$ \\
\hline 6 & $\mathrm{n}$ & 0 & -2.039290 & 1.232882 & -0.358538 & 0 & $\mathrm{~h}$ \\
\hline 7 & $\mathrm{c}$ & 0 & -3.594866 & -1.120508 & 0.356004 & 0 & $\mathrm{~h}$ \\
\hline 8 & o & 0 & -4.252801 & -2.112805 & 0.661767 & 0 & $\mathrm{~h}$ \\
\hline 9 & $\mathrm{c}$ & 0 & -2.138808 & -1.171109 & 0.128292 & 0 & $\mathrm{~m} \mathrm{~h}$ \\
\hline 10 & $\mathrm{c}$ & 0 & -1.488872 & 0.053435 & -0.218980 & 0 & $\mathrm{~m} \mathrm{~h}$ \\
\hline 11 & $\mathrm{n}$ & 0 & -0.150770 & -0.280878 & -0.371342 & 0 & $\mathrm{~m}$ \\
\hline 12 & $\mathrm{c}$ & 0 & -0.075091 & -1.629691 & -0.124453 & 0 & $\mathrm{~m}$ \\
\hline 13 & $\mathrm{~h}$ & 0 & 0.870469 & -2.153128 & -0.180300 & 0 & $\mathrm{~m}$ \\
\hline 14 & $\mathrm{n}$ & 0 & -1.247796 & -2.187331 & 0.179563 & 0 & $\mathrm{~m}$ \\
\hline 15 & $\mathrm{c}$ & 0 & 0.927361 & 0.642317 & -0.748261 & 0 & $\mathrm{~m}$ \\
\hline 16 & $\mathrm{~h}$ & 0 & 0.505385 & 1.354027 & -1.463232 & 0 & $\mathrm{~m}$ \\
\hline 17 & $\mathrm{c}$ & 0 & 1.527901 & 1.392212 & 0.454301 & 0 & $\mathrm{~m}$ \\
\hline 18 & $\mathrm{~h}$ & 0 & 0.797926 & 1.498701 & 1.268836 & 0 & $\mathrm{~m}$ \\
\hline 19 & o & 0 & 1.985479 & 2.648725 & 0.001261 & 0 & $\mathrm{~m}$ \\
\hline 20 & $\mathrm{~h}$ & 0 & 2.813747 & 2.817579 & 0.488751 & 0 & $\mathrm{~m}$ \\
\hline 21 & $\mathrm{c}$ & 0 & 2.736460 & 0.504288 & 0.817544 & 0 & $\mathrm{~m}$ \\
\hline 22 & $\mathrm{~h}$ & 0 & 2.440035 & -0.336567 & 1.452289 & 0 & $\mathrm{~m}$ \\
\hline 23 & $\mathrm{o}$ & 0 & 3.797008 & 1.252035 & 1.407343 & 0 & $\mathrm{~m}$ \\
\hline 24 & $\mathrm{~h}$ & 0 & 3.680497 & 1.243169 & 2.369868 & 0 & $\mathrm{~m}$ \\
\hline 25 & $\mathrm{c}$ & 0 & 3.180525 & -0.003124 & -0.561890 & 0 & $\mathrm{~m}$ \\
\hline 26 & $\mathrm{~h}$ & 0 & 3.841848 & 0.748716 & -1.013957 & 0 & $\mathrm{~m}$ \\
\hline 27 & o & 0 & 1.974851 & -0.101403 & -1.337058 & 0 & $\mathrm{~m}$ \\
\hline 28 & $\mathrm{c}$ & 0 & 3.901833 & -1.338543 & -0.563000 & 0 & $\mathrm{~m}$ \\
\hline 29 & $\mathrm{~h}$ & 0 & 4.894019 & -1.188247 & -0.111100 & 0 & $\mathrm{~m}$ \\
\hline 30 & $\mathrm{~h}$ & 0 & 4.040713 & -1.662989 & -1.603617 & 0 & $\mathrm{~m}$ \\
\hline 31 & o & 0 & 3.135509 & -2.283709 & 0.178120 & 0 & $\mathrm{~m}$ \\
\hline 32 & $\mathrm{~h}$ & 0 & 3.544610 & -3.156359 & 0.080746 & 0 & $\mathrm{~m}$ \\
\hline
\end{tabular}

\section{b2}

neutral molecule

Charge $=0$ Multiplicity $=1$ for low level calculation on real system.

Charge $=0$ Multiplicity $=1$ for high level calculation on model system .

Charge $=0$ Multiplicity $=1$ for low level calculation on model system . 


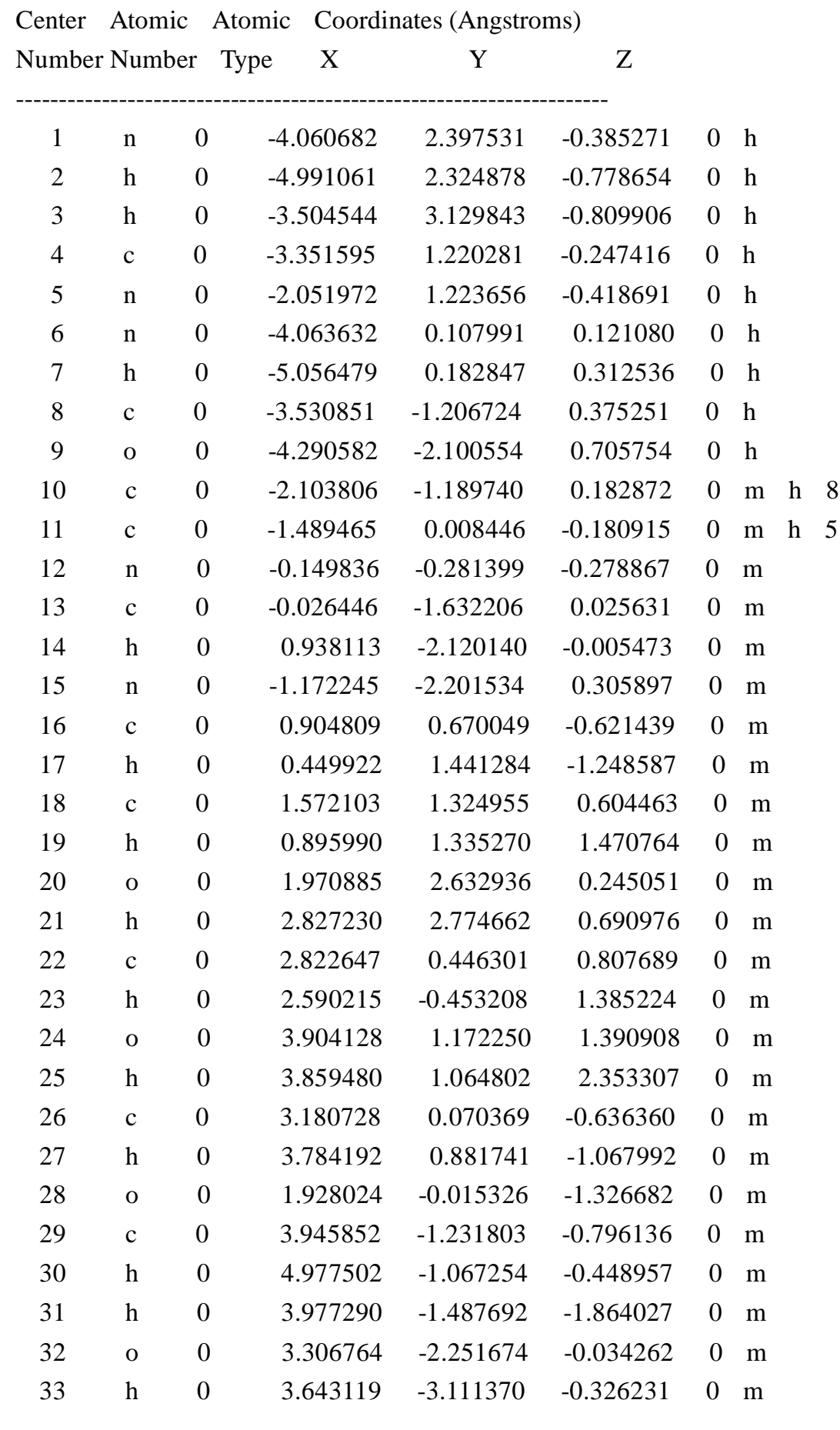

radical

Charge $=0$ Multiplicity $=2$ for low level calculation on real system.

Charge $=0$ Multiplicity $=2$ for high level calculation on model system.

Charge $=0$ Multiplicity $=2$ for low level calculation on model system. 


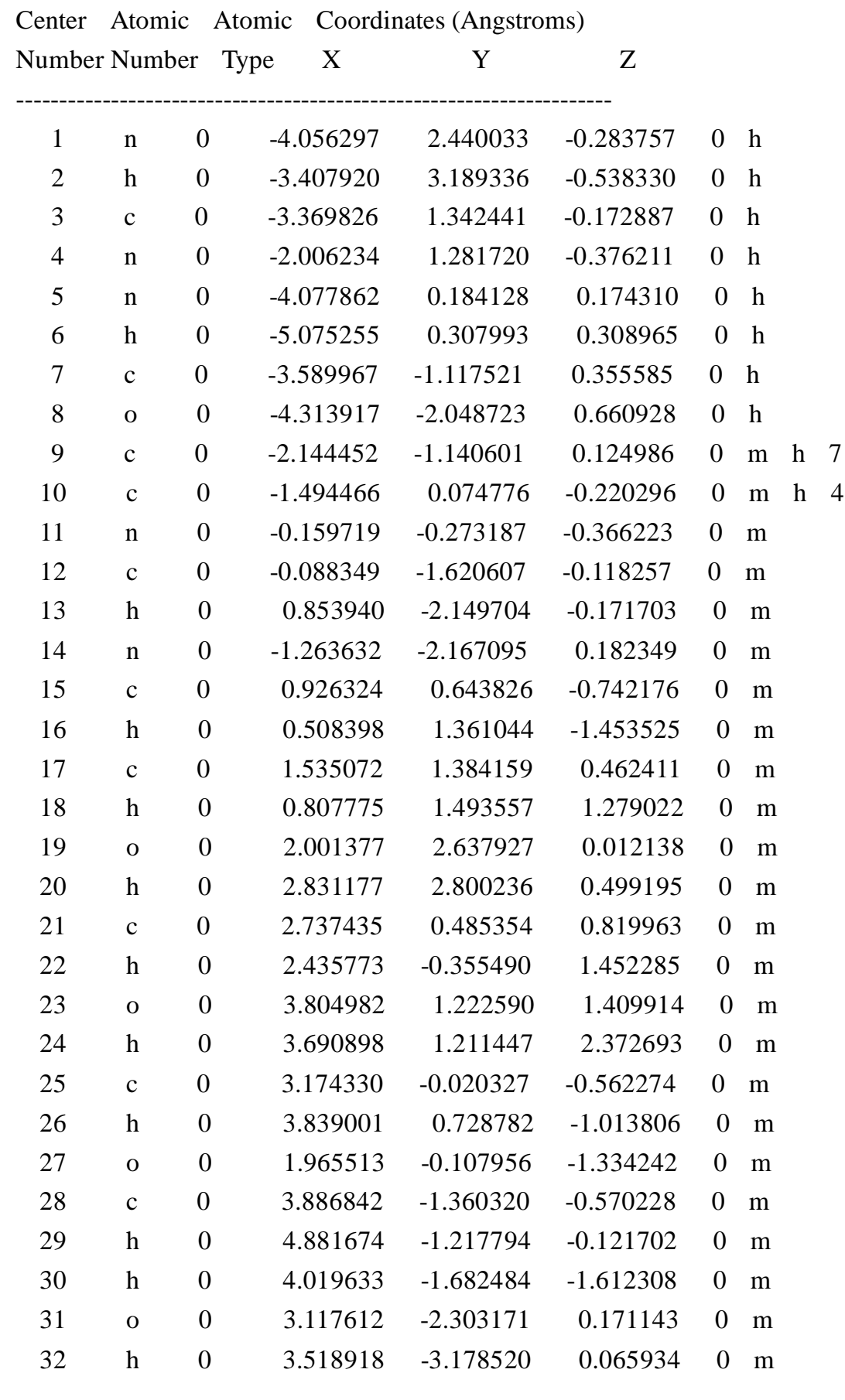

\section{b8}

neutral molecule

Charge $=0$ Multiplicity $=1$ for low level calculation on real system.

Charge $=0$ Multiplicity $=1$ for high level calculation on model system.

Charge $=0$ Multiplicity $=1$ for low level calculation on model system. 


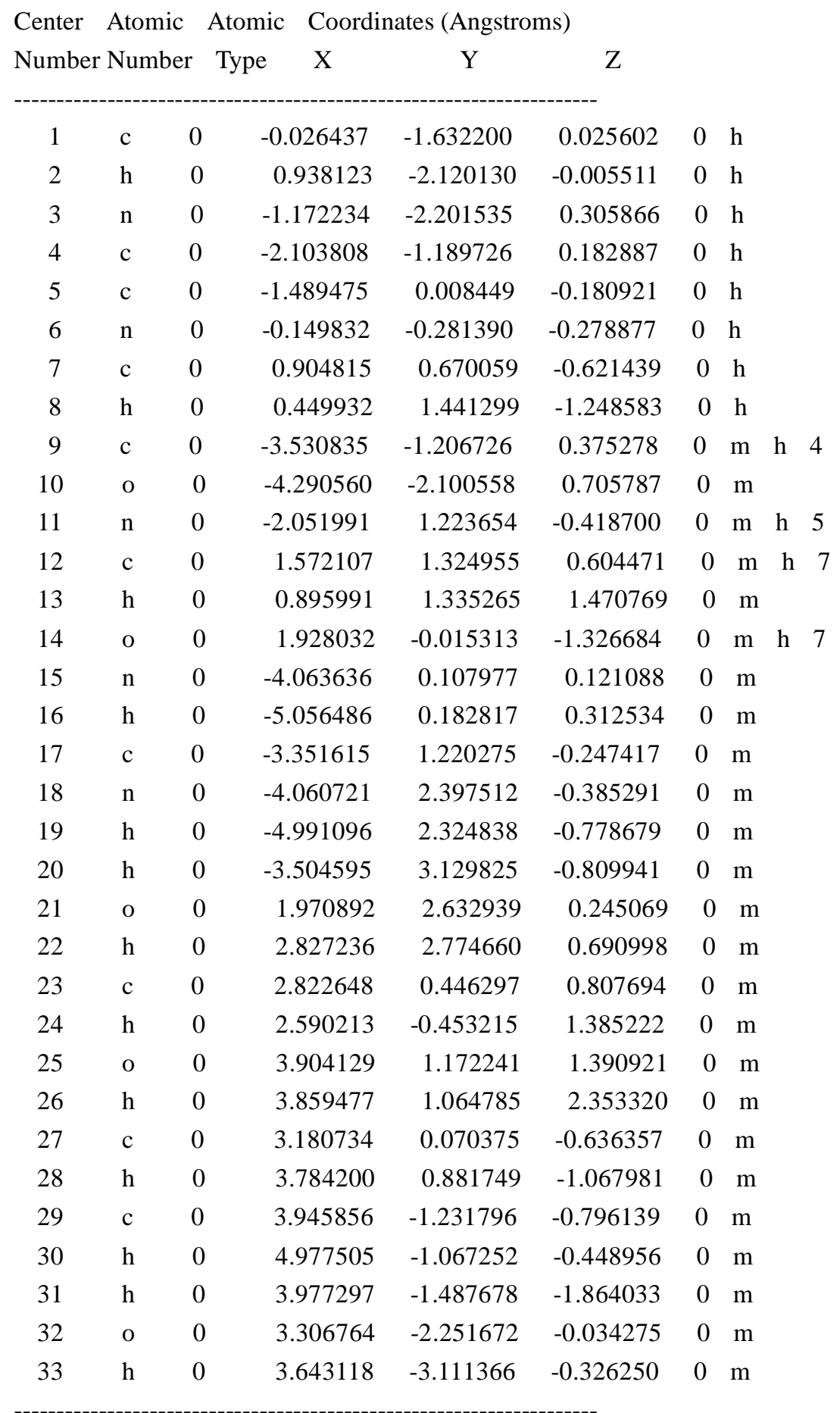

radical

Charge $=0$ Multiplicity $=2$ for low level calculation on real system.

Charge $=0$ Multiplicity $=2$ for high level calculation on model system.

Charge $=0$ Multiplicity $=2$ for low level calculation on model system. 


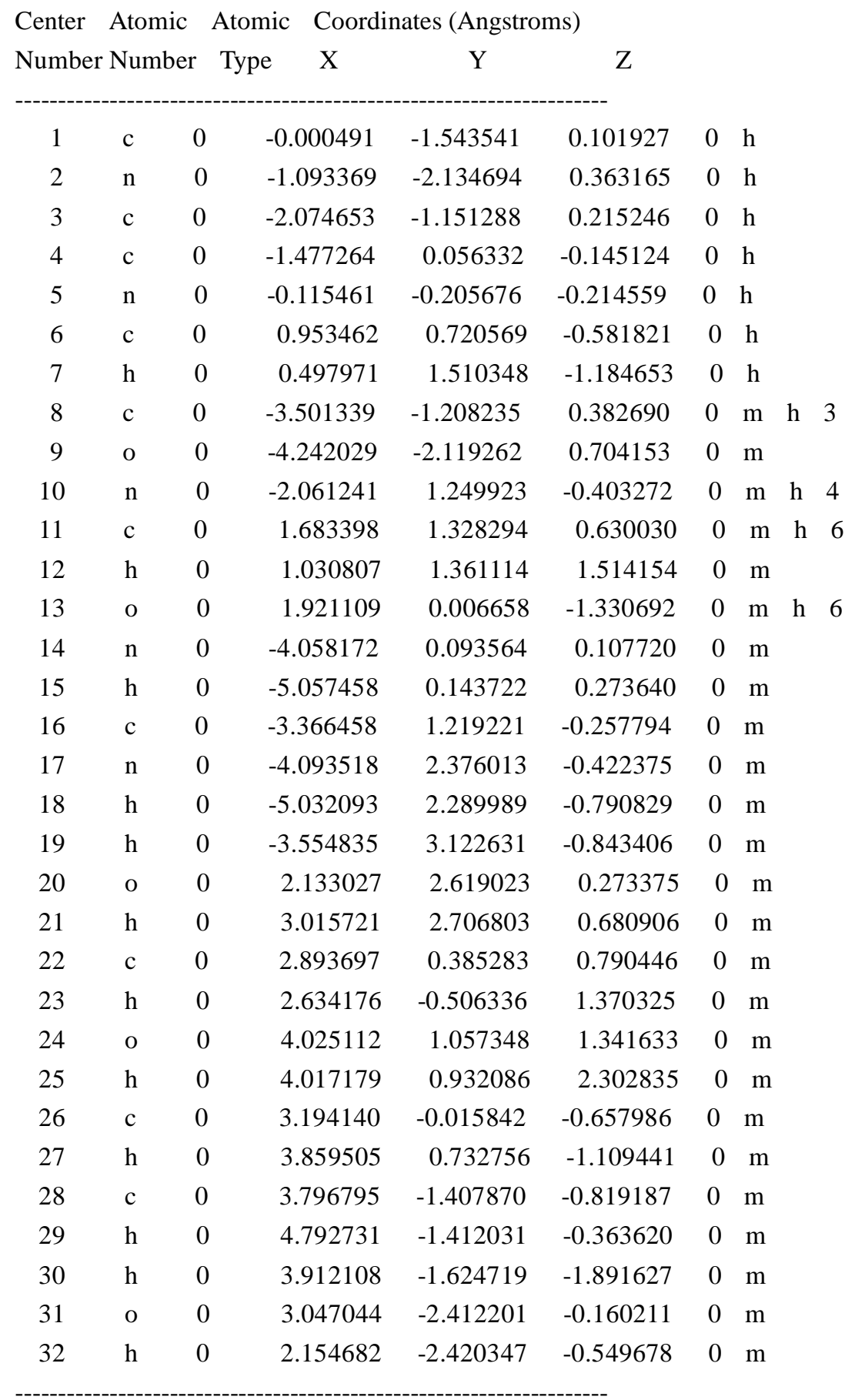




\section{s1}

neutral molecule

Charge $=0$ Multiplicity $=1$ for low level calculation on real system .

Charge $=0$ Multiplicity $=1$ for high level calculation on model system .

Charge $=0$ Multiplicity $=1$ for low level calculation on model system.

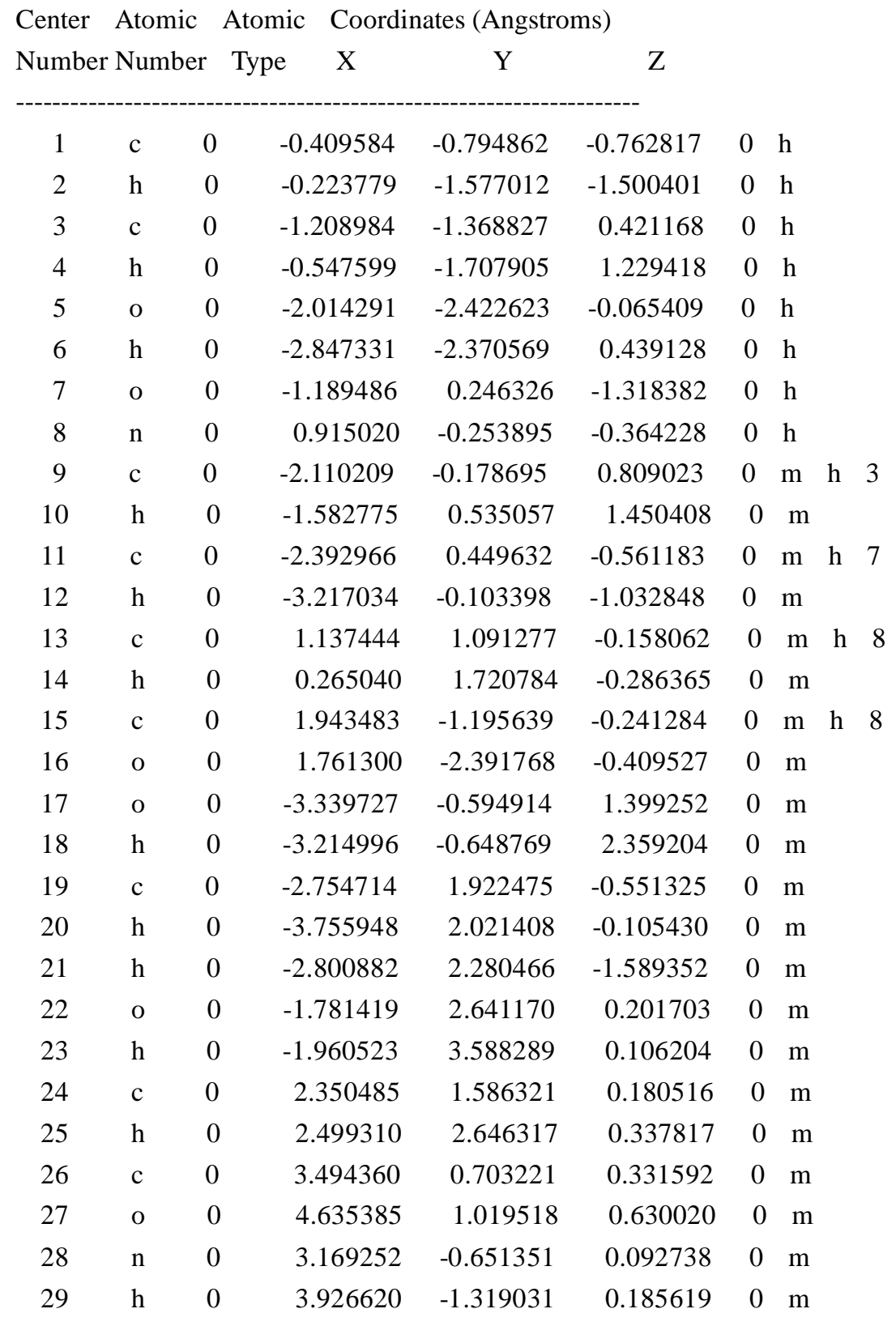


radical

Charge $=0$ Multiplicity $=2$ for low level calculation on real system.

Charge $=0$ Multiplicity $=2$ for high level calculation on model system.

Charge $=0$ Multiplicity $=2$ for low level calculation on model system.

\begin{tabular}{|c|c|c|c|c|c|c|c|c|}
\hline Center & Atomic & & Atomic & ates (Angstro & $\mathrm{ms})$ & & & \\
\hline Number & Numbe & & Type & $\mathrm{Y}$ & $\mathrm{Z}$ & & & \\
\hline 1 & $\mathrm{c}$ & 0 & -0.361961 & -0.485920 & -0.577683 & 0 & $\mathrm{~h}$ & \\
\hline 2 & $\mathrm{c}$ & 0 & -1.232385 & -1.368563 & 0.271699 & 0 & $\mathrm{~h}$ & \\
\hline 3 & $\mathrm{~h}$ & 0 & -0.691261 & -1.790105 & 1.126731 & 0 & $\mathrm{~h}$ & \\
\hline 4 & o & 0 & -1.815611 & -2.385871 & -0.527761 & 0 & $\mathrm{~h}$ & \\
\hline 5 & $\mathrm{~h}$ & 0 & -2.709926 & -2.518241 & -0.161716 & 0 & $\mathrm{~h}$ & \\
\hline 6 & o & 0 & -1.084120 & 0.558800 & -1.094885 & 0 & $\mathrm{~h}$ & \\
\hline 7 & $\mathrm{n}$ & 0 & 0.965985 & -0.123010 & -0.241759 & 0 & $\mathrm{~h}$ & \\
\hline 8 & $\mathrm{c}$ & 0 & -2.336047 & -0.355000 & 0.674665 & 0 & $\mathrm{~m}$ & h 2 \\
\hline 9 & $\mathrm{~h}$ & 0 & -2.010485 & 0.256222 & 1.523635 & 0 & $\mathrm{~m}$ & \\
\hline 10 & $\mathrm{c}$ & 0 & -2.435116 & 0.521357 & -0.582904 & 0 & $\mathrm{~m}$ & $\mathrm{~h}$ \\
\hline 11 & $\mathrm{~h}$ & 0 & -3.082347 & 0.025444 & -1.316062 & 0 & $\mathrm{~m}$ & \\
\hline 12 & $\mathrm{c}$ & 0 & 1.367512 & 1.204369 & -0.202021 & 0 & $\mathrm{~m}$ & h 7 \\
\hline 13 & $\mathrm{~h}$ & 0 & 0.558969 & 1.911681 & -0.331326 & 0 & $\mathrm{~m}$ & \\
\hline 14 & $\mathrm{c}$ & 0 & 1.897093 & -1.172280 & -0.049886 & 0 & $\mathrm{~m}$ & h 7 \\
\hline 15 & o & 0 & 1.575730 & -2.345834 & -0.062468 & 0 & $\mathrm{~m}$ & \\
\hline 16 & o & 0 & -3.599225 & -0.963299 & 0.923411 & 0 & $\mathrm{~m}$ & \\
\hline 17 & $\mathrm{~h}$ & 0 & -3.648283 & -1.188704 & 1.865030 & 0 & $\mathrm{~m}$ & \\
\hline 18 & $\mathrm{c}$ & 0 & -2.914189 & 1.942228 & -0.362356 & 0 & $\mathrm{~m}$ & \\
\hline 19 & $\mathrm{~h}$ & 0 & -3.967136 & 1.896307 & -0.043700 & 0 & $\mathrm{~m}$ & \\
\hline 20 & $\mathrm{~h}$ & 0 & -2.866827 & 2.487230 & -1.316267 & 0 & $\mathrm{~m}$ & \\
\hline 21 & o & 0 & -2.089218 & 2.550132 & 0.624903 & 0 & $\mathrm{~m}$ & \\
\hline 22 & $\mathrm{~h}$ & 0 & -2.381547 & 3.466180 & 0.741341 & 0 & $\mathrm{~m}$ & \\
\hline 23 & $\mathrm{c}$ & 0 & 2.651608 & 1.579065 & -0.003352 & 0 & $\mathrm{~m}$ & \\
\hline 24 & $\mathrm{~h}$ & 0 & 2.931004 & 2.623842 & 0.027052 & 0 & $\mathrm{~m}$ & \\
\hline 25 & $\mathrm{c}$ & 0 & 3.690880 & 0.584627 & 0.194114 & 0 & $\mathrm{~m}$ & \\
\hline 26 & o & 0 & 4.880432 & 0.788624 & 0.381201 & 0 & $\mathrm{~m}$ & \\
\hline 27 & $\mathrm{n}$ & 0 & 3.189619 & -0.736147 & 0.164205 & 0 & $\mathrm{~m}$ & \\
\hline 28 & $\mathrm{~h}$ & 0 & 3.870329 & -1.475481 & 0.299289 & 0 & $\mathrm{~m}$ & \\
\hline
\end{tabular}

s2

neutral molecule 
Charge $=0$ Multiplicity $=1$ for low level calculation on real system .

Charge $=0$ Multiplicity $=1$ for high level calculation on model system .

Charge $=0$ Multiplicity $=1$ for low level calculation on model system .

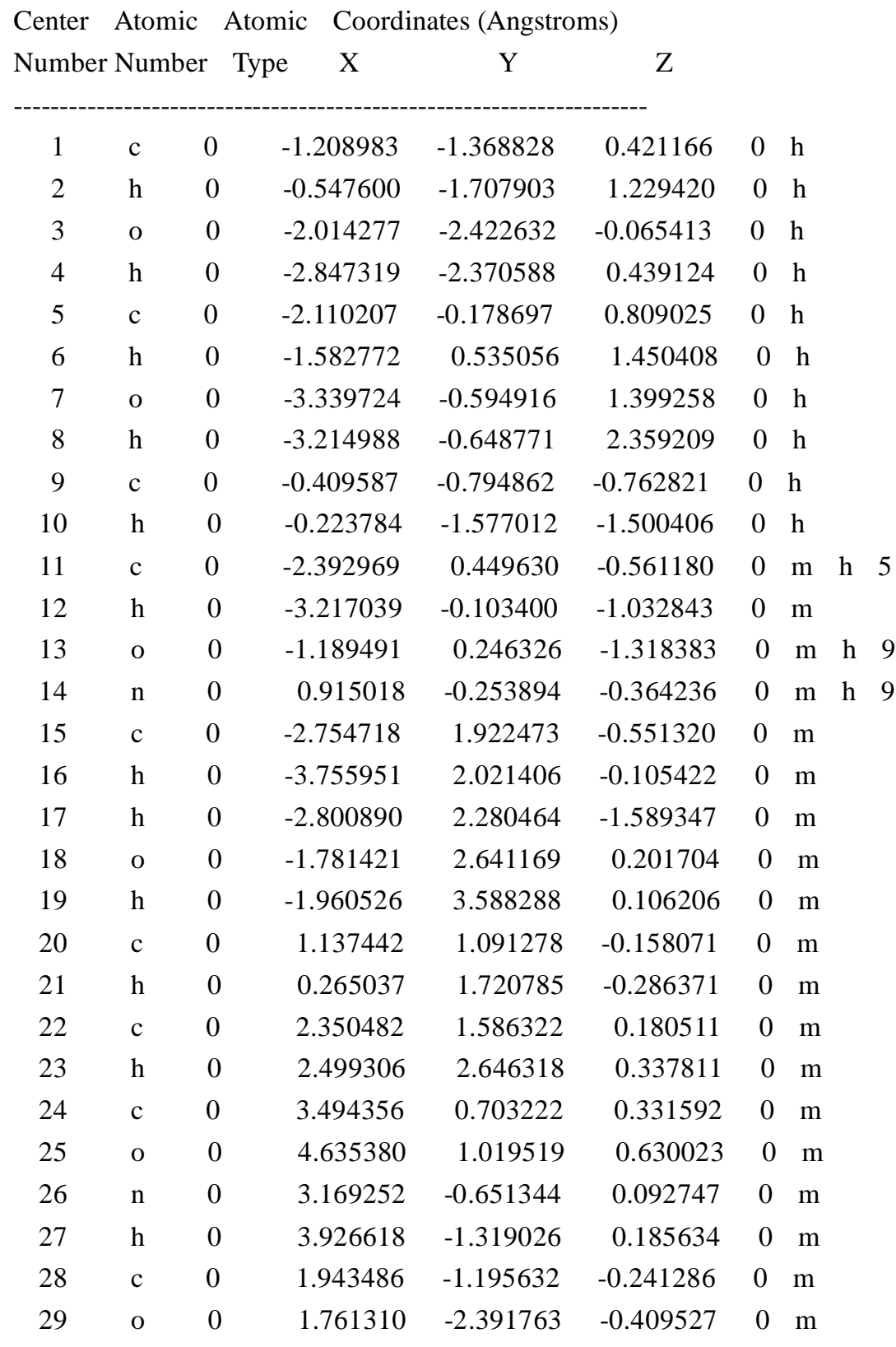

radical

Charge $=0$ Multiplicity $=2$ for low level calculation on real system.

Charge $=0$ Multiplicity $=2$ for high level calculation on model system .

Charge $=0$ Multiplicity $=2$ for low level calculation on model system. 


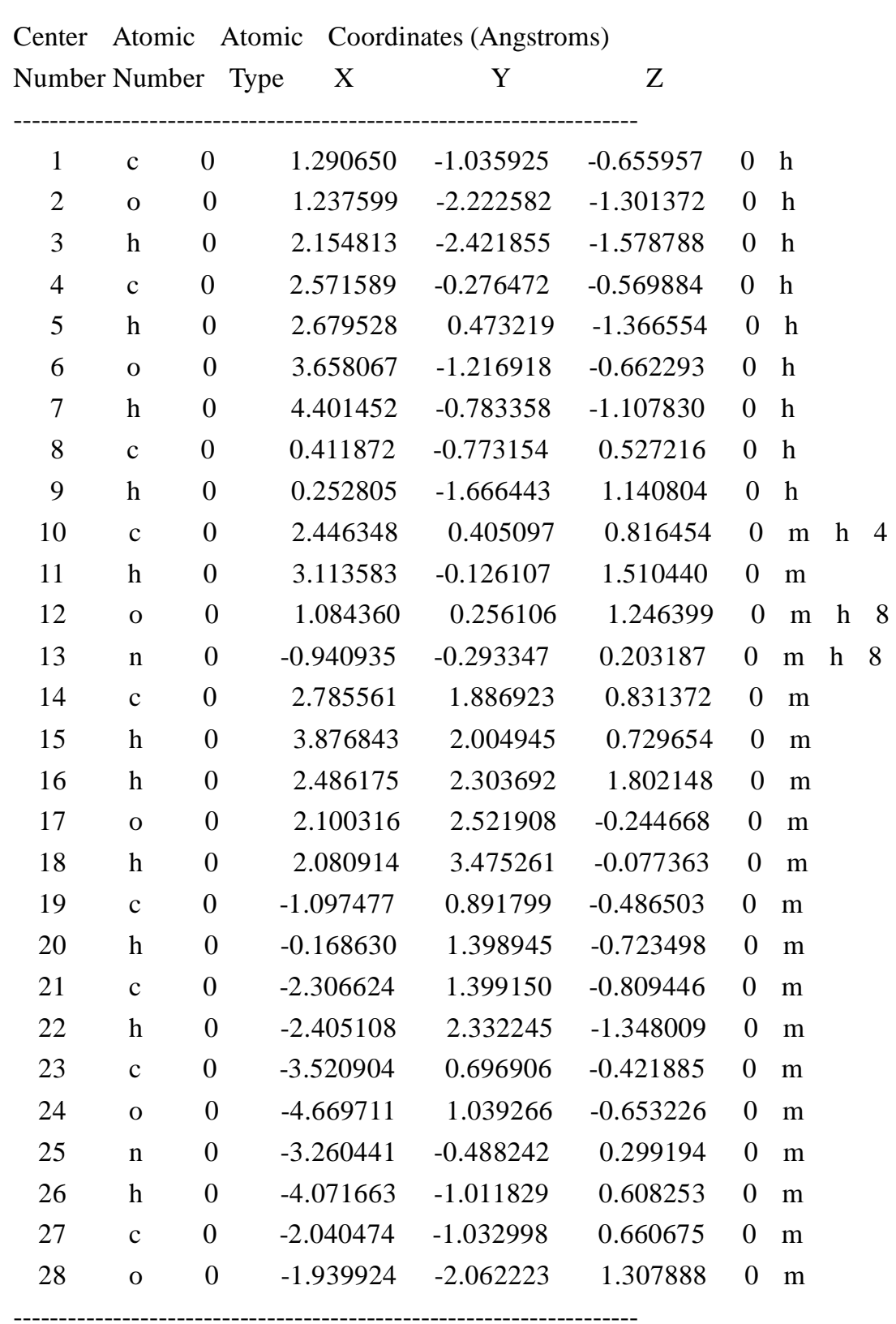

\section{s3}

neutral molecule

Charge $=0$ Multiplicity $=1$ for low level calculation on real system.

Charge $=0$ Multiplicity $=1$ for high level calculation on model system .

Charge $=0$ Multiplicity $=1$ for low level calculation on model system .

Center Atomic Atomic Coordinates (Angstroms) 


\begin{tabular}{|c|c|c|c|c|c|c|c|c|}
\hline Number & Nun & & Type & $\mathrm{Y}$ & $\mathrm{Z}$ & & & \\
\hline 1 & $\mathrm{c}$ & 0 & -2.110210 & -0.178691 & 0.809031 & 0 & $\mathrm{~h}$ & \\
\hline 2 & $\mathrm{~h}$ & 0 & -1.582782 & 0.535070 & 1.450411 & 0 & $\mathrm{~h}$ & \\
\hline 3 & $\mathrm{o}$ & 0 & -3.339725 & -0.594914 & 1.399264 & 0 & $\mathrm{~h}$ & \\
\hline 4 & $\mathrm{~h}$ & 0 & -3.214990 & -0.648768 & 2.359215 & 0 & $\mathrm{~h}$ & \\
\hline 5 & $\mathrm{c}$ & 0 & -2.392969 & 0.449625 & -0.561180 & 0 & $\mathrm{~h}$ & \\
\hline 6 & $\mathrm{~h}$ & 0 & -3.217029 & -0.103417 & -1.032845 & 0 & $\mathrm{~h}$ & \\
\hline 7 & $\mathrm{c}$ & 0 & -1.208982 & -1.368820 & 0.421173 & 0 & $\mathrm{~h}$ & \\
\hline 8 & $\mathrm{~h}$ & 0 & -0.547596 & -1.707885 & 1.229428 & 0 & $\mathrm{~h}$ & \\
\hline 9 & o & 0 & -2.014273 & -2.422626 & -0.065405 & 0 & $\mathrm{~h}$ & \\
\hline 10 & $\mathrm{~h}$ & 0 & -2.847315 & -2.370585 & 0.439131 & 0 & $\mathrm{~h}$ & \\
\hline 11 & $\mathrm{c}$ & 0 & -2.754721 & 1.922466 & -0.551333 & 0 & $\mathrm{~m}$ & h 5 \\
\hline 12 & $\mathrm{~h}$ & 0 & -3.755956 & 2.021400 & -0.105440 & 0 & $\mathrm{~m}$ & \\
\hline 13 & $\mathrm{~h}$ & 0 & -2.800890 & 2.280449 & -1.589363 & 0 & $\mathrm{~m}$ & \\
\hline 14 & $\mathrm{o}$ & 0 & -1.189490 & 0.246308 & -1.318379 & 0 & $\mathrm{~m}$ & h 5 \\
\hline 15 & $\mathrm{c}$ & 0 & -0.409588 & -0.794852 & -0.762814 & 0 & $\mathrm{~m}$ & h 7 \\
\hline 16 & $\mathrm{~h}$ & 0 & -0.223788 & -1.577000 & -1.500401 & 0 & $\mathrm{~m}$ & \\
\hline 17 & o & 0 & -1.781429 & 2.641170 & 0.201690 & 0 & $\mathrm{~m}$ & \\
\hline 18 & $\mathrm{~h}$ & 0 & -1.960536 & 3.588288 & 0.106184 & 0 & $\mathrm{~m}$ & \\
\hline 19 & $\mathrm{n}$ & 0 & 0.915020 & -0.253888 & -0.364230 & 0 & $\mathrm{~m}$ & \\
\hline 20 & $\mathrm{c}$ & 0 & 1.137447 & 1.091283 & -0.158062 & 0 & $\mathrm{~m}$ & \\
\hline 21 & $\mathrm{~h}$ & 0 & 0.265043 & 1.720792 & -0.286358 & 0 & $\mathrm{~m}$ & \\
\hline 22 & $\mathrm{c}$ & 0 & 2.350489 & 1.586322 & 0.180519 & 0 & $\mathrm{~m}$ & \\
\hline 23 & $\mathrm{~h}$ & 0 & 2.499316 & 2.646318 & 0.337822 & 0 & $\mathrm{~m}$ & \\
\hline 24 & $\mathrm{c}$ & 0 & 3.494361 & 0.703220 & 0.331594 & 0 & $\mathrm{~m}$ & \\
\hline 25 & $\mathrm{o}$ & 0 & 4.635387 & 1.019513 & 0.630024 & 0 & $\mathrm{~m}$ & \\
\hline 26 & $\mathrm{n}$ & 0 & 3.169254 & -0.651345 & 0.092746 & 0 & $\mathrm{~m}$ & \\
\hline 27 & $\mathrm{~h}$ & 0 & 3.926618 & -1.319029 & 0.185629 & 0 & $\mathrm{~m}$ & \\
\hline 28 & $\mathrm{c}$ & 0 & 1.943482 & -1.195635 & -0.241296 & 0 & $\mathrm{~m}$ & \\
\hline 29 & o & 0 & 1.761296 & -2.391763 & -0.409546 & 0 & $\mathrm{~m}$ & \\
\hline
\end{tabular}

radical

Charge $=0$ Multiplicity $=2$ for low level calculation on real system .

Charge $=0$ Multiplicity $=2$ for high level calculation on model system .

Charge $=0$ Multiplicity $=2$ for low level calculation on model system .

Center Atomic Atomic Coordinates (Angstroms)

Number Number Type $\quad X \quad$ Y $\quad$ Z

$\begin{array}{llllllll}1 & \text { c } & 0 & -2.198170 & -0.211976 & 0.707599 & 0 & \mathrm{~h} \\ 2 & \text { o } & 0 & -3.264170 & -0.268475 & 1.565917 & 0 & \mathrm{~h}\end{array}$




\begin{tabular}{|c|c|c|c|c|c|c|c|}
\hline 3 & $\mathrm{~h}$ & 0 & -2.945012 & -0.496220 & 2.454919 & 0 & $\mathrm{~h}$ \\
\hline 4 & $\mathrm{c}$ & 0 & -2.433925 & 0.377756 & -0.649425 & 0 & $\mathrm{~h}$ \\
\hline 5 & $\mathrm{~h}$ & 0 & -3.262050 & -0.142411 & -1.165994 & 0 & $\mathrm{~h}$ \\
\hline 6 & $\mathrm{c}$ & 0 & -1.234800 & -1.345070 & 0.546845 & 0 & $\mathrm{~h}$ \\
\hline 7 & $\mathrm{~h}$ & 0 & -0.588535 & -1.536810 & 1.414565 & 0 & $\mathrm{~h}$ \\
\hline 8 & o & 0 & -1.912050 & -2.550521 & 0.153560 & 0 & $\mathrm{~h}$ \\
\hline 9 & $\mathrm{~h}$ & 0 & -2.704286 & -2.632456 & 0.708683 & 0 & $\mathrm{~h}$ \\
\hline 10 & $\mathrm{c}$ & 0 & -2.758343 & 1.864587 & -0.692017 & 0 & $\mathrm{~m}$ \\
\hline 11 & $\mathrm{~h}$ & 0 & -3.780025 & 1.994639 & -0.303679 & 0 & $\mathrm{~m}$ \\
\hline 12 & $\mathrm{~h}$ & 0 & -2.744536 & 2.189254 & -1.742207 & 0 & $\mathrm{~m}$ \\
\hline 13 & o & 0 & -1.212087 & 0.099177 & -1.354452 & 0 & $\mathrm{~m} \mathrm{~h}$ \\
\hline 14 & $\mathrm{c}$ & 0 & -0.425631 & -0.868948 & -0.677711 & 0 & $\mathrm{~m} h$ \\
\hline 15 & $\mathrm{~h}$ & 0 & -0.218672 & -1.719138 & -1.328170 & 0 & $\mathrm{~m}$ \\
\hline 16 & o & 0 & -1.816283 & 2.586779 & 0.091259 & 0 & $\mathrm{~m}$ \\
\hline 17 & $\mathrm{~h}$ & 0 & -1.937601 & 3.531756 & -0.084739 & 0 & $\mathrm{~m}$ \\
\hline 18 & $\mathrm{n}$ & 0 & 0.884622 & -0.285484 & -0.323728 & 0 & $\mathrm{~m}$ \\
\hline 19 & $\mathrm{c}$ & 0 & 1.032223 & 1.052996 & -0.023164 & 0 & $\mathrm{~m}$ \\
\hline 20 & $\mathrm{~h}$ & 0 & 0.120428 & 1.637363 & -0.076884 & 0 & $\mathrm{~m}$ \\
\hline 21 & $\mathrm{c}$ & 0 & 2.226098 & 1.598699 & 0.303928 & 0 & $\mathrm{~m}$ \\
\hline 22 & $\mathrm{~h}$ & 0 & 2.316614 & 2.652083 & 0.533535 & 0 & $\mathrm{~m}$ \\
\hline 23 & $\mathrm{c}$ & 0 & 3.426886 & 0.782036 & 0.345306 & 0 & $\mathrm{~m}$ \\
\hline 24 & o & 0 & 4.558502 & 1.147768 & 0.622584 & 0 & $\mathrm{~m}$ \\
\hline 25 & $\mathrm{n}$ & 0 & 3.175258 & -0.569865 & 0.018899 & 0 & $\mathrm{~m}$ \\
\hline 26 & $\mathrm{~h}$ & 0 & 3.974647 & -1.193183 & 0.038247 & 0 & $\mathrm{~m}$ \\
\hline 27 & $\mathrm{c}$ & 0 & 1.971987 & -1.169366 & -0.301745 & 0 & $\mathrm{~m}$ \\
\hline 28 & o & 0 & 1.860078 & -2.362442 & -0.537889 & 0 & $\mathrm{~m}$ \\
\hline
\end{tabular}

\section{s4}

neutral molecule

Charge $=0$ Multiplicity $=1$ for low level calculation on real system.

Charge $=0$ Multiplicity $=1$ for high level calculation on model system.

Charge $=0$ Multiplicity $=1$ for low level calculation on model system.

\begin{tabular}{|c|c|c|c|c|c|c|}
\hline Center & Atomic & Atom & Coordi & ates (Angstr & ns) & \\
\hline Number & Numbe & r Type & $\mathrm{X}$ & $\mathrm{Y}$ & $\mathrm{Z}$ & \\
\hline 1 & $\mathrm{c}$ & 0 & -2.392971 & 0.449626 & -0.561182 & 0 \\
\hline 2 & $\mathrm{~h}$ & 0 & -3.217035 & -0.103408 & -1.032851 & 0 \\
\hline 3 & $\mathrm{c}$ & 0 & -2.754733 & 1.922465 & -0.551325 & 0 \\
\hline 4 & $\mathrm{~h}$ & 0 & -3.755970 & 2.021389 & -0.105434 & 0 \\
\hline
\end{tabular}




\begin{tabular}{|c|c|c|c|c|c|c|c|c|}
\hline 5 & $\mathrm{~h}$ & 0 & -2.800901 & 2.280456 & -1.589352 & 0 & & \\
\hline 6 & o & 0 & -1.189492 & 0.246320 & -1.318384 & 0 & $\mathrm{~h}$ & \\
\hline 7 & $\mathrm{c}$ & 0 & -2.110212 & -0.178698 & 0.809025 & 0 & $\mathrm{~h}$ & \\
\hline 8 & $\mathrm{~h}$ & 0 & -1.582784 & 0.535055 & 1.450413 & 0 & $\mathrm{~h}$ & \\
\hline 9 & o & 0 & -3.339727 & -0.594920 & 1.399258 & 0 & $\mathrm{~h}$ & \\
\hline 10 & $\mathrm{~h}$ & 0 & -3.214990 & -0.648780 & 2.359208 & 0 & $\mathrm{~h}$ & \\
\hline 11 & o & 0 & -1.781448 & 2.641170 & 0.201706 & 0 & $\mathrm{~m}$ & $\mathrm{~h}$ \\
\hline 12 & $\mathrm{~h}$ & 0 & -1.960560 & 3.588287 & 0.106207 & 0 & $\mathrm{~m}$ & \\
\hline 13 & $\mathrm{c}$ & 0 & -0.409580 & -0.794860 & -0.762820 & 0 & $\mathrm{~m}$ & $\mathrm{~h}$ \\
\hline 14 & $\mathrm{~h}$ & 0 & -0.223773 & -1.577010 & -1.500404 & 0 & $\mathrm{~m}$ & \\
\hline 15 & $\mathrm{c}$ & 0 & -1.208979 & -1.368825 & 0.421171 & 0 & $\mathrm{~m}$ & $\mathrm{~h}$ \\
\hline 16 & $\mathrm{~h}$ & 0 & -0.547592 & -1.707885 & 1.229428 & 0 & $\mathrm{~m}$ & \\
\hline 17 & o & 0 & -2.014265 & -2.422636 & -0.065405 & 0 & $\mathrm{~m}$ & \\
\hline 18 & $\mathrm{~h}$ & 0 & -2.847308 & -2.370596 & 0.439130 & 0 & $\mathrm{~m}$ & \\
\hline 19 & $\mathrm{n}$ & 0 & 0.915025 & -0.253891 & -0.364234 & 0 & $\mathrm{~m}$ & \\
\hline 20 & $\mathrm{c}$ & 0 & 1.137448 & 1.091282 & -0.158072 & 0 & $\mathrm{~m}$ & \\
\hline 21 & $\mathrm{~h}$ & 0 & 0.265044 & 1.720788 & -0.286375 & 0 & $\mathrm{~m}$ & \\
\hline 22 & $\mathrm{c}$ & 0 & 2.350488 & 1.586326 & 0.180510 & 0 & $\mathrm{~m}$ & \\
\hline 23 & $\mathrm{~h}$ & 0 & 2.499312 & 2.646323 & 0.337808 & 0 & $\mathrm{~m}$ & \\
\hline 24 & $\mathrm{c}$ & 0 & 3.494362 & 0.703227 & 0.331594 & 0 & $\mathrm{~m}$ & \\
\hline 25 & o & 0 & 4.635386 & 1.019525 & 0.630026 & 0 & $\mathrm{~m}$ & \\
\hline 26 & $\mathrm{n}$ & 0 & 3.169259 & -0.651340 & 0.092752 & 0 & $\mathrm{~m}$ & \\
\hline 27 & $\mathrm{~h}$ & 0 & 3.926624 & -1.319021 & 0.185641 & 0 & $\mathrm{~m}$ & \\
\hline 28 & $\mathrm{c}$ & 0 & 1.943489 & -1.195634 & -0.241291 & 0 & $\mathrm{~m}$ & \\
\hline 29 & o & 0 & 1.761307 & -2.391763 & -0.409536 & 0 & $\mathrm{~m}$ & \\
\hline
\end{tabular}

radical

Charge $=0$ Multiplicity $=2$ for low level calculation on real system.

Charge $=0$ Multiplicity $=2$ for high level calculation on model system.

Charge $=0$ Multiplicity $=2$ for low level calculation on model system.

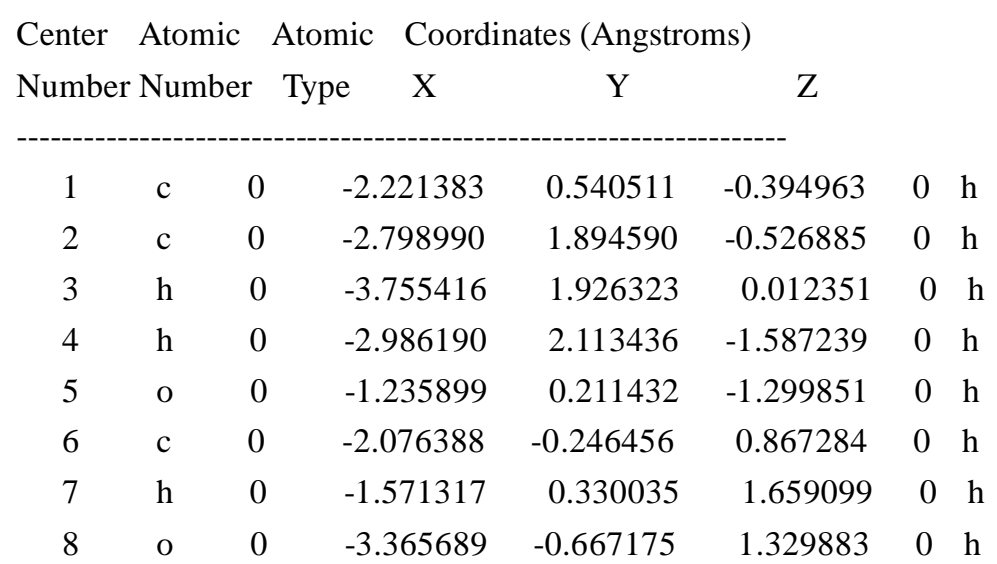




$\begin{array}{rrrrrrrrrr}9 & \mathrm{~h} & 0 & -3.296044 & -0.857462 & 2.279761 & 0 & \mathrm{~h} & & \\ 10 & \mathrm{o} & 0 & -1.866941 & 2.846812 & 0.033142 & 0 & \mathrm{~m} & \mathrm{~h} & 2 \\ 11 & \mathrm{~h} & 0 & -2.205112 & 3.739003 & -0.147275 & 0 & \mathrm{~m} & & \\ 12 & \mathrm{c} & 0 & -0.420192 & -0.832872 & -0.779866 & 0 & \mathrm{~m} & \mathrm{~h} & 5 \\ 13 & \mathrm{~h} & 0 & -0.237036 & -1.575300 & -1.554904 & 0 & \mathrm{~m} & & \\ 14 & \mathrm{c} & 0 & -1.207167 & -1.445499 & 0.394581 & 0 & \mathrm{~m} & \mathrm{~h} & 6 \\ 15 & \mathrm{~h} & 0 & -0.529128 & -1.828492 & 1.167345 & 0 & \mathrm{~m} & \\ 16 & \mathrm{o} & 0 & -2.038939 & -2.464456 & -0.105118 & 0 & \mathrm{~m} & \\ 17 & \mathrm{~h} & 0 & -2.923734 & -2.290086 & 0.269681 & 0 & \mathrm{~m} & \\ 18 & \mathrm{n} & 0 & 0.885386 & -0.262294 & -0.376672 & 0 & \mathrm{~m} & \\ 19 & \mathrm{c} & 0 & 1.080452 & 1.085189 & -0.139890 & 0 & \mathrm{~m} & \\ 20 & \mathrm{~h} & 0 & 0.207208 & 1.714776 & -0.265653 & 0 & \mathrm{~m} & \\ 21 & \mathrm{c} & 0 & 2.281574 & 1.592236 & 0.219143 & 0 & \mathrm{~m} & \\ 22 & \mathrm{~h} & 0 & 2.409335 & 2.651154 & 0.399627 & 0 & \mathrm{~m} & \\ 23 & \mathrm{c} & 0 & 3.441047 & 0.726396 & 0.359657 & 0 & \mathrm{~m} & \\ 24 & \mathrm{o} & 0 & 4.572148 & 1.057611 & 0.675731 & 0 & \mathrm{~m} & & \\ 25 & \mathrm{n} & 0 & 3.144604 & -0.628291 & 0.084949 & 0 & \mathrm{~m} & & \\ 26 & \mathrm{~h} & 0 & 3.914724 & -1.282988 & 0.166200 & 0 & \mathrm{~m} & & \\ 27 & \mathrm{c} & 0 & 1.933063 & -1.187000 & -0.271349 & 0 & \mathrm{~m} & & \\ 28 & \mathrm{o} & 0 & 1.771655 & -2.380332 & -0.474187 & 0 & \mathrm{~m} & & \end{array}$

\section{s5}

neutral molecule

Charge $=0$ Multiplicity $=1$ for low level calculation on real system.

Charge $=0$ Multiplicity $=1$ for high level calculation on model system.

Charge $=0$ Multiplicity $=1$ for low level calculation on model system .

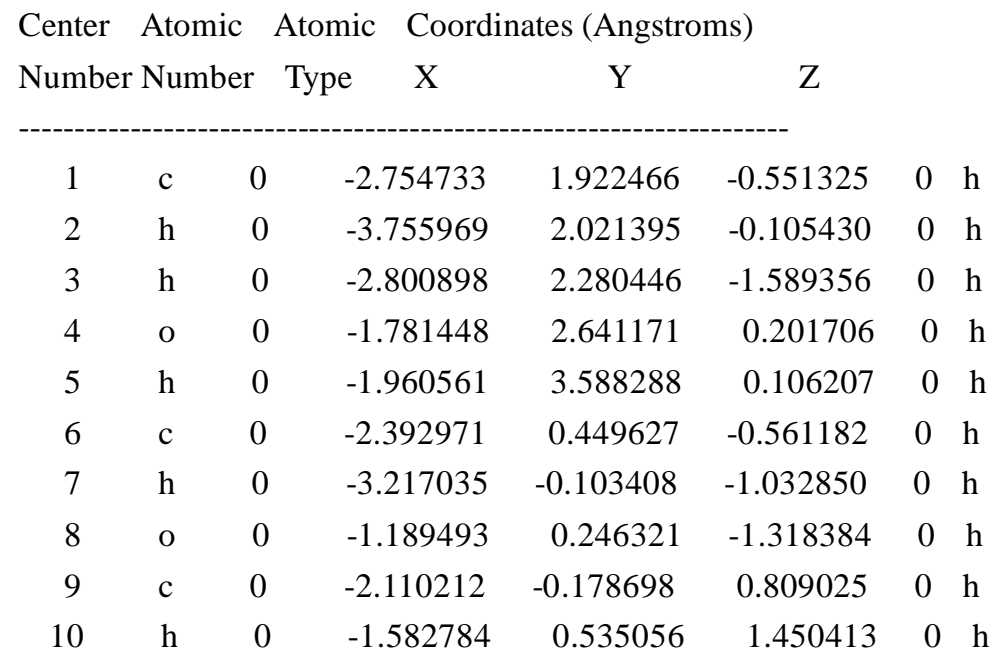




$\begin{array}{llllllllll}11 & \mathrm{c} & 0 & -0.409580 & -0.794859 & -0.762820 & 0 & \mathrm{~m} & \mathrm{~h} & 8 \\ 12 & \mathrm{~h} & 0 & -0.223773 & -1.577009 & -1.500403 & 0 & \mathrm{~m} & & \\ 13 & \mathrm{c} & 0 & -1.208979 & -1.368824 & 0.421172 & 0 & \mathrm{~m} & \mathrm{~h} & 9 \\ 14 & \mathrm{~h} & 0 & -0.547596 & -1.707888 & 1.229430 & 0 & \mathrm{~m} & & \\ 15 & \mathrm{o} & 0 & -3.339727 & -0.594924 & 1.399257 & 0 & \mathrm{~m} & \mathrm{~h} & 9 \\ 16 & \mathrm{~h} & 0 & -3.214993 & -0.648775 & 2.359208 & 0 & \mathrm{~m} & & \\ 17 & \mathrm{o} & 0 & -2.014265 & -2.422635 & -0.065406 & 0 & \mathrm{~m} & \\ 18 & \mathrm{~h} & 0 & -2.847308 & -2.370595 & 0.439129 & 0 & \mathrm{~m} & \\ 19 & \mathrm{n} & 0 & 0.915025 & -0.253891 & -0.364234 & 0 & \mathrm{~m} & \\ 20 & \mathrm{c} & 0 & 1.137448 & 1.091282 & -0.158072 & 0 & \mathrm{~m} & \\ 21 & \mathrm{~h} & 0 & 0.265044 & 1.720789 & -0.286374 & 0 & \mathrm{~m} & \\ 22 & \mathrm{c} & 0 & 2.350488 & 1.586326 & 0.180510 & 0 & \mathrm{~m} & \\ 23 & \mathrm{~h} & 0 & 2.499313 & 2.646323 & 0.337808 & 0 & \mathrm{~m} & \\ 24 & \mathrm{c} & 0 & 3.494362 & 0.703227 & 0.331593 & 0 & \mathrm{~m} & \\ 25 & \mathrm{o} & 0 & 4.635386 & 1.019524 & 0.630025 & 0 & \mathrm{~m} & \\ 26 & \mathrm{n} & 0 & 3.169259 & -0.651340 & 0.092752 & 0 & \mathrm{~m} & & \\ 27 & \mathrm{~h} & 0 & 3.926624 & -1.319022 & 0.185640 & 0 & \mathrm{~m} & & \\ 28 & \mathrm{c} & 0 & 1.943489 & -1.195634 & -0.241291 & 0 & \mathrm{~m} & & \\ 29 & \mathrm{o} & 0 & 1.761306 & -2.391763 & -0.409536 & 0 & \mathrm{~m} & & \end{array}$

radical

Charge $=0$ Multiplicity $=2$ for low level calculation on real system.

Charge $=0$ Multiplicity $=2$ for high level calculation on model system.

Charge $=0$ Multiplicity $=2$ for low level calculation on model system.

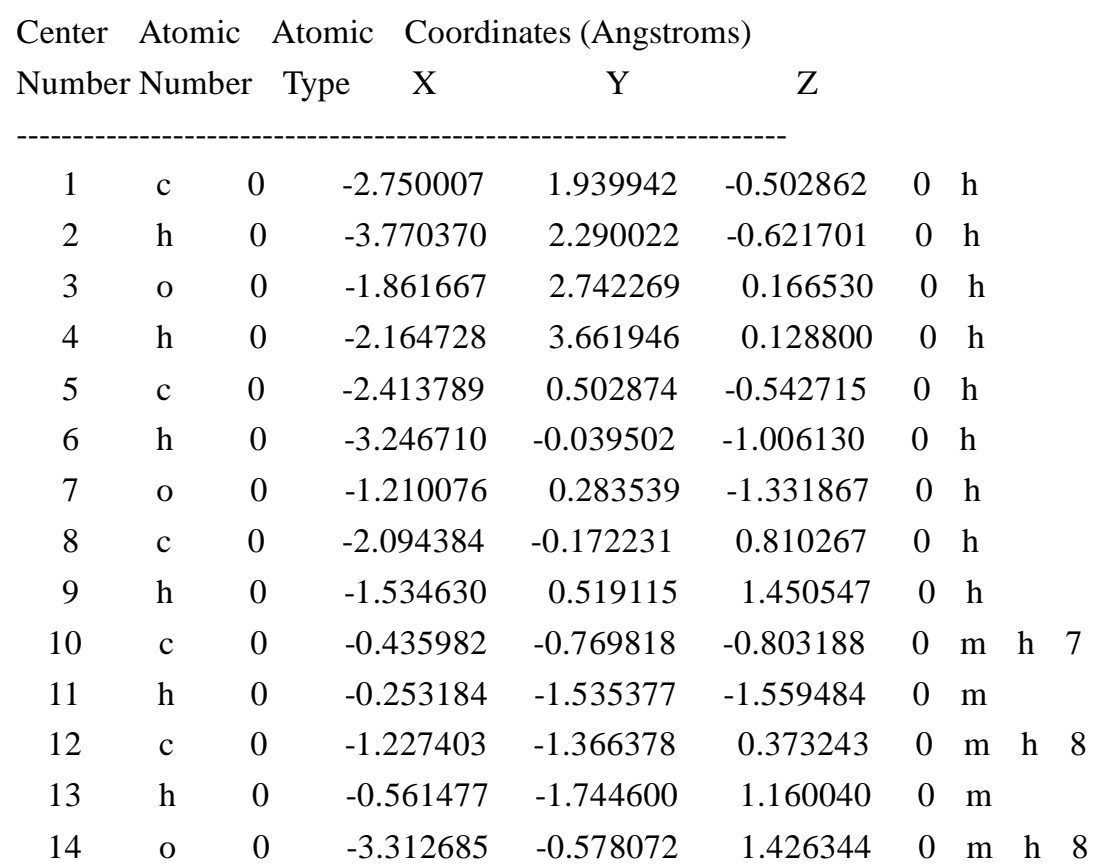




$\begin{array}{lllrrrrc}15 & \mathrm{~h} & 0 & -3.152810 & -0.686944 & 2.376482 & 0 & \mathrm{~m} \\ 16 & \mathrm{o} & 0 & -2.061197 & -2.387938 & -0.133874 & 0 & \mathrm{~m} \\ 17 & \mathrm{~h} & 0 & -2.883123 & -2.338949 & 0.388649 & 0 & \mathrm{~m} \\ 18 & \mathrm{n} & 0 & 0.894128 & -0.241451 & -0.388347 & 0 & \mathrm{~m} \\ 19 & \mathrm{c} & 0 & 1.145322 & 1.105083 & -0.240925 & 0 & \mathrm{~m} \\ 20 & \mathrm{~h} & 0 & 0.293552 & 1.745448 & -0.429576 & 0 & \mathrm{~m} \\ 21 & \mathrm{c} & 0 & 2.359892 & 1.589763 & 0.107215 & 0 & \mathrm{~m} \\ 22 & \mathrm{~h} & 0 & 2.529854 & 2.652532 & 0.216711 & 0 & \mathrm{~m} \\ 23 & \mathrm{c} & 0 & 3.478190 & 0.688871 & 0.328929 & 0 & \mathrm{~m} \\ 24 & \mathrm{o} & 0 & 4.617745 & 0.992763 & 0.645309 & 0 & \mathrm{~m} \\ 25 & \mathrm{n} & 0 & 3.128301 & -0.666987 & 0.137408 & 0 & \mathrm{~m} \\ 26 & \mathrm{~h} & 0 & 3.868765 & -1.346237 & 0.273033 & 0 & \mathrm{~m} \\ 27 & \mathrm{c} & 0 & 1.899665 & -1.197741 & -0.208828 & 0 & \mathrm{~m} \\ 28 & \mathrm{o} & 0 & 1.696485 & -2.395134 & -0.340895 & 0 & \mathrm{~m}\end{array}$

\section{b3}

neutral molecule

Charge $=0$ Multiplicity $=1$ for low level calculation on real system.

Charge $=0$ Multiplicity $=1$ for high level calculation on model system.

Charge $=0$ Multiplicity $=1$ for low level calculation on model system.

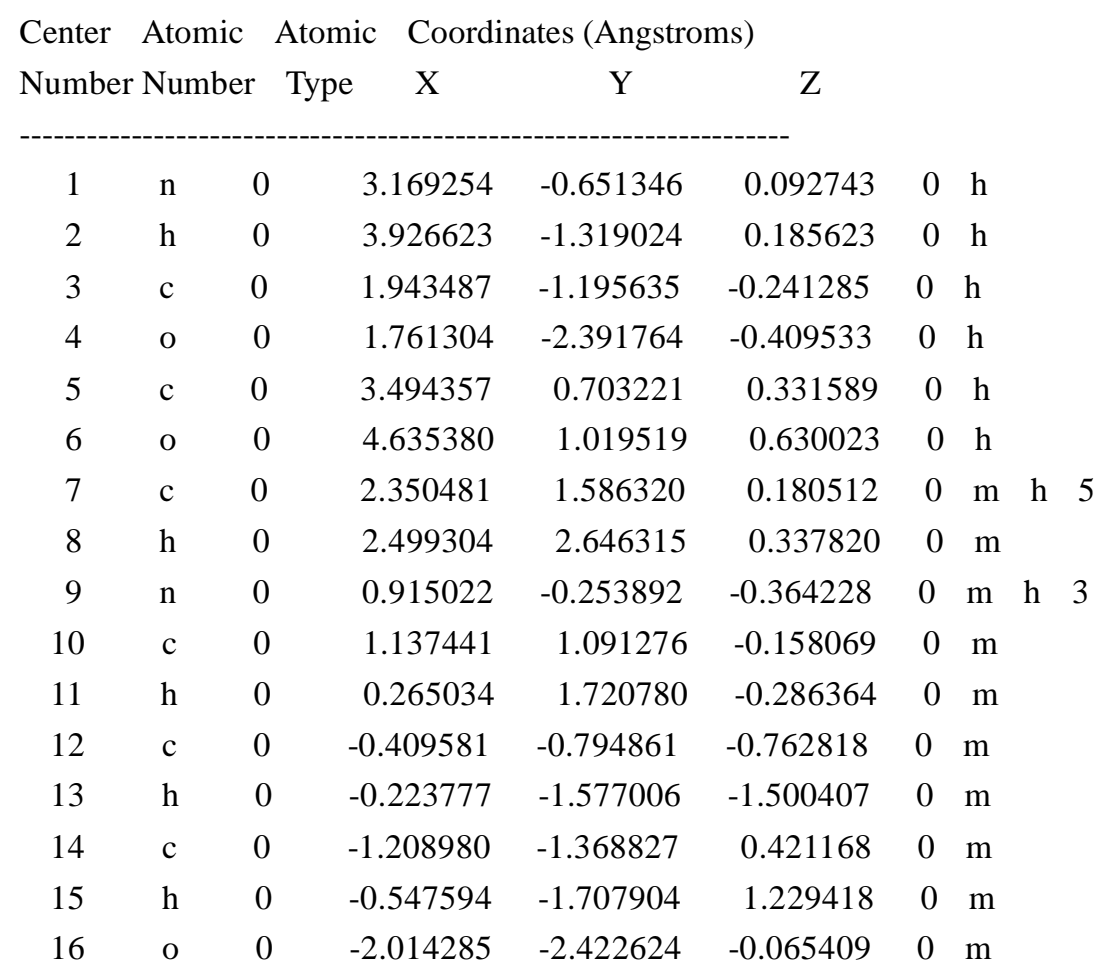




\begin{tabular}{|c|c|c|c|c|c|c|c|}
\hline 17 & $\mathrm{~h}$ & 0 & -2.847325 & -2.370572 & 0.439129 & 0 & $\mathrm{~m}$ \\
\hline 18 & c & 0 & -2.110206 & -0.178696 & 0.809023 & 0 & $\mathrm{~m}$ \\
\hline 19 & $\mathrm{~h}$ & 0 & -1.582773 & 0.535057 & 1.450408 & 0 & $\mathrm{~m}$ \\
\hline 20 & o & 0 & -3.339724 & -0.594917 & 1.399253 & 0 & $\mathrm{~m}$ \\
\hline 21 & $\mathrm{~h}$ & 0 & -3.214992 & -0.648771 & 2.359204 & 0 & $\mathrm{~m}$ \\
\hline 22 & $\mathrm{c}$ & 0 & -2.392965 & 0.449630 & -0.561183 & 0 & $\mathrm{~m}$ \\
\hline 23 & $\mathrm{~h}$ & 0 & -3.217032 & -0.103401 & -1.032848 & 0 & $\mathrm{~m}$ \\
\hline 24 & o & 0 & -1.189499 & 0.246322 & -1.318369 & 0 & $\mathrm{~m}$ \\
\hline 25 & $\mathrm{c}$ & 0 & -2.754714 & 1.922472 & -0.551325 & 0 & $\mathrm{~m}$ \\
\hline 26 & $\mathrm{~h}$ & 0 & -3.755949 & 2.021404 & -0.105431 & 0 & $\mathrm{~m}$ \\
\hline 27 & $\mathrm{~h}$ & 0 & -2.800883 & 2.280463 & -1.589353 & 0 & $\mathrm{~m}$ \\
\hline 28 & o & 0 & -1.781421 & 2.641169 & 0.201702 & 0 & $\mathrm{~m}$ \\
\hline 29 & $\mathrm{~h}$ & 0 & -1.960526 & 3.588288 & 0.106203 & 0 & \\
\hline
\end{tabular}

radical

Charge $=0$ Multiplicity $=2$ for low level calculation on real system.

Charge $=0$ Multiplicity $=2$ for high level calculation on model system.

Charge $=0$ Multiplicity $=2$ for low level calculation on model system.

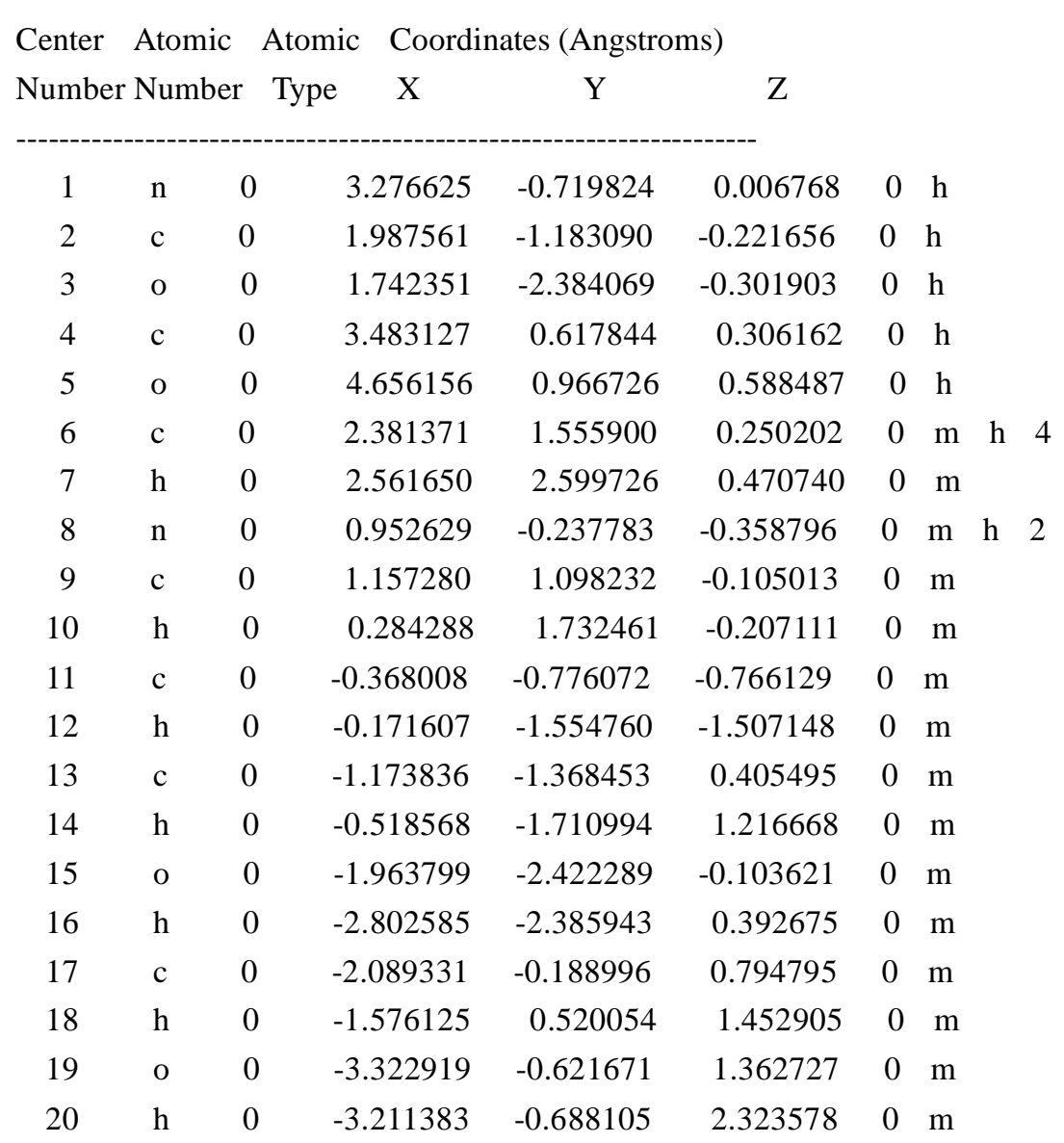




$\begin{array}{lllrrrrc}21 & \mathrm{c} & 0 & -2.359307 & 0.455805 & -0.570547 & 0 & \mathrm{~m} \\ 22 & \mathrm{~h} & 0 & -3.175898 & -0.093393 & -1.058880 & 0 & \mathrm{~m} \\ 23 & \mathrm{o} & 0 & -1.146177 & 0.266599 & -1.317577 & 0 & \mathrm{~m} \\ 24 & \mathrm{c} & 0 & -2.723927 & 1.927679 & -0.544910 & 0 & \mathrm{~m} \\ 25 & \mathrm{~h} & 0 & -3.728509 & 2.019440 & -0.105471 & 0 & \mathrm{~m} \\ 26 & \mathrm{~h} & 0 & -2.762086 & 2.299765 & -1.578179 & 0 & \mathrm{~m} \\ 27 & \mathrm{o} & 0 & -1.757409 & 2.637956 & 0.225652 & 0 & \mathrm{~m} \\ 28 & \mathrm{~h} & 0 & -1.939150 & 3.585894 & 0.143914 & 0 & \mathrm{~m}\end{array}$

\section{b5}

neutral molecule

Charge $=0$ Multiplicity $=1$ for low level calculation on real system.

Charge $=0$ Multiplicity $=1$ for high level calculation on model system.

Charge $=0$ Multiplicity $=1$ for low level calculation on model system.

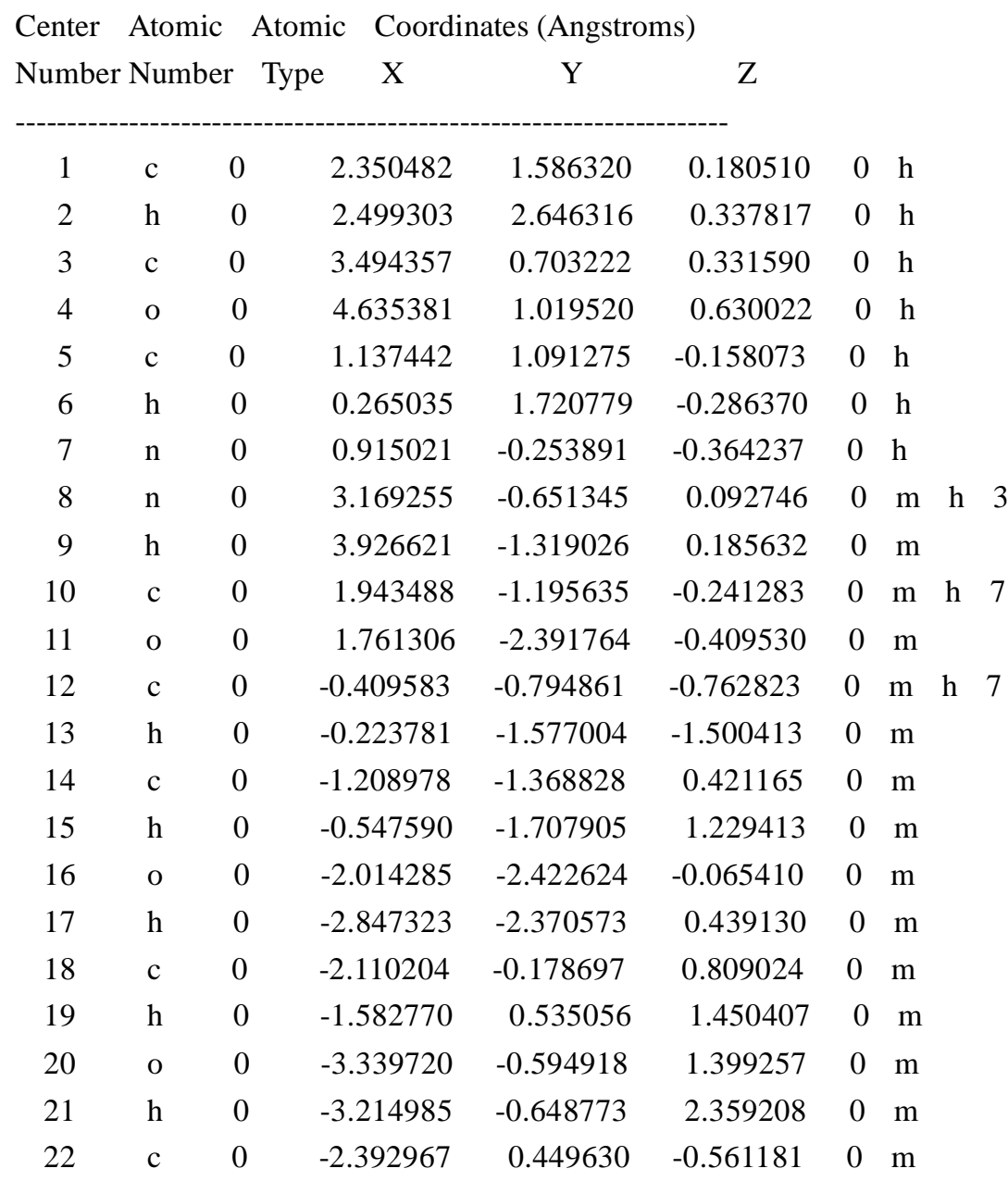




$\begin{array}{lllrrrrc}23 & \mathrm{~h} & 0 & -3.217036 & -0.103401 & -1.032844 & 0 & \mathrm{~m} \\ 24 & \mathrm{o} & 0 & -1.189503 & 0.246323 & -1.318371 & 0 & \mathrm{~m} \\ 25 & \mathrm{c} & 0 & -2.754717 & 1.922472 & -0.551321 & 0 & \mathrm{~m} \\ 26 & \mathrm{~h} & 0 & -3.755951 & 2.021403 & -0.105423 & 0 & \mathrm{~m} \\ 27 & \mathrm{~h} & 0 & -2.800889 & 2.280463 & -1.589348 & 0 & \mathrm{~m} \\ 28 & \mathrm{o} & 0 & -1.781421 & 2.641169 & 0.201703 & 0 & \mathrm{~m} \\ 29 & \mathrm{~h} & 0 & -1.960527 & 3.588287 & 0.106206 & 0 & \mathrm{~m}\end{array}$

radical

Charge $=0$ Multiplicity $=2$ for low level calculation on real system.

Charge $=0$ Multiplicity $=2$ for high level calculation on model system .

Charge $=0$ Multiplicity $=2$ for low level calculation on model system .

Center Atomic Atomic Coordinates (Angstroms)

\begin{tabular}{cccccccccc} 
Number Number & Type & X & Y & Z & & & \\
\hline 1 & $\mathrm{c}$ & 0 & 2.343765 & 1.582489 & 0.195977 & 0 & $\mathrm{~h}$ & \\
2 & $\mathrm{c}$ & 0 & 3.529335 & 0.772999 & 0.345844 & 0 & $\mathrm{~h}$ & \\
3 & $\mathrm{o}$ & 0 & 4.665288 & 1.097007 & 0.638214 & 0 & $\mathrm{~h}$ & \\
4 & $\mathrm{c}$ & 0 & 1.132282 & 1.130672 & -0.142617 & 0 & $\mathrm{~h}$ & \\
5 & $\mathrm{~h}$ & 0 & 0.256013 & 1.760408 & -0.263284 & 0 & $\mathrm{~h}$ & \\
6 & $\mathrm{n}$ & 0 & 0.926877 & -0.226023 & -0.358184 & 0 & $\mathrm{~h}$ & \\
7 & $\mathrm{n}$ & 0 & 3.199984 & -0.587170 & 0.093296 & 0 & $\mathrm{~m}$ & $\mathrm{~h}$ & 2 \\
8 & $\mathrm{~h}$ & 0 & 3.962413 & -1.252268 & 0.174583 & 0 & $\mathrm{~m}$ & \\
9 & $\mathrm{c}$ & 0 & 1.974875 & -1.145685 & -0.241679 & 0 & $\mathrm{~m}$ & $\mathrm{~h}$ & 6 \\
10 & $\mathrm{o}$ & 0 & 1.816460 & -2.344351 & -0.416124 & 0 & $\mathrm{~m}$ & \\
11 & $\mathrm{c}$ & 0 & -0.390136 & -0.781918 & -0.760553 & 0 & $\mathrm{~m}$ & $\mathrm{~h}$ & 6 \\
12 & $\mathrm{~h}$ & 0 & -0.191406 & -1.560805 & -1.498625 & 0 & $\mathrm{~m}$ & \\
13 & $\mathrm{c}$ & 0 & -1.187998 & -1.364865 & 0.419837 & 0 & $\mathrm{~m}$ & \\
14 & $\mathrm{~h}$ & 0 & -0.525860 & -1.700394 & 1.229070 & 0 & $\mathrm{~m}$ & \\
15 & $\mathrm{o}$ & 0 & -1.982960 & -2.423699 & -0.072130 & 0 & $\mathrm{~m}$ & \\
16 & $\mathrm{~h}$ & 0 & -2.819270 & -2.378298 & 0.427746 & 0 & $\mathrm{~m}$ & \\
17 & $\mathrm{c}$ & 0 & -2.099856 & -0.182761 & 0.808381 & 0 & $\mathrm{~m}$ & \\
18 & $\mathrm{~h}$ & 0 & -1.581010 & 0.531458 & 1.456178 & 0 & $\mathrm{~m}$ & \\
19 & $\mathrm{o}$ & 0 & -3.329068 & -0.611012 & 1.390190 & 0 & $\mathrm{~m}$ & \\
20 & $\mathrm{~h}$ & 0 & -3.210272 & -0.665387 & 2.350880 & 0 & $\mathrm{~m}$ & \\
21 & $\mathrm{c}$ & 0 & -2.381529 & 0.450461 & -0.560189 & 0 & $\mathrm{~m}$ & \\
22 & $\mathrm{~h}$ & 0 & -3.204087 & -0.101350 & -1.035545 & 0 & $\mathrm{~m}$ & \\
23 & $\mathrm{o}$ & 0 & -1.176738 & 0.252383 & -1.317494 & 0 & $\mathrm{~m}$ & \\
24 & $\mathrm{c}$ & 0 & -2.742496 & 1.923604 & -0.544325 & 0 & $\mathrm{~m}$ & \\
25 & $\mathrm{~h}$ & 0 & -3.742318 & 2.022855 & -0.095533 & 0 & $\mathrm{~m}$ & \\
26 & $\mathrm{~h}$ & 0 & -2.789976 & 2.285991 & -1.580617 & 0 & $\mathrm{~m}$ &
\end{tabular}




$\begin{array}{llllllll}27 & \mathrm{o} & 0 & -1.765667 & 2.636508 & 0.209688 & 0 & \mathrm{~m} \\ 28 & \mathrm{~h} & 0 & -1.930221 & 3.585480 & 0.106548 & 0 & \mathrm{~m}\end{array}$

b6

neutral molecule

Charge $=0$ Multiplicity $=1$ for low level calculation on real system.

Charge $=0$ Multiplicity $=1$ for high level calculation on model system.

Charge $=0$ Multiplicity $=1$ for low level calculation on model system .

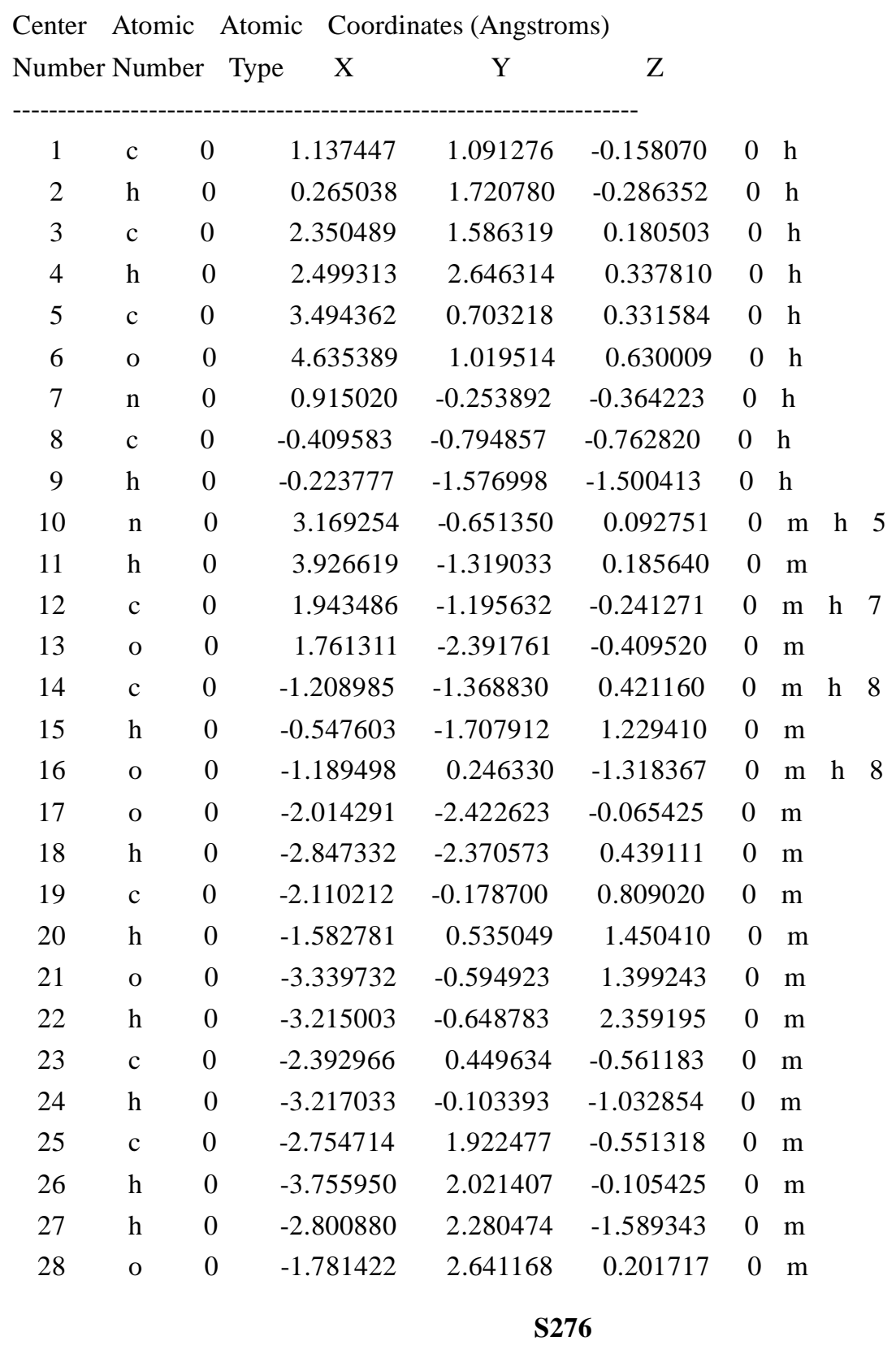


radical

Charge $=0$ Multiplicity $=2$ for low level calculation on real system.

Charge $=0$ Multiplicity $=2$ for high level calculation on model system.

Charge $=0$ Multiplicity $=2$ for low level calculation on model system.

Center Atomic Atomic Coordinates (Angstroms)

Number Number Type $\quad X \quad$ Y $\quad$ Z

\begin{tabular}{|c|c|c|c|c|c|c|c|c|}
\hline 1 & $\mathrm{c}$ & 0 & 1.111512 & 0.976565 & -0.074065 & 0 & $\mathrm{~h}$ & \\
\hline 2 & $\mathrm{c}$ & 0 & 2.272862 & 1.557847 & 0.242328 & 0 & $\mathrm{~h}$ & \\
\hline 3 & $\mathrm{~h}$ & 0 & 2.399151 & 2.611322 & 0.453143 & 0 & $\mathrm{~h}$ & \\
\hline 4 & $\mathrm{c}$ & 0 & 3.446936 & 0.677976 & 0.324692 & 0 & $\mathrm{~h}$ & \\
\hline 5 & o & 0 & 4.585297 & 1.021611 & 0.586869 & 0 & $\mathrm{~h}$ & \\
\hline 6 & $\mathrm{n}$ & 0 & 0.865268 & -0.329926 & -0.308543 & 0 & $\mathrm{~h}$ & \\
\hline 7 & $\mathrm{c}$ & 0 & -0.466241 & -0.827624 & -0.739973 & 0 & $\mathrm{~h}$ & \\
\hline 8 & $\mathrm{~h}$ & 0 & -0.289889 & -1.620912 & -1.468163 & 0 & $\mathrm{~h}$ & \\
\hline 9 & $\mathrm{n}$ & 0 & 3.143202 & -0.681286 & 0.082424 & 0 & $\mathrm{~m}$ & $\mathrm{~h}$ \\
\hline 10 & $\mathrm{~h}$ & 0 & 3.923412 & -1.326056 & 0.141880 & 0 & $\mathrm{~m}$ & \\
\hline 11 & $\mathrm{c}$ & 0 & 1.925963 & -1.260610 & -0.223151 & 0 & $\mathrm{~m}$ & h \\
\hline 12 & o & 0 & 1.760675 & -2.451888 & -0.411297 & 0 & $\mathrm{~m}$ & \\
\hline 13 & $\mathrm{c}$ & 0 & -1.314389 & -1.345204 & 0.433435 & 0 & $\mathrm{~m}$ & $\mathrm{~h}$ \\
\hline 14 & $\mathrm{~h}$ & 0 & -0.683357 & -1.716355 & 1.252900 & 0 & $\mathrm{~m}$ & \\
\hline 15 & o & 0 & -1.170132 & 0.252745 & -1.318413 & 0 & $\mathrm{~m}$ & $\mathrm{~h}$ \\
\hline 16 & o & 0 & -2.170257 & -2.354040 & -0.057768 & 0 & $\mathrm{~m}$ & \\
\hline 17 & $\mathrm{~h}$ & 0 & -3.015085 & -2.233313 & 0.415375 & 0 & $\mathrm{~m}$ & \\
\hline 18 & $\mathrm{c}$ & 0 & -2.147010 & -0.098577 & 0.800183 & 0 & $\mathrm{~m}$ & \\
\hline 19 & $\mathrm{~h}$ & 0 & -1.588260 & 0.584177 & 1.449658 & 0 & $\mathrm{~m}$ & \\
\hline 20 & $\mathrm{o}$ & 0 & -3.409861 & -0.441844 & 1.364286 & 0 & $\mathrm{~m}$ & \\
\hline 21 & $\mathrm{~h}$ & 0 & -3.316271 & -0.479025 & 2.328490 & 0 & $\mathrm{~m}$ & \\
\hline 22 & $\mathrm{c}$ & 0 & -2.364028 & 0.555267 & -0.567975 & 0 & $\mathrm{~m}$ & \\
\hline 23 & $\mathrm{~h}$ & 0 & -3.229139 & 0.086800 & -1.055921 & 0 & $\mathrm{~m}$ & \\
\hline 24 & $\mathrm{c}$ & 0 & -2.544409 & 2.068107 & -0.541408 & 0 & $\mathrm{~m}$ & \\
\hline 25 & $\mathrm{~h}$ & 0 & -3.447403 & 2.302207 & 0.031864 & 0 & $\mathrm{~m}$ & \\
\hline 26 & $\mathrm{~h}$ & 0 & -2.688990 & 2.432756 & -1.569030 & 0 & $\mathrm{~m}$ & \\
\hline 27 & o & 0 & -1.468541 & 2.733510 & 0.098288 & 0 & $\mathrm{~m}$ & \\
\hline 28 & $\mathrm{~h}$ & 0 & -0.668069 & 2.533654 & -0.417478 & 0 & $\mathrm{~m}$ & \\
\hline
\end{tabular}




\section{Part 2 Parameters Comparison}

\section{(1) Comparison Between the Parameters in the Previous Work and Mine of Deoxyribonucleosides}

Table 1. Comparison Between the Parameters of Hocquest ${ }^{[1]}$ and Mine.

\begin{tabular}{|c|c|c|c|c|}
\hline & \multicolumn{2}{|c|}{$\mathrm{dT}$} & \multicolumn{2}{|c|}{$\mathrm{dC}$} \\
\hline & Hocquest & Mine & Hocquest & Mine \\
\hline \multicolumn{5}{|c|}{ Bond Length (angstrom) } \\
\hline $\mathrm{C}_{1}{ }^{\prime}-\mathrm{C}_{2}^{\prime}$ & 1.536 & 1.535 & 1.535 & 1.534 \\
\hline $\mathrm{C}_{2}{ }^{\prime}-\mathrm{C}_{3}^{\prime}$ & 1.531 & 1.531 & 1.530 & 1.529 \\
\hline $\mathrm{C}_{3}^{\prime}-\mathrm{C}_{4}^{\prime}$ & 1.543 & 1.543 & 1.543 & 1.543 \\
\hline $\mathrm{C}_{4}^{\prime}-\mathrm{O}_{4}^{\prime}$ & 1.435 & 1.435 & 1.435 & 1.436 \\
\hline $\mathrm{O}_{4}^{\prime}-\mathrm{C}_{1}{ }^{\prime}$ & 1.423 & 1.424 & 1.423 & 1.423 \\
\hline $\mathrm{C}_{3}{ }^{\prime}-\mathrm{O}_{3}{ }^{\prime}$ & 1.425 & 1.426 & 1.426 & 1.427 \\
\hline $\mathrm{C}_{4}{ }^{\prime}-\mathrm{C}_{5}{ }^{\prime}$ & 1.522 & 1.522 & 1.522 & 1.521 \\
\hline $\mathrm{C}_{1}{ }^{\prime}-\mathrm{N}_{1}$ & 1.465 & 1.464 & 1.466 & 1.466 \\
\hline $\mathrm{C}_{5}{ }^{\prime}-\mathrm{O}_{5}{ }^{\prime}$ & 1.427 & 1.427 & 1.426 & 1.426 \\
\hline \multicolumn{5}{|c|}{ Valence Angles (degree) } \\
\hline $\mathrm{C}_{1}{ }^{\prime}-\mathrm{C}_{2}{ }^{\prime}-\mathrm{C}_{3}{ }^{\prime}$ & 102.3 & 102.3 & 102.4 & 102.4 \\
\hline $\mathrm{C}_{2}^{\prime}-\mathrm{C}_{3}^{\prime}-\mathrm{C}_{4}^{\prime}$ & 102.5 & 102.5 & 102.6 & 102.6 \\
\hline $\mathrm{C}_{3}{ }^{\prime}-\mathrm{C}_{4}{ }^{\prime}-\mathrm{O}_{4}{ }^{\prime}$ & 106.7 & 106.7 & 106.8 & 106.7 \\
\hline $\mathrm{C}_{4}{ }^{\prime}-\mathrm{O}_{4}{ }^{\prime}-\mathrm{C}_{1}{ }^{\prime}$ & 110.8 & 110.8 & 110.7 & 110.7 \\
\hline $\mathrm{O}_{4}{ }^{\prime}-\mathrm{C}_{1}{ }^{\prime}-\mathrm{C}_{2}{ }^{\prime}$ & 105.6 & 105.5 & 105.6 & 105.5 \\
\hline $\mathrm{C}_{2}{ }^{\prime}-\mathrm{C}_{3}{ }^{\prime}-\mathrm{O}_{3}{ }^{\prime}$ & 112.6 & 112.6 & 112.7 & 112.7 \\
\hline $\mathrm{C}_{4}{ }^{\prime}-\mathrm{C}_{3}{ }^{\prime}-\mathrm{O}_{3}{ }^{\prime}$ & 106.3 & 106.2 & 106.2 & 106.2 \\
\hline $\mathrm{C}_{5}{ }^{\prime}-\mathrm{C}_{4}{ }^{\prime}-\mathrm{C}_{3}{ }^{\prime}$ & 114.9 & 114.9 & 115.2 & 115.1 \\
\hline $\mathrm{C}_{5}^{\prime}-\mathrm{C}_{4}^{\prime}-\mathrm{O}_{4}^{\prime}$ & 109.7 & 109.7 & 109.5 & 109.6 \\
\hline $\mathrm{O}_{4}{ }^{\prime}-\mathrm{C}_{1}{ }^{\prime}-\mathrm{N}_{1}$ & 108.6 & 108.6 & 108.9 & 108.9 \\
\hline $\mathrm{C}_{2}{ }^{\prime}-\mathrm{C}_{1}{ }^{\prime}-\mathrm{N}_{1}$ & 114.6 & 114.6 & 114.2 & 114.2 \\
\hline $\mathrm{O}_{5}^{\prime}-\mathrm{C}_{5}^{\prime}-\mathrm{C}_{4}^{\prime}$ & 109.2 & 109.2 & 109.2 & 109.2 \\
\hline $\mathrm{C}_{1}^{\prime}-\mathrm{N}_{1}-\mathrm{C}_{2}$ & 118.1 & 118.2 & 117.4 & 117.2 \\
\hline \multicolumn{5}{|c|}{ Torsion Angels (degree) } \\
\hline $\mathrm{Y}$ & 52 & 51.2 & 54 & 54 \\
\hline$x$ & 230 & 230 & 211 & 212 \\
\hline
\end{tabular}




\section{Figure 1. Bond lengths and major Torsion angles Compared}

\section{Schaefer $^{[2]}$ to Mine}

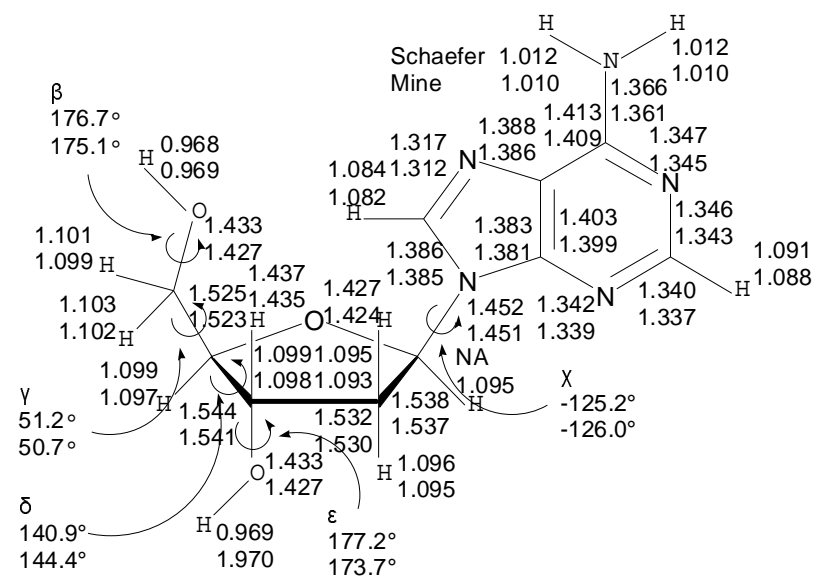

\section{(2) Comparison Between the Parameters in the Previous Work ${ }^{[1,3,4]}$ and Mine of Ribonucleosides}

\begin{tabular}{|c|c|c|c|c|c|c|c|c|c|}
\hline \multirow{2}{*}{ Bonds/Angles } & \multirow{2}{*}{ ribonucleosides $^{\mathrm{a}, \mathrm{b}}$} & \multicolumn{2}{|c|}{ rA } & \multicolumn{2}{c|}{ rC } & \multicolumn{2}{c|}{ rG } & \multicolumn{2}{c|}{ rU } \\
\cline { 3 - 10 } & & Hocquet & Mine & Hocquet & Mine & Hocquet & Mine & Hocquet & Mine \\
\hline $\mathrm{C}_{1}{ }^{\prime}-\mathrm{C}_{2}{ }^{\prime}$ & $1.529^{\mathrm{a}}$ & 1.541 & 1.541 & 1.539 & 1.538 & 1.542 & 1.542 & 1.540 & 1.540 \\
\hline $\mathrm{C}_{3}{ }^{\prime}-\mathrm{O}_{3}{ }^{\prime}$ & $1.417^{\mathrm{a}}$ & 1.426 & 1.427 & 1.426 & 1.427 & 1.426 & 1.427 & 1.425 & 1.426 \\
\hline $\mathrm{O}_{4}{ }^{\prime}-\mathrm{C}_{1}{ }^{\prime}-\mathrm{C}_{2}{ }^{\prime}$ & $107.6^{\mathrm{a}}$ & 106.8 & 106.7 & 106.7 & 106.7 & 106.8 & 106.8 & 106.9 & 106.9 \\
\hline $\mathrm{C}_{1}{ }^{\prime}-\mathrm{C}_{2}{ }^{\prime}-\mathrm{C}_{3}{ }^{\prime}$ & $101.3^{\mathrm{a}}$ & 102.1 & 102.2 & 101.8 & 101.9 & 102.3 & 102.3 & 102.1 & 102.1 \\
\hline $\mathrm{Y}$ & $54^{\mathrm{b}}$ & 49 & 49 & 52 & 52 & 48 & 48 & 51 & 51 \\
\hline $\mathrm{X}$ & $202^{\mathrm{b}}$ & 208 & 208 & 197 & 197 & 212 & 212 & 202 & 201 \\
\hline
\end{tabular}

${ }^{a}$ Observed data are those corresponding to the mean values of the sugar puckers involved in ribonucleosides as analyzed by a statistical survey of the crystal structures. ${ }^{\text {[24] }}$

${ }^{\mathrm{b}}$ The torsional angles observed in canonical B-RNA forms are indicated for comparison ${ }^{[3]}$ 


\section{Part 3 The BDEs of $56 \mathrm{C}-\mathrm{H}$ and $10 \mathrm{~N}-\mathrm{H}$ Molecules with B3LYP}

(1) The BDEs of $56 \mathrm{C}-\mathrm{H}$ Molecules with B3LYP

(1)




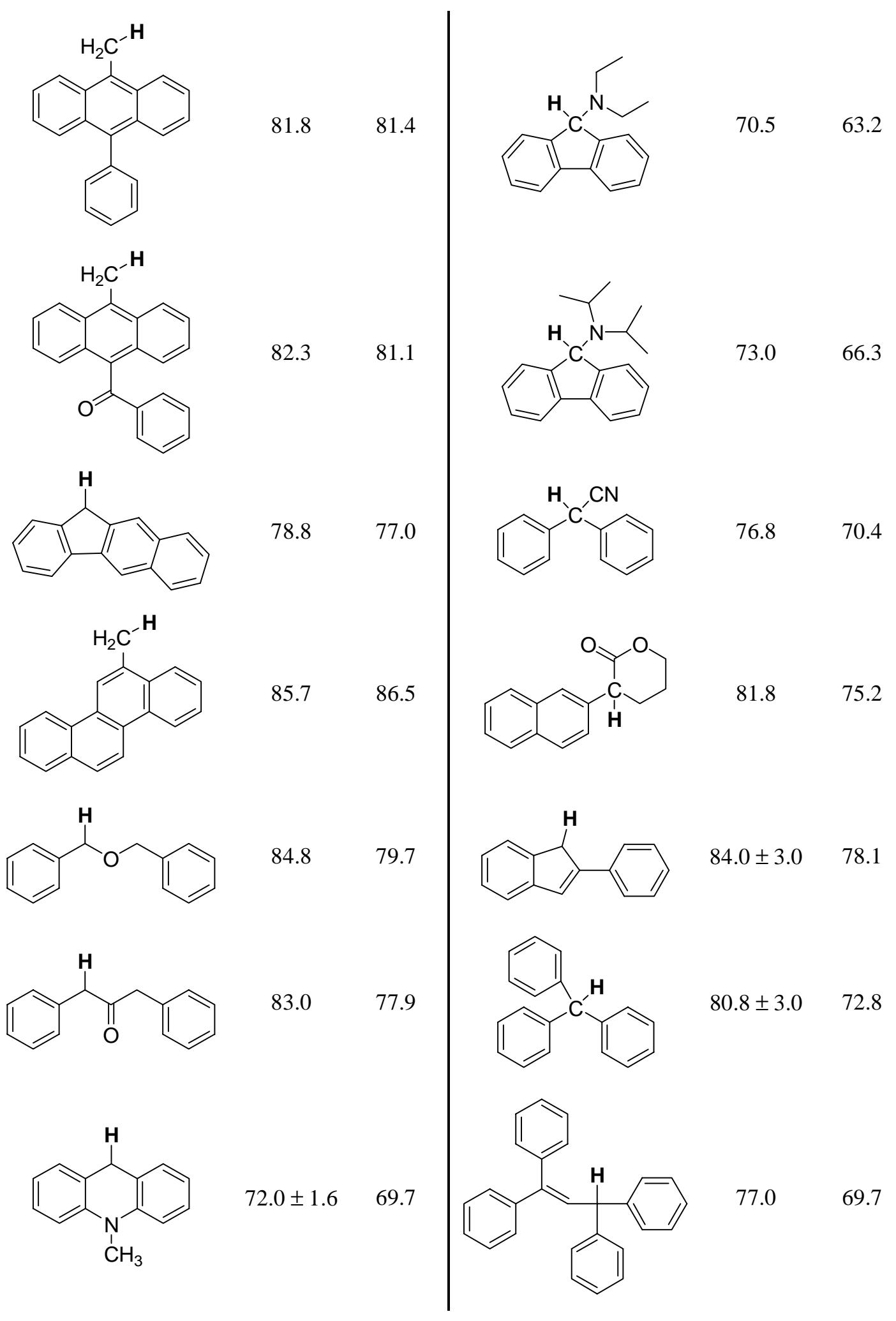




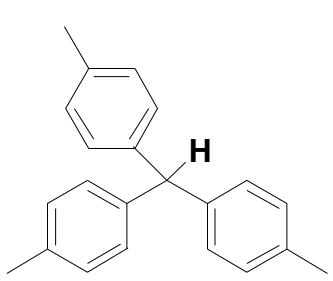<smiles>CC(c1ccccc1)c1ccccc1</smiles><smiles>N#CC(Oc1ccccc1)c1ccccc1</smiles><smiles>O=C(CCc1ccccc1)c1ccccc1</smiles>

88.5

79.5

71.3

78.6

83.9

72.2

79.5<smiles></smiles>

74.0

67.2<smiles></smiles><smiles></smiles>

82.8

80.0

78.6

80.0
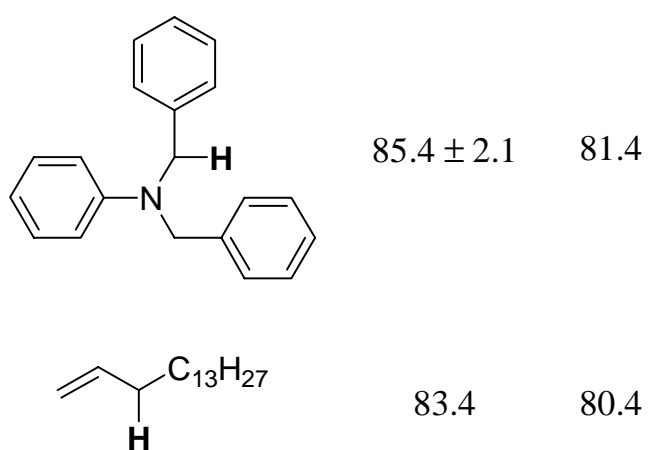

$83.4 \quad 80.4$

79.1

72.6

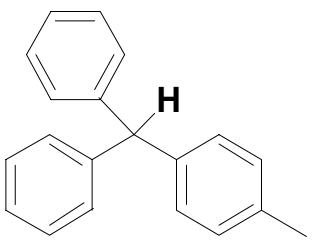

83.0

76.4<smiles>O=C(c1ccccc1)C(c1ccccc1)c1ccccc1</smiles>

$82.0 \quad 76.2$<smiles>CC(=O)C(c1ccccc1)c1ccccc1</smiles>

o

$78.1 \quad 71.3$

78.7

$93.0 \quad 92.8$ 

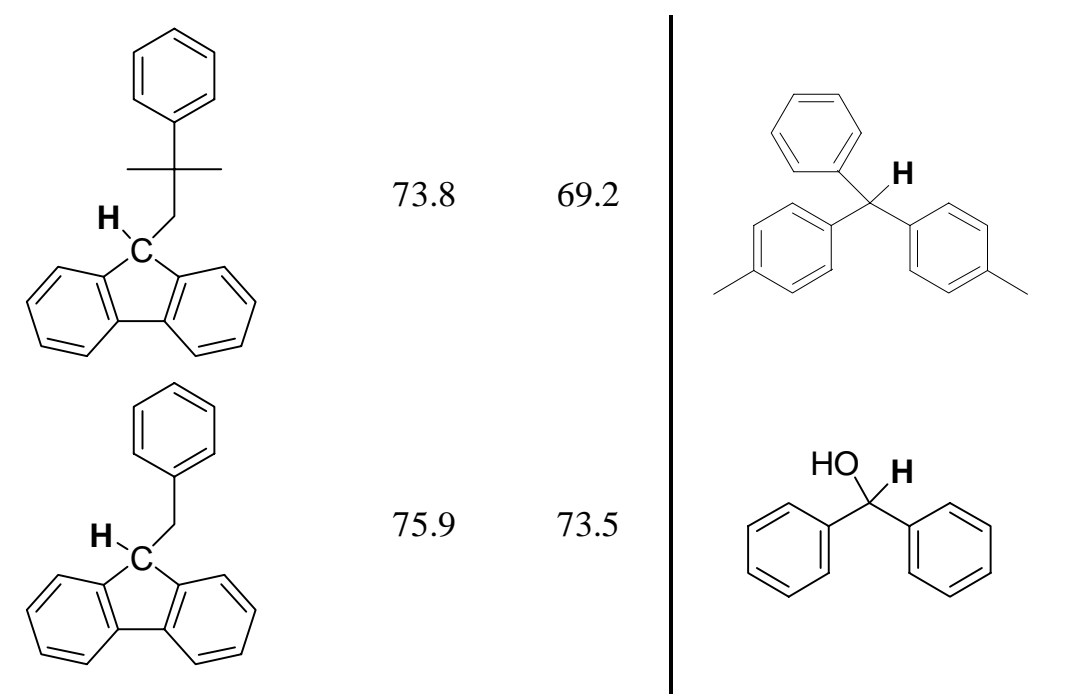

$80.3 \quad 72.5$

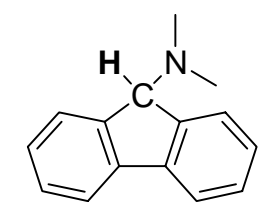

$71.5 \quad 63.6$
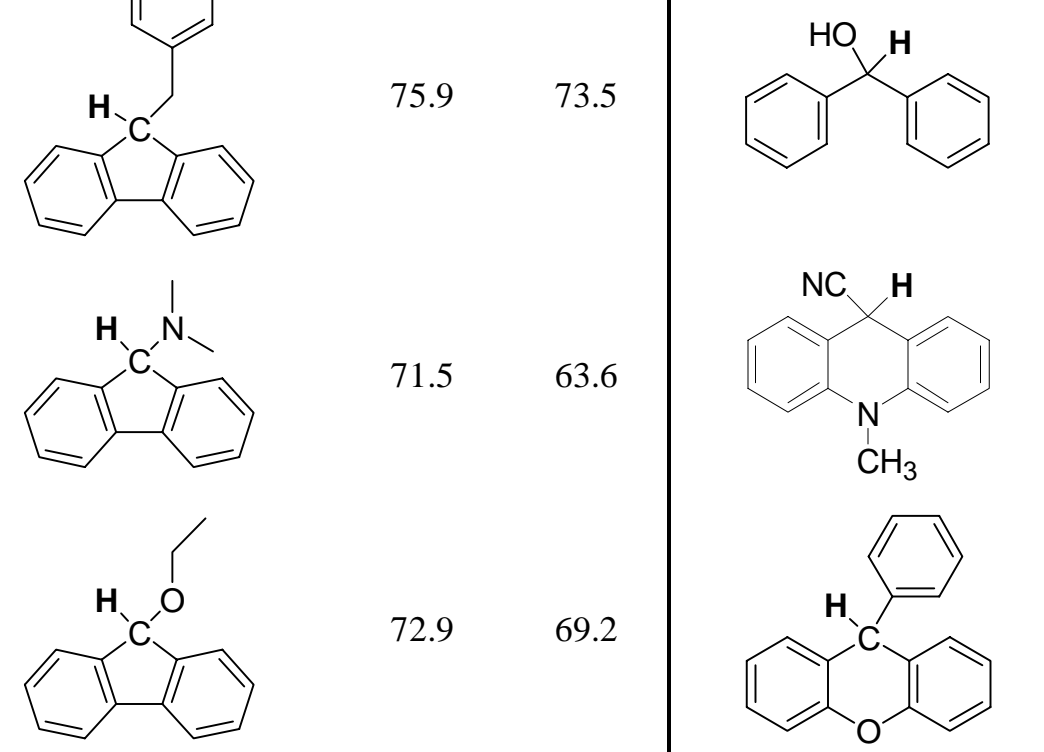

$75.4 \pm 1.5 \quad 72.3$<smiles>Oc1ccccc1</smiles><smiles>OC1c2ccccc2-c2ccccc21</smiles>

74.3

72.9

69.2

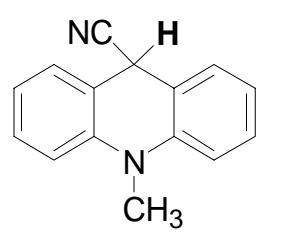

$71.3 \pm 1.5 \quad 60.3$<smiles>c1ccc(C2c3ccccc3Oc3ccccc32)cc1</smiles>

76.0 68.5

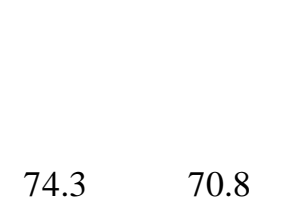<smiles>c1ccc([C]2c3ccccc3-c3ccccc32)cc1</smiles>

70.9<smiles>CCOC(=O)C(C(=O)OCC)c1ccccc1</smiles>

$85.3 \quad 76.5$<smiles>Cc1ccc(C2c3ccccc3Oc3ccccc32)cc1</smiles>

$\begin{array}{ll}75.6 & 68.5\end{array}$<smiles>COc1ccc(C2c3ccccc3Oc3ccccc32)cc1</smiles><smiles>CN(Cc1ccccc1)C1c2ccccc2-c2ccccc21</smiles>

$72.5 \quad 63.2$ 


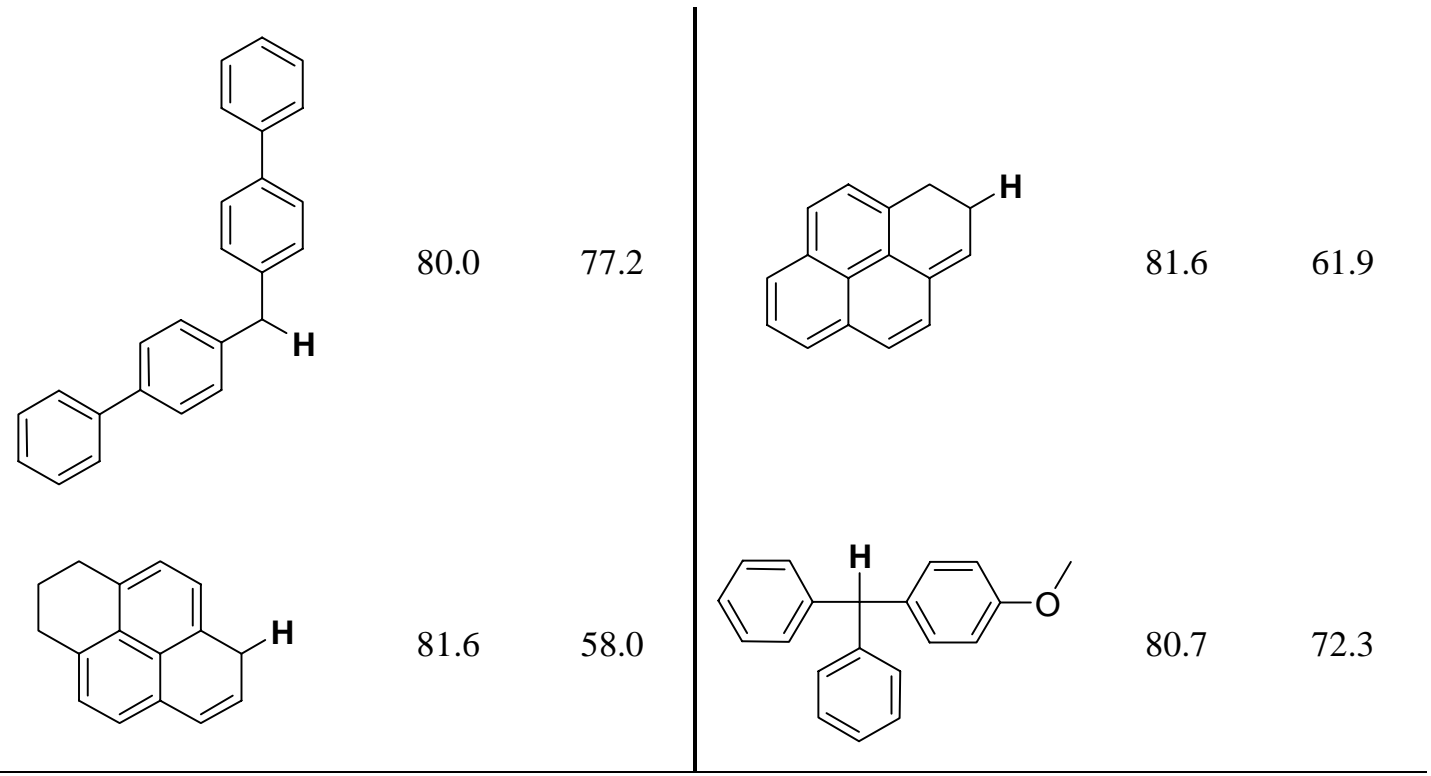

(2) The BDEs of $10 \mathrm{~N}-\mathrm{H}$ Molecules with B3LYP

Exp.




\section{Part 4 The Core Layer Division of Deoxynucleoside and Nucleoside Labeled With Red.}

(1) Deoxynucleoside

dA
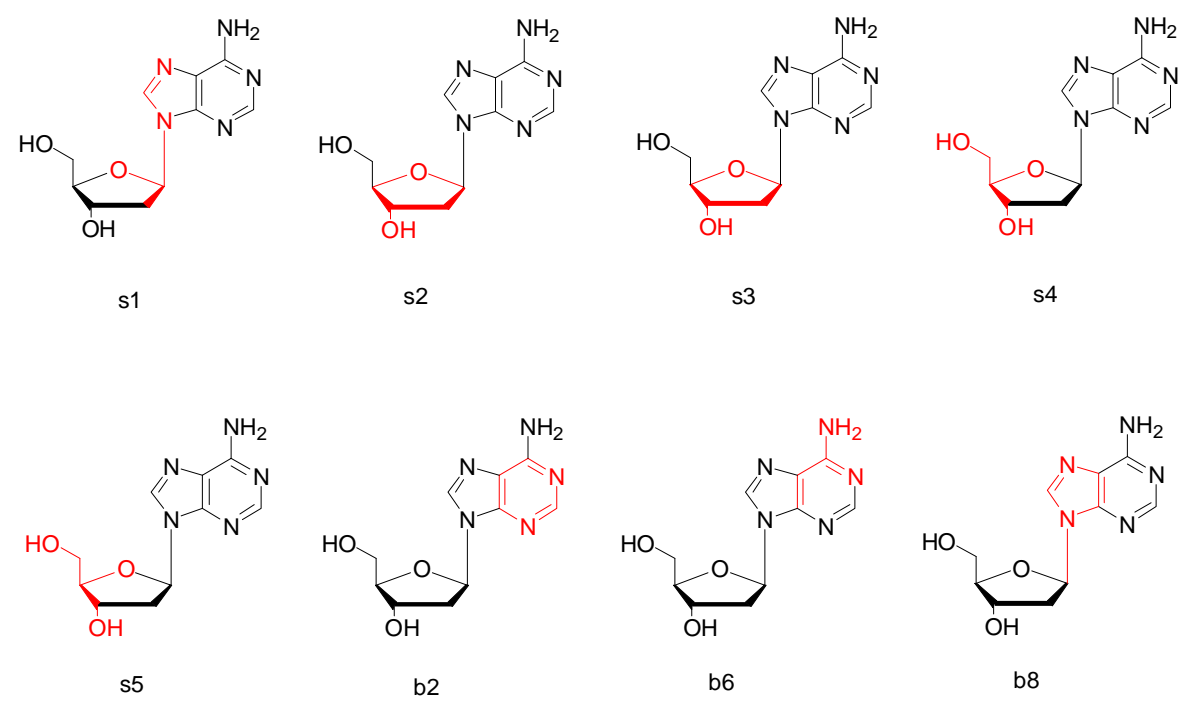

dC

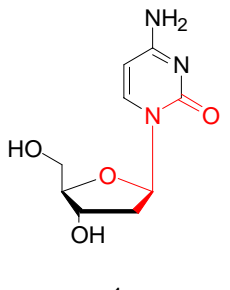

s1

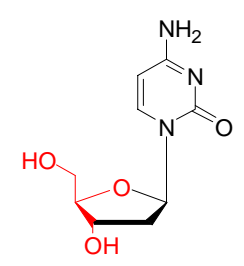

s1
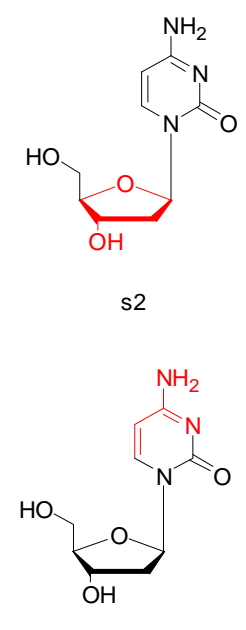

b4
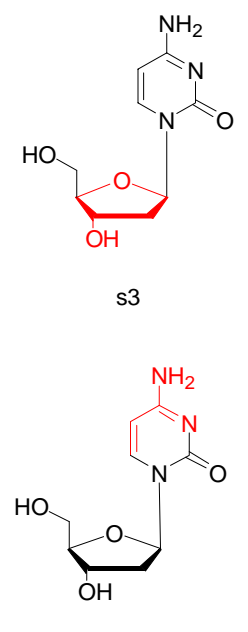

b5

S285
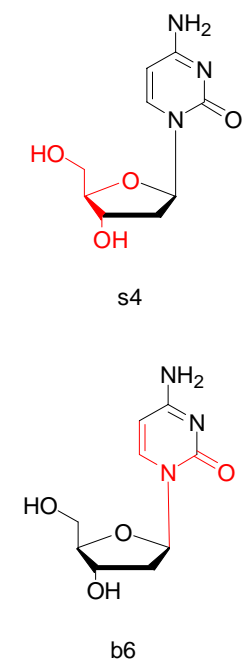

b6 


\section{dG}
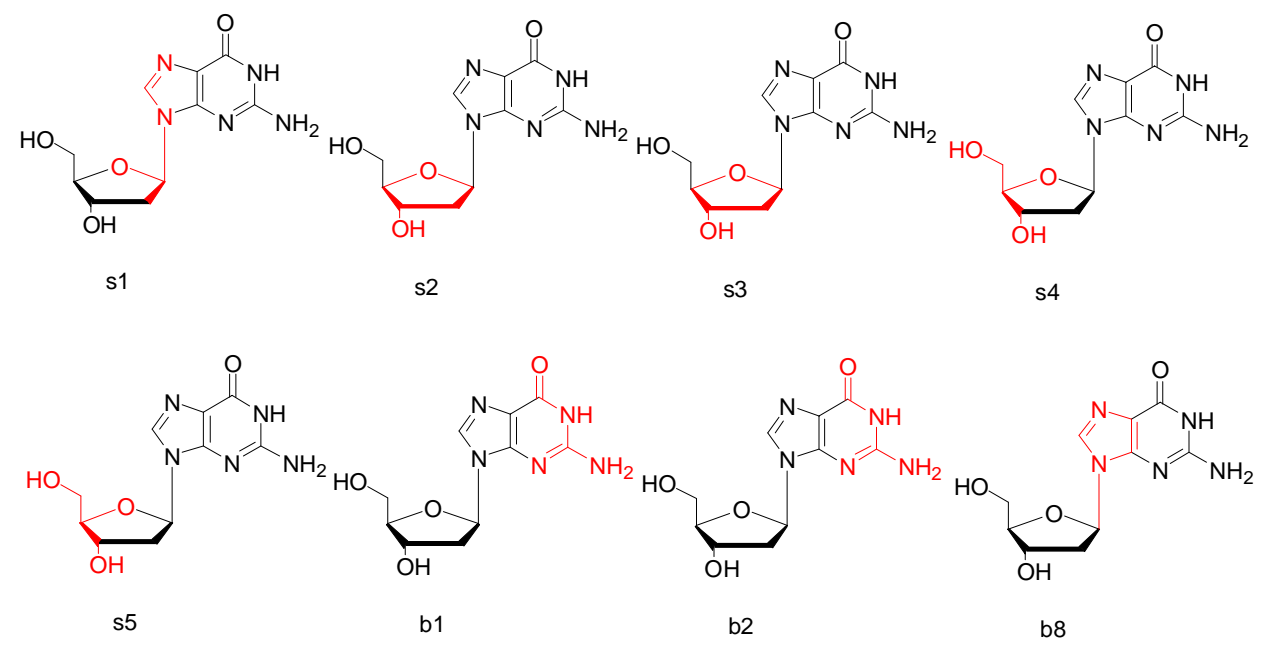

\section{dT}
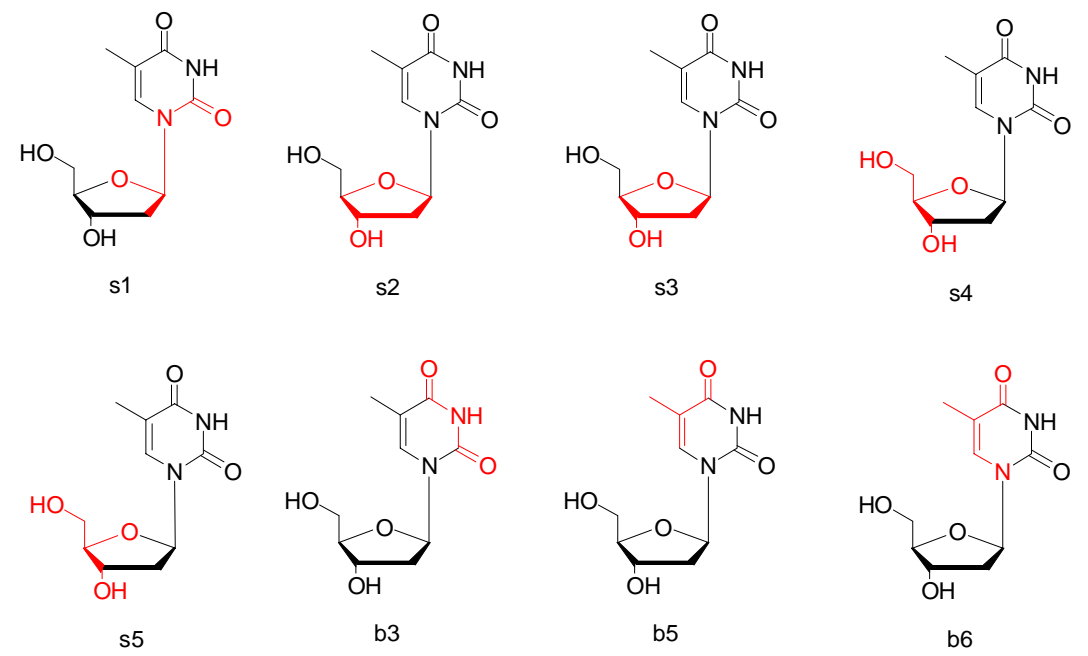


\section{(2) Nucleoside}

$\mathbf{r A}$

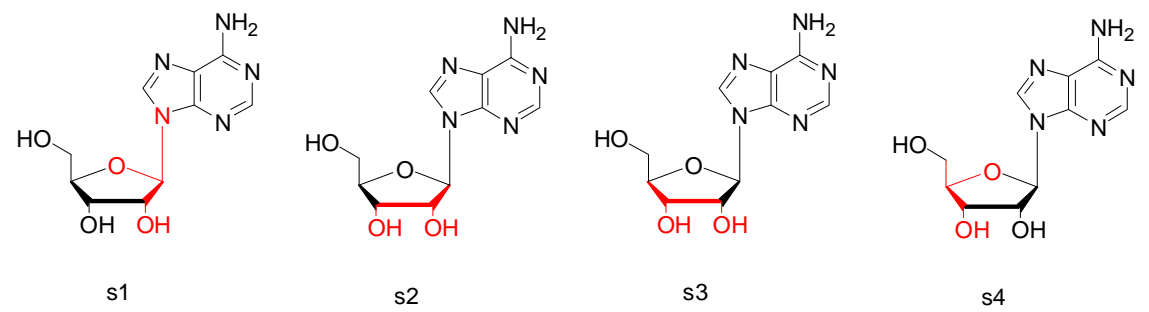

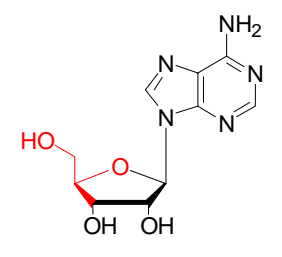

s5

\section{rC}
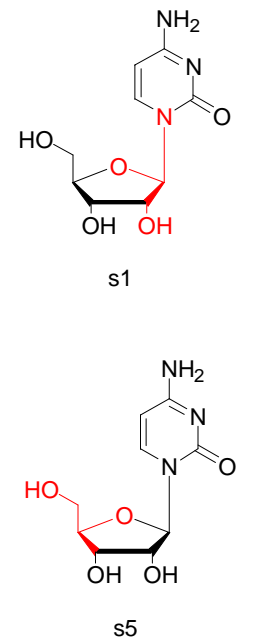
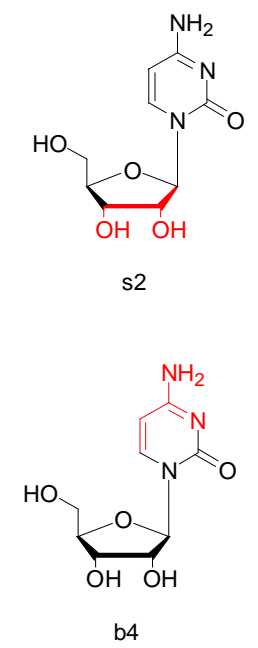
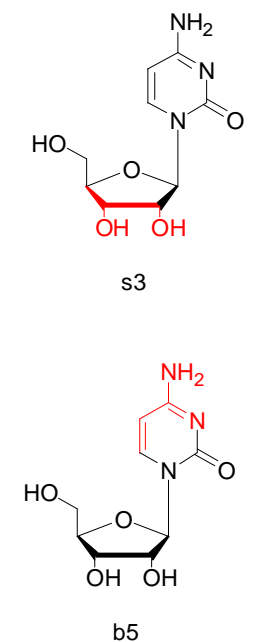
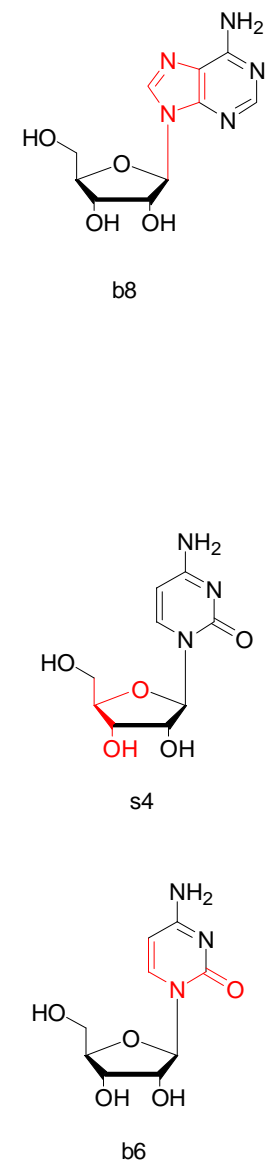


\section{rG}
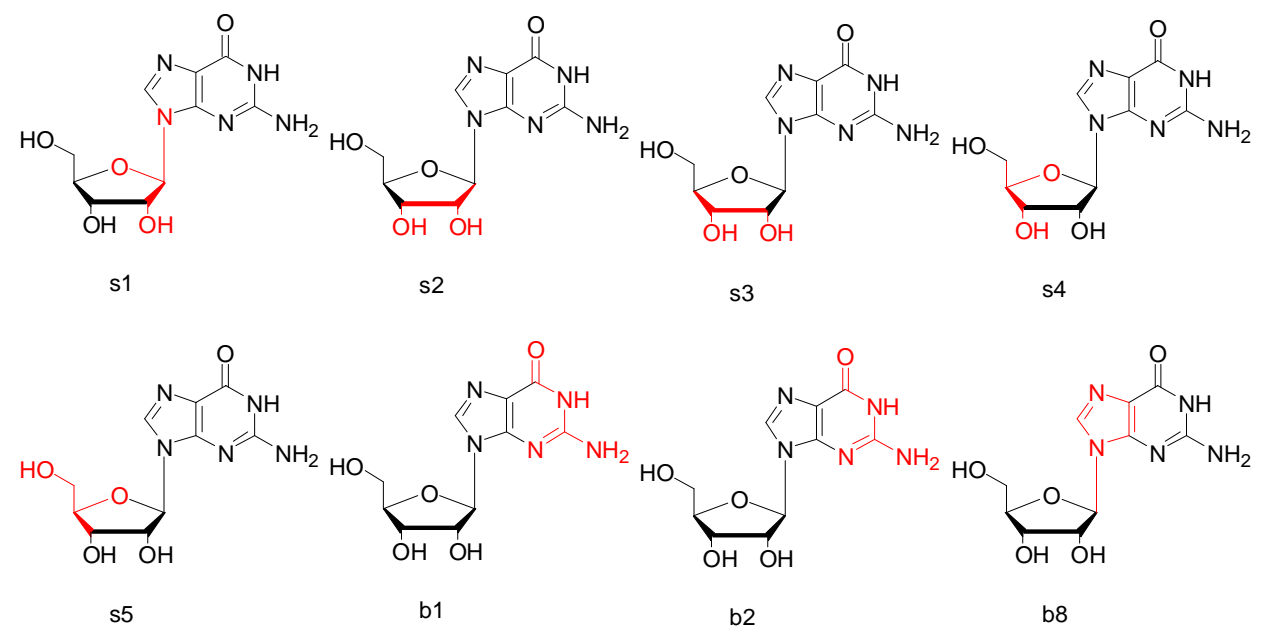

$\mathbf{r U}$

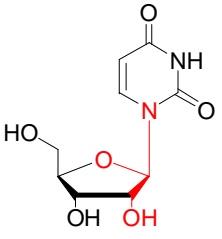

s1

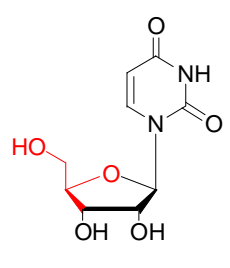

s5

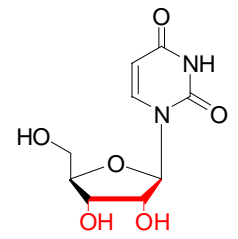

s2

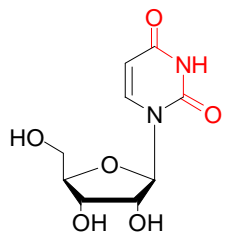

b3

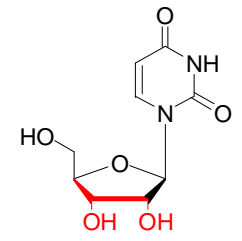

s3

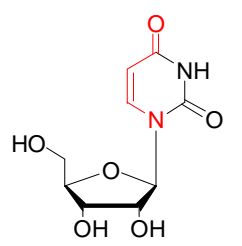

b5

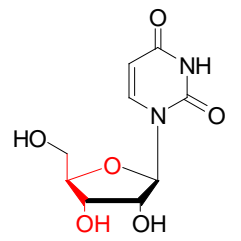

s4

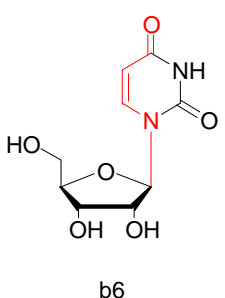

\section{References}

(1) Hocquet, A.; Leulliot, N.; Ghomi, M. J. Phys. Chem. B 2000, 104, 4560.

(2) Francesco A. Evanelista; Henry F. Schaefer III. J. Phys. Chem. A 2004, 108, 10258.

(3) Saenger, W. Principles of Nucleic Acid Structure; Springer-Verlag: New York, 1984.

(4) Gelbin, A.; Schneider, B.; Clowney, L.; Hsieh, S.; Olson, W. K.; Berman, H. M. J. Am. Chem. Soc. 1996, 118, 519. 